\title{
Structure of SWI/SNF chromatin remodeller RSC bound to a nucleosome and implications for chromatin remodelling
}

\section{Dissertation}

\author{
for the award of the degree \\ 'Doctor rerum naturalium' \\ of the Georg-August-Universität Göttingen
}

within the graduate program

Biomolecules - Structure, Function and Dynamics

of the Georg-August University School of Science (GAUSS)

submitted by

Felix Roman Wagner

from Ellwangen (Jagst), Germany

Göttingen 2019

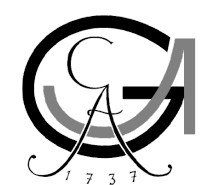

GEORG-AUGUST-UNIVERSITÄT

GÖTTINGEN

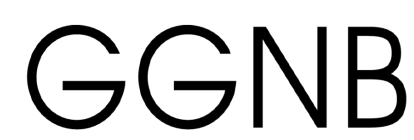

Göttingen Graduate Center for Neurosciences, Biophysics, and Molecular Biosciences 


\section{MEMBERS OF THE THESIS COMMITTEE AND THE EXAMINATION BOARD}

\section{Thesis Committee}

Prof. Dr. Patrick Cramer ( $1^{\text {st }}$ Referee)

Department of Molecular Biology

Max Planck Institute for Biophysical Chemistry, Göttingen, Germany

Prof. Dr. Holger Stark

Department of Structural Dynamics

Max Planck Institute for Biophysical Chemistry, Göttingen, Germany

PD Dr. Philipp Korber

Molecular Biology Division

Biomedical Center, LMU Munich, Planegg-Martinsried, Germany

\section{Members of the Examination Board}

Prof. Dr. Patrick Cramer (1 ${ }^{\text {st }}$ Referee)

Department of Molecular Biology

Max Planck Institute for Biophysical Chemistry, Göttingen, Germany

Prof. Dr. Henning Urlaub (2 ${ }^{\text {nd }}$ Referee)

Bioanalytical Mass Spectrometry Group

Max Planck Institute for Biophysical Chemistry, Göttingen, Germany

\section{Further Members of the Examination Board}

Prof. Dr. Holger Stark

Department of Structural Dynamics

Max Planck Institute for Biophysical Chemistry, Göttingen, Germany

PD Dr. Philipp Korber

Molecular Biology Division

Biomedical Center, LMU Munich, Planegg-Martinsried, Germany

Dr. Juliane Liepe

Research Group Quantitative and Systems Biology

Max Planck Institute for Biophysical Chemistry, Göttingen, Germany

Prof. Dr. Markus Zweckstetter

Protein structure determination using NMR

Max Planck Institute for Biophysical Chemistry, Göttingen, Germany

Date of oral examination: 29 $9^{\text {th }}$ of November 2019 
To my dear mother, you showed me devotion, curiosity and the importance of being open to new ideas. I wish you would have been with us to share this moment. 


\section{AFFIDAVIT}

Herewith I, Felix Wagner, declare that I prepared the Doctoral Thesis 'Structure of SWI/SNF chromatin remodeller RSC bound to a nucleosome and implications for chromatin remodelling' on my own and with no other sources and aids than quoted.

Göttingen, 27th of September 2019

Felix Wagner 


\section{ACKNOWLEDGEMENTS}

I would like to thank Patrick Cramer for providing me with the great opportunity to do my $\mathrm{PhD}$ in his lab, and for creating a scientific environment of trust and freedom that sparks creativity and independent ideas. Thank you for excellent scientific discussions and your supportive mentorship. I want to thank my thesis advisory committee members Holger Stark and Philipp Korber. Thank you for always taking the time and even travelling for TAC meetings. I am grateful for your important scientific insights and exceptional support along the way.

Thanks to Alexandra Stützer, Momchil Nivo, and Henning Urlaub for a great mass spectrometry collaboration which was key for the project. I want to thank Toby Barnes and Philipp Korber for a smooth collaboration on remodelling assays.

I would like to thank all past and present members of our lab, many of whom have become wonderful friends. Thank you Haibo Wang for teaching me how to work with nucleosomes and sharing your protocols. Thank you Christian Dienemann and Dmitry Tegunov for teaching me about cryo-EM, help with the microscopes and countless discussions on image processing. I want to thank Sandra Schilbach, Sarah Sainsbury, Carina Burzinsky, Svetlana Dodonova, Carrie Bernecky, Katharina Hofmann, and Tobias Gubbey for inspiring discussions and an amazing working atmosphere.

I am deeply grateful to Simon Neyer, Hauke Hillen, Paulina Seweryn, Anna Sawicka, Marco Dombrovski, Isaac Fianu, Björn Schwalb, and Saskia Gressel for all the cheerful evenings, football matches, board games, hiking tours, barbeques, ridiculous discussion, and fantastic vacations. You welcomed me in Göttingen and made it a superb place to live and work. It is a pleasure to have you as friends.

I would like to thank my dear friends Thomas Frank, Andreas Röhler, Robert Fimmen, and Georg Ladenburger for constantly staying in touch despite distances and different paths in life.

I am so very thankful for Sara Osman and Goran Kokic. Thank you for encouraging conversations, motivational coffee breaks, long walks, and just listening when things got overwhelming. Thank you for proofreading my thesis.

I love to thank my delightful big family, my dear parents Monika and Ernst, brothers Matthias, Moritz, Lorenz and my sister Ulrike. You supported me on this journey in innumerable ways. I am incredibly thankful and lucky to have you and come what may, we've always got each other's backs.

I finish by thanking you, Sara. You are an amazing woman and your passion for science is truly inspiring. Thank you for your unremitting support and endless love. 


\section{PUBLICATIONS}

Parts of this work have been submitted to a journal for publication.

(Parts of abstract, section 1.3; sections 2.11.1, 2.12, 2.13, 2.15 2.17, 2.18, 3.5 - 3.10;

figures in sections 3.1 (Figure 5), 3.4 (Figure 8); supplementary materials Table S11, Table S12).

F. R. Wagner, C. Dienemann, H. Wang, A. Stützer, D. Tegunov, H. Urlaub and P. Cramer (2019), Structure of SWI/SNF chromatin remodeller RSC bound to a nucleosome; submitted manuscript.

\section{Author contributions}

F.R.W. carried out all experiments and data analysis unless stated otherwise. C.D. assisted with data collection and model building. A.S. and H.U. carried out cross-linking and mass spectrometry analysis. H.W. helped with nucleosome biochemistry. D.T. helped with cryoEM data processing. P.C. designed and supervised the project. F.W. and P.C. wrote the manuscript, with input from all authors. 


\section{TABLE OF CONTENTS}

MEMBERS OF THE THESIS COMMITTEE AND THE EXAMINATION BOARD ......... ii

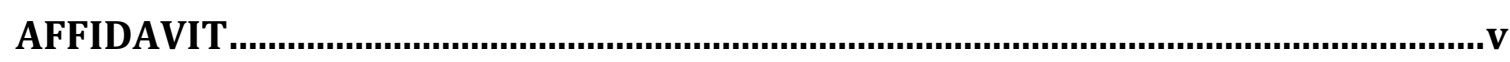

ACKNOWLEDGEMENTS ........................................................................................... vii

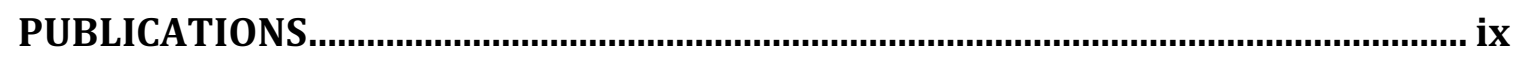

TABLE OF CONTENTS ….........................................................................................

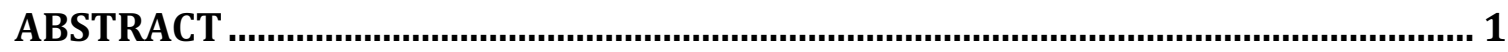

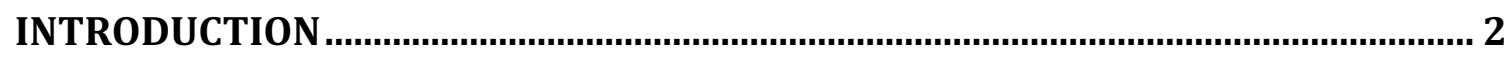

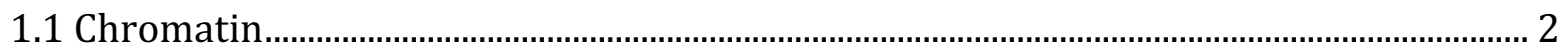

1.1.1 Nucleosomes as building blocks of chromatin ......................................................... 2

1.1.2 Nucleosomes in transcription................................................................................ 5

1.2 Four families of ATP-dependent chromatin remodellers................................................... 6

1.2.1 Chromatin remodellers and cancer ............................................................................. 7

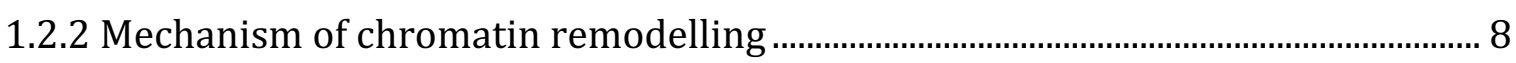

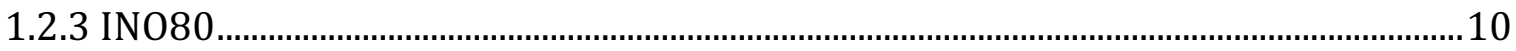

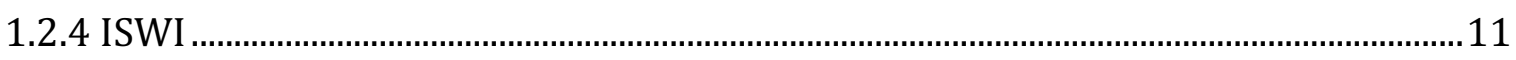

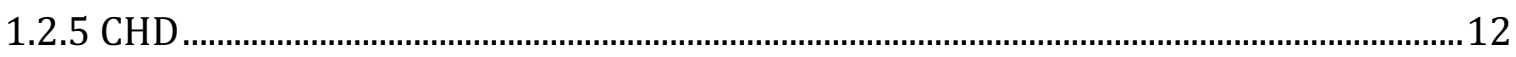

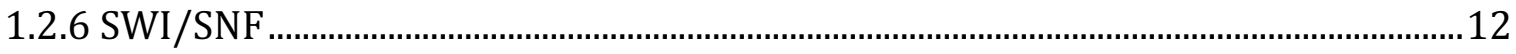

1.3 'Remodels the structure of chromatin' (RSC) complex ....................................................13

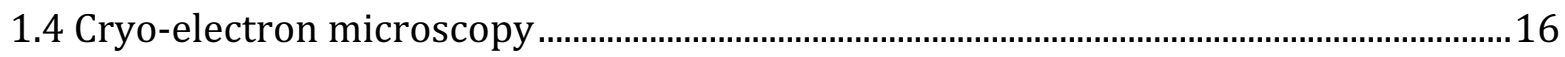

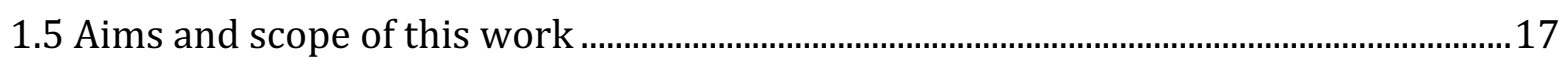

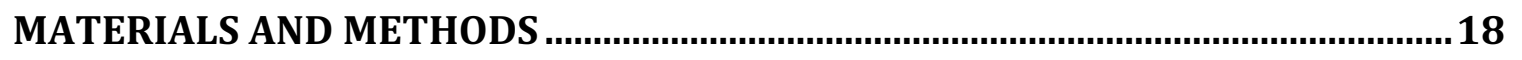

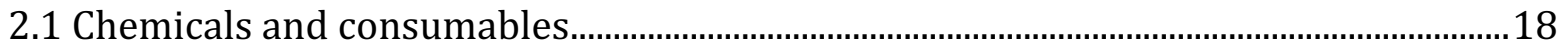

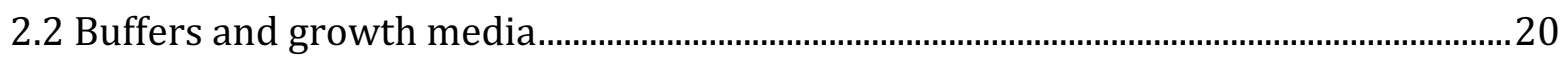

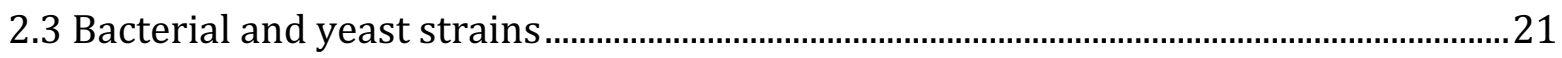

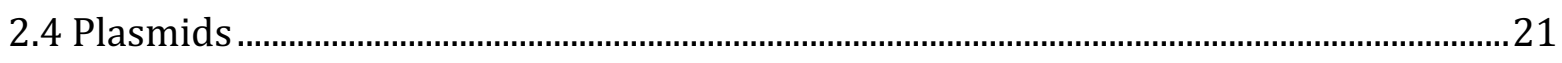

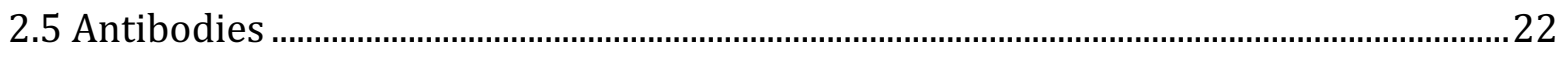

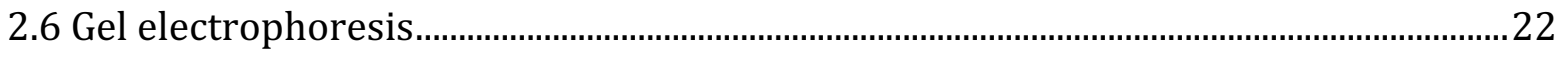

2.6.1 Sodium-dodecyl-sulfate polyacrylamide gel electrophoresis (SDS-PAGE)..........22

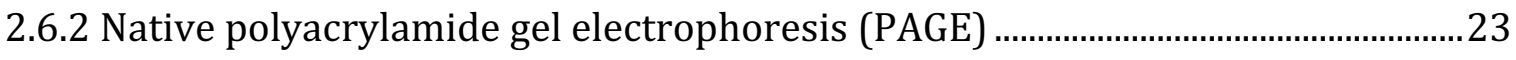

2.6.3 Electrophoretic mobility shift assay (EMSA) …….....................................................23 


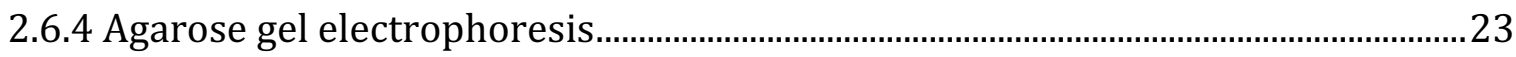

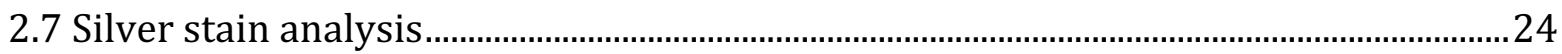

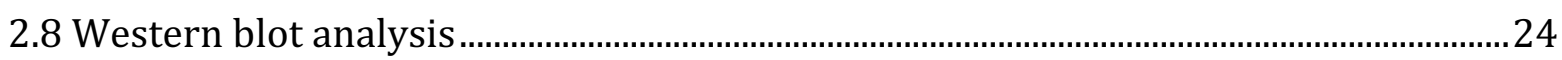

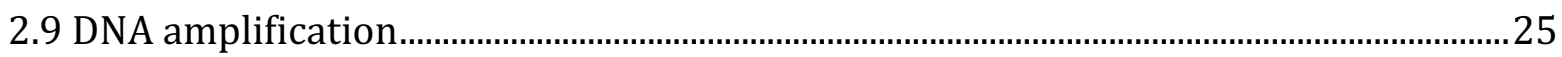

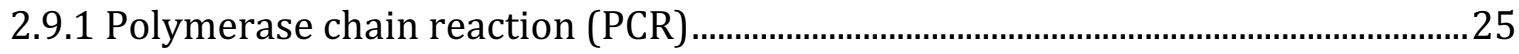

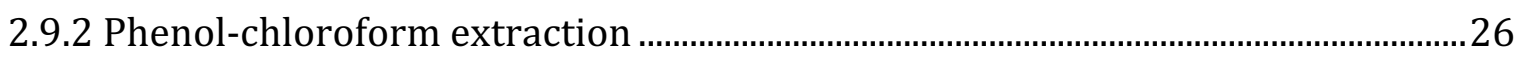

2.9.3 Size exclusion chromatography for nucleosomal scaffold DNA.............................27

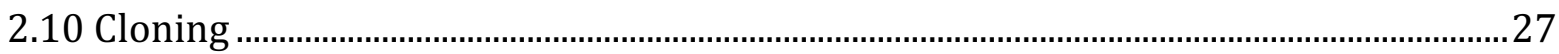

2.10.1 Transformation of chemically competent $E$. coli cells ..........................................27

2.10.2 Transformation of chemically competent $S$. cerevisiae cells.................................27

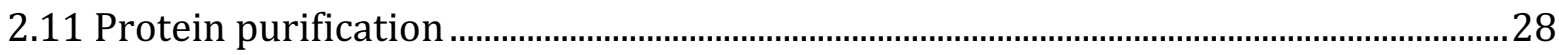

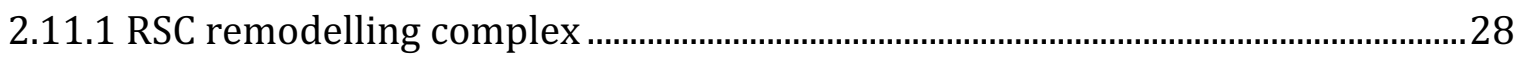

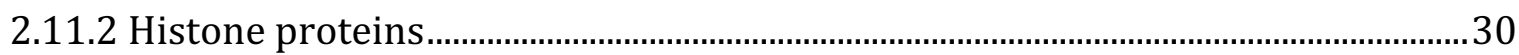

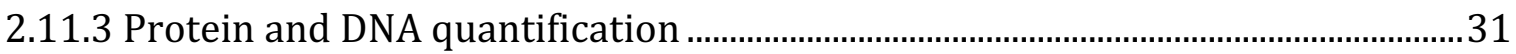

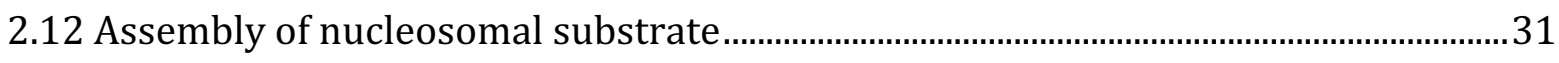

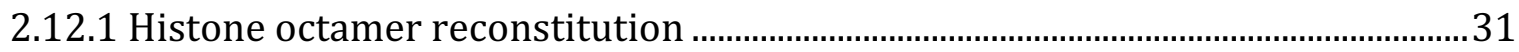

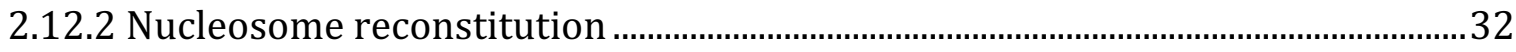

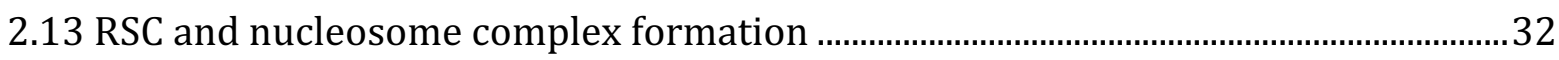

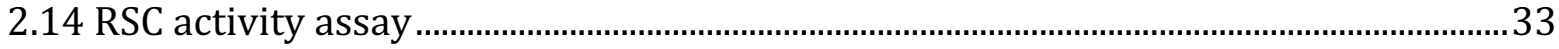

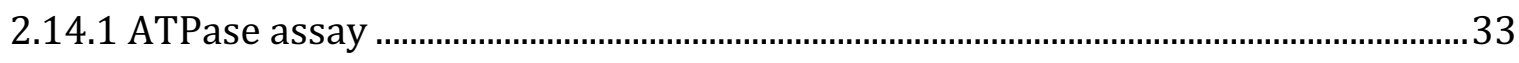

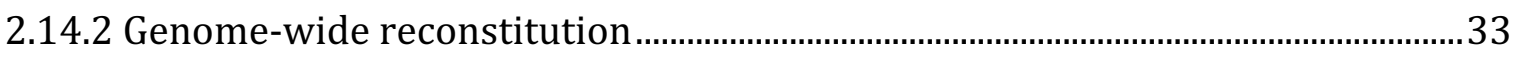

2.15 Mass-spectrometric identification of cross-linking sites ...................................................34

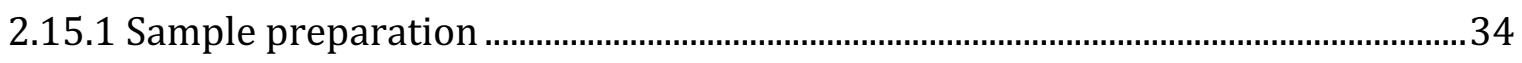

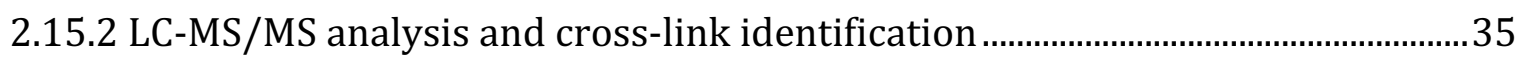

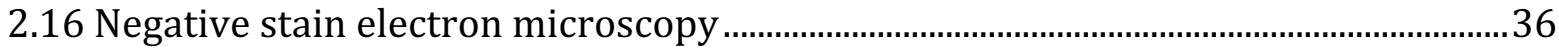

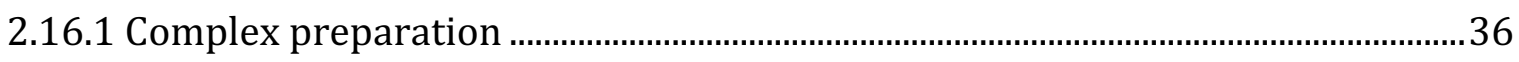

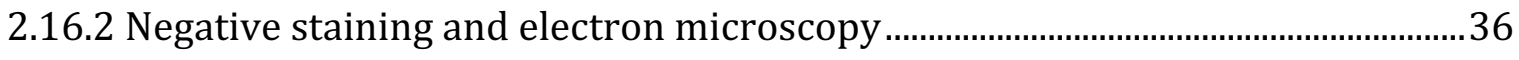

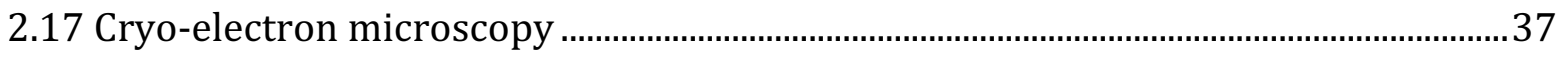

2.17.1 Cryo-electron microscopy analysis of RSC-nucleosome complex ..........................37

2.17.2 Cryo-electron microscopy analysis of the free RSC complex................................... 39

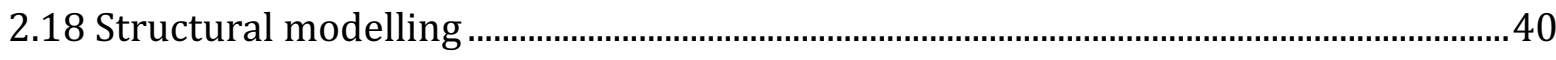

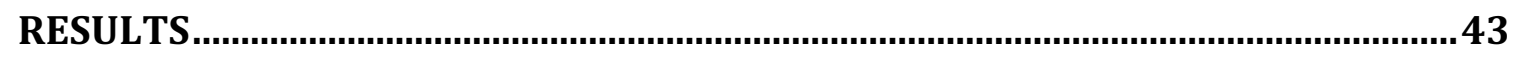

3.1 Preparation of endogenous S. cerevisiae RSC complex ……............................................. 43

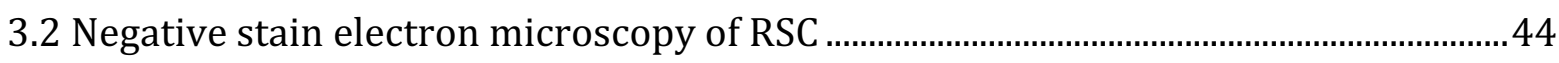




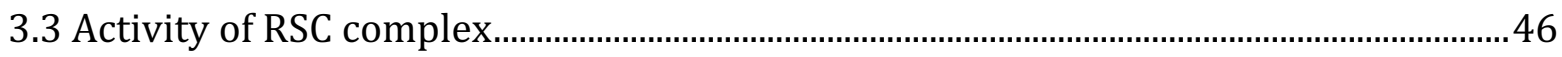

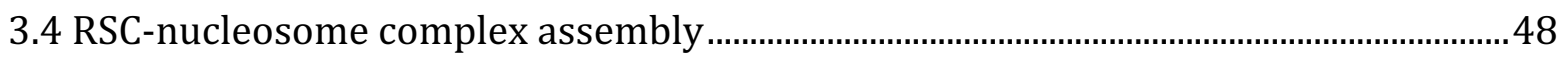

3.5 Structure of RSC-nucleosome complex...........................................................................49

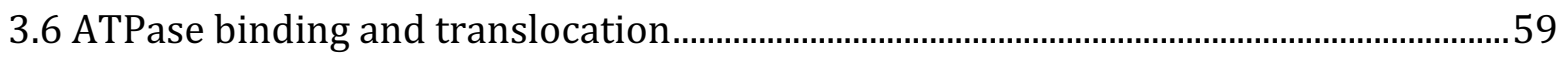

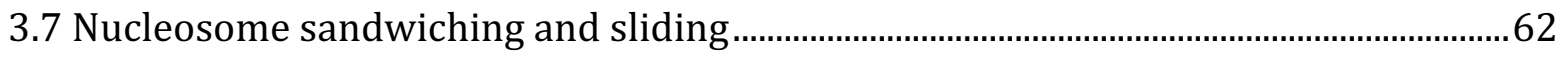

3.8 DNA recognition and NDR formation...........................................................................67

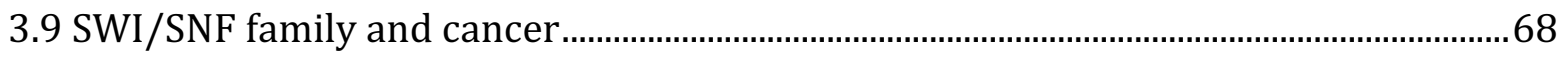

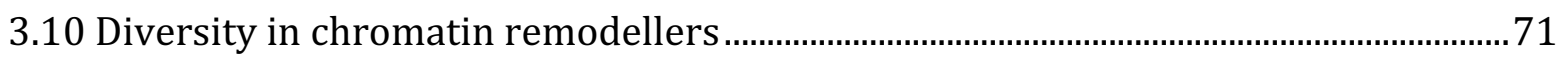

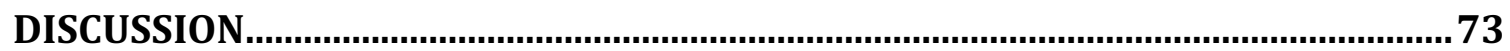

4.1 RSC in context of the four remodeller families ................................................................ 73

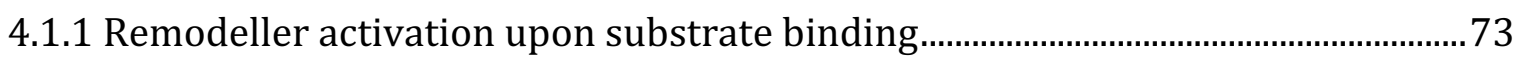

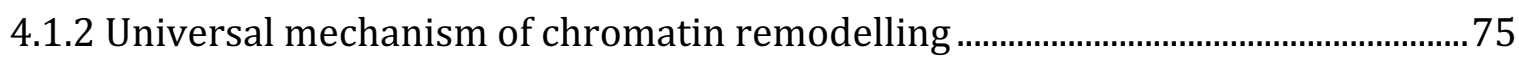

4.1.3 Interactions with extra-nucleosomal DNA ............................................................... 75

4.1.4 Multi-subunit remodellers ............................................................................................. 77

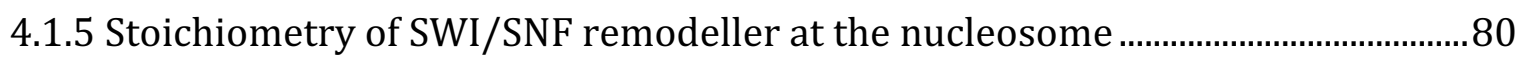

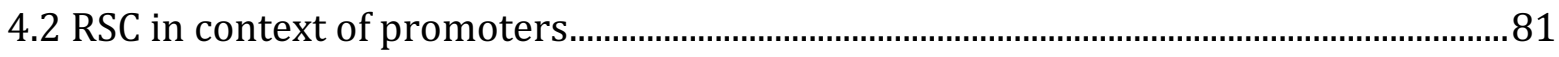

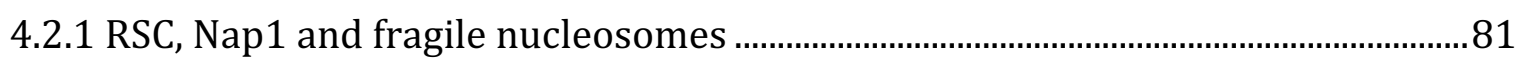

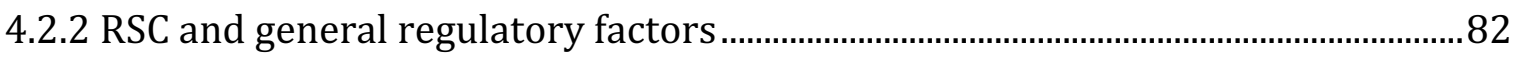

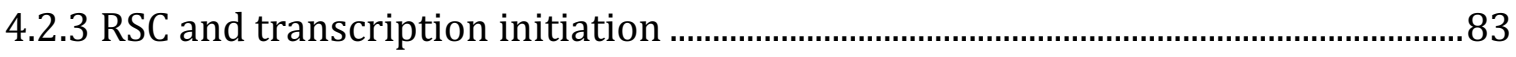

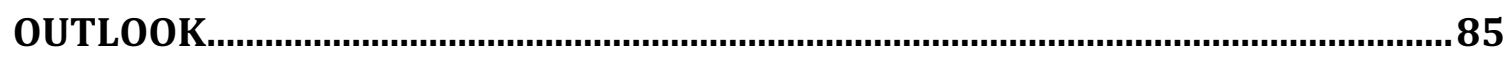

5.1 Short-term goals towards improving our understanding of RSC function..................85

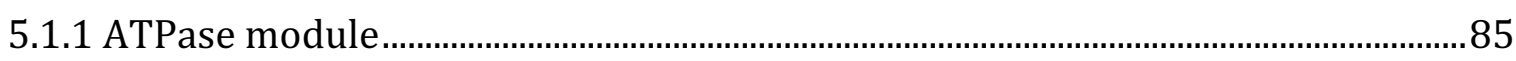

5.1.2 Differences between the RSC isoforms containing the subunits Rsc1 or Rsc2 ..86

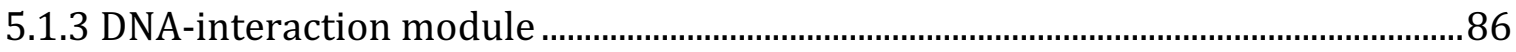

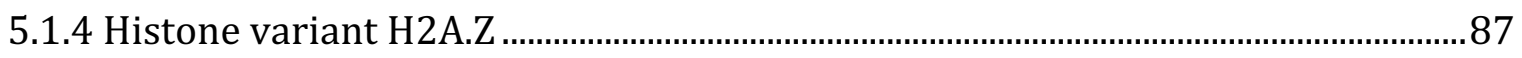

5.1.5 Post-translational histone modifications..................................................................8

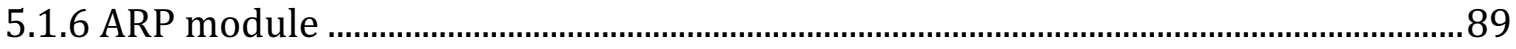

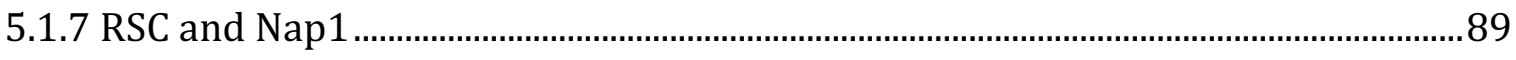

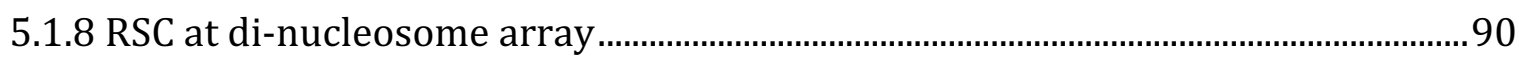

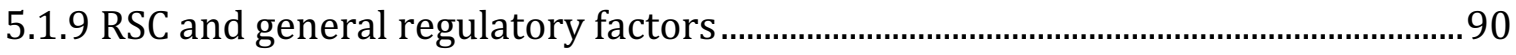

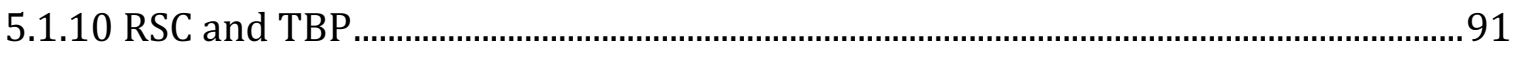

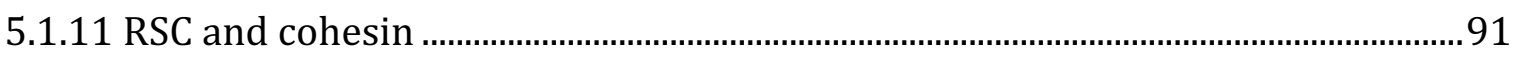

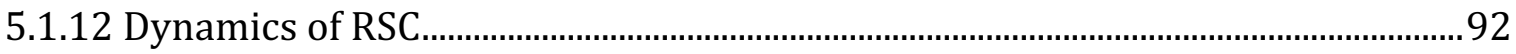

5.2 Long-term goals towards improving our understanding of RSC function ...................92

5.2.1 In vitro chromatin-based transcription system ...................................................... 92 


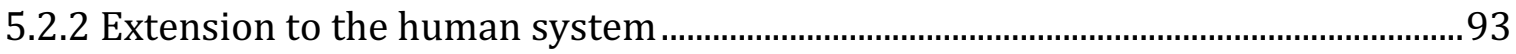

5.2.3 In vivo structure determination of complexes involved in transcription initiation at the +1 nucleosome

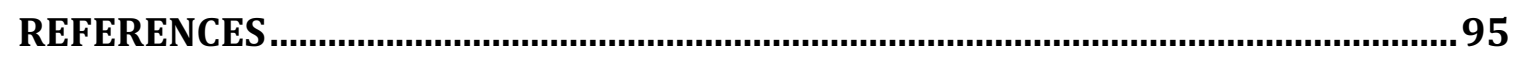

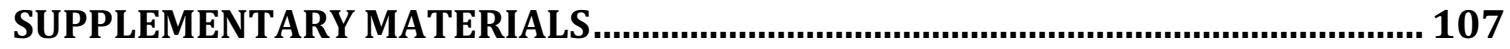

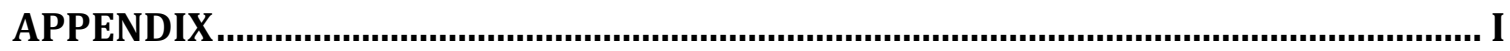

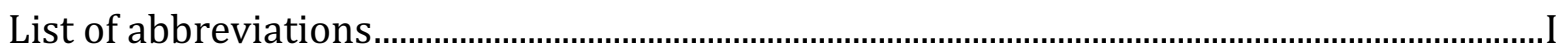

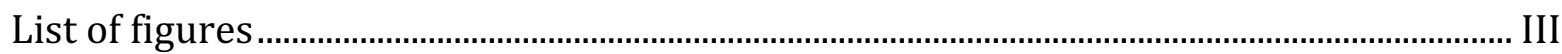

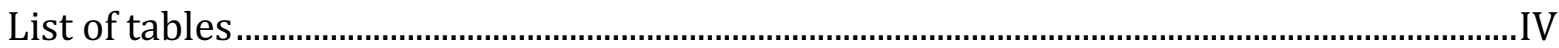




\section{ABSTRACT}

Eukaryotic cells condense their DNA into chromatin, which hampers the accessibility of genetic information. Nucleosomes are the smallest building blocks of chromatin and are comprised of an octameric core of histone proteins, around which 145 - 147 base pairs of DNA are tightly wrapped. Fundamental cellular processes such as transcription require free DNA devoid of nucleosomes. To that end, chromatin remodellers are employed to manoeuvre nucleosomes, providing the cell with means to regulate gene expression through DNA accessibility. These large molecular machines are grouped into four families, each dedicated to specific functions. While these families share a similar enzymatic subunit, they differ in auxiliary subunits, which account for most of their mass. Although recent structural and biochemical work has advanced our knowledge on the general mechanism of remodelling, the role of non-enzymatic subunits is still poorly understood. Moreover, the principles underlying their functional diversity despite utilising the structurally and functionally highly similar active subunit remain ambiguous.

The multi-subunit chromatin remodelling complexes of the SWI/SNF family are responsible for the formation of nucleosome-depleted regions and transcriptionally active promoters in the eukaryotic genome. Here, we solved the structure of a Saccharomyces cerevisiae SWI/SNF family member, the 16-subunit remodeller RSC, in complex with a nucleosome substrate. The structure reveals a modular architecture and suggests key features of the remodelling mechanism. RSC forms an intricate structure composed of the five intertwined modules: the body, arm, ARP, ATPase and DNAinteraction modules. The body provides a scaffolding base connecting the other modules. The DNA-interacting module grasps extra-nucleosomal DNA and helps recruit RSC to promoters. The ATPase and arm modules sandwich the nucleosome disc with their 'SnAC' and 'finger' elements, respectively. The dynamic ARP module bridges between the body and the ATPase modules suggesting a regulation mechanism for the remodeller. The translocase motor engages with the edge of the nucleosome at superhelical location +2 to pump DNA along the nucleosome, resulting in a sliding of the histone octamer along DNA.

The results presented here elucidate the important roles of the non-enzymatic subunits for chromatin remodelling by large, multi-subunit complexes, and shed light on the formation of nucleosome-depleted regions. Furthermore, the structure of RSC from yeast provides a basis for understanding human chromatin remodellers of the SWI/SNF family and the consequences of many cancer mutations that frequently occur in these complexes. 


\section{INTRODUCTION}

\subsection{Chromatin}

The genetic information that is required for all cellular processes is encoded in deoxyribonucleic acid (DNA). The size of genomic DNA varies between organisms, but it is in all cases large compared to the dimensions of the cell. Therefore, cells need to compact their DNA in order to fit it into the limited space. Prokaryotic cells usually contain a single circular chromosome that is condensed via DNA supercoiling and small architectural proteins, and the chromosome together with the proteins forms a nucleoid within the cytoplasm (Drlica and Rouviere-Yaniv, 1987).

Eukaryotes generally have a much larger genome than prokaryotes which imposes an even bigger challenge. For example, one copy of a human genome is made of three billion base pairs (bp) which extends to a total length of about two meters. However, the genome needs to be packed into a special organelle termed the nucleus with an average diameter of roughly $10 \mu \mathrm{m}$, in a way that still allows rapid accessibility of DNA at any given time. This compares to arranging $48 \mathrm{~km}$ of thread inside a basketball while ensuring that any part of the thread can be accessed within seconds (McGinty and Tan, 2015).

To overcome this problem, cells pack their DNA into a structure called chromatin. The smallest building block of chromatin is the nucleosome which is formed by wrapping DNA around a protein scaffold (Kornberg, 1974). This organization does not only allow for the storage of the genetic information but also offers multiple layers of spatial and temporal organization necessary for the regulatory control for higher forms of life. Although high condensation of chromatin is needed for storage, the accessibility of DNA is required for fundamental cellular processes such as transcription, DNA repair and replication.

\subsubsection{Nucleosomes as building blocks of chromatin}

The organization of DNA into nucleosomes is an elegant way for the cell to fit genetic information into the limited space of the eukaryotic nucleus. Nucleosomes are the basic 


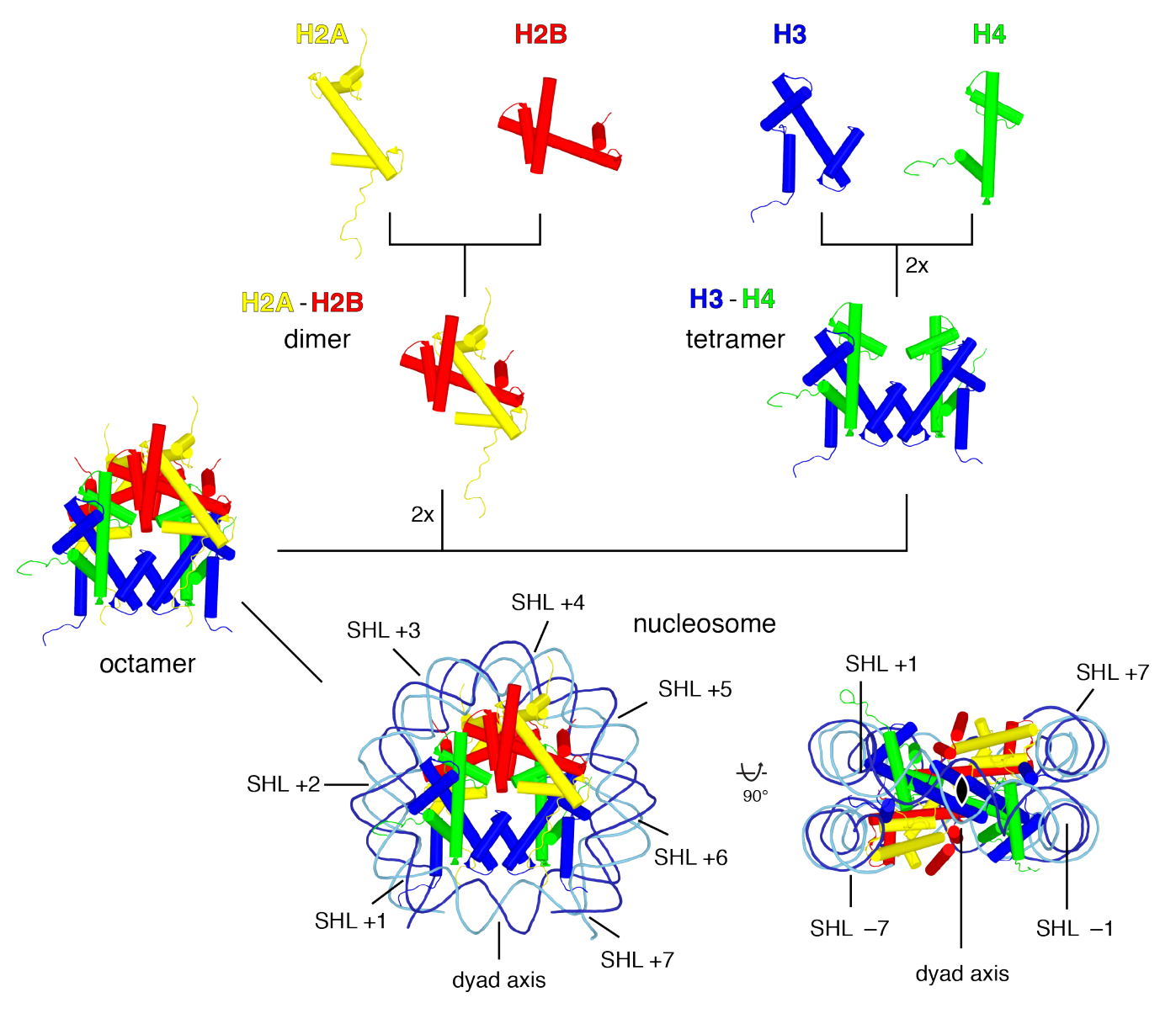

Figure 1 | Assembly pathway and architecture of the nucleosome. Histones H2A and $\mathrm{H} 2 \mathrm{~B}$ form two dimers while histones $\mathrm{H} 3$ and $\mathrm{H} 4$ assemble into a tetramer before they bind together to form the octamer. $147 \mathrm{bp}$ of DNA warp around the octamer in a left-handed superhelix. The dyad axis marks the pseudo-symmetry and the superhelical locations are numbered for the plus side and indicated for the minus side. PDB ID 3MVD.

repeating unit of chromatin and consist of an octameric complex formed by histone core proteins around which 145 - 147 base pairs (bp) of DNA are wrapped (McGinty and Tan, 2015). Since the first high resolution structure of a Xenopus laevis nucleosome was solved in 1997 (Luger et al., 1997), many additional structures of nucleosomes from various organisms have been reported on their own or bound by additional factors, showing their high architectural conservation and underlining their paramount importance throughout eukaryotic life (Armache et al., 2011; Barbera et al., 2006; Luger et al., 1997; Makde et al., 2010; McGinty et al., 2014; Tan and Davey, 2011; Tsunaka et al., 2005; Vasudevan et al., 2010; White et al., 2001) (Figure 1). 
The octameric centre of the nucleosome is formed by two copies of each of the four core histones $\mathrm{H} 2 \mathrm{~A}, \mathrm{H} 2 \mathrm{~B}, \mathrm{H} 3$ and $\mathrm{H} 4$ which share a common architecture named the histone fold (Arents et al., 1991). This fold is characterized by three $\alpha$-helices connected by two loops. The two shorter outer $\alpha$-helices pack against a central longer one. Histones also have $\mathrm{N}$ - and C-terminal extensions in addition to the central histone fold. Using this fold, two histones interact with each other in a 'handshake' motif. The octamer is assembled in a stepwise manner where $\mathrm{H} 2 \mathrm{~A}$ and $\mathrm{H} 2 \mathrm{~B}$ form two $\mathrm{H} 2 \mathrm{~A}-\mathrm{H} 2 \mathrm{~B}$ heterodimers that bind with a tetramer formed by the two $\mathrm{H} 3$ and $\mathrm{H} 4$ histones. DNA is wound around the core octamer in a left-handed superhelix comprising 1.65 turns of DNA and the DNAhistone contacts are formed by ionic interactions, direct hydrogen bonds and hydrogen bonds through water molecules, as well as non-polar interactions (Luger et al., 1997; McGinty and Tan, 2015). The two parallel DNA gyres surrounding the core are connected via a DNA stretch that follows a diagonal path on the side of the octamer where the two DNA ends exit the nucleosome. Thus, the nucleosome has a pseudo-twofold symmetry around a central axis or the dyad (Flaus et al., 1996).

The positions of the DNA around the nucleosome are termed superhelical location (SHL) with a new SHL after each full turn of the DNA duplex every $10 \mathrm{bp}$. The dyad axis is used as a central reference point with its SHL defined as 0 and the SHLs running from -7 to +7 . A characteristic feature of the nucleosome is the acidic patch, a strongly negatively charged area on each disc face of the nucleosome formed by amino acid residues of the H2A-H2B heterodimers. The acidic patch is a common interaction site for various factors operating on chromatin and many structures of nucleosome-bound factors show interactions with the acidic patch surface (Armache et al., 2011; Barbera et al., 2006; Fang et al., 2016; Makde et al., 2010; McGinty et al., 2014).

The $\mathrm{N}$ - and C-termini of histones are unstructured and extend outwards from the central nucleosomal architecture. In this way, they form a flexible platform that is a target for post-translational modifications such as phosphorylation, acetylation, methylation and ubiquitylation. These modifications are installed, detected and interpreted by protein complexes of the chromatin reader and writer/eraser classes to alter chromatin organization and bring about downstream effects (Jenuwein and Allis, 2001; Prakash and Fournier, 2018). 


\subsubsection{Nucleosomes in transcription}

Transcription of RNA polymerase II starts with the assembly of a pre-initiation complex on promoter DNA. However, access to promoter DNA is impaired by nucleosomes, which are arranged on the DNA in a 'beads-on-a-string'-like fashion. The presence of nucleosomes can inhibit initiation (Almer and Horz, 1986; Almer et al., 1986; Knezetic and Luse, 1986; Lorch et al., 1987) and obstruct the path of transcribing RNA polymerase II (Brown et al., 1996; Carey et al., 2006).

In order for transcription initiation to occur, the nucleosomes occupying the promoter must be removed or shifted to free cis promoter elements (Henikoff, 2016; Lorch and Kornberg, 2017). Indeed, several studies have shown that the nucleosomes are not evenly distributed across the genome and nucleosome-depleted regions (NDR) exist at promoters (Lantermann et al., 2010; Lee et al., 2007; Mavrich et al., 2008b; Schones et al., 2008). These regions coincide with the location of active RNA polymerase II and are flanked by specialized +1 and -1 nucleosomes on the downstream and upstream side of the NDR, respectively (Henikoff, 2016; Lorch and Kornberg, 2017).

Further downstream and upstream, the nucleosomes are arranged in a well-defined array that loses the phasing gradually with distance from the +1 and -1 nucleosome (Bai and Morozov, 2010). NDRs were shown to vary in size and, despite their name, can be occupied by 'fragile' nucleosomes that are subject to rapid disassembly and exchange as part of the additional regulatory step during transcription initiation (Kubik et al., 2015).

The architecture of gene promoters varies in different organisms. For instance, the transcription start site (TSS) in Saccharomyces cerevisiae is often located $10-15$ bp inside the +1 nucleosome, which shields the access to this element (Albert et al., 2007). In contrast, human cells usually initiate transcription from a TSS about $60 \mathrm{bp}$ upstream of the +1 nucleosome (Jiang and Pugh, 2009).

Despite these variations, NDRs are generally found throughout different species. Eukaryotic cells have evolved a large toolset of specialized protein complexes, called chromatin remodellers, to regulate the positioning of nucleosomes at promoter regions and to establish the nucleosome-depleted region (Becker and Workman, 2013; Clapier et al., 2017). 


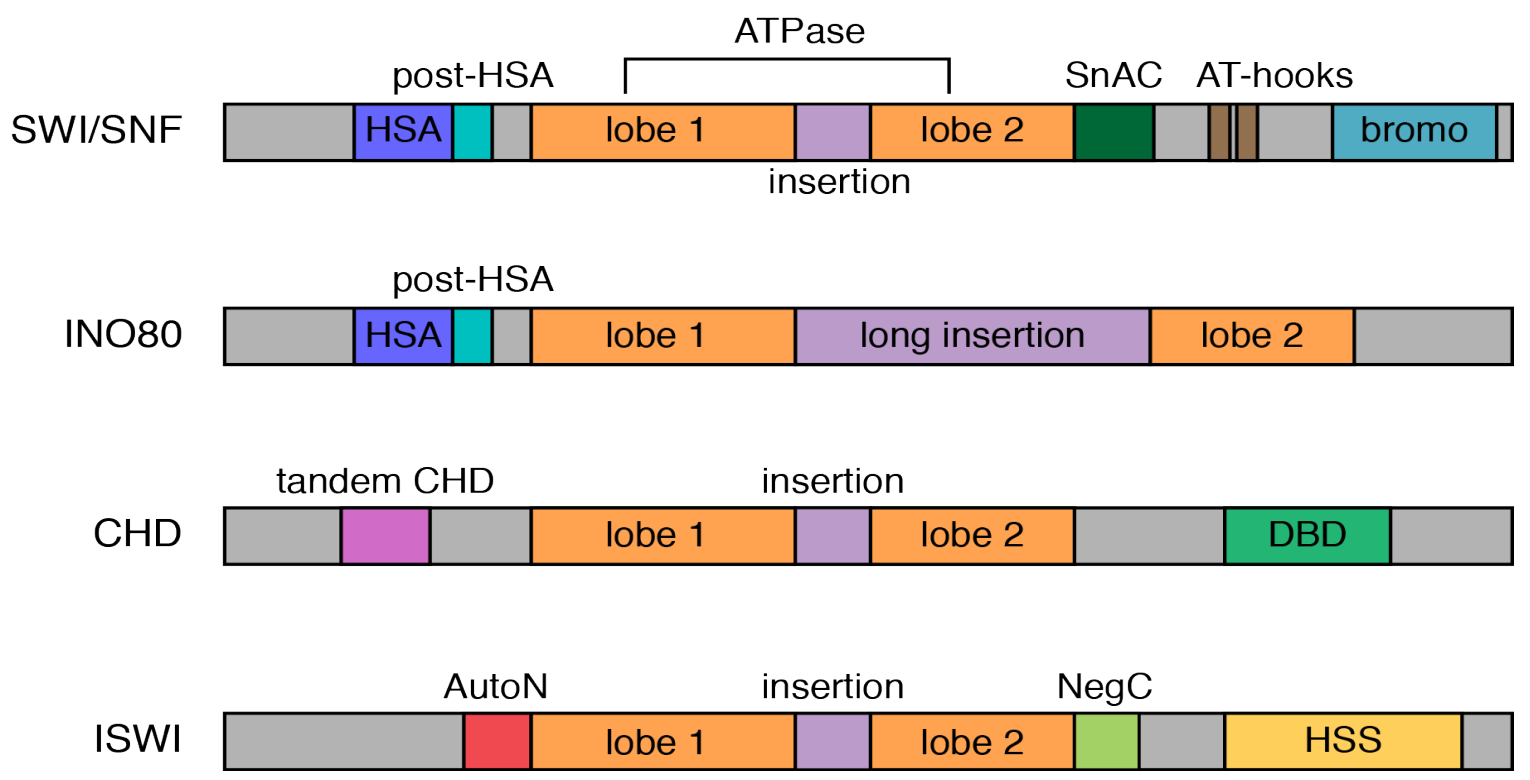

Figure 2 | ATPase domains of the four chromatin remodeller families SWI/SNF, IN080, CHD, and ISWI showing their unique domain compositions. lobe 1, 2, RecAlike ATPase lobes; HSA, helicase/SANT-associated; SnAC, Snf2 ATP coupling; bromo, bromodomain; CHD, chromodomain; DBD, DNA-binding domain; AutoN, autoinhibitory N-terminal; NegC, negative regulator of coupling; HSS, HAND-SANTSLIDE. Figure adapted from (Clapier et al., 2017).

\subsection{Four families of ATP-dependent chromatin remodellers}

The need for the genome to be highly compacted, yet readily accessible, resulted in the co-evolution of nucleosomes and chromatin remodellers. Chromatin remodellers are usually multi-subunit complexes that use the energy of ATP-hydrolysis to remodel nucleosomes and thus grant access to otherwise shielded DNA. Their action results in the movement, destabilization, rearrangement, and ejection of nucleosomes, or the exchange of histone variants (Becker and Workman, 2013; Clapier et al., 2017).

Chromatin remodellers usually contain ATPase domains that operate on nucleosomes, as well as accessory subunits that determine the specificity of remodellers and regulate their action. The ATPase domains of currently known chromatin remodellers are all helicases of the superfamily 2 and in particular members of the Snf2 family (Flaus et al., 2006). Members of this family act as double-strand DNA translocases, i.e. they bind to a double-stranded DNA substrate and utilize one of the two strands to move along the DNA minor groove in a defined direction (Singleton et al., 2007). Although 
part of the helicase family, chromatin remodellers cannot unwind the DNA because they lack a small 'pin' motif used for the separation of DNA strands (Becker and Workman, 2013; Durr et al., 2005; Saha et al., 2006).

Chromatin remodellers can be further distinguished to four different families based on the domain composition of their ATPase subunits (Figure 2). The families are named after their founding members: SWI/SNF (switch/sucrose non-fermentable) (Neigeborn and Carlson, 1984), ISWI (imitation switch) (Elfring et al., 1994), INO80 (inositol requiring mutant 80) (Ebbert et al., 1999), and CHD (chromodomain helicaseDNA binding) (Tran et al., 2000). All four families share two RecA-like lobes that are connected with an insertion and form the ATPase motor. However, the families differ in the composition of additional $\mathrm{N}$ - and C-terminal domains.

The SWI/SNF and INO80 remodellers both contain an N-terminal HSA (helicase/SANT-associated) region followed directly by a post-HSA region. In addition, the SWI/SNF family harbours a SnAC (Snf2 ATP coupling) domain inserted immediately after the ATPase lobe 2 and followed by AT-hooks and a bromodomain. The characteristics of the ISWI family are AutoN (autoinhibitory N-terminal) and NegC (negative regulator of coupling) domains that are located before and after the ATPase lobes, respectively, as well as an HSS (HAND-SANT-SLIDE) domain at the C-terminus. The CHD family is defined by a N-terminal tandem chromodomain and a DBD (DNA-binding domain) on the C-terminus. Whereas the linker connecting the two RecA-like lobes is similar in the CHD, ISWI and SWI/SNF families, the INO80 family contains a considerably larger linker between the ATPase lobes (Clapier et al., 2017).

While there is a functional overlap between the families of chromatin remodellers, with some family members having redundant or opposing roles, a dynamic interplay between them fine-tunes the arrangement of nucleosomes on the genome (Krietenstein et al., 2016; Kubik et al., 2019).

\subsubsection{Chromatin remodellers and cancer}

The crucial importance of maintaining and restructuring chromatin for cell viability resulted in the conservation of chromatin remodellers across the evolutionary scale and homologues of the four remodeller families are found in organisms ranging from yeast to humans. The increasing complexity of higher organisms is reflected in diversification and 
specialization of the chromatin remodellers for specific cell types or developmental functions (Ho and Crabtree, 2010). Their misregulation is correlated with a variety of cancers, as well as cardiac and neurodevelopmental diseases (Cairns, 2001; Hodges et al., 2016; Lafon-Hughes et al., 2008; Nair and Kumar, 2012; Sugathan et al., 2014; Tyagi et al., 2016; Weiss et al., 2016; Zhang et al., 2017). Therefore, chromatin remodellers emerged as potential targets for development of anti-cancer therapies.

\subsubsection{Mechanism of chromatin remodelling}

Efficient translocation of nucleosomes on the DNA during the remodelling process likely requires the remodeller to be anchored to the nucleosome, since the DNA interactions alone would only result in the movement of the ATPase along the DNA. Indeed, recent structures of translocases on the nucleosome show interactions with the histone H3 and the tail of histone H4 (Farnung et al., 2017; Li et al., 2019; Liu et al., 2017; Sundaramoorthy et al., 2018). As a result, the movement of the DNA is opposite to the working direction of the remodeller. As Snf2 ATPases use only one DNA strand (tracking strand) with a 3' to 5' directionality (Saha et al., 2005), this strand is translocated from 5' to 3' around the nucleosome.

The structural basis for chromatin remodelling by a Snf2 family remodeller was elucidated recently. The structure of the Snf2 ATPase bound to the nucleosome was solved in the apo state and bound to ADP or ADP beryllium fluoride (ADP-BeF 3 ) (Li et al., 2019; Liu et al., 2017). ADP- $\mathrm{BeF}_{3}$ is a transition state analogue, which mimics the ATP intermediate prior to the scission of the $\gamma$-phosphate. Analysis of the conformational changes of the ATPase lobes together with distortions on the nucleosomal DNA allowed the deduction of a two-step model for remodelling (Figure 3).

Binding of the translocase to the SHL +2 bulges out one nucleotide at this position. This causes a 1-nucleotide sliding of the phosphate groups of the tracking strand on the proximal linker side. At the same time, the opposite guiding strand moves less, and its backbone stays in register, which maintains the base pairing and stacking of the two strands. Overall, this probably results in base twisting (Bowman, 2010; Saha et al., 2006; Winger et al., 2018). ATP binding induces a closed conformation in which lobe 2 of the translocase interacts with the tracking strand and moves the DNA distortion towards the dyad axis. The guide strand follows in a conjugated movement and the double-stranded 


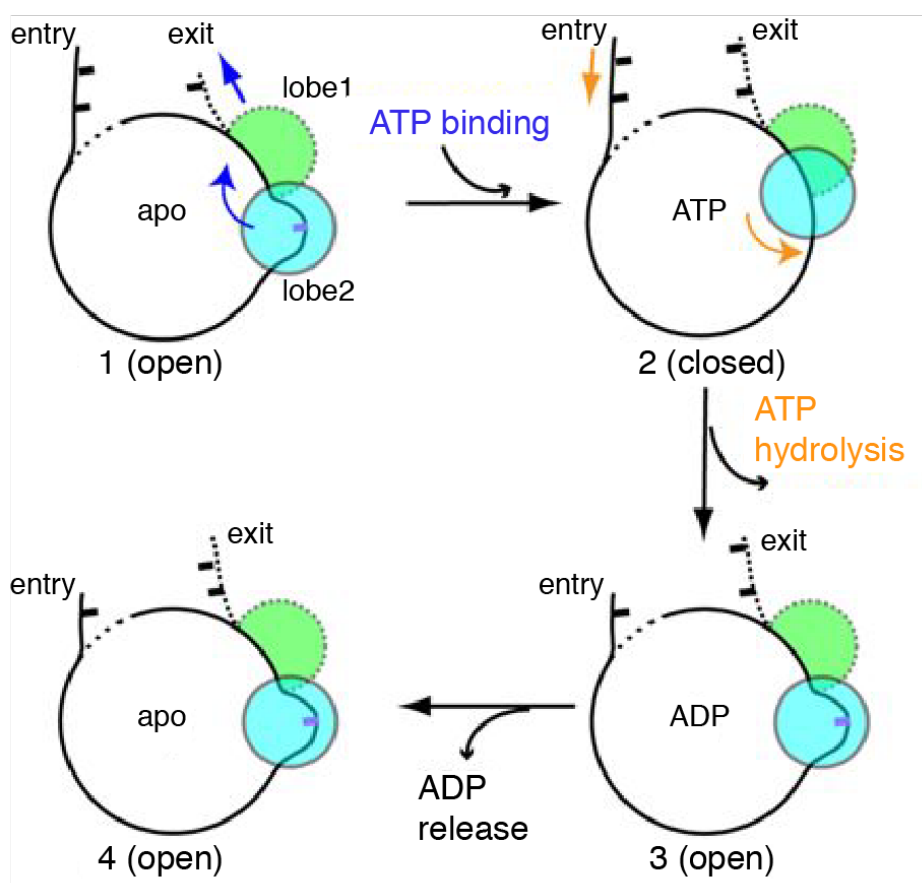

Figure 3 | Schematic overview of remodelling mechanism as suggested by the apo, $\mathrm{ADP}$ and $\mathrm{ADP}-\mathrm{BeF}_{3}$ bound structures of the Snf2 ATPase. Upon binding of the ATPase in the apo state, the nucleosomal DNA is distorted and a 1-bp bulge forms at SHL +2 (state 1). After ATP binding, the ATP lobe 2 changes conformation and pushes the DNA distortion towards the exit side, and the nucleosome adopts a relaxed form (state 2). ATP hydrolysis and phosphate release leads again to the formation of a 1-bp bulge (state 3). Release of the ADP results in the apo state without a major conformational change and primes the enzyme for the next cycle. Figure adopted from (Li et al., 2019).

DNA is translocated by 1 base pair towards the dyad axis. Upon ATP hydrolysis and phosphate release lobe 2 moves to its original position, away from dyad axis. The conformation of the ADP bound state matches the apo state and DNA is slid in again, causing a new 1 nucleotide bulge. Alternation between the ADP/apo and the ATP bound states represent the two states of the remodelling mechanism. As a result of conformational changes in the ATPase upon ATP hydrolysis, the nucleosome is translocated along the DNA (Li et al., 2019).

Differences in the remodelling outcomes of the four families of chromatin remodellers are contributed to the additional domains of the ATPase containing subunit as well as the interaction with different subunits of the corresponding remodelling complexes and additional interaction partners. 


\subsubsection{IN080}

The members of the IN080 family in S. cerevisiae are the SWR1 and the INO80 complexes, but their homologues are conserved in higher eukaryotes (Tyagi et al., 2016). Besides their ATPase, the remodellers contain 13 - 14 additional subunits and are thus among the larger chromatin remodellers (Tyagi et al., 2016). The defining characteristic of this group is a long insertion separating the two ATPase lobes (Figure 2). Using this insertion, they anchor to a heterohexameric ring formed by the ruvB-like proteins 1 and 2 (Rvb1, Rvb2) which constitutes a big part of the remodeller's mass (Wu et al., 2005). In addition, the catalytic subunits harbour an HSA (helicase/SANT-associated) region that interacts with actin-like proteins and is followed by a post-HSA region on the N-terminus (Clapier et al., 2017).

Remodellers of this family are involved in establishing and maintaining proper nucleosome spacing and the IN080 complex was shown to be necessary to set the position of the +1 and -1 nucleosomes (Krietenstein et al., 2016). Nucleosomes containing the histone H2A.Z instead of H2A are a sign for active promoter regions and are found in the nucleosomes surrounding NDRs where IN080 family of remodellers have been shown to mediate their exchange. SWR1 can insert H2A.Z histones into nucleosomes, while INO80 replaces them again with H2A (Mizuguchi et al., 2004; Papamichos-Chronakis et al., 2011). In addition, SWR1 and IN080 play a role in DNA repair pathways (van Attikum et al., 2007).

Recently, the structures of SWR1 and IN080 have been solved by cryo-electron microscopy (cryo-EM) (Ayala et al., 2018; Eustermann et al., 2018; Willhoft et al., 2018). The heterohexameric Rvb1/Rvb2 ring forms the architectural foundation of the remodellers to which other subunits attach. SWR1 and INO80 contact the nucleosome from two opposite sides using their ATPase domain and a module formed by two noncatalytic subunits. While the ATPase contributes the needed energy through ATP hydrolysis, the second module ensures the coupling of this reaction to DNA translation. Surprisingly, SWR1 binds to SHL +2 whereas INO80 engages with SHL -6. Although part of the same remodeller family, both complexes have distinct functions which might require different modes of interaction with the nucleosome. 


\subsubsection{ISWI}

The remodellers of the ISWI family are composed of two to four subunits. The three domains HAND, SLIDE, SLIDE (HSS), which bind to nucleosomal linker DNA (Dang and Bartholomew, 2007), cluster together on the C-terminal side of the ATPase domain. The two ATPase lobes are flanked by an autoinhibitory domain (AutoN) and a negative regulator of coupling domain $(\mathrm{NegC})$ on the $\mathrm{N}$ - and C-terminal sides, respectively (Clapier et al., 2017) (Figure 2).

The ISWI family of remodellers is conserved in higher eukaryotes and it is involved in nucleosome assembly and chromatin spacing, thereby regulating the accessibility of the chromatin (Tyagi et al., 2016). The remodellers in this family are important for transcription and were mostly shown to be involved in its repression, but some members also play a role in transcription activation, elongation, and termination (Tyagi et al., 2016).

S. cerevisiae has two ISWI ATPases, Isw1 and Isw2, which form three different IWSI remodellers in combination with additional subunits (Kagalwala et al., 2004; Vary et al., 2003). The AutoN, NegC and HSS domains are crucial for the function of ISWI complexes, even though they inhibit their remodelling activity. Upon nucleosome binding, 'inhibition of inhibition' takes place (Clapier and Cairns, 2012). The HSS engages with the linker DNA and inhibits the NegC. At the same time, the H4 histone tail inhibits AutoN. Together, this activates translocation and causes nucleosome sliding. In this process, the HSS domain functions as a molecular ruler to measure the distance between two adjacent nucleosomes. Once the length of the linker DNA connecting the neighbouring nucleosomes becomes too short for the HSS domain to bind, the remodelling process is terminated. In this way, the ISWI remodellers ensure correct nucleosome spacing (Clapier and Cairns, 2012; Clapier et al., 2017).

To date, only the ATPase domain has been structurally described in a complex with the nucleosome. It binds to SHL +2 and its architecture and interactions are very similar to the ATPases of the other families (Yan et al., 2019). Structural information on the auxiliary subunits in a nucleosomal context is still missing. 


\subsubsection{CHD}

The remodellers of the CHD family are characterized by a tandem chromodomain at the N-terminus, which is followed by two ATPase lobes and a DNA binding domain (DBD) on the C-terminus (Clapier et al., 2017) (Figure 2). The chromodomains bind methylated histone tails and the DBD interacts with nucleosomal linker DNA (Clapier et al., 2017; Farnung et al., 2017; Sims et al., 2005).

In $S$. cerevisiae the CHD remodellers are single subunit proteins, but in higher metazoans they can form multi-subunit complexes (Denslow and Wade, 2007; Tran et al., 2000). The CHD remodellers have a role in nucleosome spacing, and are important for transcription where they help the RNA polymerase II to transcribe through a nucleosome (Lieleg et al., 2015; Skene et al., 2014). For nucleosomal spacing, the DBD might act as a molecular ruler the same way it does for the ISWI remodellers (1.2.4).

The recently solved structures of the Chd1 and Chd4 proteins on their nucleosome target demonstrate their binding to SHL +2 and explain how DBD detaches nucleosomal DNA from SHL -5 to -7 , thereby facilitating the path of the RNA polymerase II through the nucleosome (Farnung et al., 2019; Farnung et al., 2017; Sundaramoorthy et al., 2018).

\subsubsection{SWI/SNF}

The chromatin remodellers of the SWI/SNF family are multi-subunit complexes with homologues conserved from yeast to human. The defining features of the ATPase subunits are the N-terminal helicase/SANT-associated (HSA) region followed by the postHSA region, and the C-terminal Snf2 ATP coupling (SnAC) domain and a bromodomain (Clapier et al., 2017) (Figure 2). The HSA and post-HSA regions are involved in the regulation of the remodeller through interaction with actin-like proteins (Clapier et al., 2016), whereas bromodomains recognise acetylated histone tails (Fujisawa and Filippakopoulos, 2017). The members of the SWI/SNF family generally make DNA accessible by sliding or ejecting nucleosomes (Clapier et al., 2017).

Recent structural work on the Snf2 ATPase of the SWI/SNF complex gave insights into the molecular mechanism of chromatin remodelling by the Snf2 family of remodellers (Li et al., 2019; Liu et al., 2017), but information about the whole complex is missing. 
While higher eukaryotes have several developmental- and tissue-specific SWI/SNF family subcomplexes (Ho and Crabtree, 2010), S. cerevisiae contains only two (Cairns et al., 1996): the 12 subunit SWI/SNF remodeller - after which the family was named - and the 16 subunit RSC ('remodels the structure of chromatin') complex.

\section{3 'Remodels the structure of chromatin' (RSC) complex}

The essential and abundant $S$. cerevisiae chromatin remodeller RSC is part of the SWI/SNF family. It is a multi-subunit complex that contains 17 subunits, out of which Rsc1 and Rsc2 are mutually exclusive and form two distinct RSC complexes of $1 \mathrm{MDa}$ (Cairns et al., 1996; Cairns et al., 1999). Besides multiple essential cellular processes such as DNA repair (Chambers et al., 2012; Czaja et al., 2014), cell cycle progression (Cao et al., 1997), mitochondrial function (Imamura et al., 2015), and cell wall integrity (Wang and Cheng, 2012), RSC is important for normal transcription activity (Parnell et al., 2008; Spain et al., 2014; Yu et al., 2015). Homologues of the SWI/SNF family, including RSC, are conserved and found in higher eukaryotes including fly, mouse and human (Table 1), and mutations in their subunits are linked to cancer (Henikoff, 2016; Pulice and Kadoch, 2016).

RSC is found at nucleosome-depleted regions (NDR) of gene promoters (Brahma and Henikoff, 2019; Ramachandran et al., 2015), and its loss leads to reoccupation of NDRs with nucleosomes in vitro and in vivo (Krietenstein et al., 2016; Kubik et al., 2019). RSC can bind and position the specialized +1 and -1 nucleosomes (Brahma and Henikoff, 2019; Kubik et al., 2015; Ramachandran et al., 2015) that flank NDRs on the downstream and upstream side, respectively (Henikoff, 2016; Lorch and Kornberg, 2017). RSC recognizes poly(A) and GC-rich elements in promoter DNA (Badis et al., 2008; Kubik et al., 2015; Lorch et al., 2014) and the arrangement of these elements determines the strength and asymmetric directionality of RSC action on promoter nucleosomes (Krietenstein et al., 2016; Kubik et al., 2018). RSC was shown to bind 'fragile' nucleosomes in NDRs in vivo (Brahma and Henikoff, 2019) and to cooperate with the histone chaperone Nap1 in vitro to disassemble nucleosomes (Lorch et al., 2006; Prasad et al., 2016).

The remodeller contains the ATPase Sth1 as enzymatic subunit that functions as a $3^{\prime}$ to $5^{\prime}$ translocase and it is regulated via its HSA region by the actin-like subunits (Clapier et al., 2016; Saha et al., 2002; Szerlong et al., 2008). Although Sth1 can bind the 

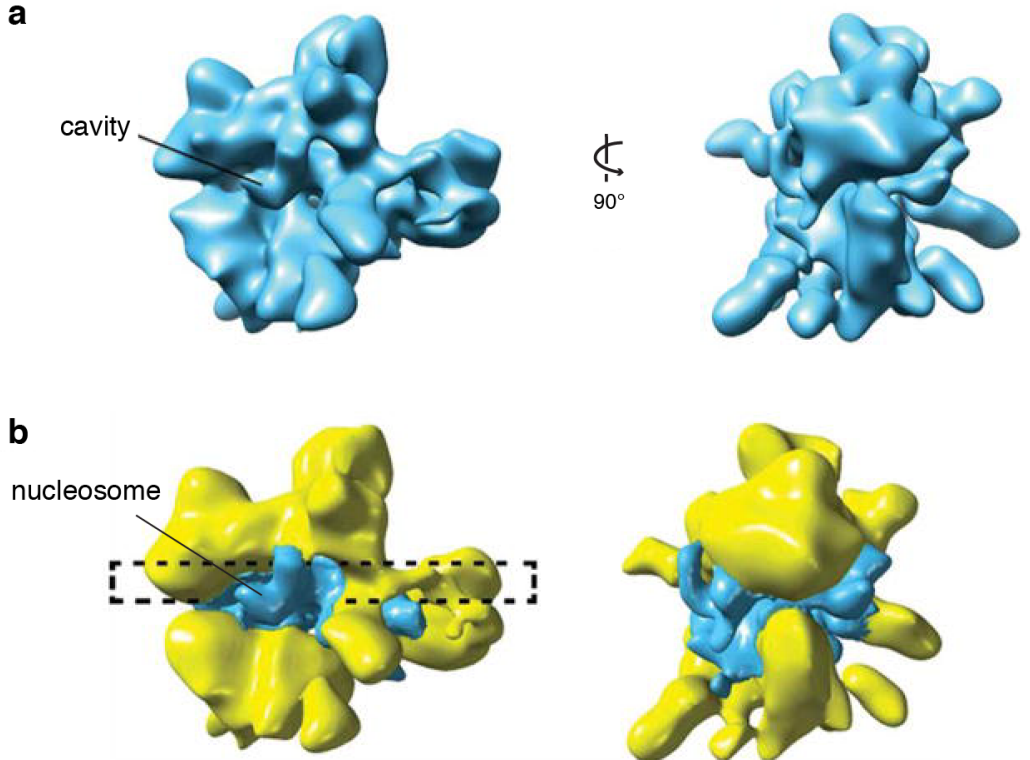

c

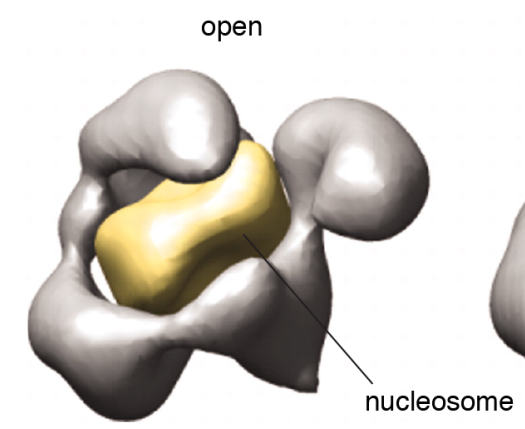

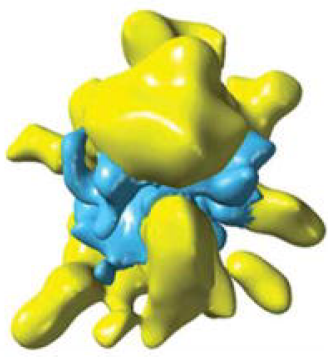

closed

Figure 4 | Two cryo-EM studies on the RSC complex. a. Free RSC complex with indicated central cavity. b. RSC-nucleosome complex from the same study shows extra density which suggests binding of the nucleosome inside the central cavity. c. Independent cryo-EM studies indicating an open and closed conformation of RSC around a cavity that would fit a manually positioned nucleosome. Figure adapted from (Chaban et al., 2008) (a+b) and (Leschziner et al., 2007) (c).

nucleosome on its own $\left(K_{D} \sim 100 \mathrm{nM}\right)$, the binding of the full RSC complex is much stronger $\left(\mathrm{K}_{\mathrm{D}} \sim 7 \mathrm{nM}\right)$ indicating that the other components of the complex are involved in nucleosome binding (Saha et al., 2005).

Electron microscopy (EM) studies of RSC showed a flexible structure with a central cavity that was suggested to bind a nucleosome (Asturias et al., 2002; Chaban et al., 2008; Leschziner et al., 2007) (Figure 4). However, these studies were limited to low resolution, which prevented mechanistic insights on a molecular level. 
Table 1 | Subunit composition of RSC and related chromatin remodelling complexes.

\begin{tabular}{|c|c|c|c|c|c|c|c|c|}
\hline \multirow[t]{2}{*}{ Module } & \multicolumn{2}{|c|}{ S. cerevisiae } & \multicolumn{2}{|c|}{ S. pombe } & \multicolumn{2}{|c|}{ D. melanogaster } & \multicolumn{2}{|c|}{ H. sapiens } \\
\hline & RSC & $\begin{array}{l}\text { SWI/ } \\
\text { SNF }\end{array}$ & RSC & $\begin{array}{l}\text { SWI/ } \\
\text { SNF }\end{array}$ & PBAP & BAP & PBAF & BAF \\
\hline $\begin{array}{l}\text { ATPase } \\
\text { module }\end{array}$ & Sth1 & Snf2 & Snf21 & Snf22 & BRM & BRM & BRG1 & $\begin{array}{c}\text { BRG1/ } \\
\text { BRM }\end{array}$ \\
\hline \multirow{3}{*}{ Arp module } & Arp9 & Arp9 & Arp9 & Arp9 & $\beta$-actin & $\beta$-actin & $\beta$-actin & $\beta$-actin \\
\hline & Arp7 & Arp7 & Arp42 & Arp42 & BAP55 & BAP55 & BAF53A/B & BAF53A/B \\
\hline & Rtt102 & Rtt102 & & & & & & \\
\hline \multirow{2}{*}{ arm module } & Sfh 1 & Snf5 & Sfh1 & Snf2 & SNR1 & SNR1 & BAF47 & BAF47 \\
\hline & Npl6 & Swp82 & Rsc7 & Snf59 & & & & \\
\hline \multirow{6}{*}{$\begin{array}{l}\text { body } \\
\text { module }\end{array}$} & Rsc6 & Swp73 & Ssr3 & Ssr3 & BAP60 & BAP60 & $\mathrm{BAF} 60 \mathrm{~A} / \mathrm{B} / \mathrm{C}$ & $\mathrm{BAF} 60 \mathrm{~A} / \mathrm{B} / \mathrm{C}$ \\
\hline & Rsc9 & Swil & Rsc9 & Sol1 & BAP170 & OSA & BAF200 & BAF250A/B \\
\hline & Htll & & & & & & & \\
\hline & Rsc58 & & Rsc58 & & & & & \\
\hline & Rsc4 & & Rsc4 & & & & & \\
\hline & $\begin{array}{c}\text { Rsc2 / } \\
\text { Rsc1 }\end{array}$ & & Rsc1 & & bromo & & BAF180 & \\
\hline $\begin{array}{l}\text { DNA-inter- } \\
\text { action } \\
\text { module }\end{array}$ & $\begin{array}{l}\text { Rsc3 } \\
\text { Rsc30 }\end{array}$ & & & & & & & \\
\hline \multirow[t]{12}{*}{ Scaffold } & $\begin{array}{l}\text { Rsc8, } \\
\text { Rsc8 }\end{array}$ & $\begin{array}{l}\text { Swi3, } \\
\text { Swi3 }\end{array}$ & $\begin{array}{l}\text { Ssr1, } \\
\text { Ssr2 }\end{array}$ & $\begin{array}{l}\text { Ssr1, } \\
\text { Ssr2 }\end{array}$ & $\begin{array}{l}\text { MOR, } \\
\text { MOR }\end{array}$ & $\begin{array}{l}\text { MOR, } \\
\text { MOR }\end{array}$ & $\begin{array}{l}\text { BAF155, } \\
\text { BAF170 }\end{array}$ & $\begin{array}{l}\text { BAF155, } \\
\text { BAF170 }\end{array}$ \\
\hline & Ldb7 & & & & & & & \\
\hline & & & & & BAP111 & BAP111 & BAF57 & BAF57 \\
\hline & & Snf11 & & & & & & \\
\hline & & Snf6 & & & & & & \\
\hline & & Taf14 & & Tfg3 & & & & \\
\hline & & & & Snf30 & & & & \\
\hline & & & & & SAYP & & BAF45A & BAF $45 \mathrm{~A} / \mathrm{B} / \mathrm{C}$ \\
\hline & & & & & & & BRD7 & BRD9 \\
\hline & & & & & & & BCL11A/B & BCL11A/B \\
\hline & & & & & & & BCL7A/B/C & BCL7A/B/C \\
\hline & & & & & & & $\begin{array}{c}\text { SS18 / } \\
\text { SS18L1 }\end{array}$ & \\
\hline
\end{tabular}

Assignment to the structural modules based on the S. cerevisiae structure of RSC presented in this work. Subunits occurring together in the complex are separated by comma, a slash indicates the use of one of the subunits. Subunits that could not be assigned to any module by homology are listed below. 


\subsection{Cryo-electron microscopy}

Despite intensive work on chromatin remodellers for more than a decade, structural insight into chromatin remodelling complexes was limited due to their size and dynamic nature (Asturias et al., 2002; Chaban et al., 2008; Leschziner et al., 2005; Leschziner et al., 2007). This changed recently with the introduction of direct electron detectors, enhanced automated data collection at the electron microscope and improved image processing software (Kuhlbrandt, 2014). These new developments dramatically increased the number of high-resolution structures of large, dynamic complexes produced by cryoelectron microscopy (cryo-EM) and enabled the study of chromatin remodellers even in the context of their nucleosome substrate (Ayala et al., 2018; Eustermann et al., 2018; Hanske et al., 2018; Willhoft et al., 2018). However, no detailed structure of the chromatin remodeller RSC is available to date. 


\subsection{Aims and scope of this work}

In the last decades, the fundamental aspects of gene regulation through transcription by RNA polymerases, in particular transcription initiation, have been intensively studied and the molecular structures of the proteins involved have strongly contributed to our knowledge of the process. However, the natural template for RNA polymerases is chromatin and not the naked DNA. Thus, to further understand gene regulation we need to shed light on chromatin organisation, particularly in the promoter regions.

Active promoters need to be nucleosome-depleted and are flanked by a stable -1 and +1 nucleosome on the upstream and downstream sides, respectively. Chromatin remodelling complexes of the highly conserved SWI/SNF family are known to be needed for the establishment and the maintenance of these regions, but despite intensive in vivo and in vitro experiments, the underlying molecular mechanism involving full multi-subunit remodelling complexes remains unknown.

Therefore, in this study we set out to solve the high-resolution structure of the essential Saccharomyces cerevisiae SWI/SNF family chromatin remodeller RSC in the context of its minimal chromatin substrate: a single nucleosome, which represents the +1 nucleosome at promoter regions.

To accomplish this goal, we needed to purify large, homogeneous amounts of RSC in a quality amenable for structural characterization. With purified complex in hand, we aimed to solve the structure of RSC in its free state and bound to a nucleosome using cross-linking mass spectrometry and cryo-electron microscopy. 


\section{MATERIALS AND METHODS}

\subsection{Chemicals and consumables}

Table 2 | List of chemicals and consumables.

\begin{tabular}{|c|c|}
\hline Chemical / Consumable & Manufacturer \\
\hline Acetic acid & Merck kGaA, Darmstadt, Germany \\
\hline $\mathrm{ADP}$ & Sigma-Aldrich Chemie GmbH, Steinheim Germany \\
\hline ATP & Sigma-Aldrich Chemie GmbH, Steinheim Germany \\
\hline Agarose, UltraPure & Thermo Fischer Scientific, Massachusetts, US \\
\hline Amberlite MB-3 & Merck kGaA, Darmstadt, Germany \\
\hline Ammonium bicarbonate & Carl Roth GmbH + Ca. KG, Karlsruhe, Germany \\
\hline Ampicillin sodium salt & Sigma-Aldrich Chemie GmbH, Steinheim Germany \\
\hline BS3 & Thermo Fischer Scientific, Massachusetts, US \\
\hline Calmodulin resin & Agilent Technologies, Santa Clara, US \\
\hline Citric acid & Carl Roth GmbH + Ca. KG, Karlsruhe, Germany \\
\hline Chloramphenicol & Carl Roth GmbH + Ca. KG, Karlsruhe, Germany \\
\hline dNTP mix & $\begin{array}{l}\text { New England BioLabs GmbH, Frankfurt on Main, } \\
\text { Germany }\end{array}$ \\
\hline DTT & Sigma-Aldrich Chemie GmbH, Steinheim Germany \\
\hline Ethanol & Merck kGaA, Darmstadt, Germany \\
\hline EDTA & Merck kGaA, Darmstadt, Germany \\
\hline EGTA & Carl Roth GmbH + Ca. KG, Karlsruhe, Germany \\
\hline Formaldehyde solution $37 \%$ & Carl Roth GmbH + Ca. KG, Karlsruhe, Germany \\
\hline Glycerol & Merck kGaA, Darmstadt, Germany \\
\hline Guanidinium hydrochloride & Carl Roth GmbH + Ca. KG, Karlsruhe, Germany \\
\hline HEPES & Sigma-Aldrich Chemie GmbH, Steinheim Germany \\
\hline HiTrap Q column & GE Healthcare, Munich, Germany \\
\hline Hydrochloric acid & Merck kGaA, Darmstadt, Germany \\
\hline IgG Sepharose 6 Fast Flow resin & GE Healthcare, Munich, Germany \\
\hline Imidazole & Merck kGaA, Darmstadt, Germany \\
\hline Instant Blue & Expedeon Inc., San Diego, US \\
\hline IPTG & Carl Roth GmbH + Ca. KG, Karlsruhe, Germany \\
\hline Kanamycin sulphate & Thermo Fischer Scientific, Massachusetts, US \\
\hline
\end{tabular}




\begin{tabular}{ll}
\hline Chemical / Consumable & Manufacturer \\
\hline Magnesium chloride hexahydrate & Merck kGaA, Darmstadt, Germany \\
Native PAGE buffer & Thermo Fischer Scientific, Massachusetts, US \\
Potassium acetate & Carl Roth GmbH + Ca. KG, Karlsruhe, Germany \\
QIAquick Gel Extraction Kit & Qiagen, Hilden, Germany \\
Silver nitrate & Carl Roth GmbH + Ca. KG, Karlsruhe, Germany \\
Slide-A-Lyzer Dialysis Cassettes & Thermo Fischer Scientific, Massachusetts, US \\
Sodium carbonate & Merck kGaA, Darmstadt, Germany \\
Sodium chloride & Merck kGaA, Darmstadt, Germany \\
Sodium hydroxide & Merck kGaA, Darmstadt, Germany \\
Sucrose & Merck kGaA, Darmstadt, Germany \\
SYBR Gold Nucleic Acid Gel Stain & Thermo Fischer Scientific, Massachusetts, US \\
TBE buffer 10x & Thermo Fischer Scientific, Massachusetts, US \\
Tetracycline hydrochloride & Carl Roth GmbH + Ca. KG, Karlsruhe, Germany \\
Urea & Merck kGaA, Darmstadt, Germany \\
VivaSpin concentrator & Sartorius AG, Goettingen, Germany \\
$\beta$-mercaptoethanol & Sigma-Aldrich Chemie GmbH, Steinheim Germany \\
\hline
\end{tabular}




\subsection{Buffers and growth media}

Table 3 | List of buffers.

\begin{tabular}{|c|c|c|}
\hline Buffer & Composition & Application \\
\hline PBS & $\begin{array}{l}137 \mathrm{mM} \mathrm{NaCl}, 2.7 \mathrm{mM} \mathrm{KCl}, 10 \mathrm{mM} \mathrm{Na}_{2} \mathrm{HPO}_{4} \\
\mathrm{pH} 7.4,1.76 \mathrm{mM} \mathrm{KH}_{2} \mathrm{PO}_{4} \mathrm{pH} 7.4\end{array}$ & $\begin{array}{l}\text { Western } \\
\text { blotting }\end{array}$ \\
\hline TBE & 89 mM Tris, 89 mM boric acid, 2 mM EDTA & EMSA assay \\
\hline TAE & $40 \mathrm{mM}$ Tris, $20 \mathrm{mM}$ acetic acid, $1 \mathrm{mM}$ EDTA & $\begin{array}{l}\text { agarose gel } \\
\text { electrophoresis }\end{array}$ \\
\hline $\begin{array}{l}\text { NuPAGE LDS } \\
\text { Sample buffer }\end{array}$ & $\begin{array}{l}141 \text { mM Tris, } 106 \text { mM Tris-HCl, } 2 \text { \% LDS, } 10 \% \\
\text { glycerol, } 0.51 \text { mM EDTA, } 0.22 \text { mM SERVA Blue } \\
\text { G250, } 0.175 \text { mM Phenol Red, pH } 8.5\end{array}$ & SDS-PAGE \\
\hline $\begin{array}{l}\text { NuPAGE MOPS SDS } \\
\text { Running buffer }\end{array}$ & $\begin{array}{l}50 \text { mM MOPS, } 50 \text { mM Tris, } 0.1 \% \text { SDS, } 1 \text { mM } \\
\text { EDTA, pH } 7.7\end{array}$ & SDS-PAGE \\
\hline $\begin{array}{l}\text { NuPAGE MES SDS } \\
\text { Running buffer }\end{array}$ & $\begin{array}{l}50 \text { mM MES, } 50 \text { mM Tris, } 0.1 \% \text { SDS, } 1 \text { mM EDTA, } \\
\text { pH } 7.3\end{array}$ & SDS-PAGE \\
\hline
\end{tabular}

Table 4 | List of growth media for E. coli and S. cerevisiae cells.

\begin{tabular}{|c|c|c|c|}
\hline Media & Composition & Supplier & Application \\
\hline LB & $\begin{array}{l}1 \%(\mathrm{w} / \mathrm{v}) \text { tryptone, } 0.5 \% \\
(\mathrm{w} / \mathrm{v}) \text { yeast extract, } 0.5 \% \\
(\mathrm{w} / \mathrm{v}) \mathrm{NaCl}\end{array}$ & $\begin{array}{l}\text { Becton } \\
\text { Dickinson, mixed } \\
\text { in house }\end{array}$ & $\begin{array}{l}\text { E. coli } \\
\text { cultures }\end{array}$ \\
\hline YPD & $\begin{array}{l}2 \%(\mathrm{w} / \mathrm{v}) \text { peptone, } 2 \% \\
(\mathrm{w} / \mathrm{v}) \text { glucose, } 1.5 \%(\mathrm{w} / \mathrm{v}) \\
\text { yeast extract }\end{array}$ & $\begin{array}{l}\text { Becton } \\
\text { Dickinson, mixed } \\
\text { in house }\end{array}$ & $\begin{array}{l}\text { S. cerevisiae } \\
\text { cultures }\end{array}$ \\
\hline YEP broth & As YPD but without dextrose & Formedium & $\begin{array}{l}\text { S. cerevisiae } \\
\text { fermentation }\end{array}$ \\
\hline $\begin{array}{l}\text { Complete supplement } \\
\text { mixture drop-out }\end{array}$ & $\begin{array}{l}\text { CSM mixture missing amino } \\
\text { acids for selection }\end{array}$ & Formedium & $\begin{array}{l}\text { S. cerevisiae } \\
\text { cultures }\end{array}$ \\
\hline
\end{tabular}


Table 5 | List of supplements and antibiotics for S. cerevisiae and E. coli cultures.

\begin{tabular}{lll}
\hline Supplement / Antibiotic & Stock concentration & Final concentration \\
\hline Kanamycin & $50 \mathrm{mg} / \mathrm{mL}$ & $50 \mu \mathrm{g} / \mathrm{mL}$ \\
Ampicillin & $100 \mathrm{mg} / \mathrm{mL}$ & $100 \mu \mathrm{g} / \mathrm{mL}$ (E. coli) \\
Chloramphenicol & $34 \mathrm{mg} / \mathrm{mL}$ & $50 \mu \mathrm{g} / \mathrm{mL}$ (S. cerevisiae) \\
Tetracycline & $12.5 \mathrm{mg} / \mathrm{mL}$ & $17 \mu \mathrm{g} / \mathrm{mL}$ \\
IPTG & $1 \mathrm{M}$ & $12.5 \mu \mathrm{g} / \mathrm{mL}$ \\
\hline
\end{tabular}

\subsection{Bacterial and yeast strains}

Table 6 | Bacterial and yeast strains used in this study.

\begin{tabular}{|c|c|c|}
\hline Strain & Genotype & Supplier \\
\hline $\begin{array}{l}\text { E. coli: } \\
\text { BL21 CodonPlus (DE3) RIL }\end{array}$ & $\begin{array}{l}\left.\text { B F ompT hsdS(r} r_{B}^{-} m_{B^{-}}\right) d c m+\text { Tet }^{r} \text { E. } \\
\text { coli gal } \lambda \text { (DE3) endA Hte [argU ileY } \\
\text { leW Camr] }\end{array}$ & Agilent \\
\hline $\begin{array}{l}\text { S. cerevisiae: } \\
\text { RSC2-TAP-HIS3 } \\
\text { (YSC1177-YLR357W) }\end{array}$ & 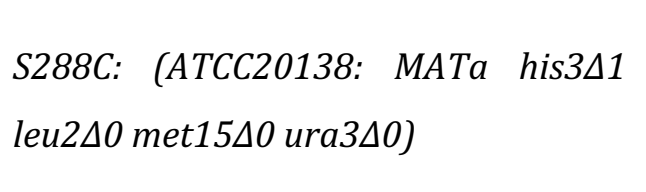 & $\begin{array}{l}\text { Dharmacon } \\
\text { (Yeast TAP-Fusion ORF } \\
\text { clones and collection) }\end{array}$ \\
\hline
\end{tabular}

\subsection{Plasmids}

Table 7 | Vectors used in this study.

\begin{tabular}{lllll}
\hline Insert gene & Vector & $\begin{array}{l}\text { Antibiotic } \\
\text { resistance }\end{array}$ & $\begin{array}{l}\text { Expression } \\
\text { system }\end{array}$ & Source \\
\hline X. laevis $\mathrm{H} 2 \mathrm{~A}$ & $\mathrm{pET3A}$ & Ampicillin & E.coli & Halic Lab \\
X. laevis $\mathrm{H} 2 \mathrm{~B}$ & $\mathrm{pET3A}$ & Ampicillin & E.coli & Halic Lab* \\
X. laevis $\mathrm{H} 3$ & $\mathrm{pET3A}$ & Ampicillin & E. coli & Halic Lab* \\
X. laevis $\mathrm{H} 4$ & $\mathrm{pET3A}$ & Ampicillin & E. coli & Halic Lab* \\
\hline
\end{tabular}

\footnotetext{
* I thank the Halic Lab from the Gene Center in Munich, Germany, for the kind gift of the expression plasmids.
} 


\subsection{Antibodies}

Table 8 | Antibodies used in this study.

\begin{tabular}{|c|c|c|c|c|}
\hline Antibody & Specificity & Dilution & $\begin{array}{l}\text { Source } \\
\text { organism }\end{array}$ & Supplier \\
\hline anti-Sth1 & Sth1 N-terminus & $1: 2000$ & goat & $\begin{array}{l}\text { Santa Cruz, sc-33289 } \\
\text { (yA-13) }\end{array}$ \\
\hline anti-Sth1 & Sth1 C-terminus & $1: 2000$ & goat & $\begin{array}{l}\text { Santa Cruz, sc-33290 } \\
\text { (yT-18) }\end{array}$ \\
\hline anti-goat-HRP & goat IgG & $1: 5000$ & donkey & Santa Cruz, sc-2750 \\
\hline anti-H2B & $\begin{array}{l}\text { Human H2B (also reacts } \\
\text { with X. laevis) }\end{array}$ & $1: 1000$ & mouse & $\begin{array}{l}\text { Thermo Fischer } \\
\text { Scientific, MA5-14835 }\end{array}$ \\
\hline anti-mouse-HRP & mouse IgG & $1: 10,000$ & goat & Sigma-Aldrich, A8786 \\
\hline TAP-HRP & tap-tag & $1: 2000$ & rabbit & Sigma-Aldrich, P1291 \\
\hline
\end{tabular}

\subsection{Gel electrophoresis}

\subsubsection{Sodium-dodecyl-sulfate polyacrylamide gel electrophoresis (SDS-PAGE)}

SDS-PAGE was applied for the analytical separation of the proteins according to their molecular weight during protein expression and purification. Protein samples were mixed with NuPAGE LDS Sample Buffer (4x, Thermo Fischer Scientific) and boiled at $\sim 95{ }^{\circ} \mathrm{C}$ for $2-5 \mathrm{~min}$. The samples were loaded into NuPAGE $4-12 \%$ gradient Bis-Tris Protein Gels (Invitrogen) together with PageRuler Prestained Protein Ladder (Thermo Fischer Scientific) as molecular weight standard. Gels were run in 1x MES or 1x MOPS buffer (Invitrogen) at $160-200 \mathrm{~V}$ for optimal separation in the lower or higher molecular weight range, respectively. Instant Blue (Invitrogen) was used for visualization of the protein bands by staining for several hours while shaking and subsequent de-staining with MilliQ $\mathrm{H}_{2} \mathrm{O}$ for removal of background staining. Gels were scanned for documentation with the Epson Perfection V800 flatbed scanner (Epson). Gels for Western blotting were not stained and transferred into MilliQ $\mathrm{H}_{2} \mathrm{O}$. 


\subsubsection{Native polyacrylamide gel electrophoresis (PAGE)}

Native PAGE was used to separate and analyse unimpaired protein complex assemblies of higher molecular weight. Native PAGE was conducted at $4{ }^{\circ} \mathrm{C}$. NuPAGE 3 - $12 \%$ Bis-Tris gels were pre-run for $30-60 \mathrm{~min}$ at $150-200 \mathrm{~V}$ in $1 \mathrm{x}$ NativePAGE buffer (20x, Novex). Wells were pre-loaded with $1 \mathrm{x}$ NativePAGE Cathode Buffer Additive (20x, Thermo Fischer Scientific) and sample supplied with $2 \%(\mathrm{v} / \mathrm{v})$ glycerol before loading if it did not already contain glycerol or sucrose. The gel was run overnight at $90 \mathrm{~V}$ or for $3-5 \mathrm{~h}$ at $120 \mathrm{~V}$ depending on the desired separation. Protein bands were visualized with Instant Blue (Expedeon Inc.) while shaking for several hours followed by de-staining with MilliQ $\mathrm{H}_{2} \mathrm{O}$ to remove background staining. Gels were documented with an Epson Perfection V800 flatbed scanner (Epson). Gels for Western blotting were not stained and transferred into MilliQ $\mathrm{H}_{2} \mathrm{O}$.

\subsubsection{Electrophoretic mobility shift assay (EMSA)}

The analysis of successful nucleosome reconstitution was done using EMSA gel analysis. Nucleosome samples were mixed with SDS-free Purple Gel Loading Dye (6x, NEB) and loaded into a NuPAGE 6 \% DNA Retardation Gel in a volume that resulted in $0.5 \mu \mathrm{g}$ of DNA per well. A 100 bp DNA Ladder (NEB) was added as size standard. Electrophoresis was conducted at $4{ }^{\circ} \mathrm{C}$ in $0.2 \mathrm{x}$ TBE buffer for $1.5 \mathrm{~h}$ at $100 \mathrm{~V}$. DNA bands were visualized by post-staining in SYBR Gold Nucleic Acid Gel Stain (Thermo Fischer Scientific) for 20 min while shaking. Gels were documented using a Typhoon FLA 9500 (GE Healthcare) imager.

\subsubsection{Agarose gel electrophoresis}

Agarose gel electrophoresis was used during cloning and DNA amplification for identification of vectors containing the insert of choice, quality control of large-scale PCR reactions, control of tag integration into yeast genome, and purification of DNA substrates for subsequent applications. Agarose gel was prepared by mixing agarose powder with TAE buffer at a usual concentration of $1 \%(w / v)$. For the separation of smaller sized DNA fragments, the agarose concentration was increased to up to $2 \%(\mathrm{w} / \mathrm{v})$. The agarose was fully dissolved by boiling and the agarose solution cooled down to approximately $50{ }^{\circ} \mathrm{C}$. 
SYBR safe DNA Gel stain (Invitrogen) was added to allow for later visualization of DNA with ultraviolet light. Agarose gels were allowed to polymerase at RT. DNA samples were mixed with 6x DNA Loading Dye (NEB), loaded into the gel together with GeneRuler DNA Ladder Mix (Thermo Fischer Scientific) and electrophoresis conducted at $100-150 \mathrm{~V}$ until desired separation of the samples. DNA was visualized using an GEL iX20 Imager system (INTAS).

\subsection{Silver stain analysis}

Silver staining is more sensitive than Coomassie staining of protein bands after SDSPAGE. It was used if the sample amount was too little to be detected with Instant Blue stain. The gels were incubated for $1 \mathrm{~h}$ in a fixation solution (40\% ethanol, $7 \%$ acetic acid) while shaking at room temperature. The gels were washed twice for 20 min with shaking using first a $25 \%$ ethanol and second a $5 \%$ ethanol solution. Subsequently, the gels were incubated in $100 \mathrm{~mL}$ reducing solution containing $35 \mu \mathrm{M}$ DTT, followed by incubation in silver nitrate solution ( $1 \mathrm{mg} / \mathrm{mL}$ silver nitrate, $0.37 \%(\mathrm{v} / \mathrm{v})$ formaldehyde) while shaking. The gels were rinsed twice with cold MilliQ $\mathrm{H}_{2} \mathrm{O}$ and slowly developed using ice cold sodium carbonate solution $\left(30 \mathrm{mg} / \mathrm{mL} \mathrm{Na}_{2} \mathrm{CO}_{3}, 1.85 \%\right.$ (v/v) formaldehyde) until protein bands were visible. The developing reaction was stopped by the application of citric acid powder and incubation for 10 min while shaking. Gels were washed with MilliQ $\mathrm{H}_{2} \mathrm{O}$ and documented using an Epson Perfection V800 flatbed scanner (Epson).

\subsection{Western blot analysis}

Western blot and antibody staining were performed to analysis the presence of specific protein subunits during RSC complex purification and to additionally verify the successful assembly of the RSC-nucleosome complex. After standard SDS-PAGE or Native PAGE gel electrophoresis, the unstained gels were briefly washed with MilliQ $\mathrm{H}_{2} \mathrm{O}$ and proteins transferred onto a polyvinylidene difluoride (PVDF) membrane using the Trans-Blot Turbo Blotting System (Bio-Rad). Ready-to-use pre-assembled Trans-Blot Turbo Mini PVDF Transfer Packs (Bio-Rad) were used for the blotting sandwich according to the manufacturer's instructions. Blotting was done with the pre-installed high molecular 
weight settings (10 min, $25 \mathrm{~V}, 2.5 \mathrm{~A}$ ). After transfer, the membranes were blotted with $2 \%$ or $5 \%(\mathrm{w} / \mathrm{v})$ milk powder / 1x PBS-T solution in for $60 \mathrm{~min}$ or $30 \mathrm{~min}$, respectively, while shaking. Subsequently, the appropriate primary antibody was supplied in $10 \mathrm{~mL}$ $2 \%\left((\mathrm{w} / \mathrm{v})\right.$ milk powder / $1 \mathrm{x}$ PBS-T solution and incubated overnight at $4{ }^{\circ} \mathrm{C}$ with shaking. Antibody solution was removed by washing 3x with $30 \mathrm{~mL} 1 \mathrm{x}$ PBS-T and shaking for 5 - $10 \mathrm{~min}$. If the primary antibody was HRP-coupled the membranes were analysed directly. Otherwise the corresponding HRP-coupled secondary antibody was added in $10 \mathrm{~mL} 2 \%(\mathrm{w} / \mathrm{v})$ milk powder / 1x PBS-T solution and incubated for $1 \mathrm{~h}$ at room temperature while shaking. Membranes were washed again 3x with $30 \mathrm{~mL} 1 \mathrm{x}$ PBS-T and shaking for 5 - $10 \mathrm{~min}$. HRP-coupled antibodies were visualised using the Pierce Enhance Chemi-Luminescence Western Blotting Substrate kit (Thermo Fischer Scientific) and documented with the Advanced Fluorescent Imager (Intas).

\subsection{DNA amplification}

\subsubsection{Polymerase chain reaction (PCR)}

Standard PCR reactions were used for the amplification of genes and tag-constructs, and large-scale DNA amplification for nucleosome assembly. Phusion High-Fidelity DNA polymerase (NEB) was used for the DNA amplification. A typical $50 \mu \mathrm{l}$ PCR reaction contained $200 \mu \mathrm{M}$ dNTPs, $0.5 \mu \mathrm{M}$ forward primer, $0.5 \mu \mathrm{M}$ reverse primer, 50 ng template DNA, 1 unit Phusion DNA polymerase and 1x Phusion HF buffer (NEB). The PCR was performed using a Professional TRIO Thermocycler (Biometra) with a program according to Table 9. After the program was finished, reactions were supplemented with $1 \mathrm{x}$ DNA Loading Dye (6x, NEB) and analysed by agarose gel electrophoresis with appropriate molecular weight standard to confirm the production of a PCR product of the correct size. If DNA from the PCR reaction was not only analysed to ensure successful PCR reactions, the DNA was further extracted from the gels in order to send it for sequencing. The DNA bands were visualized with a BST-20G-D2R BlueLED Bio Transilluminator (Biostep) and the DNA of interest was cut out within a small surrounding gel piece. The weight of the gel piece was determined, and the DNA purified from the gel using a QIAquick Gel Extraction kit (Quigen) according to the manufacturer's instructions. 
DNA fragments for nucleosome reconstruction were prepared by PCR as previously described (Maskell et al., 2015). The large-scale DNA amplification for nucleosomal scaffold DNA was performed in a total volume of $42 \mathrm{ml}$, split into $80 \mu \mathrm{l}$ reactions each containing 300 pg template gBlock DNA (IDT) resembling the 145-bp Widom 601 sequence (Lowary and Widom, 1998) with a 55 bp overhang on the 5 '-end and a $37 \mathrm{bp}$ extension on the 3'-end. Several PCR reactions were analysed with agarose gel electrophoresis to ensure the successful production of the scaffold DNA. PCR reactions were pooled, and DNA products recovered by phenol-chloroform-extraction. The primers and final nucleosome scaffold sequence were as follows:

Forward primer: TCATTACCCAGCCCGCCTAG

Reverse primer: CCTACGGACCGGATATCTTCCCTG

Full DNA scaffold with underlined nucleosome positioning sequence:

TCATTACCCAGCCCGCCTAGTTTTAAAGGCGAAAAAAACCGACGAAAAGAGTTAAATCGATGT ATATATCTGACACGTGCCTGGAGACTAGGGAGTAATCCCCTTGGCGGTTAAAACGCGGGGGAC AGCGCGTACGTGCGTTTAAGCGGTGCTAGAGCTGTCTACGACCAATTGAGCGGCCTCGGCACCG GGATTCTGATGGAAACCCATACACAGGGAAGATATCCGGTCCGTAGG

Table 9 | PCR program used for DNA amplification.

\begin{tabular}{lll}
\hline Step & Temperature & Time \\
\hline Initial denaturation & $98{ }^{\circ} \mathrm{C}$ & $30 \mathrm{~s}$ \\
Melting & $98{ }^{\circ} \mathrm{C}$ & $10 \mathrm{~s}$ \\
Annealing & $62-70^{\circ} \mathrm{C}$ & $30 \mathrm{~s}$ \\
Elongation & $72{ }^{\circ} \mathrm{C}$ & $25 \mathrm{~s} / \mathrm{kb}$ \\
Final elongation & $72{ }^{\circ} \mathrm{C}$ & $10 \mathrm{~min}$ \\
Hold & $4{ }^{\circ} \mathrm{C}$ & - \\
\hline
\end{tabular}

\subsubsection{Phenol-chloroform extraction}

The DNA containing PCR reaction volume was supplemented with an equal volume of phenol-chloroform solution (Sigma-Aldrich) and thoroughly mixed by shaking for $15 \mathrm{~s}$. The mixture was centrifuged using a SIGMA 2-16KL centrifuge (SIGMA) with 14,000x g for $30 \mathrm{~min}$ at $4{ }^{\circ} \mathrm{C}$. After centrifugation, the watery phase was carefully isolated and supplemented with cold $3 \mathrm{M}$ sodium acetate $\mathrm{pH} 5.2$ solution ( $0.1 \mathrm{x}$ of the sample volume). Cold, pure $100 \%$ ethanol was added (2.5x of the sample volume), mixed and incubated 
overnight at $-20^{\circ} \mathrm{C}$ for precipitation. The sample was centrifuged again using a SIGMA 216KL centrifuge (SIGMA) with $14,000 \mathrm{x}$ g for $30 \mathrm{~min}$ at $4{ }^{\circ} \mathrm{C}$, supernatant ethanol was removed, and the pellet washed twice with $70 \%$ cold ethanol. The pellet was air dried, resuspended in MilliQ $\mathrm{H}_{2} \mathrm{O}$ and stored at $-20^{\circ} \mathrm{C}$.

\subsubsection{Size exclusion chromatography for nucleosomal scaffold DNA}

PCR amplified nucleosomal DNA was purified from small DNA fragments and dNTPs using size exclusion chromatography. The nucleosomal scaffold DNA was mixed with buffer stock solution to a final concentration of 20 mM Na-HEPES pH 7.5, $200 \mathrm{mM} \mathrm{NaCl}, 1$ mM EDTA applied to a Superpose 6 10/100 Increase column (GE Healthcare) preequilibrated in gel filtration buffer (20 mM HEPES pH 7.5, $200 \mathrm{mM} \mathrm{NaCl}, 1 \mathrm{mM}$ EDTA). DNA containing peak fractions were collected and merged. Sample size was reduced to $10 \%$ of its original volume using a Concentrator plus (Eppendorf) speed vacuum system. The DNA concentration was measured using a Nanodrop-2000 spectrometer (Thermo Fischer Scientific) and sample stored at $-20^{\circ} \mathrm{C}$.

\subsection{Cloning}

\subsubsection{Transformation of chemically competent $E$. coli cells}

Frozen chemically competent E. coli cells $(100 \mu \mathrm{L})$ were thawed on ice, plasmid DNA was added and carefully mixed. Cells were incubated for 20 min on ice, heat-shocked for $45 \mathrm{~s}$ at $42{ }^{\circ} \mathrm{C}$ and transferred back to ice for $2 \mathrm{~min}$. $1 \mathrm{~mL}$ plain LB medium was added and cells recovered for $1 \mathrm{~h}$ at $37^{\circ} \mathrm{C}$ in a Thermomixer (Eppendorf) with shaking. Cells were plated on LB agar plate supplemented with the appropriate antibiotics and incubated overnight at $37^{\circ} \mathrm{C}$.

\subsubsection{Transformation of chemically competent $S$. cerevisiae cells}

On overnight culture of the desired yeast strain was used to inoculate $50 \mathrm{~mL}$ YPD medium and grown to $\mathrm{OD}_{600}$ of $0.4-0.6$. The cell number was roughly calculated $\left(\mathrm{OD}_{660}=\right.$ 
$1.0 \equiv 1.85 \times 10^{7}$ cells $/ \mathrm{mL}$ ) and a volume containing $10^{8}$ cells transferred to a $50 \mathrm{~mL}$ falcon tube. Cells were checked for viability using a light microscope and pelleted for $2 \mathrm{~min}$ at room temperature with $3000 \mathrm{rpm}$ (Eppendorf 5810R). Supernatant was discarded, cells washed with $15 \mathrm{~mL}$ sterile water, centrifuged again, and transferred with $1 \mathrm{~mL}$ sterile water into a $1.5 \mathrm{~mL}$ Eppendorf tube. Cells were pelleted for $2 \mathrm{~min}$ with $4400 \mathrm{rpm}$ at room temperature (Eppendorf 5424R). The pellet was washed with $1 \mathrm{~mL}$ LiAc-TE (100 mM lithium acetate, $10 \mathrm{mM}$ Tris pH 7.5, 1 mM EDTA) and after pelleting resuspended in 100 $\mu \mathrm{L}$ LiAc-TE. Briefly boiled salmon carrier DNA $(2.5 \mu \mathrm{L}, 10 \mathrm{mg} / \mathrm{mL})$ together with up to $15 \mu \mathrm{L}$ of the DNA to be transformed (500 - $2000 \mathrm{ng}$ ) were added and mixed gently. After incubation for $10 \mathrm{~min}$ at room temperature, $260 \mu \mathrm{l}$ LiAc-TE-PEG (LiAc-TE $+40 \%(\mathrm{w} / \mathrm{v})$ PEG4000) were added, mixed gently, and incubated again for $30 \mathrm{~min}$ at room temperature. $43 \mu \mathrm{M}$ DMSO were supplemented, mixed gently, and heat-shocked for 5 min at $42{ }^{\circ} \mathrm{C}$. The cells were recovered for $3 \mathrm{~h}$ at $30{ }^{\circ} \mathrm{C}$ in plain YPD medium with shaking. After recovery, cells were pelleted for $3 \mathrm{~min}$ at $4400 \mathrm{rpm}$ (Eppendorf 5424R), supernatant discarded, and the cells resuspended in $100 \mu \mathrm{L}$ YPD medium. The transformed cells were plated on appropriate selection medium agar plates supplemented - if necessary - with the appropriate antibiotics and colonies grown for 2 4 days at $30^{\circ} \mathrm{C}$.

\subsection{Protein purification}

\subsubsection{RSC remodelling complex}

Rsc2-TAP tagged yeast cells were grown on YP agar plates supplemented with $2 \%$ glucose (w/v) from a frozen glycerol stock. A single colony was used to inoculate a $100 \mathrm{~mL}$ overnight culture in YPD medium with $50 \mu \mathrm{g} / \mathrm{mL}$ ampicillin sodium salt and $12.5 \mu \mathrm{g} / \mathrm{mL}$ tetracyclin-hydrochloride. The next pre-culture was grown from the overnight culture in $2 \mathrm{~L}$ YPD with antibiotics to an $\mathrm{OD}_{600}$ of 1.6. Cells were fermented in $250 \mathrm{~L}$ of $3 \%$ YEP broth (w/v, Formedium) supplemented with $2 \%$ glucose, $250 \mathrm{~mL}$ Antifoam C Emulsion (Sigma-Aldrich), $50 \mathrm{~g} / \mathrm{L}$ ampicillin sodium salt and $12.5 \mathrm{~g} / \mathrm{L}$ tetracyclin-hydrochloride at pH 6.6 (stirring $250 \mathrm{rpm}$, air flow $20 \mathrm{Nl} / \mathrm{h}$ ). The fermentation was started with an $\mathrm{OD}_{600}$ of 0.006 and cells were harvested at an $\mathrm{OD}_{600}$ of 10. The yeast pellet was resuspended in cold 2x lysis buffer (100 mM K-HEPES, pH 7.6, 
$20 \%$ glycerol (v/v), 1.4 M KAc, $2 \mathrm{mM} \mathrm{MgCl} 2,2 \mathrm{mM}$ DTT and 3x protease inhibitor (100x: $0.028 \mathrm{mg} / \mathrm{mL}$ leupeptin, $0.137 \mathrm{mg} / \mathrm{mL}$ pepstatin A, $17 \mathrm{mg} / \mathrm{mL}$ PMSF, $33 \mathrm{mg} / \mathrm{mL}$ benzamidine)), frozen in liquid $\mathrm{N}_{2}$ to pea-sized granules and stored at $-80{ }^{\circ} \mathrm{C}$.

RSC was purified based on the tap-tag purification strategy with modifications (Lorch and Kornberg, 2004; Rigaut et al., 1999). All purification procedures were performed at $4{ }^{\circ} \mathrm{C}$ unless stated otherwise. $600 \mathrm{~g}$ of yeast granules were lysed by cryomilling (Spex Freezer/Mill 6875D) and stored at $-80{ }^{\circ} \mathrm{C}$ until further purification. Yeast powder was slowly thawed at $30{ }^{\circ} \mathrm{C}$, diluted with $100 \mathrm{~mL} 1 \mathrm{x}$ lysis buffer and cleared by centrifugation $(25,200 \mathrm{x}$ g). The supernatant was incubated for $6 \mathrm{~h}$ with $10 \mathrm{~mL}$ IgG Sepharose 6 Fast Flow resin (GE Healthcare) pre-equilibrated in lysis buffer. The resin was recovered by centrifugation (3200x g) and washed with $100 \mathrm{~mL}$ TEV-elution buffer (50 mM K-HEPES, pH 7.6, 150 mM KAc, 10 \% glycerol (v/v), 3 mM CaCl 2,1 mM imidazole, $1 \mathrm{mM}$ DTT, 0.5x protease inhibitor). IgG resin was resuspended in $10 \mathrm{~mL}$ TEV-elution buffer, mixed with $2 \mathrm{~mL}$ calmodulin resin (Agilent Technologies) pre-equilibrated in TEVelution buffer and supplied with catalytic amounts of TEV protease for cleavage from IgG and binding to calmodulin resin overnight. The resin was washed with $100 \mathrm{~mL}$ TEVelution buffer (without protease inhibitor) and protein eluted with $50 \mathrm{~mL}$ elution buffer (50 mM K-HEPES, pH 7.6, 150 mM KAc, 10 \% glycerol (v/v), 3 mM EGTA, 1 mM imidazole, $1 \mathrm{mM}$ DTT). Elution was applied to a HiTrap Q $1 \mathrm{~mL}$ HP column (GE Healthcare) preequilibrated with Q-150 buffer (50 mM K-HEPES, pH 7.6, 150 mM KAc, $10 \%$ glycerol, $1 \mathrm{mM}$ DTT) and the column was washed with $10 \mathrm{CV}$ Q-150 buffer. Protein was eluted with a linear gradient from 0 - $100 \%$ buffer Q-1500 (50 mM K-HEPES, pH 7.6, 1.5 M KAc, $10 \%$ glycerol (v/v), 1 mM DTT) over 50 CV. RSC-containing fractions were concentrated using a Vivaspin 6 MWCO 30,000 device (Sartorius), dialysed overnight to dialysis buffer A (50 mM K-HEPES, pH 7.6, 150 mM KAc, $10 \%$ glycerol, 5 mM MgCl 2,1 mM DTT) and immediately used for cryo-EM sample preparation. Typical yields were $0.2-0.3 \mathrm{mg}$ from $300 \mathrm{~g}$ of yeast pellet.

For activity assays the protein was additionally purified after concentration by application to a $10-30 \%(\mathrm{w} / \mathrm{v})$ sucrose gradient for $16 \mathrm{~h}$ at 32,000 rpm in a SW $60 \mathrm{Ti}$ swinging-bucket rotor (Beckmann) at $4{ }^{\circ} \mathrm{C}$. The gradient was generated with a BioComp Gradient Master 108 (BioComp Instruments) from a $10 \%$ sucrose light solution (10 \% sucrose (w/v), 50 mM K-HEPES, pH 7.6, 150 mM KAc, 5 \% glycerol, 1 mM DTT) and a $30 \%$ sucrose heavy solution (30 \% sucrose (w/v), 50 mM K-HEPES, pH 7.6, 150 mM KAc, 
$5 \%$ glycerol, $1 \mathrm{mM}$ DTT). The gradient was fractionated manually into $200 \mu \mathrm{L}$ fractions and RSC-containing fractions were dialysed to dialysis buffer B (50 mM K-HEPES, pH 7.6, 150 mM KAc, 5 \% glycerol, 1 mM DTT) using Slide-A-Lyzer 10K MWCO 0.5 mL cassettes (Thermo Fisher Scientific) before freezing in liquid $\mathrm{N}_{2}$ and storage at $-80{ }^{\circ} \mathrm{C}$.

\subsubsection{Histone proteins}

Xenopus leavis histones were expressed and purified as described previously (Dyer et al., 2003; Luger et al., 1999). Briefly, histones H2A, H2B, H3 and H4 were expressed as inclusion bodies from pET3a vectors introduced into E. coli BL21 CodonPlus (DE3) RIL cells. Bacteria were grown in LB medium (ingredients from Becton Dickinson) supplemented with $50 \mu \mathrm{g} / \mathrm{mL}$ ampicillin sodium salt and $17 \mu \mathrm{g} / \mathrm{mL}$ chloramphenicol to an $\mathrm{OD}_{600}$ of 0.4 , induced with $0.5 \mathrm{mM}$ IPTG, harvested after $3 \mathrm{~h}$ by centrifugation and pellets frozen in liquid $\mathrm{N}_{2}$ for storage at $-80^{\circ} \mathrm{C}$. All purification steps were performed at $4{ }^{\circ} \mathrm{C}$ unless stated otherwise. Cells from $6 \mathrm{~L}$ were thawed in wash buffer ( $50 \mathrm{mM}$ Tris- $\mathrm{HCl}$, 7.5, $200 \mathrm{mM} \mathrm{NaCl}, 1 \mathrm{mM} \beta$-mercaptoethanol, 1x protease inhibitor), lysed by sonication, inclusion bodies separated by centrifugation (15,000 rpm, $20 \mathrm{~min}$ ) and washed four times with $70 \mathrm{~mL}$ wash buffer using a manual Dounce tissue grinder (Sigma-Aldrich) and centrifugation. Inclusion bodies were resuspended in $50 \mathrm{~mL}$ unfolding buffer-A $(50 \mathrm{mM}$ Tris-HCl, pH7.5, $200 \mathrm{mM} \mathrm{NaCl}, 6 \mathrm{M}$ guanidinium hydrochloride, $1 \mathrm{mM} \beta$-mercaptothanol) supplemented with $2 \mathrm{~mL}$ DMSO for $5 \mathrm{~h}$ at room temperature. After removal of contamination by centrifugation $(25,000 \mathrm{rpm}, 20 \mathrm{~min})$ the supernatant was dialysed against $6 \mathrm{~L}$ of dialysis buffer $(50 \mathrm{mM}$ Tris- $\mathrm{HCl}, \mathrm{pH}$ 7.5, $200 \mathrm{mM} \mathrm{NaCl}, 6 \mathrm{M}$ urea (treated with ion exchanger Amberlite MB-3, Merck), $2.5 \mathrm{mM} \beta$-mercaptoethanol) in three steps using Spectra/Por snake skin MWCO 3.5K (Thermo Fischer Scientific).

Histones were further purified using ion exchange chromatography by loading them on a HiTrap SP $5 \mathrm{~mL}$ HP column through a HiTrap Q $5 \mathrm{~mL}$ HP column, both preequilibrated in buffer A-200 (50 mM Tris- $\mathrm{HCl}, \mathrm{pH}$ 7.5, $200 \mathrm{mM} \mathrm{NaCl}, 8 \mathrm{M}$ urea (treated with ion exchanger Amberlite MB-3), $1 \mathrm{mM} \beta$-mercaptoethanol). The histones bound to the HiTrap SP column were washed with $6 \mathrm{CV}$ buffer A-200 and eluted with a linear gradient of buffer B-600 (50 mM Tris- $\mathrm{HCl}, \mathrm{pH}$ 7.5, $600 \mathrm{mM} \mathrm{NaCl}, 8 \mathrm{M}$ urea (treated with ion exchanger Amberlite MB-3), $1 \mathrm{mM} \beta$-mercaptoethanol) over $20 \mathrm{CV}$ before dialysis against two times $4.5 \mathrm{~L}$ of MilliQ $\mathrm{H}_{2} \mathrm{O}$ (Merck) supplemented with $5 \mathrm{mM} \beta$ - 
mercaptoethanol followed by lyophilisation (Alpha 1-2 LDplus, Christ) and storage at $-80^{\circ} \mathrm{C}$.

\subsubsection{Protein and DNA quantification}

The purified protein and DNA samples were measured using a NanoDrop-200 spectrophotometer (Thermo Fischer Scientific) at a wavelength of $260 \mathrm{~nm}$ and $280 \mathrm{~nm}$. The absorption at $260 \mathrm{~nm}$ was used for the quantification of DNA and the absorption at $280 \mathrm{~nm}$ was used for the quantification of the protein samples. The contamination of the RSC purification with nucleic acids was shown by the ratio of the two wavelengths. After the HiTrap Q ion exchange step the RSC sample was considered free of DNA or RNA. The extinction coefficients for the individual proteins and the multi-subunit complex were calculated using the Expasy ProtParam software (Artimo et al., 2012).

\subsection{Assembly of nucleosomal substrate}

\subsubsection{Histone octamer reconstitution}

For octamer preparation lyophilised histones were resuspended for $30 \mathrm{~min}$ at $4{ }^{\circ} \mathrm{C}$ in unfolding buffer-B (20 mM Na-HEPES, pH 7.5, 7 M guanidinium hydrochloride, $10 \mathrm{mM}$ DTT) to a concentration of $3 \mathrm{mg} / \mathrm{mL}$. H2A, H2B, H3 and H4 were combined at a molar ratio of 1.2:1.2:1:1 and dialysed against $2 \mathrm{~L}$ of refolding buffer (10 mM Na-HEPES, pH 7.5, $2 \mathrm{M} \mathrm{NaCl}, 1 \mathrm{mM}$ EDTA, $2.5 \mathrm{mM}$ DTT) twice, for a total of $12 \mathrm{~h}$ at $4{ }^{\circ} \mathrm{C}$. Sample was concentrated using Vivaspin 500 MWCO 10,000 device (Sartorius) and applied to a Superdex 200 Increase 10/300 size exclusion column pre-equilibrated with refolding buffer. Peak fractions were pooled and frozen in liquid $\mathrm{N}_{2}$ at a concentration of $1.34 \mathrm{mg} / \mathrm{mL}$. 


\subsubsection{Nucleosome reconstitution}

Nucleosome reconstitution was performed as described (Dyer et al., 2003), with minor modifications. DNA and histone octamer were mixed at a 1:0.9 - 1:1.3 molar ration in high salt buffer (20 mM Na-HEPES, pH 7.5, $2 \mathrm{M} \mathrm{NaCl}, 1 \mathrm{mM}$ EDTA, $2 \mathrm{mM}$ DTT) and incubated for $30 \mathrm{~min}$ on ice. Sample was transferred to a Slide-A-Lyzer 3.5K MWCO MINI device in a beaker with $500 \mathrm{~mL}$ high salt buffer and gradient dialysed against $2 \mathrm{~L}$ of low salt buffer (20 mM Na-HEPES, pH 7.5, 20 mM NaCl, 1 mM EDTA, 2 mM DTT) over 22 h. After a heat shift for $30 \mathrm{~min}$ at $50{ }^{\circ} \mathrm{C}$, the sample was recovered and analysed for successful nucleosome assembly using EMSA assays. Nucleosomes from the reaction with the best reconstitution (usually the 1:1.2 ratio) were then immediately used for complex formation with freshly prepared, unfrozen RSC protein. The concentration of the DNA in the sample determined using a Nanodrop-2000 spectrometer (Thermo Fischer Scientific) and used for further calculations.

\subsection{RSC and nucleosome complex formation}

RSC complex was mixed with $\mathrm{ADP}-\mathrm{BeF}_{3}$ at a final concentration of $1 \mathrm{mM}$ and incubated on ice for $30 \mathrm{~min}$. A 1.6-fold molecular excess of nucleosome substrate was added, the mixture incubated for $15 \mathrm{~min}$ at $30{ }^{\circ} \mathrm{C}$ and transferred back on ice. RSC-nucleosome complex was cross-linked using the GraFix method (Kastner et al., 2008). The sample was applied to a gradient generated from a $10 \%$ sucrose light solution $(10 \%$ sucrose $(\mathrm{w} / \mathrm{v})$, 50 mM K-HEPES, 7.6, 150 mM KAc, 5 \% glycerol (v/v), 2 mM MgCl, 1 mM DTT, 0.5 mM $\mathrm{ADP}_{-} \mathrm{BeF}_{3}$ ) and a $25 \%$ sucrose heavy solution (25\% sucrose (w/v), $50 \mathrm{mM} \mathrm{K-HEPES,} \mathrm{7.6,}$ $150 \mathrm{mM} \mathrm{KAc}, 5 \%$ glycerol (v/v), $2 \mathrm{mM} \mathrm{MgCl}_{2}, 1 \mathrm{mM}$ DTT, $0.5 \mathrm{mM} \mathrm{ADP}-\mathrm{BeF}_{3}$ ) containing $0.2 \%$ glutaraldehyde cross-linker with a BioComp Gradient Master 108 (BioComp Instruments). Centrifugation was executed for $16 \mathrm{~h}$ at $32,000 \mathrm{rpm}$ in a SW $60 \mathrm{Ti}$ swinging-bucket rotor (Beckmann) at $4{ }^{\circ} \mathrm{C}$. $200 \mu \mathrm{L}$ fractions were collected manually and quenched with aspartate (pH 7.5) at a final concentration of $50 \mathrm{mM}$. Fractions containing RSC-nucleosome complex were dialysed for $8 \mathrm{~h}$ at $4{ }^{\circ} \mathrm{C}$ to freezing buffer $(20 \mathrm{mM} \mathrm{K}$ HEPES, pH 7.6, 150 mM KAc, 1 \% glycerol (v/v), 3 mM MgCl, 1 mM DTT) in Slide-A-Lyzer 20K MWCO $2 \mathrm{~mL}$ devices (Thermo Fischer Scientific) to remove sucrose and applied to cryo-EM grids. 


\subsection{RSC activity assay}

\subsubsection{ATPase assay}

ATP hydrolysis by RSC was assessed as previously described (Forne et al., 2012). Briefly, the ATPase assay was conducted in a reaction buffer of final composition $25 \mathrm{mM}$ Tris- $\mathrm{HCl}$ pH 8.0, 100 mM NaCl, 1.5 mM MgOAc, 0.1 mM Na-EDTA pH 8.0, $10 \%$ glycerol (v/v), $0.2 \mathrm{mg} / \mathrm{mL}$ BSA, $1 \mathrm{mM}$ DTT, $2 \mathrm{mM} \mathrm{Mg}{ }^{2+}$ ATP, $500 \mathrm{ng}$ circular plasmid DNA (pJet1.2), $15.5 \mathrm{U} / \mathrm{mL}$ pyruvate kinase, $3 \mathrm{mM}$ phosphoenopyrovate, $15.5 \mathrm{U} / \mathrm{mL}$ lactate dehydrogenase, 0.6 mM NADH and 27 nM RSC in duplicates. A Biotek PowerWave HT 384 well plate reader was used to measure $\mathrm{NADH}$ absorption at $340 \mathrm{~nm}$ over $30 \mathrm{~min}$ in flatbottom, uncoated 384 well plates (Greiner).

\subsubsection{Genome-wide reconstitution}

Genome-wide reconstitution of RSC activity was performed as previously described with minor modifications (Krietenstein et al., 2016). Salt-gradient dialysed (SGD) chromatin was formed by first mixing 10 ug yeast plasmid library (Jones et al., 2008) at the quantity of Drosophila embryo histone octamers that yielded an assembly degree comparable to previous work (Krietenstein et al., 2016), as assessed by MNase digestion and MNase ladder extent. Reconstitution reactions were performed in $100 \mathrm{uL}$, with final buffer conditions of $1 \mathrm{mM}$ Tris-HCl pH 7.6, 2 mM K-HEPES pH 7.5, 19.6 mM Na-HEPES pH 7.5, $13 \%$ glycerol, $2.7 \mathrm{mM}$ DTT, $3 \mathrm{mM} \mathrm{MgCl}_{2}$, $0.6 \mathrm{mM}$ EGTA, $0.1 \mathrm{mM}$ EDTA, $85.5 \mathrm{mM} \mathrm{NaCl}$, $8 \mathrm{mM} \mathrm{KCl}, 0.005$ \% Tween, $10 \mathrm{mM}\left(\mathrm{NH}_{4}\right)_{2} \mathrm{SO}_{4}, 2.5 \mathrm{mM}$ ATP, $10 \mathrm{mM}$ creatine phosphate, $20 \mathrm{ng} / \mu \mathrm{L}$ creatine kinase, $\sim 80 \mathrm{ng} / \mathrm{uL}$ SGD chromatin and if used, $10 \mathrm{nM}$ RSC. Reactions were incubated at $30{ }^{\circ} \mathrm{C}$ for $2 \mathrm{~h}$, before treatment with $200 \mathrm{mU}$ apyrase for $15 \mathrm{~min}$ to halt remodelling. MNase digestion was performed by supplementing $\mathrm{CaCl}_{2}$ to $1.5 \mathrm{mM}$ final concentration, then adding 100 units MNase and incubating at $30{ }^{\circ} \mathrm{C}$ for exactly 5 min. MNase digestion was halted by addition of EDTA (10 mM final) and SDS (0.2\% final). After proteinase $\mathrm{K}$ digestion (100 ug, $45 \mathrm{~min}$ at $37^{\circ} \mathrm{C}$ ), the resulting DNA was purified by first supplementing glycogen (40 ug), then performing classical ethanol precipitation. Purified DNA was separated on an agarose gel (1.5\%, 1x TAE, $100 \mathrm{~V}, 70 \mathrm{~min})$ and the mono-nucleosomal band was excised and purified by gel extraction. Libraries were 
prepared for Illumina sequencing using standard NEBNext manufacturer's protocols and sequenced on a HiSeq 1500, single-end mode and 50 bp reads.

\subsection{Mass-spectrometric identification of cross-linking sites}

\subsubsection{Sample preparation}

RSC-nucleosome complex was prepared as described above. The cross-linking reaction was performed with BS3 (bis(sulfosuccinimidyl(suberate))) cross-linker (Thermo Fischer Scientific) at a final concentration of $1 \mathrm{mM}$ on ice for $30 \mathrm{~min}$ before quenching with Tris- $\mathrm{HCl} \mathrm{pH} \mathrm{7.5,} \mathrm{and} \mathrm{ammonium} \mathrm{bicarbonate} \mathrm{at} \mathrm{a} \mathrm{final} \mathrm{concentration} \mathrm{of} 100 \mathrm{mM}$ and $20 \mathrm{mM}$, respectively. The cross-linked sample was applied to a $10 \%-25 \%$ sucrose gradient as described above (no glutaraldehyde in the heavy solution) and protein containing fractions were pooled $(\sim 800 \mu \mathrm{L}, \sim 50 \mu \mathrm{g}$ complex $)$ and applied to in-solution digest. $150 \mu \mathrm{L}$ of urea buffer (8 $\mathrm{M}$ urea, $50 \mathrm{mM} \mathrm{NH}_{4} \mathrm{HCO}_{3} \mathrm{pH}$ 8) and $60 \mu \mathrm{L} 0.1 \mathrm{M}$ DTT (in $50 \mathrm{mM} \mathrm{NH}_{4} \mathrm{HCO}_{3} \mathrm{pH}$ 8) were added to reduce the sample for $30 \mathrm{~min}$ at $37{ }^{\circ} \mathrm{C}, 300 \mathrm{rpm}$. The sample was alkylated with $60 \mu \mathrm{L} 0.4 \mathrm{M}$ iodoacetamide (in $50 \mathrm{mM} \mathrm{NH}_{4} \mathrm{HCO}_{3} \mathrm{pH}$ 8) for $30 \mathrm{~min}$ at $37^{\circ} \mathrm{C}, 300 \mathrm{rpm}$, in the dark. The reaction was quenched by addition of $60 \mu \mathrm{L}$ $0.1 \mathrm{M}$ DTT (in $50 \mathrm{mM} \mathrm{NH}_{4} \mathrm{HCO}_{3} \mathrm{pH}$ 8). The sample was digested for $30 \mathrm{~min}$ at $37{ }^{\circ} \mathrm{C}$ with $0.5 \mu \mathrm{L}$ Pierce Universal Nuclease $(250 \mathrm{U} / \mu \mathrm{L})$ in presence of $1 \mathrm{mM} \mathrm{MgCl} 2$. The final sample volume was adjusted to $1200 \mu \mathrm{L}$ with $50 \mathrm{mM} \mathrm{NH} \mathrm{NHCO}_{3} \mathrm{pH} 8$ resulting in a final urea concentration of $1 \mathrm{M}$. Trypsin digest was performed overnight at $37{ }^{\circ} \mathrm{C}$ with $2.5 \mu \mathrm{g}$ trypsin (Promega, V5111). Tryptic peptides were desalted with C18 spin columns (Harvard Apparatus 74-4601), lyophilized and dissolved in $30 \%$ (v/v) acetonitrile, $0.1 \%$ $(\mathrm{v} / \mathrm{v})$ trifluoroacetic acid. The peptide mixture was separated on a Superdex Peptide 3.2/300 (GE Healthcare) column run at $50 \mu \mathrm{L} / \mathrm{min}$ with $30 \%(\mathrm{v} / \mathrm{v}$ ) acetonitrile, $0.1 \%$ (v/v) trifluoroacetic acid. Cross-linked species are enriched by size exclusion chromatography based on their higher molecular weight compared to linear peptides. $50 \mu \mathrm{L}$ fractions were collected from $1.0 \mathrm{~mL}$ post-injection. Fractions from $1.0-1.6 \mathrm{~mL}$ post-injection were dried in a speed-vac and dissolved in $5 \%(\mathrm{v} / \mathrm{v})$ acetonitrile, $0.05 \%$ (v/v) trifluoroacetic acid and subjected to LC-MS/MS. 


\subsubsection{LC-MS/MS analysis and cross-link identification}

LC-MS/MS analyses were performed on a Q Exactive HF-X hybrid quadrupole-orbitrap mass spectrometer (Thermo Scientific) coupled to a Dionex Ultimate 3000 RSLCnano system. The peptide mixtures from in-solution digest were loaded on a Pepmap 300 C18 column (Thermo Fisher) at a flow rate of $10 \mu \mathrm{L} / \mathrm{min}$ in buffer A (0.1\% (v/v) formic acid) and washed for 3 min with buffer A. The sample was separated on an in-house packed C18 column (30 cm; ReproSil-Pur $120 \AA$, $1.9 \mu \mathrm{m}, \mathrm{C} 18-\mathrm{AQ}$; inner diameter, $75 \mu \mathrm{m}$ ) at a flow rate of $300 \mathrm{nl} / \mathrm{min}$. Sample separation was performed over $120 \mathrm{~min}$ using a buffer system consisting of $0.1 \%(\mathrm{v} / \mathrm{v}$ ) formic acid (buffer $\mathrm{A}$ ) and $80 \%(\mathrm{v} / \mathrm{v}$ ) acetonitrile, $0.08 \%(\mathrm{v} / \mathrm{v}$ ) formic acid (buffer B). The main column was equilibrated with $5 \%$ buffer B, followed by sample application and a wash with $5 \%$ buffer B. Peptides were eluted by a linear gradient from 15 - 48 \% buffer B. The gradient was followed by a wash step at $95 \%$ buffer B and re-equilibration at $5 \%$ buffer B. Eluting peptides were analysed in positive mode using a data-dependent top 30-acquisition methods. MS1 and MS2 resolution were set to 120,000 and 30,000 FWHM, respectively. Precursors selected for MS2 were fragmented using $30 \%$ normalized, higher-energy collision-induced dissociation (HCD) fragmentation. Allowed charge states of selected precursors were +3 to +7 . Further MS/MS parameters were set as follows: isolation width, $1.4 \mathrm{~m} / \mathrm{z}$; dynamic exclusion, 10 s; max. injection time (MS1/MS2), 60 ms / 200 ms. The lock mass option $(\mathrm{m} / \mathrm{z}$ 445.12002) was used for internal calibration. All measurements were performed in duplicates.

The .raw files of all replicates were searched by the software pLink 2, version 2.3.1 (Yang et al., 2012) against a customized protein database containing the expressed proteins and protein-protein cross-links were filtered with 1 \% FDR (Table S12). Crosslinks appearing less than three times were excluded during evaluation to increase confidence and plotted using xVis (Grimm et al., 2015) and xiNET (Combe et al., 2015). 


\subsection{Negative stain electron microscopy}

\subsubsection{Complex preparation}

Freshly purified RSC complex was applied to gradient centrifugation either without cross-linker or cross-linked using the GraFix method (Kastner et al., 2008). The sample was applied to a gradient generated from a $10 \%$ sucrose light solution $10 \%$ sucrose (w/v), 50 mM K-HEPES, 7.6, 150 mM KAc, $10 \%$ glycerol (v/v), 1 mM DTT) and a $30 \%$ sucrose heavy solution (30 \% sucrose (w/v), 50 mM K-HEPES, 7.6, 150 mM KAc, $10 \%$ glycerol (v/v), $1 \mathrm{mM}$ DTT) either with or without $0.1 \%$ glutaraldehyde cross-linker with a BioComp Gradient Master 108 (BioComp Instruments). Centrifugation was executed for $16 \mathrm{~h}$ at 32,000 rpm in a SW $60 \mathrm{Ti}$ swinging-bucket rotor (Beckmann) at $4{ }^{\circ} \mathrm{C} .200 \mu \mathrm{L}$ fractions were collected manually and cross-linker containing fractions quenched with lysine (pH 7.5) at a final concentration of $50 \mathrm{mM}$. Fractions containing RSC complex were dialysed for overnight at $4{ }^{\circ} \mathrm{C}$ to dialysis buffer (50 mM K-HEPES, pH 8, $150 \mathrm{mM} \mathrm{KAc,} 5 \%$ glycerol (v/v), 1 mM DTT) in Slide-A-Lyzer 20K MWCO 2 mL devices (Thermo Fischer Scientific) to remove sucrose.

\subsubsection{Negative staining and electron microscopy}

Negative stain solution was prepared by dissolving uranyl formate in double distilled $\mathrm{H}_{2} \mathrm{O}$ at a concentration of $2 \%(\mathrm{w} / \mathrm{v})$. The solution was thoroughly vortexed for $15-20 \mathrm{~min}$ and undissolved uranyl formate precipitation removed by centrifugation for $30 \mathrm{~min}$ at full speed at $4{ }^{\circ} \mathrm{C}$. The staining solution was protected from light and kept on ice. Carbon coated copper mesh grids (S160-4, Plano) were prepared by glow discharging with a PELCO easiGlow System (Ted Pello) for $30 \mathrm{~s}$ with $25 \mathrm{~mA}$ at 0.39 mbar. $4 \mu \mathrm{l}$ sample was applied to the grids and incubated for various times in order to allow the adsorption of the particles to the carbon foil. The liquid sample was blotted with filter paper and the grids washed twice by floating on top of two $120 \mu \mathrm{l}$ drops of double distilled $\mathrm{H}_{2} \mathrm{O}$ for $10 \mathrm{~s}$ each. Subsequently, $40 \mu \mathrm{l}$ of uranyl formate solution was applied to the grids and incubated for 60 - 90 s. Staining solution was blotted with filter paper and the grids allowed to air dry at room temperature. The grids were stored at room temperature and protected from light. 
Negative stain grids were imaged at room temperature on a CM200 FEG electron microscope (Phillips) operated at $160 \mathrm{~V}$, equipped with a $4 \mathrm{k} \times 4 \mathrm{k}$ CCD camera (TVIPS) with two-fold binning of pixels. The images were acquired with the Spot scan software at a nominal magnification of $88,000 \mathrm{x}$, resulting in a calibrated pixel size of $2.51 \AA /$ pix. Particles were picked using the EMAN2 software package in a semi-automated way. Subsequently, the particles were processed using the software CryoSPARC (Structure Biotechnology Inc.) in order to get an $a b$ initio 3D reconstruction. Using this 3D reconstruction as an initial reference, the particles were again processed, including CTF correction, 2D and 3D classifications as well as 3D refinements, using the software package RELION-2 (Kimanius et al., 2016; Scheres, 2012).

\subsection{Cryo-electron microscopy}

\subsubsection{Cryo-electron microscopy analysis of RSC-nucleosome complex}

The RSC-nucleosome complex was prepared as described in section 2.13. RSCnucleosome complex was absorbed to a thin carbon film before plunge freezing as described before (Stark, 2010) with minor modifications. Carbon was evaporated onto freshly cleaved mica using a Leica EM ACE600 high vacuum sputter coater (Leica) to create a thin $\sim 3.1 \mathrm{~nm}$ carbon layer. A small piece was cut from the mica sheet and the carbon floated onto a $50 \mu \mathrm{L}$ drop of sample such that the side of the carbon film previously attached to the mica was facing the sample surface. After incubation of 2 3 min the carbon film was recovered with copper R2/1 or R3.5/1 grids (Quantifoil) and vitrified by plunge-freezing in liquid ethane using a Vitrobot Mark IV (FEI) operated at $4{ }^{\circ} \mathrm{C}$ and $100 \%$ humidity.

Electron micrographs were acquired on an FEI Titan Krios G2 transmission election microscope operated at $300 \mathrm{keV}$ in EFTEM mode, equipped with a Quantum LS 967 energy filter (Gatan), zero loss mode, $30 \mathrm{eV}$ slit width, and a K2 Summit direct electron detector (Gatan) in counting mode. Automated data acquisition was done using the FEI EPU software package at a nominal magnification of $130,000 x$, resulting in a calibrated pixel size of $1.05 \AA$ /pix. Micrographs for the two datasets were collected at a dose rate of $4.78 \mathrm{e}^{-} / \AA^{2} / \mathrm{s}$ over $10 \mathrm{~s}$ resulting in a total dose of $47.8 \mathrm{e}^{-} / \AA^{2}$ and at a dose 
rate of $5.67 \mathrm{e}^{-} / \AA^{2} / \mathrm{s}$ over $8 \mathrm{~s}$ resulting in a total dose of $45.4 \mathrm{e}^{-} / \AA^{2}$, respectively. Both datasets were dose fractionated over 40 frames.

Dose weighting, CTF estimation and motion correction were carried out during data collection using Warp (Tegunov and Cramer, 2018). Automated particle picking by Warp resulted in 112,657 particles from the first dataset (4404 micrographs) and $1,119,875$ particles from the second dataset $(19,415$ micrographs). Particle coordinates were exported, combined, extracted and processed using RELION 3.0 (Zivanov et al., 2018). Removal of bad particles through global 3D classifications with a negative stain reconstruction of the RSC complex as reference resulted in high-quality particles that could be refined to an overall map of the RSC remodeller together with the nucleosome (Map 1) at a resolution of $\sim 15 \AA$. Further processing of the particles revealed great flexibility and dynamics which could not be resolved by focused 3D classifications and refinements.

The particles corresponding to the RSC-nucleosome map were re-extracted centred on the nucleosome with a box mainly including the nucleosome and the ATPase module. Global 3D classification resulted in a good class that revealed the Sth1 subunit bound to the nucleosome. Focused 3D refinement excluding the Sth1 density and postprocessing with automatic B-factor determination in RELION provided a nucleosome map (Map 2) (Figure 9) at a resolution of $3.6 \AA$ (gold-standard Fourier shell correlation 0.143 criterion) and a B-factor of $-155 \AA^{2}$. Improvement of the Sth1 density turned out to be very difficult and showed its highly dynamic nature in this sample. A strategy of focused 3D classification without image alignment on the Sth1 part, followed by a global 3D refinement and additional focused 3D classification on the combined Sth1nucleosome density led to the best results. A focused 3D classification and postprocessing with automatic B-factor determination in RELION resulted in an overall resolution of the Sth1-nuclesome map of $4.3 \AA$ (FSC 0.143 criterion) and B-factor of $-186 \AA^{2}$ (map 3) (Figure 9). Final focused maps were combined using the Frankenmap tool distributed with Warp (Figure 9). Masks encompassing the regions of interest were created with UCSF Chimera (Pettersen et al., 2004) and RELION. 


\subsubsection{Cryo-electron microscopy analysis of the free RSC complex}

Freshly purified RSC complex was mixed with $\mathrm{ADP}^{-B \mathrm{~F}_{3}}$ to a final concentration of $1 \mathrm{mM}$ and incubated for $15 \mathrm{~min}$ on ice. BS3 (bis(sulfosuccinimidyl(suberate))) cross-linker (Thermo Fischer Scientific) was added to a final concentration of $1 \mathrm{mM}$, incubated on ice for $30 \mathrm{~min}$ before quenching with Tris- $\mathrm{HCl} \mathrm{pH} \mathrm{7.5,} \mathrm{and} \mathrm{ammonium} \mathrm{bicarbonate} \mathrm{at} \mathrm{a} \mathrm{final}$ concentration of $100 \mathrm{mM}$ and $20 \mathrm{mM}$, respectively. After size exclusion chromatography using a Sepharose 6 Increase 3.2/300 column (GE Healthcare) pre-equilibrated in gel filtration buffer (50 mM K-HEPES pH 7.6, $150 \mathrm{mM} \mathrm{KAc,} 4 \mathrm{mM} \mathrm{MgCl}$, $1 \mathrm{mM}$ DTT), peak fractions were immediately applied to cryo-EM grids. $4 \mu \mathrm{L}$ of sample were applied to glow discharged (Pelco easiGlow) R2/2 gold grids (Quantifoil). Grids were blotted and vitrified as described above.

Cryo-EM data was collected as described above, with small modifications. The energy filter slit width was set to $20 \mathrm{eV}$. Micrographs for the two $0^{\circ}$ tilt datasets were collected at a dose rate of $4.88 \mathrm{e}^{-} / \AA \AA \mathrm{s}$ for $8 \mathrm{~s}$ resulting in a total dose of $39 \mathrm{e}^{-} / \AA^{2}$ and at a dose rate of $5.02 \mathrm{e}^{-} / \AA^{2} / \mathrm{s}$ over $9 \mathrm{~s}$ resulting in a total dose of $45.2 \mathrm{e}^{-} / \AA^{2}$, respectively, and fractionated over 40 frames. The third, $25^{\circ}$ tilted dataset was acquired in 44 frames at a dose rate of $4.99 \mathrm{e}^{-} / \AA^{2} / \mathrm{s}$ for $11 \mathrm{~s}$ resulting in a total dose of $54.9 \mathrm{e}^{-} / \AA^{2}$.

Pre-processing and particle picking were carried out as described above and resulted in 363,824 particles from the first dataset (3284 micrographs), 170,028 particles from the second dataset (1216 micrographs) and 475,168 particles from the tilted dataset (3158 micrographs). Particles were processed with global 3D classifications using RELION-3 (Zivanov et al., 2018) and a negative stain reconstruction of the RSC complex as a first reference to obtain an improved initial reference. All 1,009,020 particles were newly extracted, and bad particles were sorted out in multiple rounds of global 3D classifications in combination with global 3D refinements. The best resulting class was refined with a mask excluding the flexible DNA-interaction module (DIM). Particles corresponding to this reconstruction were subjected to CTF refinement and Bayesian polishing in RELION. Using focused 3D refinements, the maps for the arm module, and body 2 and body 1 submodules were further improved. Postprocessing with automatic Bfactor determination in RELION resulted in overall resolutions of $3.8 \AA$, $3.6 \AA$ and $3.6 \AA$, respectively, and B-factors of $-136 \AA^{2},-100 \AA^{2}$ and $-103 \AA^{2}$, respectively (Figure 10). Final focused maps were combined with Warp (Figure 10). 


\subsection{Structural modelling}

The lower resolution cryo-EM map 1 of the RSC-nucleosome complex was used to align the individually generated cryo-EM maps $2-8$. The combined cryo-EM map 7 was used for model building of the Sth1 subunit bound to the nucleosome. The final map was created with the local resolution tool from RELION and a B-factor of $-150 \AA^{2}$. The structure of the yeast Snf2 bound to the nucleosome in the ADP-BeF 3 state (PDB code 5Z3U) (Li et al., 2019) was used as basis for modelling. Published data together with the close homology between Sth1 and Snf2 (Figure 25) suggest that Sth1 also binds at SHL +2 . The remodeller and the nucleosome part were fitted separately. The Xenopus laevis histones and Widom 601 sequence of PDB 5Z3U were the same as used in our study. The nucleosome structure was rigid-body fitted into our cryo-EM map in UCSF Chimera (Pettersen et al., 2004) and the entry side DNA and histone tails trimmed according to the density in COOT (Emsley et al., 2010). Due to lower resolution, amino acid side chains of residues 15 - 22 of $\mathrm{H} 4$ (chain B) were stubbed in COOT. The nucleosome structure was flexibly fitted using Namdinator (Kidmose et al., 2019) and real space refined in PHENIX (Adams et al., 2010) with secondary structure restraints (including base paring and base stacking restraints).

High conservation of amino acids between Sth1 and Snf2 (Figure 25) allowed for generation of a Sth1 homology model with Rosetta (Raman et al., 2009; Song et al., 2013). The homology model was trimmed according to the density in COOT, Brace-II helix was removed, and amino acid side chains were stubbed owing to the lower resolution of the map area before rigid-body docking using UCSF Chimera. Additional real space refinement with secondary structure restraints (including base paring and base stacking restraints) was performed in PHENIX. The overhanging exit side DNA was modelled by generating a bend B-DNA following the density in map 1 in 3D-DART (van Dijk and Bonvin, 2009). The DNA duplex was connected to the nucleosomal Widom 601 DNA and geometry optimized with base pairing and base stacking restraints in PHENIX.

Map 1 allowed for the rigid-body docking of the crystal structure of the ARP module bound to the Snf2 HSA region (PDB code 4I6M) (Schubert et al., 2013) using UCSF Chimera. The amino acid residues of the Snf2 HSA helix were mutated to the ones from Sth1 according to sequence alignment (Figure 25) starting at the C-terminus and ignoring gaps. The model for the Sth1 HSA helix is thus an extrapolation based on the strong $\alpha$ - 
helical secondary structure prediction and the register might differ slightly (Schubert et al., 2013).

The combined cryo-EM map 8 and the focused refined maps $4-6$ were used for model building. SWISS-MODEL (Bienert et al., 2017; Waterhouse et al., 2018) was used to generate homology models for several domains of RSC subunits. Homology models were generated for the Rsc58 N-terminal bromodomain, the Rsc6 SWIB domain, the Rsc8 SWIRM, SANT and ZZ zinc finger domains, the Rsc9 armadillo-like domain and the Sfh1 RPT1 and RPT2 domains. The models were derived from the Homo sapiens crystal structure of a bromodomain of Polybromo (PDB code 3LJW) (Charlop-Powers et al., 2010), the Mus musculus BAF60a SWIB domain NMR structure (PDB code 1UHR), the Saccharomyces cerevisiae Swi3 SWIRM domain crystal structure (PDB code 2FQ3) (Da et al., 2006), the NMR structure of the Homo sapiens SANT domain of BAF155 (PDB code 2YUS), the Mus musculus CBP ZZ domain NMR structure (PDB code 1TOT) (Legge et al., 2004), the crystal structure of the de novo created armadillo repeat protein (PDB code 4V3Q) (Reichen et al., 2016) and the Homo sapiens RPT1 domain NMR structure of BAF47 (PDB code 6AX5), respectively. The homology models were rigid-body placed using UCSF Chimera (Pettersen et al., 2004) and manually adjusted and re-build in COOT (Emsley et al., 2010).

The quality of the maps allowed for de novo building of the other model parts (Table S11). Modelling was guided and validated by BS3 cross-linking data visualized with xVis (Grimm et al., 2015) and secondary structure predictions performed with Quick2D (Zimmermann et al., 2018) and PSIPRED (Buchan and Jones, 2019; Jones, 1999). Amino acid residues connecting the domains of the two Rsc8 subunits could not be modelled. For clarification, they were placed into a single chain (chain L) clustered by proximity. The Sfh1 C-terminal finger helix was built into the density of map 7. A polyalanine model was placed into density that could not be assigned to any RSC subunit (chain X). Bulky amino acid side chain density in the maps $4-8$ enabled us to assign the sequence registers, however in some regions register shifts cannot be entirely excluded. The modelled RSC subunits Rsc4, Rsc58, Rsc6, Rsc8, Rsc9, Npl6, Htl1, Sfh1 and Sth1 (residues 48 - 293) together with the poly-alanine chain were applied to several rounds of real space refinement and geometry optimisation using PHENIX (Adams et al., 2010), and flexible fitting with Namdinator (Kidmose et al., 2019) against the combined map 8. MolProbity (Williams et al., 2018) was used to flip and optimise Asn, Gln and His side 
chains. The C-terminal helix of Sfh1 was real space refined with PHENIX against map 7. The final structure displayed excellent stereochemistry as shown by MolProbity (Table 10). Figures were created using PyMol (Schrodinger, 2015),UCSF Chimera (Pettersen et al., 2004) and UCSF ChimeraX (Goddard et al., 2018). The angular distribution plots were generated using the AngularDistribution tool distributed with Warp (Tegunov and Cramer, 2018).

Sites of mutations found in human cancers were derived from the cBio cancer genomics portal (cBioPortal) (Cerami et al., 2012; Gao et al., 2013) and mapped onto the RSC structure for residues that are identical in its human counterpart PBAF using MSAProbs (Liu et al., 2010; Zimmermann et al., 2018). MSAPobs and Aline (Bond and Schuttelkopf, 2009) were used to map conservation between RSC and PBAF. 


\section{RESULTS}

\subsection{Preparation of endogenous S. cerevisiae RSC complex}

For our structural studies we decided to work with the endogenous RSC complex from $S$. cerevisiae. Endogenous RSC is assembled in its natural organism, carries all the potentially needed post-translational modifications, and occurs in the right subunit stoichiometry. RSC is very abundant in yeast cells (Cairns et al., 1996) which can be fermented in large amounts in order to get enough cell mass for purifications. In addition, some information on the purification of endogenous RSC was available (Cairns et al., 1996; Lorch and Kornberg, 2004; Saha et al., 2002).

With this information integrated, we established an optimised fermentation and purification strategy for Rsc2-tagged RSC complex (section 2.11.1). Rsc1 and Rsc2 are mutually exclusive and form two RSC complexes. Thus, tagging the complex through one of the two subunits removes this heterogeneity. The purification via TAP-tag was suggested (Lorch and Kornberg, 2004; Saha et al., 2002), but since this tagging strategy did not yield sufficient amounts of RSC, several large, modular affinity tags were stably integrated into the yeast genome at different RSC subunits and screened for a higher yield. However, none of the new tags could improve the purification.

Nevertheless, the experiments revealed a crucial bottle neck for the RSC preparation. During the lysis of the yeast cells, the lysate needs to stay cool to prevent RSC degradation. Therefore, the cell lysis through cryogenic grinding was established. An additional increase in yield was achieved by combining the TEV elution and the calmodulin binding step, and remaining DNA had to be removed by ion exchange chromatography to prevent RSC aggregation. The last cleaning step via sucrose gradient centrifugation or size exclusion chromatography resulted in sufficient amounts of pure, homogeneous RSC complex (Figure 5). When RSC was bound to the nucleosome, this last purification step was applied after the assembly of the RSC-nucleosome complex. Typical yields were $0.2-0.3 \mu \mathrm{g}$ from $300 \mathrm{~g}$ of yeast pellet. 
a

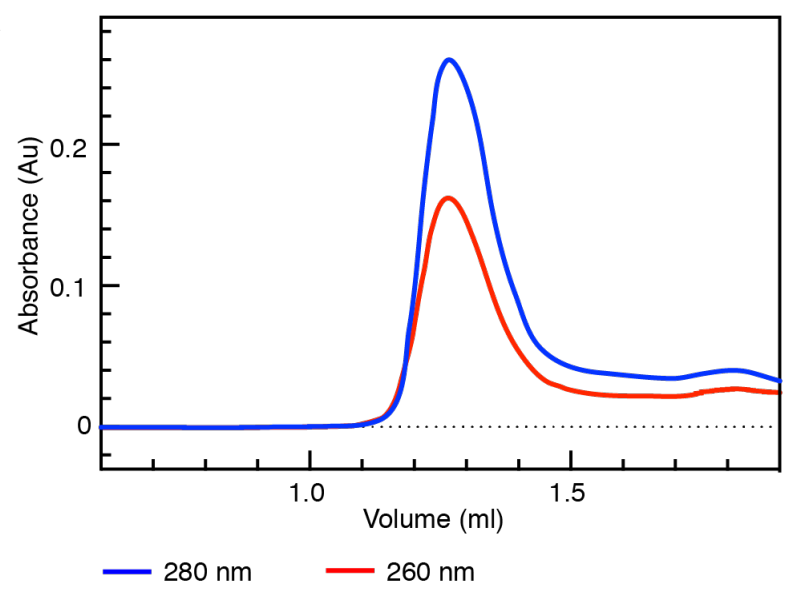

b

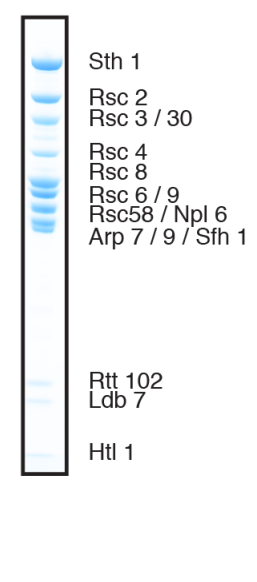

C

\begin{tabular}{l|l}
\hline RSC & kDa \\
\hline Sth1 & 157 \\
Rsc2 & $102^{*}$ \\
Rsc3 & 102 \\
Rsc30 & 101 \\
Rsc4 & 72 \\
Rsc9 & 65 \\
Rsc8 & 63 \\
Rsc58 & 58 \\
Rsc6 & 54 \\
Arr7 & 54 \\
Arp9 & 53 \\
Npl6 & 50 \\
Sth1 & 49 \\
Ldb7 & 20 \\
Rtt102 & 18 \\
Ht11 & 9 \\
\hline *108 kDa due to \\
residual TAP-tag
\end{tabular}

Figure 5 | Preparation of endogenous Rsc2-containing isoform of the RSC complex from $S$. cerevisiae. a,b. Analysis of purified RSC by size-exclusion chromatography (a) and SDS-PAGE showed high purity and homogeneity with stoichiometric subunits as assessable by Coomassie stain (b). c. Subunit identity was confirmed by mass spectrometry. The table shows the expected molecular weights of the RSC subunits.

\subsection{Negative stain electron microscopy of RSC}

We analysed the purified RSC complex with negative stain electron microscopy (EM) to validate its integrity and to generate a reliable, unbiased reference for further cryo-EM studies (section 2.16). The RSC complex disintegrated during sample preparation, but cross-linking of the complex with glutaraldehyde during sucrose gradient ultracentrifugation (Kastner et al., 2008) ensured its integrity (Figure 6a-d). We collected a small dataset on uranyl formate stained sample and reconstructed an ab initio RSC structure (Figure 6e). This procedure did not allow any bias to be introduced in the reconstruction. The obtained RSC structure showed the previously described modular architecture and overall resembled the low-resolution structures available (Asturias et al., 2002; Chaban et al., 2008; Leschziner et al., 2007) demonstrating a successful sample preparation. 


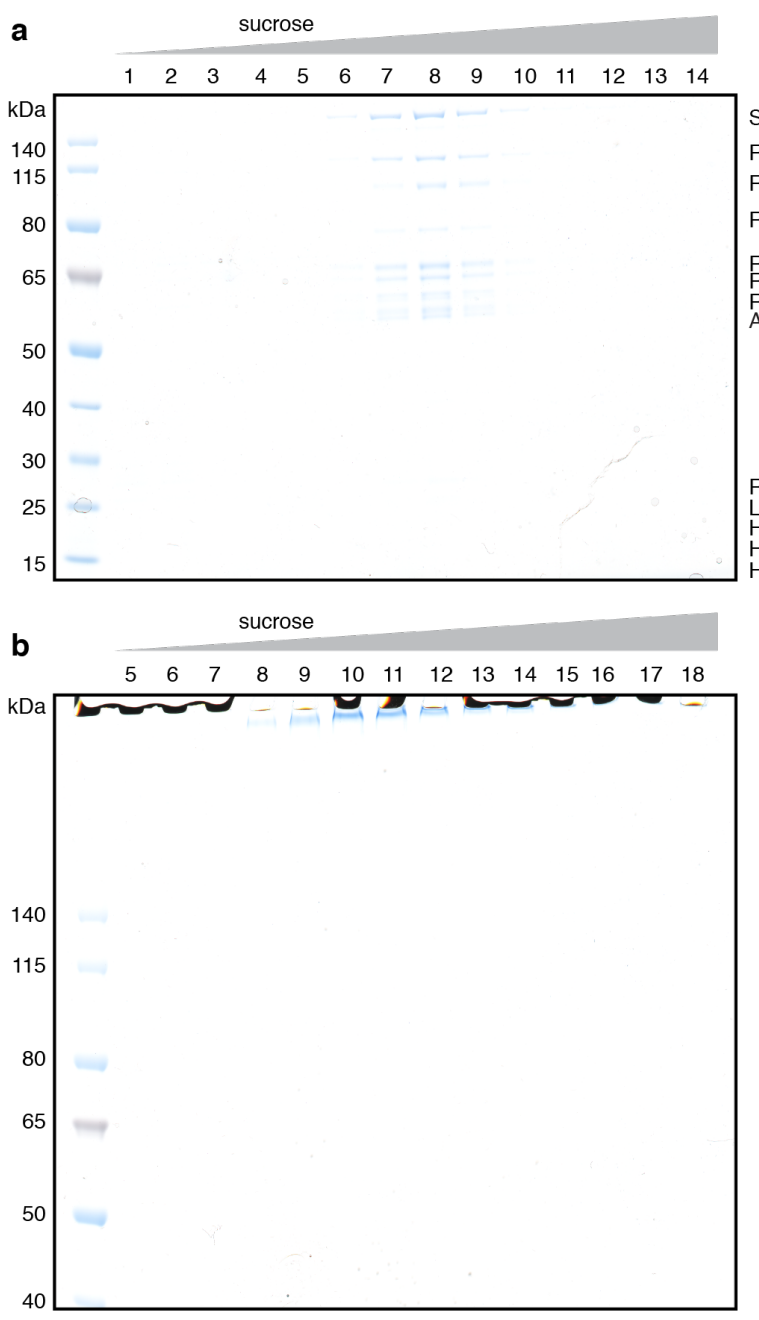

c
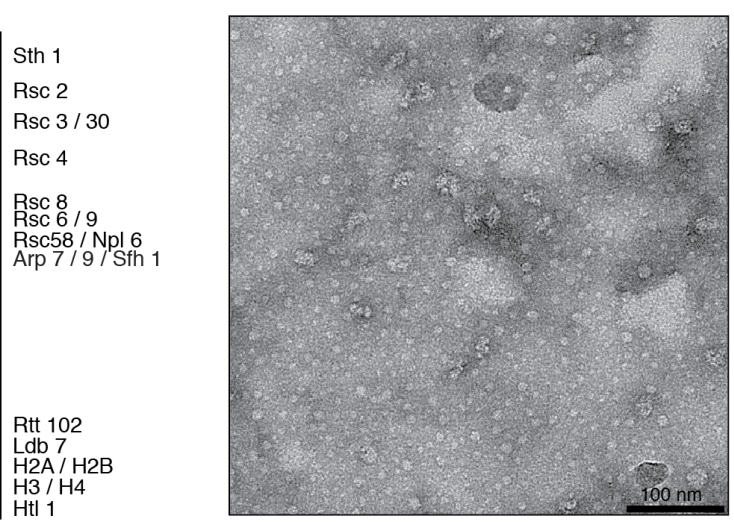

d

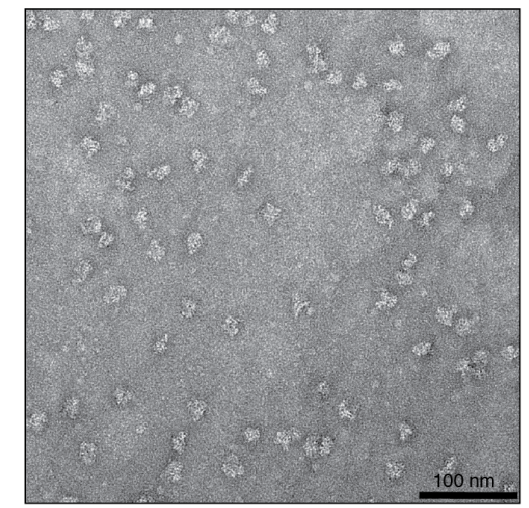

e

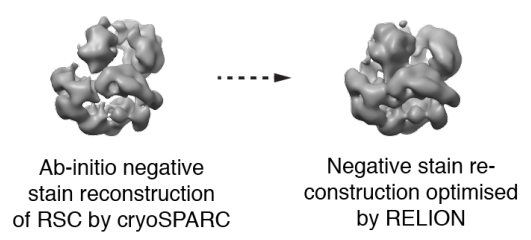

Figure 6 | Negative stain electron microscopy of the RSC complex. a,b. Sucrose gradient ultra-centrifugation without (a) and with glutaraldehyde cross-linking (b) c,d. Exemplary negative stain micrographs of RSC. Without stabilization through cross-linking, the complex disassembled during sample preparation (c), but mild cross-linking resulted in stabile RSC (d). e. A small negative stain dataset was collected, and an unbiased ab initio structure was reconstructed using cryoSPARC and RELION software packages. The structure showed a modular architecture and overall resembled the published low-resolution structures (Asturias et al., 2002; Chaban et al., 2008; Leschziner et al., 2007). 


\subsection{Activity of RSC complex*}

We further validated the quality of the RSC preparation by assaying the enzymatic activity of RSC. The ATPase activity was measured in a coupled ATP-NADH assay (section 2.14.1). ADP that gets produced by the activity of RSC is regenerated to ATP by the two enzymes, pyruvate kinase and lactate dehydrogenase, through oxidation of NADH to $\mathrm{NAD}^{+}$. The decrease in NADH is reflected by a decrease in the absorbance at a wavelength of $340 \mathrm{~nm}$. The reaction was monitored over $30 \mathrm{~min}$. The average reduction in absorbance between the linear portion across all samples between 10 - 20 min was measured to 0.097 absorbance units and significantly higher than the negative control without enzyme where basically no reduction in absorbance was measured. This experiment proved that the ATPase activity of the remodeller was functional.

Next, we tested the integrity of the remodeller with an in vitro genome-wide reconstitution assay that monitors the remodelling activity of RSC (section 2.14.2). Chromatin is reconstituted from Drosophila melanogaster embryo histones and yeast plasmid DNA using salt-gradient dialysis (SGD). Subsequently, the chromatin is digested with MNase. The DNA which was protected by nucleosomes is isolated and sequenced. Mapping of the DNA reads against a reference genome results in the positional information of the nucleosomes. In the assay RSC and ATP were added to reconstituted chromatin and the nucleosome positions after remodelling were compared to a negative control without RSC and to the positions measured in vivo on extracted chromatin (Figure 7). The chromatin from the negative control (SGD) shows a small nucleosome-depleted region (NDR) similar to the in vivo situation. Upon addition of RSC and ATP, the NDR region becomes wider and deeper. This amplification of the NDR demonstrates that the here purified RSC complex exhibits not only ATPase activity, but is a functional remodeller as described before (Krietenstein et al., 2016). 


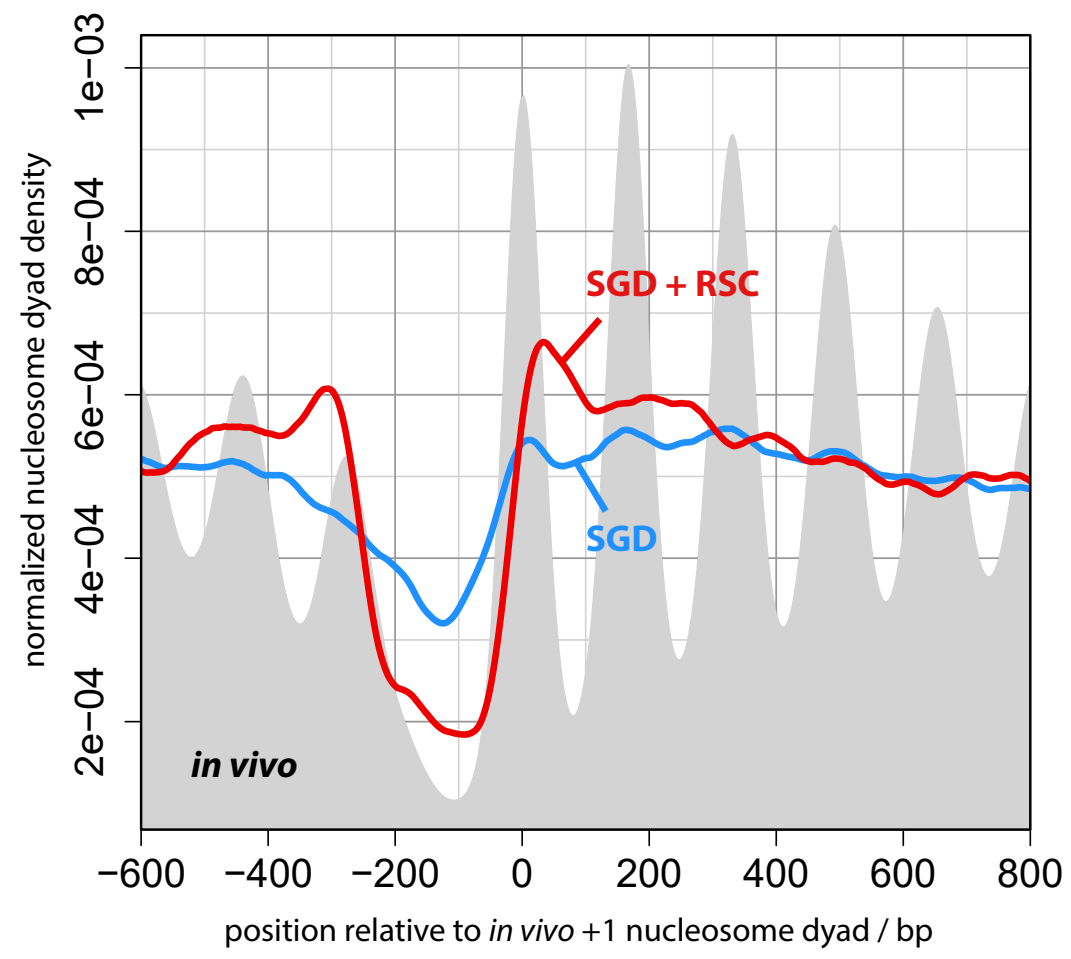

Figure 7 | Remodelling activity of RSC in a genome-wide reconstitution assay. Nucleosomes assembled onto plasmid DNA via salt-gradient dialysis (SGD) (blue) show a small NDR region similar to the in vivo situation (grey in background). Upon addition of the here purified RSC complex and ATP (red), the NDR becomes deeper and wider demonstrating active remodelling by RSC.

* The activity of the RSC complex was assayed in collaboration with Toby Barnes from the group of PD Dr. Philipp Korber at the Molecular Biology Division of the Biomedical Center of the LMU Munich in PlaneggMartinsried, Germany. RSC was purified by Felix Wagner and shipped to the Korber laboratory where Toby Barnes carried out the assays. 


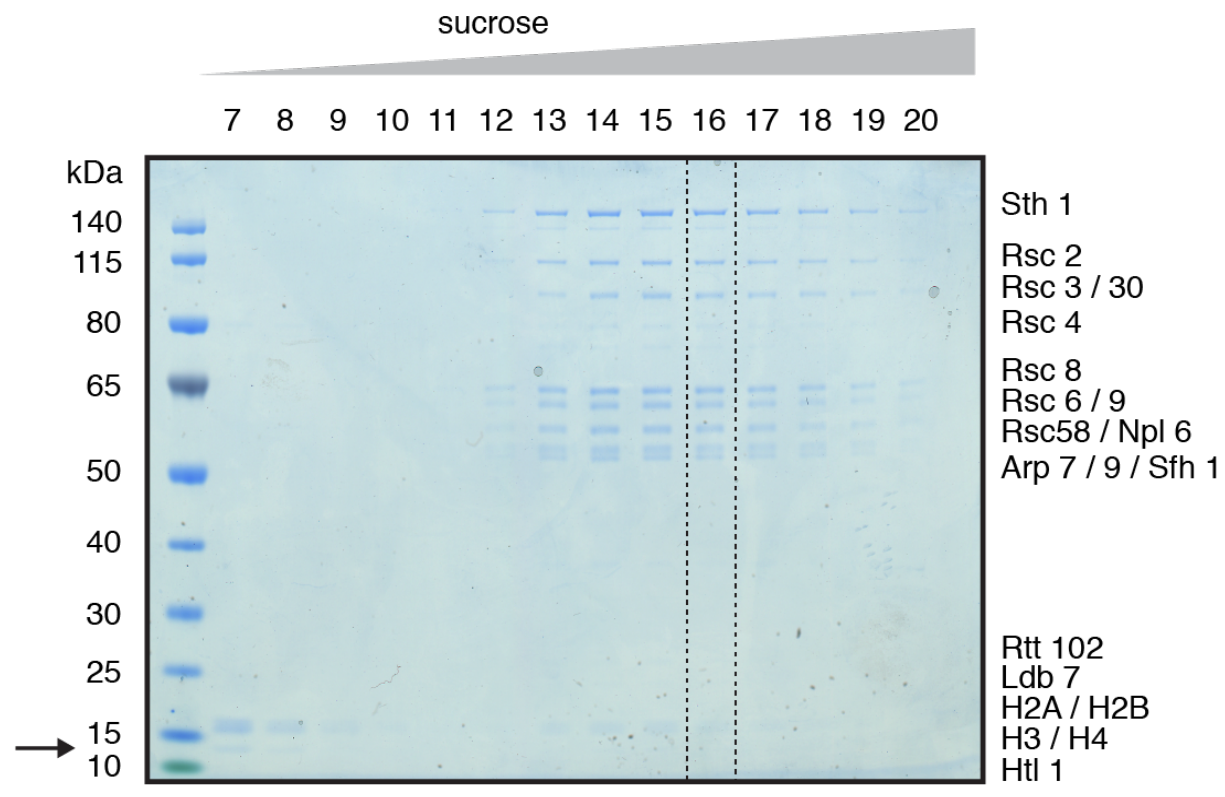

Figure 8 | Assembly of the RSC-nucleosome complex. SDS-PAGE analysis of the fractions $7-20$ of a $10-25 \%$ sucrose gradient ultracentrifugation. Complex formation was successful as demonstrated by the co-migration of histones with the RSC complex. The unbound over-stoichiometric nucleosomes only migrated to fraction 7 and 8 (black arrow). Fraction 16, but from a glutaraldehyde cross-linked RSC-nucleosome complex, was used for cryo-EM grid preparation (dashed box).

\subsection{RSC-nucleosome complex assembly}

We reconstituted nucleosomes from Xenopus laevis histones and a DNA fragment containing the 145-bp Widom 601 sequence (Lowary and Widom, 1998) extended by 55 bp on the 5 '-end and 37 bp on the 3 '-end (section 2.11.2, 2.12). Since RSC can bind free DNA, the overhangs were designed to offer RSC additional binding interfaces presumably resulting in a more stable RSC-nucleosome complex. RSC purification and nucleosome preparation were timed in order to have fresh sample available for complex assembly on the same day (section 2.13). RSC was saturated with nucleosome substrate in the presence of the ATP transition state analogue ADP beryllium fluoride ( $\mathrm{ADP}-\mathrm{BeF}_{3}$ ). RSCnucleosome complex was separated from unbound nucleosomes and mildly cross-linked for stabilization using the GraFix method (Kastner et al., 2008) (Figure 8). 


\subsection{Structure of RSC-nucleosome complex}

Cryo-EM analysis resulted in a medium-resolution reconstruction of the RSC-nucleosome complex (Figure 9). Focused 3D classification enabled modelling of the nucleosome and associated ATPase with the use of a related structure (Li et al., 2019) (Table 10), and placement of an adapted ARP module structure (Schubert et al., 2013) (Figure 9). We also subjected the free RSC complex to cryo-EM analysis, and resolved the body and arm modules at resolutions of $3.6 \AA$ and $3.8 \AA ̊$, respectively (Figure 10) (Table 10).

The medium-resolution reconstruction revealed the nucleosome, four turns of DNA exiting from one side of the nucleosome, and five RSC modules that we refer to as ATPase, ARP, body, arm, and DNA-interaction module (DIM) (Figure 11a). The structural model built with the densities of the arm and body (Figure 12) modules reveals the intricate architecture of RSC (Figure 11). It only lacks the DIM module and agrees with lysine-lysine cross-linking information (Figure 13).

The body module contains subunits Rsc4, Rsc6, Rsc8, Rsc9, Rsc58, Htl1, and the Nterminal region of Sth1 (Figure 14, Table 1). The ARP module is flexibly tethered to the body and comprises the helicase-SANT associated (HSA) region of Sth1, the actin-related proteins Arp7 and Arp9, and subunit Rtt102. The C-terminal region of Sth1 extends from the HSA region and forms the ATPase module (Figure 15a). The arm module protrudes from the body and contains subunit Sfh1 and parts of Rsc8, Npl6, and Rsc9 (Figure 14). The arm and body modules are tightly connected by two copies of Rsc 8 that adopt different structures (Figure 15b). The N-terminal SWIRM domains of Rsc8 reside in the arm, whereas the SANT domains and one of the ZZ zinc finger domains reside in the body, as do the long C-terminal helices. The RSC structure and observed subunit interactions explain the requirement of the Rsc4 C-terminal region for cell growth (Kasten et al., 2004), the known interaction between Rsc6 and Rsc8 (Treich et al., 1998), mutations in Htl1 that lead to its dissociation (Florio et al., 2007), and lethal effects of Rsc58 truncation (Taneda and Kikuchi, 2004). 
a
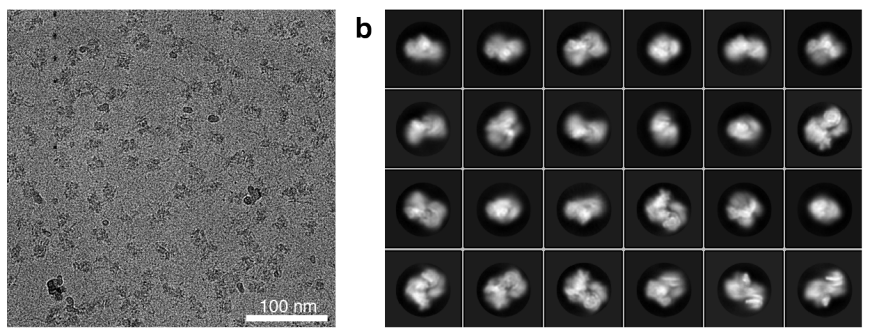

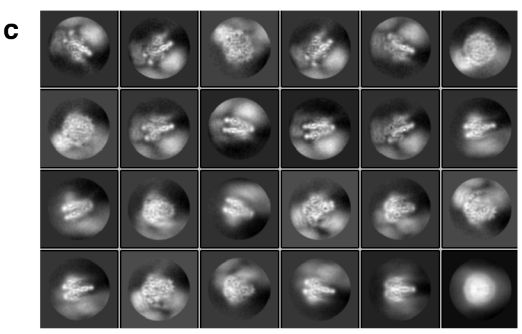

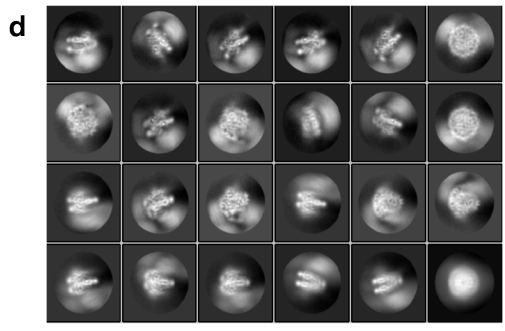
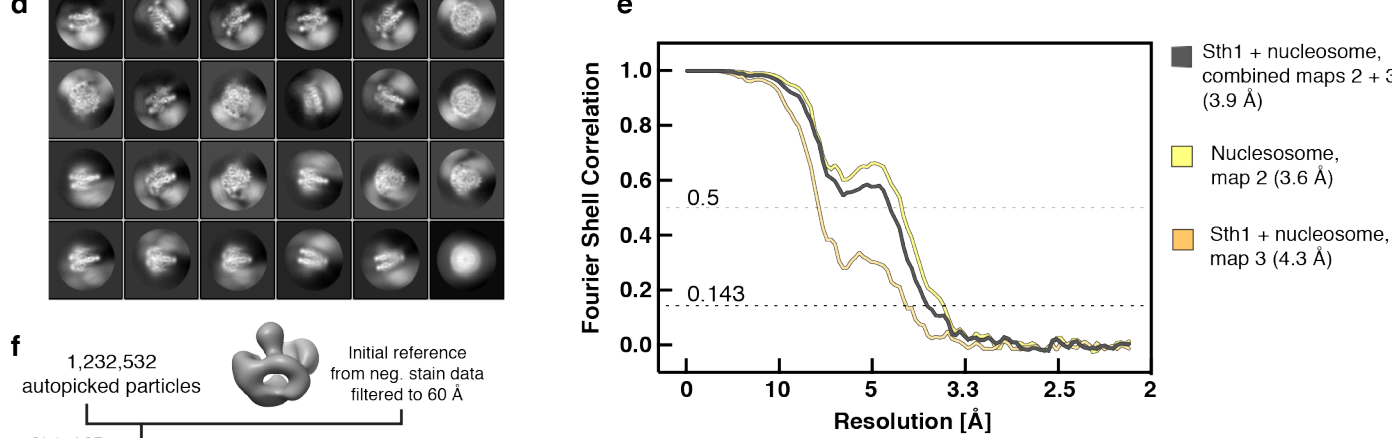

Sth1 + nucleosome, $(3.9 \AA)$

Nuclesosome, map $2(3.6 \AA)$

Sth1 + nucleosome map $3(4.3 \AA)$

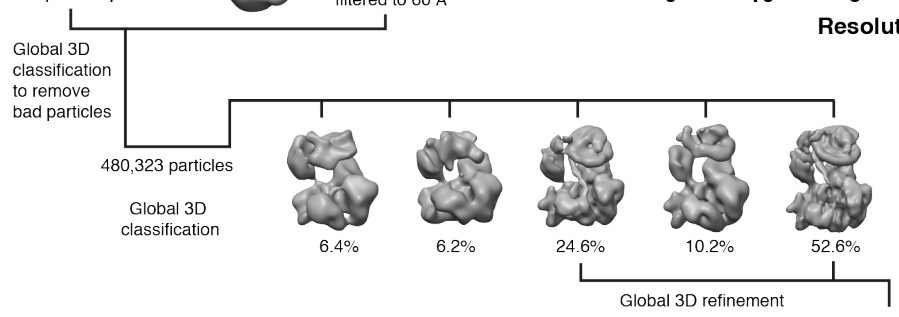
Re-extraction of patricles centered on
the nucleosome with box including
nucleosome and Sth1
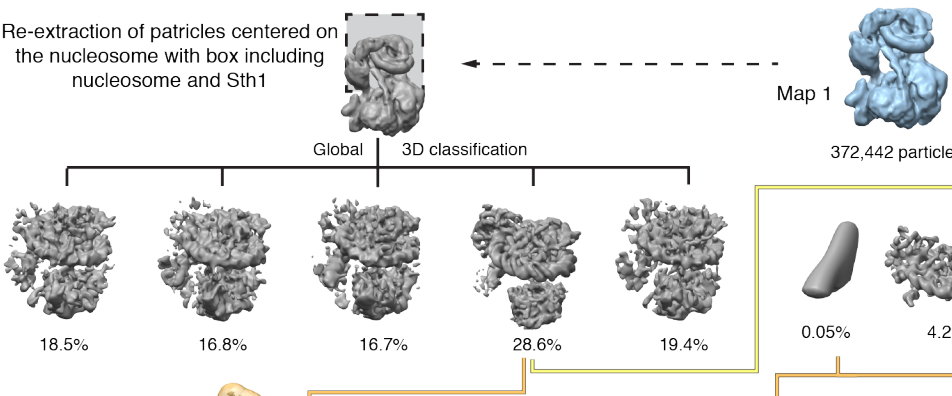

Focused 3D refinement, postprocessing
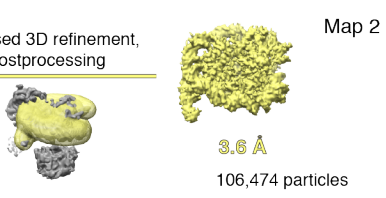

106,474 particles

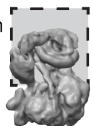

ATPase module

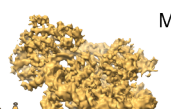

4.3 A 35,972 particles

\begin{tabular}{l|l}
442 particles & Focused $3 \mathrm{D}$ refinement \\
\hline
\end{tabular}

postprocessing

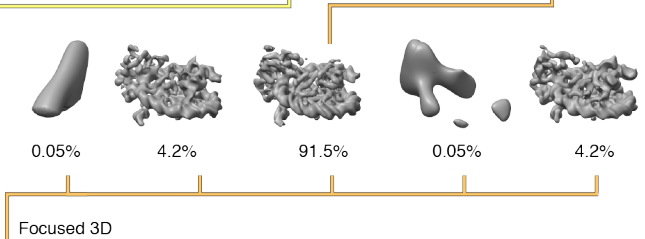

Global 3D refinement,
focused 3D classification

h
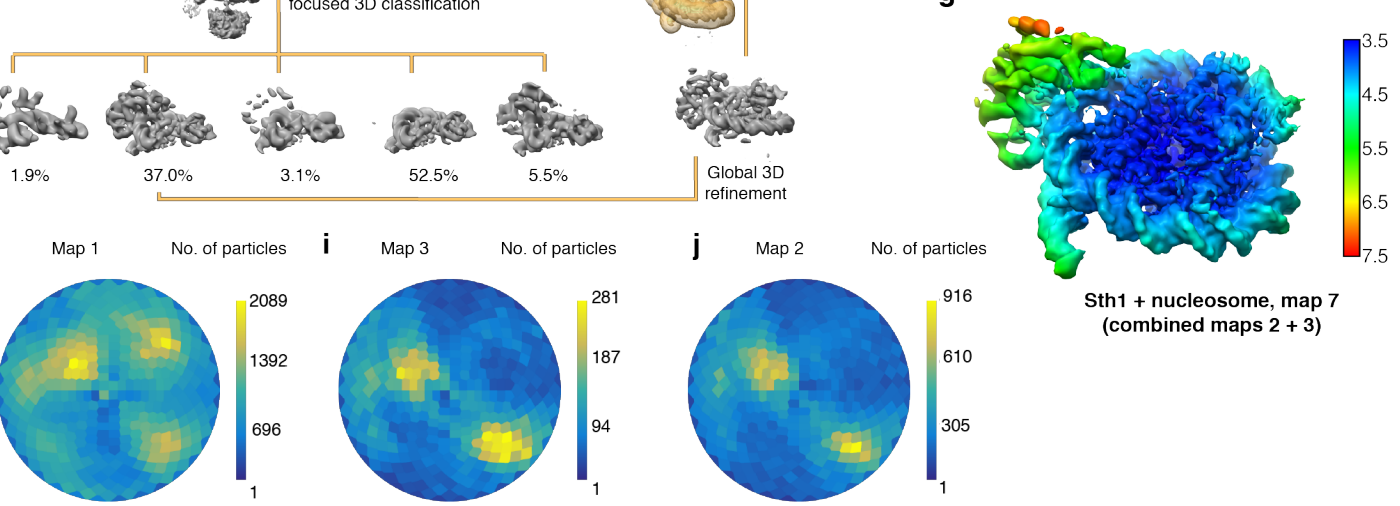

Sth1 + nucleosome, map 7 (combined maps $2+3$ )

Figure 9 | Cryo-EM analysis of the RSC-nucleosome complex. See next page for caption. 
Figure 9 | Cryo-EM analysis of the RSC-nucleosome complex. Figure on previous page. a. Representative cryo-EM micrograph of the RSC-nucleosome complex shows homogeneously distributed individual particles. b-d. 2D class averages of the RSC-nucleosome (b), the Sth1-nucleosome subcomplex (c) and the nucleosome subcomplex (d). e. Fourier shell correlation plots reveal the overall resolutions of the cryo-EM reconstructions. f. Cryo-EM processing workflow for the reconstructions of the RSC-nucleosome, the Sth1-nucleosome subcomplex, and the nucleosome subcomplex. Particle distribution after 3D classifications is indicated below the corresponding map. The final maps are shown in colours. The masks used for focused classifications and refinements are colour coded corresponding to the final maps they were used for. Views are generally rotated by $180^{\circ}$ with respect to Figure 11c, left. g. Local resolution estimation of the combined Sth1-nucleosome map as implemented in RELION (Zivanov et al., 2018). We note that the resolution of the peripheral area with the Sth1 subunit is overestimated. h-j. Angular distribution plot for all particles contributing to the final reconstructions of the RSC-nucleosome (h), the Sth1-nucleosome (i) and the nucleosome complex (j).

Figure 10 | Cryo-EM analysis of the free RSC complex. Figure on next page. a. Representative cryo-EM micrograph of the free RSC complex shows homogeneously spaced individual particles. b. 2D class averages of the free RSC complex. c. Cryo-EM processing workflow for the reconstruction of the free RSC complex. Particle distribution after 3D classifications is indicated below the corresponding map. The final maps after focused 3D refinement and masks are depicted in colour. Views are generally rotated by $180^{\circ}$ with respect to Figure $11 \mathrm{c}$, right. d. Angular distribution plot for all particles contributing to the final reconstruction of the free RSC complex. e. Two views of the combined RSC core map coloured according to the local resolution as implemented in RELION (Zivanov et al., 2018). f. Fourier shell correlation plots of the maps used for model building of the RSC core complex. 
a

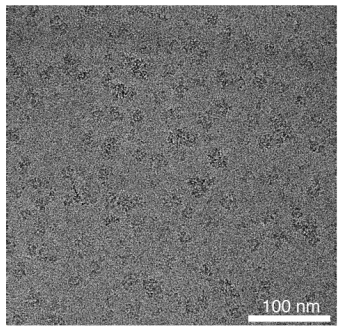

b

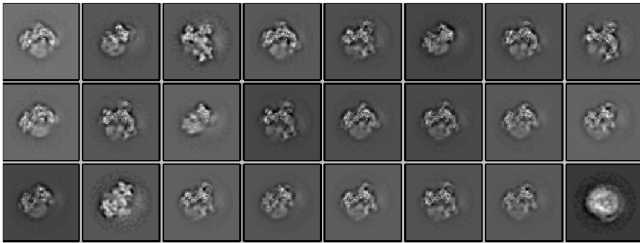

c

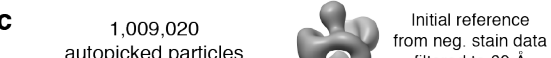
tered to $60 \AA$
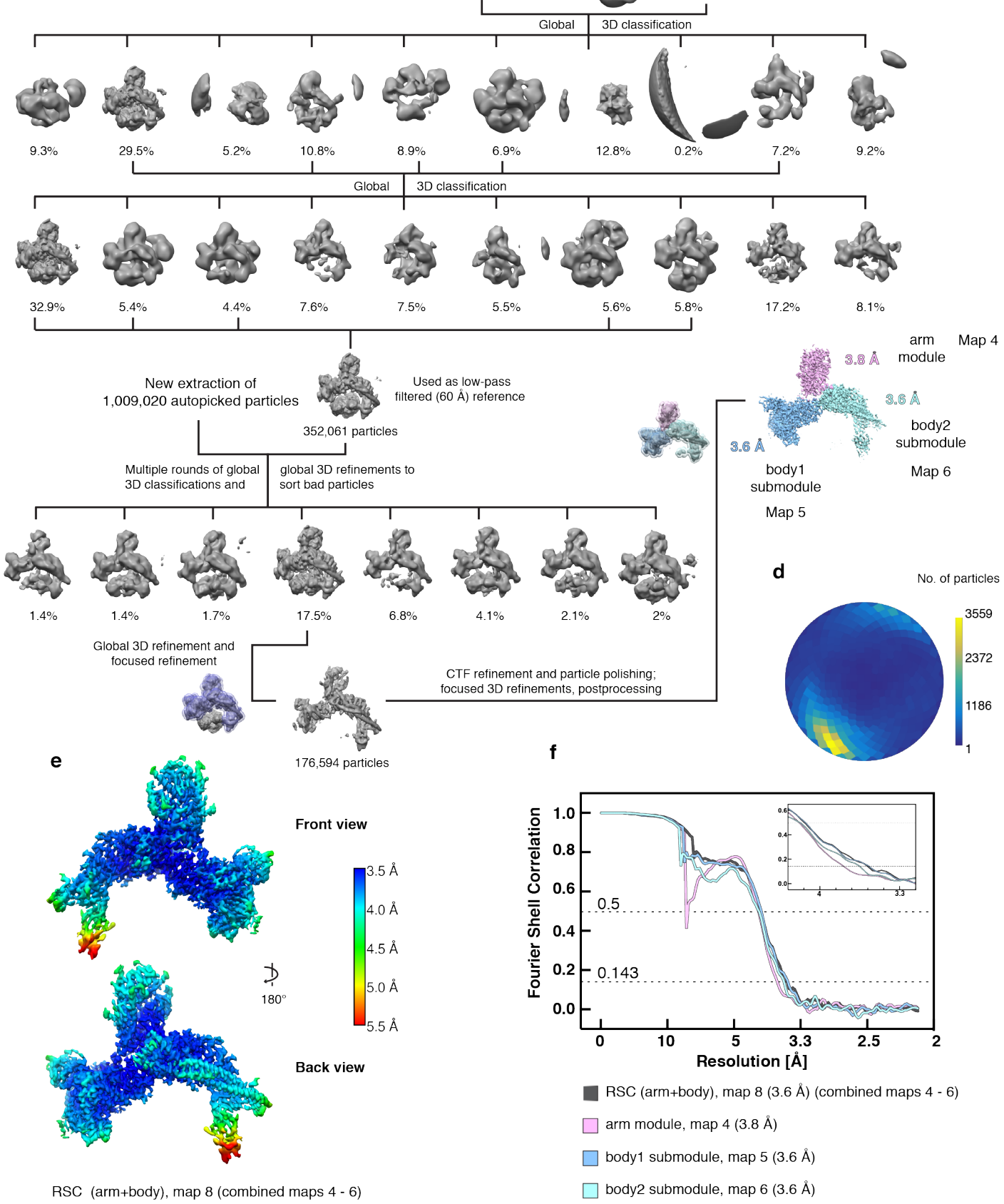

Figure 10 | Cryo-EM analysis of the free RSC complex. See previous page for caption. 
Table 10 | Cryo-EM data collection, refinement and validation statistics.

\begin{tabular}{|c|c|c|}
\hline & $\begin{array}{l}\text { Sth1-nucleosome structure } \\
\text { (map 7) } \\
\text { (EMDB-xxxx) } \\
\text { (PDB xxxx) }\end{array}$ & $\begin{array}{l}\text { RSC core structure } \\
\text { (map 8) } \\
(\text { EMDB-xxxx) } \\
(\text { PDB } x x x x)\end{array}$ \\
\hline \multicolumn{3}{|l|}{ Data collection and processing } \\
\hline Magnification & 130,000 & 130,000 \\
\hline Voltage $(\mathrm{kV})$ & 300 & 300 \\
\hline Electron exposure $\left(\mathrm{e}-/ \AA^{2}\right)$ & $47.8 / 45.4^{*}$ & $39.0^{\dagger} / 45.02^{\dagger} / 54.9^{\ddagger}$ \\
\hline Defocus range $(\mu \mathrm{m})$ & $0.4-2.9$ & $0.8-4.8$ \\
\hline Pixel size $(\AA)$ & 1.05 & 1.05 \\
\hline Symmetry imposed & $\mathrm{C} 1$ & $\mathrm{C} 1$ \\
\hline Initial particle images (no.) & $1,232,532$ & $1,009,020$ \\
\hline Final particle images (no.) & $106,474 / 35,972$ & 176,594 \\
\hline Map resolution $(\AA)$ & 3.9 & 3.6 \\
\hline FSC threshold & 0.143 & 0.143 \\
\hline Map resolution range $(\AA)$ & $3.5-7.5$ & $3.5-5.5$ \\
\hline \multicolumn{3}{|l|}{ Refinement } \\
\hline Initial model used (PDB code) & $5 Z 3 U$ & $\begin{array}{l}\text { 3LJW, 1UHR, 2FQ3, } \\
\text { 2YUS, 1TOT, 4V3Q, } \\
\text { 6AX5 }\end{array}$ \\
\hline Model resolution $(\AA)$ & 3.63 & 3.76 \\
\hline FSC threshold & 0.5 & 0.5 \\
\hline Map sharpening $B$ factor $\left(\AA^{2}\right)$ & -150 & -105.7 \\
\hline \multicolumn{3}{|l|}{ Model composition } \\
\hline Non-hydrogen atoms & 13,655 & 20,545 \\
\hline Protein / Nucleotide residues & $1,274 / 246$ & $2,678 /-$ \\
\hline Ligands & 0 & 1x Zn \\
\hline \multicolumn{3}{|l|}{$B$ factors $\left(\AA^{2}\right)$} \\
\hline Protein & 106.53 & 53.27 \\
\hline Ligand & & 30.00 \\
\hline Nucleotide & 119.35 & \\
\hline \multicolumn{3}{|l|}{ R.m.s. deviations } \\
\hline Bond lengths $(\AA)$ & 0.004 & 0.004 \\
\hline Bond angles $\left({ }^{\circ}\right)$ & 0.748 & 0.931 \\
\hline \multicolumn{3}{|l|}{ Validation } \\
\hline MolProbity score & 1.19 & 1.57 \\
\hline Clashscore & 4.07 & 4.30 \\
\hline Poor rotamers (\%) & 0.00 & 0.14 \\
\hline \multicolumn{3}{|l|}{ Ramachandran plot } \\
\hline Favored $(\%)$ & 98.07 & 94.84 \\
\hline Allowed (\%) & 1.93 & 5.16 \\
\hline Disallowed (\%) & 0.00 & 0.00 \\
\hline
\end{tabular}

* Dataset from two collections were combined

Datasets from three collections were combined, ${ }^{\dagger}$ not tilted, ${ }^{\ddagger} 25^{\circ}$ tilt 

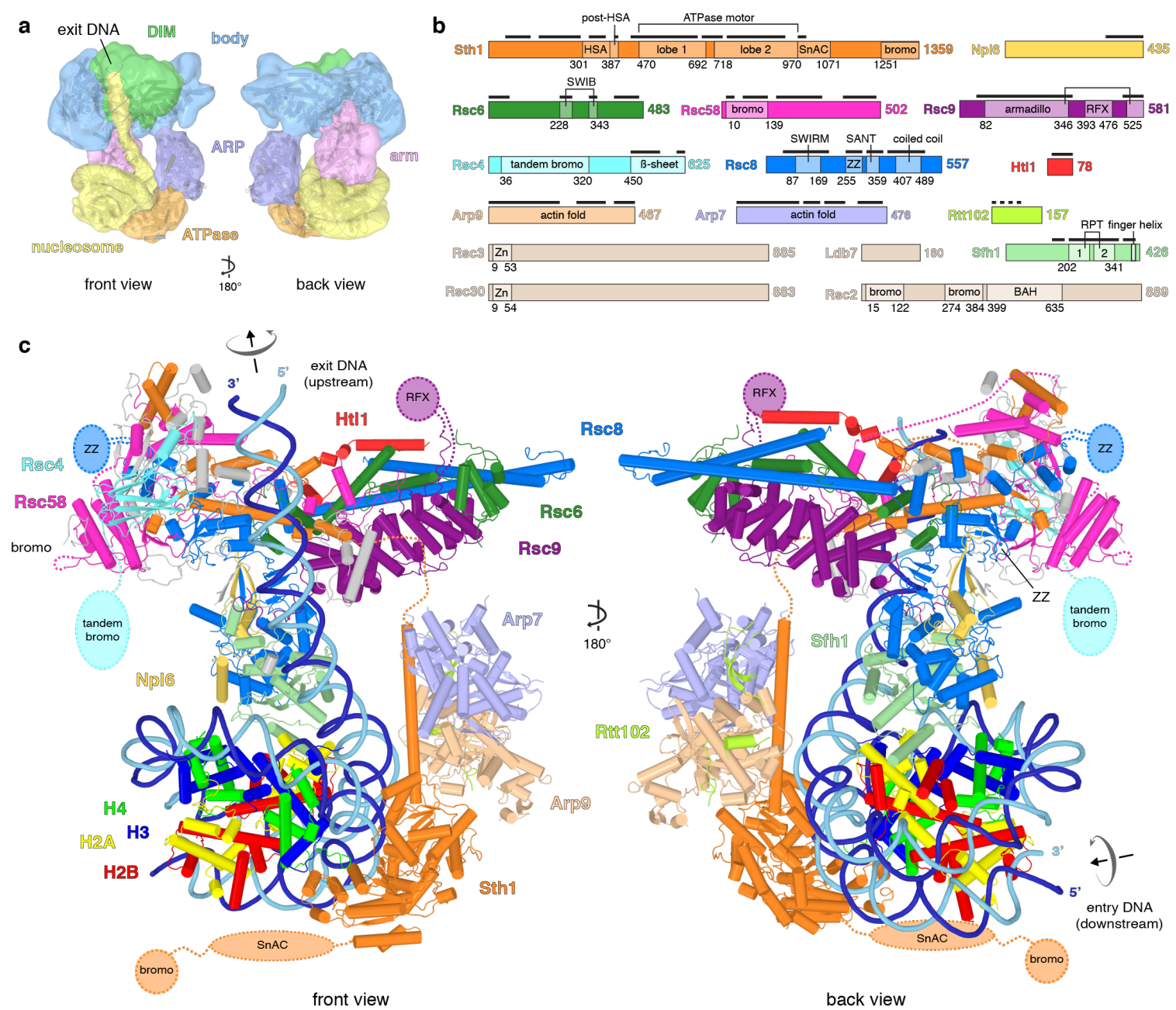

Figure 11 | Structure of RSC-nucleosome complex. a. Overall architecture presented in two views of the low pass-filtered cryo-EM density. The five RSC modules are in different colours. Colour code for modules used throughout. The nucleosome substrate with exit DNA is in yellow. DIM, DNA-interaction module. b. Schematic of RSC subunit domain architecture. Colour code for subunits used throughout. Domain boundaries are marked with residue numbers and black bars indicate modelled regions. HSA, helicase-SANT-associated; SnAC, Snf2 ATP coupling; bromo, bromodomain; armadillo, armadillo repeat fold; RFX, DNAbinding RFX-type winged-helix; SWIRM, Swi3 Rsc8 Moira; ZZ, ZZ-type zinc finger; SANT, Swi3 Ada N-Cor TFIIIB; coiled coil, C-terminal helix forming coiled coil-like structure; Zn, Zn(2)-C6 fungal-type zinc finger; RPT, repeat; BAH, bromo-adjacent homology. c. Cartoon representation of the RSC-nucleosome complex structure. Unassigned elements shown in grey. Mobile domains depicted schematically. Arrows indicate directionality of DNA translocation. 

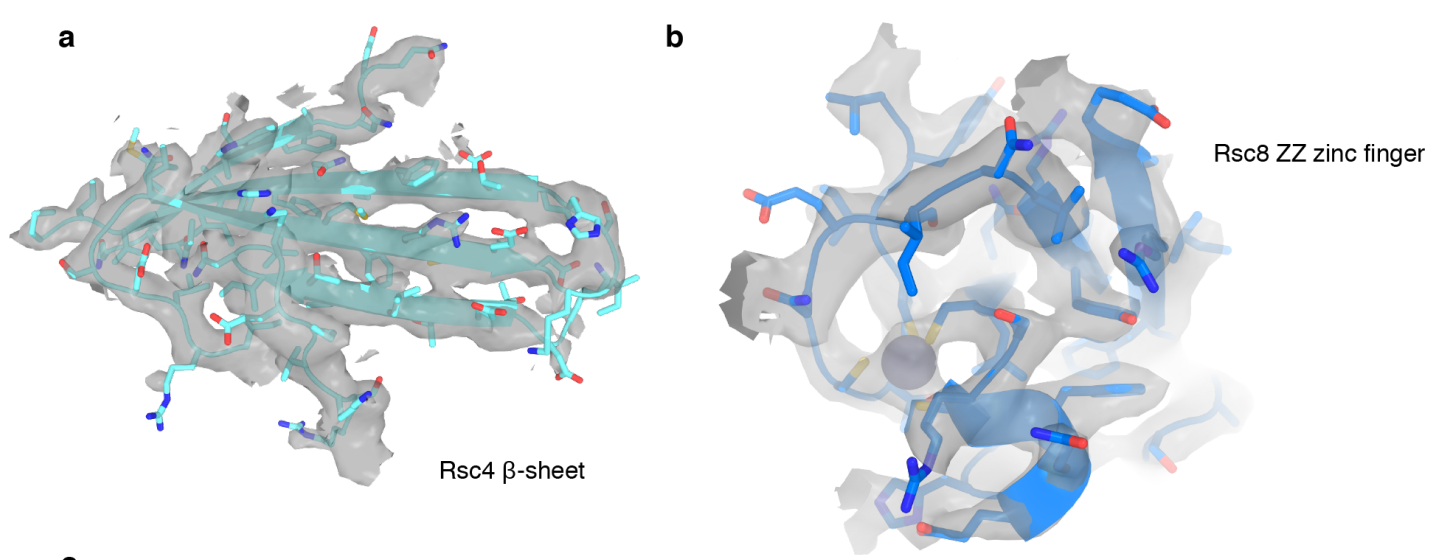

C

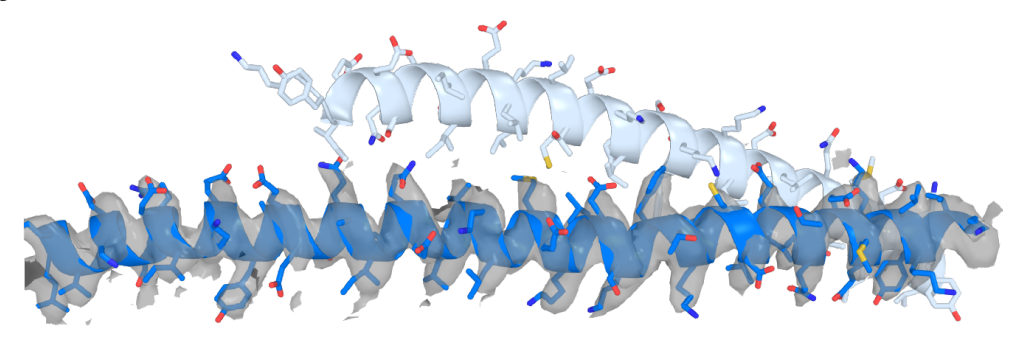

Rsc8 coiled coil region

Figure 12 | Cryo-EM densities for selected RSC regions as examples of map quality. a. Close-up of the Rsc4 $\beta$-sheet shows clear separation of individual strands. $\mathbf{b}$. The high quality of the map for the ZZ zinc finger of Rsc8 allowed backbone tracing and placement of side chains as well as for the zinc ion. c. Coiled coil helices of the two Rsc8 subunits with density for one helix.

RSC also contains six domains that are implicated in interactions with histone tails. The $\mathrm{N}$-terminal bromodomain in Rsc58 locates to the surface of the body (Figure 15c). The five other domains are mobile, and include a bromodomain in Sth1, two bromodomains in Rsc2, a BAH domain in Rsc2 that binds histone H3 (Chambers et al., 2013), and a tandem bromodomain in Rsc4 that interacts with acetylated H3 tails (Kasten et al., 2004), in particular acetylated lysine K14 (Kasten et al., 2004; VanDemark et al., 2007). RSC also contains five putative DNA-binding domains, of which four are mobile. These include the zinc finger domains in subunits Rsc3 and Rsc30, an RFX domain in subunit Rsc9, and a ZZ finger domain in one of the two Rsc8 subunits. In summary, RSC consists of five modules and nine flexibly connected domains, of which some are suggested to be involved in substrate selection via the recognition of histone modifications and DNA sequence features. 
a

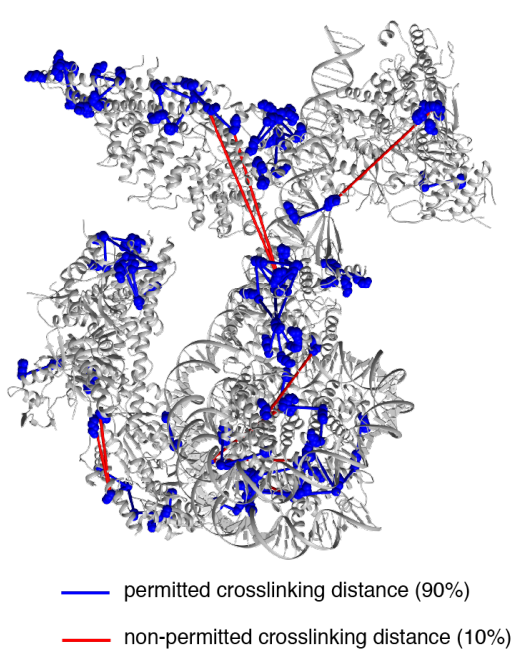

b

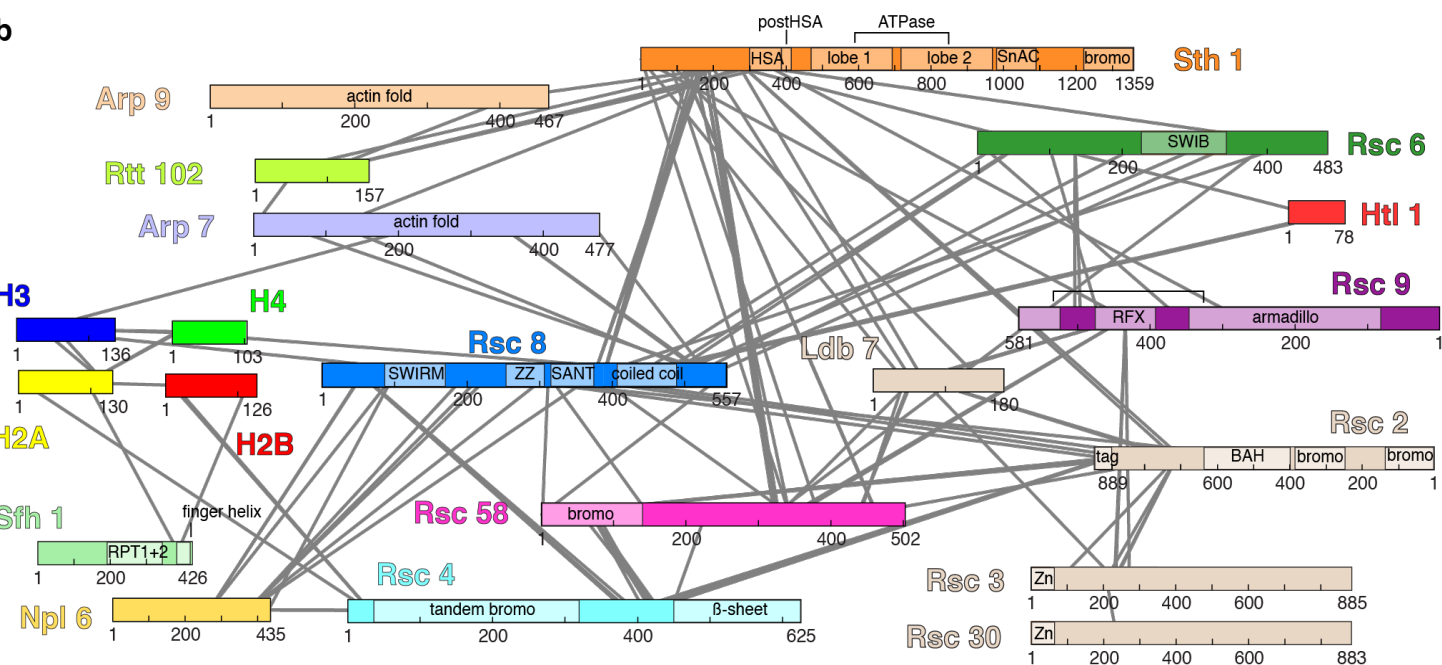

Figure 13 | Cross-linking network of the RSC nucleosome complex. a. Location of cross-linking sites mapped onto the structure. BS3 cross-links that appeared at least in triplicates were mapped onto the RSC-nucleosome structure. Lysine residues involved in the cross-linking network are shown as blue spheres and cross-linked residues are connected with lines indicating permitted (blue) and non-permitted (red) cross-linking distances. $90 \%$ of the mapped cross-links are within the permitted cross-linking distance which was set to $30 \AA$. The remaining $10 \%$ of nonpermitted cross-links likely reflect ambiguity caused by the presence of two identical Rsc8 subunits in the structure as well as flexibility of the complex in the buffer conditions or arise from technical errors. b. Cross-linking network between subunits of the RSC-nucleosome complex. Subunits are coloured as in Figure 11. Cross-links with a score above 2.5 are shown. A comprehensive list of cross-links can be found in the Table S12. 


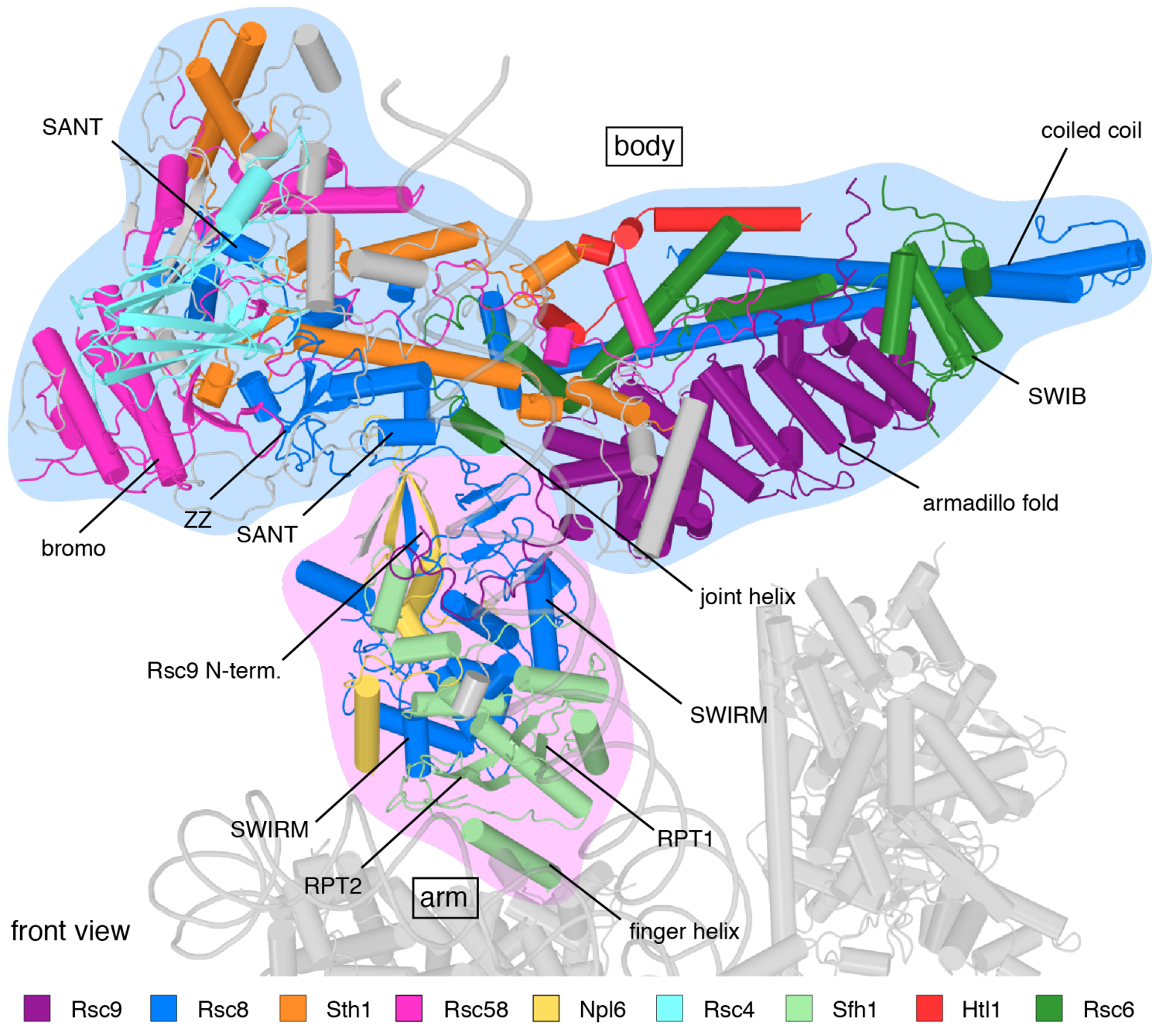

Figure 14 | Structure of RSC body and arm modules. Cartoon representation viewed as in Figure 11. The coloured structural elements were built de novo and with the use of homology models (Table S11). Important structural elements are labelled. The joint helix of Rsc6 resides in the middle of the arm and body interface. bromo, bromodomain; SWIRM, Swi3 Rsc8 Moira; ZZ, ZZ-type zinc finger; SANT, Swi3 Ada NCor TFIIIB; coiled coil, C-terminal helix forming coiled coil-like structure; RPT, repeat. 

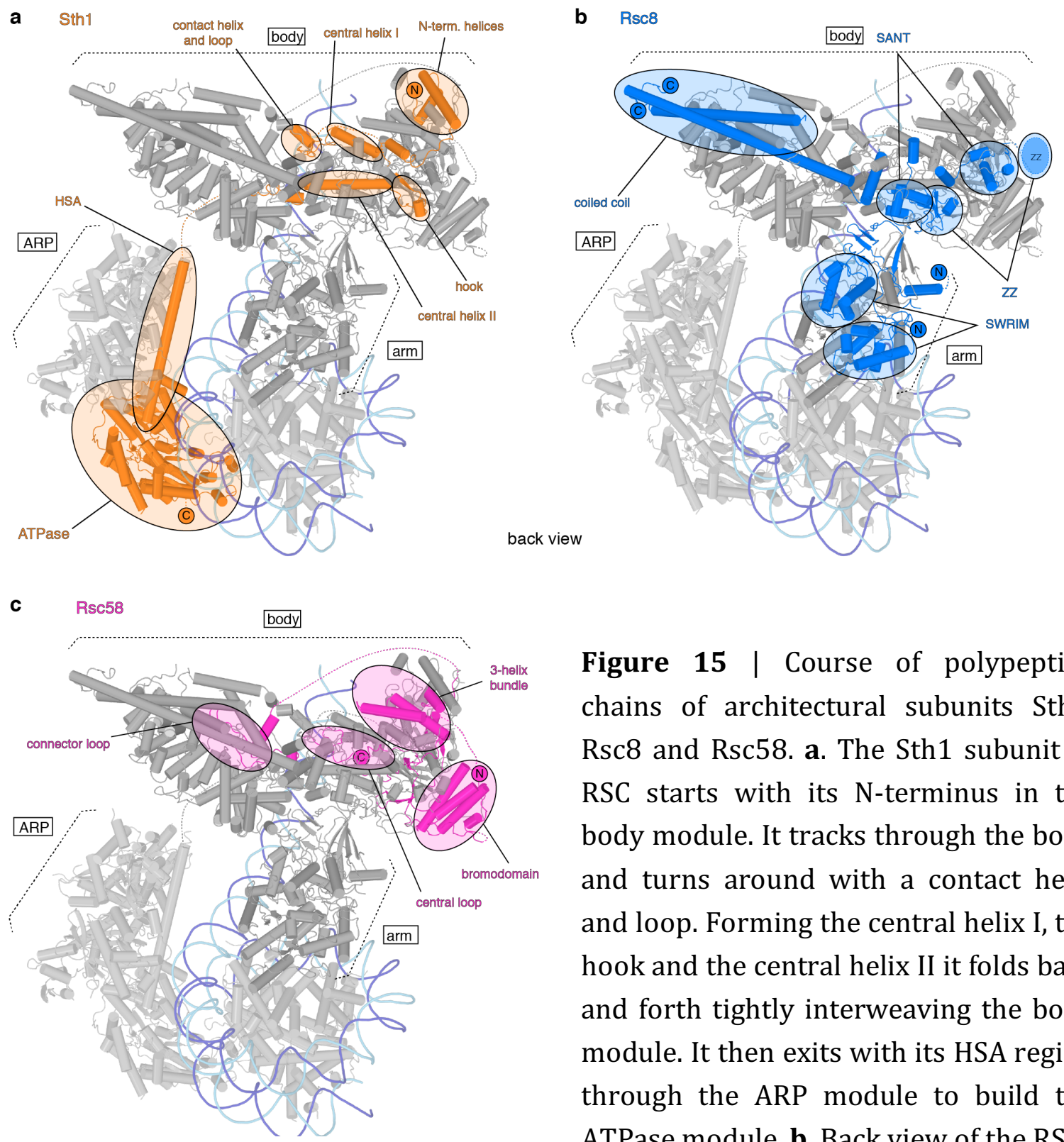

Figure 15 | Course of polypeptide chains of architectural subunits Sth1, Rsc8 and Rsc58. a. The Sth1 subunit of RSC starts with its N-terminus in the body module. It tracks through the body and turns around with a contact helix and loop. Forming the central helix I, the hook and the central helix II it folds back and forth tightly interweaving the body module. It then exits with its HSA region through the ARP module to build the ATPase module. b. Back view of the RSC

remodeller with the domains of the two Rsc8 subunits highlighted in blue. Both Rsc8 subunits start with $\mathrm{N}$-terminal SWIRM domains in the arm module where they both support the two repeat domains of Sfh 1 in the same way. They then follow distinct paths through the arm towards the body module where they integrate with both their SANT and ZZ zinc finger domains. Here the two domains of each subunit form different contacts with various interactions partners. Whereas one ZZ zinc finger domain is tightly packed at the body and DNA-interaction module interface, the other seems to extend from the body, presumably as additional interaction surface. Both Rsc8 subunits unite again with their C-terminal long helices in a coiled coil fold on the opposite side of the body module. c. Rsc58 N-terminal bromodomain attaches to the top of the body module. Then, Rsc58 follows an interwound path through the body module via the central and connector loop. It turns back docking to the body with a 3-helix bundle and stabilizing the module with its C-terminal end. 


\subsection{ATPase binding and translocation}

RSC engages in multivalent interactions with its substrate, contacting both DNA and histones (Figure 11). The ATPase and arm modules interact with the nucleosome, whereas the DIM module engages with DNA exiting from the nucleosome. The ATPase module binds the edge of the nucleosome, contacting both DNA gyres in a conformation poised for translocation activity (Figure 16a). The two lobes of the ATPase motor domain contact one gyre at superhelical location (SHL) +2 and adopt the same relative orientation as in the structure of the related SWI/SNF ATPase Snf2 bound to a nucleosome (Li et al., 2019). The N-terminal ATPase lobe 1 also binds the second DNA gyre around SHL -6 (Figure 16b), a location where the N-terminal tail of histone H3 is expected to protrude (Figure 17). Considering the known directionality of the translocase (Saha et al., 2005), we arrive at the model that the RSC ATPase motor pumps DNA towards the nucleosome dyad and along the octamer surface in the exit direction, which corresponds to the upstream direction of transcription, thus liberating more promoter DNA.

The ARP module couples RSC ATPase activity to DNA translocation and regulates the remodelling activity (Cairns et al., 1998; Clapier et al., 2016; Schubert et al., 2013). Our results suggest that this regulation involves changes in the position of the mobile ARP module that influence the conformation and mobility of the ATPase lobe 1 and its interactions with both DNA gyres (Figure 16b). These changes are likely transmitted through the hinge region between the HSA region and lobe 1 that includes the post-HSA region of Sth1. Mutations of the post-HSA region increase ATPase activity and DNA translocation, suggesting that the hinge acts as a throttle for the ATPase (Clapier et al., 2016; Saha et al., 2002; Szerlong et al., 2008). The ARP module adopts a defined position in the RSC-nucleosome complex, but it is mobile in the free RSC structure. Based on these results, we propose that the position of the mobile ARP module can influence the conformation and motility of the bilobal ATPase motor and thereby control the translocation activity of RSC (Figure 16b). 


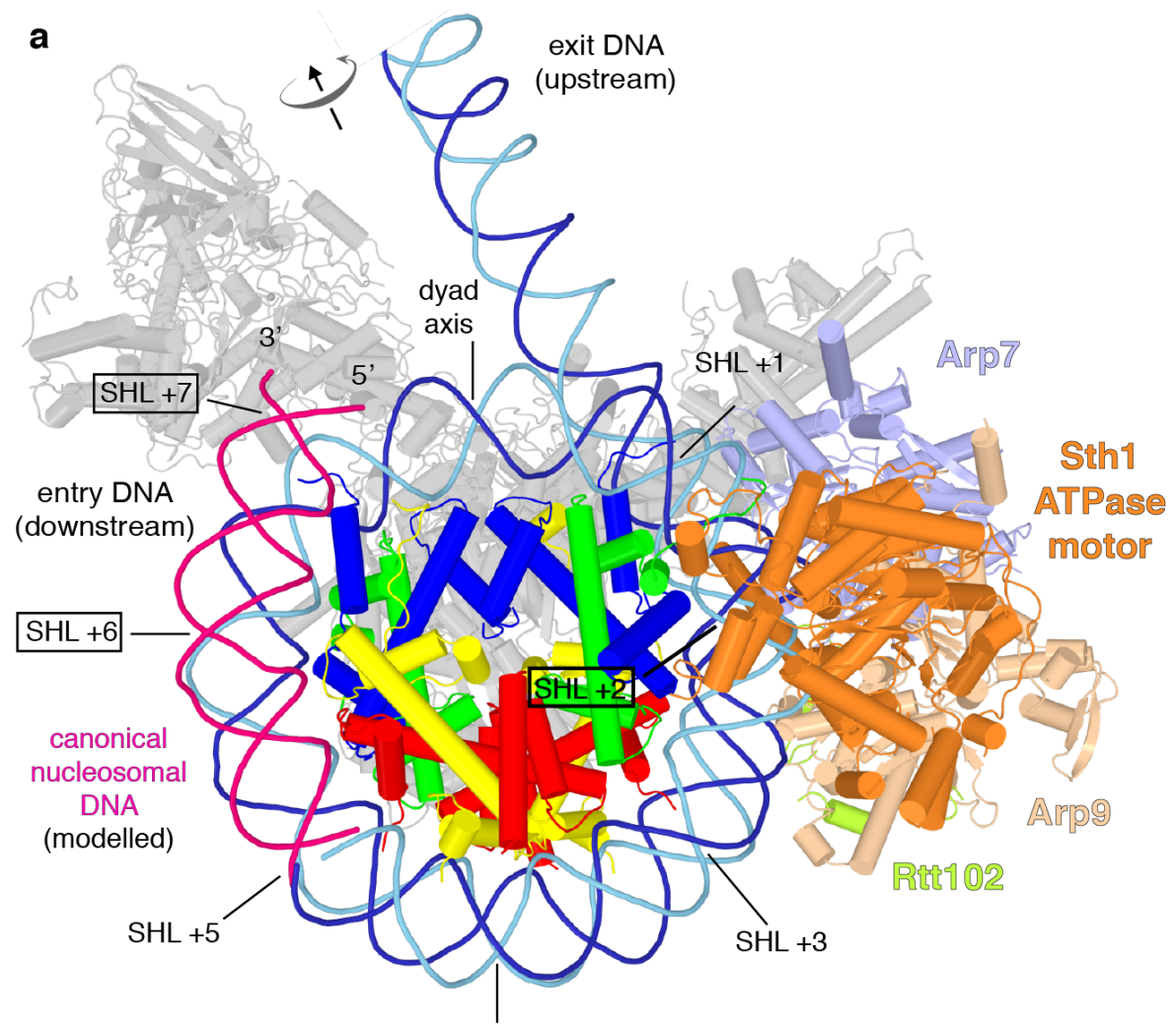

$A D$

$90^{\circ} 180^{\circ}$

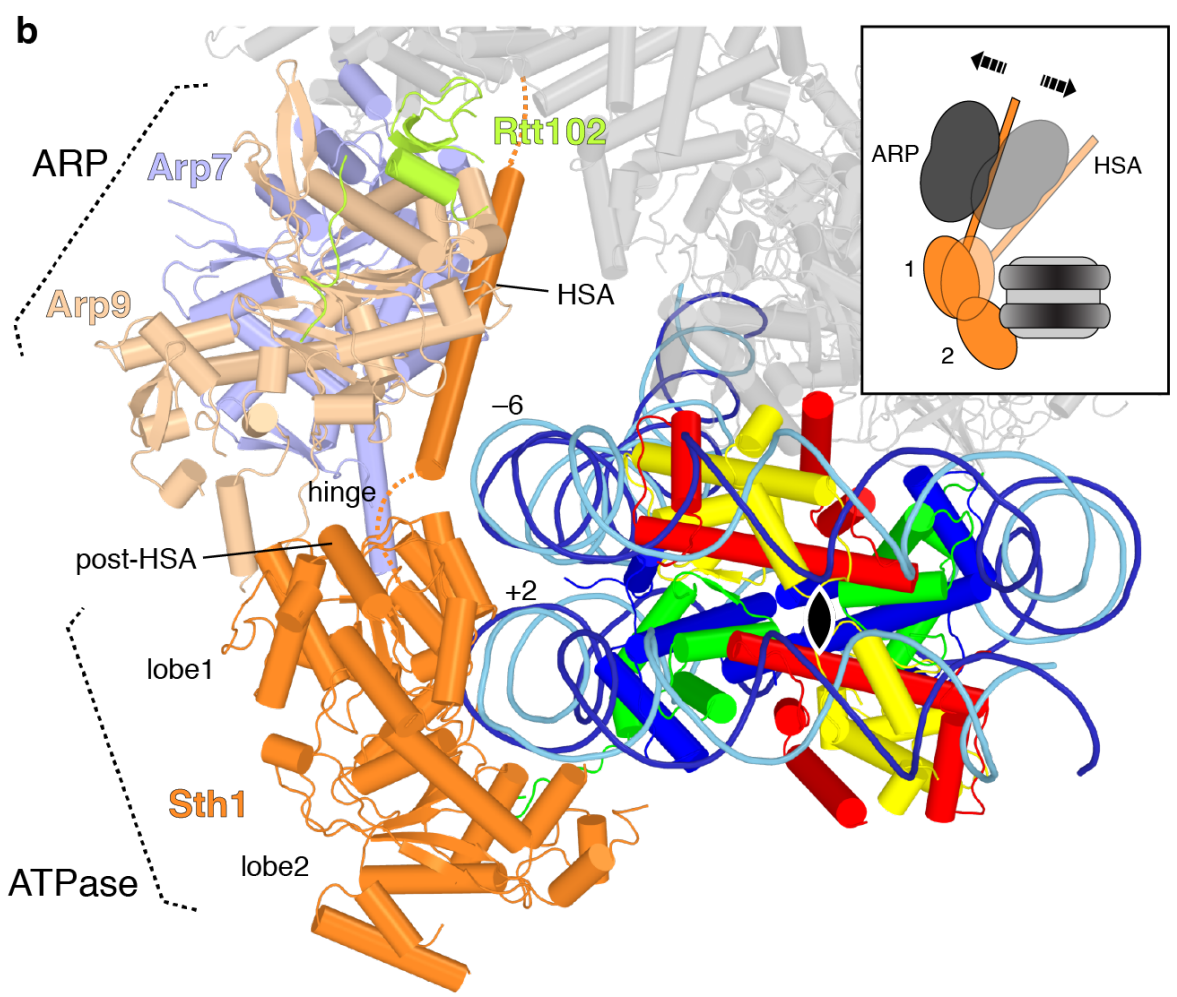

Figure 16 | ATPase-nucleosome interactions. See next page for caption. 
Figure 16 | ATPase-nucleosome interactions. Figure on previous page. a. Contacts of Sth1 ATPase motor (orange) with the nucleosome. View as in Figure 11c, left, but rotated by $45^{\circ}$ around a horizontal axis. Arrows indicate directionality of DNA translocation. Density for about $20 \mathrm{bp}$ from SHL +7 to +5 was missing and modelled with canonical nucleosomal DNA for illustration (magenta). b. View along the nucleosome dyad (black oval). View is as in Figure 11c, right, but rotated by $45^{\circ}$ around a horizontal axis. The Sth1 ATPase domain binds to the first gyre at superhelical location +2 and also interacts with its N-terminal lobe 1 with the second gyre at superhelical location -6 . The translocation and remodelling activity of the ATPase are regulated by the ARP module (Cairns et al., 1998; Clapier et al., 2016; Schubert et al., 2013). Our results suggest that the dynamic ARP module influences the position and mobility of the ATPase lobe 1 at the first DNA gyre (schematic in the box) thereby controlling the translocation activity likely via the post-HSA region.

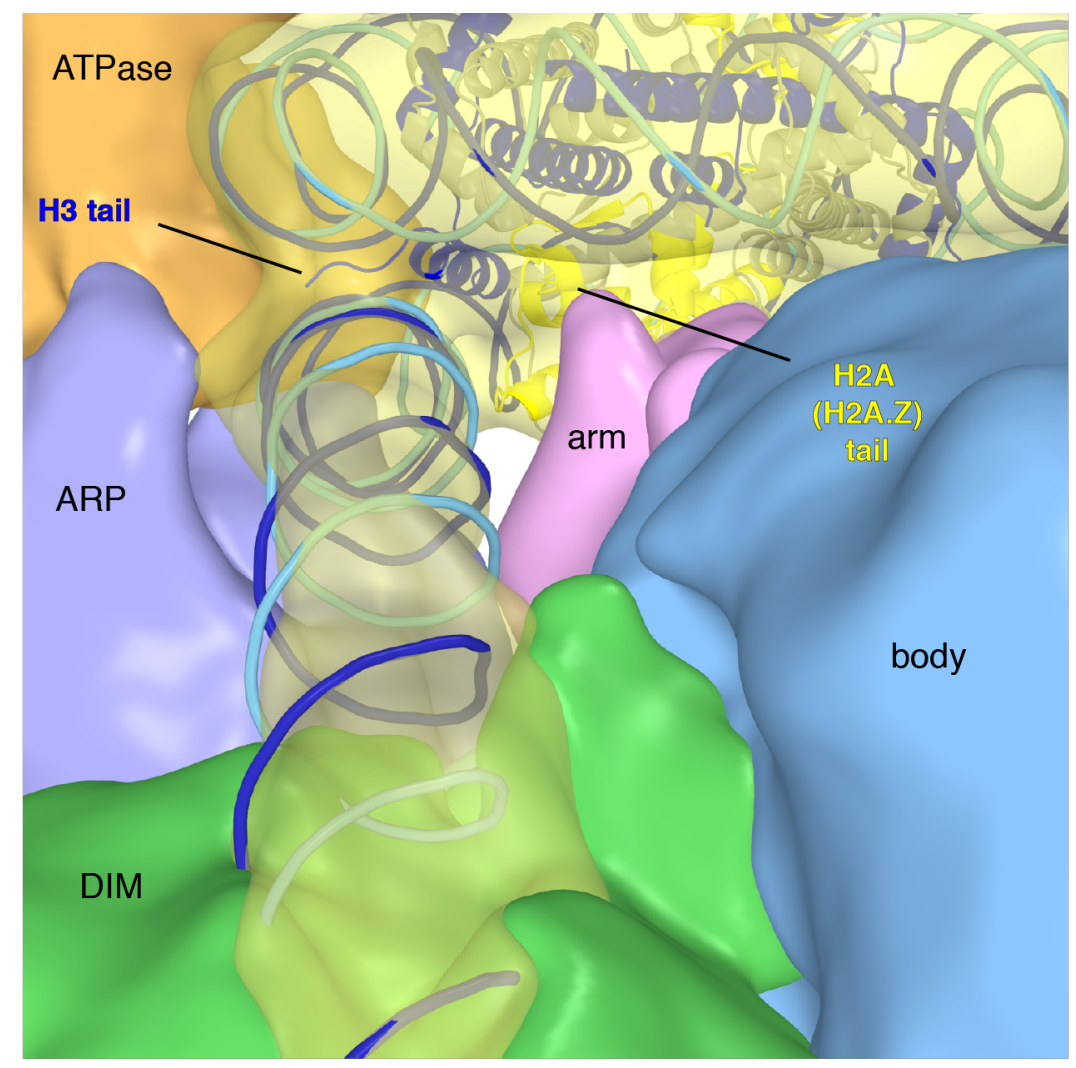

Figure 17 | Position of H2A and H3 histone tails. View along the exit DNA in the direction of the nucleosome showing the low pass-filtered maps for the modules ATPase, ARP, DIM, arm, body, and the nucleosome. At the site where the H2A Cterminal tail protrudes from the nucleosome, low resolution density connecting the arm module and the nucleosome is visible. This would also be the position where the tail of the H2A.Z variant would protrude from the nucleosome. Density bridging form the ARP module to the exit DNA close to the H3 histone tail can be observed. 


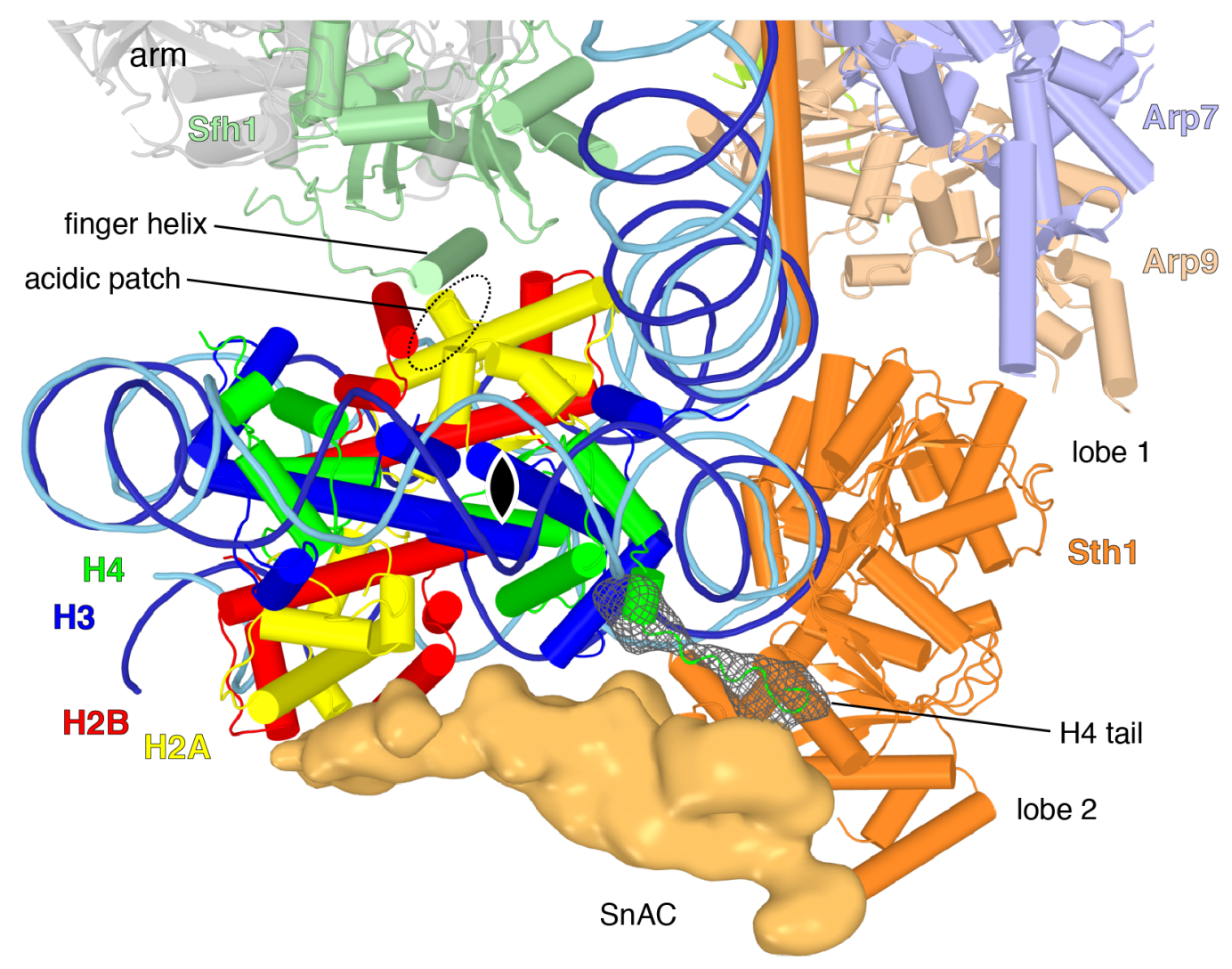

Figure 18 | RSC sandwiches the nucleosome. RSC-nucleosome interactions viewed along the nucleosome dyad (black oval). On the outer face of the histone octamer, densities for the Sth1 SnAC domain and the histone H4 tail are shown as an orange surface and a green mesh, respectively. On the inner face, the arm module and Sfh1 finger helix are depicted.

\subsection{Nucleosome sandwiching and sliding}

The structure also suggests a model for how RSC can slide nucleosomes along DNA. RSC contacts the nucleosome disc not only at the edge, but also binds both of its faces. The SnAC domain in subunit Sth1 binds the outer face of the histone octamer, whereas the arm module binds the inner face (Figure 18). Sandwiching interactions would retain the histone octamer and enable the ATPase motor to pump DNA around it, effectively sliding the octamer downstream on DNA. Consistent with this model, the SnAC domain in the SWI/SNF homologue Snf2 is important for remodelling in vivo and biochemical data suggested that it acts as a histone anchor that is required for nucleosome sliding (Sen et al., 2013). The strength of the SnAC-histone octamer contact may be influenced by the N- 


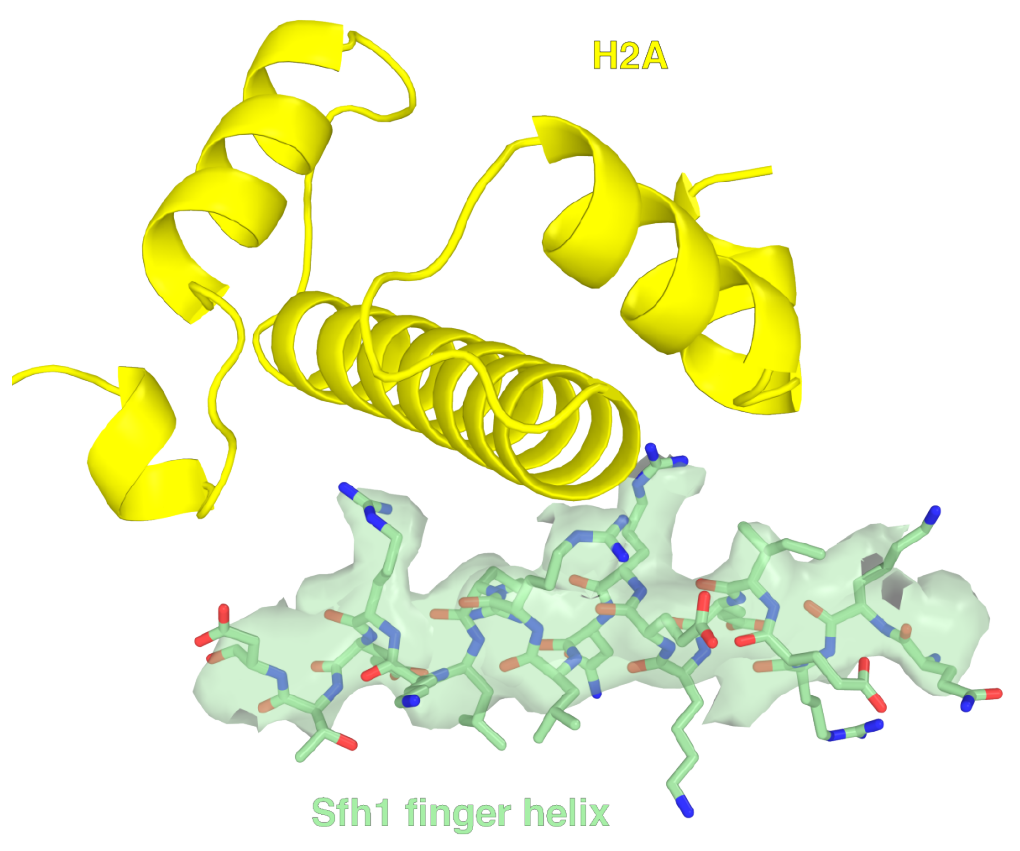

Figure 19 | Density representing the finger helix (green) at the acidic patch of the nucleosome (indicated by $\mathrm{H} 2 \mathrm{~A}$ in yellow). Side chain density is visible for conserved arginine residues.

terminal tail of histone $\mathrm{H} 4$, which binds at the interface of the SnAC and ATPase motor of Sth1 (Figure 18). Since histone acetylation can impair octamer transfer by RSC to the histone chaperone Nap1 (Lorch et al., 2018), this leads to the intriguing model that histone acetylation may strengthen the sandwiching contacts, thereby impairing octamer eviction and favouring nucleosome sliding. Binding of the arm module to the inner face of the histone octamer is mediated by an exposed 'finger' helix, which resides in the Cterminal region of subunit Sfh1 that is required for normal cell growth (Cao et al., 1997) (Figure 13b, Figure 18, Figure 19). The finger helix contains four arginine residues (R397, R400, R401 and R404) that contact the acidic patch of the octamer (Figure 20a). Three of these arginines are known to be mutated in human cancers (Figure 20a), pointing to the functional significance of the finger helix-acidic patch interaction. The finger helix and its arginine residues are highly conserved in Sfh1 homologues throughout eukaryotes (Figure 20b). The SnAC domain is also conserved over species and between SWI/SNF complexes (Sen et al., 2011), suggesting that the sandwiching mechanism of nucleosome sliding is used by all SWI/SNF family complexes. 

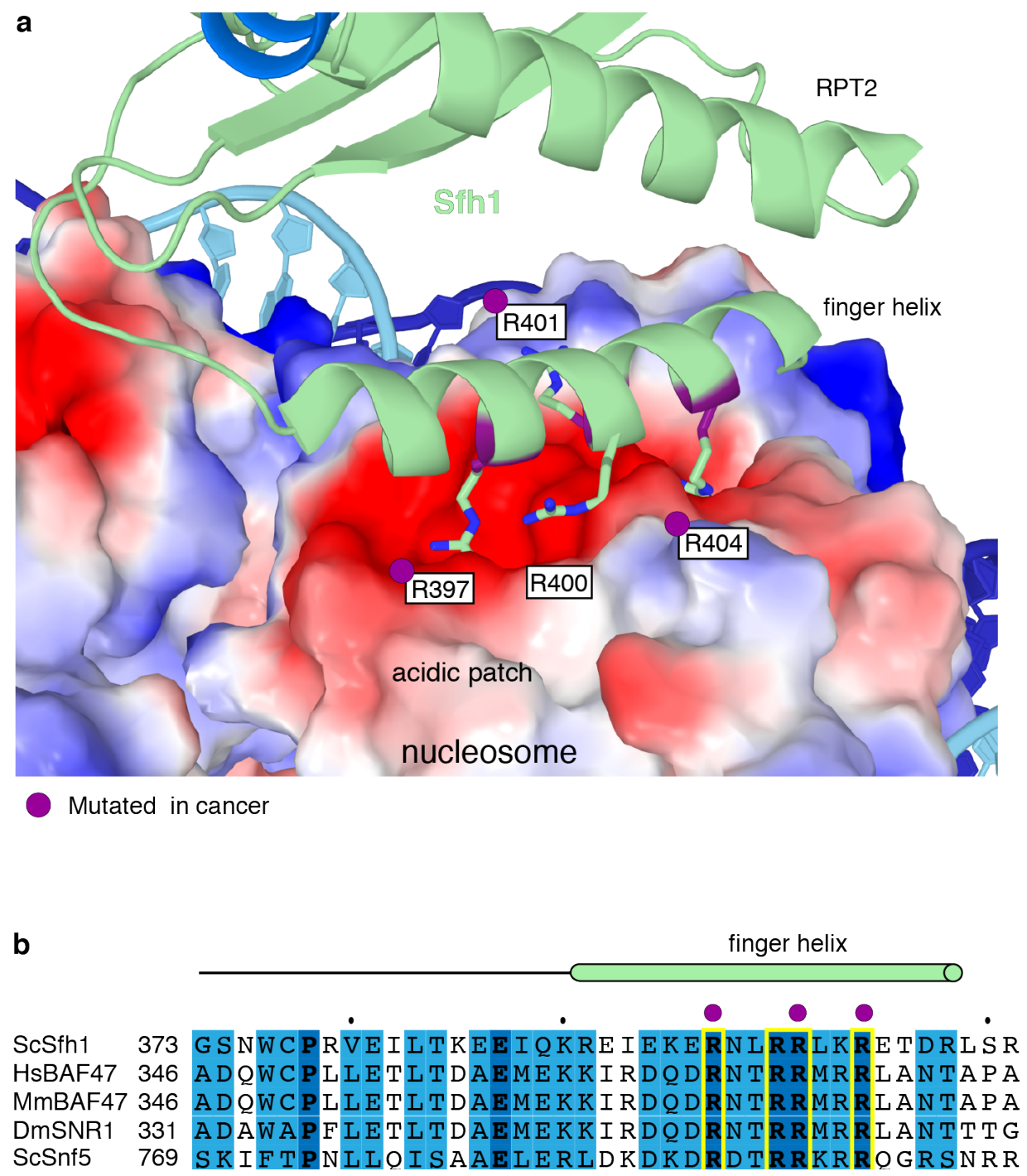

Figure 20 | Interactions of the Sfh1 finger helix with the acidic patch of the nucleosome. a. Interaction of the Sfh1 finger helix with the acidic patch of the inner face of the histone octamer (surface representation coloured by electrostatic charge; red, negative; blue, positive). Conserved arginine residues are shown with side chains. Residues mutated in human cancer (Methods) are highlighted in purple. b. Sequence alignment of the finger helix region (green cylinder) in $S$. cerevisiae (Sc) Sfh1 with its homologues H. sapiens (Hs) BAF47, M. musculus (Mm) BAF47, D. melanogaster (Dm) SNR1 and ScSnf5. Invariant and conserved residues in dark and light blue, respectively. Arginine residues shown in (a) highlighted in yellow. Purple dots mark residues mutated in cancer (section 2.18). 


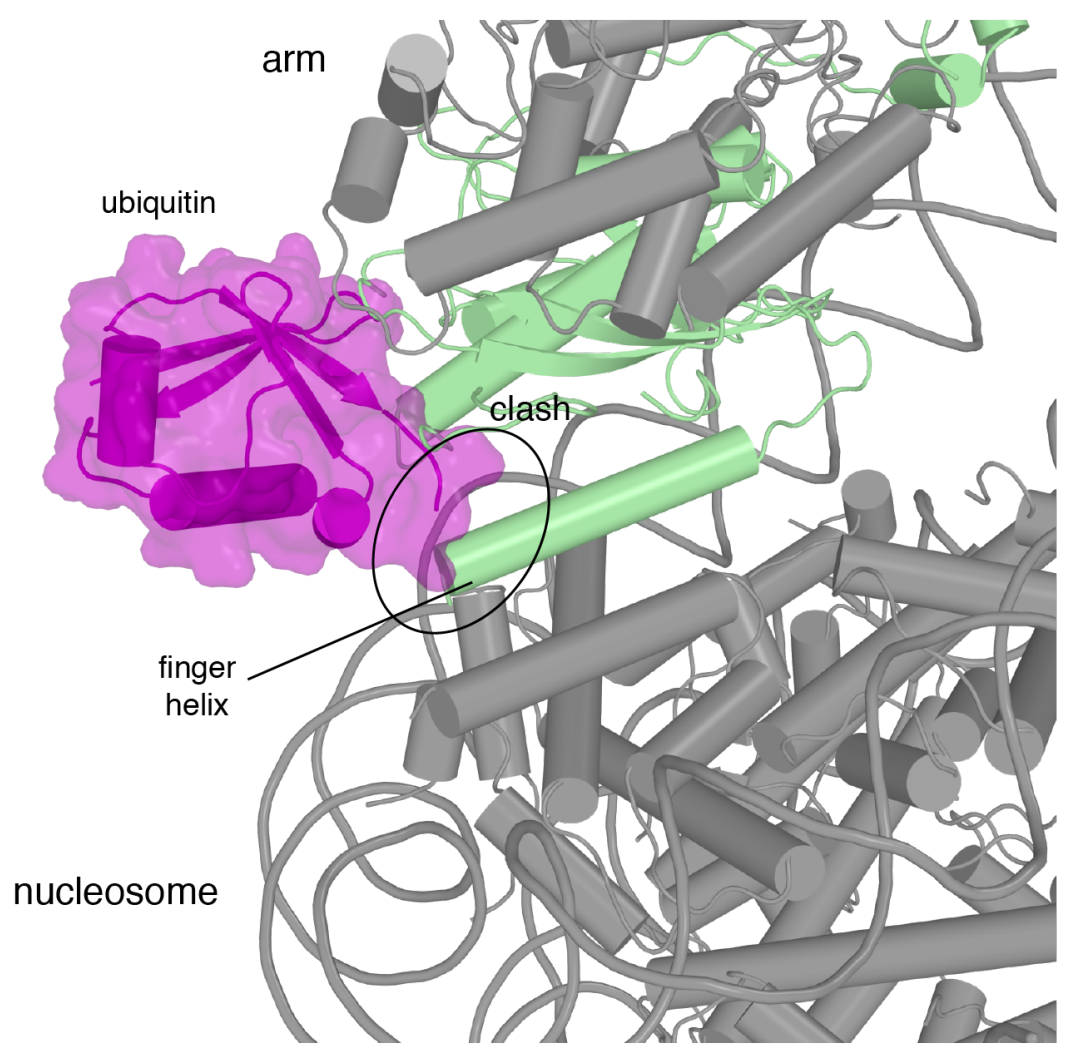

Figure 21 | Interaction of RSC with the nucleosome is sterically impaired by H2B ubiquitylation at K120. The Sfh1 finger helix and the ubiquitin moiety (ubiquitylated nucleosome PDB code 6NOG) (Worden et al., 2019) overlap after superposition of nucleosomes.

The arm module and its finger helix may also contribute to substrate selection. RSC preferentially recognizes nucleosomes that contain the histone variant H2A.Z (Cakiroglu et al., 2019). Such nucleosomes show a more extended acidic patch (Suto et al., 2000) and may have increased affinity for the basic RSC finger. The arm module may also contact the C-terminal tail of H2A.Z (Figure 17) that differs in ten amino acid residues from the tail of H2A in yeast. The observed arm-octamer interaction may also explain why ubiquitination of histone H2B counteracts RSC function (Materne et al., 2016). The ubiquitin moiety attached to $\mathrm{H} 2 \mathrm{~B}$ residue K123 (human K120) is predicted to sterically interfere with the arm-octamer interaction (Figure 21). 


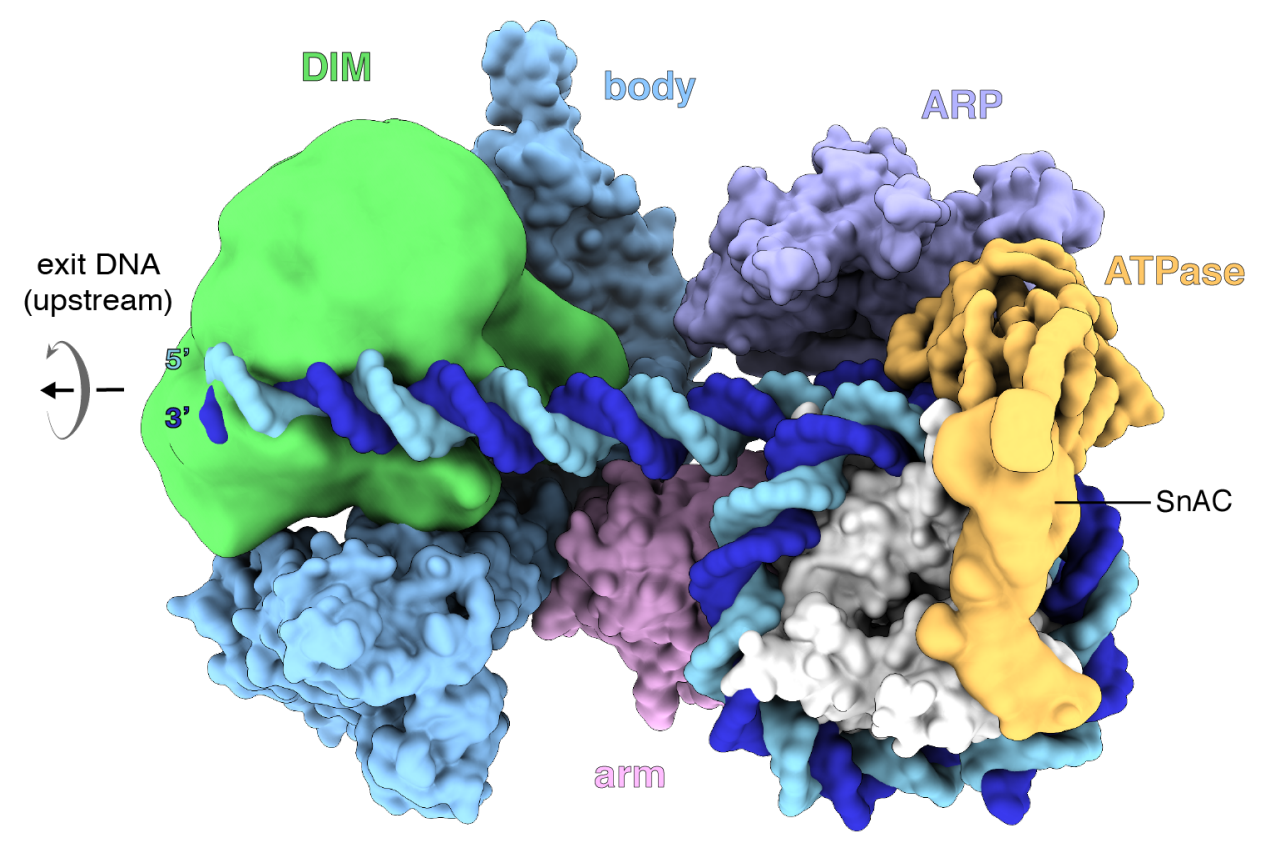

$9-$

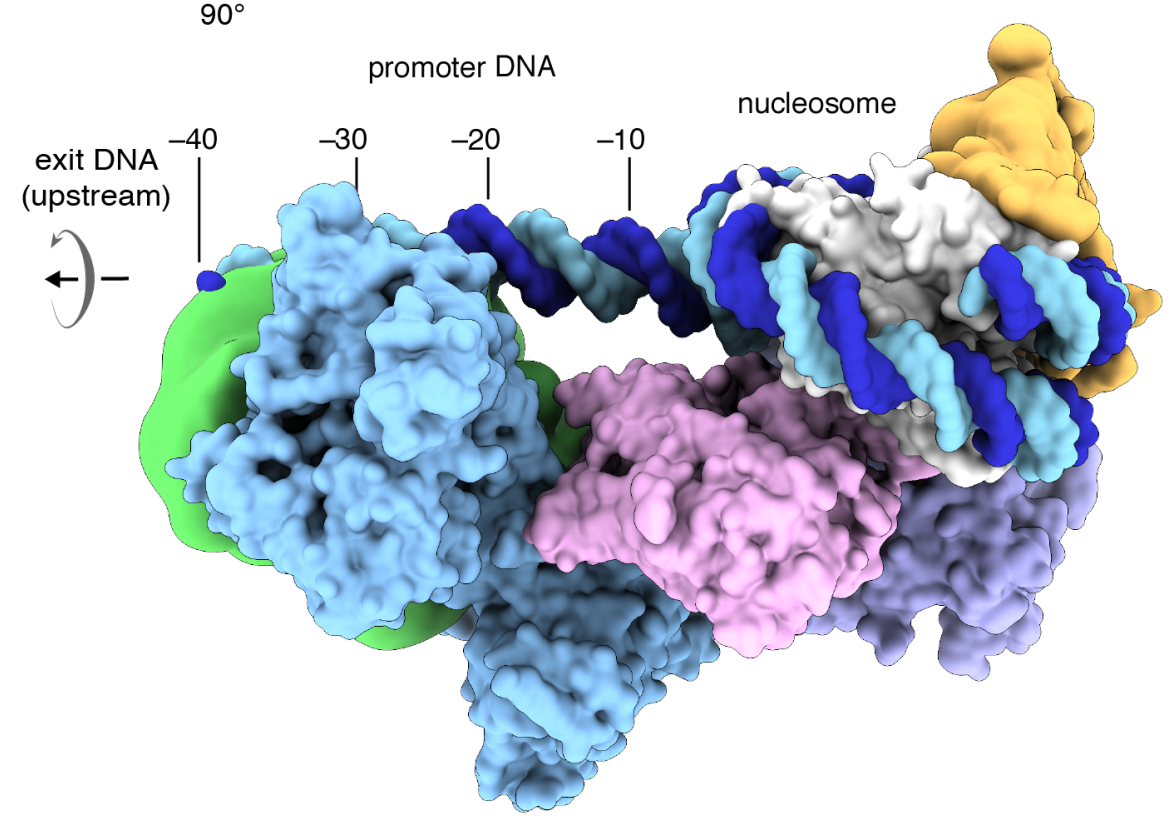

Figure 22 | DNA recognition and NDR formation. Space-filling RSC-nucleosome structure with DIM (green) and SnAC (orange) densities. View on the top as in Figure $11 \mathrm{c}$, left, but rotated by $90^{\circ}$. Arrows indicate directionality of DNA translocation. Number of upstream DNA base pairs relative to SHL -7 is provided. The exiting, upstream DNA spans the central cavity of RSC. 


\subsection{DNA recognition and NDR formation}

RSC not only binds the nucleosome, but also DNA that exits from it (Figure 22). The DIM module contacts exiting DNA $\sim 20-40 \mathrm{bp}$ upstream of SHL -7 of the nucleosome. This is in agreement with RSC protecting $\sim 50$ bp of extra-nucleosomal DNA from nuclease digestion (Brahma and Henikoff, 2019). The DIM-DNA contact also explains how RSC recognizes specific DNA elements that are enriched in promoters (Badis et al., 2008; Kubik et al., 2015; Kubik et al., 2018; Lorch et al., 2014). Consistent with cross-linking information, the RSC subunits Rsc2, Rsc3 and Rsc30 are located in the DIM (Figure 13b). Rsc3 and Rsc30 are known to interact (Angus-Hill et al., 2001) and recognize a CGCG DNA element located upstream of the transcription start site (Badis et al., 2008). They may bind DNA via their N-terminal zinc cluster domains (Angus-Hill et al., 2001; Badis et al., 2008). It remains to be seen to what extent promoter targeting by RSC depends on its binding to DNA sequence, histone modifications, and the presence of histone variant H2A.Z.

The results also elucidate the formation of NDRs. In $S$. cerevisiae, the DNA linker length between two nucleosomes is only $\sim 23$ bp on average (Brogaard et al., 2012). Steric considerations predict that RSC can enter chromatin only at sites where the length of the DNA linking two nucleosomes is at least 40 - 50 bp (Figure 23). This can explain why RSC is targeted only to promoter regions, which are intrinsically nucleosome-depleted to some extent. NDR formation involves sliding of both flanking nucleosomes away from the NDR centre (Krietenstein et al., 2016). Here we have interpreted the RSC-nucleosome structure to describe RSC action at the +1 nucleosome, but the structure can equally describe RSC action at the -1 nucleosome. In the latter case, DNA exits in downstream direction, rather than upstream, the ATPase engages with SHL -2 , rather than SHL +2, and DNA translocation slides the nucleosome upstream, rather than downstream. Provided that RSC remains bound to both flanking nucleosomes after remodelling, a minimum NDR size of $\sim 100$ bp would result (Figure 23). However, larger NDRs can be formed when RSC evicts a nucleosome (Kubik et al., 2015). 


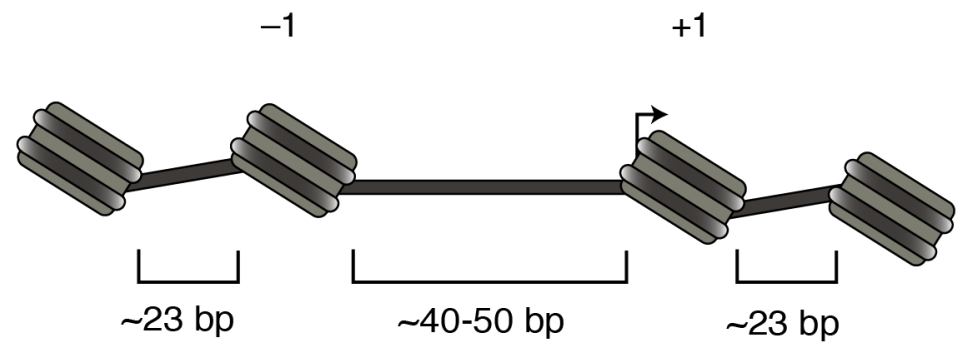

\section{NDR formation}
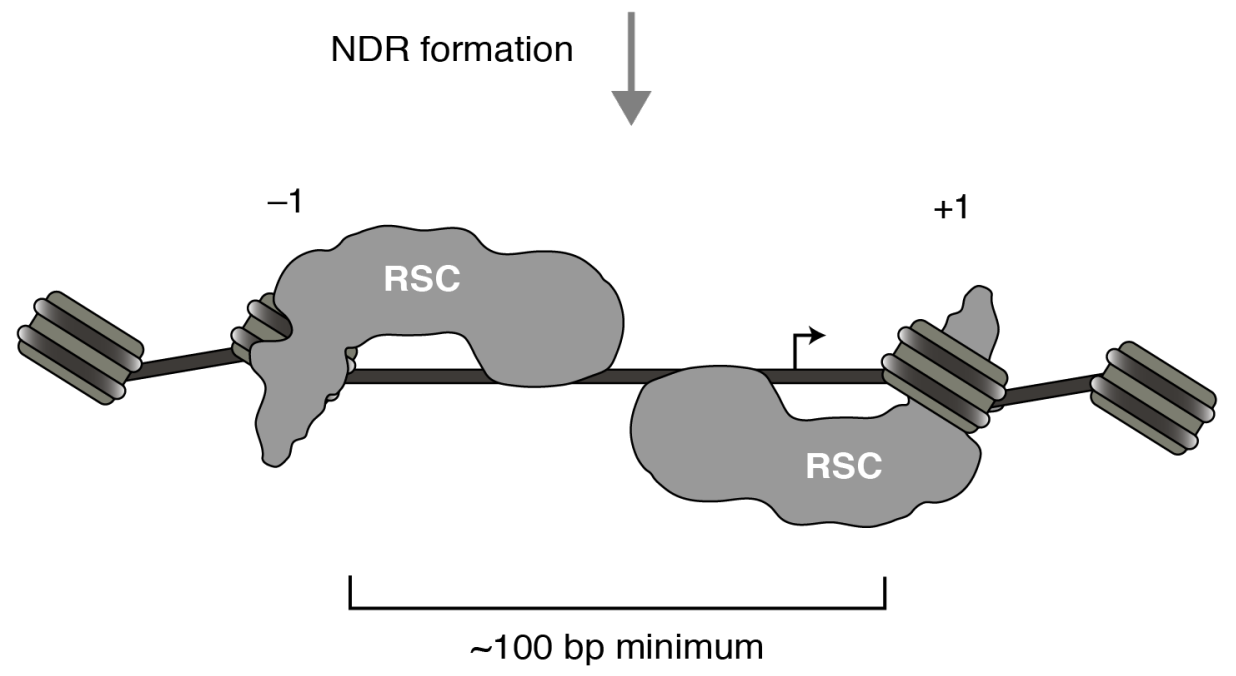

Figure 23 | Model of RSC remodelling at a promoter. Schematic of a promoter before (top) and after (bottom) RSC remodelling. RSC binds to the +1 nucleosome at SHL +2 and the -1 nucleosome at SHL -2 . In both positions it translocates the DNA towards the centre, thereby increasing the NDR by sliding the flanking -1 and +1 nucleosomes away. Arrows indicate the transcription start site.

\subsection{SWI/SNF family and cancer}

The PBAF complex is the human counterpart of RSC and contains subunits homologous to Sth1, Rsc6, the Rsc8 dimer, Sfh1, Arp7 and Arp9 (Table 1). In addition, the PBAF subunit BAF200 (Yan et al., 2005) contains an armadillo repeat fold (Waterhouse et al., 2018) and likely corresponds to Rsc9. Further, the BAF180 subunit comprises regions that resemble Rsc2 and Rsc4 (Xue et al., 2000). Only the small RSC subunits Rsc58, Rtt102 and Htl1 lack obvious counterparts. Therefore, the yeast RSC structure is a good model for human PBAF. Projection of the homologous regions onto the RSC structure reveals that the ATPase, ARP and arm modules are well conserved in PBAF, and that the body is at least partially conserved (Figure 24). The DIM differs substantially in PBAF because human counterparts of subunits Rsc3 and Rsc30 are not known. However, PBAF subunits contain 


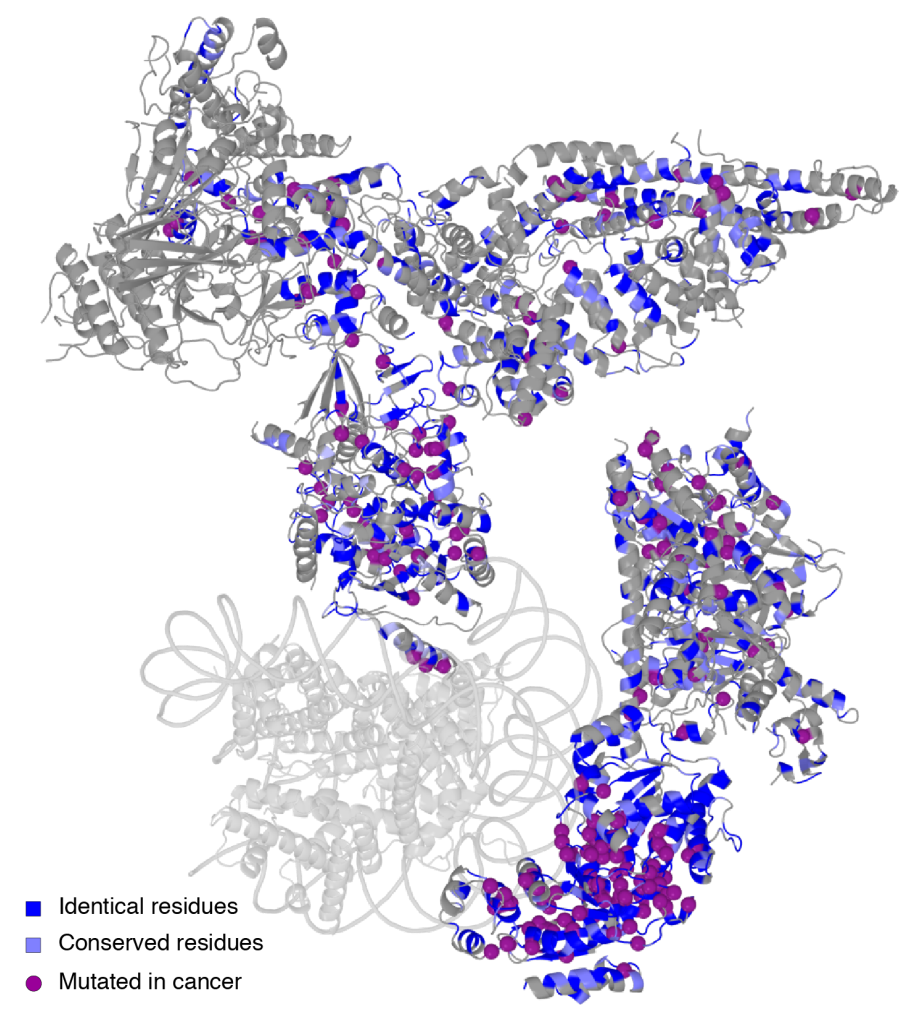

Figure 24 | Conservation between SWI/SNF complexes RSC (yeast) and PBAF (human). Residues that are identical (blue) or conserved (light blue) in human PBAF highlighted on the RSC structure (grey). Purple spheres depict identical residues that show missense mutations in various cancers (section 2.18).

12 putative DNA-binding domains located in subunits BAF180 (HMG box) (Nicolas and Goodwin, 1996), BAF200 (AT-rich domain, two C2H2 zinc fingers, RFX domain) (Yan et al., 2005), BAF57 (HMG box) (Wang et al., 1998) and BCL11A/B (six C2H2 zinc fingers) (Satterwhite et al., 2001) that may mediate DNA recognition.

The RSC structure also suggests the architecture of the related yeast SWI/SNF complex and its human counterpart BAF. Based on subunit composition and sequence homologies (Figure 25), the yeast SWI/SNF complex contains RSC-related ATPase, ARP, and arm modules, whereas its body module is apparently smaller. The human BAF complex contains counterparts of RSC subunits Sth1, Sfh1, Arp7, Arp9, Rsc6, and Rsc8. The BAF subunit BAF250a is predicted to contain five armadillo repeats (Sandhya et al., 2018), and is likely the counterpart of Rsc9. Thus, BAF also contains the ATPase, ARP and arm modules, and a body module that is at least partially conserved. 
a ScSth1 38 $\operatorname{ScSnf2} \quad 666$
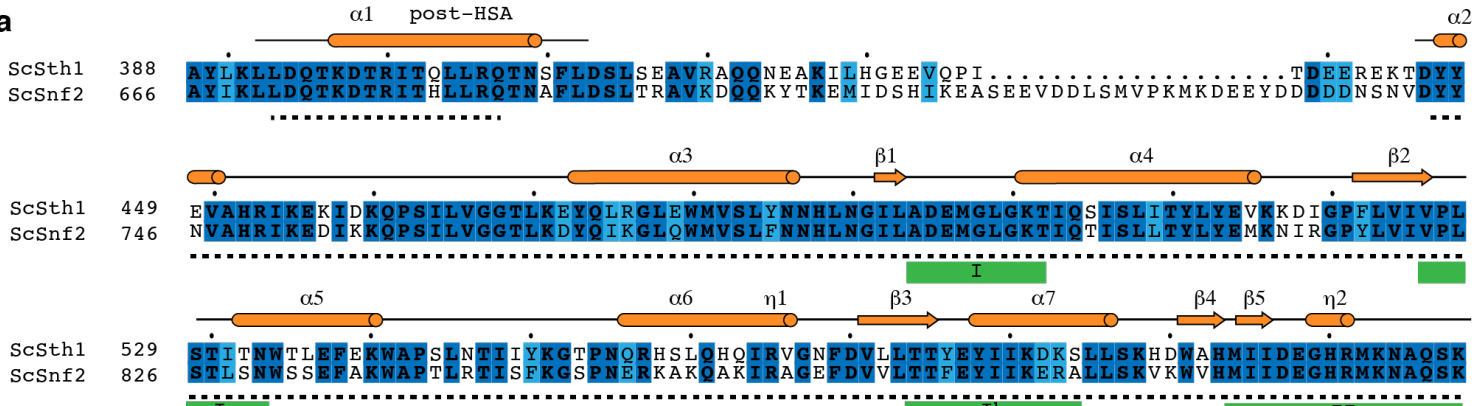

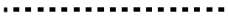

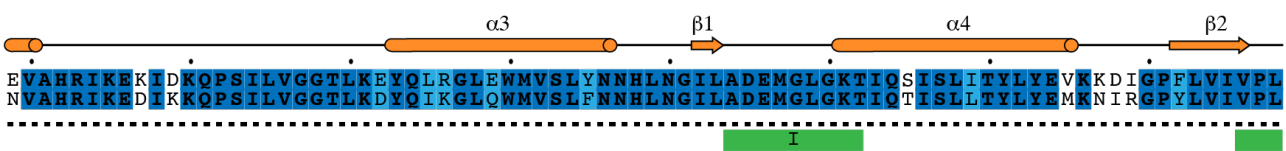

$\alpha 5$ $\beta 3 \quad \alpha 7$ $\underbrace{\beta 4} \underbrace{\beta 5} \overbrace{}^{2}$ ScSnf2 826
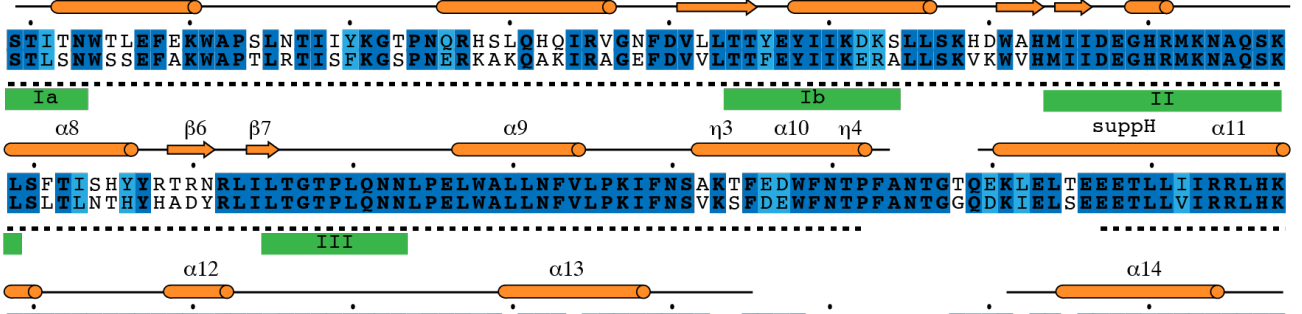

ScSth1 609 ScSnf2 906

$\begin{array}{ll} & \\ \text { ScSth1 } & 689 \\ \text { ScSnf2 } & 986\end{array}$

vic$$
\begin{array}{ll}
\text { ScSth1 } & 769 \\
\text { ScSnf2 } & 1065
\end{array}
$$

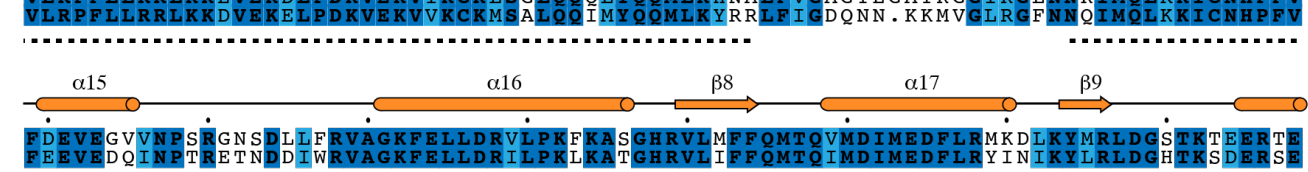
$\operatorname{ScSnf2} 1065$

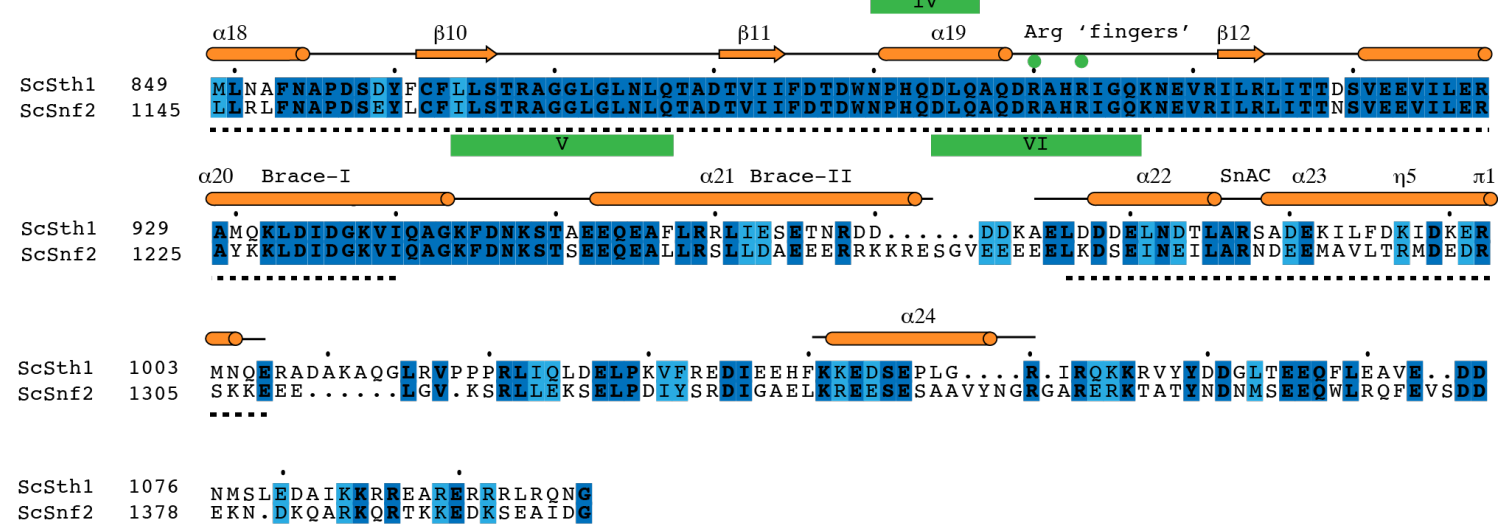

b

HSA

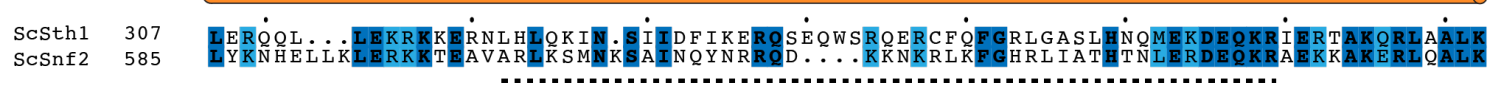

Figure 25 | Sequence alignments for the Sth1 ATPase domain and HSA region. a. Sequence alignment of the $S$. cerevisiae Sth1 ATPase domain to the homologous Snf2 ATPase domain of the same organism. Secondary structure elements are represented in orange according to the cryo-EM structure of the Snf2 ATPase (PDB entry 5Z3U) (Li et al., 2019). Residues modelled in the Snf2 structure are topped by a back line with helical regions shown as cylinders and sheet regions as arrows. The Sth1 residues modelled in this work are indicated with a black dashed line below. ATPase motifs are underlined in green. Invariant residues are coloured in dark blue and conserved residues in light blue. The alignment was generated with MSAProbs (Liu et al., 2010) within the MPI Bioinformatics Toolkit (Zimmermann et al., 2018) and visualized using ESPript (Robert and Gouet, 2014). b. Sequence alignment of the HSA regions from $S$. cerevisiae homologues Sth1 and Snf2. Illustration and generation of the alignment as in (a). 

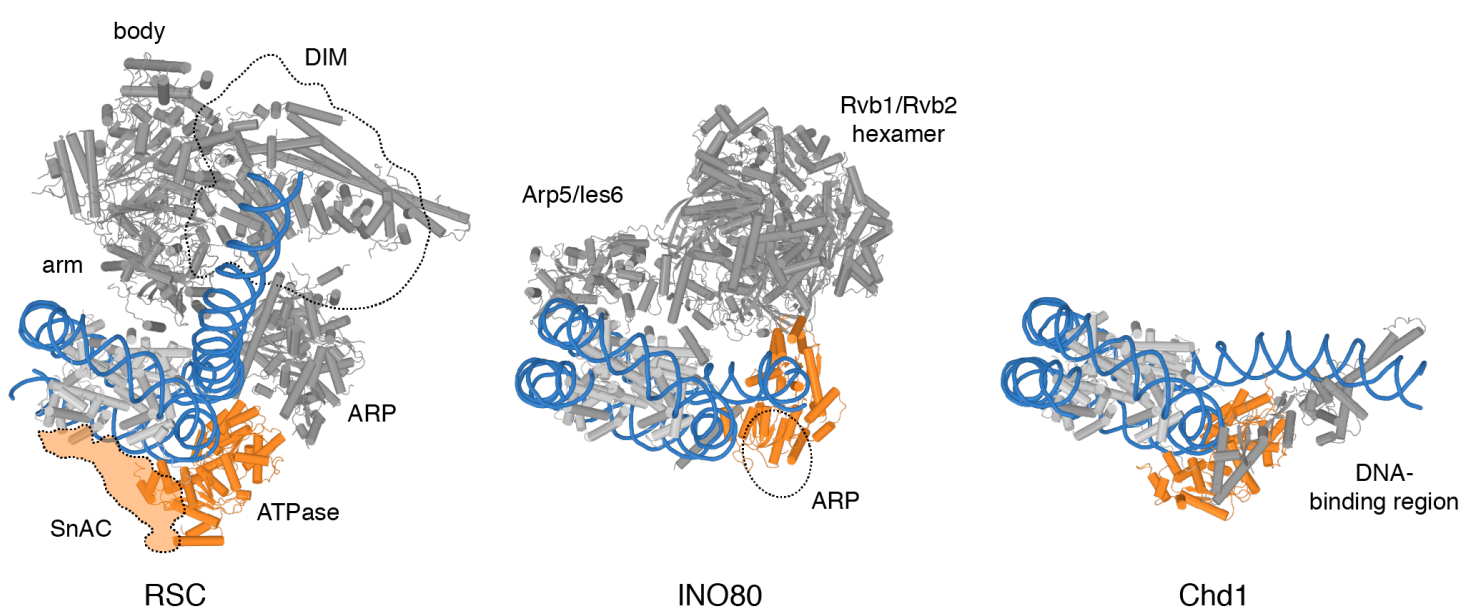

Chd1

Figure 26 | Comparison of overall structure of RSC with complexes of CHD (yeast Chd1 (Farnung et al., 2017)) and INO80 (yeast INO80 (Eustermann et al., 2018)) families. ATPase motor domains are shown in orange, DNA in blue.

Due to these homologies, the RSC structure can be used to locate protein sites in PBAF that are known to be mutated in human cancers. This analysis shows that cancerassociated mutations are scattered throughout the remodelling complex (Figure 24). Most mapped mutations are located inside the structured modules and are predicted to destabilize protein folds. However, mutations are particularly enriched within the ATPase, ARP and arm modules that surround and contact the nucleosome, suggesting that they cause functional defects.

\subsection{Diversity in chromatin remodellers}

Finally, we compared the RSC-nucleosome structure with nucleosome complex structures of the three other families of chromatin remodelling factors (Figure 26). Whereas RSC is a good model for the SWI/SNF family, factors of the ISWI, CHD and INO80 families are clearly distinct. With respect to the ISWI family, the ATPase motor binds SHL +2 (Yan et al., 2016; Yan et al., 2019), but other interactions have not been structurally resolved.

With respect to the CHD family, the ATPase motor of yeast Chd1 also binds SHL +2, but its DNA-binding region engages with exiting DNA in close proximity to the nucleosome, leading to a different trajectory of exit DNA (Farnung et al., 2017; Sundaramoorthy et al., 2018). 
With regard to the IN080 family, the ATPase motor of the SWR1 complex also binds SHL +2 (Willhoft et al., 2018), whereas the ATPase of the INO80 complex binds SHL -6 (Ayala et al., 2018; Eustermann et al., 2018). IN080 also contains an ARP module (Knoll et al., 2018), which however contacts exit DNA in a manner that is distinct from the DIM-DNA contacts observed for RSC. The INO80 complex also contains flexible protein extensions, called the 'Arp5 grappler' and the 'Ies RAR', which can contact both faces of the histone octamer (Eustermann et al., 2018), but these contacts differ substantially from the sandwiching interactions formed by RSC. In conclusion, the RSC-nucleosome structure provides mechanistic insights into the fourth family of chromatin remodelling complexes, and provides a basis for investigating the targeting, regulation, and cellular roles of SWI/SNF family complexes. 


\section{DISCUSSION}

\subsection{RSC in context of the four remodeller families}

The various chromatin remodellers were classified by their ATPase subunits into the ISWI, CHD, INO80 and SWI/SNF families (Figure 2) (Clapier and Cairns, 2009). The ATPase subunits of all four families share a common ATPase motor domain formed by two RecA-like lobes, but they differ strongly in the accompanying $\mathrm{N}$ - and C-terminal domains. In addition, the number and type of supplementary subunits which compose a complete remodeller vary. Reflecting these differences, the tasks fulfilled by the four remodeller families in the cell are distinct (Clapier and Cairns, 2009; Clapier et al., 2017). However, based on the conserved motor domain, a similar basic remodelling mechanism has been suggested for all remodellers and the specific remodelling outcome would be directed through the additional set of domains and subunits of each remodeller type (Clapier et al., 2017).

The recent developments in cryo-electron microscopy resulted in structures of chromatin remodellers bound to their nucleosome substrate and mechanistic insights. These structures include the Chaetomium thermophilum (Eustermann et al., 2018) and human (Ayala et al., 2018) IN080 complex, the yeast SWR1 complex (Willhoft et al., 2018), the human CHD4 (Farnung et al., 2019) and yeast Chd1 remodellers (Farnung et al., 2017), the yeast Isw1 ATPase subunit (Yan et al., 2019), as well as the yeast Snf2 ATPase subunit (Li et al., 2019; Liu et al., 2017). The structure of the RSC remodeller presented here extends this collection significantly. It is the first SWI/SNF family structure encompassing additional subunits and therefore elucidates the organisation of this multi-subunit family beyond the single ATPase.

\subsubsection{Remodeller activation upon substrate binding}

It is important for cells to control the activity of chromatin remodellers and only allow them to work at their dedicated substrate. The structures of the past years revealed a common mechanism to inactivate free, not substrate bound, remodellers. In the free 
state, their ATPase lobes are oriented in an inactive open state (Hauk et al., 2010; Xia et al., 2016; Yan et al., 2016).

In CHD remodellers the open state is stabilized by the tandem chromodomain which packs against the DNA-binding surface of the ATPase (Hauk et al., 2010). Upon binding of the remodeller to the nucleosome at SHL +2 , the tandem chromodomain binds to the DNA at SHL +1 thereby freeing the ATPase lobes. As a result, lobe 2 rotates about $40^{\circ}$ forming an active closed conformation with lobe 1 (Farnung et al., 2017).

For the ISWI family, the two RecA-like ATPase lobes of Isw1 are enclosed by the AutoN and NegC domains which both inhibit the remodeller when not bound to the nucleosome (Clapier and Cairns, 2012). Both NegC and AutoN bind to lobe 2 and keep it in an inactive conformation (Yan et al., 2016). Binding of the Isw1 ATPase to SHL +2 causes a $\sim 148^{\circ}$ rotation of lobe 2 towards lobe 1 (Yan et al., 2019). NegC is antagonised and disordered by Isw1's HAND-SANT-SLIDE (HSS) DNA-binding domain. The N-terminal AutoN packs against protrusion 1 (also termed SuppH (Yan et al., 2016; Yan et al., 2019)) and couples ATP hydrolysis to DNA translocation.

An interesting aspect is that the position of the AutoN superimposes with the postHSA region of Snf2 and Sth1 of the SWI/SNF and RSC remodellers, respectively. The protrusion 1 helix, the post-HSA helix and the brace helix are very important regulatory elements in Sth1 and Snf2 ATPases that couple ATP hydrolysis to nucleosome sliding (Clapier et al., 2016; Li et al., 2019; Liu et al., 2017).

SWI/SNF family ATPases lack a tandem chromodomain or the AutoN and NegC elements, but a similar mode of activation has been observed. The Snf2 ATPase lobes are rotated into an inactive conformation which is stabilized by stacking interactions between the two lobes (Xia et al., 2016). Binding to the nucleosome causes lobe 2 to rotate $\sim 80^{\circ}$ towards lobe 1 forming an active enzyme (Liu et al., 2017).

RSC's ATPase module could not be resolved in the free state likely due to its flexibility with respect to the arm and body modules. Therefore, structural information on orientation of the free ATPase lobes 1 and 2 of Sth1 are missing. However, the extremely high conservation between Snf2 and Sth1 (Figure 25) strongly suggest the same inactive conformation. In addition, the structure of the Sth1 ATPase module bound the nucleosome and inhibited by $\mathrm{ADP}-\mathrm{BeF}_{3}$ presented here resembles the active mode of the ATPase lobes 1 and 2. 


\subsubsection{Universal mechanism of chromatin remodelling}

The structural studies, including the here presented RSC, as well as biochemical experiments indeed strongly support a unifying basic ATPase mechanism of translocation for all remodeller families (Clapier et al., 2017). The ATPase motor interacts with the DNA minor groove and ATP hydrolysis causes a conformational change between the two RecA-like lobes which leads to translocation in the $3^{\prime}$ to $5^{\prime}$ direction (section 1.2.2). Hydrolysis of one ATP molecule results in a single base pair step. Anchoring of the motor to the histone octamer results in an effective movement of the DNA around the nucleosome in the $5^{\prime}$ to 3 ' direction.

The motor of the IN080 complex was found to bind at the nucleosomal superhelical position (SHL) -6 to pump linker DNA into the nucleosome (Ayala et al., 2018; Eustermann et al., 2018). To date, this is the exception compared to all other remodeller ATPases, which bind to SHL +2 , but also translocate DNA from the proximal linker towards the dyad into the nucleosome (Farnung et al., 2019; Farnung et al., 2017; Li et al., 2019; Liu et al., 2017; Willhoft et al., 2018; Yan et al., 2019).

This observation underlines the important nature of the auxiliary domains and subunits that distinguish the four families and add a layer of diversification to the mechanism of remodelling.

\subsubsection{Interactions with extra-nucleosomal DNA}

An important task carried out by the additional subunits is the interaction with extranucleosomal DNA. The nature of these interactions varies for each remodeller family and reflects the different functions.

Although not structurally resolved for the ISWI remodeller, Isw1's HAND-SANTSLIDE (HSS) DNA-binding domain functions as a ruler that measures extra-nucleosomal DNA and allows remodelling by freeing the NegC inhibition only if the linker DNA is of sufficient length (Clapier and Cairns, 2012; Yan et al., 2019). This feature likely allows the ISWI remodeller to measure inter-nucleosomal distances and enables it to organise the spacing of the nucleosomal array.

Chd1 contains a C-terminal SANT-SLIDE DNA-binding domain which binds to proximal linker DNA. This interaction brakes histone DNA contacts at H2A, H2B and H3, 
and alters the trajectory of the exit DNA from SHL -7 to -5 by lifting it off the octamer surface (Farnung et al., 2017). The detachment of the DNA is believed to facilitate the path of a transcribing RNA polymerase II through a nucleosome. In addition, similar to the ISWI family, Chd1 is involved in nucleosome spacing and the DNA-binding domain could be used as a ruler to measure nucleosome distances (Clapier et al., 2017). Human CHD4 lacks the DNA-binding domain and no interactions with linker DNA were observed (Farnung et al., 2019).

With regard to the IN080 family, interactions with extra-nucleosomal DNA were also observed via the ARP module of INO80 (Knoll et al., 2018). In addition, binding of its ATPase subunit Ino80 to SHL -6 causes about 15 bp of canonical DNA to unwrap from the nucleosome between SHL -5.5 and -7 (Eustermann et al., 2018). In SWR1 the subunits Arp6 and Swc6 engage the nucleosomal DNA distal to the ATPase module and cause the exposure of $\sim 2.5$ turns of canonical DNA by bending it $\sim 65^{\circ}$ (Willhoft et al., 2018). In both cases, the unwrapping liberates histone DNA interactions and likely facilitates the histone variant exchange reported for both the INO80 and SWR1 complexes (Brahma et al., 2017; Eustermann et al., 2018; Mizuguchi et al., 2004; Papamichos-Chronakis et al., 2011; Willhoft et al., 2018).

The structure of RSC bound to a nucleosome solved in this study showed that RSC binds upstream extra-nucleosomal DNA via its DNA-interaction module (DIM) 20 - 40 base pairs (bp) upstream of the SHL -7. In contrast to the other remodellers, this interaction does not cause a deviation of the DNA trajectory from the canonical path around the nucleosome. Since the DIM binds upstream of the +1 nucleosome in the nucleosome depleted regions (NDRs) opposite to the remodelling direction of RSC (which shifts the nucleosome in the downstream direction), it is likely not used to measure internucleosome distances during remodelling. In agreement with this, RSC was not reported to establish chromatin arrays. However, the DIM highly likely contains the subunits Rsc3 and Rsc30 which can recognise DNA binding elements (Badis et al., 2008). RSC action is directed by poly(A) and GC-rich elements and those elements cluster in the NDR (Badis et al., 2008; Kubik et al., 2015; Kubik et al., 2018; Lorch et al., 2014). An intriguing possibility is that the DIM is responsible for the read-out of these elements and its flexible nature might be needed to adapt to their spacing and distance. Further research is needed to disentangle this additional level of regulation. 
In addition, density for $\sim 20$ bp of DNA on the downstream side of the nucleosome is missing from SHL +7 to +5 . Nucleosomal DNA close to the exit sites has been reported to undergo dynamic breathing, which is enhanced in experimental conditions of higher salt (Culkin et al., 2017; Kelbauskas et al., 2008; Koopmans et al., 2009). Although the RSC-nucleosome complex was prepared in $150 \mathrm{mM}$ potassium acetate, it is surprising that consistently $\sim 20 \mathrm{bp}$ are missing and thus this might have a functional relevance. Keeping the entry side DNA detached would reduce the histone-DNA contacts that need to be broken during the remodelling mechanism and thus facilitate the process. RSC can also evict nucleosomes (Lorch et al., 2006; Prasad et al., 2016), and partially freeing the H2A-H2B dimer might prime the remodeller for nucleosome disassembly if needed. Further experiments are needed to resolve possible interactions of RSC with the DNA that might have been too flexible in the structure presented here. This problem may be overcome by using a DNA construct containing the DIM recognition sequence elements.

\subsubsection{Multi-subunit remodellers}

CHD family members are all single subunit remodellers and for the multi-subunit ISWI remodeller family, only the ATPase domain has been structurally resolved so far. Therefore, until now structural information on the organisation of the auxiliary domains of multi-subunit remodellers were only available for the IN080 family. The structure of the full RSC remodeller in complex with the nucleosomes solved here delivers insights into the SWI/SNF family. This enables us to appreciate how energy gained by the same basic remodelling motor is transformed into very different remodelling activities based on the specific subunit combination.

Both families use an extensive multi-subunit body as an assembly scaffold. While in the IN080 family the scaffold is formed by the heterohexameric Rvb1/Rvb2 complex to which the other subunits are attached, the RSC backbone is made mainly by the two Rsc8 proteins intertwined with the other subunits. One example is the anchoring of the ATPase subunits. Whereas the N-terminal part of Sth1 largely contributes to the scaffolding network of RSC, the Ino80 ATPase is anchored to the heterohexamer via the long insertion between the two ATPase lobes. Interestingly, the shorter linker between the ATPase lobes in Sth1 forms the protrusion 1 region that plays a role in the regulation of the enzyme. 
All multi-subunit remodellers show several, yet very distinct interactions with their nucleosome substrate. In addition to the ATPase module, INO80 contacts the nucleosome at SHL -2 and -3 with a module formed by the Arp5 and Ies6 subunits (Ayala et al., 2018; Eustermann et al., 2018). These interactions are mirrored by the Arp5-Swc6 proteins in SWR1 (Willhoft et al., 2018). During remodelling, DNA gets translocated from the linker into the nucleosome against those modules in small steps. SWR1 uses a restrained translocation against the block to induce histone distortions that facilitate the exchange of histone variant $\mathrm{H} 2 \mathrm{~A}$ to $\mathrm{H} 2 \mathrm{AZ}$, and no net translocation is observed (Willhoft et al., 2018). IN080 likely also uses the created internal DNA loop to facilitate the exchange back to H2A histones, but the accumulated DNA gets released in larger ratchet steps of 10 - $20 \mathrm{bp}$ to achieve nucleosome sliding (Eustermann et al., 2018).

Since RSC is not involved in the exchange of histone variants no such roadblocklike interaction with the nucleosome would be expected. Indeed, the RSC structure only shows contacts to the nucleosomal DNA with the ATPase module.

However, RSC extensively interacts with both sides of the octamer face via the finger helix and the SnAC domain. While the engagement of the finger helix with the acidic patch of the inner octamer face could be resolved in the presented RSC-nucleosome structure, how exactly the SnAC domain interacts with the outer octamer face is still elusive. The density suggests a helical structure at the acidic patch as well, but higher resolution structures are still needed to determine that with certainty. The extended octamer interaction interface of the SnAC domain inspires the fascinating model that this interaction might contribute to the differential recognition of H2A.Z and H2A variant containing nucleosomes. In this way, specific targeting of RSC to the promoter nucleosomes (which are enriched in $\mathrm{H} 2 \mathrm{AZ}$ ), but not to other nucleosomes along the gene body, may be achieved. Similar models have been suggested for the IN080's sensor foot (Eustermann et al., 2018).

The HSA region of INO80 is involved in remodeller control (Clapier et al., 2016; Knoll et al., 2018), and is bound by the ARP modules of the corresponding remodeller. The INO80 ARPIN080 module is composed of Arp8, N-actin and Arp4 proteins and binds to a segmented HSA helix (Knoll et al., 2018). In contrast, the RSC ARPRSC module is built by Arp7, Arp9 and the non-actin-related protein Rtt102 that together bind to a long, unsegmented HSA helix (Schubert et al., 2013). Given these similarities, the HSA regions and ARP modules of both families are found at very different positions (Figure 27). The 

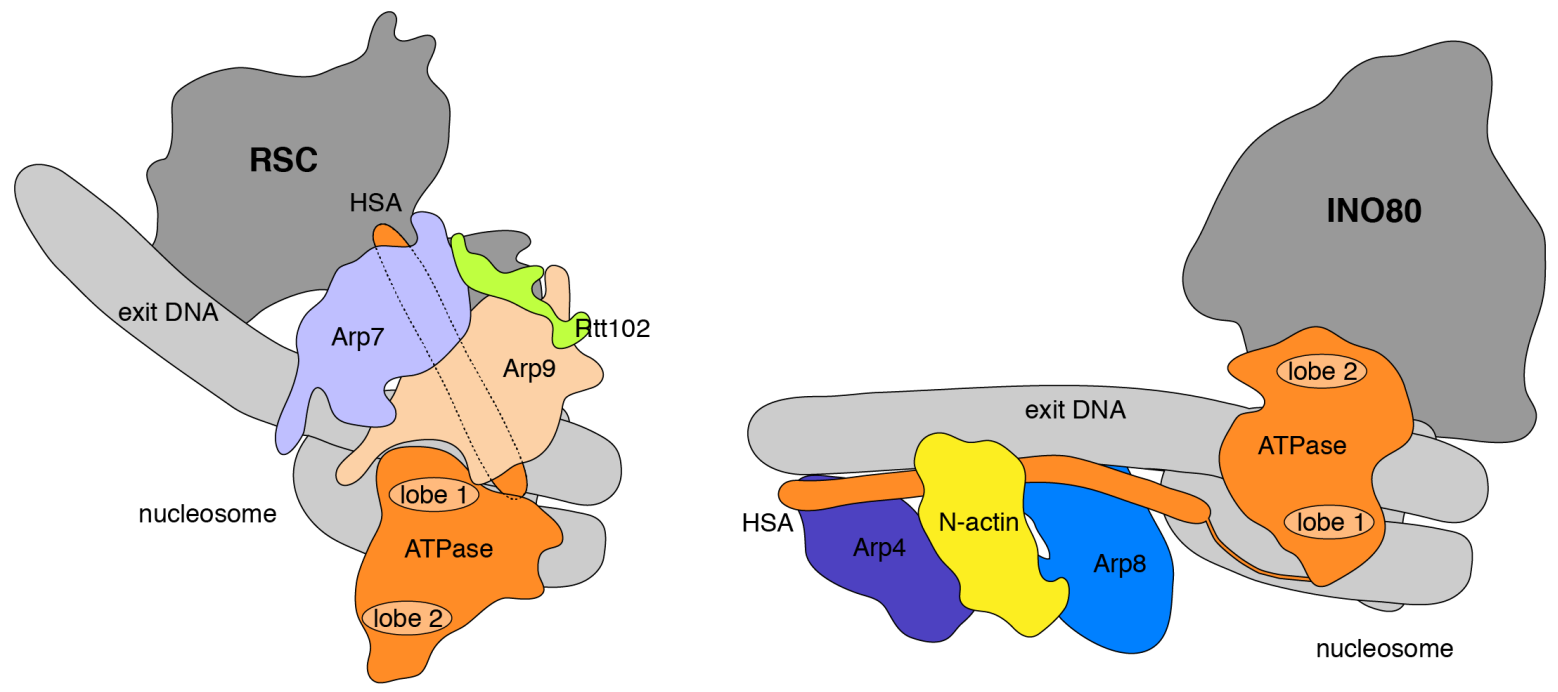

Figure 27 | Schematic representation of the RSC complex and the INO80 complex showing the location of the HSA region and ARP module of the remodellers. ATPase module and HSA region in orange, nucleosome and extra-nucleosomal DNA in light grey and other remodeller subunits in dark grey. lobe 1, 2, RecA-like ATPase lobes 1, 2. The schematic representation of the INO80 complex with the ARP module adapted from figure 5 in (Knoll et al., 2018).

HSA region of RSC extends from the ATPase module towards the body module causing the ARP module to bind in between them. INO80's ARP module binds to the HSA helix which binds to the exit DNA and follows its trajectory away from the rest of the remodeller (Knoll et al., 2018).

This explains why the two remodellers are regulated in a different way by their ARP modules. INO80 efficiently translocates DNA only if the linker length of DNA is $>40$ bp (Zhou et al., 2018) which matches the footprint of the ARPINO80 module on the linker DNA (Knoll et al., 2018). Therefore, the ARPINO80 module was suggested to increase nucleosome sliding by preventing DNA 'back-slippage' during remodelling (Knoll et al., 2018; Zhou et al., 2018). However, it is possible that the ARPIN080 module in addition applies a similar regulation mechanism as proposed here for RSC (section 3.6). The shortening of the DNA linker would lead to less stable interactions with the ARPINO80 module resulting in increased dynamics of the module and causing a conformational change of the bilobal ATPase transmitted through the HSA and post-HSA region.

The ARPRSC module seems to regulate the ATPase independent of DNA as suggested by its location in the RSC-nucleosome complex. The dynamics necessary for the proposed mechanism would be in accordance with the observed flexibility of the ARP 
module. Understanding the interactions of the ARPRSC with the HSA and post-HSA regions and possibly the ATPase lobes in context of the RSC-nucleosome complex is key to a more comprehensive model of RSC remodelling. Therefore, further research needs to be done to decipher the exact molecular nature of RSC regulation through ARPRSC.

\subsubsection{Stoichiometry of SWI/SNF remodeller at the nucleosome}

Cryo-EM investigations of the human SWI/SNF family SNF2h ATPase module have resolved single nucleosomes bound by two SNF2h ATPases simultaneously at SHL -2 and +2 (Armache et al., 2019). It was suggested that binding two ATPase modules might serve a regulatory role and an allosteric mode of control through the nucleosome (Armache et al., 2019). In this model, specific histone contacts get disordered resulting in an asymmetric octamer. This causes a deformation of the acidic patch region which only allows one of the two ATPase modules to be active and would avoid a tug of war situation between the two motors.

However, the ATPase module does not operate in isolation but rather in context of all the auxiliary factors. The RSC-nucleosome structure presented here uncovers an expansive interaction surface of not only of the ATPase motor but also the attached SnAC domain as well as the additional subunit Sfh1 to the nucleosome octamer. Such a binding topology clearly allows for only one full RSC complex to be accommodated per nucleosome, avoiding the tug of war situation through steric hinderance. This would indicate that the binding of two ATPase modules in the case of SNF2h may have been an experimental artefact that might not be reproduced if the complete complex is used instead.

Nevertheless, the observed octamer distortions via the SNF2h ATPase might indeed be important for remodelling in another context. Thus, while we learn a lot by looking at isolated parts of the remodelling machinery, this finding demonstrates the importance of investigating the multi-subunit complexes as a whole to fully understand their mechanisms. 


\subsection{RSC in context of promoters}

While we focused on the interactions of RSC with a single nucleosome in this study, RSC does not function as a standalone machine, but has been shown to interact with various other factors present at gene promoters such as TATA-binding protein (TBP) (Kubik et al., 2018; Sanders et al., 2002), RNA polymerase II (Soutourina et al., 2006), general regulatory factors ABF-binding factor 1 (Abf1), DNA-binding protein Reb1 (Reb1), Repressor/activator site-binding protein 1 (Rap1) (Krietenstein et al., 2016; Kubik et al., 2015; Kubik et al., 2018), nucleosome assembly protein 1 (Nap1) (Hsu et al., 2019; Lorch et al., 2006; Prasad et al., 2016) and cohesin (Maya-Miles et al., 2019; Munoz et al., 2019).

These factors collaborate with RSC to result in promoter nucleosome clearance or set the stage for the assembly of the transcription initiation machinery. The interaction of RSC with these factors will be discussed in the following sections.

\subsubsection{RSC, Nap1 and fragile nucleosomes}

Evidence is mounting for the existence of nucleosome-depleted regions (NDR) with fragile nucleosomes (FNs) between the stable -1 and +1 nucleosomes (Brahma and Henikoff, 2019; Kubik et al., 2015; Xi et al., 2011). A recent study shows that FNs have partially unwrapped DNA and are bound by RSC and general regulatory factors (GRF) (Brahma and Henikoff, 2019). The histone chaperone Nap1 was shown to cooperate with RSC in nucleosome disassembly and histone transfer from the octamer (Hsu et al., 2019; Lorch et al., 2006; Prasad et al., 2016). Two different models for that process were proposed and are supported by two different lines of evidence.

RSC was shown to completely disassemble mono-nucleosomes in the presence of ATP and Nap1 in a stepwise manner with one H2A-H2B dimer being removed first which generates hexameric nucleosome intermediates (Lorch et al., 2006). However, on a dinucleosome template, RSC seems to remodel one of the two nucleosomes while the other one gets disassembled with the help of Nap1 (Prasad et al., 2016).

While this seems to be contradictive, it is possible that the discrepancy is due to the specific experimental setups that were used. It can be envisioned that RSC remodelling of two nucleosomes into each other destabilizes the second one and partially unwraps the DNA from its surface, and therefore creates a binding surface for Nap1 to the 
H2A-H2B dimer. However, in situations where this is not possible due to restriction through roadblocks or the stabilization of the second nucleosome through binding of other factors, a disassembly of a single nucleosome by RSC and Nap1 may be needed.

In the RSC-nucleosome complex, about 20 bp of entry DNA from SHL +5 to +7 are unwrapped and the histone dimer H2A-H2B is partially freed. RSC's SnAC domain contacts the octamer from the outer face and thus keeps the nucleosome together during remodelling and sliding. The structure suggests a possible mode of interplay between RSC and Nap1 for single nucleosome disassembly. The partially freed DNA already primes the detachment of the H2A-H2B dimer, but it is held in place by the SnAC domain. Nap1 might displace the SnAC domain and remove the H2A-H2B dimer generating a hexameric histone core as an intermediate during eviction. Thus, the interaction of SnAC with Nap1 would contribute to determining whether RSC performs nucleosome sliding or eviction.

\subsubsection{RSC and general regulatory factors}

General regulatory factors such as Abf1, Reb1, or Rap1 interact with RSC to free the promoter region from nucleosomes and create NDRs (Brahma and Henikoff, 2019; Krietenstein et al., 2016; Kubik et al., 2015; Kubik et al., 2018). How their interaction regulates NDR formation and how the interplay between these factors and RSC works is still not fully understood.

A possible scenario is that GRFs bind first to chromatin and recruit the RSC complex. RSC not only interacts with the nucleosome but also flanks the upstream exit with its body module and contacts it with the DNA-interaction module (DIM). This architecture provides a large potential interaction surface to contact GRFs that are already bound to DNA. On the other hand, RSC could also bind first, free nucleosomal DNA and allow GRF binding (Brahma and Henikoff, 2019).

Therefore, the RSC-nucleosome structure presents a starting point to nucleate the investigation of whether and how physical interactions between chromatin remodellers and GRFs regulate NDR formation. 


\subsubsection{RSC and transcription initiation}

Gene promoters need to be freed from nucleosomes to allow for transcription by RNA polymerase II (Almer et al., 1986; Knezetic and Luse, 1986; Lorch et al., 1987). RSC can slide or evict nucleosomes and widen the nucleosome-depleted region around promoters (Brahma and Henikoff, 2019; Henikoff, 2016; Krietenstein et al., 2016; Kubik et al., 2015; Kubik et al., 2018; Lorch and Kornberg, 2017). The here presented RSC-nucleosome structure is a good model for the remodeller bound to the +1 nucleosome and poised for action (also the -1 nucleosome as explained above in section 3.8).

However, the initiation of transcription is achieved by an interplay of many factors and molecular machines that all need to share the limited promoter space. In this crowded environment, it is easy to imagine that the remodellers - especially RSC which opens the NDR and makes the promoters accessible - and the transcription factors communicate via physical interactions, and handovers of the shared binding areas take place.

Indeed, RSC action frees TATA elements protected within the nucleosomal borders and facilitates the binding of TBP to promoters (Kubik et al., 2018). In addition, 13 RSC subunits were co-immunoprecipitated using anti-TBP IgG antibodies and identified by DALPC mass-spectrometry (Sanders et al., 2002). The co-purification was performed in the presence of ethidium bromide to rule out DNA as a bridging molecule but indirect interaction between RSC and TBP via other protein factors cannot be entirely excluded.

Binding of RSC's DNA-interaction module to the upstream DNA causes a modest bend in the DNA path and TBP has a 100 - 300 times higher affinity to slightly pre-bent DNA (Parvin et al., 1995). It is appealing to imagine that RSC not only frees the TATA element but also prepares the DNA for facilitated TBP binding. This interplay could also coordinate and determine the TBP binding site for TATA-less promoters.

According to current models of transcription initiation, TBP is delivered to its binding site by either transcription factor II D (TFIID) or the Spt-Ada-Gcn5 (SAGA) complex (Rhee and Pugh, 2012). Although co-immunoprecipitation experiments showed an association between RSC and TBP independent of TFIID (Sanders et al., 2002), a handover of TBP between RSC and TFIID, or RSC and SAGA might still happen and support TBP binding and prime initiation assembly. The binding interactions could be too transient to be measured under the experimental conditions applied by (Sanders et al., 2002). More research will be needed to give answers to these questions. 
Interestingly, the C-terminus of Rsc4 was shown to interact with the Rbp5 subunit of the RNA polymerase II (Soutourina et al., 2006). In the RNA polymerase II structure, Rpb5 is positioned next to the exit side of the downstream DNA. This side would face RSC bound to the +1 nucleosome when the transcription machinery assembles. The Cterminus of Rsc4 forms part of the $\beta$-sheet that anchors the subunit to the upstream facing side of the body module of RSC. Therefore, direct interactions between the transcription machinery and RSC are possible and worth investigating further.

The chromatin remodellers of the four families are conserved from yeast to human and the underlying principles of their function are most likely the same in all organisms. Regarding RSC, such common functional principles include the clearance of nucleosomes from the NDRs through sliding or eviction, sandwich-like engagement of the nucleosome, interaction with the upstream exit DNA, recognition of specific DNA binding sites and interactions with GRF and the transcription machinery.

Given the differences between yeast and higher organisms such as human, the precise regulation and functional need for the remodellers may differ. This is already reflected by the fact that the human SWI/SNF family forms cell-type specific subcomplexes (Lessard et al., 2007; Wang et al., 1996). In yeast, the transcription start site (TSS) is usually found $\sim 13 \mathrm{bp}$ inside the borders of the +1 nucleosome and the TATA box is often scattered across the border of the -1 nucleosome (Albert et al., 2007). In metazoans, the +1 nucleosome is generally located $\sim 60 \mathrm{bp}$ downstream of the TSS (Barski et al., 2007; Mavrich et al., 2008a; Valouev et al., 2008). This is around the area where the RNA polymerase II pausing happens (Guenther et al., 2007; Muse et al., 2007; Rahl et al., 2010; Zeitlinger et al., 2007).

Therefore, RNA polymerase II in yeast encounters the +1 nucleosome already during initiation whereas in metazoans the +1 nucleosome is likely involved in regulating the pausing of an already initiated RNA polymerase II. Thus, while our studies in yeast lay an invaluable foundation for our understanding of the general functions of the chromatin remodellers such as RSC, it will need carefully conducted experiments in each metazoan system to learn more about the specific regulatory roles of chromatin remodellers. The RSC-nucleosome structure presents an exciting starting point to achieve this goal. 


\section{OUTLOOK}

The structure of the RSC chromatin remodeller in complex with its nucleosome substrate, presented in this work, elucidates for the first time the intricate architecture of the multisubunit SWI/SNF remodeller family and the role of the non-enzymatic subunits. However, we are only beginning to understand these sophisticated machines and their function in chromatin organisation. The structure of the RSC-nucleosome complex provides an excellent starting point for further investigation, as outlined below in form of short- and long-term goals.

\subsection{Short-term goals towards improving our understanding of RSC function}

\subsubsection{ATPase module}

Given the strong conservation between the Snf2 and Sth1 ATPase domains, it is highly likely that they fold into identical structures and employ the very same ATP-based remodelling mechanism. Nevertheless, increasing the resolution of the Sth1 ATPase module on the nucleosome in its apo, $\mathrm{ADP}$ and $\mathrm{ADP}-\mathrm{BeF}_{3}$ bound state will be needed to test this assumption which would either corroborate the model of the universal basic remodelling mechanism, or allow us to correct and improve it.

This can be done by purifying the RSC ATPase module in isolation and solving its structure in complex with the nucleosome, to bypass the high conformational heterogeneity of the complete RSC complex. The S. cerevisiae Snf2 ATPase module could be recombinantly expressed and purified in sufficient amounts from E. coli cells (Li et al., 2019; Liu et al., 2017). Therefore, a similar approach would be recommended for the RSC ATPase. 


\subsubsection{Differences between the RSC isoforms containing the subunits Rsc1 or Rsc2}

Two subcomplexes of RSC exist in S. cerevisiae. They are defined by containing one of the two mutually exclusive subunits Rsc1 or Rsc2 and are otherwise identical in subunit composition (Cairns et al., 1999). Earlier studies showed the Rsc1 and Rsc2 isoforms differ in their function during sporulation (Bungard et al., 2004; Yukawa et al., 2002) and that Rsc2, but not Rsc1, is needed for $2 \mu \mathrm{m}$ plasmid maintenance in S. cerevisiae (Wong et al., 2002). However, both subcomplexes seem to have indistinguishable binding profiles on the genome (Ng et al., 2002).

The Rsc1 and Rsc2 proteins have 47\% sequence identity and both comprise the same domains - two bromodomains and a BAH domain - at almost the same positions within the primary amino acid sequence. From a structural perspective, it is a conundrum why two copies of such similar proteins are needed and how they perform their different function. However, one would expect the loss of a redundant remodeller isoform during evolution, again pointing towards their requirement for different functions.

System wide studies with methods such as ChIP-seq, CUT\&RUN, and CUT\&RUN.ChIP will find the potentially different targets for the two Rsc1- and Rsc2containing subcomplexes and thereby give insights into their distinct functional roles.

On the structural side, the endogenous purification strategy established in this work could be used to purify the Rsc1-TAP tagged subcomplex for cryo-EM studies. Comparison of the structures of the Rsc1 and Rsc2 subcomplexes in a free state along with the subcomplexes bound to a nucleosome might already solve some of the posed questions. Optimisation of the sample preparation strategy as discussed below will probably be needed.

\subsubsection{DNA-interaction module}

The DNA-interaction module showed the greatest flexibility of all five RSC modules. While this observation might imply some of its functions, it could also be a result of imperfect sample preparation. In any case, the structure of the DIM needs to be resolved at a molecular level to learn more about its interactions and the role it fulfils in the RSC complex. 
The results of this work strongly suggest that the DIM is formed by the subunits Rsc3 and Rsc30, and at least by parts of Rsc2 (or Rsc1 in the other RSC isoform). Both Rsc3 and Rsc30 contain a zinc finger domain which they likely use to recognise a specific CGCG DNA element (Badis et al., 2008). Interestingly, RSC is regulated by the presence of a poly(A) stretch and a GC-element and their relative position and orientation (Krietenstein et al., 2016; Kubik et al., 2018). This implies that elucidating the molecular nature of the DIM will directly lead to a better understanding of RSC's remodelling mechanism and its regulation.

From a structural perspective, there are two ways to tackle this problem, either by $\mathrm{X}$-ray crystallography of the isolated DIM module or by cryo-EM analysis of the free or nucleosome-bound RSC complex prepared in varying, optimized conditions. Since we know the subunits that likely constitute the DIM, its recombinant expression in E. coli or the baculovirus expression system should be feasible in a reasonable time. While crystallization of the DIM on its own would already shed light on its function, understanding it fully would require its co-crystallization with bound DNA. For that reason, it will be critical to vary the length of the DNA and to try combinations of GC-rich or poly(A) elements. Similarly, changing the length and sequence of the upstream exit DNA would be the first thing to try to stabilise the DIM in cryo-EM. RSC also binds strongly to free DNA which would be a way of stabilising the DIM.

Using recombinant DIM in experiments such as electromobility shift assays or fluorescent anisotropy would be a fast way to screen for DNA sequences that tightly bind to it and represent good candidates for structural work. Since it is possible that the dynamic nature of the DIM in the full complex does not allow for high-resolution structure determination, it might be advisable to try both approaches in parallel. This would allow the integration of the crystal structure into a medium resolution cryo-EM map.

\subsubsection{Histone variant H2A.Z}

RSC was found to bind preferentially to nucleosomes carrying the histone variant H2A.Z (Cakiroglu et al., 2019). However, it is still unknown how the complex discriminates between nucleosomes with H2A or H2A.Z. We observed a strong interaction of the Sfh1 finger helix with the acidic patch of the nucleosome. The SnAC module apparently also 
extends towards the acidic patch on the opposite face of the nucleosome. H2A.Z containing nucleosomes were shown to have an extended acidic patch (Suto et al., 2000) and the resulting increased interactions might help to attract the remodeller. Nevertheless, a more defined interaction that reads out amino acid differences of the two histone variants for discrimination is possible and was suggested for the INO80 and SWR1 remodellers that also contact the acidic patch on the nucleosome (Eustermann et al., 2018; Willhoft et al., 2018).

Improving the resolution of the finger helix interaction as well as the SnAC module might give important insights into this question. Therefore, the RSC-nucleosome complex would need to be assembled on a H2A.Z containing nucleosome for cryo-EM studies. In another approach the recombinantly purified SnAC domain and finger helix could be used to resolve these interactions on a structural level.

In addition, we observed density of the H2A tail extending from the nucleosome towards the arm module of RSC. There are several amino acid variations in the H2A.Z tail compared to the tail of the canonical $\mathrm{H} 2 \mathrm{~A}$ and it is also imaginable that the arm module can sense them. Binding RSC to the H2A.Z carrying nucleosome for cryo-EM experiments will resolve this question. Since RSC seems to preferentially bind nucleosomes carrying H2A.Z, there is a fair chance that this complex shows a better stability and is less dynamic.

\subsubsection{Post-translational histone modifications}

The H4 tail interacts with the ATPase module and could strengthen the sandwiching contacts between RSC and the nucleosome. The acetylation of H4's lysine 16 was described to be involved in regulation of nucleosome eviction (Lorch et al., 2018). Therefore, this modification is a good candidate to be tested and might help increase the stability of the RSC-nucleosome complex.

Of the six RSC domains that are implicated in histone tail interactions, only the bromodomain of Rsc58 appeared in our structure bound to the body module. The nucleosome used in this study did not contain any post-translational modifications, particularly the acetylation of H3K14, which might be needed to establish stable interactions to these domains. These modifications can be introduced with acetyl 
transferases such as Gcn5 or by chemical cross-linking of modified histone tails to the core histone (Chatterjee and Muir, 2010).

However, there is also the possibility that the bromodomains and the BAH domain are attached to RSC on flexible linkers and used to sense for the flexible, modified histone tails. If the tethering works in this way, it might be very challenging to resolve the domains in the experimental setup used here due to intrinsic dynamics.

\subsubsection{ARP module}

The ARP module likely influences the interactions between the post-HSA, protrusion 1 and brace helices and thus regulates RSC. Nonetheless, how exactly the ARP module influences RSC is still unresolved and needs to be answered to fully understand RSC remodelling. For this purpose, the sample preparation conditions need to be optimised to trap the ARP module in a stable conformation. Our structure does not inform, whether the ARP module could not be resolved at higher resolution because it is not stably bound, or because it is stably bound but highly dynamic. Recombinant preparation and overstoichiometric addition of the ARP module to the complex might improve the stability.

Interplay with another factor could be responsible for different conformations of the ARP module in vivo. Nap1 disassembles nucleosomes together with an active RSC complex and its binding to the remodeller might influence the dynamics of the ARP module.

\subsubsection{RSC and Nap1}

It is interesting to speculate that Nap1 physically interacts with RSC in a handover mechanism in order to disassemble nucleosomes (section 4.2.1). A first step to answer this question would be the recombinant purification of Nap1 and subsequent electromobility shift assays or pull-down assays with RSC to probe for physical interactions. In addition, the recombinant SnAC domain, which is the anticipated Nap1 interaction partner, could be investigated. Also, interactions with the ARP module are possible and should be studied. Should physical interactions be found, structural studies would follow. 


\subsubsection{RSC at di-nucleosome array}

The NDR is flanked by stable -1 and +1 nucleosomes which are both manipulated by RSC to expand the NDR. RSC binds to SHL +2 and this interaction was never shown to depend on a specific sequence motif. The RSC-nucleosome structure reported here was solved with a mono-nucleosome that had linker DNA overhangs on both sides. In such an experimental setup, the mono-nucleosome represents the -1 and the +1 nucleosome at the same time and the positions SHL -2 and SHL +2 are indistinguishable for the remodeller. Therefore, as discussed above (section 3.8), the presented structure of RSC at the +1 nucleosome also reflects the situation on the -1 nucleosome.

In order to learn more about RSC acting on both sides of the NDR simultaneously, a di-nucleosomal array with no DNA overhangs on the outside would be needed. The length and sequence of the linker connecting the two nucleosomes can be varied. Using binding assays, such as the electromobility shift assays, one could test the linker length needed for two RSC complexes to bind to the array and if sequence variations - such as poly(A) or GC-rich elements - make a difference in affinity.

After establishing stable conditions, it would be of great interest to analyse the sample with cryo-EM. This way we would learn whether and how the two RSC complexes physically interact and communicate for establishing an NDR.

\subsubsection{RSC and general regulatory factors}

RSC and the general regulatory factors Abf1, Reb1 and Rap1 are involved in establishing NDRs (Krietenstein et al., 2016; Kubik et al., 2015; Kubik et al., 2018). A physical interaction during their operation on chromatin is probable and should be investigated. All three factors can be purified recombinantly and could be tested with pull-down and electromobility shift assays for their binding to RSC. It is conceivable that a stable interaction requires the presence of the nucleosome, nucleosomal DNA or free DNA. In this case, the DNA sequence might play an important role because all GRFs and RSC have special DNA recognition sequences (Badis et al., 2008), and their spacing could be crucial.

Since it is possible that RSC and GRFs are only tethered together by DNA, a smart experimental design is needed to exclude a false positive result - e.g. orthogonal tagging strategies could be employed. In this approach, not only the full length GRFs could be 
tested, but also the protein interaction domains independently of the DNA binding domains. Deciphering the structures of RSC with GRFs will not only shed light on their interactions but will also make it possible to draw conclusions of their sequential binding order. Thus, those experiments will reveal an important mechanism of chromatin organisation at the NDRs.

\subsubsection{RSC and TBP}

A RSC-TBP complex is plausible (section 4.2.3) and would be a good candidate for structural investigation. In a similar way as for the GRFs discussed above (section 5.1.9), the binding conditions of TBP to RSC should be probed before conducting cryo-EM experiments. Results of these experiments would be a big step forward in understanding the initiation of transcription.

\subsubsection{RSC and cohesin}

Cohesin plays a central role in sister chromatid cohesion, and it is important for DNA repair and transcription regulation. Cohesin is conserved and forms a large ring-shaped complex that holds DNA together by topologically encircling chromatin (Makrantoni and Marston, 2018). In order for this to happen, the cohesin ring has to be loaded onto chromatin by the cohesin loader complex comprised of the protein dimer Scc2-Scc4 (Makrantoni and Marston, 2018). Recent studies have indicated the involvement of RSC in cohesin function (Huang et al., 2004; Liefshitz and Kupiec, 2011; Maya-Miles et al., 2019; Prasad et al., 2019) and showed direct interactions between RSC and the cohesin loader complex (Munoz et al., 2019). Understanding the role of cohesin in chromatin organisation and transcription regulation requires more knowledge about the first step of cohesin-chromatin engagement and its regulation. The large-scale RSC purification and cryo-EM imaging conditions established here are a solid foundation for structural investigations of this process. 


\subsubsection{Dynamics of RSC}

The experiments leading to the RSC-nucleosome structure have shown a great flexibility and high intrinsic dynamics of free RSC as well as in complex with the nucleosome. Given RSC's modular topology and the specific interactions and functions of the modules, many conformational states of the remodeller are expected. In a simple brute force approach, the collection of a very large dataset combined with smart intensive 3D classification might resolve several highly occupied RSC conformations. For this approach only a slight sample optimisation towards higher complex density on the grid would be necessary.

The endogenous RSC complex might be post-translationally modified at specific phosphorylation sites. Probing for such modifications by mass-spectrometry of endogenous sample might support the search for stable RSC conformations. In addition, the ideas described above (section 5.1) to improve the quality of distinct parts of RSC will presumably also help to trap and stabilise different conformations of RSC.

\subsection{Long-term goals towards improving our understanding of RSC function}

\subsubsection{In vitro chromatin-based transcription system}

Establishing an in vitro system where all factors are controllable would be invaluable to elucidate and disentangle the multiple steps necessary to prepare a promoter for transcription initiation.

For this purpose, it might be beneficial to also establish a fully recombinant expression system for RSC, and the baculovirus expression system would be recommended. Recombinant preparation of RSC would allow for mutational modifications that are not feasible with the endogenous source. The structure presented here shows how RSC's subunits and domains are assembled and hence will be important to guide the cloning and expression design.

Finding appropriate endogenous promoters or designing artificial DNA sequences for this purpose would be vital. The promoters will not only need to allow for the biochemical reconstitution of transcription from a chromatin template, but importantly 
also have to be compatible with structural studies that require strong binding of dynamically trapped complexes.

With such a system at hand, our knowledge of transcription initiation will be dramatically broadened. Regarding RSC, many questions about its function in a more sophisticated background could be answered. For example, does RSC stay stably bound to the nucleosome, or does it dynamically engage the nucleosome with high on and off dissociation rates? How does RSC know when to stop remodelling?

Interestingly, the +1 and +2 nucleosomes as well as the -1 and -2 nucleosomes are spaced in an orderly fashion and no clashes are observed in in vivo experiments (Krietenstein et al., 2016; Kubik et al., 2019; Kubik et al., 2015; Kubik et al., 2018). This means that a simple steric clash with the following nucleosome in an array of nucleosomes cannot be used as a model to explain the termination of remodelling. RSC does not seem to contain an element with which it can measure the distance to the next downstream (at the +1 ) or upstream (at the -1) nucleosome. Therefore, physical constraints and the interplay with regulatory factors might determine remodelling termination. Regulatory factors are probably already known RSC interactors, such as GRFs, TFIID, SAGA or the pre-initiation complex (PIC).

Using the in vitro chromatin-based transcription system, one could also learn in more detail about the binding order of the aforementioned factors to start transcription from an inactive promoter. Using nucleosomes with post-translationally modified histones would inform us on the role of modifications during chromatin opening and transcription initiation.

The knowledge gained from a well-established in vitro chromatin-based transcription system will form the foundation of many important structural studies leading to a better understanding of gene regulation.

\subsubsection{Extension to the human system}

The multi-subunit complex PBAF is the human homologue of the yeast SWI/SNF family remodeller RSC. However, the human system has a higher degree of complexity reflected in cell-type specific PBAF subcomplexes (Lessard et al., 2007; Wang et al., 1996). Nevertheless, the structure of the RSC complex in this work is a good model for PBAF in a similar way as discussed for yeast RSC (section 3.9), and it can guide the establishment of 
the recombinant expression of the human PBAF subcomplexes in the insect cell system. Using the human complexes, the structural and consequently functional differences of the subcomplexes could be studied. It would also be the first step to get insight into the specific different roles of SWI/SNF remodellers during transcription in yeast and human as discussed in section 4.2.3.

\subsubsection{In vivo structure determination of complexes involved in transcription initiation at the +1 nucleosome}

Complexes involved in the establishment of transcription at the +1 nucleosome are multisubunit assemblies in the size range of $\geq 1 \mathrm{MDa}$ and thus possible targets for studies using correlative light-electron microscopy (CLEM) combined with cryo-electron tomography (cryo-ET). In CLEM, fluorescently tagging the factor of interest with proteins such as green fluorescent protein (GFP) allows for detection of its location in the cell. In subsequent cryo-ET the coordinates are used to direct data collection at the correct position of the cell. In case of yeast cells, a cryo-focused ion beam milling step would be needed before tomography acquisition to generate a sample with a thickness applicable for cryo-ET.

Once this elaborate workflow would be established, orthogonal tagging of other factors participating in transcription initiation at the +1 nucleosome, such as SAGA, TFIID or the PIC would allow to detect locations where several of these factors reside at the same time.

Integrating structural knowledge gained with techniques such as X-ray crystallography, nuclear magnetic resonance, cryo-EM and biochemical assays, would reveal structures of these large assemblies at molecular resolution. Solving these structures in vivo will give important clues into the process of chromatin opening for transcription initiation.

Consequently, the structure of the RSC-nucleosome complex presented in this work is one of many puzzle pieces on an exciting journey to our understanding of cellular chromatin organisation and transcription. 


\section{REFERENCES}

Adams, P.D., Afonine, P.V., Bunkoczi, G., Chen, V.B., Davis, I.W., Echols, N., Headd, J.J., Hung, L.W., Kapral, G.J., Grosse-Kunstleve, R.W., et al. (2010). PHENIX: a comprehensive Python-based system for macromolecular structure solution. Acta Crystallogr D Biol Crystallogr 66, 213-221.

Albert, I., Mavrich, T.N., Tomsho, L.P., Qi, J., Zanton, S.J., Schuster, S.C., and Pugh, B.F. (2007). Translational and rotational settings of H2A.Z nucleosomes across the Saccharomyces cerevisiae genome. Nature 446, 572-576.

Almer, A., and Horz, W. (1986). Nuclease hypersensitive regions with adjacent positioned nucleosomes mark the gene boundaries of the PHO5/PHO3 locus in yeast. EMBO J 5, 2681-2687.

Almer, A., Rudolph, H., Hinnen, A., and Horz, W. (1986). Removal of positioned nucleosomes from the yeast PHO5 promoter upon PHO5 induction releases additional upstream activating DNA elements. EMBO J 5, 2689-2696.

Angus-Hill, M.L., Schlichter, A., Roberts, D., Erdjument-Bromage, H., Tempst, P., and Cairns, B.R. (2001). A Rsc3/Rsc30 zinc cluster dimer reveals novel roles for the chromatin remodeler RSC in gene expression and cell cycle control. Mol Cell 7, 741751.

Arents, G., Burlingame, R.W., Wang, B.C., Love, W.E., and Moudrianakis, E.N. (1991). The nucleosomal core histone octamer at 3.1 A resolution: a tripartite protein assembly and a left-handed superhelix. Proc Natl Acad Sci U S A 88, 10148-10152.

Armache, J.P., Gamarra, N., Johnson, S.L., Leonard, J.D., Wu, S., Narlikar, G.J., and Cheng, Y. (2019). Cryo-EM structures of remodeler-nucleosome intermediates suggest allosteric control through the nucleosome. Elife 8, 213.

Armache, K.J., Garlick, J.D., Canzio, D., Narlikar, G.J., and Kingston, R.E. (2011). Structural basis of silencing: Sir3 BAH domain in complex with a nucleosome at $3.0 \mathrm{~A}$ resolution. Science 334, 977-982.

Artimo, P., Jonnalagedda, M., Arnold, K., Baratin, D., Csardi, G., de Castro, E., Duvaud, S., Flegel, V., Fortier, A., Gasteiger, E., et al. (2012). ExPASy: SIB bioinformatics resource portal. Nucleic Acids Res 40, W597-603.

Asturias, F.J., Chung, W.H., Kornberg, R.D., and Lorch, Y. (2002). Structural analysis of the RSC chromatin-remodeling complex. Proc Natl Acad Sci U S A 99, 13477-13480.

Ayala, R., Willhoft, O., Aramayo, R.J., Wilkinson, M., McCormack, E.A., Ocloo, L., Wigley, D.B., and Zhang, X. (2018). Structure and regulation of the human IN080nucleosome complex. Nature 556, 391-395.

Badis, G., Chan, E.T., van Bakel, H., Pena-Castillo, L., Tillo, D., Tsui, K., Carlson, C.D., Gossett, A.J., Hasinoff, M.J., Warren, C.L., et al. (2008). A library of yeast transcription factor motifs reveals a widespread function for Rsc3 in targeting nucleosome exclusion at promoters. Mol Cell 32, 878-887.

Bai, L., and Morozov, A.V. (2010). Gene regulation by nucleosome positioning. Trends Genet 26, 476-483.

Barbera, A.J., Chodaparambil, J.V., Kelley-Clarke, B., Joukov, V., Walter, J.C., Luger, K., and Kaye, K.M. (2006). The nucleosomal surface as a docking station for Kaposi's sarcoma herpesvirus LANA. Science 311, 856-861. 
Barski, A., Cuddapah, S., Cui, K., Roh, T.Y., Schones, D.E., Wang, Z., Wei, G., Chepelev, I., and Zhao, K. (2007). High-resolution profiling of histone methylations in the human genome. Cell 129, 823-837.

Becker, P.B., and Workman, J.L. (2013). Nucleosome remodeling and epigenetics. Cold Spring Harb Perspect Biol 5.

Bienert, S., Waterhouse, A., de Beer, T.A., Tauriello, G., Studer, G., Bordoli, L., and Schwede, T. (2017). The SWISS-MODEL Repository-new features and functionality. Nucleic Acids Res 45, D313-D319.

Bond, C.S., and Schuttelkopf, A.W. (2009). ALINE: a WYSIWYG protein-sequence alignment editor for publication-quality alignments. Acta Crystallogr D Biol Crystallogr 65, 510-512.

Bowman, G.D. (2010). Mechanisms of ATP-dependent nucleosome sliding. Curr Opin Struct Biol 20, 73-81.

Brahma, S., and Henikoff, S. (2019). RSC-Associated Subnucleosomes Define MNaseSensitive Promoters in Yeast. Mol Cell 73, 238-249 e233.

Brahma, S., Udugama, M.I., Kim, J., Hada, A., Bhardwaj, S.K., Hailu, S.G., Lee, T.H., and Bartholomew, B. (2017). INO80 exchanges H2A.Z for H2A by translocating on DNA proximal to histone dimers. Nat Commun 8, 15616.

Brogaard, K., Xi, L., Wang, J.P., and Widom, J. (2012). A map of nucleosome positions in yeast at base-pair resolution. Nature 486, 496-501.

Brown, S.A., Imbalzano, A.N., and Kingston, R.E. (1996). Activator-dependent regulation of transcriptional pausing on nucleosomal templates. Genes Dev 10, 1479-1490.

Buchan, D.W.A., and Jones, D.T. (2019). The PSIPRED Protein Analysis Workbench: 20 years on. Nucleic Acids Res 47, W402-W407.

Bungard, D., Reed, M., and Winter, E. (2004). RSC1 and RSC2 are required for expression of mid-late sporulation-specific genes in Saccharomyces cerevisiae. Eukaryot Cell 3, 910-918.

Cairns, B.R. (2001). Emerging roles for chromatin remodeling in cancer biology. Trends Cell Biol 11, S15-21.

Cairns, B.R., Erdjument-Bromage, H., Tempst, P., Winston, F., and Kornberg, R.D. (1998). Two actin-related proteins are shared functional components of the chromatinremodeling complexes RSC and SWI/SNF. Mol Cell 2, 639-651.

Cairns, B.R., Lorch, Y., Li, Y., Zhang, M., Lacomis, L., Erdjument-Bromage, H., Tempst, P., Du, J., Laurent, B., and Kornberg, R.D. (1996). RSC, an essential, abundant chromatinremodeling complex. Cell 87, 1249-1260.

Cairns, B.R., Schlichter, A., Erdjument-Bromage, H., Tempst, P., Kornberg, R.D., and Winston, F. (1999). Two functionally distinct forms of the RSC nucleosomeremodeling complex, containing essential AT hook, BAH, and bromodomains. Mol Cell 4, 715-723.

Cakiroglu, A., Clapier, C.R., Ehrensberger, A.H., Darbo, E., Cairns, B.R., Luscombe, N.M., and Svejstrup, J.Q. (2019). Genome-wide reconstitution of chromatin transactions reveals that RSC preferentially disrupts H2AZ-containing nucleosomes. Genome Res 29, 988-998.

Cao, Y., Cairns, B.R., Kornberg, R.D., and Laurent, B.C. (1997). Sfh1p, a component of a novel chromatin-remodeling complex, is required for cell cycle progression. Mol Cell Biol 17, 3323-3334.

Carey, M., Li, B., and Workman, J.L. (2006). RSC exploits histone acetylation to abrogate the nucleosomal block to RNA polymerase II elongation. Mol Cell 24, 481-487.

Cerami, E., Gao, J., Dogrusoz, U., Gross, B.E., Sumer, S.O., Aksoy, B.A., Jacobsen, A., Byrne, C.J., Heuer, M.L., Larsson, E., et al. (2012). The cBio cancer genomics portal: an open 
platform for exploring multidimensional cancer genomics data. Cancer Discov 2, 401-404.

Chaban, Y., Ezeokonkwo, C., Chung, W.H., Zhang, F., Kornberg, R.D., Maier-Davis, B., Lorch, Y., and Asturias, F.J. (2008). Structure of a RSC-nucleosome complex and insights into chromatin remodeling. Nat Struct Mol Biol 15, 1272-1277.

Chambers, A.L., Brownlee, P.M., Durley, S.C., Beacham, T., Kent, N.A., and Downs, J.A. (2012). The two different isoforms of the RSC chromatin remodeling complex play distinct roles in DNA damage responses. PLoS One 7, e32016.

Chambers, A.L., Pearl, L.H., Oliver, A.W., and Downs, J.A. (2013). The BAH domain of Rsc2 is a histone $\mathrm{H} 3$ binding domain. Nucleic Acids Res 41, 9168-9182.

Charlop-Powers, Z., Zeng, L., Zhang, Q., and Zhou, M.M. (2010). Structural insights into selective histone H3 recognition by the human Polybromo bromodomain 2. Cell Res 20, 529-538.

Chatterjee, C., and Muir, T.W. (2010). Chemical approaches for studying histone modifications. J Biol Chem 285, 11045-11050.

Clapier, C.R., and Cairns, B.R. (2009). The biology of chromatin remodeling complexes. Annu Rev Biochem 78, 273-304.

Clapier, C.R., and Cairns, B.R. (2012). Regulation of ISWI involves inhibitory modules antagonized by nucleosomal epitopes. Nature 492, 280-284.

Clapier, C.R., Iwasa, J., Cairns, B.R., and Peterson, C.L. (2017). Mechanisms of action and regulation of ATP-dependent chromatin-remodelling complexes. Nat Rev Mol Cell Biol 18, 407-422.

Clapier, C.R., Kasten, M.M., Parnell, T.J., Viswanathan, R., Szerlong, H., Sirinakis, G., Zhang, Y., and Cairns, B.R. (2016). Regulation of DNA Translocation Efficiency within the Chromatin Remodeler RSC/Sth1 Potentiates Nucleosome Sliding and Ejection. Mol Cell 62, 453-461.

Combe, C.W., Fischer, L., and Rappsilber, J. (2015). xiNET: cross-link network maps with residue resolution. Mol Cell Proteomics 14, 1137-1147.

Culkin, J., de Bruin, L., Tompitak, M., Phillips, R., and Schiessel, H. (2017). The role of DNA sequence in nucleosome breathing. Eur Phys J E Soft Matter 40, 106.

Czaja, W., Mao, P., and Smerdon, M.J. (2014). Chromatin remodelling complex RSC promotes base excision repair in chromatin of Saccharomyces cerevisiae. DNA Repair (Amst) 16, 35-43.

Da, G., Lenkart, J., Zhao, K., Shiekhattar, R., Cairns, B.R., and Marmorstein, R. (2006). Structure and function of the SWIRM domain, a conserved protein module found in chromatin regulatory complexes. Proc Natl Acad Sci U S A 103, 2057-2062.

Dang, W., and Bartholomew, B. (2007). Domain architecture of the catalytic subunit in the ISW2-nucleosome complex. Mol Cell Biol 27, 8306-8317.

Denslow, S.A., and Wade, P.A. (2007). The human Mi-2/NuRD complex and gene regulation. Oncogene $26,5433-5438$.

Drlica, K., and Rouviere-Yaniv, J. (1987). Histonelike proteins of bacteria. Microbiol Rev 51, 301-319.

Durr, H., Korner, C., Muller, M., Hickmann, V., and Hopfner, K.P. (2005). X-ray structures of the Sulfolobus solfataricus SWI2/SNF2 ATPase core and its complex with DNA. Cell 121, 363-373.

Dyer, P.N., Edayathumangalam, R.S., White, C.L., Bao, Y., Chakravarthy, S., Muthurajan, U.M., and Luger, K. (2003). Reconstitution of Nucleosome Core Particles from Recombinant Histones and DNA. In Methods in Enzymology (Academic Press), pp. 23-44. 
Ebbert, R., Birkmann, A., and Schuller, H.J. (1999). The product of the SNF2/SWI2 paralogue IN080 of Saccharomyces cerevisiae required for efficient expression of various yeast structural genes is part of a high-molecular-weight protein complex. Mol Microbiol 32, 741-751.

Elfring, L.K., Deuring, R., McCallum, C.M., Peterson, C.L., and Tamkun, J.W. (1994). Identification and characterization of Drosophila relatives of the yeast transcriptional activator SNF2/SWI2. Mol Cell Biol 14, 2225-2234.

Emsley, P., Lohkamp, B., Scott, W.G., and Cowtan, K. (2010). Features and development of Coot. Acta Crystallogr D Biol Crystallogr 66, 486-501.

Eustermann, S., Schall, K., Kostrewa, D., Lakomek, K., Strauss, M., Moldt, M., and Hopfner, K.P. (2018). Structural basis for ATP-dependent chromatin remodelling by the IN080 complex. Nature 556, 386-390.

Fang, Q., Chen, P., Wang, M., Fang, J., Yang, N., Li, G., and Xu, R.M. (2016). Human cytomegalovirus IE1 protein alters the higher-order chromatin structure by targeting the acidic patch of the nucleosome. Elife 5.

Farnung, L., Ochmann, M., and Cramer, P. (2019). Nucleosome-CHD4 chromatin remodeller structure explains human disease mutations. bioRxiv.

Farnung, L., Vos, S.M., Wigge, C., and Cramer, P. (2017). Nucleosome-Chd1 structure and implications for chromatin remodelling. Nature 550, 539-542.

Flaus, A., Luger, K., Tan, S., and Richmond, T.J. (1996). Mapping nucleosome position at single base-pair resolution by using site-directed hydroxyl radicals. Proc Natl Acad Sci U S A 93, 1370-1375.

Flaus, A., Martin, D.M., Barton, G.J., and Owen-Hughes, T. (2006). Identification of multiple distinct Snf2 subfamilies with conserved structural motifs. Nucleic Acids Res 34, 2887-2905.

Florio, C., Moscariello, M., Ederle, S., Fasano, R., Lanzuolo, C., and Pulitzer, J.F. (2007). A study of biochemical and functional interactions of Htl1p, a putative component of the Saccharomyces cerevisiae, Rsc chromatin-remodeling complex. Gene 395, 72-85.

Forne, I., Ludwigsen, J., Imhof, A., Becker, P.B., and Mueller-Planitz, F. (2012). Probing the conformation of the ISWI ATPase domain with genetically encoded photoreactive crosslinkers and mass spectrometry. Mol Cell Proteomics 11, M111 012088.

Fujisawa, T., and Filippakopoulos, P. (2017). Functions of bromodomain-containing proteins and their roles in homeostasis and cancer. Nat Rev Mol Cell Biol 18, 246262.

Gao, J., Aksoy, B.A., Dogrusoz, U., Dresdner, G., Gross, B., Sumer, S.O., Sun, Y., Jacobsen, A., Sinha, R., Larsson, E., et al. (2013). Integrative analysis of complex cancer genomics and clinical profiles using the cBioPortal. Sci Signal 6, pl1.

Goddard, T.D., Huang, C.C., Meng, E.C., Pettersen, E.F., Couch, G.S., Morris, J.H., and Ferrin, T.E. (2018). UCSF ChimeraX: Meeting modern challenges in visualization and analysis. Protein Sci 27, 14-25.

Grimm, M., Zimniak, T., Kahraman, A., and Herzog, F. (2015). xVis: a web server for the schematic visualization and interpretation of crosslink-derived spatial restraints. Nucleic Acids Res 43, W362-369.

Guenther, M.G., Levine, S.S., Boyer, L.A., Jaenisch, R., and Young, R.A. (2007). A chromatin landmark and transcription initiation at most promoters in human cells. Cell 130, 77-88.

Hanske, J., Sadian, Y., and Muller, C.W. (2018). The cryo-EM resolution revolution and transcription complexes. Curr Opin Struct Biol 52, 8-15. 
Hauk, G., McKnight, J.N., Nodelman, I.M., and Bowman, G.D. (2010). The chromodomains of the Chd1 chromatin remodeler regulate DNA access to the ATPase motor. Mol Cell 39, 711-723.

Henikoff, S. (2016). Mechanisms of Nucleosome Dynamics In Vivo. Cold Spring Harb Perspect Med 6.

Ho, L., and Crabtree, G.R. (2010). Chromatin remodelling during development. Nature 463, 474-484.

Hodges, C., Kirkland, J.G., and Crabtree, G.R. (2016). The Many Roles of BAF (mSWI/SNF) and PBAF Complexes in Cancer. Cold Spring Harb Perspect Med 6, a026930.

Hsu, K.W., Chow, S.Y., Su, B.Y., Lu, Y.H., Chen, C.J., Chen, W.L., Cheng, M.Y., and Fan, H.F. (2019). The synergy between RSC, Nap1 and adjacent nucleosome in nucleosome remodeling. Biochim Biophys Acta Gene Regul Mech 1862, 129-140.

Huang, J., Hsu, J.M., and Laurent, B.C. (2004). The RSC nucleosome-remodeling complex is required for Cohesin's association with chromosome arms. Mol Cell 13, 739-750.

Imamura, Y., Yu, F., Nakamura, M., Chihara, Y., Okane, K., Sato, M., Kanai, M., Hamada, R., Ueno, M., Yukawa, M., et al. (2015). RSC Chromatin-Remodeling Complex Is Important for Mitochondrial Function in Saccharomyces cerevisiae. PLoS One 10, e0130397.

Jenuwein, T., and Allis, C.D. (2001). Translating the histone code. Science 293, 1074-1080.

Jiang, C., and Pugh, B.F. (2009). Nucleosome positioning and gene regulation: advances through genomics. Nat Rev Genet 10, 161-172.

Jones, D.T. (1999). Protein secondary structure prediction based on position-specific scoring matrices. J Mol Biol 292, 195-202.

Jones, G.M., Stalker, J., Humphray, S., West, A., Cox, T., Rogers, J., Dunham, I., and Prelich, G. (2008). A systematic library for comprehensive overexpression screens in Saccharomyces cerevisiae. Nat Methods 5, 239-241.

Kagalwala, M.N., Glaus, B.J., Dang, W., Zofall, M., and Bartholomew, B. (2004). Topography of the ISW2-nucleosome complex: insights into nucleosome spacing and chromatin remodeling. EMBO J 23, 2092-2104.

Kasten, M., Szerlong, H., Erdjument-Bromage, H., Tempst, P., Werner, M., and Cairns, B.R. (2004). Tandem bromodomains in the chromatin remodeler RSC recognize acetylated histone H3 Lys14. EMBO J 23, 1348-1359.

Kastner, B., Fischer, N., Golas, M.M., Sander, B., Dube, P., Boehringer, D., Hartmuth, K., Deckert, J., Hauer, F., Wolf, E., et al. (2008). GraFix: sample preparation for singleparticle electron cryomicroscopy. Nat Methods 5, 53-55.

Kelbauskas, L., Chan, N., Bash, R., DeBartolo, P., Sun, J., Woodbury, N., and Lohr, D. (2008). Sequence-dependent variations associated with $\mathrm{H} 2 \mathrm{~A} / \mathrm{H} 2 \mathrm{~B}$ depletion of nucleosomes. Biophys J 94, 147-158.

Kidmose, R.T., Juhl, J., Nissen, P., Boesen, T., Karlsen, J.L., and Pedersen, B.P. (2019). Namdinator - automatic molecular dynamics flexible fitting of structural models into cryo-EM and crystallography experimental maps. IUCrJ 6, 526-531.

Kimanius, D., Forsberg, B.O., Scheres, S.H., and Lindahl, E. (2016). Accelerated cryo-EM structure determination with parallelisation using GPUs in RELION-2. Elife 5.

Knezetic, J.A., and Luse, D.S. (1986). The presence of nucleosomes on a DNA template prevents initiation by RNA polymerase II in vitro. Cell 45, 95-104.

Knoll, K.R., Eustermann, S., Niebauer, V., Oberbeckmann, E., Stoehr, G., Schall, K., Tosi, A., Schwarz, M., Buchfellner, A., Korber, P., et al. (2018). The nuclear actin-containing Arp8 module is a linker DNA sensor driving IN080 chromatin remodeling. Nat Struct Mol Biol 25, 823-832. 
Koopmans, W.J., Buning, R., Schmidt, T., and van Noort, J. (2009). spFRET using alternating excitation and FCS reveals progressive DNA unwrapping in nucleosomes. Biophys J 97, 195-204.

Kornberg, R.D. (1974). Chromatin structure: a repeating unit of histones and DNA. Science 184, 868-871.

Krietenstein, N., Wal, M., Watanabe, S., Park, B., Peterson, C.L., Pugh, B.F., and Korber, P. (2016). Genomic Nucleosome Organization Reconstituted with Pure Proteins. Cell 167, 709-721 e712.

Kubik, S., Bruzzone, M.J., Challal, D., Dreos, R., Mattarocci, S., Bucher, P., Libri, D., and Shore, D. (2019). Opposing chromatin remodelers control transcription initiation frequency and start site selection. Nat Struct Mol Biol 26, 744-754.

Kubik, S., Bruzzone, M.J., Jacquet, P., Falcone, J.L., Rougemont, J., and Shore, D. (2015). Nucleosome Stability Distinguishes Two Different Promoter Types at All ProteinCoding Genes in Yeast. Mol Cell 60, 422-434.

Kubik, S., O'Duibhir, E., de Jonge, W.J., Mattarocci, S., Albert, B., Falcone, J.L., Bruzzone, M.J., Holstege, F.C.P., and Shore, D. (2018). Sequence-Directed Action of RSC Remodeler and General Regulatory Factors Modulates +1 Nucleosome Position to Facilitate Transcription. Mol Cell 71, 89-102 e105.

Kuhlbrandt, W. (2014). Biochemistry. The resolution revolution. Science 343, 1443-1444.

Lafon-Hughes, L., Di Tomaso, M.V., Mendez-Acuna, L., and Martinez-Lopez, W. (2008). Chromatin-remodelling mechanisms in cancer. Mutat Res 658, 191-214.

Lantermann, A.B., Straub, T., Stralfors, A., Yuan, G.C., Ekwall, K., and Korber, P. (2010). Schizosaccharomyces pombe genome-wide nucleosome mapping reveals positioning mechanisms distinct from those of Saccharomyces cerevisiae. Nat Struct Mol Biol 17, 251-257.

Lee, W., Tillo, D., Bray, N., Morse, R.H., Davis, R.W., Hughes, T.R., and Nislow, C. (2007). A high-resolution atlas of nucleosome occupancy in yeast. Nat Genet 39, 1235-1244.

Legge, G.B., Martinez-Yamout, M.A., Hambly, D.M., Trinh, T., Lee, B.M., Dyson, H.J., and Wright, P.E. (2004). ZZ domain of CBP: an unusual zinc finger fold in a protein interaction module. J Mol Biol 343, 1081-1093.

Leschziner, A.E., Lemon, B., Tjian, R., and Nogales, E. (2005). Structural studies of the human PBAF chromatin-remodeling complex. Structure 13, 267-275.

Leschziner, A.E., Saha, A., Wittmeyer, J., Zhang, Y., Bustamante, C., Cairns, B.R., and Nogales, E. (2007). Conformational flexibility in the chromatin remodeler RSC observed by electron microscopy and the orthogonal tilt reconstruction method. Proc Natl Acad Sci U S A 104, 4913-4918.

Lessard, J., Wu, J.I., Ranish, J.A., Wan, M., Winslow, M.M., Staahl, B.T., Wu, H., Aebersold, R., Graef, I.A., and Crabtree, G.R. (2007). An essential switch in subunit composition of a chromatin remodeling complex during neural development. Neuron 55, 201-215.

Li, M., Xia, X., Tian, Y., Jia, Q., Liu, X., Lu, Y., Li, M., Li, X., and Chen, Z. (2019). Mechanism of DNA translocation underlying chromatin remodelling by Snf2. Nature 567, 409-413.

Liefshitz, B., and Kupiec, M. (2011). Roles of RSC, Rad59, and cohesin in double-strand break repair. Mol Cell Biol 31, 3921-3923.

Lieleg, C., Ketterer, P., Nuebler, J., Ludwigsen, J., Gerland, U., Dietz, H., Mueller-Planitz, F., and Korber, P. (2015). Nucleosome spacing generated by ISWI and CHD1 remodelers is constant regardless of nucleosome density. Mol Cell Biol 35, 15881605.

Liu, X., Li, M., Xia, X., Li, X., and Chen, Z. (2017). Mechanism of chromatin remodelling revealed by the Snf2-nucleosome structure. Nature 544, 440-445. 
Liu, Y., Schmidt, B., and Maskell, D.L. (2010). MSAProbs: multiple sequence alignment based on pair hidden Markov models and partition function posterior probabilities. Bioinformatics 26, 1958-1964.

Lorch, Y., and Kornberg, R.D. (2004). Isolation and assay of the RSC chromatinremodeling complex from Saccharomyces cerevisiae. Methods Enzymol 377, 316322.

Lorch, Y., and Kornberg, R.D. (2017). Chromatin-remodeling for transcription. Q Rev Biophys 50, e5.

Lorch, Y., LaPointe, J.W., and Kornberg, R.D. (1987). Nucleosomes inhibit the initiation of transcription but allow chain elongation with the displacement of histones. Cell 49, 203-210.

Lorch, Y., Maier-Davis, B., and Kornberg, R.D. (2006). Chromatin remodeling by nucleosome disassembly in vitro. Proc Natl Acad Sci U S A 103, 3090-3093.

Lorch, Y., Maier-Davis, B., and Kornberg, R.D. (2014). Role of DNA sequence in chromatin remodeling and the formation of nucleosome-free regions. Genes Dev 28, 24922497.

Lorch, Y., Maier-Davis, B., and Kornberg, R.D. (2018). Histone Acetylation Inhibits RSC and Stabilizes the +1 Nucleosome. Mol Cell 72, 594-600 e592.

Lowary, P.T., and Widom, J. (1998). New DNA sequence rules for high affinity binding to histone octamer and sequence-directed nucleosome positioning. J Mol Biol 276, 1942.

Luger, K., Mader, A.W., Richmond, R.K., Sargent, D.F., and Richmond, T.J. (1997). Crystal structure of the nucleosome core particle at 2.8 A resolution. Nature 389, 251-260.

Luger, K., Rechsteiner, T.J., and Richmond, T.J. (1999). Expression and purification of recombinant histones and nucleosome reconstitution. Methods Mol Biol 119, 1-16.

Makde, R.D., England, J.R., Yennawar, H.P., and Tan, S. (2010). Structure of RCC1 chromatin factor bound to the nucleosome core particle. Nature 467, 562-566.

Makrantoni, V., and Marston, A.L. (2018). Cohesin and chromosome segregation. Curr Biol 28, R688-R693.

Maskell, D.P., Renault, L., Serrao, E., Lesbats, P., Matadeen, R., Hare, S., Lindemann, D., Engelman, A.N., Costa, A., and Cherepanov, P. (2015). Structural basis for retroviral integration into nucleosomes. Nature 523, 366-369.

Materne, P., Vazquez, E., Sanchez, M., Yague-Sanz, C., Anandhakumar, J., Migeot, V., Antequera, F., and Hermand, D. (2016). Histone H2B ubiquitylation represses gametogenesis by opposing RSC-dependent chromatin remodeling at the ste11 master regulator locus. Elife 5 .

Mavrich, T.N., Ioshikhes, I.P., Venters, B.J., Jiang, C., Tomsho, L.P., Qi, J., Schuster, S.C., Albert, I., and Pugh, B.F. (2008a). A barrier nucleosome model for statistical positioning of nucleosomes throughout the yeast genome. Genome Res 18, 10731083.

Mavrich, T.N., Jiang, C., Ioshikhes, I.P., Li, X., Venters, B.J., Zanton, S.J., Tomsho, L.P., Qi, J., Glaser, R.L., Schuster, S.C., et al. (2008b). Nucleosome organization in the Drosophila genome. Nature 453, 358-362.

Maya-Miles, D., Andujar, E., Perez-Alegre, M., Murillo-Pineda, M., Barrientos-Moreno, M., Cabello-Lobato, M.J., Gomez-Marin, E., Morillo-Huesca, M., and Prado, F. (2019). Crosstalk between chromatin structure, cohesin activity and transcription. Epigenetics Chromatin 12, 47.

McGinty, R.K., Henrici, R.C., and Tan, S. (2014). Crystal structure of the PRC1 ubiquitylation module bound to the nucleosome. Nature 514,591-596. 
McGinty, R.K., and Tan, S. (2015). Nucleosome structure and function. Chem Rev 115, 2255-2273.

Mizuguchi, G., Shen, X., Landry, J., Wu, W.H., Sen, S., and Wu, C. (2004). ATP-driven exchange of histone H2AZ variant catalyzed by SWR1 chromatin remodeling complex. Science 303, 343-348.

Munoz, S., Minamino, M., Casas-Delucchi, C.S., Patel, H., and Uhlmann, F. (2019). A Role for Chromatin Remodeling in Cohesin Loading onto Chromosomes. Mol Cell 74, 664673 e665.

Muse, G.W., Gilchrist, D.A., Nechaev, S., Shah, R., Parker, J.S., Grissom, S.F., Zeitlinger, J., and Adelman, K. (2007). RNA polymerase is poised for activation across the genome. Nat Genet 39, 1507-1511.

Nair, S.S., and Kumar, R. (2012). Chromatin remodeling in cancer: a gateway to regulate gene transcription. Mol Oncol 6, 611-619.

Neigeborn, L., and Carlson, M. (1984). Genes affecting the regulation of SUC2 gene expression by glucose repression in Saccharomyces cerevisiae. Genetics 108, 845858.

Ng, H.H., Robert, F., Young, R.A., and Struhl, K. (2002). Genome-wide location and regulated recruitment of the RSC nucleosome-remodeling complex. Genes Dev 16, 806-819.

Nicolas, R.H., and Goodwin, G.H. (1996). Molecular cloning of polybromo, a nuclear protein containing multiple domains including five bromodomains, a truncated HMG-box, and two repeats of a novel domain. Gene 175, 233-240.

Papamichos-Chronakis, M., Watanabe, S., Rando, O.J., and Peterson, C.L. (2011). Global regulation of H2A.Z localization by the INO80 chromatin-remodeling enzyme is essential for genome integrity. Cell 144, 200-213.

Parnell, T.J., Huff, J.T., and Cairns, B.R. (2008). RSC regulates nucleosome positioning at Pol II genes and density at Pol III genes. EMBO J 27, 100-110.

Parvin, J.D., McCormick, R.J., Sharp, P.A., and Fisher, D.E. (1995). Pre-bending of a promoter sequence enhances affinity for the TATA-binding factor. Nature 373,724 727.

Pettersen, E.F., Goddard, T.D., Huang, C.C., Couch, G.S., Greenblatt, D.M., Meng, E.C., and Ferrin, T.E. (2004). UCSF Chimera--a visualization system for exploratory research and analysis. J Comput Chem 25, 1605-1612.

Prakash, K., and Fournier, D. (2018). Evidence for the implication of the histone code in building the genome structure. Biosystems 164, 49-59.

Prasad, P., Sanyal, K., and Ghosh, S.K. (2019). Sth1, the Key Subunit of the RSC Chromatin Remodeling Complex, Is Essential in Maintaining Chromosomal Integrity and Mediating High Fidelity Chromosome Segregation in the Human Fungal Pathogen Candida albicans. Front Microbiol 10, 1303.

Prasad, R., D'Arcy, S., Hada, A., Luger, K., and Bartholomew, B. (2016). Coordinated Action of Nap1 and RSC in Disassembly of Tandem Nucleosomes. Mol Cell Biol 36, 22622271.

Pulice, J.L., and Kadoch, C. (2016). Composition and Function of Mammalian SWI/SNF Chromatin Remodeling Complexes in Human Disease. Cold Spring Harb Symp Quant Biol 81, 53-60.

Rahl, P.B., Lin, C.Y., Seila, A.C., Flynn, R.A., McCuine, S., Burge, C.B., Sharp, P.A., and Young, R.A. (2010). c-Myc regulates transcriptional pause release. Cell 141, 432-445.

Ramachandran, S., Zentner, G.E., and Henikoff, S. (2015). Asymmetric nucleosomes flank promoters in the budding yeast genome. Genome Res 25, 381-390. 
Raman, S., Vernon, R., Thompson, J., Tyka, M., Sadreyev, R., Pei, J., Kim, D., Kellogg, E., DiMaio, F., Lange, O., et al. (2009). Structure prediction for CASP8 with all-atom refinement using Rosetta. Proteins 77 Suppl 9, 89-99.

Reichen, C., Madhurantakam, C., Hansen, S., Grutter, M.G., Pluckthun, A., and Mittl, P.R. (2016). Structures of designed armadillo-repeat proteins show propagation of interrepeat interface effects. Acta Crystallogr D Struct Biol 72, 168-175.

Rhee, H.S., and Pugh, B.F. (2012). Genome-wide structure and organization of eukaryotic pre-initiation complexes. Nature 483, 295-301.

Rigaut, G., Shevchenko, A., Rutz, B., Wilm, M., Mann, M., and Seraphin, B. (1999). A generic protein purification method for protein complex characterization and proteome exploration. Nat Biotechnol 17, 1030-1032.

Robert, X., and Gouet, P. (2014). Deciphering key features in protein structures with the new ENDscript server. Nucleic Acids Res 42, W320-324.

Saha, A., Wittmeyer, J., and Cairns, B.R. (2002). Chromatin remodeling by RSC involves ATP-dependent DNA translocation. Genes Dev 16, 2120-2134.

Saha, A., Wittmeyer, J., and Cairns, B.R. (2005). Chromatin remodeling through directional DNA translocation from an internal nucleosomal site. Nat Struct Mol Biol 12, 747755.

Saha, A., Wittmeyer, J., and Cairns, B.R. (2006). Chromatin remodelling: the industrial revolution of DNA around histones. Nat Rev Mol Cell Biol 7, 437-447.

Sanders, S.L., Jennings, J., Canutescu, A., Link, A.J., and Weil, P.A. (2002). Proteomics of the eukaryotic transcription machinery: identification of proteins associated with components of yeast TFIID by multidimensional mass spectrometry. Mol Cell Biol $22,4723-4738$.

Sandhya, S., Maulik, A., Giri, M., and Singh, M. (2018). Domain architecture of BAF250a reveals the ARID and ARM-repeat domains with implication in function and assembly of the BAF remodeling complex. PLoS One 13, e0205267.

Satterwhite, E., Sonoki, T., Willis, T.G., Harder, L., Nowak, R., Arriola, E.L., Liu, H., Price, H.P., Gesk, S., Steinemann, D., et al. (2001). The BCL11 gene family: involvement of BCL11A in lymphoid malignancies. Blood 98, 3413-3420.

Scheres, S.H. (2012). RELION: implementation of a Bayesian approach to cryo-EM structure determination. J Struct Biol 180, 519-530.

Schones, D.E., Cui, K., Cuddapah, S., Roh, T.Y., Barski, A., Wang, Z., Wei, G., and Zhao, K. (2008). Dynamic regulation of nucleosome positioning in the human genome. Cell 132, 887-898.

Schrodinger, LLC (2015). The PyMOL Molecular Graphics System, Version 1.8.

Schubert, H.L., Wittmeyer, J., Kasten, M.M., Hinata, K., Rawling, D.C., Heroux, A., Cairns, B.R., and Hill, C.P. (2013). Structure of an actin-related subcomplex of the SWI/SNF chromatin remodeler. Proc Natl Acad Sci U S A 110, 3345-3350.

Sen, P., Ghosh, S., Pugh, B.F., and Bartholomew, B. (2011). A new, highly conserved domain in Swi2/Snf2 is required for SWI/SNF remodeling. Nucleic Acids Res 39, 9155-9166.

Sen, P., Vivas, P., Dechassa, M.L., Mooney, A.M., Poirier, M.G., and Bartholomew, B. (2013). The SnAC domain of SWI/SNF is a histone anchor required for remodeling. Mol Cell Biol 33, 360-370.

Sims, R.J., 3rd, Chen, C.F., Santos-Rosa, H., Kouzarides, T., Patel, S.S., and Reinberg, D. (2005). Human but not yeast CHD1 binds directly and selectively to histone H3 methylated at lysine 4 via its tandem chromodomains. J Biol Chem 280, 4178941792. 
Singleton, M.R., Dillingham, M.S., and Wigley, D.B. (2007). Structure and mechanism of helicases and nucleic acid translocases. Annu Rev Biochem 76, 23-50.

Skene, P.J., Hernandez, A.E., Groudine, M., and Henikoff, S. (2014). The nucleosomal barrier to promoter escape by RNA polymerase II is overcome by the chromatin remodeler Chd1. Elife 3, e02042.

Song, Y., DiMaio, F., Wang, R.Y., Kim, D., Miles, C., Brunette, T., Thompson, J., and Baker, D. (2013). High-resolution comparative modeling with RosettaCM. Structure 21, 17351742.

Soutourina, J., Bordas-Le Floch, V., Gendrel, G., Flores, A., Ducrot, C., Dumay-Odelot, H., Soularue, P., Navarro, F., Cairns, B.R., Lefebvre, O., et al. (2006). Rsc4 connects the chromatin remodeler RSC to RNA polymerases. Mol Cell Biol 26, 4920-4933.

Spain, M.M., Ansari, S.A., Pathak, R., Palumbo, M.J., Morse, R.H., and Govind, C.K. (2014). The RSC complex localizes to coding sequences to regulate Pol II and histone occupancy. Mol Cell 56, 653-666.

Stark, H. (2010). GraFix: stabilization of fragile macromolecular complexes for single particle cryo-EM. Methods Enzymol 481, 109-126.

Sugathan, A., Biagioli, M., Golzio, C., Erdin, S., Blumenthal, I., Manavalan, P., Ragavendran, A., Brand, H., Lucente, D., Miles, J., et al. (2014). CHD8 regulates neurodevelopmental pathways associated with autism spectrum disorder in neural progenitors. Proc Natl Acad Sci U S A 111, E4468-4477.

Sundaramoorthy, R., Hughes, A.L., El-Mkami, H., Norman, D.G., Ferreira, H., and OwenHughes, T. (2018). Structure of the chromatin remodelling enzyme Chd1 bound to a ubiquitinylated nucleosome. Elife 7.

Suto, R.K., Clarkson, M.J., Tremethick, D.J., and Luger, K. (2000). Crystal structure of a nucleosome core particle containing the variant histone H2A.Z. Nat Struct Biol 7, 1121-1124.

Szerlong, H., Hinata, K., Viswanathan, R., Erdjument-Bromage, H., Tempst, P., and Cairns, B.R. (2008). The HSA domain binds nuclear actin-related proteins to regulate chromatin-remodeling ATPases. Nat Struct Mol Biol 15, 469-476.

Tan, S., and Davey, C.A. (2011). Nucleosome structural studies. Curr Opin Struct Biol 21, 128-136.

Taneda, T., and Kikuchi, A. (2004). Genetic analysis of RSC58, which encodes a component of a yeast chromatin remodeling complex, and interacts with the transcription factor Swi6. Mol Genet Genomics 271, 479-489.

Tegunov, D., and Cramer, P. (2018). Real-time cryo-EM data pre-processing with Warp. bioRxiv.

Tran, H.G., Steger, D.J., Iyer, V.R., and Johnson, A.D. (2000). The chromo domain protein chd1p from budding yeast is an ATP-dependent chromatin-modifying factor. EMBO J 19, 2323-2331.

Treich, I., Ho, L., and Carlson, M. (1998). Direct interaction between Rsc6 and Rsc8/Swh3, two proteins that are conserved in SWI/SNF-related complexes. Nucleic Acids Res 26, 3739-3745.

Tsunaka, Y., Kajimura, N., Tate, S., and Morikawa, K. (2005). Alteration of the nucleosomal DNA path in the crystal structure of a human nucleosome core particle. Nucleic Acids Res 33, 3424-3434.

Tyagi, M., Imam, N., Verma, K., and Patel, A.K. (2016). Chromatin remodelers: We are the drivers!! Nucleus 7, 388-404.

Valouev, A., Ichikawa, J., Tonthat, T., Stuart, J., Ranade, S., Peckham, H., Zeng, K., Malek, J.A., Costa, G., McKernan, K., et al. (2008). A high-resolution, nucleosome position map of 
C. elegans reveals a lack of universal sequence-dictated positioning. Genome Res 18, 1051-1063.

van Attikum, H., Fritsch, O., and Gasser, S.M. (2007). Distinct roles for SWR1 and IN080 chromatin remodeling complexes at chromosomal double-strand breaks. EMBO J 26, 4113-4125.

van Dijk, M., and Bonvin, A.M. (2009). 3D-DART: a DNA structure modelling server. Nucleic Acids Res 37, W235-239.

VanDemark, A.P., Kasten, M.M., Ferris, E., Heroux, A., Hill, C.P., and Cairns, B.R. (2007). Autoregulation of the rsc4 tandem bromodomain by gcn5 acetylation. Mol Cell 27, 817-828.

Vary, J.C., Jr., Gangaraju, V.K., Qin, J., Landel, C.C., Kooperberg, C., Bartholomew, B., and Tsukiyama, T. (2003). Yeast Isw1p forms two separable complexes in vivo. Mol Cell Biol 23, 80-91.

Vasudevan, D., Chua, E.Y.D., and Davey, C.A. (2010). Crystal structures of nucleosome core particles containing the '601' strong positioning sequence. J Mol Biol 403, 1-10.

Wang, S.L., and Cheng, M.Y. (2012). The defects in cell wall integrity and G2-M transition of the htl1 mutant are interconnected. Yeast 29, 45-57.

Wang, W., Chi, T., Xue, Y., Zhou, S., Kuo, A., and Crabtree, G.R. (1998). Architectural DNA binding by a high-mobility-group/kinesin-like subunit in mammalian SWI/SNFrelated complexes. Proc Natl Acad Sci U S A 95, 492-498.

Wang, W., Xue, Y., Zhou, S., Kuo, A., Cairns, B.R., and Crabtree, G.R. (1996). Diversity and specialization of mammalian SWI/SNF complexes. Genes Dev 10, 2117-2130.

Waterhouse, A., Bertoni, M., Bienert, S., Studer, G., Tauriello, G., Gumienny, R., Heer, F.T., de Beer, T.A.P., Rempfer, C., Bordoli, L., et al. (2018). SWISS-MODEL: homology modelling of protein structures and complexes. Nucleic Acids Res 46, W296-W303.

Weiss, K., Terhal, P.A., Cohen, L., Bruccoleri, M., Irving, M., Martinez, A.F., Rosenfeld, J.A., Machol, K., Yang, Y., Liu, P., et al. (2016). De Novo Mutations in CHD4, an ATPDependent Chromatin Remodeler Gene, Cause an Intellectual Disability Syndrome with Distinctive Dysmorphisms. Am J Hum Genet 99, 934-941.

White, C.L., Suto, R.K., and Luger, K. (2001). Structure of the yeast nucleosome core particle reveals fundamental changes in internucleosome interactions. EMBO J 20, 5207-5218.

Willhoft, O., Ghoneim, M., Lin, C.L., Chua, E.Y.D., Wilkinson, M., Chaban, Y., Ayala, R., McCormack, E.A., Ocloo, L., Rueda, D.S., et al. (2018). Structure and dynamics of the yeast SWR1-nucleosome complex. Science 362.

Williams, C.J., Headd, J.J., Moriarty, N.W., Prisant, M.G., Videau, L.L., Deis, L.N., Verma, V., Keedy, D.A., Hintze, B.J., Chen, V.B., et al. (2018). MolProbity: More and better reference data for improved all-atom structure validation. Protein Sci 27, 293-315.

Winger, J., Nodelman, I.M., Levendosky, R.F., and Bowman, G.D. (2018). A twist defect mechanism for ATP-dependent translocation of nucleosomal DNA. Elife 7.

Wong, M.C., Scott-Drew, S.R., Hayes, M.J., Howard, P.J., and Murray, J.A. (2002). RSC2, encoding a component of the RSC nucleosome remodeling complex, is essential for 2 microm plasmid maintenance in Saccharomyces cerevisiae. Mol Cell Biol 22, 42184229.

Worden, E.J., Hoffmann, N.A., Hicks, C.W., and Wolberger, C. (2019). Mechanism of Crosstalk between H2B Ubiquitination and H3 Methylation by Dot1L. Cell 176, 1490-1501 e1412.

Wu, W.H., Alami, S., Luk, E., Wu, C.H., Sen, S., Mizuguchi, G., Wei, D., and Wu, C. (2005). Swc2 is a widely conserved H2AZ-binding module essential for ATP-dependent histone exchange. Nat Struct Mol Biol 12, 1064-1071. 
Xi, Y., Yao, J., Chen, R., Li, W., and He, X. (2011). Nucleosome fragility reveals novel functional states of chromatin and poises genes for activation. Genome Res 21, 718724.

Xia, X., Liu, X., Li, T., Fang, X., and Chen, Z. (2016). Structure of chromatin remodeler Swi2/Snf2 in the resting state. Nat Struct Mol Biol 23, 722-729.

Xue, Y., Canman, J.C., Lee, C.S., Nie, Z., Yang, D., Moreno, G.T., Young, M.K., Salmon, E.D., and Wang, W. (2000). The human SWI/SNF-B chromatin-remodeling complex is related to yeast rsc and localizes at kinetochores of mitotic chromosomes. Proc Natl Acad Sci U S A 97, 13015-13020.

Yan, L., Wang, L., Tian, Y., Xia, X., and Chen, Z. (2016). Structure and regulation of the chromatin remodeller ISWI. Nature 540, 466-469.

Yan, L., Wu, H., Li, X., Gao, N., and Chen, Z. (2019). Structures of the ISWI-nucleosome complex reveal a conserved mechanism of chromatin remodeling. Nat Struct Mol Biol 26, 258-266.

Yan, Z., Cui, K., Murray, D.M., Ling, C., Xue, Y., Gerstein, A., Parsons, R., Zhao, K., and Wang, W. (2005). PBAF chromatin-remodeling complex requires a novel specificity subunit, BAF200, to regulate expression of selective interferon-responsive genes. Genes Dev 19, 1662-1667.

Yang, B., Wu, Y.J., Zhu, M., Fan, S.B., Lin, J., Zhang, K., Li, S., Chi, H., Li, Y.X., Chen, H.F., et al. (2012). Identification of cross-linked peptides from complex samples. Nat Methods 9, 904-906.

Yu, F., Imamura, Y., Ueno, M., Suzuki, S.W., Ohsumi, Y., Yukawa, M., and Tsuchiya, E. (2015). The yeast chromatin remodeler Rsc1-RSC complex is required for transcriptional activation of autophagy-related genes and inhibition of the TORC1 pathway in response to nitrogen starvation. Biochem Biophys Res Commun 464, 1248-1253.

Yukawa, M., Koyama, H., Miyahara, K., and Tsuchiya, E. (2002). Functional differences between RSC1 and RSC2, components of a for growth essential chromatinremodeling complex of Saccharomyces cerevisiae, during the sporulation process. FEMS Yeast Res 2, 87-91.

Zeitlinger, J., Stark, A., Kellis, M., Hong, J.W., Nechaev, S., Adelman, K., Levine, M., and Young, R.A. (2007). RNA polymerase stalling at developmental control genes in the Drosophila melanogaster embryo. Nat Genet 39, 1512-1516.

Zhang, S., Zhou, B., Wang, L., Li, P., Bennett, B.D., Snyder, R., Garantziotis, S., Fargo, D.C., Cox, A.D., Chen, L., et al. (2017). INO80 is required for oncogenic transcription and tumor growth in non-small cell lung cancer. Oncogene 36, 1430-1439.

Zhou, C.Y., Johnson, S.L., Lee, L.J., Longhurst, A.D., Beckwith, S.L., Johnson, M.J., Morrison, A.J., and Narlikar, G.J. (2018). The Yeast IN080 Complex Operates as a Tunable DNA Length-Sensitive Switch to Regulate Nucleosome Sliding. Mol Cell 69, 677-688 e679.

Zimmermann, L., Stephens, A., Nam, S.Z., Rau, D., Kubler, J., Lozajic, M., Gabler, F., Soding, J., Lupas, A.N., and Alva, V. (2018). A Completely Reimplemented MPI Bioinformatics Toolkit with a New HHpred Server at its Core. J Mol Biol 430, 2237-2243.

Zivanov, J., Nakane, T., Forsberg, B.O., Kimanius, D., Hagen, W.J., Lindahl, E., and Scheres, S.H. (2018). New tools for automated high-resolution cryo-EM structure determination in RELION-3. Elife 7. 


\section{SUPPLEMENTARY MATERIALS}

Table S11 | RSC subunit modelling. Modelling details for the RSC complex. Density that could not be assigned to a subunit was modelled with a poly alanine backbone in chain $\mathrm{X}$. The domains of the two Rsc8 subunits are combined in one chain (L) spaced by 1000 amino acid offset.

\begin{tabular}{|c|c|c|c|c|}
\hline $\begin{array}{l}\text { Subunit / } \\
\text { Chain ID }\end{array}$ & Domain & $\begin{array}{l}\text { Residue } \\
\text { range }\end{array}$ & $\begin{array}{c}\text { PDB entry of } \\
\text { homology model / } \\
\text { Modelling algorithm }\end{array}$ & $\begin{array}{c}\text { Changes to } \\
\text { homology model }\end{array}$ \\
\hline $\begin{array}{c}H 2 A / \\
C+G \\
H 2 B / \\
D+H \\
H 3 / \\
A+E \\
H 4 / \\
B+F\end{array}$ & $\begin{array}{l}\text { Histone } \\
\text { Histone }\end{array}$ & $\begin{array}{l}15-116 \\
15-116 \\
30-121 \\
30-121 \\
39-134 \\
40-134 \\
15-101 \\
21-102\end{array}$ & $\begin{array}{l}5 Z 3 U \text { / Used as } \\
\text { deposited, } \\
\text { without chain O }\end{array}$ & $\begin{array}{l}\text { UCSF Chimera, } \\
\text { Namdinator, } \\
\text { PHENIX real space } \\
\text { refinement } \\
\text { H4 (B) 15-22 } \\
\text { stubbed }\end{array}$ \\
\hline $\begin{array}{l}\text { DNA / I } \\
\text { DNA / J }\end{array}$ & $\begin{array}{l}\text { Nucl. DNA } \\
\text { Nucl. DNA }\end{array}$ & $\begin{array}{l}2-124 \\
24-146\end{array}$ & & $\begin{array}{l}\text { Removed residues } \\
\text { (see residue range) }\end{array}$ \\
\hline $\begin{array}{l}\text { DNA / I } \\
\text { DNA / J }\end{array}$ & $\begin{array}{l}\text { Upstream DNA } \\
\text { Upstream DNA }\end{array}$ & $\begin{array}{c}-35-1 \\
125-183\end{array}$ & B-DNA / 3D-DART & $\begin{array}{l}\text { UCSF Chimera, } \\
\text { PHENIX geometry } \\
\text { minimization }\end{array}$ \\
\hline Sfh1 / K & $\mathrm{N}$-term. & $1-150$ & not modelled & \\
\hline Sfh1 / K & & $151-186$ & $\begin{array}{l}\text { COOT, density guided } \\
\text { de novo modelling }\end{array}$ & \\
\hline Sfh1 / K & & $187-198$ & not modelled & \\
\hline Sfh1 / K & & $198-201$ & $\begin{array}{l}\text { COOT, density guided } \\
\text { de novo modelling }\end{array}$ & \\
\hline Sfh1 / K & RPT1 & $202-267$ & $6 a x 5$ / SWISS & $\begin{array}{l}\text { UCSF Chimera, } \\
\text { COOT, density + } \\
\text { model guided } \\
\text { building }\end{array}$ \\
\hline Sfh1 / K & & $268-274$ & $\begin{array}{l}\text { COOT, density guided } \\
\text { de novo modelling }\end{array}$ & \\
\hline Sfh1 / K & RPT2 & $275-341$ & 6ax5 / SWISS & $\begin{array}{l}\text { UCSF Chimera, } \\
\text { COOT, density + } \\
\text { model guided } \\
\text { building }\end{array}$ \\
\hline
\end{tabular}




\begin{tabular}{|c|c|c|c|c|}
\hline $\begin{array}{l}\text { Subunit I } \\
\text { Chain ID }\end{array}$ & Domain & $\begin{array}{l}\text { Residue } \\
\text { range }\end{array}$ & $\begin{array}{c}\text { PDB entry of } \\
\text { homology model / } \\
\text { Modelling algorithm }\end{array}$ & $\begin{array}{c}\text { Changes to } \\
\text { homology model }\end{array}$ \\
\hline Sfh1 / K & & $342-346$ & $\begin{array}{l}\text { COOT, density guided } \\
\text { de novo modelling }\end{array}$ & \\
\hline Sfh1 / K & Poly-alanine & $347-360$ & $\begin{array}{l}\text { COOT, density guided } \\
\text { de novo modelling }\end{array}$ & \\
\hline Sfh1 / K & & $361-372$ & not modelled & \\
\hline Sfh1 / K & Docking helix & $373-408$ & $\begin{array}{l}\text { COOT, density guided } \\
\text { de novo modelling }\end{array}$ & \\
\hline Sfh1 / K & C-term. & $408-426$ & not modelled & \\
\hline Rsc8 / L & N-term. & $1-58$ & not modelled & \\
\hline Rsc8 / L & & $59-86$ & $\begin{array}{l}\text { COOT, density guided } \\
\text { de novo modelling }\end{array}$ & \\
\hline Rsc8 / L & SWIRM & $87-169$ & 2FQ3 / SWISS & $\begin{array}{l}\text { UCSF Chimera, } \\
\text { COOT, density + } \\
\text { model guided } \\
\text { building }\end{array}$ \\
\hline Rsc8 / L & & $170-200$ & $\begin{array}{l}\text { COOT, density guided } \\
\text { de novo modelling }\end{array}$ & \\
\hline Rsc8 / L & & $201-254$ & not modelled & \\
\hline Rsc8 / L & Zn finger & $255-307$ & 1TOT / SWISS & $\begin{array}{l}\text { UCSF Chimera, } \\
\text { COOT, density + } \\
\text { model guided } \\
\text { building }\end{array}$ \\
\hline Rsc8 / L & & $308-314$ & not modelled & \\
\hline Rsc8 / L & SANT & $315-359$ & 2YUS / SWISS & $\begin{array}{l}\text { UCSF Chimera, } \\
\text { COOT, density + } \\
\text { model guided } \\
\text { building }\end{array}$ \\
\hline Rsc8 / L & & $360-368$ & $\begin{array}{l}\text { COOT, density guided } \\
\text { de novo modelling }\end{array}$ & \\
\hline Rsc8 / L & & $369-385$ & not modelled & \\
\hline Rsc8 / L & Long helix & $386-492$ & $\begin{array}{l}\text { COOT, density guided } \\
\text { de novo modelling }\end{array}$ & \\
\hline Rsc8 / L & C-term. & $493-557$ & not modelled & \\
\hline Rsc8 / L & N-term. & $1001-1081$ & not modelled & \\
\hline Rsc8 / L & & $1082-1086$ & $\begin{array}{l}\text { COOT, density guided } \\
\text { de novo modelling }\end{array}$ & \\
\hline Rsc8 / L & SWIRM & $1087-1169$ & 2FQ3 / SWISS & $\begin{array}{l}\text { UCSF Chimera, } \\
\text { COOT, density + } \\
\text { model guided } \\
\text { building }\end{array}$ \\
\hline
\end{tabular}




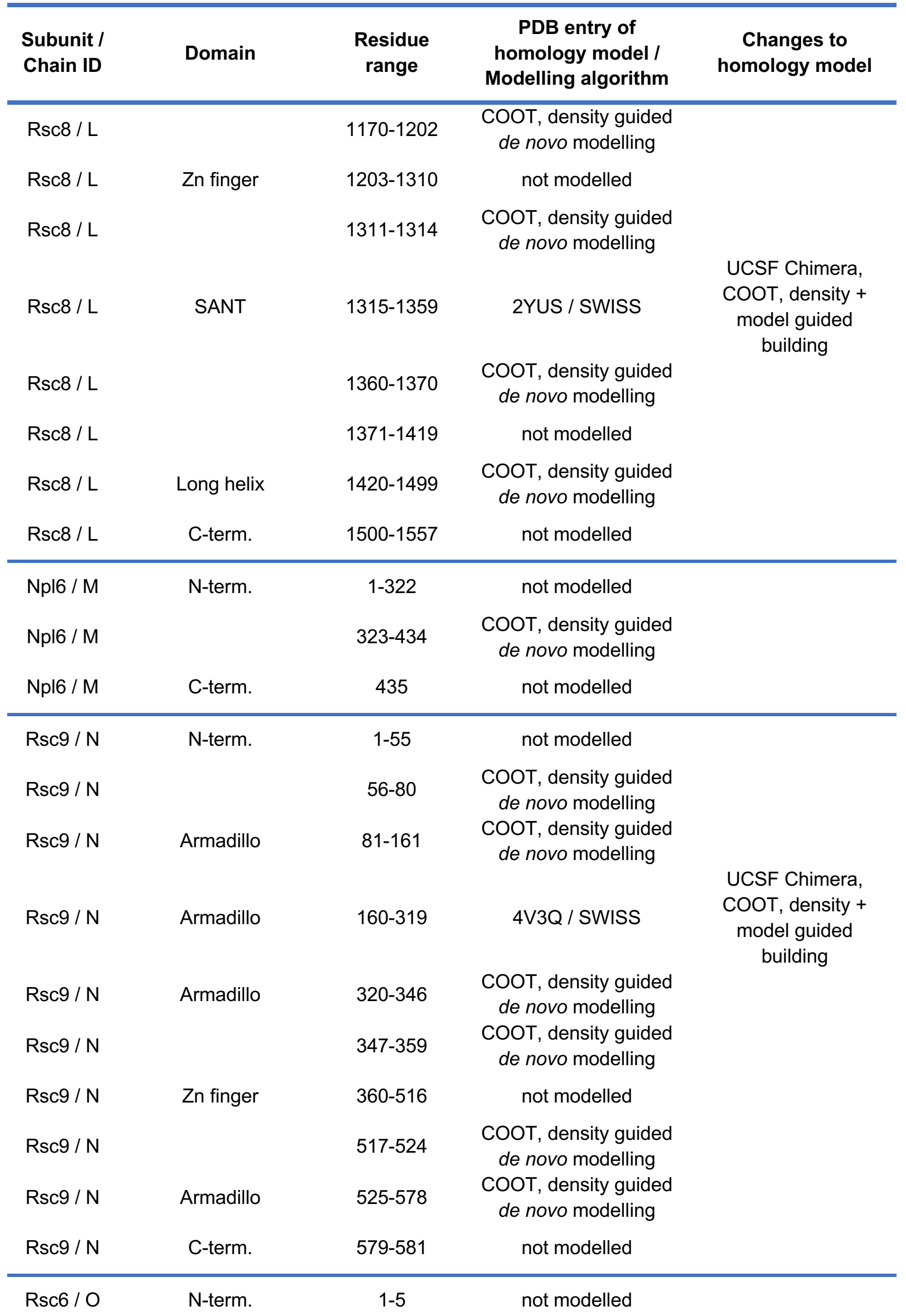




\begin{tabular}{|c|c|c|c|c|}
\hline $\begin{array}{l}\text { Subunit } / \\
\text { Chain ID }\end{array}$ & Domain & $\begin{array}{l}\text { Residue } \\
\text { range }\end{array}$ & $\begin{array}{c}\text { PDB entry of } \\
\text { homology model / } \\
\text { Modelling algorithm }\end{array}$ & $\begin{array}{c}\text { Changes to } \\
\text { homology model }\end{array}$ \\
\hline Rsc6 / O & & $6-61$ & $\begin{array}{l}\text { COOT, density guided } \\
\text { de novo modelling }\end{array}$ & \\
\hline Rsc6 / O & & $62-227$ & not modelled & \\
\hline Rsc6 / O & SWIB & 228-269 & 1UHR / SWISS & $\begin{array}{l}\text { UCSF Chimera, } \\
\text { COOT, density + } \\
\text { model guided } \\
\text { building }\end{array}$ \\
\hline Rsc6 / O & & $270-312$ & not modelled & \\
\hline Rsc6 / O & SWIB & $313-343$ & 1UHR / SWISS & $\begin{array}{l}\text { UCSF Chimera, } \\
\text { COOT, density + } \\
\text { model guided } \\
\text { building }\end{array}$ \\
\hline Rsc6 / O & & $344-391$ & not modelled & \\
\hline Rsc6 / O & & $392-480$ & $\begin{array}{l}\text { COOT, density guided } \\
\text { de novo modelling }\end{array}$ & \\
\hline Rsc6 / O & C-term. & $481-483$ & not modelled & \\
\hline Rsc58 / P & N-term. & $1-9$ & not modelled & \\
\hline Rsc58 / P & Bromo & $10-36$ & $\begin{array}{l}\text { COOT, density guided } \\
\text { de novo modelling }\end{array}$ & \\
\hline Rsc58 / P & Bromo & $37-71$ & not modelled & \\
\hline Rsc58 / P & Bromo & $72-121$ & 3LJW / SWISS & $\begin{array}{l}\text { UCSF Chimera, } \\
\text { COOT, density + } \\
\text { model guided } \\
\text { building }\end{array}$ \\
\hline Rsc58 / P & Bromo & $122-139$ & $\begin{array}{l}\text { COOT, density guided } \\
\text { de novo modelling }\end{array}$ & \\
\hline Rsc58 / P & & $140-167$ & not modelled & \\
\hline Rsc58 / P & & 168-316 & $\begin{array}{l}\text { COOT, density guided } \\
\text { de novo modelling }\end{array}$ & \\
\hline Rsc58 / P & & $317-387$ & not modelled & \\
\hline Rsc58 / P & & $388-491$ & $\begin{array}{l}\text { COOT, density guided } \\
\text { de novo modelling }\end{array}$ & \\
\hline Rsc58 / P & C-term. & $492-502$ & not modelled & \\
\hline $\mathrm{Ht} \mid 1 / \mathrm{Q}$ & N-term. & $1-16$ & not modelled & \\
\hline $\mathrm{Ht} \mid 1 / \mathrm{Q}$ & & $17-74$ & $\begin{array}{l}\text { COOT, density guided } \\
\text { de novo modelling }\end{array}$ & \\
\hline $\mathrm{Ht} 1$ 1 / Q & C-term. & $75-78$ & not modelled & \\
\hline
\end{tabular}




\begin{tabular}{|c|c|c|c|c|}
\hline $\begin{array}{l}\text { Subunit / } \\
\text { Chain ID }\end{array}$ & Domain & $\begin{array}{l}\text { Residue } \\
\text { range }\end{array}$ & $\begin{array}{c}\text { PDB entry of } \\
\text { homology model / } \\
\text { Modelling algorithm }\end{array}$ & $\begin{array}{c}\text { Changes to } \\
\text { homology model }\end{array}$ \\
\hline Rsc4 / R & $\begin{array}{c}\mathrm{N} \text {-term. / Tandem } \\
\text { bromo }\end{array}$ & $1-443$ & not modelled & \\
\hline Rsc4 / R & Sheet bundle 1 & $444-536$ & $\begin{array}{l}\text { COOT, density guided } \\
\text { de novo modelling }\end{array}$ & \\
\hline Rsc4 / R & & $537-587$ & not modelled & \\
\hline Rsc4 / R & Sheet bundle 2 & $588-625$ & $\begin{array}{l}\text { COOT, density guided } \\
\text { de novo modelling }\end{array}$ & \\
\hline Sth1 / S & $\mathrm{N}$-term. & $1-47$ & not modelled & \\
\hline Sth1 / S & & $48-134$ & $\begin{array}{l}\text { COOT, density guided } \\
\text { de novo modelling }\end{array}$ & \\
\hline Sth1 / S & & $135-156$ & not modelled & \\
\hline Sth1 / S & & $157-293$ & $\begin{array}{l}\text { COOT, density guided } \\
\text { de novo modelling }\end{array}$ & \\
\hline Sth1 / S & & $294-321$ & not modelled & \\
\hline \multirow{3}{*}{ Sth1 / S } & \multirow{3}{*}{ HSA } & \multirow{3}{*}{$322-369$} & \multirow{3}{*}{$\begin{array}{c}4 \mathrm{I} 6 \mathrm{M}: \mathrm{C} / \\
\text { Used as deposited }\end{array}$} & $\begin{array}{c}\text { UCSF Chimera, } \\
\text { rigid-body docking } \\
\text { together with ARP } \\
\text { module }\end{array}$ \\
\hline & & & & $\begin{array}{l}\text { Removed residues } \\
\text { (see residue range) }\end{array}$ \\
\hline & & & & $\begin{array}{c}\text { Amino acid residues } \\
\text { mutated to Sth1- } \\
\text { HSA (see Methods) }\end{array}$ \\
\hline Sth1 / S & & $370-392$ & not modelled & \\
\hline Sth1 / S & ATPase & $\begin{array}{c}\text { modelled: } \\
393-407 \\
447-662 \\
677-734 \\
754-940 \\
976-1006 \\
\text { not modelled: } \\
408-446 \\
663-676 \\
735-753 \\
941-975\end{array}$ & 5Z3U:O / Rosetta & $\begin{array}{l}\text { UCSF Chimera, } \\
\text { PHENIX real space } \\
\text { refinement } \\
\text { Side chains stubbec } \\
\text { Removed residues } \\
\text { (see residue range) }\end{array}$ \\
\hline Sth1 / S & $\begin{array}{l}\text { Bromo, } \\
\text { C-term. }\end{array}$ & $1007-1359$ & not modelled & \\
\hline
\end{tabular}




$\begin{array}{lccc}\text { Arp7 } / \mathrm{T} & & \text { Same as in } & 416 \mathrm{M} / \text { Used as } \\ \text { Arp9 / U } & \text { ARP module } & \begin{array}{c}\text { PDB entry } \\ \text { deposited }\end{array} & \begin{array}{c}\text { UCSF Chimera, } \\ \text { rigid-body docking }\end{array}\end{array}$

Rtt102 / V

Chain $\mathrm{X}$

poly-alanine

COOT, density guided

de novo modelling 
Table S12 | List of all BS3 cross-links detected for the RSC-nucleosome complex. Intra- and inter-subunit lysine-lysine cross-links as identified by LC-MS analyses and subsequent database search using pLink 2 . The respective scores of cross-link identification are listed as well as the number of CSMs (cross-linked spectra matches). R1, residue 1; R2, residue 2; S, score.

\begin{tabular}{|c|c|c|c|c|c|c|c|}
\hline PEPTIDE & PROTEINS & CSM & PROTEIN1 & PROTEIN2 & R1 & R2 & $\mathbf{S}$ \\
\hline SAPAPKK(6)-KAVTK(1) & sp|P02281|H2B11_XENLA (8)-sp|P02281|H2B11_XENLA (13)/ & 12 & H2B11_XENLA & H2B11_XENLA & 8 & 13 & 14.09 \\
\hline TQKKDGK(3)-KAVTK(1) & sp|P02281|H2B11 XENLA (20)-sp|P02281|H2B11 XENLA (13)/ & 2 & H2B11_XENLA & H2B11_XENLA & 20 & 13 & 8.01 \\
\hline KESYAIYVYK(1)-KGNYAER(1) & sp|P02281|H2B11_XENLA (31)-tr|Q6AZJ8|H2A_XĒNLA (36)/ & 14 & H2B11_XENLA & H2A_X XENLA & 31 & 36 & 5.49 \\
\hline QVHPDTGISSKAMSIMNSFVNDVFER(11)-KESYAIYVYK(1) & sp|P02281|H2B11 XENLA (54)-sp|P02281|H2B11 XENLA (31)/ & 8 & H2B11_XENLA & H2B 111 XENLA & 54 & 31 & 5.21 \\
\hline AVTKYTSAK(4)-GKQGGK(2) & sp|P02281|H2B11_XENLA (117)-tr|Q6AZJ8|H2A_XEENLA (5)/ & 2 & H2B11_XENLA & H2A_XĒNLA & 117 & 5 & 5.18 \\
\hline SAPAPKKGSK(7)-KAVTK(1) & sp|P02281|H2B11 XENLA (9)-sp|P02281|H2B11 XENLA (13)/ & 1 & H2B11_XENLA & H2B 111 XENLA & 9 & 13 & 4.14 \\
\hline SAPAPKKGSK(6)-KAVTK(1) & sp|P02281|H2B11_XENLA (8)-sp|P02281|H2B11 XENLA (13)/ & 12 & H2B11_XENLA & H2B11_XENLA & 8 & 13 & 3.72 \\
\hline AKSAPAPK(2)-KAVTK(1) & sp|P02281|H2B11_XENLA (2)-sp|P02281|H2B11_XENLA (13)/ & 1 & H2B11_XENLA & H2B11_XENLA & 2 & 13 & 3.51 \\
\hline HAVSEGTKAVTK(8)-YTSAK(5) & sp|P02281|H2B11_XENLA (113)-sp|P02281|H2B̄11_XENLA (122)/ & 6 & H2B11_XENLA & H2B11_XENLA & 113 & 122 & 3.24 \\
\hline QVHPDTGISSKAMSIMNSFVNDVFER(11)-TRKESYAIYVYK(3) & sp|P02281|H2B11_XENLA (54)-sp|P02281|H2B11_ẌENLA (31)/ & 8 & H2B11_XENLA & H2B11_XENLA & 54 & 31 & 3.05 \\
\hline $\begin{array}{l}\text { QVHPDTGISSKAMSIMNSFVNDVFER(11)-AKSAPAPK(1) } \\
\text { Q10 }\end{array}$ & sp|P02281|H2B11 XENLA (54)-sp|P02281|H2B11_XENLA (1)/ & 2 & H2B11_XENLA & H2B11_XENLA & 54 & 1 & 2.96 \\
\hline LAHYNKR(6)-KGNYAER(1) & sp|P02281|H2B11_XENLA (82)-tr|Q6AZJ8|H2A_XĒNLA (36)/ & 3 & H2B11_XENLA & H2A_XENLA & 82 & 36 & 2.31 \\
\hline LLLPGELAKHAVSEGTK(9)-NDEELNKLLGR(7) & sp|P02281|H2B11_XENLA (105)-tr|Q6AZJ8|H2A___XENLA (95)/ & 37 & H2B11_XENLA & H2A_XENLA & 105 & 95 & 2.28 \\
\hline HAVSEGTKAVTK(8)-LAGDPIDKSAR(8) & sp|P02281|H2B11_XENLA (113)-sp|P32832|NPL6_YEAST (267)/ & 5 & H2B11_XENLA & NPL__YEAST & 113 & 267 & 2.18 \\
\hline TQKKDGK(4)-KAVTK(1) & sp|P02281|H2B11_XENLA (21)-sp|P02281|H2B11_XENLA (13)/ & 1 & H2B11_XENLA & H2B11_XENLA & 21 & 13 & 2.18 \\
\hline QVHPDTGISSKAMSIMNSFVNDVFER(11)-TKQTAR(2) & sp|P02281|H2B11_XENLA (54)-sp|P84233|H32_XĒNLA (4)/ & 3 & H2B11_XENLA & H32_XĒNLA & 54 & 4 & 2.08 \\
\hline LLLPGELAKHAVSEGTK(9)-GLGKGGAKR(4) & sp|P02281|H2B11_XENLA (105)-sp|P62799|H4_-XENLA (12)/ & 6 & H2B11_XENLA & H4_X̄ENLA & 105 & 12 & 1.99 \\
\hline KAVTKTQK(5)-KGSKK(1) & sp|P02281|H2B11_XENLA (17)-sp|P02281|H2B11_XENLA (9)/ & 1 & H2B11_XENLA & H2B̄B11_XENLA & 17 & 9 & 1.83 \\
\hline LLLPGELAKHAVSEGTK(9)-TKQTAR(2) & sp|P02281|H2B11_XENLA (105)-sp|P84233|H32_XENLA (4)/ & 8 & H2B11_XENLA & H32_XENNLA & 105 & 4 & 1.69 \\
\hline LLLPGELAKHAVSEGTK(9)-GKGGK(2) & sp|P02281|H2B11_XENLA (105)-sp|P62799|H4_XENLA (5)/ & 13 & H2B11_XENLA & H4_X̄ENLA & 105 & 5 & 1.64 \\
\hline LLLPGELAKHAVSEGTK(9)-ADAKAQGLR(4) & sp|P02281|H2B11_XENLA (105)-sp|P32597|ST̄̄1_YEAST (1011)/ & 4 & H2B11_XENLA & STT̄'1_YEAST & 105 & 1011 & 1.54 \\
\hline TRKESYAIYVYK(3)-KGNYAER(1) & sp|P02281|H2B11_XENLA (31)-tr|Q6AZJ8|H2A_XENLA (36)/ & 14 & H2B11_XENLA & H2A_X̄ENLA & 31 & 36 & 1.50 \\
\hline QVHPDTGISSKAMSIMNSFVNDVFER(11 & 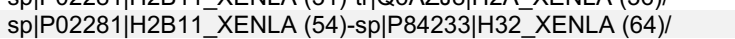 & 13 & H2B11_XENLA & H32_XENLA & 54 & 64 & 1.39 \\
\hline QVHPDTGISSKAMSIMNSFVNDVFER(11)-KSTGGK(1) & sp|P02281|H2B11_XENLA (54)-sp|P84233|H32_XENLA (9)/ & 2 & H2B11_XENLA & H32_XENLA & 54 & 9 & 1.37 \\
\hline AVTKTQK(4)-SAPAPKK(6) & sp|P02281|H2B11_XENLA (17)-sp|P02281|H2B11_XENLA (8)/ & 2 & H2B11_XENLA & H2B11_XENLA & 17 & 8 & 1.26 \\
\hline LLLPGELAKHAVSEGTK(9)-VEILTKEEIQKR(6) & sp|P02281|H2B11_XENLA (105)-sp|Q06168|SFH1_YEAST (385)/ & 1 & H2B11_XENLA & SFH1_YEAST & 105 & 385 & 1.14 \\
\hline $\begin{array}{l}\text { HAVSEGTKAVTK(8)-AKVSKPKR(2) } \\
\text { HAKR (2) }\end{array}$ & sp|P02281|H2B11_XENLA (113)-sp|P38210|LDB7_YEAST (54)/ & 1 & H2B11_XENLA & LDB7_YEAST & 113 & 54 & 1.06 \\
\hline LLLPGELAKHAVSEGTK(9)-EIAQDFKTDLR(7) & sp|P02281|H2B11_XENLA (105)-sp|P84233|H32_XEENLA (79)/ & 6 & H2B11_XENLA & H32_X̄ENLA & 105 & 79 & 1.04 \\
\hline LLLPGELAKHAVSEGTK(9)-GKGGKGLGK(5) & sp|P02281|H2B11_XENLA (105)-sp|P62799|H4_XENLA (8)/ & 10 & H2B11_XENLA & H4_XENLA & 105 & 8 & 0.88 \\
\hline LLLPGELAKHAVSEGTK(9)-GGAKR(4) & sp|P02281|H2B11_XENLA (105)-sp|P62799|H4_XENLA (16)/ & 7 & H2B11_XENLA & H4_XENLA & 105 & 16 & 0.78 \\
\hline LLLPGELAKHAVSEGTK(9)-KPLRDNR(1) & sp|P02281|H2B11_XENLA (105)-sp|Q06488|RSC2_YEAST (234)/ & 2 & H2B11_XENLA & RSC2_YEAST & 105 & 234 & 0.77 \\
\hline LLLPGELAKHAVSEGTK(9)-GKGGKGLGK(2) & sp|P02281|H2B11_XENLA (105)-sp & 13 & H2B11_XENLA & H4_XENLA & 105 & 5 & 0.71 \\
\hline AVTKYTSAK(4)-AKAKTR(2) & sp|P02281|H2B11_XENLA (117)-tr|Q6AZJ8|H2A__XENLA (13)/ & 2 & H2B11_XENLA & H2A_XENLA & 117 & 13 & 0.67 \\
\hline LLLPGELAKHAVSEGTK(9)-IRQKK(4) & sp|P02281|H2B11_XENLA (105)-sp|P32597|STH1_YEAST (1054)/ & 3 & H2B11 XENLA & STH1_YEAST & 105 & 1054 & 0.66 \\
\hline LLLPGELAKHAVSEGTK(9)-GLGKGGAK(4) & sp|P02281|H2B11_XENLA (105)-sp|P62799|H4_XENLA (12)/ & 6 & H2B11_XENLA & H4 XENLA & 105 & 12 & 0.62 \\
\hline LLLPGELAKHAVSEGTK(9)-GGKGLGK( & sp|P02281|H2B11_XENLA (105)- & 10 & H2B11_XENLA & H4_XENLA & 105 & 8 & 0.50 \\
\hline
\end{tabular}




\begin{tabular}{|c|c|c|c|c|c|c|c|}
\hline PEPTIDE & PROTEINS & CSM & PROTEIN1 & PROTEIN2 & R1 & R2 & $\mathbf{s}$ \\
\hline KAVTKTQKK(5)-SAPAPKKGSK(6) & sp|P02281|H2B11_XENLA (17)-sp|P02281|H2B11_XENLA (8)/ & 2 & H2B11_XENLA & H2B11_XENLA & 17 & 8 & 0.80 \\
\hline HAVSEGTKAVTK(8)-ADAKAQGLR(4) & sp|P02281|H2B11_XENLA (113)-sp|P32597|STH1__YEAST (1011)/ & 1 & H2B11_XENLA & STH1_Y̌EAST & 113 & 1011 & 0.41 \\
\hline AKSAPAPK(1)-KAVTK(1) & sp|P02281|H2B11 XENLA (1)-sp|P02281|H2B11 X̄ENLA (13)/ & 2 & H2B11 XENLA & H2B1̄ XENLA & 1 & 13 & 0.67 \\
\hline AKSAPAPK(1)-KGSKK(1) & sp|P02281|H2B11 XENLA (1)-sp|P02281|H2B11 XENLA (9)/ & 1 & H2B11_XENLA & H2B11_XENLA & 1 & 9 & 0.59 \\
\hline AVTKTQKK(4)-KGSKK(4) & sp|P02281|H2B11_XENLA (17)-sp|P02281|H2B11_XENLA (12)/ & 2 & H2B11_XENLA & H2B11_XENLA & 17 & 12 & 0.53 \\
\hline AVTKTQK(4)-KDGKK(1) & sp|P02281|H2B11_XENLA (17)-sp|P02281|H2B11_XENLA (21)/ & 1 & H2B11_XENLA & H2B11_XENLA & 17 & 21 & 0.52 \\
\hline $\begin{array}{l}\text { VTQTNPVPVTYPTDAYIPTYLPDDKVSNLADLKK(1)- } \\
\text { SKECSUIFATIPNEISYNATPDYHUKHK(30) }\end{array}$ & sp|P25632|RSC6_YEAST (2)-sp|Q03124|RSC9_YEAST (358)/ & 27 & RSC6_YEAST & RSC9_YEAST & 2 & 358 & 10.13 \\
\hline $\begin{array}{l}\text { SKECSLILEATLPNLLSYNIATPDYHLLQKHK(30) } \\
\text { DTTYGATTLDVDVSHILHQPQPQPNLQKEEETDAEDTAK(28)- } \\
\text { VTQTNPVPVTYPTDAYIPTYLPDDKVSNLADLKK(1) }\end{array}$ & sp|P25632|RSC6_YEAST (387)-sp|P25632|RSC6_YEAST (2)/ & 4 & RSC6_YEAST & RSC6_YEAST & 387 & 2 & 9.74 \\
\hline $\begin{array}{l}\text { VTQTNPVPVTYPTDAYIPTYLPDDKVSNLADLKK(1)- } \\
\text { ECSSILEATIPNLISYNIATPDYHLIOKHK(28) }\end{array}$ & sp|P25632|RSC6_YEAST (2)-sp|Q03124|RSC9_YEAST (358)/ & 27 & RSC6_YEAST & RSC9_YEAST & 2 & 358 & 9.61 \\
\hline LVSPLPPIILDYTIDLSKDTTYGATTLDVDVSHILHQPQPQPNLQKEE & & & & & & & \\
\hline $\begin{array}{l}\text { ETDAEDTAK(18)- } \\
\text { VTQTNPVPVTYPTDAYIPTYLPDDKVSNLADLKK(1) }\end{array}$ & sp|P25632|RSC6_YEAST (359)-sp|P25632|RSC6_YEAST (2)/ & 1 & RSC6_YEAST & RSC6_YEAST & 359 & 2 & 6.82 \\
\hline LVSPLPPIILDYTIDLSKDTTYGATTLDVDVSHILHQPQPQPNLQKEE & & & & & & & \\
\hline $\begin{array}{l}\text { ETDAEDTAK(46)- } \\
\text { VTQTNPVPVTYPTDAYIPTYLPDDKVSNLADLKK(1) }\end{array}$ & sp|P25632|RSC6_YEAST (387)-sp|P25632|RSC6_YEAST (2)/ & 4 & RSC6_YEAST & RSC6_YEAST & 387 & 2 & 6.79 \\
\hline SDSGTPADSGKTTWTLR(11)-FVIKGIQPR(4) & sp|P25632|RSC6_YEAST (98)-sp|Q03124|RSC9_YEAST (470)/ & 12 & RSC6_YEAST & RSC9_YEAST & 98 & 470 & 6.64 \\
\hline $\begin{array}{l}\text { GVDGGKVQYSPNLATLIGMQTGSVNDAVYSIYK(6)- } \\
\text { RISTINLPTNTKTK(13) }\end{array}$ & sp|P25632|RSC6_YEAST (231)-sp|P25632|RSC6_YEAST (63)/ & 19 & RSC6_YEAST & RSC6_YEAST & 231 & 63 & 5.62 \\
\hline TKDHPPNKEMLR(2)-LSLSKQILDLNK(5) & sp|P25632|RSC6 YEAST (65)-sp|P43609|RSC8_YEAST (477)/ & 7 & RSC6 YEAST & RSC8 YEAST & 65 & 477 & 5.40 \\
\hline LDSLLQKVLDTNAAHLPLMNVVQTVNK(7)-QILDLNKSLEELNVSK(7) & sp|P25632|RSC6_YEAST (321)-sp|P43609|RSC苂_YEAST (484)/ & 20 & RSC6_YEAST & RSC8_YEAST & 321 & 484 & 4.61 \\
\hline $\begin{array}{l}\text { GVDGGKVQYSPNLATLIGMQTGSVNDAVYSIYK(6)- } \\
\text { TKDHPPNKEMLR(2) }\end{array}$ & sp|P25632|RSC6_YEAST (231)-sp|P25632|RSC6_YEAST (65)/ & 42 & RSC6_YEAST & RSC6_YEAST & 231 & 65 & 4.51 \\
\hline LKPLGMGK(2)-QGKDNLR(3) & sp|P25632|RSC6_YEAST (135)-sp|P25632|RSC6_YEAST (213)/ & 17 & RSC6_YEAST & RSC6_YEAST & 135 & 213 & 4.26 \\
\hline EITKLALQLNSSAQK(4)-IGYMDLYEKNEKK(9) & sp|P25632|RSC6_YEAST (404)-sp|Q07979|RSC58_YEAST (322)/ & 13 & RSC6_YEAST & RSC58_YEAST & 404 & 322 & 4.09 \\
\hline $\begin{array}{l}\text { EEIVDALEWNYDENNVVEFDGIDIKR(25)- } \\
\text { LKPPAPKEPPNLSEDLFOQLFK(7) }\end{array}$ & sp|P25632|RSC6_YEAST (209)-sp|Q03124|RSC9_YEAST (373)/ & 1 & RSC6_YEAST & RSC9_YEAST & 209 & 373 & 3.82 \\
\hline $\begin{array}{l}\text { LKPPAPKEPPNNLSEDLFQQLFK(7) } \\
\text { EITKLALQLNSSAQK(4)-IGYMDLYEKNEK(9) }\end{array}$ & sp|P25632|RSC6_YEAST (404)-sp|Q07979|RSC58_YEAST (322)/ & 13 & RSC6_YEAST & RSC58_YEAST & 404 & 322 & 3.65 \\
\hline $\begin{array}{l}\text { GVDGGKVQYSPNLATLIGMQTGSVNDAVYSIYK(6)- } \\
\text { TKDHPPNKEMLR(8) }\end{array}$ & sp|P25632|RSC6_YEAST (231)-sp|P25632|RSC6_YEAST (71)/ & 39 & RSC6_YEAST & RSC6_YEAST & 231 & 71 & 3.61 \\
\hline LLHESANGKHPFSEFLEGVAVDFKR(9)-TKDHPPNKEMLR(2) & sp|P25632|RSC6_YEAST (117)-sp|P25632|RSC6_YEAST (65)/ & 17 & RSC6_YEAST & RSC6_YEAST & 117 & 65 & 3.56 \\
\hline $\begin{array}{l}\text { GVDGGKVQYSPNLATLIGMQTGSVNDAVYSIYK(6)- } \\
\text { LDTSINLPTNTKTKDHPPNKEMLR(12) }\end{array}$ & sp|P25632|RSC6_YEAST (231)-sp|P25632|RSC6_YEAST (63)/ & 19 & RSC6_YEAST & RSC6_YEAST & 231 & 63 & 3.53 \\
\hline $\begin{array}{l}\text { LVSPLPPIILDYTIDLSKDTTYGATTLDVDVSHILHQPQPQPNLQKEE } \\
\text { ETDAEDTAK(18)-VGKEGEEVGEGDSIAK(3) }\end{array}$ & sp|P25632|RSC6_YEAST (359)-sp|P43609|RSC8_YEAST (533)/ & 7 & RSC6_YEAST & RSC8_YEAST & 359 & 533 & 3.49 \\
\hline $\begin{array}{l}\text { GVDGGKVQYSPNLATLIGMQTGSVNDAVYSIYK(6)- } \\
\text { LDTSINLPTNTKTK(12) }\end{array}$ & sp|P25632|RSC6_YEAST (231)-sp|P25632|RSC6_YEAST (63)/ & 19 & RSC6_YEAST & RSC6_YEAST & 231 & 63 & 3.38 \\
\hline $\begin{array}{l}\text { ETLTHYLWSSKQNELVLQGDQYFNEDAAR(11)- } \\
\text { EVVGSTLNGKGGDSR(10) }\end{array}$ & sp|P25632|RSC6_YEAST (438)-sp|P43609|RSC8_YEAST (375)/ & 5 & RSC6_YEAST & RSC8_YEAST & 438 & 375 & 3.37 \\
\hline LLHESANGKHPFSEFLEGVAVDFKR(9)-RLDTSINLPTN & sp|P25632|RSC6_YEAST (117)-sp|P25632|RSC6_YEAST (63)/ & 1 & RSC6_YEAST & RSC6_YEAST & 117 & 63 & 3.32 \\
\hline $\begin{array}{l}\text { GVDGGKVQYSPNLATLIGMQTGSVNDAVYSIYK(6)- } \\
\text { DHPPNKEMLR(6) }\end{array}$ & sp|P25632|RSC6_YEAST (231)-sp|P25632|RSC6_YEAST (71)/ & 39 & RSC6_YEAST & RSC6_YEAST & 231 & 71 & 3.32 \\
\hline $\begin{array}{l}\text { GVDGGKVQYSPNLATLIGMQTGSVNDAVYSIYK(6)- } \\
\text { RLDTSINLPTNTKTKDHPPNK(13) }\end{array}$ & sp|P25632|RSC6_YEAST (231)-sp|P25632|RSC6_YEAST (63)/ & 19 & RSC6_YEAST & RSC6_YEAST & 231 & 63 & 3.31 \\
\hline SDSGTPADSGKTTWTLR(11)-QGKDNLR(3) & sp|P25632|RSC6_YEAST (98)-sp|P25632|RSC6_YEAST (213)/ & 21 & RSC6_YEAST & RSC6_YEAST & 98 & 213 & 3.19 \\
\hline $\begin{array}{l}\text { VTQTNPVPVTYPTDAYIPTYLPDDKVSNLADLKK(1)- } \\
\text { EKSNLNADNTTANK(2) }\end{array}$ & sp|P25632|RSC6_YEAST (2)-sp|P38781|RSC30_YEAST (234)/ & 1 & RSC6_YEAST & RSC30_YEAST & 2 & 234 & 2.90 \\
\hline VTQTNPVPVTYPTDAYIPTYLPDDKVSNLADLK(1)- & sp|P25632|RSC6_YEAST (2)-sp|Q03124|RSC9_YEAST (358)/ & 27 & RSC6_YEAST & RSC9_YEAST & 2 & 358 & 2.89 \\
\hline
\end{tabular}




\begin{tabular}{|c|c|c|c|c|c|c|c|}
\hline PEPTIDE & PROTEINS & CSM & PROTEIN1 & PROTEIN2 & R1 & R2 & $\mathbf{s}$ \\
\hline \multicolumn{8}{|l|}{ ECSLILEATLPNLLSYNIATPDYHLLQKHK(28) } \\
\hline $\begin{array}{l}\text { VTQTNPVPVTYPTDAYIPTYLPDDKVSNLADLKK(1)- } \\
\text { QNSIPEVKKFPQELSDVSK(8) }\end{array}$ & sp|P25632|RSC6_YEAST (2)-sp|Q03124|RSC9_YEAST (518)/ & 1 & RSC6_YEAST & RSC9_YEAST & 2 & 518 & 2.88 \\
\hline $\begin{array}{l}\text { GVDGGKVQYSPNLATLIGMQTGSVNDAVYSIYK(6)- } \\
\text { LDTSINLPTNTKTKDHPPNK(12) }\end{array}$ & sp|P25632|RSC6_YEAST (231)-sp|P25632|RSC6_YEAST (63)/ & 19 & RSC6_YEAST & RSC6_YEAST & 231 & 63 & 2.84 \\
\hline $\begin{array}{l}\text { ETLTHYLWSSKQNELVLQGDQYFNEDAAR(11)- } \\
\text { SLGLESKFSNATK(7) }\end{array}$ & sp|P25632|RSC6_YEAST (438)-sp|P32597|STH1_YEAST (177)/ & 9 & RSC6_YEAST & STH1_YEAST & 438 & 177 & 2.75 \\
\hline $\begin{array}{l}\text { LLHESANGKHPFSEFLEGVAVDFKR(9)-TKDHPPNKEMLR(8) } \\
\text { RQGKDNLR(4)-LKPLGMGK(2) }\end{array}$ & $\begin{array}{l}\text { sp|P25632|RSC6_YEAST (117)-sp|P25632|RSC6_YEAST (71)/ } \\
\text { sp|P25632|RSC6_YEAST (213)-sp|P25632|RSC6_YEAST (135)/ }\end{array}$ & $\begin{array}{c}68 \\
1\end{array}$ & $\begin{array}{l}\text { RSC6_YEAST } \\
\text { RSC6_YEAST }\end{array}$ & $\begin{array}{l}\text { RSC6_YEAST } \\
\text { RSC6_YEAST }\end{array}$ & $\begin{array}{l}117 \\
213\end{array}$ & $\begin{array}{c}71 \\
135\end{array}$ & $\begin{array}{l}2.72 \\
2.71\end{array}$ \\
\hline $\begin{array}{l}\text { LVSPLPPIILDYTIDLSKDTTYGATTLDVDVSHILHQPQPQPNLQK(18) } \\
\text {-VGKEGEEVGEGDSIAK(3) }\end{array}$ & sp|P25632|RSC6_YEAST (359)-sp|P43609|RSC8_YEAST (533)/ & 7 & RSC6_YEAST & RSC8_YEAST & 359 & 533 & 2.66 \\
\hline RLDTSINLPTNTKTK(13)-YVKESEK(3) & sp|P25632|RSC6_YEAST (63)-sp|P43609|RSC8_YEAST (463)/ & 13 & RSC6_YEAST & RSC8_YEAST & 63 & 463 & 2.62 \\
\hline $\begin{array}{l}\text { lDSLLQKVLDTNAAHLPLMNVVQTVNK(7)- } \\
\text { QILDLNKSLEELNVSKK(7) }\end{array}$ & sp|P25632|RSC6_YEAST (321)-sp|P43609|RSC8_YEAST (484)/ & 20 & RSC6_YEAST & RSC8_YEAST & 321 & 484 & 2.54 \\
\hline TKDHPPNKEMLR(8)-YVKESEK(3) & sp|P25632|RSC6_YEAST (71)-sp|P43609|RSC8_YEAST (463)/ & 8 & RSC6_YEAST & RSC8_YEAST & 71 & 463 & 2.42 \\
\hline TKDHPPNKEMLR(2)-YVKESEK(3) & sp|P25632|RSC6_YEAST (65)-sp|P43609|RSC8_YEAST (463)/ & 12 & RSC6_YEAST & RSC8_YEAST & 65 & 463 & 2.33 \\
\hline LLHESANGKHPFSEFLEGVAVDFKR(9)-TKDHPPNK(2) & sp|P25632|RSC6_YEAST (117)-sp|P25632|RSC6_YEAST (65)/ & 17 & RSC6_YEAST & RSC6_YEAST & 117 & 65 & 2.32 \\
\hline TKDHPPNKEMLR(2)-LKYVK(2) & sp|P25632|RSC6_YEAST (65)-sp|P43609|RSC8_Y̌EAST (460)/ & 6 & RSC6_YEAST & RSC8_YEAST & 65 & 460 & 2.32 \\
\hline $\begin{array}{l}\text { GVDGGKVQYSPNLATLIGMQTGSVNDAVYSIYK(6)- } \\
\text { RRLDTSINLPTNTKTK(14) }\end{array}$ & sp|P25632|RSC6_YEAST (231)-sp|P25632|RSC6_YEAST (63)/ & 19 & RSC6_YEAST & RSC6_YEAST & 231 & 63 & 2.28 \\
\hline $\begin{array}{l}\text { GVDGGKVQYSPNLATLIGMQTGSVNDAVYSIYK(6)- } \\
\text { DQEGDDEDGNTATGHGVKR(18) }\end{array}$ & sp|P25632|RSC6_YEAST (231)-sp|P43609|RSC8_YEAST (529)/ & 2 & RSC6_YEAST & RSC8_YEAST & 231 & 529 & 2.21 \\
\hline $\begin{array}{l}\text { GVDGGKVQYSPNLATLIGMQTGSVNDAVYSIYK(6)- } \\
\text { RLDTSINLPTNTKTTKDHPPNK(15) }\end{array}$ & sp|P25632|RSC6_YEAST (231)-sp|P25632|RSC6_YEAST (65)/ & 42 & RSC6_YEAST & RSC6_YEAST & 231 & 65 & 2.18 \\
\hline $\begin{array}{l}\text { GVDGGKVQYSPNLATLIGMQTGSVNDAVYSIYK(6)- } \\
\text { ESEKMLNDR(4) }\end{array}$ & sp|P25632|RSC6_YEAST (231)-sp|P43609|RSC8_YEAST (467)/ & 1 & RSC6_YEAST & RSC8_YEAST & 231 & 467 & 2.09 \\
\hline $\begin{array}{l}\text { DKPELGEVKLDSLLQKVLDTNAAHLPLMNVVQTVNK(16)- } \\
\text { KLVLISEQVDSGIQLVKDQEGDDEDGNTATGHGVK(18) }\end{array}$ & sp|P25632|RSC6_YEAST (321)-sp|P43609|RSC8_YEAST (511)/ & 1 & RSC6_YEAST & RSC8_YEAST & 321 & 511 & 2.08 \\
\hline $\begin{array}{l}\text { DKPELGEVKLDSLLQKVLDTNAAHLPLMNVVQTVNK(16)- } \\
\text { QILDLNKSLEELNVSK(7) }\end{array}$ & sp|P25632|RSC6_YEAST (321)-sp|P43609|RSC8_YEAST (484)/ & 20 & RSC6_YEAST & RSC8_YEAST & 321 & 484 & 1.99 \\
\hline GVDGGKVQYSPNLATLIGMQTGSVNDAVYSIYK(6)-TKDHPPNK(2) & sp|P25632|RSC6_YEAST (231)-sp|P25632|RSC6_YEAST (65)/ & 42 & RSC6_YEAST & RSC6_YEAST & 231 & 65 & 1.85 \\
\hline LKPLGMGKK(2)-QGKDNLR(3) & sp|P25632|RSC6_YEAST (135)-sp|P25632|RSC6_YEAST (213)/ & 17 & RSC6_YEAST & RSC6_YEAST & 135 & 213 & 1.66 \\
\hline LKPLGMGKK(8)-QGKDNLR(3) & sp|P25632|RSC6_YEAST (141)-sp|P25632|RSC6_YEAST (213)/ & 2 & RSC6_YEAST & RSC6_YEAST & 141 & 213 & 1.65 \\
\hline LLHESANGKHPFSEFLEGVAVDFK(9)-TKDHPPNKEMLR(8) & $\begin{array}{l}\text { sp|P25632|RSC6_YEAST (117)-sp|P25632|RSC6_YEAST (71)/ } \\
\text { (1) }\end{array}$ & 68 & RSC6_YEAST & RSC6_YEAST & 117 & 71 & 1.57 \\
\hline TKDHPPNK(2)-LKYVK(2) & sp|P25632|RSC6_YEAST (65)-sp|P43609|RSC8_Y̌EAST (460)/ & 6 & RSC6_YEAST & RSC8_YEAST & 65 & 460 & 1.45 \\
\hline $\begin{array}{l}\text { VTQTNPVPVTYYTDAYIPTYLPDDKVSNLADLKK(1)- } \\
\text { LLELQEITGKK(10) }\end{array}$ & sp|P25632|RSC6_YEAST (2)-sp|Q06639|RSC3_YEAST (198)/ & 1 & RSC6_YEAST & RSC3_YEAST & 2 & 198 & 1.41 \\
\hline $\begin{array}{l}\text { DKPELGEVKLDSLLQKVLDTNAAHLPLMNVVQTVNK(16)- } \\
\text { KLVLISEQVDSGIQLVEK(1) }\end{array}$ & sp|P25632|RSC6_YEAST (321)-sp|P43609|RSC8_YEAST (494)/ & 2 & RSC6_YEAST & RSC8_YEAST & 321 & 494 & 1.34 \\
\hline LLHESANGKHPFSEFLEGVAVDFKR(9)-DHPPNKEMLR(6) & sp|P25632|RSC6_YEAST (117)-sp|P25632|RSC6_YEAST (71)/ & 68 & RSC6_YEAST & RSC6_YEAST & 117 & 71 & 1.31 \\
\hline RLDTSINLPTNTKTK(13)-DHPPNKEMLR(6) & sp|P25632|RSC6_YEAST (63)-sp|P25632|RSC6_Y'YEAST (71)/ & 2 & RSC6_YEAST & RSC6_YEAST & 63 & 71 & 1.12 \\
\hline LREITKLALQLNSSAQK(6)-IGYMDLYEKNEK(9) & sp|P25632|RSC6_YEAST (404)-sp|Q07979|RSC58_YEAST (322)/ & 13 & RSC6_YEAST & RSC58_YEAST & 404 & 322 & 1.10 \\
\hline TKDHPPNKEMLR(8)-ESEKMLND & sp|P25632|RSC6_YEAST (71)-sp|P43609|RSC8_YEAST (467)/ & 8 & RSC6_YEAST & RSC8_Y̌EAST & 71 & 467 & 1.08 \\
\hline LDTSINLPTNTKTK(12)-YVKESEK(3) & sp|P25632|RSC6_YEAST (63)-sp|P43609|RSC8_YEAST (463)/ & 13 & RSC6_YEAST & RSC8_YEAST & 63 & 463 & 1.05 \\
\hline $\begin{array}{l}\text { VTQTNPVPVTYPTDAYIPTYLPDDKVSNLADLKK(25)- } \\
\text { IVEGLNKSSICDSK(7) }\end{array}$ & sp|P25632|RSC6_YEAST (26)-sp|Q03124|RSC9_YEAST (143)/ & 1 & RSC6_YEAST & RSC9_YEAST & 26 & 143 & 1.04 \\
\hline $\begin{array}{l}\text { DKPELGEVKLDSLLQKVLDTNAAHLPLMNVVQTVNK(16)- } \\
\text { QILDLNKSLEELNVSKK(7) }\end{array}$ & sp|P25632|RSC6_YEAST (321)-sp|P43609|RSC8_YEAST (484)/ & 20 & RSC6_YEAST & RSC8_YEAST & 321 & 484 & 1.02 \\
\hline $\begin{array}{l}\text { DTTYGATTLDVDVSHILHQPQPQPNLQKEEETDAEDTAK(28)- } \\
\text { SKECSLILEATLPNLLSYNIATPDYHLLQKHK(30) }\end{array}$ & sp|P25632|RSC6_YEAST (387)-sp|Q03124|RSC9_YEAST (358)/ & 3 & RSC6_YEAST & RSC9_YEAST & 387 & 358 & 0.97 \\
\hline
\end{tabular}




\begin{tabular}{|c|c|c|c|c|c|c|c|}
\hline PEPTIDE & PROTEINS & CSM & PROTEIN1 & PROTEIN2 & R1 & $\mathbf{R 2}$ & $\mathbf{S}$ \\
\hline $\begin{array}{l}\text { DKPELGEVKLDSLLQKVLDTNAAHLPLMNVVQTVNK(16)- } \\
\text { LSLSKQILDLNK(5) }\end{array}$ & sp|P25632|RSC6_YEAST (321)-sp|P43609|RSC8_YEAST (477)/ & 6 & RSC6_YEAST & RSC8_YEAST & 321 & 477 & 0.97 \\
\hline $\begin{array}{l}\text { DTTYGATTLDVDVSHILHQPQPQPNLQKEEETDAEDTAK(28)- } \\
\text { FTKLCDLETQLEMEK(3) }\end{array}$ & sp|P25632|RSC6_YEAST (387)-sp|P43609|RSC8_YEAST (446)/ & 1 & RSC6_YEAST & RSC8_YEAST & 387 & 446 & 0.90 \\
\hline $\begin{array}{l}\text { LDSLLQKVLDTNAAHLPLMNVVQTVNK(7)- } \\
\text { KLVLISEQVDSGIQLVEK(1) }\end{array}$ & sp|P25632|RSC6_YEAST (321)-sp|P43609|RSC8_YEAST (494)/ & 2 & RSC6_YEAST & RSC8_YEAST & 321 & 494 & 0.76 \\
\hline $\begin{array}{l}\text { VTQTNPPVPVTYPTDAYIPTYLPDDKVSNLADLK(25)- } \\
\text { TLECKAALETHDVFLAGAGQLSLPFQSHIESPR(5) }\end{array}$ & sp|P25632|RSC6_YEAST (26)-sp|P38210|LDB7_YEAST (116)/ & 1 & RSC6_YEAST & LDB7_YEAST & 26 & 116 & 0.69 \\
\hline $\begin{array}{l}\text { MVTQTNPVPVTYPTDAYIPTYLPDDKVSNLADLK(1)- } \\
\text { NLYSQATKPLLGSR(8) }\end{array}$ & sp|P25632|RSC6_YEAST (1)-sp|Q02206|RSC4_YEAST (468)/ & 1 & RSC6_YEAST & RSC4_YEAST & 1 & 468 & 0.68 \\
\hline $\begin{array}{l}\text { LVSPLPPIILDYTIDLSKDTTYGATTLDVDVSHILHQPQPQPNLQKEE } \\
\text { ETDAEDTAK(18)-RVGKEGEEVGEGDSIAK(4) }\end{array}$ & sp|P25632|RSC6_YEAST (359)-sp|P43609|RSC8_YEAST (533)/ & 7 & RSC6_YEAST & RSC8_YEAST & 359 & 533 & 0.55 \\
\hline LLHESANGKHPFSEFLEGVAVDFK(9)-DHPPNKEMLR(6) & sp|P25632|RSC6_YEAST (117)-sp|P25632|RSC6_YEAST (71)/ & 68 & RSC6_YEAST & RSC6_YEAST & 117 & 71 & 0.82 \\
\hline QGKDNLR(3)-EKYNMK(2) & sp|P25632|RSC6_YEAST (213)-sp|Q05123|ARP9_YEAST (453)/ & 1 & RSC6_YEAST & ARP9_YEAST & 213 & 453 & 0.49 \\
\hline SDSGTPADSGKTTWTLR(11)-RQGKDNLR(4) & sp|P25632|RSC6_YYAST (98)-sp|P25632|RSC6_ȲEAST (213)/ & 21 & RSC6_YEAST & RSC6_YEAST & 98 & 213 & 0.75 \\
\hline RQGKDNLR(4)-LKPLGMGKK(8) & 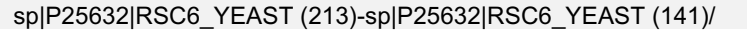 & 1 & RSC6_YEAST & RSC6_YEAST & 213 & 141 & 0.75 \\
\hline $\begin{array}{l}\text { MVTQTNPVPVTYYTDAYIPTYLPDDKVSNLADLKK(1)- } \\
\text { LAHYNKRSTITSR(6) }\end{array}$ & sp|P25632|RSC6_YEAST (1)-sp|P02281|H2B11_XENLA (82)/ & 1 & RSC6_YEAST & H2B11_XENLA & 1 & 82 & 0.39 \\
\hline LLHESANGKHPFSEFLEGVAVDFK(9)-LAGDPIDKSAR(8) & sp|P25632|RSC6_YEAST (117)-sp|P32832|NPL6_YEAST (267)/ & 1 & RSC6_YEAST & NPL6_YEAST & 117 & 267 & 0.36 \\
\hline LDSLLQKVLDTNAAHLPLMNVVQTVNK(7)-LSLSKQILDLNK(5) & sp|P25632|RSC6_YEAST (321)-sp|P43609|RSC8_YEAST (477)/ & 6 & RSC6_YEAST & RSC8_YEAST & 321 & 477 & 0.34 \\
\hline FSNATKTALGDPDTEIR(6)-AKSLGLESK(2) & sp|P32597|STH1_YEAST (183)-sp|P32597|STH1_YEAST (170)/ & 22 & STH1_YEAST & STH1_YEAST & 183 & 170 & 15.23 \\
\hline HNALFVGAGTEGATKGGIK(15)-KEDSEPLGR(1) & sp|P32597|STH1_YEAST (746)-sp|P32597|STH1_YEAST (1042)/ & 4 & STH1_YEAST & STH1_YEAST & 746 & 1042 & 7.35 \\
\hline ILFDKIDKER(8)-ADAKAQGLR(4) & sp|P32597|STH1_YEAST (1000)-sp|P32597|STH1_YEAST (1011)/ & 34 & STH1_YEAST & STH1_YEAST & 1000 & 1011 & 7.27 \\
\hline KICNHPFVFDEVEGVVNPSR(1)-HNALFVGAGTEGATKGGIK(15) & sp|P32597|STH1_YEAST (761)-sp|P32597|STH1_Y̌EAST (746)/ & 15 & STH1_YEAST & STH1_YEAST & 761 & 746 & 5.92 \\
\hline NAQSKLSFTISHYYR(5)-DKSLLSK(2) & sp|P32597|STH1_YEAST (608)-sp|P32597|STH1_YEAST (583)/ & 7 & STH1_YEAST & STH1_YEAST & 608 & 583 & 5.75 \\
\hline $\begin{array}{l}\text { DTDVPDYFLDLPDTKNDNTTAIEVDYSEKKPIK(15)- } \\
\text { DGSVSGSKLMECVNDAVQTLLQGDDKLGK(8) }\end{array}$ & sp|P32597|STH1_YEAST (142)-sp|P43609|RSC8_YEAST (388)/ & 4 & STH1_YEAST & RSC8_YEAST & 142 & 388 & 5.59 \\
\hline IKEKIDKQPSILVGGTLK(4)-FDNKSTAEEQEAFLRR(4) & sp|P32597|STH1_YEAST (457)-sp|P32597|STH1_YEAST (948)/ & 2 & STH1_YEAST & STH1_YEAST & 457 & 948 & 5.31 \\
\hline ILFDKIDKER(5)-FKASGHR(2) & sp|P32597|STH1_YEAST (997)-sp|P32597|STH1_YEAST (804)/ & 49 & STH1_YEAST & STH1_YEAST & 997 & 804 & 5.17 \\
\hline VIQAGKFDNKSTAEEQEAFLRR(10)-IKEKIDKQPSILVGGTLK(4) & sp|P32597|STH1_YEAST (948)-sp|P32597|STH1_YEAST (457)/ & 8 & STH1_YEAST & STH1_YEAST & 948 & 457 & 5.15 \\
\hline QKLINNVASQAHHNIPYLR(2)-LQQQQHQYQQQKR(12) & sp|P32597|STH1_YEAST (260)-sp|Q06488|RSC2_YEAST (713)/ & 39 & STH1_YEAST & RSC2_YEAST & 260 & 713 & 5.14 \\
\hline $\begin{array}{l}\text { LSGLQQQLYQQMLKHNALFVGAGTEGATK(14)- } \\
\text { EDIEEHFKKEDSEPLGR(9) }\end{array}$ & sp|P32597|STH1_YEAST (731)-sp|P32597|STH1_YEAST (1042)/ & 3 & STH1_YEAST & STH1_YEAST & 731 & 1042 & 5.07 \\
\hline SIDKGFQYDEDLLNK(4)-KVSKVPINNVR(1) & sp|P32597|STH1_YEAST (106)-sp|Q07979|RSC58_YEAST (464)/ & 13 & STH1_YEAST & RSC58_YEAST & 106 & 464 & 5.05 \\
\hline LGASLHNQMEKDEQKR(15)-TAKQR(3) & sp|P32597|STH1_YEAST (368)-sp|P32597|STH1_YEAST (375)/ & 20 & STH1_YEAST & STH1_ȲEAST & 368 & 375 & 5.03 \\
\hline NLHLQKINSIIDFIK(6)-QQLLEKR(6) & sp|P32597|STH1_YEAST (326)-sp|P32597|STH1_YEAST (315)/ & 5 & STH1_YEAST & STH1_YEAST & 326 & 315 & 4.84 \\
\hline LIQLDELPKVFR(9)-KEDSEPLGR(1) & sp|P32597|STH1_YEAST (1030)-sp|P32597|STH1_YEAST (1042)/ & 11 & STH1_YEAST & STH1_YEAST & 1030 & 1042 & 4.76 \\
\hline IKEKIDKQPSILVGGTLK(4)-FDNKSTAEEQEAFLR(4) & sp|P32597|STH1_YEAST (457)-sp|P32597|STH1_Y̌EAST (948)/ & 2 & STH1_YEAST & STH1_YEAST & 457 & 948 & 4.62 \\
\hline NGTYKTLEEVR(5)-SKSLGIFPTVEK(2) & sp|P32597|STH1_YEAST (1313)-sp|P32597|STH1__YEAST (1247)/ & 12 & STH1_YEAST & STH1_YEAST & 1313 & 1247 & 4.29 \\
\hline AIQNHPKENKLEIK(7)-NSEAKR(5) & sp|P32597|STH1_YEAST (70)-sp|P38210|LDB7_YEAST (149)/ & 8 & STH1_YEAST & LDB7_YEAST & 70 & 149 & 4.21 \\
\hline LNKTTVLENNDGKR(3)-LTVKIK(4) & sp|P32597|STH1_YEAST (1212)-sp|P32597|STH1_YYEAST (1207)/ & 18 & STH1_YEAST & STH1_YEAST & 1212 & 1207 & 4.14 \\
\hline VIQAGKFDNKSTAEEQEAFLR(10)-IKEKIDKQPSILVGGTLK(4) & sp|P32597|STH1_YEAST (948)-sp|P32597|STH1_ȲEAST (457)/ & 8 & STH1_YEAST & STH1_YEAST & 948 & 457 & 4.10 \\
\hline $\begin{array}{l}\text { LSGLQQQLYQQMLKHNALFVGAGTEGATK(14)- } \\
\text { VFREDIEEHFKKEDSEPLGR(11) }\end{array}$ & sp|P32597|STH1_YEAST (731)-sp|P32597|STH1_YEAST (1041)/ & 3 & STH1_YEAST & STH1_YEAST & 731 & 1041 & 4.04 \\
\hline QKLINNVASQAHHNIPYLR(2)-ISHRGDPESIKISNILEK(11) & sp|P32597|STH1_YEAST (260)-sp|Q06488|RSC2_YEAST (366)/ & 1 & STH1_YEAST & RSC2_YEAST & 260 & & 4.01 \\
\hline LAALKSNDEEAYLK(5)-LLDQTKDTR(6) & H1_Y_YEAST (382)-sp|P32597|STH1_YEAST (397)/ & 7 & STH1_YEAST & STH1_YEAST & 382 & 397 & \\
\hline DYPDYFKVIEKPMAIDIILK(7)-LPSKR(4) & sp|P32597|STH1_YEAST (1292)-sp|P32597|STH1̄_YEAST (1284 & 14 & STH1_YE & STH1_YE & 1292 & 1284 & 3.89 \\
\hline LNKTTVLENNDGKR(3)-AEEKPESKSPAKK(8) & sp|P32597|STH1_YEAST (1212)-sp|P32597|STH1_YEAST (1231)/ & 10 & STH1_YEAST & STH1_YEAST & 1212 & 1231 & 3.89 \\
\hline $\begin{array}{l}\text { MLQEQSELMSTVMNNTPTTVAALAAVAAASETNGKLGSEEQPEITI } \\
\text { PKPR(1)-SSQVVFSATELQQTQQSHATDKGIEGSENSK(23) }\end{array}$ & sp|P32597|STH1_YEAST (1)-sp|P38210|LDB7_YEAST (43)/ & 5 & STH1_YEAST & LDB7_YEAST & 1 & 43 & 3.86 \\
\hline
\end{tabular}




\begin{tabular}{|c|c|c|c|c|c|c|c|}
\hline PEPTIDE & PROTEINS & CSM & PROTEIN1 & PROTEIN2 & R1 & R2 & $\mathbf{s}$ \\
\hline EDIEEHFKKEDSEPLGR(9)-LIQLDELPKVFR(9) & sp|P32597|STH1_ YEAST (1042)-sp|P32597|STH1_ YEAST (1030)/ & 14 & STH1_YEAST & STH1 YEAST & 1042 & 1030 & 3.67 \\
\hline SSSVEIINGSESKK(13)-IKLNK(2) & sp|P32597|STH1_YEAST (1198)-sp|P32597|STH1_YEAST (1209)/ & 6 & STH1_YEAST & STH1_YEAST & 1198 & 1209 & 3.62 \\
\hline GLNNKIMQLR(5)-KEDSEPLGR(1) & sp|P32597|STH1_YEAST (755)-sp|P32597|STH1_YEAST (1042)/ & 6 & STH1_YEAST & STH1_YEAST & 755 & 1042 & 3.61 \\
\hline ILFDKIDK(5)-FKASGHR(2) & sp|P32597|STH1_YEAST (997)-sp|P32597|STH1_YEAST (804)/ & 49 & STH1_YEAST & STH1_YEAST & 997 & 804 & 3.55 \\
\hline $\begin{array}{l}\text { VFREDIEEHFKKEDSEPLGR(11)-HNALFVGAGTEGATKGGIK(15) }\end{array}$ & $\begin{array}{l}\text { sp|P32597|STH1 YEAST (1041)-sp|P32597|STH1 YEAST (746)/ }\end{array}$ & 3 & STH1_YEAST & STH1 YEAST & 1041 & 746 & 3.54 \\
\hline SNDEEAYLKLLDQTK(9)-KSLMTSHTK(1) & sp|P32597|STH1_YEAST (391)-sp|P53330|RT102_YEAST (94)/ & 1 & STH1_YEAST & RT102_YEAST & 391 & 94 & 3.51 \\
\hline $\begin{array}{l}\text { VFREDIEEHFKKEDSEPLGR(12)-HNALFVGAGTEGATKGGIK(15) }\end{array}$ & $\begin{array}{l}\text { sp|P32597|STH1 YEAST (1042)-sp|P32597|STH1_ YEAST (746)/ }\end{array}$ & 5 & STH1_YEAST & STH1 YEAST & 1042 & 746 & 3.50 \\
\hline VIQAGKFDNK(6)-KEVEK(1) & sp|P32597|STH1_YEAST (944)-sp|P32597|STH1_YEAST (700)/ & 3 & STH1_YEAST & STH1_YEAST & 944 & 700 & 3.49 \\
\hline LTVKIK(4)-KKKPK(1) & sp|P32597|STH1_YEAST (1207)-sp|P32597|STH1_YEAST (1199)/ & 4 & STH1_YEAST & STH1_YEAST & 1207 & 1199 & 3.46 \\
\hline $\begin{array}{l}\text { MLQEQSELMSTVMNNTPTTVAALAAVAAASETNGK(1)- } \\
\text { KQNDENNKNVDGK(1) }\end{array}$ & sp|P32597|STH1_YEAST (1)-sp|Q07979|RSC58_YEAST (343)/ & 3 & STH1_YEAST & RSC58_YEAST & 1 & 343 & 3.42 \\
\hline $\begin{array}{l}\text { MLQEQSELMSTVMNNTPTTVAALAAVAAASETNGK(1)- } \\
\text { NVSNAFNNAAAIVITDPVTGKK(21) }\end{array}$ & sp|P32597|STH1_YEAST (1)-sp|Q03124|RSC9_YEAST (464)/ & 1 & STH1_YEAST & RSC9_YEAST & 1 & 464 & 3.42 \\
\hline 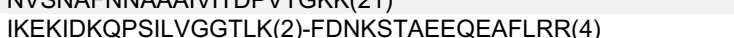 & sp|P32597|STH1 YEAST (455)-sp|P32597|STH1 YEAST (948)/ & 1 & STH1 YEAST & STH1 YEAST & 455 & 948 & 3.41 \\
\hline FDNKSTAEEQEAFLRR(4)-EVEKDLPDKVEK(4) & sp|P32597|STH1_YEAST (948)-sp|P32597|STH1_YEAST (704)/ & 29 & STH1 YEAST & STH1 YEAST & 948 & 704 & 3.39 \\
\hline ADAKAQGLR(4)-IDKER(3) & sp|P32597|STH1_YEAST (1011)-sp|P32597|STH1_YEAST (1000)/ & 18 & STH1_YEAST & STH1_YEAST & 1011 & 1000 & 3.30 \\
\hline VIEKPMAIDIILKNCK(13)-LPSKR(4) & sp|P32597|STH1_YEAST (1305)-sp|P32597|STH1_YEAST (1284)/ & 30 & STH1 YEAST & STH1 YEAST & 1305 & 1284 & 3.28 \\
\hline TSIFEKLPSKR(6)-NCK & p|P32597|STH1_YEAST (1280)-sp|P32597|STH1_YEAST (1308)/ & 8 & STH1_YEAST & STH1_YEAST & 1280 & 1308 & 3.25 \\
\hline SSSVEIINGSESKK(13)-ADAKAQGLR(4) & sp|P32597|STH1 YEAST (1198)-sp|P32597|STH1 YEAST (1011) & 7 & STH1 YEAST & STH1 YEAST & 1198 & 1011 & 3.24 \\
\hline NDNTTAIEVDYSEKKPIK(14)-AKSLGLESK(2) & sp|P32597|STH1_YEAST (156)-sp|P32597|STH1_Y̌EAST (170)/ & 12 & STH1_YEAST & STH1_YEAST & 156 & 170 & 3.23 \\
\hline VIQAGKFDNK(6)-AMQKLDIDGK(4) & sp|P32597|STH1_YEAST (944)-sp|P32597|STH1_YEAST (932)/ & 15 & STH1_YEAST & STH1_YEAST & 944 & 932 & 3.23 \\
\hline MLQEQSELMSTVMNNTPTTVAALAAVAAASETNGK(1)- & sp|P32597|STH1_YEAST (1)-sp|P38210|LDB7_YEAST (43)/ & 5 & STH1_YEAST & LDB7_YEAST & 1 & 43 & 3.19 \\
\hline $\begin{array}{l}\text { KPIKISADFNAK(4)-AKSLGLESK(2) } \\
\text { KPIIST }\end{array}$ & sp|P32597|STH1_YEAST (160)-sp|P32597|STH1_YEAST (170)/ & 7 & STH1 YEAST & STH1 YEAST & 160 & 170 & 3.18 \\
\hline KSIDKGFQYDEDLLNK(5)-KVSKVPINNVR(1) & sp|P32597|STH1_YEAST (106)-sp|Q07979|RSC58 YEAST (464)/ & 13 & STH1_YEAST & RSC58 YEAST & 106 & 464 & 3.16 \\
\hline SLMTSHTK(1) & sp|P32597|STH1_YEAST (444)-sp|P53330|RT102_YEAST (94)// & 3 & STH1_YEAST & RT102_YEAST & 444 & 94 & 3.15 \\
\hline $\begin{array}{l}\text { FSNATKTALGDPDTEIR(6)-EVVGSTLNGKGGDSR(10) } \\
\text { (10) }\end{array}$ & sp|P32597|STH1_YEAST (183)-sp|P43609|RSC8 YEAST (375)/ & 21 & STH1_YEAST & RSC8 YEAST & 183 & 375 & 3.11 \\
\hline SADEKILFDKIDKER(5)-EVEKDLPDKVEKVIK(12) & sp|P32597|STH1_YEAST (992)-sp|P32597|STH1_YEAST (712)/ & 7 & STH1_YEAST & STH1_YEAST & 992 & 712 & 3.10 \\
\hline ILHGEEVQPITDEEREKTDYYEVAHR(17)- & sp|P32597|STH1_YEAST (444)-sp|P53330|RT102_YEAST (107)/ & 2 & STH1_YEAST & RT102_YEAST & 444 & 107 & 3.10 \\
\hline SIDKGFQYDEDLLNK(4)-ILKEVILAKK(9) & sp|P32597|STH1_YEAST (106)-sp|Q07979|RSC58_YEAST (463)/ & 8 & STH1_YEAST & RSC58_YEAST & 106 & 463 & 3.07 \\
\hline DDDDKAELDDDELNDTLAR(5)-MKNAQSK(2) & sp|P32597|STH1_YEAST (973)-sp|P32597|STH1_Y̌EAST (603)/ & 3 & STH1_YEAST & STH1_Y̌YAST & 973 & 603 & 3.07 \\
\hline FSNATKTALGL & sp|P32597|STH1 YEAST (183)-sp|Q07979|RSC58 YEAST (343)/ & 1 & STH1 YEAST & RSC58 YEAST & 183 & 343 & 3.06 \\
\hline VFREDIEEHFKK(11) & sp|P32597|STH1 YEAST (746)-sp|P32597|STH1 YEAST (1041)/ & 2 & STH1_YEAST & STH1 YEAST & 746 & 1041 & 3.02 \\
\hline LAALKSNDEEAYLK(5)-TAKQR(3) & $\begin{array}{l}\text { sp|P32597|STH1_YEAST (382)-sp|P32597|STH1_YEAST (375)/ }\end{array}$ & 8 & STH1_YEAST & STH1_YEAST & 382 & 375 & 3.00 \\
\hline MLQEQSELMSTVMNNTPTTVAALAAVAAASETNGK(1)- & sp|P32597|STH1_YEAST (1)-sp|Q06639|RSC3_YEAST (845)/ & 1 & STH1_YEAST & RSC3_YEAST & 1 & 845 & 3.00 \\
\hline IDKQPSILVGGTLKEYQLR(14)-IGQKNEVR(4) & sp|P32597|STH1_YEAST (471)-sp|P32597|STH1_YEAST (907)/ & 30 & STH1_YEAST & STH1_YEAST & 471 & 907 & 2.99 \\
\hline $\begin{array}{l}\text { MLQEQSELMSTVMNNTPTTVAALAAVAAASETNGK(1)- } \\
\text { VKQEFUNTNEEGINR(2) }\end{array}$ & sp|P32597|STH1_YEAST (1)-sp|Q07979|RSC58_YEAST (328)/ & 1 & STH1_YEAST & RSC58_YEAST & 1 & 328 & 2.99 \\
\hline FSNATKTALGDPDTEIR(6)-IGYMDLYEKNEKK(9) & sp|P32597|STH1_YEAST (183)-sp|Q07979|RSC58_YEAST (322)/ & 4 & STH1_YEAST & RSC58_YEAST & 183 & 322 & 2.96 \\
\hline $\begin{array}{l}\text { LSGLQQQLYQQMLKHNALFVGAGTEGATK(14)- } \\
\text { VEREDIFFHEKKFEDSFPI GR(1) }\end{array}$ & sp|P32597|STH1_YEAST (731)-sp|P32597|STH1_YEAST (1042)/ & 3 & STH1_YEAST & STH1_YEAST & 731 & 1042 & 2.94 \\
\hline $\begin{array}{l}\text { VFREDIEEHFKKEDSEPLGR(12) } \\
\text { SIDKGFQYDEDLLNK(4)-EVILAKK(6) }\end{array}$ & sp|P32597|STH1_YEAST (106)-sp|Q07979|RSC58_YEAST (463)/ & 8 & STH1_YEAST & RSC58_YEAST & 106 & 463 & 2.93 \\
\hline MLQEQSELMSTVMNNTPTTVAALAAVAAASETNGK(1)- & sp|P32597|STH1_YEAST (1)-sp|Q06639|RSC3_YEAST (231)/ & 1 & STH1_YEAST & RSC3_YEAST & 1 & 231 & 2.90 \\
\hline $\begin{array}{l}\text { KEDSEPLGR(1)-GGIKGLNNK(4) } \\
\text { EDIEEHFKKEDSEPLGR(9)-HNALFVGAGTEGATKGGIK(15) }\end{array}$ & $\begin{array}{l}\text { sp|P32597|STH1_YYEAST (1042)-sp|P32597|STH1_YEAST (750)/ } \\
\text { sp|P32597|STH1_YEAST (1042)-sp|P32597|STH1_YEAST (746)/ }\end{array}$ & $\begin{array}{l}6 \\
5\end{array}$ & $\begin{array}{l}\text { STH1_YEAST } \\
\text { STH1_YEAST }\end{array}$ & $\begin{array}{l}\text { STH1_YEAST } \\
\text { STH1_YEAST }\end{array}$ & $\begin{array}{l}1042 \\
1042\end{array}$ & $\begin{array}{l}750 \\
746\end{array}$ & $\begin{array}{l}2.87 \\
2.85\end{array}$ \\
\hline DTDVPDYFLDLPDTKNDNTTAIEVDYSEKKPIK(15)- & sp|P32597|STH1_YEAST (142)-sp|Q07979|RSC58_YEAST (343)/ & 3 & STH1_YEAST & RSC58_YEAST & 142 & 343 & 2.83 \\
\hline
\end{tabular}




\begin{tabular}{|c|c|c|c|c|c|c|c|}
\hline PEPTIDE & PROTEINS & CSM & PROTEIN1 & PROTEIN2 & R1 & R2 & $\mathbf{s}$ \\
\hline EVEKDLPDKVEK(4)-VIQAGKFDNK(6) & sp|P32597|STH1 YEAST (704)-sp|P32597|STH1_YEAST (944)/ & 18 & STH1_YEAST & STH1_YEAST & 704 & 944 & 2.82 \\
\hline HNALFVGAGTEGATKGGIK(15)-NDEELNKLLGR(7) & sp|P32597|STH1_YEAST (746)-tr|Q6AZJ8|H2A_XENLA (95)/ & 1 & STH1_YEAST & H2A_X̄ENLA & 746 & 95 & 2.81 \\
\hline EKTDYYEVAHR(2)-LHKVLRPFLLR(3) & sp|P32597|STH1_YEAST (444)-sp|P32597|STH1_YEAST (688)/ & 12 & STH1_YEAST & STH1_YEAST & 444 & 688 & 2.81 \\
\hline VIQAGKFDNKSTAEEQEAFLRR(10)-EVEKDLPDKVEK(4) & sp|P32597|STH1_YEAST (948)-sp|P32597|STH1_YYAST (704)/ & 29 & STH1_YEAST & STH1_YEAST & 948 & 704 & 2.81 \\
\hline $\begin{array}{l}\text { MLQEQSELMSTVMNNTPTTVAALAAVAAASETNGKLGSEEQPEITI } \\
\text { PKPR(1)-KLLPAVEFIK(1) }\end{array}$ & sp|P32597|STH1_YEAST (1)-sp|Q03124|RSC9_YEAST (434)/ & 9 & STH1_YEAST & RSC9_YEAST & 1 & 434 & 2.78 \\
\hline LLDQTKDTR(6)-KSLMTSHTK(1) & sp|P32597|STH1_YEAST (397)-sp|P53330|RT102_YEAST (94)/ & 6 & STH1_YEAST & RT102_YEAST & 397 & 94 & 2.75 \\
\hline FSNATKTALGDPDTEIR(6)-AKVSKPK(2) & sp|P32597|STH1_YYEAST (183)-sp|P38210|LDB7_Y̌EAST (54)/ & 10 & STH1_YEAST & LDB7_ȲYEAST & 183 & 54 & 2.74 \\
\hline $\begin{array}{l}\text { DTDVPDYFLDLPDTKNDNTTAIEVDYSEK(15)- } \\
\text { DGSVSGSKLMECVNDAVQTLLGDKLGK(8) }\end{array}$ & sp|P32597|STH1_YEAST (142)-sp|P43609|RSC8_YEAST (388)/ & 4 & STH1_YEAST & RSC8_YEAST & 142 & 388 & 2.74 \\
\hline $\begin{array}{l}\text { MLQEQSELMSTVMNNTPTTVAALAAVAAASETNGK(1)- } \\
\text { TNDANEISEQTDIHSNNDNSKR(21) }\end{array}$ & sp|P32597|STH1_YEAST (1)-sp|Q06639|RSC3_YEAST (744)/ & 2 & STH1_YEAST & RSC3_YEAST & 1 & 744 & 2.74 \\
\hline LNKTTVLENNDGKR(3)-AEEKPESK(4) & sp|P32597|STH1_YEAST (1212)-sp|P32597|STH1_YEAST (1227)/ & 7 & STH1_YEAST & STH1_YEAST & 1212 & 1227 & 2.67 \\
\hline SKSLGIFPTVEK(2)-KTAAK(1) & sp|P32597|STH1_YEAST (1247)-sp|P32597|STH1_YEAST (1236)/ & 3 & STH1_YEAST & STH1_YEAST & 1247 & 1236 & 2.66 \\
\hline LNKTTVLENNDGKR(3)-SSSVEIINGSESKK(13) & sp|P32597|STH1_YEAST (1212)-sp|P32597|STH1_YEAST (1198)/ & 2 & STH1_YEAST & STH1_YEAST & 1212 & 1198 & 2.66 \\
\hline LLDQTKDTR(6)-KAVTK(1) & sp|P32597|STH1_YEAST (397)-sp|P02281|H2B11_XENLA (13)/ & 1 & STH1_YEAST & H2B11__XENLA & 397 & 13 & 2.65 \\
\hline NDNTTAIEVDYSEKKPIK(15)-AKSLGLESK(2) & sp|P32597|STH1_YEAST (157)-sp|P32597|STH1_Y̌EAST (170)/ & 2 & STH1_YEAST & STH1_Y̌YEAST & 157 & 170 & 2.63 \\
\hline EKTDYYEVAHR(2)-LLDQTKDTR(6) & sp|P32597|STH1_YEAST (444)-sp|P32597|STH1_YEAST (397)/ & 8 & STH1_YEAST & STH1_YEAST & 444 & 397 & 2.57 \\
\hline FDNKSTAEEQEAFLR(4)-AMQKLDIDGK(4) & sp|P32597|STH1_YEAST (948)-sp|P32597|STH1_YEAST (932)/ & 23 & STH1_YEAST & STH1_YEAST & 948 & 932 & 2.57 \\
\hline VIQAGKFDNKSTAEEQEAFLRR(10)-KEVEKDLPDKVEK(5) & sp|P32597|STH1_YEAST (948)-sp|P32597|STH1_YEAST (704)/ & 29 & STH1_YEAST & STH1__YEAST & 948 & 704 & 2.56 \\
\hline FDNKSTAEEQEAFLRR(4)-AMQKLDIDGK(4) & sp|P32597|STH1_YEAST (948)-sp|P32597|STH1_YEAST (932)/ & 23 & STH1_YEAST & STH1_YEAST & 948 & 932 & 2.54 \\
\hline AEEKPESKSPAKK(8)-TTVLENNDGKR(10) & sp|P32597|STH1_YEAST (1231)-sp|P32597|STH1_YEAST (1222)/ & 7 & STH1_YEAST & STH1_YEAST & 1231 & 1222 & 2.52 \\
\hline FDNKSTAEEQEAFLR(4)-EVEKDLPDKVEK(4) & sp|P32597|STH1_YEAST (948)-sp|P32597|STH1_Y̌EAST (704)/ & 29 & STH1_YEAST & STH1_YEAST & 948 & 704 & 2.51 \\
\hline AEEKPESK(4)-LTVKIK(4) & sp|P32597|STH1_YEAST (1227)-sp|P32597|STH1__YEAST (1207)/ & 2 & STH1_YEAST & STH1_-YEAST & 1227 & 1207 & 2.46 \\
\hline EDIEEHFKKEDSEPLGR(9)-HAVSEGTKAVTK(8) & sp|P32597|STH1_YEAST (1042)-sp|P02281|H2B11_XENLA (113)/ & 4 & STH1_YEAST & H2B11_XENLA & 1042 & 113 & 2.44 \\
\hline $\begin{array}{l}\text { SKSLGIFPTVEK(2)-KTKTK(3) } \\
\text { SKST }\end{array}$ & sp|P32597|STH1_YEAST (1247)-sp|P32597|STH1_YEAST (1243)/ & 7 & STH1_YEAST & STH1_YEAST & 1247 & 1243 & 2.42 \\
\hline SSSVEIINGSESKK(13)-KKPKLTVK(4) & sp|P32597|STH1_YEAST (1198)-sp|P32597|STH1_YEAST (1203)/ & 9 & STH1_YEAST & STH1_YEAST & 1198 & 1203 & 2.42 \\
\hline VFREDIEEHFKKEDSEPLGR(11)-HAVSEGTKAVTK(8) & sp|P32597|STH1_YEAST (1041)-sp|P02281|H2B11_XENLA (113)/ & 5 & STH1_YEAST & H2B11_XENLA & 1041 & 113 & 2.40 \\
\hline VGNFDVLLTTYYEYIIKDK(16)-IDKER(3) & sp|P32597|STH1_YEAST (581)-sp|P32597|STH1_YEAST (1000)/ & 2 & STH1_YEAST & STH1_Y YEAST & 581 & 1000 & 2.39 \\
\hline VFREDIEEHFKK(11)-GLNNKIMQLR(5) & sp|P32597|STH1_YEAST (1041)-sp|P32597|STH1_YEAST (755)/ & 16 & STH1_YEAST & STH1_YEAST & 1041 & 755 & 2.38 \\
\hline VIQAGKFDNKSTAEEQEAFLR(10)-EVEKDLPDKVEK(4) & sp|P32597|STH1_YEAST (948)-sp|P32597|STH1_Y YEAST (704)/ & 29 & STH1_YEAST & STH1_YEAST & 948 & 704 & 2.36 \\
\hline VFREDIEEHFKKEDSEPLGR(12)-HAVSEGTKAVTK(8) & sp|P32597|STH1_YYAST (1042)-sp|P02281|H2B11_XENLA (113)/ & 4 & STH1_YEAST & H2B11_XENLA & 1042 & 113 & 2.34 \\
\hline RLHKVLRPFLLR(4)-EKTDYYEVAHR(2) & sp|P32597|STH1_YEAST (688)-Sp|P32597|STH1_YEAST (444)/ & 5 & STH1_YEAST & STH1_Y YEAST & 688 & 444 & 2.33 \\
\hline SKSLGIFPTVEK(2)-NCKNGTYK(3) & sp|P32597|STH1_YYAST (1247)-sp|P32597|STH1_YEAST (1308)/ & 9 & STH1_YEAST & STH1_YEAST & 1247 & 1308 & 2.33 \\
\hline VIEKPMAIDIILK(4)-LPSKR(4) & sp|P32597|STH1_YEAST (1296)-sp|P32597|STH1_YEAST (1284)/ & 10 & STH1_YEAST & STH1_YEAST & 1296 & 1284 & 2.32 \\
\hline $\begin{array}{l}\text { MLQEQSELMSTVMNNTPTTVAALAAVAAASETNGK(1)- } \\
\text { AMLLSDYTKNCK(10) }\end{array}$ & sp|P32597|STH1_YEAST (1)-sp|Q06639|RSC3_YEAST (768)/ & 1 & STH1_YEAST & RSC3_YEAST & 1 & 768 & 2.32 \\
\hline $\begin{array}{l}\text { IAMLLSDYTKNCK(10) } \\
\text { FSNATKTALGDPDTEIR(6)-IGYMDLYEKNEK(9) }\end{array}$ & sp|P32597|STH1_YEAST (183)-sp|Q07979|RSC58_YEAST (322)/ & 4 & STH1_YEAST & RSC58_YEAST & 183 & 322 & 2.31 \\
\hline AIQNHPKENKLEIK(7)-KVIICK(1) & sp|P32597|STH1_YEAST (70)- & 2 & STH1_YEAST & LDB7_YEAST & 70 & 153 & 2.30 \\
\hline ADAKAQGLRVPPPR(4)-ILFDKIDKER(8) & sp|P32597|STH1_YYAST (1011)-sp|P32597|STH1_YEAST (1000)/ & 18 & STH1_YEAST & STH1_YEAST & 1011 & 1000 & 2.30 \\
\hline LSGLQQQLYQQMLKHNALFVGAGTEGATK(14)-KEDSEPLGR(1) & sp|P32597|STH1_YEAST (731)-sp|P32597|STH1_Y̌YEAST (1042)/ & 3 & STH1_YEAST & STH1_YEAST & 731 & 1042 & 2.28 \\
\hline SSSVEIINGSESKK(13)-LTVKIK(4) & sp|P32597|STH1_YEAST (1198)-sp|P32597|STH1_YEAST (1207)/ & 4 & STH1_YEAST & STH1_YEAST & 1198 & 1207 & 2.28 \\
\hline VIQAGKFDNKSTAE & sp|P32597|STH1_YEAST (948)-sp|P32597|STH1_Y̌EAST (932)/ & 23 & STH1_YEAST & STH1_YEAST & 948 & 932 & 2.27 \\
\hline PDKVEK(4)-IGQK & sp|P32597|STH1_YEAST (704)-sp|P32597|STH1_YYAST (907)/ & 4 & STH1_YEAST & STH1_YEAST & 704 & 907 & 2.25 \\
\hline AEEKPESKSPAK(8)-KTAAKK $(1$ & sp|P32597|STH1_YEAST (1231)-sp|P32597|STH1_YEAST (1236)/ & 11 & STH1_YEAST & STH1_YEAST & 1231 & 1236 & 2.25 \\
\hline MKDLKYMR(2)-FKASGHR(2) & Sp|P32597|STH1_YEAST (830)-sp|P32597|STH1_YEAST (804)/ & 1 & STH1_YEAST & STH1_YEAST & 830 & 804 & 2.24 \\
\hline ENKLEIK(3)-NSEAKR(5) & sp|P32597|STH1_YEAST (73)-sp|P38210|LDB7_YEAST (149)/ & 3 & STH1_YEAST & LDB7_YEAST & 73 & 149 & 2.22 \\
\hline LAALKSNDEEAYLK(5)-KAVTK(1) & Sp|P32597|STH1_YEAST (382)-sp|P02281|H2B11_XENLA (13)/ & 10 & STH1_YEAST & H2B11_XENLA & 382 & 13 & 2.22 \\
\hline VIEKPMAIDIILKNCK(13)-SKSLGIFPTVEK(2) & sp|P32597|STH1_YEAST (1305)-sp|P32597|STH1_YEAST (1247)/ & 4 & STH1_YEAST & STH1_YEEAST & 1305 & 1247 & 2.18 \\
\hline EKTDYYEVAHR(2)-SLMTSHTKGDTSK(8) & sp|P32597|STH1 1 YEAST (444)-sp|P53330|RT102 YEAST (102)/ & 7 & STH1 YEAST & RT102 YEAST & 444 & 102 & 2.11 \\
\hline
\end{tabular}




\begin{tabular}{|c|c|c|c|c|c|c|c|}
\hline PEPTIDE & PROTEINS & CSM & PROTEIN1 & PROTEIN2 & R1 & R2 & $\mathbf{s}$ \\
\hline 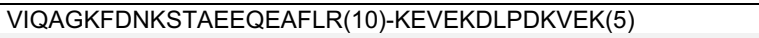 & sp|P32597|STH1_YEAST (948)-sp|P32597|STH1_YEAST (704)/ & 29 & STH1_YEAST & STH1_YEAST & 948 & 704 & 2.09 \\
\hline LLDQTKDTR(6)-VKNVK(2) & sp|P32597|STH1_YEAST (397)-sp|Q05123|ARP9_YEAST (272)/ & 8 & STH1_YEAST & ARP9_YEAST & 397 & 272 & 2.09 \\
\hline LLDQTKDTR(6)-GQKVK(3) & sp|P32597|STH1_YEAST (397)-sp|Q05123|ARP9_YEAST (270)/ & 6 & STH1_YEAST & ARP9_YEAST & 397 & 270 & 2.09 \\
\hline AEEKPESKSPAK(8)-KTAAK(1) & sp|P32597|STH1_YEAST (1231)-sp|P32597|STH1_YEAST (1236)/ & 11 & STH1_YEAST & STH1_YEAST & 1231 & 1236 & 2.05 \\
\hline $\begin{array}{l}\text { DTDVPDYFLDLPDTKNDNTTAIEVDYSEKKPIK(15)- } \\
\text { EVVGSTLNGKGGDSR(10) }\end{array}$ & sp|P32597|STH1_YEAST (142)-sp|P43609|RSC8_YEAST (375)/ & 7 & STH1_YEAST & RSC8_YEAST & 142 & 375 & 2.03 \\
\hline EKIDKQPSILVGGTLKEYQLR(16)-IGQKNEVR(4) & sp|P32597|STH1_YEAST (471)-sp|P32597|STH1_YEAST (907)/ & 30 & STH1_YEAST & STH1_YEAST & 471 & 907 & 2.00 \\
\hline LNKTTVLENNDGKR(3)-AEEKPESKSPAK(8) & sp|P32597|STH1 YEAST (1212)-sp|P32597|STH1 YEAST (1231)/ & 10 & STH1 YEAST & STH1 YEAST & 1212 & 1231 & 1.99 \\
\hline VIQAGKFDNK(6)-GGIKGLNNK(4) & sp|P32597|STH1_YEAST (944)-sp|P32597|STH1_Y'YEAST (750)/ & 4 & STH1_YEAST & STH1_YEAST & 944 & 750 & 1.99 \\
\hline LNKTTVLENNDGKR(3)-KKKPK(2) & sp|P32597|STH1_YEAST (1212)-sp|P32597|STH1_YEAST (1200)/ & 7 & STH1_YEAST & STH1_YEAST & 1212 & 1200 & 1.93 \\
\hline AEEKPESKSPAK(8)-TTVLENNDGKR(10) & sp|P32597|STH1_Y_YAST (1231)-sp|P32597|STH1_YEAST (1222)/ & 7 & STH1_YEAST & STH1_YEAST & 1231 & 1222 & 1.91 \\
\hline VFREDIEEHFKKEDSEPLGR(12)-GLNNKIMQLR(5) & sp|P32597|STH1_YEAST (1042)-sp|P32597|STH1_YEAST (755)/ & 7 & STH1 YEAST & STH1 YEAST & 1042 & 755 & 1.90 \\
\hline 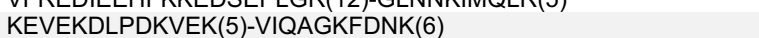 & $\begin{array}{l}\text { sp|P32597|STH1 YEAST (704)-sp|P32597|STH1 Y YEAST (944)/ }\end{array}$ & 18 & STH1 YEAST & STH1 YEAST & 704 & 944 & 1.87 \\
\hline EVEKDLPDKVEKVIK(12)-SADEKILFDKIDK(5) & sp|P32597|STH1 YEAST (712)-sp|P32597|STH1 YEAST (992) & 4 & STH1 YEAST & STH1 YEAST & 712 & 992 & 1.86 \\
\hline FSNATKTALGDPDTEIR(6)-IGYMDLYEKNEKK(12) & sp|P32597|STH1_YEAST (183)-sp|Q07979|RSC58_YEAST (325)/ & 1 & STH1_YEAST & RSC58_YEAST & 183 & 325 & 1.85 \\
\hline EVEKDLPDKVEK(9)-IGQKNEVR(4) & sp|P32597|STH1_YEAST (709)-sp|P32597|STH1_YEAST (907)/ & 5 & STH1_YEAST & STH1_Y̌EAST & 709 & 907 & 1.85 \\
\hline 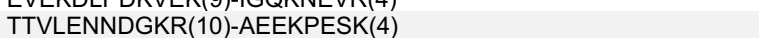 & sp|P32597|STH1-YEAST (1222)-sp|P32597|STH1 YEAST (1227)/ & 10 & STH1 YEAST & STH1 YEAST & 1222 & 1227 & 1.84 \\
\hline EDIEEHFKKEDSEPLGR(9)-GLNNKIMQLR(5) & sp|P32597|STH1 YEAST (1042)-sp|P32597|STH1 YEAST (755)/ & 7 & STH1 YEAST & STH1 YEAST & 1042 & 755 & 1.82 \\
\hline EVEKDLPDKVEKVIK(12)-SADEKILFDK(5) & sp|P32597|STH1_YEAST (712)-sp|P32597|STH1_VYEAST (992)/ & 4 & STH1_YEAST & STH1_YEAST & 712 & 992 & 1.80 \\
\hline TTVLENNDGKR(10)-IKLNK(2) & sp|P32597|STH1_YEAST (1222)-sp|P32597|STH1_YEAST (1209)/ & 6 & STH1_YEAST & STH1_YEAST & 1222 & 1209 & 1.80 \\
\hline SLGIFPTVEKLVEEMREQLDEVDSHPR(10)-KTAAK(1) & sp|P32597|STH1_YEAST (1257)-sp|P32597|STH1 YEAST (1236)/ & 4 & STH1_YEAST & STH1 YEAST & 1257 & 1236 & 1.78 \\
\hline KPIKISADFNAK(4)-VSDKSR(4) & sp|P32597|STH1_YEAST (160)-sp|P43609|RSC8_YEAST (413)/ & 8 & STH1_YEAST & RSC8_YEAST & 160 & 413 & 1.78 \\
\hline LNKTTVLENNDGKR(3)-ADAKAQGLR(4) & sp|P32597|STH1_YYEAST (1212)-sp|P32597|STH1_YEAST (1011)/ & 4 & STH1_YEAST & STH1_YEAST & 1212 & 1011 & 1.75 \\
\hline GLNNKIMQLR(5)-EDIEEHFKK(8) & sp|P32597|STH1_YEAST (755)-sp|P32597|STH1_YEAST (1041)/ & 2 & STH1_YEAST & STH1_YEAST & 755 & 1041 & 1.75 \\
\hline EDIEEHFKKEDSEPLGR(9)-LLLPGELAKHAVSEGTK(9) & sp|P32597|STH1__YEAST (1042)-sp|P02281|H2B11_XENLA (105)/ & 4 & STH1_YEAST & H2B11_XENLA & 1042 & 105 & 1.74 \\
\hline DTDVPDYFLDLPDTKNDNTTAIEVDYSEK(15)- & sp|P32597|STH1_YEAST (142)-sp|P43609|RSC8_YEAST (375)/ & 7 & STH1_YEAST & RSC8_YEAST & 142 & 375 & 1.74 \\
\hline $\begin{array}{l}\text { EVVGSTLNGKGGDSR(10) } \\
\text { VEEEVKSSSVEIINGSESK(6)-KKKPK(2) }\end{array}$ & sp|P32597|STH1 YEAST (1185)-sp|P32597|STH1 YEAST (1200)/ & 4 & STH1 YEAST & STH1 YEAST & 1185 & 1200 & 1.74 \\
\hline $\begin{array}{l}\text { SLGIFPTVEKLVEEMR(10)-KTAAK(1) } \\
\text { STIT) }\end{array}$ & sp|P32597|STH1_YEAST (1257)-sp|P32597|STH1_YEAST (1236)/ & 4 & STH1_YEAST & STH1_YEAST & 1257 & 1236 & 1.73 \\
\hline MLQEQSELMSTVMNNTPTTVAALAAVA & sp|P32597|STH1_YEAST (1)-sp|Q07979|RSC58_YEAST (350)/ & 2 & STH1_YEAST & RSC58_YEAST & 1 & 350 & 1.70 \\
\hline $\begin{array}{l}\text { QNDENNKNVDGK(7) } \\
\text { HNALFVGAGTEGATKGGIK(15)-VIQAGKFDNK(6) }\end{array}$ & sp|P32597|STH1 YEAST (746)-sp|P32597|STH1 YEAST (944)/ & 3 & STH1 YEAST & STH1 YEAST & 746 & 944 & 1.70 \\
\hline HNALFVGAGTEGATKGGIK(15)-GLNNKIMQLRK(5) & sp|P32597|STH1_YEAST (746)-sp|P32597|STH1_YEAST (755)/ & 8 & YEAST & STH1_YEAST & 746 & 755 & 1.69 \\
\hline LGASLHNQMEKDEQK(11)-TAKQR(3) & p|P32597|STH1_YEAST (364)-sp|P32597|STH1_YEAST (375)/ & 4 & STH1_YEAST & STH1_YEAST & 364 & 375 & 1.67 \\
\hline VEEEVKSSSVEIINGSESK(6)-KKKPK(1) & sp|P32597|STH1_YEAST (1185)-sp|P32597|STH1_YEAST (1199)/ & 2 & STH1_YEAST & STH1_YEAST & 1185 & 1199 & 1.67 \\
\hline LAALKSNDEEAYLK(5) & sp|P32597|STH1_YEAST (382)-sp|P02281|H2B11_XENLA (9)/ & 2 & STH1_YEAST & H2B11_XENLA & 382 & 9 & 1.66 \\
\hline $\begin{array}{l}\text { SADEKILFDKIDKER(5)-DLPDKVEKVIK(8) } \\
\text { SADEF }\end{array}$ & sp|P32597|STH1_YEAST (992)-sp|P32597|STH1_YEAST (712)/ & 7 & STH1_YEAST & STH1_YEAST & 992 & 712 & 1.62 \\
\hline LSGLQQQLYQQMLKHNALFVGAGTEGATK(14)-GLNNKIMQLR(5) & sp|P32597|STH1 YEAST (731)-sp|P32597|STH1 YEAST (755)/ & 6 & STH1 YEAST & STH1 YEAST & 731 & 755 & 1.61 \\
\hline VIQAGKFDNKSTAEEQEAFLR(10)-AMQKLDIDGK(4) & sp|P32597|STH1_YEAST (948)-sp|P32597|STH1_YEAST (932)/ & 23 & STH1_YEAST & STH1_YEAST & 948 & 932 & 1.54 \\
\hline $\begin{array}{l}\text { MLQEQSELMSTVMNNTPTTVAALAAVAAASETNGKLGSEEQPEITI } \\
\text { PKPR(1)-LNHELSKISESLIK(7) }\end{array}$ & sp|P32597|STH1_YEAST (1)-sp|Q06639|RSC3_YEAST (830)/ & 4 & STH1_YEAST & RSC3_YEAST & 1 & 830 & 1.53 \\
\hline NDNTTAIEVDYSEKKPIK(15)-VSDKSR(4) & sp|P32597|STH1_YEAST (157)-sp|P43609|RSC8_YEAST (413)/ & 6 & STH1_YEAST & RSC8_YEAST & 157 & 413 & 1.52 \\
\hline HNALFVGAGTEGATKGGIK(15)-LLLPGELAKHAVSEGTK(9) & sp|P32597|STH1_YEAST (746)-sp|P02281|H2B11_XENLA (105)/ & 6 & STH1_YEAST & H2B11_XENLA & 746 & 105 & 1.51 \\
\hline $\begin{array}{l}\text { MLQEQSELMSTVMNNTPTTVAALAAVAAASETNGK(1)- } \\
\text { AlQNHPKENKLEIK(7) }\end{array}$ & sp|P32597|STH1_YEAST (1)-sp|P32597|STH1_YEAST (70)/ & 3 & STH1_YEAST & STH1_YEAST & 1 & 70 & 1.48 \\
\hline LAALKSNDEEAYLK(5)-LKKEVEK(2) & sp|P32597|STH1_YEAST (382)-sp|P32597|STH1_YEAST (699)/ & 1 & STH1_YEAST & STH1_YEAST & 382 & 699 & 1.48 \\
\hline MLQEQSELMSTVMNNTPTTVAALAAVAAASETNGK(1)- & sp|P32597|STH1_YEAST (1)-sp|P32597|STH1_YEAST (170)/ & 1 & STH1_YEAST & STH1_YEAST & 1 & 170 & 1.47 \\
\hline VFREDIEEHFKKEDSEPLGR(12)-GGIKGLNNK(4) & p|P32597|STH1_YEA & 6 & ST & STH1_YEAST & 1042 & 750 & 1.47 \\
\hline VFREDIEEHFKKEDS & OP32597|STH1_YEA & 1 & EAST & H32_X̄ENLA & 1041 & 9 & 1.46 \\
\hline
\end{tabular}




\begin{tabular}{|c|c|c|c|c|c|c|c|}
\hline PEPTIDE & PROTEINS & CSM & PROTEIN1 & PROTEIN2 & R1 & $\mathbf{R 2}$ & $\mathbf{s}$ \\
\hline $\begin{array}{l}\text { MLQEQSELMSTVMNNTPTTVAALAAVAAASETNGK(1)- } \\
\text { KLLPAVEFIK(1) }\end{array}$ & sp|P32597|STH1_YEAST (1)-sp|Q03124|RSC9_YEAST (434)/ & 9 & STH1_YEAST & RSC9_YEAST & 1 & 434 & 1.45 \\
\hline LGSEEQPEITIPKPR(13)-VLKPTTEER(3) & sp|P32597|STH1_YEAST (48)-sp|Q07979|RSC58_YEAST (440)/ & 1 & STH1_YEAST & RSC58_YEAST & 48 & 440 & 1.45 \\
\hline ADAKAQGLR(4)-IKLNK(2) & sp|P32597|STH1_YEAST (1011)-sp|P32597|STH1_YEAST (1209)/ & 2 & STH1_YEAST & STH1_Y̌EAST & 1011 & 1209 & 1.44 \\
\hline AQQNEAKILHGEEVQPITDEER(7)-LGASLHNQMEKDEQKR(11) & sp|P32597|STH1_YEAST (427)-sp|P32597|STH1_Y̌EAST (364)/ & 1 & STH1_YEAST & STH1_YEAST & 427 & 364 & 1.41 \\
\hline EDIEEHFKKEDSEPLGR(8)-GLNNKIMQLR(5) & sp|P32597|STH1_YEAST (1041)-sp|P32597|STH1__YEAST (755)/ & 16 & STH1_YEAST & STH1_Y_YEAST & 1041 & 755 & 1.41 \\
\hline IKLNKTTVLENNDGKR(5)-AEEKPESKSPAKK(8) & sp|P32597|STH1_YEAST (1212)-sp|P32597|STH1_YEAST (1231)/ & 10 & STH1_YEAST & STH1_YEAST & 1212 & 1231 & 1.39 \\
\hline LLDQTKDTR(6)-TAKQR(3) & sp|P32597|STH1_YEAST (397)-sp|P32597|STH1_Y̌EAST (375)/ & 2 & STH1_YEAST & STH1_YEEAST & 397 & 375 & 1.39 \\
\hline SKSLGIFPTVEK(2)-TAAKK(4) & sp|P32597|STH1_YEAST (1247)-sp|P32597|STH1_YEAST (1240)/ & 2 & STH1_YEAST & STH1_YEAST & 1247 & 1240 & 1.36 \\
\hline LAALKSNDEEAYLK(5)-VIQAGKFDNK(6) & sp|P32597|STH1_YEAST (382)-sp|P32597|STH1_Y'YEAST (944)/ & 1 & STH1_YEAST & STH1_Y_YEAST & 382 & 944 & 1.36 \\
\hline $\begin{array}{l}\text { LRQNGTKENEIETLENTPEASETSLIENNSFTAAVDEETNADKETTA } \\
\text { SR(7)-ADAKAQGLR(4) }\end{array}$ & sp|P32597|STH1_YEAST (1101)-sp|P32597|STH1_YEAST (1011)/ & 2 & STH1_YEAST & STH1_YEAST & 1101 & 1011 & 1.35 \\
\hline QPSILVGGTLKEYQLR(11)-IGQKNEVR(4) & sp|P32597|STH1_YEAST (471)-sp|P32597|STH1_YEAST (907)/ & 30 & STH1_YEAST & STH1_YEAST & 471 & 907 & 1.35 \\
\hline SIDKGFQYDEDLLNKHLVALQLLEK(4)-KVSKVPINNVR(1) & sp|P32597|STH1_YEAST (106)-sp|Q07979|RSC58_YEAST (464)/ & 13 & STH1_YEAST & RSC58_YEAST & 106 & 464 & 1.35 \\
\hline KICNHPFVFDEVEGVVNPSR(1)-GGIKGLNNK(4) & sp|P32597|STH1_YEAST (761)-sp|P32597|STH1_YEEAST (750)/ & 6 & STH1_YEAST & STH1_Y̌EAST & 761 & 750 & 1.34 \\
\hline KEVEKDLPDKVEK(1)-VIQAGKFDNK(6) & sp|P32597|STH1_YEAST (700)-sp|P32597|STH1_YEAST (944)/ & 2 & STH1_YEAST & STH1_Y_YEAST & 700 & 944 & 1.32 \\
\hline MLQEQSELMSTVMNNTPTTVAALAAVAAASETNGK(1)-KVIICK(1) & sp|P32597|STH1_YEEAST (1)-sp|P38210|LDB7_YEAST (153)/ & 2 & STH1_YEAST & LDB7_YEAST & 1 & 153 & 1.31 \\
\hline LNKTTVLENNDGKR(3)-KKPKLTVK(4) & sp|P32597|STH1_YEAST (1212)-sp|P32597|STHH1_YEAST (1203)/ & 3 & STH1_YEAST & STH1_YEAST & 1212 & 1203 & 1.29 \\
\hline DTDVPDYFLDLPDTKNDNTTAIEVDYSEKKPIK(29)- & sp|P32597|STH1_YEAST (156)-sp|P43609|RSC8_YEAST (388)/ & 6 & STH1_YEAST & RSC8_YEAST & 156 & 388 & 1.28 \\
\hline EDIEEHFKKEDSEPLGR(8)-LIQLDELPKVFR(9) & sp|P32597|STH1 YEAST (1041)-sp|P32597|STH1 YEAST (1030)/ & 2 & STH1 YEAST & STH1 YEAST & 1041 & 1030 & 1.28 \\
\hline $\begin{array}{l}\text { TTVLENNDGKR(10)-SPAKK(4) } \\
\text { TISTE }\end{array}$ & sp|P32597|STH1_YEAST (1222)-sp|P32597|STH1_YEAST (1235)/ & 2 & STH1_YEAST & $1-\mathrm{YE}$ & 1222 & 1235 & 1.28 \\
\hline LNKTTVLENNDGKR(3)-KKKPK(1) & sp|P32597|STH1_YEAST (1212)-sp|P32597|STH1_YEAST (1199)/ & 3 & STH1_YEAST & STH1_YEAST & 1212 & 1199 & 1.27 \\
\hline LTVKIK(4)-KKKPK(2) & sp|P32597|STH1_YEAST (1207)-sp|P32597|STH1_YEAST (1200)/ & 2 & STH1_YEAST & STH1_-YEAST & 1207 & 1200 & 1.24 \\
\hline PIKISADFNAK(3)-QQLLEKR(6) & sp|P32597|STH1_YEAST (160)-sp|P32597|STH1_Y̌EAST (315)/ & 1 & STH1_YEAST & STH1_YEAST & 160 & 315 & 1.24 \\
\hline KKPKLTVK(4)-IKLNK(2) & sp|P32597|STH1_YEAST (1203)-sp|P32597|STH1_YEAST (1209)/ & 3 & STH1_YEAST & STH1_YEAST & 1203 & 1209 & 1.21 \\
\hline MLQEQSELMSTVMNNTPTTVAALAAVAAASETNGK(1)- & sp|P32597|STH1_YEAST (1)-sp|Q06639|RSC3_YEAST (830)/ & 4 & STH1_YEAST & RSC3_YEAST & 1 & 830 & 1.21 \\
\hline $\begin{array}{l}\text { LNHELSKISESLIK(7) } \\
\text { LAALKSNDEEAYLK(5)-KGSKK(4) }\end{array}$ & sp|P32597|STH1_YEAST (382)-sp|P02281|H2B11_XENLA (12)/ & 1 & STH1_YEAST & H2B11_XENLA & 382 & 12 & 1.18 \\
\hline LAALKSNDEEAYLK(5)-AVTKTQK(4) & sp|P32597|STH1_YEAST (382)-sp|P02281|H2B11_XENLA (17)/ & 3 & STH1_YE. & $\mathrm{H} 2$ & 382 & 17 & 1.17 \\
\hline EVEKDLPDKVEK(9)-AMQKLDIDGK(4) & sp|P32597|STH1_YEAST (709)-sp|P32597|STH1_YEAST (932)/ & 4 & STH1_YEAST & STH1_Y YEAST & 709 & 932 & 1.17 \\
\hline IKLNKTTVLENNDGKR(5)-KKKPK(1) & sp|P32597|STH1_YEAST (1212)-sp|P32597|STH1__YEAST (1199)/ & 3 & STH1_YEAST & STH1_YEEAST & 1212 & 1199 & 1.17 \\
\hline $\begin{array}{l}\text { MLQEQSELMSTVMNNTPTTVAALAAVAAASETNGKLGSEEQPEITI } \\
\text { PKPR(1)-FNKLFIK(3) }\end{array}$ & sp|P32597|STH1_YEAST (1)-sp|P32597|STH1_YEAST (73)/ & 6 & STH1_YEAST & STH1_YEAST & 1 & 73 & 1.12 \\
\hline KPKLTVK(3)-IKLNK(2) & sp|P32597|STH1_YEAST (1203)-sp|P32597|STH1_YEAST (1209)/ & 3 & STH1_YEAST & STH1_YEAST & 1203 & 1209 & 1.11 \\
\hline LIQLDELPKVFR(9)-EDIEEHFKK(8) & sp|P32597|STH1_YYAST (1030)-sp|P32597|STH1_YEAST (1041)/ & 2 & STH1_YEAST & 1_YEAST & 1030 & 1041 & 1.09 \\
\hline SKSLGIFPTVEK(2)-AEEKPESKSPAK(4) & sp|P32597|STH1_YEAST (1247)-sp|P32597|STH1_YEAST (1227)/ & 1 & STH1_YEAST & STH1_YEAST & 1247 & 1227 & 1.09 \\
\hline 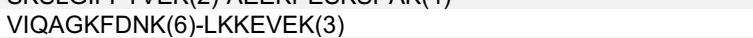 & sp|P32597|STH1_YEAST (944)-sp|P32597|STH1_YEAST (700)/ & 3 & STH1_YEAST & STH1_YEAST & 944 & 700 & 1.08 \\
\hline VIQAGKFDNK(6)-TAKQR(3) & sp|P32597|STH1_YEAST (944)-sp|P32597|STH1_YEAST (375)/ & 1 & STH1_YEAST & STH1_YEAST & 944 & 375 & 1.08 \\
\hline EKTDYYEVAHR(2)-VKNVK(2) & sp|P32597|STH1_YEAST (444)-sp|Q05123|ARP9_YEAST (272)/ & 5 & STH1_YE & 9_YEA & 444 & 272 & 1.10 \\
\hline ILHGEEVQPITDEEREKTDYYEVAHR(17)-SLMTSHTKGDTSK(8) & sp|P32597|STH1_YEAST (444)-sp|P53330|RT102_YEAST (102)/ & 7 & STH1_YEAST & RT102_YEAST & 444 & 102 & 1.09 \\
\hline VFREDIEEHFKKEDSEPLGR(11)-LLLPGELAKHAVSEGTK(9) & sp|P32597|STH1_YEAST (1041)-sp|P02281|H2B11_XENLA (105)/ & 1 & STH1_YEAST & H2B11 XENLA & 1041 & 105 & 1.08 \\
\hline LGASLHNQMEKDEQKR(15)-KGSKK(1) & sp|P32597|STH1_YEAST (368)-Sp|P02281|H2B11_X̂XENLA (9)/ & 2 & STH1_YEAST & H2B11_XENLA & 368 & 9 & 1.04 \\
\hline LAALKSNDEEAYLK(5)-KAVTKTQK(1) & sp|P32597|STH1_YEAST (382)-sp|P02281|H2B11_XENLA (13)/ & 10 & STH1_YEAST & H2B11 XENLA & 382 & 13 & 0.93 \\
\hline $\begin{array}{l}\text { MLQEQSELMSTVMNNTTPTTVAALAAVAAASETNGK(1)- } \\
\text { GIEGSENSKAK(9) }\end{array}$ & sp|P32597|STH1_YEAST (1)-sp|P38210|LDB7_YEAST (52)/ & 1 & STH1_YEAST & LDB7_YEAST & 1 & 52 & 0.90 \\
\hline SKSLGIFPTVEK(2)-SAKSK(5) & 32597|STH1_YEAST (1247)-tr|Q6AZJ8|H & 2 & STH1_YE & $\mathrm{H}_{2}$ & 1247 & 129 & 0.89 \\
\hline VFREDIEEHFKKEDSEPLGR(12)-LLLPGELAKHAVSEGTK(9) & sp|P32597|STH1_YEAST (1042)- & 4 & ST & $\mathrm{H}_{2}$ & 1042 & 105 & 0.88 \\
\hline SIDKGFQYDEDLLNK(4)-TGENSKKKGK(7) & sp|P32597|STH1_YEAST (106)-sp|P38781|RSC30_YEAST (256)/ & 1 & STH1_YEAST & RSC30_YEAST & 106 & 256 & 0.87 \\
\hline FSNATKTALGDPDTEIR(6)-VSKPKR(3) & sp|P32597|STH1 YEAST (183)-sp|P38210|LDB7 YEAST (57)/ & 4 & STH1 YEAST & LDB7 YEAST & 183 & 57 & 0.84 \\
\hline
\end{tabular}




\begin{tabular}{|c|c|c|c|c|c|c|c|}
\hline PEPTIDE & PROTEINS & CSM & PROTEIN1 & PROTEIN2 & R1 & $\mathbf{R 2}$ & $\mathbf{s}$ \\
\hline $\begin{array}{l}\text { VIQAGKFDNKSTAEEQEAFLRR(6)-EVEKDLPDKVEK(4) } \\
\end{array}$ & $\begin{array}{l}\text { sp|P32597|STH1 YEAST (944)-sp|P32597|STH1_ YEAST (704)/ } \\
\end{array}$ & 2 & STH1 YEAST & STH1_YEAST & 944 & 704 & 1.07 \\
\hline AEEKPESKSPAK(8)-KTAAKK(5) & sp|P32597|STH1_YEAST (1231)-Sp|P32597|STH1_YEAST (1240)/ & 2 & STH1_YEAST & STH1_YEAST & 1231 & 1240 & 1.07 \\
\hline MLQEQSELMSTVMNNTPTTVAALAAVAAASETNGK(1)-ENKLEIK(3) & sp|P32597|STH1_YEAST (1)-sp|P32597|STH1_YEAST (73)/ & 6 & STH1_YEAST & STH1_YEAST & 1 & 73 & 1.06 \\
\hline TISIVTAEDKENTQEESTSQENGGAKVEEEVK(26)-ADAKAQGLR(4) & sp|P32597|STH1_YEAST (1179)-sp|P32597|STH1_YYEAST (1011)/ & 3 & STH1_YEAST & STH1_YEAST & 1179 & 1011 & 1.02 \\
\hline SSSVEIINGSESKKK(13)-KPKLTVK(3) & sp|P32597|STH1_YEAST (1198)-sp|P32597|STH1_YYEAST (1203)/ & 9 & STH1_YEAST & STH1_YEAST & 1198 & 1203 & 0.99 \\
\hline ILFDKIDKER(5)-ADAKAQGLR(4) & sp|P32597|STH1_YEAST (997)-sp|P32597|STH1_Y̌EAST (1011)/ & 1 & STH1_YEAST & STH1_YEAST & 997 & 1011 & 0.99 \\
\hline AEEKPESKSPAKK(4)-TTVLENNDGKR(10) & sp|P32597|STH1_YEAST (1227)-sp|P32597|STH1__YEAST (1222)/ & 1 & STH1_YEAST & STH1_YEAST & 1227 & 1222 & 0.97 \\
\hline VIEKPMAIDIILKNCK(13)-TSIFEKLPSKR(6) & sp|P32597|STH1_YEAST (1305)-sp|P32597|STH1_YYEAST (1280)/ & 2 & STH1_YEAST & STH1_YEAST & 1305 & 1280 & 0.97 \\
\hline $\begin{array}{l}\text { MLQEQSELMSTVMNNTPTTVAALAAVAAASETNGK(1)- } \\
\text { SYESKFGQPVR(5) }\end{array}$ & sp|P32597|STH1_YEAST (1)-sp|Q03124|RSC9_YEAST (423)/ & 2 & STH1_YEAST & RSC9_YEAST & 1 & 423 & 0.77 \\
\hline $\begin{array}{l}\text { MLQEQSELMSTVMNNTPTTVAALAAVAAASETNGK(1)- } \\
\text { LWKTPDGK(3) }\end{array}$ & sp|P32597|STH1_YEAST (1)-sp|Q06488|RSC2_YEAST (437)/ & 2 & STH1_YEAST & RSC2_YEAST & 1 & 437 & 0.74 \\
\hline ADAKAQGLR(4)-GKGGK(2) & sp|P32597|STH1_YEAST (1011)-sp|P62799|H4_XENLA (5)/ & 1 & STH1_YEAST & H4_XENLA & 1011 & 5 & 0.73 \\
\hline LGASLHNQMEKDEQKR(15)-DGKKR(3) & sp|P32597|STH1_YEAST (368)-Sp|P02281|H2B11_XENLA (24)/ & 2 & STH1_YEAST & H2B11_XENLA & 368 & 24 & 0.71 \\
\hline LLDQTKDTR(6)-ERGQKVK(5) & sp|P32597|STH1_YEAST (397)-sp|Q05123|ARP9_YEAST (270)/ & 6 & STH1_YEAST & ARP9 Y YEAST & 397 & 270 & 0.67 \\
\hline LAALKSNDEEAYLK(5)-GKGGK(2) & sp|P32597|STH1_YEAST (382)-sp|P62799|H4_XENLA (5)/ & 2 & STH1_YEAST & H4_XĒNLA & 382 & 5 & 0.67 \\
\hline NDNTTAIEVDYSEKKPIK(14)-VSDKSR(4) & sp|P32597|STH1_YEAST (156)-sp|P43609|RSC8_YEAST (413)/ & 1 & STH1_YEAST & RSC8_YEAST & 156 & 413 & 0.66 \\
\hline 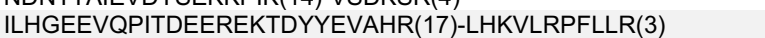 & sp|P32597|STH1_YEAST (444)-sp|P32597|STH1_YEAST (688)/ & 12 & STH1_YEAST & STH1_YEAST & 444 & 688 & 0.97 \\
\hline LAALKSNDEEAYLK(5)-QGGKTR(4) & sp|P32597|STH1_YEAST (382)-tr|Q6AZJ8|H2A_X̄ENLA (9)/ & 2 & STH1_YEAST & H2A_X̄ENLA & 382 & 9 & 0.66 \\
\hline LGASLHNQMEKDEQKR(15)-KEFPVNLTIKK(1) & sp|P32597|STH1_YEAST (368)-sp|P43609|RSC-8_YEAST (222)/ & 1 & STH1_YEAST & RSC-̄_YEAST & 368 & 222 & 0.66 \\
\hline LAALKSNDEEAYLK(5)-SAPAPKK(6) & sp|P32597|STH1_YEAST (382)-sp|P02281|H2B11_XENLA (8)/ & 1 & STH1_YEAST & H2B11_XENLA & 382 & 8 & 0.65 \\
\hline $\begin{array}{l}\text { DTDVPDYFLDLPDTKNDNTTAIEVDYSEKKPIK(15)- } \\
\text { IISNMHIFYSITFNFIFPIKSIK(20) }\end{array}$ & sp|P32597|STH1_YEAST (142)-sp|P38781|RSC30_YEAST (681)/ & 1 & STH1_YEAST & RSC30_YEAST & 142 & 681 & 0.62 \\
\hline $\begin{array}{l}\text { QNGTKENEIETLENTPEASETSLIENNSFTAAVDEETNADKETTASR } \\
\text { (5)-LLLPGELAKHAVSEGTK(9) }\end{array}$ & sp|P32597|STH1_YEAST (1101)-sp|P02281|H2B11_XENLA (105)/ & 1 & STH1_YEAST & H2B11_XENLA & 1101 & 105 & 0.55 \\
\hline ILHGEEVQPITDEEREKTDYYEVAHR(17)-ERGQKVK(5) & sp|P32597|STH1_YEAST (444)-sp|Q05123|ARP9_YEAST (270)/ & 1 & STH1_YEAST & ARP9_YEAST & 444 & 270 & 0.54 \\
\hline SLLSKHDWAHMIIDEGHR(5)-TKQTAR(2) & sp|P32597|STH1_YEAST (588)-sp|P84233|H32_XENLA (4)/ & 1 & STH1_YEAST & H32_XEENLA & 588 & 4 & 0.54 \\
\hline HNALFVGAGTEGATKGGIK(15)-GLNNKIMQLR(5) & sp|P32597|STH1_YEAST (746)-sp|P32597|STH1__YEAST (755)/ & 8 & STH1_YEAST & STH1_YEAST & 746 & 755 & 0.94 \\
\hline KKKPK(1)-IKLNK(2) & sp|P32597|STH1_YEAST (1199)-sp|P32597|STH1_YEAST (1209)/ & 1 & STH1_YEAST & STH1_YEAST & 1199 & 1209 & 0.92 \\
\hline $\begin{array}{l}\text { ILHGEEVQPITDEEREKTDYYEVAHR(17)-RLHKVLRPFLLR(4) } \\
\text { (L) }\end{array}$ & sp|P32597|STH1_YEAST (444)-sp|P32597|STH1_Y̌AST (688)/ & 12 & STH1_YEAST & STH1_YEAST & 444 & 688 & 0.92 \\
\hline EDIEEHFKKEDSEPLGR(9)-GGIKGLNNK(4) & sp|P32597|STH1_YEAST (1042)-sp|P32597|STH1_YEAST (750)/ & 6 & STH1_YEAST & STH1_YEAST & 1042 & 750 & 0.91 \\
\hline AMQKLDIDGK(4)-LKKEVEK(3) & sp|P32597|STH1_YEAST (932)-sp|P32597|STH1_'̄EAST (700)/ & 1 & STH1_YEAST & STH1_YEAST & 932 & 700 & 0.91 \\
\hline AMQKLDIDGK(4)-VIKCK(3) & sp|P32597|STH1_YEAST (932)-sp|P32597|STH1_YEAST (715)/ & 1 & STH1_YEAST & STH1_YEAST & 932 & 715 & 0.90 \\
\hline LNKTTVLENNDGKR(3)-KKPKLTVK(2) & sp|P32597|STH1_YEAST (1201)/ & 2 & STH1_YEAST & STH1_YEAST & 1212 & 1201 & 0.89 \\
\hline LNKTTVLENNDGKR(3)-KTAAK(1) & sp|P32597|STH1_YEAST (1212)-sp|P32597|STH1_YEAST (1236)/ & 1 & STH1_YEAST & STH1_YEAST & 1212 & 1236 & 0.89 \\
\hline LIESETNRDDDDKAELLDDELNDTLAR(13)-ADAKAQGLR(4) & sp|P32597|STH1_YYAST (973)-sp|P32597|STH1_Y̌EAST (1011)/ & 2 & STH1_YEAST & STH1_YEAST & 973 & 1011 & 0.87 \\
\hline AQQNEAKILHGEEVQPITDEER(7)-EKTDYYEVAHR(2) & sp|P32597|STH1_YEAST (427)-Sp|P32597|STH1_YEAST (444)/" & 3 & STH1_YEAST & STH1_YEAST & 427 & 444 & 0.87 \\
\hline IGQKNEVR(4)-DLPDKVEK(5) & sp|P32597|STH1_YEAST (907)-Sp|P32597|STH1_YEAST (709)/ & 1 & STH1_YEAST & STH1_YEAST & 907 & 709 & 0.87 \\
\hline $\begin{array}{l}\text { MLQEQSELMSTVMNNTPTTVAALAAVAAASETNGKLGSEEQPEITI } \\
\text { PKPR( (5) }\end{array}$ & sp|P32597|STH1_YEAST (35)-sp|P32597|STH1_YEAST (70)/ & 1 & STH1_YEAST & STH1_YEAST & 35 & 70 & 0.86 \\
\hline $\begin{array}{l}\text { PKPR(35)-AIQNHPKENKLEIK(7) } \\
\text { IDKQPSILVGGTLKEYQLR(14)-EVEKDLPDKVEK(9) }\end{array}$ & sp|P32597|STH1_YEAST (471)-sp|P32597|STH1_YEAST (709)/ & 1 & STH1_YEAST & STH1_YEAST & 471 & 709 & 0.86 \\
\hline $\begin{array}{l}\text { TISIVTAEDKENTQEESTSQENGGAKVEEEVK(26)- } \\
\text { SSSVEIINGSESKK(13) }\end{array}$ & sp|P32597|STH1_YEAST (1179)-sp|P32597|STH1_YEAST (1198)/ & 1 & STH1_YEAST & STH1_YEAST & 1179 & 1198 & 0.85 \\
\hline LIQLDELPKVFREDIEEHFK(9)-KEDSEPLGR(1) & sp|P32597|STH1_YEAST (1030)-sp|P32597|STH1_YEAST (1042)/ & 11 & YEAST & I-YE & 1030 & 1042 & 0.83 \\
\hline RAEEKPESKSPAK(9)-KTAAK(1) & sp|P32597|STH1_YEAST (1231)-sp|P32597|STH1_YEAST (1236)/ & 11 & STH1_YEAST & STH1_YEAST & 1231 & 1236 & 0.82 \\
\hline SADEKILFDKIDK(5)-DLPDKVEKVIK(8) & sp|P32597|STH1_YEAST (992)-sp|P32597|STH1_Y̌EAST (712)/ & 7 & STH1_YEAST & STH1_YEAST & 992 & 712 & 0.81 \\
\hline LAALKSNDEEAYLK(5)-AVTKTQKK(4) & sp|P32597|STH1_YEAST (382)-sp|P02281|H2B11_XENLA (17)/ & 3 & STH1_YEAST & H2B11_XENLA & 382 & 17 & 0.50 \\
\hline $\begin{array}{l}\text { TISIVTAEDKENTQEESTSQENGGAKVEEEVK(26)- } \\
\text { KSAPATGGVVKPHR(1) }\end{array}$ & sp|P32597|STH1_YEAST (1179)-sp|P84233|H32_XENLA (27)/ & 2 & STH1_YEAST & H32_XENLA & 1179 & 27 & 0.47 \\
\hline TISIVTAEDKENTQEESTSQENGGAKVEEEVK(10)-ADAKAQGLR(4) & sp|P32597|STH1 YEAST (1163)-sp|P32597|STH1 YEAST (1011)/ & 1 & STH1 YEAST & STH1 YEAST & 1163 & 1011 & 0.78 \\
\hline
\end{tabular}




\begin{tabular}{|c|c|c|c|c|c|c|c|}
\hline PEPTIDE & PROTEINS & CSM & PROTEIN1 & PROTEIN2 & R1 & R2 & $\mathbf{s}$ \\
\hline TISIVTAEDKENTQEESTSQENGGAKVEEEVK(26)-TKQTAR(2) & sp|P32597|STH1_YEAST (1179)-sp|P84233|H32_XENLA (4)/ & 1 & STH1_YEAST & H32_XENLA & 1179 & 4 & 0.46 \\
\hline $\begin{array}{l}\text { VEEEVKSSSVEIINGSESKK(6)-KKPKLTVK(4) } \\
\text { VEST) }\end{array}$ & sp|P32597|STH1_YEAST (1185)-sp|P32597|STH1_YEAST (1203)/ & 1 & STH1_YEAST & STH1_YEAST & 1185 & 1203 & 0.76 \\
\hline SKSLGIFPTVEK(2)-SPAKK(4) & sp|P32597|STH1_YEAST (1247)-sp|P32597|STH1_YEAST (1235)/ & 1 & STH1_YEAST & STH1_YEAST & 1247 & 1235 & 0.75 \\
\hline AMQKLDIDGK(4)-VIQAGKFDNK(6) & sp|P32597|STH1_YEAST (932)-sp|P32597|STH1_YEAST (944)/ & 1 & STH1_YEAST & STH1_YEAST & 932 & 944 & 0.74 \\
\hline MKDLKYMR(5)-FKASGHR(2) & sp|P32597|STH1_YEAST (833)-sp|P32597|STH1_YEAST (804)/ & 1 & STH1_YEAST & STH1_YEAST & 833 & 804 & 0.74 \\
\hline $\begin{array}{l}\text { MLQEQSELMSTVMNNTPTTTVAALAAVAAASETNGK(1)- } \\
\text { NSEAKRK(5) }\end{array}$ & sp|P32597|STH1_YEAST (1)-sp|P38210|LDB7_YEAST (149)/ & 2 & STH1_YEAST & LDB7_YEAST & 1 & 149 & 0.45 \\
\hline FSNATKTALGDPDTEIR(6)-VSKPK(3) & sp|P32597|STH1_YEAST (183)-sp|P38210|LDB7_YEAST (57)/ & 4 & STH1_YEAST & LDB7_YEAST & 183 & 57 & 0.45 \\
\hline TISIVTAEDKENTQEESTSQENGGAKVEEEVK(26)-GKGGK(2) & sp|P32597|STH1_YEAST (1179)-sp|P62799|H4_X'XENLA (5)/ & 1 & STH1_YEAST & H4_XĒENLA & 1179 & 5 & 0.43 \\
\hline 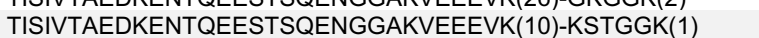 & sp|P32597|STH1 YEAST (1163)-sp|P84233|H32 XENLA (9)/ & 1 & STH1_YEAST & H32 XENLA & 1163 & 9 & 0.42 \\
\hline SKSLGIFPTVEK(2)-GKGGKGLGKGGAK(5) & sp|P32597|STH1_YEAST (1247)-sp|P62799|H4_X̌XENLA (8)/ & 1 & STH1_YEAST & H4_X̄XNLA & 1247 & 8 & 0.41 \\
\hline YRAIQNHPKENK(9)-ISADFNAKAK(8) & sp|P32597|STH1_YEAST (70)-sp|P32597|STH1_YEAST (168)/ & 2 & STH1_YEAST & STH1_YEAST & 70 & 168 & 0.72 \\
\hline LEIKAIEDTFR(4)-AIQNHPKENK(7) & sp|P32597|STH1_YEEAST (77)-sp|P32597|STH1_YEAST (70)/ & 1 & STH1_YEAST & STH1_YEAST & 77 & 70 & 0.71 \\
\hline 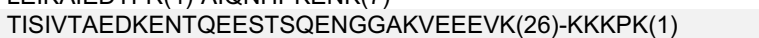 & sp|P32597|STH1_YEAST (1179)-sp|P32597|STH1_YEAST (1199)/ & 1 & STH1_YEAST & STH1_YEAST & 1179 & 1199 & 0.71 \\
\hline SIDKGFQYDEDLLNKHLVALQLLEK(4)-EVILAKK(6) & sp|P32597|STH1_YEAST (106)-sp|Q07979|RSC58_YEAST (463)/ & 8 & STH1_YEAST & RSC58_YEAST & 106 & 463 & 0.40 \\
\hline AQQNEAKILHGEEVQPITDEEREK(7)-VKNVK(2) & sp|P32597|STH1_YEAST (427)-sp|Q05123|ARP9_YEAST (272)/ & 2 & STH1_YEAST & ARP9 YEAST & 427 & 272 & 0.40 \\
\hline NDNTTAIEVDYSEKKPIKISADFNAK(18)-VSDKSR(4) & sp|P32597|STH1_YEAST (160)-sp|P43609|RSC8_YEAST (413)/ & 8 & STH1_YEAST & RSC8_YEAST & 160 & 413 & 0.40 \\
\hline $\begin{array}{l}\text { LIESETNRDDDDKAELDDDELNDTLAR(13)- } \\
\text { FVFKDIPDKVEKVIK(12) }\end{array}$ & sp|P32597|STH1_YEAST (973)-sp|P32597|STH1_YEAST (712)/ & 2 & STH1_YEAST & STH1_YEAST & 973 & 712 & 0.69 \\
\hline LDIDGKVIQAGK(6)-LKKEVEK(2) & sp|P32597|STH1_YEAST (938)-sp|P32597|STH1_YEAST (699)/ & 1 & STH1_YEAST & YEAST & 938 & 699 & 0.65 \\
\hline LAALKSNDEEAYLK(5)-LKKEVEK(3) & sp|P32597|STH1 YEAST (382)-sp|P32597|STH1 YEAST (700)/ & 1 & STH1 YEAST & STH1 YEAST & 382 & 700 & 0.65 \\
\hline $\begin{array}{l}\text { EVEKDLPDKVEK(9)-VIKCK(3) } \\
\text { EVET) }\end{array}$ & sp|P32597|STH1_YEAST (709)-sp|P32597|STH1_YEAST (715)/ & 1 & STH1_YEAST & STH1_YEAST & 709 & 715 & 0.64 \\
\hline LNKTTVLENNDGK(3)-KKKPK(2) & sp|P32597|STH1_YEAST (1212)-sp|P32597|STH1_YEAST (1200)/ & 7 & STH1_YEAST & STH1_YE & 1212 & 1200 & 0.62 \\
\hline AQQNEAKILHGEEVQPITDEER(7)-VKNVK(2) & sp|P32597|STH1_YEAST (427)-sp|Q05123|ARP9_YEAST (272)/ & 2 & STH1_YEAST & ARP9_YEAST & 427 & 272 & 0.38 \\
\hline $\begin{array}{l}\text { QNGTKENEIETLENTPEASETSLIENNSFTAAVDEETNADKETTASR } \\
\text { (5)-ADAKAQGLR(4) }\end{array}$ & sp|P32597|STH1_YEAST (1101)-sp|P32597|STH1_YEAST (1011)/ & 2 & STH1_YEAST & STH1_YEAST & 1101 & 1011 & 0.62 \\
\hline LNKTTVLENNDGKR(3)-KKKPK(3) & sp|P32597|STH1_YEAST (1212)-sp|P32597|STH1_YEAST (1201)/ & 2 & AST & ST & 1212 & 1201 & 0.61 \\
\hline TTVLENNDGKR(10)-LTVKIK(4) & sp|P32597|STH1_YEAST (1222)-sp|P32597|STH1_YEAST (1207)/ & 1 & STH1_YEAST & STH1_-YEAST & 1222 & 1207 & 0.60 \\
\hline $\begin{array}{l}\text { MLQEQSELMSTVMNNTPTTVAALAAVAAASETNGKLGSEEQPEITI } \\
\text { PKPR (35)-ENKLEIK(3) }\end{array}$ & sp|P32597|STH1_YEAST (35)-sp|P32597|STH1_YEAST (73)/ & 1 & STH1_YEAST & STH1_YEAST & 35 & 73 & 0.60 \\
\hline SSSVEIINGSESKKKK(15)-PKLTVK(2) & sp|P32597|STH1_YEAST (1200)-sp|P32597|STH1_YEAST (1203)/ & 1 & STH1_YEAST & STH1_YEAST & 1200 & 1203 & 0.58 \\
\hline IDKERMNQERADAK(3)-AEEKPESKSPAK(4) & sp|P32597|STH1_YYEAST (1000)-sp|P32597|STH1_YEAST (1227)/ & 1 & STH1_YEAST & STH1_YEAST & 1000 & 1227 & 0.58 \\
\hline SKSLGIFPTVEKLVEEMR(12)-QQLLEKRKK(6) & sp|P32597|STH1_YEAST (1257)-sp|P32597|STH1_YEAST (315)/ & 1 & STH1_YEAST & STH1_YEAST & 1257 & 315 & 0.58 \\
\hline VIQAGKFDNK(6)-LKKEVEK(2) & sp|P32597|STH1 YEAST (944)-sp|P32597|STH1 YEAST (699)/ & 1 & STH1 YEAST & STH1 YEAST & 944 & 699 & 0.58 \\
\hline VFREDIEEHFKKEDSEPLGR(11)-C & Sp|P32597|STH1_YEAST (1041)-sp|P32597|STH1_YEAST (755)/ & 16 & STH1_YEAST & STH1_YEAST & 1041 & 755 & 0.58 \\
\hline VGNFDVLLTTYEYIIKDK(16)-GNRKFMLATEPAR(4) & sp|P32597|STH1_YEAST (581)-sp|P32832|NPL6_YEAST (163)/ & 1 & STH1_YEAST & NPL6_YEAST & 581 & 163 & 0.38 \\
\hline AMQKLDIDGK(4)-LKKEVEK(2) & sp|P32597|STH1_YEAST (932)-sp|P32597|STH1_YEAST (699)/ & 1 & STH1_YEAST & STH1_YEAST & 932 & 699 & 0.57 \\
\hline IKLNKTTVLENNDGKR(5)-KKKPK(2) & sp|P32597|STH1 YEAST (1212)-sp|P32597|STH1 YEAST (1200)/ & 7 & STH1 YEAST & STH1 YEAST & 1212 & 1200 & 0.55 \\
\hline DTDVPDYFLDLPDTKNDNTTAIEVDYSEKKPIK(29)-AKSLGLESK(2) & sp|P32597|STH1_YEAST (156)-sp|P32597|STH1_Y̌EAT (170)/ & 12 & STH1_YEAST & STH1_YEAST & 156 & 170 & 0.55 \\
\hline DYPDYFKVIEK(7)-DLPDKVEKVIK(8) & sp|P32597|STH1_YEAST (1292)-sp|P32597|STH1_YEAST (712)/ & 1 & STH1_YEAST & STH1_YEAST & 1292 & 712 & 0.53 \\
\hline $\begin{array}{l}\text { LSGLQQQLYQQMLKHNALFVGAGTEGATK(14)- } \\
\text { VFRFDFFHFKK(11) }\end{array}$ & sp|P32597|STH1_YEAST (731)-sp|P32597|STH1_YEAST (1041)/ & 3 & STH1_YEAST & STH1_YEAST & 731 & 1041 & 0.52 \\
\hline RAEEKPESKSPAK(9)-KTAAKK(1) & sp|P32597|STH1 YEAST (1231)-sp|P32597|STH1 YEAST (1236)/ & 11 & STH1 YEAST & STH1 YEAST & 1231 & 1236 & 0.51 \\
\hline MLQEQSELMSTVMNNTPTTVAALAAVAAASETNGK(1)-NSEAKR(5) & P38210|LDB7_YEAST (149)/ & 2 & YEAST & LDB7 YEAST & 1 & 149 & 0.36 \\
\hline HNALFVGAGTEGATKGGIK(15)-IRQKK(4) & sp|P32597|STH1_YEAST (746)-sp|P32597|STḦ1_YEAST (1054)/ & 1 & STH1_YEAST & STH1_YEAST & 746 & 1054 & 0.49 \\
\hline MLQEQSELMSTVMNNTPTTVAALAAVAAASETNGK(1)- & sp|P32597|STH1_YEAST (1)-sp|P32597|STH1_YEAST (70)/ & 3 & STH1_YEAST & STH1_YEAST & 1 & 70 & 0.49 \\
\hline $\begin{array}{l}\text { AIQNHPKENK(7) } \\
\text { NGTYKTLEEVR(5)-TAAKK(4) }\end{array}$ & sp|P32597|STH1 YEAST (1313)-sp|P32597|STH1 YEAST (1240)/ & 2 & & & 1313 & 1240 & 0.48 \\
\hline 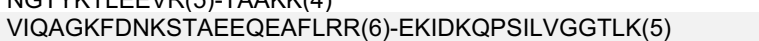 & sp|P32597|STH1_YEAST (944)-Sp|P32597|STH1_YEAST (460)// & 1 & STH1 YEAST & STH1 YEAST & 944 & 460 & 0.47 \\
\hline DTDVPDYFLDLPDTKNDNTTAIEVDYSEKKPIK $(30)$ & sp|P32597|STH1 YEAST (157)-sp|P32597|STH1 YEAST (168)/ & 1 & STH1 YEAST & STH1 YEAST & 157 & 168 & 0.45 \\
\hline
\end{tabular}




\begin{tabular}{|c|c|c|c|c|c|c|c|}
\hline PEPTIDE & PROTEINS & CSM & PROTEIN1 & PROTEIN2 & R1 & $\mathbf{R 2}$ & $\mathbf{s}$ \\
\hline \multicolumn{8}{|l|}{ ISADFNAKAKSLGLESK(8) } \\
\hline LGASLHNQMEKDEQK(11)-NGTYKTLEEVR(5) & sp|P32597|STH1_YEAST (364)-sp|P32597|STH1_YEAST (1313)/ & 1 & STH1_YEAST & STH1_YEAST & 364 & 1313 & 0.45 \\
\hline $\begin{array}{l}\text { TISIVTAEDKENTQEESTSQENGGAKVEEEVK(26)- } \\
\text { KSAPATGGVK(1) }\end{array}$ & sp|P32597|STH1_YEAST (1179)-sp|P84233|H32_XENLA (27)/ & 2 & STH1_YEAST & H32_XENLA & 1179 & 27 & 0.34 \\
\hline LGASLHNQMEKDEQKR(15)-QGGKTR(4) & sp|P32597|STH1_YEAST (368)-tr|Q6AZJ8|H2A_XENLA (9)/ & 2 & STH1_YEAST & H2A_XENLA & 368 & 9 & 0.33 \\
\hline LEIKAIEDTFR(4)-GLNNKIMQLRK(5) & sp|P32597|STH1_YEAST (77)-sp|P32597|STH1_YEAST (755)/ & 1 & STH1_YEAST & STH1̄_YEAST & 77 & 755 & 0.43 \\
\hline VFREDIEEHFKKEDSEPLGR(12)-IRQKK(4) & sp|P32597|STH1_YYAST (1042)-sp|P32597|STT̄1_YEAST (1054)/ & 1 & STH1_YEAST & STH1_YEAST & 1042 & 1054 & 0.43 \\
\hline LNKTTVLENNDGK(3)-KKKPK(1) & sp|P32597|STH1_YYEAST (1212)-sp|P32597|STH1_YEAST (1199)/ & 3 & STH1_YEAST & STH1_YEAST & 1212 & 1199 & 0.42 \\
\hline ADAKAQGLR(4)-LPSKR(4) & sp|P32597|STH1_YYEAST (1011)-sp|P32597|STH1_YEAST (1284)/ & 1 & STH1_YEAST & STH1_YEAST & 1011 & 1284 & 0.42 \\
\hline $\begin{array}{l}\text { LRQNGTKENEIETLENTPEASETSLIENNSFTAAVDEETNADKETTA } \\
\text { SR(7)-SGRGK(1) }\end{array}$ & $\begin{array}{l}\text { sp|P32597|STH1_YEAST (1101)-tr|Q6AZJ8|H2A_XENLA } \\
\text { (1)/sp|P32597|STH1_YEAST (1101)-sp|P62799|'̄H4_XENLA (1)/ }\end{array}$ & 1 & STH1_YEAST & $\begin{array}{l}\text { H2A_X̄ENLA;sp|P } \\
62799 \mid H 4 \_X E N L A\end{array}$ & 1101 & $1 ; 1$ & 0.95 \\
\hline LGASLHNQMEKDEQKR(11)-SGRGK(1) & $\begin{array}{l}\text { sp|P32597|STH1_YEAST (364)-tr|Q6AZJ8||H2A_XĒNLA } \\
\text { (1)/sp|P32597|STH1 YEAST (364)-sp|P62799|H4 XENLA (1)/ }\end{array}$ & 1 & STH1_YEAST & $\begin{array}{l}\text { H2A XENLA;sp|P } \\
62799 \mid \text { H } 4 \text { XENLA }\end{array}$ & 364 & $1 ; 1$ & 0.55 \\
\hline $\begin{array}{l}\text { TKGNVIEGK(2)-EFGAKIIR(5) } \\
\text { LAGDPIDKSAR(8)-TKGNVIEGK(2) }\end{array}$ & $\begin{array}{l}\text { sp|P32832|NPL6_YEAST (252)-sp|P32832|NPL6_YEAST (232)/ } \\
\text { sp|P32832|NPL6_YEAST (267)-sp|P32832|NPL6_YEAST (252)/ }\end{array}$ & $\begin{array}{l}18 \\
11\end{array}$ & $\begin{array}{l}\text { NPL6_YEAST } \\
\text { NPL6_YEAST }\end{array}$ & $\begin{array}{l}\text { NPL6_YEAST } \\
\text { NPL6_YEAST }\end{array}$ & $\begin{array}{l}252 \\
267\end{array}$ & $\begin{array}{l}232 \\
252\end{array}$ & $\begin{array}{l}6.33 \\
6.30\end{array}$ \\
\hline $\begin{array}{l}\text { SKNDSPSDTSIVYETVIHDNDLNKPK(2)- } \\
\text { KNVYDSAQDFNALQDESR(1) }\end{array}$ & sp|P32832|NPL6_YEAST (375)-sp|P43609|RSC8_YEAST (232)/ & 6 & NPL6_YEAST & RSC8_YEAST & 375 & 232 & 6.03 \\
\hline SKFKKPVFPGIDDAEENLNPLK(4)-HVDEEEDLSEDKGVTR(12) & sp|P32832|NPL6_YEAST (101)-sp|P32832|NPL6_YEAST (89)/ & 4 & NPL6_YEAST & NPL6_YEAST & 101 & 89 & 5.14 \\
\hline SKNDSPSDTSIVYETVIHDNDLNKPK(2)-LENNGNSVKK(9) & sp|P32832|NPL6_YEAST (375)-sp|P43609|RSC8_YEAST (312)/ & 3 & NPL6_YEAST & RSC8_YEAST & 375 & 312 & 5.08 \\
\hline GGKHITDDYYASELR(3)-TKGNVIEGK(2) & sp|P32832|NPL6_YEAST (238)-sp|P32832|NPL6_YEAST (252)/ & 8 & NPL6_YEAST & NPL6_YEAST & 238 & 252 & 4.76 \\
\hline KPVFPGIDDAEENLNPLK(1)-HVDEEEDLSEDKGVTR(12) & sp|P32832|NPL6_YEAST (102)-sp|P32832|NPL6_YEAST (89)/ & 3 & NPL6_YEAST & NPL6_YEAST & 102 & 89 & 4.68 \\
\hline GGKHITDDYYASELR(3)-LAGDPIDKSAR(8) & sp|P32832|NPL6_YEAST (238)-sp|P32832|NPL6_YEAST (267)/ & 22 & NPL6_YEAST & NPL6_YEAST & 238 & 267 & 4.52 \\
\hline FKKPVFPGIDDAEENLNPLK(2)-HVDEEEDLSEDKGVTR(12) & sp|P32832|NPL6_YEAST (101)-sp|P32832|NPL6_YEAST (89)/ & 4 & NPL6_YEAST & NPL6_YEAST & 101 & 89 & 4.22 \\
\hline GNVIEGKLAGDPIDK(7)-EFGAKIIR(5) & sp|P32832|NPL6_YEAST (259)-sp|P32832|NPL6_YEAST (232)/ & 26 & NPL6_YEAST & NPL6_YEAST & 259 & 232 & 4.20 \\
\hline LAGDPIDKSAR(8)-EFGAKIIR(5) & sp|P32832|NPL6_YEAST (267)-sp|P32832|NPL6_YEAST (232)/ & 12 & NPL6_YEAST & NPL6_YEAST & 267 & 232 & 3.95 \\
\hline AITEQQNFEKCNEYI(10)-IHEIEKR(6) & sp|P32832|NPL6_YEAST (430)-sp|P43609|RSC8_YEAST (99)/ & 9 & NPL6_YEAST & RSC8_YEAST & 430 & 99 & 3.87 \\
\hline TKGNVIEGKLAGDPIDK(9)-GVFKEFGAKIIR(9) & sp|P32832|NPL6_YEAST (259)-sp|P32832|NPL6_YEAST (232)/ & 26 & NPL6_YEAST & NPL6_YEAST & 259 & 232 & 3.58 \\
\hline SKNDSPSDTSIVYETVIHDNDLNKPK(2)-EVVGSTLNGKGGDSR(10) & sp|P32832|NPL6_YEAST (375)-sp|P43609|RSC8_YEAST (375)/ & 7 & NPL6_YEAST & RSC8_YEAST & 375 & 375 & 3.56 \\
\hline TGLSEIPKEIYEDVVDEDVLR(8)-AITEQQNFEKCNEYI(10) & sp|P32832|NPL6_YEAST (407)-sp|P32832|NPL6_YEAST (430)/ & 8 & NPL6_YEAST & NPL6_YEAST & 407 & 430 & 3.54 \\
\hline TKGNVIEGKLAGDPIDK(9)-GGKHITDDYYASELR(3) & sp|P32832|NPL6 YEAST (259)-sp|P32832|NPL6 YEAST (238)/ & 5 & NPL6 YEAST & NPL6 YEAST & 259 & 238 & 3.52 \\
\hline SKNDSPSDTSIVYETVIHDNDLNKPK(2)-QEVEGGDGAEPQVKK(14) & sp|P32832|NPL6_YEAST (375)-sp|P43609|RSC8_YEAST (221)/ & 3 & NPL6_YEAST & RSC8_YEAST & 375 & 221 & 3.44 \\
\hline GGKHITDDYYASELR(3)-GNVIEGKLAGDPIDK(7) & sp|P32832|NPL6_YEAST (238)-sp|P32832|NPL6_YEAST (259)/ & 12 & NPL6_YEAST & NPL6_YEAST & 238 & 259 & 3.42 \\
\hline GVFKEFGAKIIR(9)-TKGNVIEGK(2) & sp|P32832|NPL6_YEAST (232)-sp|P32832|NPL6_YEAST (252)/ & 14 & NPL6_YEAST & NPL6_YEAST & 232 & 252 & 3.35 \\
\hline RSKNDSPSDTSIVYETVIHDNDLNKPK(3)- & sp|P32832|NPL6_YEAST (375)-sp|P43609|RSC8_YEAST (232)/ & 6 & NPL6_YEAST & RSC8_YEAST & 375 & 232 & 3.05 \\
\hline $\begin{array}{l}\text { KNVYDSAQDFNALQDESR(1) } \\
\text { KPVFPGIDDAEENLNPLK(1)-EFGAKIIR(5) }\end{array}$ & sp|P32832|NPL6_YEAST (102)-sp|P32832|NPL6_YEAST (232)/ & 1 & NPL6 YEAST & NPL6 YEAST & 102 & 232 & 2.88 \\
\hline KPVFPGIDDAEENLNPLK(1)-TKGNVIEGK(2) & sp|P32832|NPL6_YEAST (102)-sp|P32832|NPL6_YEAST (252)/ & 2 & NP & NPL6_YEAST & 102 & 252 & 2.77 \\
\hline LAGDPIDKSAR(8)-IHEIEKR(6) & sp|P32832|NPL6 YEAST (267)-sp|P43609|RSC8 YEAST (99)/ & 15 & NPL6 YEAST & RSC8 YEAST & 267 & 99 & 2.74 \\
\hline RHVDEEEDLSEDKGVTR(13)-KPVFPGIDDAEENLNPLK(1) & sp|P32832|NPL6_YEAST (89)-sp|P32832|NPL6_Y̌ EAST (102)/ & 1 & NPL6_YEAST & NPL6_YEAST & 89 & 102 & 2.73 \\
\hline LAGDPIDKSAR(8)-ADAKAQGLR(4) & sp|P32832|NPL6_YEAST (267)-sp|P32597|STH1_YEAST (1011)/ & 2 & NPL6_YEAST & STH1_YEAST & 267 & 1011 & 2.53 \\
\hline SKFKKPPVFPGIDDAEENLNPLK(4)-RHVDEEEDLSEDKGVTR(13) & sp|P32832|NPL6_YEAST (101)-sp|P32832|NPL6_YEAST (89)/ & 4 & NPL6_YEAST & NPL6_YEAST & 101 & 89 & 2.51 \\
\hline GNVIEGKLAGDPIDK(7)-GVFKEFGAKIIR(9) & sp|P32832|NPL6_YEAST (259)-sp|P32832|NPL6_YEAST (232)/ & 26 & NPL6_YEAST & NPL6_YEAST & 259 & 232 & 2.41 \\
\hline AITEQQNFEKCNEYI(10)-AYKDTR(3) & sp|P32832|NPL6_YEAST (430)-sp & 4 & NPL6_YEA & RSC8_YEAST & 430 & 119 & 2.35 \\
\hline TKGNVIEGKLAGDPIDK(9)-EFGAKIIR(5) & sp|P32832|NPL6_YEAST (259)-sp|P32832|NPL6_YEAST (232)/ & 26 & NPL6_YEAST & NPL6_YEAST & 259 & 232 & 2.31 \\
\hline GVFKEFGAKIIR(9)-LAGDPIDKSAR(8) & sp|P32832|NPL6_YEAST (232)-sp|P32832|NPL6_YEAST (267)/ & 10 & NPL6_YEAST & NPL6_YEAST & 232 & 267 & 2.21 \\
\hline KPVFPGIDDAEENLNPLK(1)-TFTLTEKGNRK(7) & sp|P32832|NPL6_YEAST (102)-sp|P32832|NPL6_YEAST (159)/ & 2 & NPL6_YEAST & NPL6_YEAST & 102 & 159 & 2.15 \\
\hline HVDEEEDLSEDKGVTR(12)-TFTLTEKGNRK(7) & YEAST (159)/ & 5 & NPL6_YEAST & NPL6_YEAST & 89 & 159 & 1.62 \\
\hline LAGDPIDKSAR(8)-AYKDTR(3) & sp|P32832|NPL6_YEAST (267)-sp|P43609|RSC8_YEAST (119)/ & 7 & NPL6_YEAST & RSC8_YEAST & 267 & 119 & 1.59 \\
\hline HVDEEEDLSEDKGVTR(12)-EFGAKIIR(5) & sp|P32832|NPL6_YEAST (89)-sp|P32832|NPL6_YYEAST (232)/ & 1 & NPL6_YEAST & NPL6_YEAST & 89 & 232 & 1.52 \\
\hline RHVDEEEDLSEDKGVTR(13)-TFTLTEKG। & sp|P32832|NPL6_YEAST (89)-sp|P32832|NPL6_YEAST (159)/ & 5 & NPL6_YEAST & NPL6_YEAST & 89 & 159 & 1.47 \\
\hline
\end{tabular}




\begin{tabular}{|c|c|c|c|c|c|c|c|}
\hline PEPTIDE & PROTEINS & CSM & PROTEIN1 & PROTEIN2 & R1 & R2 & $\mathbf{s}$ \\
\hline FKKPVFPGIDDAEENLNPLK(2)-TFTLTEKGNRK(7) & sp|P32832|NPL6_YEAST (101)-sp|P32832|NPL6_YEAST (159)/ & 2 & NPL6_YEAST & NPL6_YEAST & 101 & 159 & 1.41 \\
\hline AITEQQNFEKCNEYI(10)-FKTPKAYKDTR(5) & sp|P32832|NPL6_YEAST (430)-sp|P43609|RSC8_YEAST (116)/ & 7 & NPL6_YEAST & RSC8_YEAST & 430 & 116 & 1.41 \\
\hline AITEQQNFEKCNEYI(10)-FKTPKAYK(5) & sp|P32832|NPL6_YEAST (430)-sp|P43609|RSC8_YEAST (116)/ & 7 & NPL6_YEAST & RSC8_YEAST & 430 & 116 & 1.29 \\
\hline $\begin{array}{l}\text { KPVFPGIDDAEENLNPLKVVNEEYVLPDDPEGETK(18)- } \\
\text { TFTLTEKGNRK(7) }\end{array}$ & sp|P32832|NPL6_YEAST (119)-sp|P32832|NPL6_YEAST (159)/ & 3 & NPL6_YEAST & NPL6_YEAST & 119 & 159 & 1.28 \\
\hline $\begin{array}{l}\text { VVNEEYVLPDDPEGETKITADGDLLGGR(17)- } \\
\text { SKFKKPVFPGIDDAEENLNPLK(4) }\end{array}$ & sp|P32832|NPL6_YEAST (136)-sp|P32832|NPL6_YEAST (101)/ & 1 & NPL6_YEAST & NPL6_YEAST & 136 & 101 & 1.17 \\
\hline $\begin{array}{l}\text { FKKPVFFGIDDAEENLNPLKVVNEEYVLPDDPEGETK(20)- } \\
\text { TFTLTEKGNRK(7) }\end{array}$ & sp|P32832|NPL6_YEAST (119)-sp|P32832|NPL6_YEAST (159)/ & 3 & NPL6_YEAST & NPL6_YEAST & 119 & 159 & 1.12 \\
\hline $\begin{array}{l}\text { VKVLLVDQQGLR(2)-GKGGK(2) } \\
\text { GGKHITDDYYASELR(3)-EESATLGAEVAHKK(13) }\end{array}$ & $\begin{array}{l}\text { sp|P32832|NPL6_YEAST (340)-sp|P62799|H4_XENLA (5)/ } \\
\text { sp|P32832|NPL6 YEAST (238)-sp|P43609|RSC8 YEAST (54)/ }\end{array}$ & $\begin{array}{l}1 \\
2\end{array}$ & $\begin{array}{l}\text { NPL6_YEAST } \\
\text { NPL6 YEAST }\end{array}$ & $\begin{array}{l}\text { H4_XENLA } \\
\text { RSC8 YEAST }\end{array}$ & $\begin{array}{l}340 \\
238\end{array}$ & $\begin{array}{c}5 \\
54\end{array}$ & $\begin{array}{l}0.98 \\
0.95\end{array}$ \\
\hline 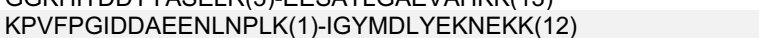 & sp|P32832|NPL6 YEAST (102)-sp|Q07979|RSC58 YEAST (325)/ & 2 & NPL6 YEAST & RSC58 YEAST & 102 & $\begin{array}{l}54 \\
325\end{array}$ & 0.95 \\
\hline 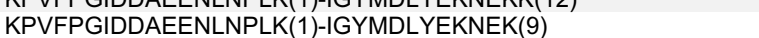 & sp|P32832|NPL6 YEAST (102)-sp|Q07979|RSC58- YEAST (322)/ & 1 & NPL6 YEAST & RSC58 YEAST & 102 & 322 & 0.67 \\
\hline GNVIEGKLAGDPIDK(7)-IHEIEKR(6) & sp|P32832|NPL6_YEAST (259)-sp|P43609|R & 1 & NPL6 YEAST & RSC8 YEAST & 259 & 99 & 0.65 \\
\hline RHVDEEEDLSEDKGVTR(13)-IHEIEKR(6) & sp|P32832|NPL6_YEAST (89)-sp|P43609|RSC8_YEAST (99)/ & 2 & NPL6_YEAST & RSC8_YEAST & 89 & 99 & 0.64 \\
\hline 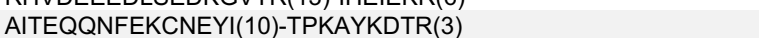 & sp|P32832|NPL6 YEAST (430)-sp|P43609|RSC 8 YEAST (116)/ & 7 & NPL6 YEAST & RSC8 YEAST & 430 & 116 & 0.60 \\
\hline VKVLLVDQQGL & sp|P32832|NPL6 YEAST (3 & 1 & NPL6 YEAST & H4 XENLA & 340 & 16 & 0.59 \\
\hline HVDEEEDLSEDKGVTR(12)-IHEIEKR(6) & sp|P32832|NPL6_YYEAST (89)-sp|P43609|RSC8_YEAST (99)/ & 2 & NPL6_YEAST & RSC8_YEAST & 89 & 99 & 0.49 \\
\hline $\begin{array}{l}\text { KPVFPGIDDAEENLNPLKVVNEEYVLPDDPEGETK(18)- } \\
\text { SIDKGFOYDEDUNKHLVALOLEK(15) }\end{array}$ & sp|P32832|NPL6_YEAST (119)-sp|P32597|STH1_YEAST (117)/ & 1 & NPL6_YEAST & STH1_YEAST & 119 & 117 & 0.37 \\
\hline EFGAKIIR(5)-ADAKAQGLR(4) & sp|P32832|NPL6_YEAST (232)-sp|P32597|STH1_YEAST (1011)/ & 1 & NPL6_YEAST & STH1_YEAST & 232 & 1011 & 0.34 \\
\hline TKGNVIEGK(2)-IHEIEKR(6) & sp|P32832|NPL6_YEAST (252)-sp|P43609|RSC8_YEAST (99)/ & 1 & NPL6_YEAST & RSC8_YEAST & 252 & 99 & 0.32 \\
\hline $\begin{array}{l}\text { VAVHGYLGGKVSLADAAQVEYEVGHSLLGSYVPR(10)- } \\
\text { LQQQQHQYQQQKR(12) }\end{array}$ & sp|P38210|LDB7_YEAST (70)-sp|Q06488|RSC2_YEAST (713)/ & 17 & LDB7_YEAST & RSC2_YEAST & 70 & 713 & 13.65 \\
\hline $\begin{array}{l}\text { KVIICK(1)-NSEAKR(5) } \\
\text { KVIICKR(1)-NSEAKR(5) }\end{array}$ & $\begin{array}{l}\text { sp|P38210|LDB7_YEAST (153)-sp|P38210|LDB7_YEAST (149)/ } \\
\text { sp|P38210|LDB7_YEAST (153)-sp|P38210|LDB7_YEAST (149)/ }\end{array}$ & $\begin{array}{l}13 \\
13\end{array}$ & $\begin{array}{l}\text { LDB7_YEAST } \\
\text { LDB7_YEAST }\end{array}$ & $\begin{array}{l}\text { LDB7_YEAST } \\
\text { LDB7_YEAST }\end{array}$ & $\begin{array}{l}153 \\
153\end{array}$ & $\begin{array}{l}149 \\
149\end{array}$ & $\begin{array}{l}6.53 \\
6.17\end{array}$ \\
\hline $\begin{array}{l}\text { VAVHGYLGGKVSLADAAQVEYEVGHSLLGSYVPR(10)- } \\
\text { QKLINNVASQAHHNIPYLR(2) }\end{array}$ & sp|P38210|LDB7_YEAST (70)-sp|P32597|STH1_YEAST (260)/ & 9 & LDB7_YEAST & STH1_YEAST & 70 & 260 & 6.03 \\
\hline $\begin{array}{l}\text { VAVHGYLGGKVSLADAAQVEYEVGHSLLGSYVPR(10)- } \\
\text { KITGVNLDLKQDSSAQMQSSHSNR(10) }\end{array}$ & sp|P38210|LDB7_YEAST (70)-sp|Q06639|RSC3_YEAST (208)/ & 1 & LDB7_YEAST & RSC3_YEAST & 70 & 208 & 5.85 \\
\hline $\begin{array}{l}\text { VAVHGYLGGKVSLADDAAQVEYEVGHSLLGSYVPR(10)- } \\
\text { KLSEDGVTDGDGKPIPESER(1) }\end{array}$ & sp|P38210|LDB7_YEAST (70)-sp|Q06639|RSC3_YEAST (234)/ & 2 & LDB7_YEAST & RSC3_YEAST & 70 & 234 & 5.42 \\
\hline SSQVVFSATELQQLTQQSHATDKGIEGSENSK(23)-VLKPTTEER(3) & sp|P38210|LDB7_YEAST (43)-sp|Q07979|RSC58_YEAST (440)/ & 18 & LDB7_YEAST & RSC58_YEAST & 43 & 440 & 4.86 \\
\hline $\begin{array}{l}\text { VAVHGYLGGKVSLADAAQVEYEVGHSLLGSYVPR(10)- } \\
\text { VGSPGAGGPLTVQGLKQHQLQR(16) }\end{array}$ & sp|P38210|LDB7_YEAST (70)-sp|Q06488|RSC2_YEAST (695)/ & 9 & LDB7_YEAST & RSC2_YEAST & 70 & 695 & 4.05 \\
\hline $\begin{array}{l}\text { VAVHGYLGGKVSLADAAQVEYEVGHSLLGSYVPR(10)- } \\
\text { NQEEFLTIKK(9) }\end{array}$ & sp|P38210|LDB7_YEAST (70)-sp|Q06639|RSC3_YEAST (231)/ & 4 & LDB & RSC3_ & 70 & 231 & 3.34 \\
\hline $\begin{array}{l}\text { SSQVVFSATELQQLTQQSHATDKGIEGSENSK(23)- } \\
\text { KQNDENNKNVDGK(1) }\end{array}$ & sp|P38210|LDB7_YEAST (43)-sp|Q07979|RSC58_YEAST (343)/ & 5 & LDB7_YEAST & RSC58_YEAST & 43 & 343 & 2.32 \\
\hline SSQVVFSATELQQLTQQSHATDKGIEGSENSK(23)-AKVSKPK(2) & sp|P38210|LDB7_YEAST (43)-sp|P38210|LDB7_YEAST (54)/ & 10 & LDB7_YEAST & LDB7_YEAST & 43 & 54 & 2.21 \\
\hline $\begin{array}{l}\text { TLECKAALETHDVFLAGAGQLSLPFQSHIESPR(5)- } \\
\text { VGSPGAGGPLTVQGLKQHQLQR(16) }\end{array}$ & sp|P38210|LDB7_YEAST (116)-sp|Q06488|RSC2_YEAST (695)/ & 2 & LDB7_YEAST & RSC2_YEAST & 116 & 695 & 2.19 \\
\hline $\begin{array}{l}\text { SSQVVFSATELQQLTQQSHATDKGIEGSENSK(23)- } \\
\text { ILNKVLKPTTEER(4) }\end{array}$ & sp|P38210|LDB7_YEAST (43)-sp|Q07979|RSC58_YEAST (437)/ & 4 & LDB7_YEAST & RSC58_YEAST & 43 & 437 & 2.01 \\
\hline $\begin{array}{l}\text { SSQVVFSATELQQLTQQSHATDKGIEGSENSKAK(32)- } \\
\text { KQNDENNKNVDGK(1) }\end{array}$ & sp|P38210|LDB7_YEAST (52)-sp|Q07979|RSC58_YEAST (343)/ & 3 & LDB7_YEAST & RSC58_YEAST & 52 & 343 & 1.77 \\
\hline GIEGSENSKAK(9)-VLKPTTEER(3) & sp|P38210|LDB7_YEAST (52)-sp|Q07979|RSC58_YEAST (440)/ & 4 & LDB7_YEAST & RSC58_YEAST & 52 & 440 & 1.61 \\
\hline SSQVVFSATELQQLTQQSHATDKG & sp|P38210|LDB7_YEAST (43)-sp|Q07979|RSC58_YEAST (350)/ & 5 & LDB7_YEAST & RSC58_YEAST & 43 & 350 & 1.54 \\
\hline TLECKAALETHDVFLAGAGQLSLPFQSHIESPR(5)-VLKPTTEER(3) & sp|P38210|LDB7_YEAST (116)-sp|Q07979|RSC58_YEAST (440)/ & 12 & LDB7_YEAST & RSC58_YEAST & 116 & 440 & 1.46 \\
\hline
\end{tabular}




\begin{tabular}{|c|c|c|c|c|c|c|c|}
\hline PEPTIDE & PROTEINS & CSM & PROTEIN1 & PROTEIN2 & R1 & $\mathbf{R 2}$ & $\mathbf{S}$ \\
\hline VAVHGYLGGKVSLADAAQVEYEVGHSLLGSYVPR(10)-VSKPKR(3) & $\begin{array}{l}\text { sp|P38210|LDB7_YEAST (70)-sp|P38210|LDB7_YEAST (57)/ } \\
\end{array}$ & 4 & LDB7_YEAST & LDB7_YEAST & 70 & 57 & 1.45 \\
\hline $\begin{array}{l}\text { SSQVVFSATELQQLTQQSHATDKGIEGSENSK(23)- } \\
\text { LNHELSKISESLIK(7) }\end{array}$ & sp|P38210|LDB7_YEAST (43)-sp|Q06639|RSC3_YEAST (830)/ & 4 & LDB7_YEAST & RSC3_YEAST & 43 & 830 & 1.42 \\
\hline $\begin{array}{l}\text { VAVHGYLGGKVSLADAAQVEYEVGHSLLGSYVPR(10)- } \\
\text { ISNILEKTFTSLAR(7) }\end{array}$ & sp|P38210|LDB7_YEAST (70)-sp|Q06488|RSC2_YEAST (373)/ & 1 & LDB7_YEAST & RSC2_YEAST & 70 & 373 & 1.40 \\
\hline $\begin{array}{l}\text { SSQVVFSATELQQLTQQSHATDKGIEGSENSK(23)- } \\
\text { QNDENNKNVDGK(7) }\end{array}$ & sp|P38210|LDB7_YEAST (43)-sp|Q07979|RSC58_YEAST (350)/ & 5 & LDB7_YEAST & RSC58_YEAST & 43 & 350 & 1.29 \\
\hline VAVHGYLGGKVSLADAAQVEYEVGHSLLGSYVPR(10)-VSKPKR(5) & sp|P38210|LDB7_YEAST (70)-sp|P38210|LDB7_YEAST (59)/ & 9 & LDB7_YEAST & LDB7_YEAST & 70 & 59 & 1.28 \\
\hline $\begin{array}{l}\text { SSQVVFSATELQQLTQQSHATDKGIEGSENSK(23)- } \\
\text { TNDANEISEQTDIHSNNDNSKR(21) }\end{array}$ & sp|P38210|LDB7_YEAST (43)-sp|Q06639|RSC3_YEAST (744)/ & 8 & LDB7_YEAST & RSC3_YEAST & 43 & 744 & 1.27 \\
\hline $\begin{array}{l}\text { SSQVVFSATELQQQLTQQSHATDKGIEGSENSK(23)- } \\
\text { VLKPTTEERELYFK(3) }\end{array}$ & sp|P38210|LDB7_YEAST (43)-sp|Q07979|RSC58_YEAST (440)/ & 18 & LDB7_YEAST & RSC58_YEAST & 43 & 440 & 1.23 \\
\hline RKVIICK(2)-NSEAKR(5) & sp|P38210|LDB7_YEAST (153)-sp|P38210|LDB7_YEAST (149)/ & 13 & LDB7_YEAST & LDB7_YEAST & 153 & 149 & 1.21 \\
\hline $\begin{array}{l}\text { VAVHGYLGGKVSLADAAQVEYEVGHSLLGSYVPR(10)- } \\
\text { KPLININPYTR(1) }\end{array}$ & sp|P38210|LDB7_YEAST (70)-sp|Q06488|RSC2_YEAST (223)/ & 1 & LDB7_YEAST & RSC2_YEAST & 70 & 223 & 1.18 \\
\hline $\begin{array}{l}\text { SSQVVFSATELQQLTQQSHATDKGIEGSENSK(23)- } \\
\text { LGSEEQPEITIPKPR(13) }\end{array}$ & sp|P38210|LDB7_YEAST (43)-sp|P32597|STH1_YEAST (48)/ & 2 & LDB7_YEAST & STH1_YEAST & 43 & 48 & 0.86 \\
\hline $\begin{array}{l}\text { SSQVVFSATELQQLTQQSHATDKGIEGSENSK(23)- } \\
\text { NGTPDKLVSDSLLKLKR(14) }\end{array}$ & sp|P38210|LDB7_YEAST (43)-sp|Q07979|RSC58_YEAST (425)/ & 2 & LDB7_YEAST & RSC58_YEAST & 43 & 425 & 0.80 \\
\hline $\begin{array}{l}\text { SSQVVFSATELQQLTQQSHATDKGIEGSENSK(23)- } \\
\text { LQQQQHQYQQQRR(12) }\end{array}$ & sp|P38210|LDB7_YEAST (43)-sp|Q06488|RSC2_YEAST (713)/ & 2 & LDB7_YEAST & RSC2_YEAST & 43 & 713 & 0.72 \\
\hline SGSNMGYYDVLAGLSALEK(1)-YKDIFK(2) & sp|P38210|LDB7_YEAST (2)-sp|Q02206|RSC4_YEAST (74)/ & 1 & LDB7_YEAST & RSC4_YEAST & 2 & 74 & 0.66 \\
\hline $\begin{array}{l}\text { SSQVVFSATELQQLTQQSHATDKGIEGSENSKAK(32)- } \\
\text { VLKPTTEER(3) }\end{array}$ & sp|P38210|LDB7_YEAST (52)-sp|Q07979|RSC58_YEAST (440)/ & 4 & LDB7_YEAST & RSC58_YEAST & 52 & 440 & 0.63 \\
\hline $\begin{array}{l}\text { RVAVHGYLGGKVSLADAAQVEYEVGHSLLGSYVPR(11)- } \\
\text { LQQQQHQYQQQKR(12) }\end{array}$ & sp|P38210|LDB7_YEAST (70)-sp|Q06488|RSC2_YEAST (713)/ & 17 & LDB7_YEAST & RSC2_YEAST & 70 & 713 & 0.62 \\
\hline $\begin{array}{l}\text { SSQVVFSATELQQLTQ' } \\
\text { ILNKVLKPTATDKGIEGSENSKAK(32)- }\end{array}$ & sp|P38210|LDB7_YEAST (52)-sp|Q07979|RSC58_YEAST (437)/ & 1 & LDB7_YEAST & RSC58_YEAST & 52 & 437 & 0.52 \\
\hline $\begin{array}{l}\text { VAVHGYLGGKVSLADAAQVEYEVGHSLLGSYVPR(10)- } \\
\text { AKVSKPKR(5) }\end{array}$ & sp|P38210|LDB7_YEAST (70)-sp|P38210|LDB7_YEAST (57)/ & 4 & LDB7_YEAST & LDB7_YEAST & 70 & 57 & 0.76 \\
\hline $\begin{array}{l}\text { SSQVVFSATELQQLTQQSHATDKGIEGSENSKAK(32)-VSKPKR(5) } \\
\text { SSOVVFSATELOQLTQQSHATDKGIEGSENSK(23)-AKVSKPKR(2) }\end{array}$ & $\begin{array}{l}\text { sp|P38210|LDB7_YEAST (52)-sp|P38210|LDB7_YEAST (59)/ } \\
\text { sp|P38210|LDB7-YEAST (43)-splP38210|LDB7-YEAST (54)/ }\end{array}$ & $\begin{array}{c}1 \\
10\end{array}$ & $\begin{array}{l}\text { LDB7_YEAST } \\
\text { LDB7 YEAST }\end{array}$ & LDB7_YEAST & $\begin{array}{l}52 \\
43\end{array}$ & 59 & 0.60 \\
\hline $\begin{array}{l}\text { SSQVVFSATELQQLTQQSHATDKGIEGSENSK(23)-AKVSKPKR(2) } \\
\text { GIEGSENSKAK(9)-VSKPKR(3) }\end{array}$ & $\begin{array}{l}\text { sp|P38210|LDB7_YEAST (43)-sp|P38210|LDB7_YEAST (54)/ } \\
\text { sp|P38210|LDB7_YEAST (52)-sp|P38210|LDB7_YEAST (57)/ }\end{array}$ & $\begin{array}{c}10 \\
1\end{array}$ & $\begin{array}{l}\text { LDB7_YEAST } \\
\text { LDB7_YEAST }\end{array}$ & $\begin{array}{l}\text { LDB7-YEAST } \\
\text { LDB7_YEAST }\end{array}$ & $\begin{array}{l}43 \\
52\end{array}$ & $\begin{array}{l}54 \\
57\end{array}$ & $\begin{array}{l}0.54 \\
0.49\end{array}$ \\
\hline $\begin{array}{l}\text { PKRVAVHGYLGGKVSLADAAQVEYEVGHSLLGSYVPR(13)- } \\
\text { DEMNSNGSFKSIKGFNK(10) }\end{array}$ & sp|P38210|LDB7_YEAST (70)-sp|P38781|RSC30_YEAST (777)/ & 1 & LDB7_YEAST & RSC30_YEAST & 70 & 777 & 0.32 \\
\hline $\begin{array}{l}\text { TGENSKK(6)-GKVDGKR(2) } \\
\text { NSDNNNVTDYSOSAKNK(15)-NVLLKFPVSELNR(5) }\end{array}$ & $\begin{array}{l}\text { sp|P38781|RSC30_YEAST (255)-sp|P38781|RSC30_YEAST (258)/ } \\
\text { sp|P38781RSSC30_YEAST (819-sDP3878|RSC30 Y YEAST (826)/ }\end{array}$ & $\begin{array}{l}1 \\
9\end{array}$ & $\begin{array}{l}\text { RSC30_YEAST } \\
\text { RSC30_YEAST }\end{array}$ & $\begin{array}{l}\text { RSC30_YEAST } \\
\text { RSC30_YEAST }\end{array}$ & $\begin{array}{l}255 \\
819\end{array}$ & $\begin{array}{l}258 \\
826\end{array}$ & $\begin{array}{l}4.41 \\
3.01\end{array}$ \\
\hline $\begin{array}{l}\text { HSLLGQSSFMVPAAENLSPIPASIDTNDIPLIANDLKLLETQAK(37)- } \\
\text { NVSNAFNNAAAIVITDPVTGKK(21) }\end{array}$ & sp|P38781|RSC30_YEAST (557)-sp|Q03124|RSC9_YEAST (464)/ & 2 & RSC30_YEAST & RSC9_YEAST & 557 & 464 & 2.64 \\
\hline $\begin{array}{l}\text { EKSNLNADNTTANK(2)-INKTGENSK(3) } \\
\text { SNLNADNTTANKINK(12)-TGENSKKGK(7) }\end{array}$ & $\begin{array}{l}\text { sp|P38781|RSC30_YEAST (234)-sp|P38781|RSC30_YEAST (249)/ } \\
\text { sp|P38781|RSC30-YEAST (246-s|P38781|RSC30 Y YEAST (256) }\end{array}$ & $\begin{array}{l}3 \\
3\end{array}$ & $\begin{array}{l}\text { RSC30_YEAST } \\
\text { RSC30_YEAST }\end{array}$ & $\begin{array}{l}\text { RSC30_YEAST } \\
\text { RSC30-YEAST }\end{array}$ & 234 & 249 & $\begin{array}{l}2.02 \\
1.95\end{array}$ \\
\hline SLKEKSNLNADNTTANK(5)-INKTGENSK(3) & $\begin{array}{l}\text { sp|P38781|RSC30_YEAST (234)-sp|P38781|RSC30_YEAST (249)/ } \\
\text { (PEST) }\end{array}$ & 3 & RSC30_YEAST & RSC30_YEAST & 234 & 249 & 1.61 \\
\hline SNLNADNTTANKINK(12)-TGENSKK(6) & sp|P38781|RSC30_YEAST (246)-sp|P38781|RSC30_YEAST (255)/ & 4 & RSC30_YEAST & RSC30_YEAST & 246 & 255 & 1.59 \\
\hline $\begin{array}{l}\text { HSLLGQSSFMVPAAENLSPIPASIDTNDIPLIANDLKLLETQAK(37)- } \\
\text { FVIKGIQPR(4) }\end{array}$ & sp|P38781|RSC30_YEAST (557)-sp|Q03124|RSC9_YEAST (470)/ & 3 & RSC30_YEAST & RSC9_YEAST & 557 & 470 & 1.27 \\
\hline SNLNADNTTANKINK(12)-VSKPKR(5) & sp|P38781|RSC30_YEAST (246)-sp|P38210|LDB7_YEAST (59)/ & 1 & RSC30_YEAST & LDB7_YEAST & 246 & 59 & 1.24 \\
\hline & SPI TSO & 1 & & & 657 & 838 & 1.22 \\
\hline $\begin{array}{l}\text { SNLNADNTTANKINK(12)-SLKEK(3) } \\
\text { SNLNADNTTANKINK(12)-GKOGGK(2) }\end{array}$ & $\begin{array}{l}\text { sp|P38781|RSC30-YVAST (246)-sp|P38781|RSC30-YEAST (232)/ } \\
\text { sp|P38781|RSC30 YEAST (246)-tr|Q6AZJ8|H2A XENLA (5)// }\end{array}$ & $\begin{array}{l}4 \\
1\end{array}$ & $\begin{array}{l}\text { RSC30_YEAST } \\
\text { RSC30 YEAST }\end{array}$ & $\begin{array}{l}\text { RSC30-YEAST } \\
\text { H2A XENLA }\end{array}$ & $\begin{array}{l}246 \\
246\end{array}$ & $\begin{array}{c}232 \\
5\end{array}$ & $\begin{array}{l}1.16 \\
1.00\end{array}$ \\
\hline 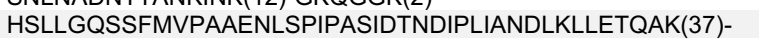 & sp|P38781|RSC30_YEAST (557)-sp|Q03124|RSC9_YEAST (496)/ & 1 & RSC30_YEAST & RSC9_YEAST & 557 & 496 & 0.98 \\
\hline
\end{tabular}


SUPPLEMENTARY MATERIALS

\begin{tabular}{|c|c|c|c|c|c|c|c|}
\hline PEPTIDE & PROTEINS & CSM & PROTEIN1 & PROTEIN2 & R1 & R2 & $\mathbf{s}$ \\
\hline \multicolumn{8}{|l|}{ ESQVPISALKSK(10) } \\
\hline KLVLISEQVDSGIQLVEKDQEGDDEDGNTATGHGVKR(36)- & sp|P43609|RSC8_YEAST (529)-sp|P43609|RSC8_YEAST (533)/ & 26 & RSC8_YEAST & RSC8_YEAST & 529 & 533 & 13.60 \\
\hline KNVYDSAQDFNALQDESR(1)-LENNGNSVKK(9) & sp|P43609|RSC8_YEAST (232)-sp|P43609|RSC8_YEAST (312)/ & 18 & RSC8_YEAST & RSC8_YEAST & 232 & 312 & 11.09 \\
\hline KINYEQEAQKLEEK(1)-KEFPVNLTIKK(1) & sp|P43609|RSC8_YEAST (55)-sp|P43609|RSC8_Y YEAST (222)/ & 56 & RSC8_YEAST & RSC8_YEAST & 55 & 222 & 9.85 \\
\hline $\begin{array}{l}\text { TKPSLIGPSFTGHFQVVLDTPQGLKPFLPENVIKQEVEGGDGAEPQ } \\
\text { VK(34)-ETLTHYLWSSKQNELVLQGDQYFNEDAAR(11) }\end{array}$ & sp|P43609|RSC8_YEAST (207)-sp|P25632|RSC6_YEAST (438)/ & 2 & RSC8_YEAST & RSC6_YEAST & 207 & 438 & 9.38 \\
\hline $\begin{array}{l}\text { TKPSLIGPSFTGHFQVVLDTPQGLKPFLPENVIKQEVEGGDGAEPQ } \\
\text { VK(25)-ETLTHYLWSSKQNELVLQGDQYFNEDAAR(11) }\end{array}$ & sp|P43609|RSC8_YEAST (198)-sp|P25632|RSC6_YEAST (438)/ & 2 & RSC8_YEAST & RSC6_YEAST & 198 & 438 & 9.07 \\
\hline $\begin{array}{l}\text { KLVLISEQVDSGIQLVEKDQEGDDEDGNTATGHGVKR(36)- } \\
\text { GVDGGKVQYSPNLATLIGMQTGSVNDAVYSIYK(6) }\end{array}$ & sp|P43609|RSC8_YEAST (529)-sp|P25632|RSC6_YEAST (231)/ & 2 & RSC8_YEAST & RSC6_YEAST & 529 & 231 & 9.01 \\
\hline KEFPVNLTIK(1)-LENNGNSVKK(9) & sp|P43609|RSC8 YEAST (222)-sp|P43609|RSC8 YEAST (312)/ & 18 & RSC8_YEAST & RSC8 YEAST & 222 & 312 & 8.96 \\
\hline KINYEQEAQKLEEK(1)-KEFPVNLTIK(1) & sp|P43609|RSC8 YEAST (55)-sp|P43609|RSC8 YEAST (222)/ & 56 & RSC8 YEAST & RSC8-YEAST & 55 & 222 & 8.81 \\
\hline KEFPVNLTIKK(1)-KINYEQEAQK(1) & sp|P43609|RSC8_YEAST (222)-sp|P43609|RSC8_YEAST (55)/ & 12 & RSC8_YEAST & RSC8_YEAST & 222 & 55 & 8.32 \\
\hline LENNGNSVKK(9)-TESVGGNK(1) & sp|P43609|RSC8 YEAST (312)-sp|Q07979|RSC58 YEAST (2)/ & 4 & RSC8 YEAST & RSC58 YEAST & 312 & 2 & 7.51 \\
\hline VEDCIEKFLSLPIEDNYIR(7)-KEFPVNLTIKK(1) & sp|P43609|RSC8_YEAST (353)-sp|P43609|RSC8_YEAST (222)/ & 12 & RSC8_YEAST & RSC8_Y̌EAST & 353 & 222 & 6.78 \\
\hline TKPSLIGPSFTGHFQVVLDTPQGLKPFLPENVIK(25)- & sp|P43609|RSC8_YEAST (198)-sp|P32832|NPL6_YEAST (375)/ & 12 & RSC8_YEAST & NPL6_YEAST & 198 & 375 & 6.71 \\
\hline VEDCIEKFLSLPIEDNYIR(7)-KEFPVNLTIK(1) & sp|P43609|RSC8_YEAST (353)-sp|P43609|RSC8_YEAST (222)/ & 12 & RSC8_YEAST & RSC8_YEAST & 353 & 222 & 5.96 \\
\hline LQPQVYKPWSL(7)-SGRGKGGK(1) & sp|P43609|RSC8 YEAST (553)-sp|P62799|H4 XENLA (1)/ & 3 & RSC8 YEAST & H4 XĒNLA & 553 & 1 & 5.90 \\
\hline KINYEQEAQK(1)-KEFPVNLTIK(1) & sp|P43609|RSC8 YEAST (55)-sp|P43609|RSC8 YEAST (222)/ & 56 & RSC8 YEAST & RSC8 YEAST & 55 & 222 & 5.77 \\
\hline EISEKYIEESQAIIQELVK(5)-MSLEEMKK(7) & sp|P43609|RSC8_YEAST (420)-sp|Q9URQ5|HTL1_YEAST (66)/ & 13 & RSC8_YEAST & HTL1_YEAST & 420 & 66 & 5.73 \\
\hline $\begin{array}{l}\text { VGKEGEEVGEGDSIAKLQPQVYKPWSL(16)- } \\
\text { VSPEELPLVITMPATNGKPDMAILER(18) }\end{array}$ & sp|P43609|RSC8_YEAST (546)-sp|Q12406|ARP7_YEAST (114)/ & 1 & RSC8_YEAST & ARP7_YEAST & 546 & 114 & 5.43 \\
\hline $\begin{array}{l}\text { EESATLGAEVAHKK(13)-KEFPVNLTIKK(1) } \\
\text { KINYEQEAQKLEEK(1)-KLIAKPETVQSEVK(1) }\end{array}$ & $\begin{array}{l}\text { sp|P43609|RSC8_YEAST (54)-sp|P43609|RSC8_YEAST (222)/ } \\
\text { sp|P43609|RSC8 YEAST (55)-sp|Q02206|RSC4 YEAST (386)/ }\end{array}$ & $\begin{array}{c}12 \\
2\end{array}$ & $\begin{array}{l}\text { RSC8_YEAST } \\
\text { RSC8 YEAST }\end{array}$ & $\begin{array}{l}\text { RSC8_YEAST } \\
\text { RSC4 YEAST }\end{array}$ & $\begin{array}{l}54 \\
55\end{array}$ & $\begin{array}{l}222 \\
386\end{array}$ & $\begin{array}{l}5.36 \\
5.30\end{array}$ \\
\hline $\begin{array}{l}\text { KLVLISEQVDSGIQLVEKDQEGDDEDGNTATGHGVK(1)- } \\
\text { QILDLNKSLEELNVSK(7) }\end{array}$ & sp|P43609|RSC8_YEAST (494)-sp|P43609|RSC8_YEAST (484)/ & 5 & RSC8_YEAST & RSC8_YEAST & 494 & 484 & 5.29 \\
\hline EESATLGAEVAHKK(13)-KEFPVNLTIK(1) & sp|P43609|RSC8_YEAST (54)-sp|P43609|RSC8_YEAST (222)/ & 12 & RSC8_YEAST & RSC8_YEAST & 54 & 222 & 5.24 \\
\hline $\begin{array}{l}\text { KLVLISEQVDSGIQLVEKDQEGDDEDGNTATGHGVKR(36)- } \\
\text { VSPEELPLVITMPATNGKPDMAILER(18) }\end{array}$ & sp|P43609|RSC8_YEAST (529)-sp|Q12406|ARP7_YEAST (114)/ & 3 & RSC8_YEAST & ARP7_YEAST & 529 & 114 & 5.22 \\
\hline $\begin{array}{l}\text { KNWSDQEMLLLLEGIEMYEDQWEKIADHVGGHKR(24)- } \\
\text { TESVGGNK(1) }\end{array}$ & sp|P43609|RSC8_YEAST (336)-sp|Q07979|RSC58_YEAST (2)/ & 2 & RSC8_YEAST & RSC58_YEAST & 336 & 2 & 5.03 \\
\hline EVVGSTLNGKGGDSR(10)-KEFPVNLTIK(1) & sp|P43609|RSC8_YEAST (375)-sp|P43609|RSC8_YEAST (222)/ & 32 & RSC8_YEAST & RSC8_YEAST & 375 & 222 & 4.95 \\
\hline QEVEGGDGAEPQVKK(14)-LENNGNSVKK(9) & sp|P43609|RSC8_YEAST (221)-sp|P43609|RSC8_YEAST (312)/ & 8 & RSC8_YEAST & RSC8_YEAST & 221 & 312 & 4.89 \\
\hline LENNGNSVKK(9)-WFTTNKK(6) & sp|P43609|RSC8_YEAST (312)-sp|Q06488|RSC2 YEAST (823)/ & 10 & RSC8_YEAST & RSC2 YEAST & 312 & 823 & 4.80 \\
\hline KINYEQEAQKLEEK(1)-QEVEGGDGAEPQVKK(14) & sp|P43609|RSC8_YEAST (55)-sp|P43609|RSC8_YEAST (221)/ & 6 & RSC8_YEAST & RSC8_YEAST & 55 & 221 & 4.80 \\
\hline KINYEQEAQKLEEK(1)-LAGDPIDKSAR(8) & sp|P43609|RSC8_YEAST (55)-sp|P32832|NPL6_YEAST (267)/ & 11 & RSC8_YEAST & NPL6_YEAST & 55 & 267 & 4.69 \\
\hline EVVGSTLNGKGGDSR(10)-KEFPVNLTIKK(1) & sp|P43609|RSC8 YEAST (375)-sp|P43609|RSC8 YEAST (222)/ & 32 & RSC8 YEAST & RSC8 YEAST & 375 & 222 & 4.69 \\
\hline QEVEGGDGAEPQVKK(14)-EVVGSTLNGKGGDSR(10) & sp|P43609|RSC8_YEAST (221)-sp|P43609|RSC8_YEAST (375)/ & 10 & RSC8_YEAS & RSC8_YEAST & 221 & 375 & 4.64 \\
\hline KEFPVNLTIKK(1)-LENNGNSVKK(9) & sp|P43609|RSC8 YEAST (222)-sp|P43609|RSC8 YEAST (312)/ & 18 & RSC8 YEAST & RSC8 YEAST & 222 & 312 & 4.49 \\
\hline KINYEQEAQK(1)-LAGDPIDKSAR(8) & sp|P43609|RSC8_YEAST (55)-sp|P32832|NPL6_YEAST (267)/ & 11 & RSC8_YEAST & NPL6_YEAST & 55 & 267 & 4.41 \\
\hline VGKEGEEVGEGDSIAKLQPQVYKPWSL(16)- & sp|P43609|RSC8_YEAST (546)-sp|Q12406|ARP7_YEAST (183)/ & 2 & RSC8_YEAST & ARP7_YEAST & 546 & 183 & 4.32 \\
\hline FLAKQTHPVIIPSFASWFDISK(4)-INYEQEAQKLEEKALR(13) & sp|P43609|RSC8_YEAST (75)-sp|P43609|RSC8_YEAST (68)/ & & RSC8_YEAST & RSC8_YEAST & & 68 & 4.32 \\
\hline DTR(3) & sp|P43609|RSC8_YEAST (99)-sp|P43609|RSC8_YEAST (119)/ & 50 & RSC8_YEAST & RSC8_YEAST & 99 & 119 & 4.24 \\
\hline KINYEQEAQKLEEK(1)-LIAKPETVQSEVK(4) & sp|P43609|RSC8_YEAST (55)-sp|Q02206|RSC4_YEAST (390)/ & 6 & RSC8_YEAST & RSC4_YEAST & 55 & 390 & 4.17 \\
\hline KLVLISEQVDSGIQLVEKDQEGDDEDGNTATGHGVKR(18)- & sp|P43609|RSC8_YEAST (511)-sp|P25632|RSC6_YEAST (231)/ & 5 & RSC8_YEAST & RSC6_YEAST & 511 & 231 & 4.14 \\
\hline $\begin{array}{l}\text { GVDGGKVGYSPNLAILGIMQ IGSVNDAVYSTYK( } \\
\text { DGSVSGSKMECVNDAVQTLLQGDDLGK)- }\end{array}$ & sp|P43609|RSC8 YEAST (388)-sp|P32597|STH1 YEAST (177)/ & 14 & RSC8 YEAST & STH1 YEAST & 388 & 177 & 4.03 \\
\hline
\end{tabular}




\begin{tabular}{|c|c|c|c|c|c|c|c|}
\hline PEPTIDE & PROTEINS & CSM & PROTEIN1 & PROTEIN2 & R1 & $\mathbf{R 2}$ & $\mathbf{s}$ \\
\hline \multicolumn{8}{|l|}{ SLGLESKFSNATK(7) } \\
\hline VGKEGEEVGEGDSIAK(3)-KAGASINSMK(1) & sp|P43609|RSC8_YEAST (533)-sp|Q12406|ARP7_YEAST (339)/ & 13 & RSC8_YEAST & ARP7_YEAST & 533 & 339 & 4.02 \\
\hline $\begin{array}{l}\text { DGSVSGSKLMECVNDAVQTLLQGDDKLGK(8)- } \\
\text { NDNTTAIEVDYSEKKPIK(14) }\end{array}$ & sp|P43609|RSC8_YEAST (388)-sp|P32597|STH1_YEAST (156)/ & 6 & RSC8_YEAST & STH1_YEAST & 388 & 156 & 3.99 \\
\hline $\begin{array}{l}\text { KLVLISEQVDSGIQLVEKDQEGDDEDGNTATGHGVK(1)- } \\
\text { LDSLLQKVLDTNAAHLPLMNVVQTVNK(7) }\end{array}$ & sp|P43609|RSC8_YEAST (494)-sp|P25632|RSC6_YEAST (321)/ & 2 & RSC8_YEAST & RSC6_YEAST & 494 & 321 & 3.99 \\
\hline EGEEVGEGDSIAKLQPQVYKPWSL(13)- & sp|P43609|RSC8_YEAST (546)-sp|Q12406|ARP7_YEAST (37)/ & 3 & RSC8_YEAST & ARP7_YEAST & 546 & 37 & 3.97 \\
\hline $\begin{array}{l}\text { LQPQVYKPWSL(7)-MTESVGGNK(1) } \\
\text { LAGOSG }\end{array}$ & sp|P43609|RSC8 YEAST (553)-sp|Q07979|RSC58 YEAST (1)/ & 4 & RSC8 YEAST & RSC58 YEAST & 553 & 1 & 3.95 \\
\hline EESATLGAEVAHKK(13)-LAGDPIDKSAR(8) & sp|P43609|RSC8 YEAST (54)-sp|P32832|NPL6 YEAST (267)/ & 7 & RSC8 YEAST & NPL6 YEAST & 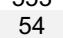 & 267 & 3.80 \\
\hline DQEGDDEDGNTATGHGVKR(18)-KAGASINSMK(1) & sp|P43609|RSC8_YEAST (529)-sp|Q12406|ARP7_YEAST (339)/ & 7 & RSC8_YEAST & ARP7_YEAST & 529 & 339 & 3.78 \\
\hline TPKAYKDTR(3)-IHEIEKR(6) & sp|P43609|RSC8_YEAST (116)-sp|P43609|RSC8_YEAST (99)/ & 27 & RSC8_YEAST & RSC8_YEAST & 116 & 99 & 3.61 \\
\hline $\begin{array}{l}\text { DGSVSGSKLMECVNDAVQTLLQGDDKLGK(8)- } \\
\text { EVVGSTLNGKGGDSR(10) }\end{array}$ & sp|P43609|RSC8_YEAST (388)-sp|P43609|RSC8_YEAST (375)/ & 12 & RSC8_YEAST & RSC8_YEAST & 388 & 375 & 3.56 \\
\hline INYEQEAQKLEEK(9)-EESATLGAEVAHKK(13) & sp|P43609|RSC8_YEAST (64)-sp|P43609|RSC8_YEAST (54)/ & 10 & RSC8_YEAST & RSC8_YEAST & 64 & 54 & 3.45 \\
\hline $\begin{array}{l}\text { KLVLISEQVDSGIQLVEKDQEGDDEDGNTATGHGVKR(1)- } \\
\text { LDSLLQKVLDTNAAHLPLMNVVQTVNK(7) }\end{array}$ & sp|P43609|RSC8_YEAST (494)-sp|P25632|RSC6_YEAST (321)/ & 2 & RSC8_YEAST & RSC6_YEAST & 494 & 321 & 3.42 \\
\hline INYEQEAQKLEEKALR(13)-EESATLGAEVAHKK(13) & sp|P43609|RSC8_YEAST (68)-sp|P43609|RSC8_YEAST (54)/ & 2 & RSC8_YEAST & RSC8_YEAST & 68 & 54 & 3.39 \\
\hline $\begin{array}{l}\text { KLVLISEQVDSGIQLVEKDQEGDDEDGNTATGHGVKR(1)- } \\
\text { QILDLNKSLEELNVSK(7) }\end{array}$ & sp|P43609|RSC8_YEAST (494)-sp|P43609|RSC8_YEAST (484)/ & 5 & RSC8_YEAST & RSC8_YEAST & 494 & 484 & 3.36 \\
\hline FLAKQTHPVIIPSFASWFDISK(4)-KINYEQEAQKLEEKALR(14) & sp|P43609|RSC8_YEAST (75)-sp|P43609|RSC8_YEAST (68)/ & 8 & RSC8_YEAST & RSC8_YEAST & 75 & 68 & 3.36 \\
\hline YIEESQAIIQELVKLTMEK(14)-KLIEMDSR(1) & sp|P43609|RSC8_YEAST (434)-sp|P25632|RSC6_YEAST (35)/ & 7 & RSC8 YEAST & RSC6_YEAST & 434 & 35 & 3.34 \\
\hline KEFPVNLTIKK(1)-IPGLINPWKR(9) & sp|P43609|RSC8_YEAST (222)-sp|Q06488|RSC2_YEAST (900)/ & 2 & RSC8_YEAST & RSC2_YEAST & 222 & 900 & 3.31 \\
\hline KLVLISEQVDSGIQLVEKDQEGDDEDGNTATGHGVKR(36)- & sp|P43609|RSC8_YEAST (529)-sp|Q12406|ARP7_YEAST (339)/ & 7 & RSC8_YEAST & ARP7_YEAST & 529 & 339 & 3.29 \\
\hline 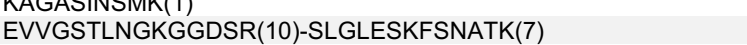 & sp|P43609|RSC8_YEAST (375)-sp|P32597|STH1_YEAST (177)/ & 31 & RSC8_YEAST & STH1_YEAST & 375 & 177 & 3.27 \\
\hline EVVGSTLNGKGGDSR(10)-AKSLGLESK(2) & sp|P43609|RSC8_YEAST (375)-sp|P325 & 12 & RSC8_YEA & STH1_YE & & 170 & 3.26 \\
\hline DGSVSGSKLMECVNDAVQTLLQGDDKLGK(8)-KPIKISADFNAK(4) & $\begin{array}{l}\text { sp|P43609|RSC8_YEAST (388)-sp|P32597|STH1_YEAST (160)/ } \\
\text { s. }\end{array}$ & 2 & RSC8_YEAST & STH1_YEAST & 388 & 160 & 3.13 \\
\hline VEDCIEKFLSLPIEDNYIR(7)-QEVEGGDGAEPQVKK(14) & sp|P43609|RSC8_YEAST (353)-sp|P43609|RSC8_YEAST (221)/ & 1 & RSC8_YEAST & RSC8_YEAST & 353 & 221 & 3.07 \\
\hline RVEDCIEKFLSLPIEDNYIR(8)-KEFPVNLTIKK(1) & sp|P43609|RSC8_YEAST (353)-sp|P43609|RSC8_YEAST (222)/ & 12 & RSC8_YEAST & RSC8_YEAST & 353 & 222 & 3.00 \\
\hline LVLISEQVDSGIQLVEKDQEGDDEDGNTATGHGVKR(35)- & sp|P43609|RSC8_YEAST (529)-sp|P43609|RSC8_YEAST (533)/ & 26 & RSC8_YEAST & RSC8_YEAST & 529 & 533 & 2.98 \\
\hline $\begin{array}{l}\text { FTKLCDLETQLEMEK(3)-LCDLETQLEMEKLK(12) } \\
\text { FTLELE }\end{array}$ & sp|P43609|RSC8 YEAST (446)-sp|P43609|RSC8 YEAST (458)/ & 2 & RSC8 YEAST & RSC8 YEAST & 446 & 458 & 2.93 \\
\hline KINYEQEAQK(1)-LEEKALR(4) & sp|P43609|RSC8_YEAST (55)-sp|P4360 & 3 & RSC8_YEAST & YEAST & 55 & 68 & 2.92 \\
\hline KINYEQEAQKLEEK(1)-ADAKAQGLR(4) & sp|P43609|RSC8_YEAST (55)-sp|P32597|STH1_YEAST (1011)/ & 1 & RSC8_YEAST & STH1_YEAST & 55 & 1011 & 2.90 \\
\hline VGKEGEEVGEGDSIAK(3)-KQSQATNATN(1) & sp|P43609|RSC8_YEAST (533)-sp|Q12406|ARP7_YEAST (468)/ & 16 & RSC8_YEAST & ARP7_YEAST & 533 & 468 & 2.88 \\
\hline DGSVSGSKLMECVNDAVQTLLQGDDKLGK(8)-AKSLGLESK(2) & sp|P43609|RSC8_YEAST (388)-sp|P32597|STH1_YEAST (170)/ & 5 & RSC8_YEAST & STH1_YEAST & 388 & 170 & 2.82 \\
\hline $\begin{array}{l}\text { KNWSDQEMLLLLEGIEMYEDQWEKIADHVGGHK(24)- } \\
\text { TFSVGGNK(1) }\end{array}$ & sp|P43609|RSC8_YEAST (336)-sp|Q07979|RSC58_YEAST (2)/ & 2 & RSC8_YEAST & RSC58_YEAST & 336 & 2 & 2.72 \\
\hline RVGKEGEEVGEGDSIAK(4)-KQSQATNATN(1) & sp|P43609|RSC8_YEAST (533)-sp|Q12406|ARP7_YEAST (468)/ & 16 & RSC8_YEAST & ARP7_YEAST & 533 & 468 & 2.72 \\
\hline KINYEQEAQKLEEK(1)-AYKDTR(3) & sp|P43609|RSC8_YEAST (55)-sp|P436C & 7 & RSC8_YEA & RSC8_YEAST & 55 & 119 & 2.72 \\
\hline EISEKYIEESQAIIQELVK(5)-QRMSLEEMKK(9) & sp|P43609|RSC8_YEAST (420)-sp|Q9URQ5|HTL1_YEAST (66)/ & 13 & RSC8 YEAST & HTL1_YEAST & 420 & 66 & 2.71 \\
\hline KINYEQEAQKLEEK(1)-IHEIEKR(6) & sp|P43609|RSC8 YEAST (55)-sp|P43609|RSC8 YEAST (99)/ & 2 & RSC8 YEAST & RSC8 YEAST & 55 & 99 & 2.61 \\
\hline EESATLGAEVAHKK(13)-IHEIEKR(6) & sp|P43609|RSC8_YEAST (54)-sp|P43609|RSC8_YEAST (99)/ & 5 & RSC8_YEAST & RSC8_YEAST & 54 & 99 & 2.60 \\
\hline $\begin{array}{l}\text { VGKEGEEVGEGDSIAKLQPPVYKPWSL(16)- } \\
\text { TVAGFSNVELPQCIIPSSYIKR(21) }\end{array}$ & sp|P43609|RSC8_YEAST (546)-sp|Q12406|ARP7_YEAST (37)/ & 3 & RSC8_YEAST & ARP7_YEAST & 546 & 37 & 2.58 \\
\hline LENNGNSVKK(9)-WFTTNKKR(7) & sp|P43609|RSC8_YEAST (312)-sp|Q06488|RSC2_YEAST (824)/ & 1 & RSC8_YEAST & RSC2_YEAST & 312 & 824 & 2.57 \\
\hline $\begin{array}{l}\text { NWSDQEMLLLLEGIEMYEDQWEKIADHVGGHKR(23)- } \\
\text { LQQQQHQYQQQKR(12) }\end{array}$ & sp|P43609|RSC8_YEAST (336)-sp|Q06488|RSC2_YEAST (713)/ & 3 & RSC8_YEAST & RSC2_YEAST & 336 & 713 & 2.54 \\
\hline YIEESQAIIQELVKLTMEK(14)-MLDALKNER(6) & sp|P43609|RSC8_YEAST (434)-sp|Q9URQ5|HTL1_YEAST (73)/ & 15 & RSC8_YEAST & HTL1_YEAST & 434 & 73 & 2.54 \\
\hline
\end{tabular}




\begin{tabular}{|c|c|c|c|c|c|c|c|}
\hline PEPTIDE & PROTEINS & CSM & PROTEIN1 & PROTEIN2 & R1 & $\mathbf{R 2}$ & $\mathbf{s}$ \\
\hline IHEIEKR(6)-TPKAYK(3) & 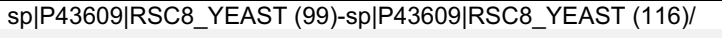 & 7 & RSC8_YEAST & RSC8_YEAST & 99 & 116 & 2.51 \\
\hline $\begin{array}{l}\text { KLVLISEQVDSGIQLVEKDQEGDDEDGNTATGHGVK(18)- } \\
\text { GVDGGKVOYSPNLATLIGMOTGSVNDAVYSIYK(6) }\end{array}$ & sp|P43609|RSC8_YEAST (511)-sp|P25632|RSC6_YEAST (231)/ & 5 & RSC8_YEAST & RSC6_YEAST & 511 & 231 & 2.49 \\
\hline RVGKEGEEVGEGDSIAK(4)-KAGASINSMK(1) & sp|P43609|RSC8_YEAST (533)-sp|Q12406|ARP7_YEAST (339)/ & 13 & RSC8_YEAST & ARP7_YEAST & 533 & 339 & 2.45 \\
\hline QILDLNKSLEELNVSK(7)-SLEELNVSKK(9) & sp|P43609|RSC8_YEAST (484)-sp|P43609|RSC8_YEAST (493)/ & 4 & RSC8_YEAST & RSC8_YEAST & 484 & 493 & 2.45 \\
\hline LSLSKQILDLNK(5)-SLEELNVSKK(9) & sp|P43609|RSC8_YEAST (477)-sp|P43609|RSC8_YEAST (493)/ & 10 & RSC8_YEAST & RSC8_YEAST & 477 & 493 & 2.42 \\
\hline $\begin{array}{l}\text { LMECVNDAVQTLLQGDDKLGKVSDK(21)- } \\
\text { EISEKYIEESQAIIQELVK(5) }\end{array}$ & sp|P43609|RSC8_YEAST (409)-sp|P43609|RSC8_YEAST (420)/ & 1 & RSC8_YEAST & RSC8_YEAST & 409 & 420 & 2.41 \\
\hline KINYEQEAQKLEEK(1)-GNVIEGKLAGDPIDK(7) & sp|P43609|RSC8_YEAST (55)-sp|P32832|NPL6_YEAST (259)/ & 1 & RSC8_YEAST & NPL6_YEAST & 55 & 259 & 2.36 \\
\hline $\begin{array}{l}\text { KLVLISEQVDSGIQLVEKDQEGDDEDGNTATGHGVKR(18)- } \\
\text { KAGASINSMK(1) }\end{array}$ & sp|P43609|RSC8_YEAST (511)-sp|Q12406|ARP7_YEAST (339)/ & 2 & RSC8_YEAST & ARP7_YEAST & 511 & 339 & 2.36 \\
\hline $\begin{array}{l}\text { LMECVNDAVQTLLQGDDKLGK(18)-NDNTTAIEVDYSEKKPIK(14) } \\
\text { INYEQEAOKLEEK(9)-KLIAKPETVQSEVK(1) }\end{array}$ & sp|P43609|RSC8_YEAST (406)-sp|P32597|STH1_YEAST (156)/ & 4 & RSC8_YEAST & STH1_YEAST & 406 & 156 & 2.35 \\
\hline $\begin{array}{l}\text { INYEQEAQKLEEK(9)-KLIAKPETVQSEVK(1) } \\
\text { DQEGDDEDGNTATGHGVKR(18)-VGKEGEEVGEGDSIAK(3) }\end{array}$ & sp|P43609|RSC8_YEAST (64)-sp|Q02206|RSC4_YEAST (386)/ & $\begin{array}{c}3 \\
26\end{array}$ & RSC8_YEAST & RSC4_YEAST & $\begin{array}{c}64 \\
529\end{array}$ & $\begin{array}{l}386 \\
533\end{array}$ & $\begin{array}{l}2.34 \\
2.31\end{array}$ \\
\hline $\begin{array}{l}\text { DQEGDDEDGNTATGHGVKR(18)-VGKEGEEVGEGDSIAK(3) } \\
\text { DGSVSGSKLMECVNDAVQTLLQGDDK(8)- }\end{array}$ & sp|P43609|RSC8_YEAST (529)-sp|P43609|RSC8_YEAST (533)/ & 26 & RSC8_YEAST & C8_YEAST & 529 & 533 & 2.31 \\
\hline NDNTTAIEVDYSEKKPIK(14) & sp|P43609|RSC8_YEAST (388)-sp|P32597|STH1_YEAST (156)/ & 6 & RSC8_YEAST & STH1_YEAST & 388 & 156 & 2.27 \\
\hline $\begin{array}{l}\text { DVPMVDSHEATEEPPTTSTNTPSFPHLAQEQAKEESATLGAEVAH } \\
\text { K(33)-TPKAYK(3) }\end{array}$ & sp|P43609|RSC8_YEAST (41)-sp|P43609|RSC8_YEAST (116)/ & 2 & RSC8_YEAST & RSC8_YEAST & 41 & 116 & 2.18 \\
\hline INYEQEAQKLEEK(9)-AYKDTR(3) & sp|P43609|RSC8_YEAST (64)-sp|P43609|RSC8_YEAST (119)/ & 2 & RSC8_YEAST & RSC8_YEAST & 64 & 119 & 2.17 \\
\hline LENNGNSVKK(9)-WFTTNKKR(6) & sp|P43609|RSC8_YEAST (312)-sp|Q06488|RSC2_YEAST (823)/ & 10 & RSC8_YEAST & RSC2_YEAST & 312 & 823 & 2.12 \\
\hline QTHPVIIPSFASWFDISKIHEIEK(18)-AYKDTR(3) & sp|P43609|RSC8_YEAST (93)-sp|P43609|RSC8_YEAST (119)/ & 21 & RSC8_YEAST & RSC8_YEAST & 93 & 119 & 2.07 \\
\hline DGSVSGSKLMECVNDAVQTLLQGDDK(8)-SLGLESKFSNATK(7) & sp|P43609|RSC8_YEAST (388)-sp|P32597|STH1_YEAST (177)/ & 14 & RSC8_YEAST & STH1_YEAST & 388 & 177 & 2.06 \\
\hline $\begin{array}{l}\text { VGKEGEEVGEGDSIAK(3)-TKDHPPNKEMLR(8) } \\
\text { ESEKMLNDR(4)-LKYVK(2) }\end{array}$ & sp|P43609|RSC8_YEAST (533)-sp|P25632|RSC6_YEAST (71)i & $\begin{array}{c}13 \\
5\end{array}$ & RSC8_YEAST & RSC6_YEAST & 533 & 71 & 2.06 \\
\hline ESEKMLNDR(4)-LKYVK(2) & sp|P43609|RSC8_YEAST (467)-sp|P43609|RSC8_YEAST (460)/ & 5 & RSC8_YEAST & RSC8_YEAST & 467 & 460 & 2.04 \\
\hline $\begin{array}{l}\text { TKPSLIGPSFTGHFQVVLDTPQGLKPFLPENVIKQEVEGGDGAEPQ } \\
\text { VK(25)-SKNDSPSDTSIVYETVIHDNDLNKPK(2) }\end{array}$ & sp|P43609|RSC8_YEAST (198)-sp|P32832|NPL6_YEAST (375)/ & 12 & RSC8_YEAST & NPL6_YEAST & 198 & 375 & 2.01 \\
\hline LQPQVYKPWSL(7)-FKALGIADGER(2) & sp|P43609|RSC8_YEAST (553)-sp|Q03124|RSC9_YEAST (477)/ & 4 & RSC8_YEAST & RSC9_YEAST & 553 & 477 & 2.00 \\
\hline $\begin{array}{l}\text { KNWSDQEMLLLLEGIEMYEDQWEKIADHVGGHKR(24)- } \\
\text { KNFIAVSAANR(1) }\end{array}$ & sp|P43609|RSC8_YEAST (336)-sp|Q06488|RSC2_YEAST (905)/ & 4 & RSC8_YEAST & RSC2_YEAST & 336 & 905 & 1.94 \\
\hline $\begin{array}{l}\text { LSLSKQILDLNK(5)-TKDHPPNK(2) } \\
\text { VGKEGEEVGEGDSIAK(3)-DRKQSQATNATN(3) }\end{array}$ & $\begin{array}{l}\text { sp|P43609|RSC8_YEAST (477)-sp|P25632|RSC6_YEAST (65)/ } \\
\text { sp|P43609|RSC8_YEAST (533)-sp|Q12406|ARP7_YEAST (468)/ }\end{array}$ & $\begin{array}{l}13 \\
16\end{array}$ & $\begin{array}{l}\text { RSC8_YEAST } \\
\text { RSC8_YEAST }\end{array}$ & $\begin{array}{l}\text { RSC6_YEAST } \\
\text { ARP7_YEAST }\end{array}$ & $\begin{array}{l}477 \\
533\end{array}$ & $\begin{array}{c}65 \\
468\end{array}$ & $\begin{array}{l}1.93 \\
1.92\end{array}$ \\
\hline $\begin{array}{l}\text { DGSVSGSKLMECVNDAVQTLLQGDDK(8)- } \\
\text { EVVGSTLNGKGGDSR(10) }\end{array}$ & sp|P43609|RSC8_YEAST (388)-sp|P43609|RSC8_YEAST (375)/ & 12 & RSC8_YEAST & RSC8_YEAST & 388 & 375 & 1.91 \\
\hline FLAKQTHPVIIPSFASWFDISK(4)-LAGDPIDKSAR(8) & sp|P43609|RSC8_YEAST (75)-sp|P32832|NPL6_YEAST (267)/ & 1 & RSC8_YEAST & NPL6_YEAST & 75 & 267 & 1.80 \\
\hline KINYEQEAQKLEEK(1)-EFGAKIIR(5) & sp|P43609|RSC8_YEAST (55)-sp|P32832|NPL6_YEAST (232)/ & 3 & RSC8_YEAST & YEAST & 55 & 232 & 1.75 \\
\hline VEDCIEKFLSLPIEDNYIR(7)-LENNGNSVKK(9) & sp|P43609|RSC8_YEAST (353)-sp|P43609|RSC8_YEAST (312)/ & 2 & RSC8_YEAST & RSC8_YEAST & 353 & 312 & 1.68 \\
\hline LMECVNDAVQTLLQGDDKLGK( & sp|P43609|RSC8_YEAST (406)-sp|Q03124|RSC9_YEAST (143)/ & 1 & RSC8_YEAST & RSC9_YEAST & 406 & 143 & 1.68 \\
\hline EESATLGAEVAHKK(13)-LIAKPETVQSEVK(4) & sp|P43609|RSC8_YEAST (54)-sp|Q02206|RSC4_Y̌EAST (390)/ & 3 & RSC8_YEAST & RSC4_YEAST & 54 & 390 & 1.66 \\
\hline $\begin{array}{l}\text { KNWSDQEMLLLLEGIEMYEDQWEKIADHVGGHKR(24)- } \\
\text { LQQQQHQYQQQKR(12) }\end{array}$ & sp|P43609|RSC8_YEAST (336)-sp|Q06488|RSC2_YEAST (713)/ & 3 & RSC8_YEAST & RSC2_YEAST & 336 & 713 & 1.65 \\
\hline EGEEVGEGDSIAKLQPQVYKPWSL(13)-SKFGGDFLDFQVHER(2) & sp|P43609|RSC8_YEAST (546)-sp|Q12406|ARP7_YEAST (183)/ & 2 & RSC8_YEAST & ARP7_YEAST & 546 & 183 & 1.62 \\
\hline $\begin{array}{l}\text { LVLISEQVDSGIQLVEKDQEGDDEDGNTATGHGVKR(35)- } \\
\text { GVDGGKVQYSPNLATLIGMQTGSVNDAVYSIYK(6) }\end{array}$ & sp|P43609|RSC8_YEAST (529)-sp|P25632|RSC6_YEAST (231)/ & 2 & RSC8_YEAST & RSC6_YEAST & 529 & 231 & 1.60 \\
\hline $\begin{array}{l}\text { KLVLISEQVDSGIQLVEKDQEGDDEDGNTATGHGVKR(1)- } \\
\text { GVDGGKVQYSPNLATLIGMQTGSVNDAVYSIYK(6) }\end{array}$ & sp|P43609|RSC8_YEAST (494)-sp|P25632|RSC6_YEAST (231)/ & 1 & RSC8_YEAST & RSC6_YEAST & 494 & 231 & 1.60 \\
\hline $\begin{array}{l}\text { KLVLISEQVDSGIQLVEKDQEGDDEDGNTATGHGVKR(18)- } \\
\text { VGKEGEEVGEGDSIAKLQPQVYKPWSL(16) }\end{array}$ & sp|P43609|RSC8_YEAST (511)-sp|P43609|RSC8_YEAST (546)/ & 1 & RSC8_YEAST & RSC8_YEAST & 511 & 546 & 1.56 \\
\hline FKTPKAYK(5)-IHEIEKR(6) & sp|P43609|RSC8_YEAST (116)-sp|P43609|RSC8_YEAST (99)/ & 27 & RSC8_YEAST & RSC8_YEAST & 116 & 99 & 1.55 \\
\hline $\begin{array}{l}\text { NWSDQEMLLLLEGIEMYEDQWEKIADHVGGHKR(23)- } \\
\text { VGSPGAGGPLTVQGLKQHQLQR(16) }\end{array}$ & sp|P43609|RSC8_YEAST (336)-sp|Q06488|RSC2_YEAST (695)/ & 2 & RSC8_YEAST & RSC2_YEAST & 336 & 695 & 1.54 \\
\hline
\end{tabular}




\begin{tabular}{|c|c|c|c|c|c|c|c|}
\hline PEPTIDE & PROTEINS & CSM & PROTEIN1 & PROTEIN2 & R1 & $\mathbf{R 2}$ & $\mathbf{s}$ \\
\hline $\begin{array}{l}\text { KNVYDSAQDFNALQDESR(1)-EPVKSR(4) } \\
\text { QTHPVIIPSFASWFDISKIHEIEKR(18)-AYKDTR(3) }\end{array}$ & $\begin{array}{l}\text { sp|P43609|RSC8_YEAST (232)-sp|Q02206|RSC4_YEAST (534)/ } \\
\text { sp|P43609|RSC8_YEAST (93)-sp|P43609|RSC8_YEAST (119)/ }\end{array}$ & $\begin{array}{c}1 \\
21\end{array}$ & $\begin{array}{l}\text { RSC8_YEAST } \\
\text { RSC8_YEAST }\end{array}$ & $\begin{array}{l}\text { RSC4_YEAST } \\
\text { RSC8_YEAST }\end{array}$ & $\begin{array}{l}232 \\
93\end{array}$ & $\begin{array}{l}534 \\
119\end{array}$ & $\begin{array}{l}1.52 \\
1.52\end{array}$ \\
\hline $\begin{array}{l}\text { TKPSLIGPSFTGHFQVVLDTPQGLKPFLPENVIKQEVEGGDGAEPQ } \\
\text { VK(25)-KINYEQEAQKLEEK(1) }\end{array}$ & sp|P43609|RSC8_YEAST (198)-sp|P43609|RSC8_YEAST (55)/ & 1 & RSC8_YEAST & RSC8_YEAST & 198 & 55 & 1.50 \\
\hline $\begin{array}{l}\text { TKPSLIGPSFTGHFQVVLDTPQGLKPFLPENVIKQEVEGGDGAEPQ } \\
\text { VK(34)-KINYEQEAQK(1) }\end{array}$ & sp|P43609|RSC8_YEAST (207)-sp|P43609|RSC8_YEAST (55)/ & 2 & RSC8_YEAST & RSC8_YEAST & 207 & 55 & 1.50 \\
\hline QTHPVIIPSFASWFDISKIHEIEKR(18)-AITEQQNFEKCNEYI(10) & sp|P43609|RSC8_YEAST (93)-sp|P32832|NPL6_YEAST (430)/ & 3 & RSC8_YEAST & NPL6_YEAST & 93 & 430 & 1.50 \\
\hline $\begin{array}{l}\text { LVLISEQVDSGIQLVEKDQEGDDEDGNTATGHGVKR(35)- } \\
\text { KAGASINSMK(1) }\end{array}$ & sp|P43609|RSC8_YEAST (529)-sp|Q12406|ARP7_YEAST (339)/ & 7 & RSC8_YEAST & ARP7_YEAST & 529 & 339 & 1.46 \\
\hline $\begin{array}{l}\text { EVVGSTLNGKGGDSR(10)-EVVGSTLNGKGGDSR(10) } \\
\text { FKTPKAYK(2)-JHFIFKR(1) }\end{array}$ & sp|P43609|RSC8_YEAST (375)-sp|P43609|RSC8_YEAST (375)/ & $\begin{array}{l}6 \\
5\end{array}$ & RSC8_YEAST & $\begin{array}{l}\text { RSC8_YEAST } \\
\text { RSC8_YFAST }\end{array}$ & 375 & 375 & $\begin{array}{l}1.45 \\
1.39\end{array}$ \\
\hline $\begin{array}{l}\text { FKTPKAYK(2)-IHEIEKR(6) } \\
\text { INYEQEAQKLEEKALR(9)-EESATLGAEVAHKK(13) }\end{array}$ & $\begin{array}{l}\text { sp|P43609|RSC8_YEAST (113)-sp|P43609|RSC8 _YEAST (99)/ } \\
\text { sp|P43609|RSC8_YEAST (64)-sp|P43609|RSC8_YEAST (54)/ }\end{array}$ & $\begin{array}{c}5 \\
10\end{array}$ & $\begin{array}{l}\text { RSC8_YEAST } \\
\text { RSC8 YEAST }\end{array}$ & $\begin{array}{l}\text { RSC8_YEAST } \\
\text { RSC8 YEAST }\end{array}$ & $\begin{array}{l}113 \\
64\end{array}$ & $\begin{array}{l}99 \\
54\end{array}$ & $\begin{array}{l}1.39 \\
1.39\end{array}$ \\
\hline FKTPKAYKDTR(2)-IHEIEKR(6) & sp|P43609|RSC8_YEAST (113)-sp|P43609|RSC年_YEAST (99)/ & 5 & RSC8_YEAST & RSC8_YEAST & 113 & 99 & 1.38 \\
\hline $\begin{array}{l}\text { LVLISEQVDSGIQLVEKDQEGDDEDGNTATGHGVKR(17)- } \\
\text { GVDGGKVQYSPNLATLIGMQTGSVNDAVYSIYK(6) }\end{array}$ & sp|P43609|RSC8_YEAST (511)-sp|P25632|RSC6_YEAST (231)/ & 5 & RSC8_YEAST & RSC6_YEAST & 511 & 231 & 1.37 \\
\hline $\begin{array}{l}\text { IHAFLEKWGLINYQIDPR(7)-TPKAYKDTR(3) } \\
\text { KNVYDSAQDFNALQDESR(1)-AKTRSSR(2) }\end{array}$ & $\begin{array}{l}\text { sp|P43609|RSC8_YEAST (162)-sp|P43609|RSC8_YEAST (116)/ } \\
\text { sp|P43609|RSC8_YEAST (232)-tr|Q6AZJ8|H2A_XENLA (15)/ }\end{array}$ & $\begin{array}{l}1 \\
2\end{array}$ & $\begin{array}{l}\text { RSC8_YEAST } \\
\text { RSC8_YEAST }\end{array}$ & $\begin{array}{l}\text { RSC8_YEAST } \\
\text { H2A_XENLA }\end{array}$ & $\begin{array}{l}162 \\
232\end{array}$ & $\begin{array}{c}116 \\
15\end{array}$ & $\begin{array}{l}1.31 \\
1.28\end{array}$ \\
\hline $\begin{array}{l}\text { TKPSLIGPSFTGHFQVVLDTPQGLKPFLPENVIKQEVEGGDGAEPQ } \\
\text { VK(34)-KEFPVNLTIKK(1) }\end{array}$ & sp|P43609|RSC8_YEAST (207)-sp|P43609|RSC8_YEAST (222)/ & 2 & RSC8_YEAST & RSC8_YEAST & 207 & 222 & 1.27 \\
\hline $\begin{array}{l}\text { LMECVNDAVQTLLQGDDKLGKVSDK(18)- } \\
\text { NDNTTAIEVDYSEKKPIK(14) }\end{array}$ & sp|P43609|RSC8_YEAST (406)-sp|P32597|STH1_YEAST (156)/ & 4 & RSC8_YEAST & STH1_YEAST & 406 & 156 & 1.24 \\
\hline $\begin{array}{l}\text { TKPSLIGPSFTGHFQVVLDTPQGLKPFLPENVIK(25)- } \\
\text { MEMALLSGIPEEIKWSLKK(14) }\end{array}$ & sp|P43609|RSC8_YEAST (198)-sp|Q03124|RSC9_YEAST (98)/ & 2 & RSC8_YEAST & RSC9_YEAST & 198 & 98 & 1.21 \\
\hline $\begin{array}{l}\text { NWSDQEMLLLLEGIEMYEDQWEKIADHVGGHKR(23)- } \\
\text { KNFIAVSAANR(1) }\end{array}$ & sp|P43609|RSC8_YEAST (336)-sp|Q06488|RSC2_YEAST (905)/ & 4 & RSC8_YEAST & RSC2_YEAST & 336 & 905 & 1.18 \\
\hline LMECVNDAVQTLLQGDDKLGK(18)-LGKVSDK(3) & sp|P43609|RSC8_YEAST (406)-sp|P43609|RSC8_YEAST (409)/ & 1 & RSC8_YEAST & RSC8_YEAST & 406 & 409 & 1.14 \\
\hline FKTPKAYKDTR(5)-IHEIEKR(6) & sp|P43609|RSC8_YEAST (116)-sp|P43609|RSC8_YEAST (99)/ & 27 & RSC8_YEAST & RSC8_YEAST & 116 & 99 & 1.09 \\
\hline $\begin{array}{l}\text { TKPSLIGPSFTGHFQVVLDTPQGLKPFLPENVIKQEVEGGDGAEPQ } \\
\text { VK(25)-SSICDSKAMDSLQMGLNALLILR(7) }\end{array}$ & sp|P43609|RSC8_YEAST (198)-sp|Q03124|RSC9_YEAST (150)/ & 1 & RSC8_YEAST & RSC9_YEAST & 198 & 150 & 1.07 \\
\hline $\begin{array}{l}\text { KLVLISEQVDSGIQLVEKDQEGDDEDGNTATGHGVKR(18)- } \\
\text { VSPEELPLVITMPATNGKPDMAILER(18) }\end{array}$ & sp|P43609|RSC8_YEAST (511)-sp|Q12406|ARP7_YEAST (114)/ & 1 & RSC8_YEAST & ARP7_YEAST & 511 & 114 & 1.05 \\
\hline $\begin{array}{l}\text { YIEESQAAIIQELVKLTMEKLESK(14)-MLDALKNER(6) } \\
\text { INYEQEAQKLEEKALR(13)-EFGAKIIR(5) }\end{array}$ & $\begin{array}{l}\text { sp|P43609|RSC8_YEAST (434)-sp|Q9URQ5|HTL1_YEAST (73)/ } \\
\text { sp|P43609|RSC8_YEAST (68)-sp|P32832|NPL6_YEAST (232)/ }\end{array}$ & $\begin{array}{c}15 \\
1\end{array}$ & $\begin{array}{l}\text { RSC8_YEAST } \\
\text { RSC8_YEAST }\end{array}$ & $\begin{array}{l}\text { HTL1_YEAST } \\
\text { NPL6_YEAST }\end{array}$ & $\begin{array}{l}434 \\
68\end{array}$ & $\begin{array}{c}73 \\
232\end{array}$ & $\begin{array}{l}1.00 \\
0.99\end{array}$ \\
\hline $\begin{array}{l}\text { TKPSLIGPSFTGHFQVVLDTPQGLKPFLPENVIK(25)- } \\
\text { MEMALLSGIPEEIKWSLKK(18) }\end{array}$ & sp|P43609|RSC8_YEAST (198)-sp|Q03124|RSC9_YEAST (102)/ & 1 & RSC8_YEAST & RSC9_YEAST & 198 & 102 & 0.98 \\
\hline KNVYDSAQDFNALQDESR(1)-RIPGLINPWKR(10) & sp|P43609|RSC8_YEAST (232)-sp|Q06488|RSC2_YEAST (900)/ & 1 & RSC8_YEAST & RSC2_YEAST & 232 & 900 & 0.93 \\
\hline $\begin{array}{l}\text { MSDTEKDKDVPMVDSHEATEEPPTTSTNTPSFPHLAQEQAK(1)- } \\
\text { KLSEDGVTDGDGK(1) }\end{array}$ & sp|P43609|RSC8_YEAST (1)-sp|Q06639|RSC3_YEAST (234)/ & 1 & RSC8_YEAST & RSC3_YEAST & 1 & 234 & 0.92 \\
\hline $\begin{array}{l}\text { KLVLISEQVDSGIQLVEKDQEGDDEDGNTATGHGVK(1)- } \\
\text { TKDHPPNKEMLR(8) }\end{array}$ & sp|P43609|RSC8_YEAST (494)-sp|P25632|RSC6_YEAST (71)/ & 2 & RSC8_YEAST & RSC6_YEAST & 494 & 71 & 0.82 \\
\hline EISEKYIEESQAIIQELVK(5)-YLPGKHPK(5) & sp|P43609|RSC8_YEAST (420)-sp|Q02206|RSC4_YEAST (25)/ & 2 & RSC8_YEAST & RSC4_YEAST & 420 & 25 & 0.80 \\
\hline $\begin{array}{l}\text { LVLISEQVDSGIQLVEKDQEGDDEDGNTATGHGVK(17)- } \\
\text { RVGKEGEEVGEGDSIAK(4) }\end{array}$ & sp|P43609|RSC8_YEAST (511)-sp|P43609|RSC8_YEAST (533)/ & 5 & RSC8_YEAST & RSC8_YEAST & 511 & 533 & 1.01 \\
\hline $\begin{array}{l}\text { KLVLISEQVDSGIQLVEKDQEGDDEDGNTATGHGVKR(36)- } \\
\text { KQSQATNATN(1) }\end{array}$ & sp|P43609|RSC8_YEAST (529)-sp|Q12406|ARP7_YEAST (468)/ & 2 & RSC8_YEAST & ARP7_YEAST & 529 & 468 & 0.77 \\
\hline $\begin{array}{l}\text { TKPSLIGPSFTGHFQVVLDTPQGLKPFLPENVIKQEVEGGDGAEPQ } \\
\text { VK(25)-RSKNDSPSDTSIVYETVIHDNDLNKPK(3) }\end{array}$ & sp|P43609|RSC8_YEAST (198)-sp|P32832|NPL6_YEAST (375)/ & 12 & RSC8_YEAST & NPL6_YEAST & 198 & 375 & 0.77 \\
\hline YVKESEKMLNDR(7)-TKDHPPNKEMLR(8) & 8_YEAST (467)-sp|P25632 & 1 & RS & RS & 467 & 71 & 0.75 \\
\hline $\begin{array}{l}\text { KNVYDSAQDFNALQDESR(1)-VLKPTTEER(3) } \\
\text { RVGKEGEEVGEGDSIAK(4)-TKDHPPNKEMLR(8) }\end{array}$ & $\begin{array}{l}\text { sp|P43609|RSC8_YEAST (232)-sp|Q07979|RSC58_YEAST (440)/ } \\
\text { sp|P43609|RSC8_YEAST (533)-sp|P25632|RSC6_YEAST (71)/ }\end{array}$ & $\begin{array}{c}4 \\
13\end{array}$ & $\begin{array}{l}\text { RSC8_YEAST } \\
\text { RSC8YYEAST }\end{array}$ & $\begin{array}{l}\text { RSC } 5 \overline{8} \text { YYAAST } \\
\text { RSC6 YEAST }\end{array}$ & $\begin{array}{l}232 \\
533\end{array}$ & $\begin{array}{c}440 \\
71\end{array}$ & $\begin{array}{l}0.73 \\
0.73\end{array}$ \\
\hline
\end{tabular}




\begin{tabular}{|c|c|c|c|c|c|c|c|}
\hline PEPTIDE & PROTEINS & CSM & PROTEIN1 & PROTEIN2 & R1 & $\mathbf{R 2}$ & $\mathbf{s}$ \\
\hline RVGKEGEEVGEGDSIAK(4)-DRKQSQATNATN(3) & sp|P43609|RSC8_YEAST (533)-sp|Q12406|ARP7_YEAST (468)/ & 16 & RSC8_YEAST & ARP7_YEAST & 533 & 468 & 0.72 \\
\hline $\begin{array}{l}\text { KLVLISEQVDSGIQLVEKDQEGDDEDGNTATGHGVKR(18)- } \\
\text { TKDHPPNKEMLR(8) }\end{array}$ & sp|P43609|RSC8_YEAST (511)-sp|P25632|RSC6_YEAST (71)/ & 3 & RSC8_YEAST & RSC6_YEAST & 511 & 71 & 0.69 \\
\hline VGKEGEEVGEGDSIAK(3)-DHPPNKEMLR(6) & sp|P43609|RSC8_YEAST (533)-sp|P25632|RSC6_YEAST (71)/ & 13 & $\begin{array}{l}\text { RSC8_YEAST } \\
\text { RSC8 YEAST }\end{array}$ & $\begin{array}{l}\text { RSC6_YEAST } \\
\text { STH1YYEAST }\end{array}$ & $\begin{array}{l}533 \\
388\end{array}$ & $\begin{array}{l}71 \\
170\end{array}$ & 0.66 \\
\hline $\begin{array}{l}\text { DGSVSGSKLMECVNDAVQTLLQGDDK(8)-AKSLGLESK(2) } \\
\text { LVLISEQVDSGIQLVEKDQEGDDEDGNTATGHGVK(17)- }\end{array}$ & sp|P43609|RSC8_YEAST (388)-sp|P32597|STH1_YEAST (170)/ & 5 & RSC8_YEAST & YEAST & 388 & 170 & 0.66 \\
\hline $\begin{array}{l}\text { TKDHPPNKEMLR(8) } \\
\text { TKLEDE }\end{array}$ & sp|P43609|RSC8_YEAST (511)-sp|P25632|RSC6_YEAST (71)/ & 3 & RSC8_YEAST & RSC6_YEAST & 511 & 71 & 0.66 \\
\hline DGSVSGSKLMECVNDAVQTLLQGDDKLGK(8)-ISADFNAKAK(8) & sp|P43609|RSC8_YEAST (388)-sp|P32597|STH1_YEAST (168)/ & 1 & RSC8_YEAST & STH1_YEAST & 388 & 168 & 0.63 \\
\hline $\begin{array}{l}\text { LVLISEQVDSGIQLVEKDQEGDDEDGNTATGHGVK(17)- } \\
\text { TKDHPPNK(2) }\end{array}$ & sp|P43609|RSC8_YEAST (511)-sp|P25632|RSC6_YEAST (65)/ & 1 & RSC8_YEAST & RSC6_YEAST & 511 & 65 & 0.58 \\
\hline EVVGSTLNGKGGDSRDGSVSGSK(10)-SLGLESKFSNATK(7) & sp|P43609|RSC8_YEAST (375)-sp|P32597|STH1_YEAST (177)/ & 31 & RSC8_YEAST & STH1_YEAST & 375 & 177 & 0.57 \\
\hline $\begin{array}{l}\text { KLVLISEQVDSGIQLVEKDQEGDDEDGNTATGHGVK(18)- } \\
\text { RVGKEGEEVGEGSIAK(4) }\end{array}$ & sp|P43609|RSC8_YEAST (511)-sp|P43609|RSC8_YEAST (533)/ & 5 & RSC8_YEAST & RSC8_YEAST & 511 & 533 & 0.87 \\
\hline FLAKQTHPVIIPSFASWFDISK(4)-INYEQEAQKLEEK $(9$ & sp|P43609|RSC8_YEAST (75)-sp|P43609|RSC8_YEAST (64)/ & 3 & RSC8_YEAST & RSC8_YEAST & 75 & 64 & 0.85 \\
\hline FLAKQTHPVIIPSFASWFDISK(4)-KINYEQEA & sp|P43609|RSC8_YEAS & 3 & EAST & RSC & 75 & 64 & 0.84 \\
\hline KYIEESQAUl & sp|P43609|RSC8_YEAST (420)-sp|P43609|RSC文_YEAST (119)/ & 3 & RSC8_YEAST & YEAST & 420 & 119 & 0.83 \\
\hline DQEGDDEDGNTATGHGVKR(18)-DHPPNKEMLR(6) & sp|P43609|RSC8_YEAST (529)-sp|P25632|RSC6_YEAST (71)/ & 2 & RSC8_YEAST & RSC6_YEAST & 529 & 71 & 0.48 \\
\hline $\begin{array}{l}\text { PFLPENVIKQEVEGGDGAEPQVKKEFPVNLTIK(23)- } \\
\text { RSKNDSPSDTSIVYETVIHDNDLNKPK(3) }\end{array}$ & sp|P43609|RSC8_YEAST (221)-sp|P32832|NPL6_YEAST (375)/ & 1 & RSC8_YEAST & NPL6_YEAST & 221 & 375 & 0.47 \\
\hline $\begin{array}{l}\text { TKPSLIGPSFTGHFQVVLDTPQGLKPFLPENVIKQEVEGGDGAEPQ } \\
\text { VK(34)-KINYEQEAQKLEEK(1) }\end{array}$ & sp|P43609|RSC8_YEAST (207)-sp|P43609|RSC8_YEAST (55)/ & 2 & RSC8_YEAST & RSC8_YEAST & 207 & 55 & 0.78 \\
\hline $\begin{array}{l}\text { LMECVNDAVQTLLQGDDKLGKVSDK(21)- } \\
\text { LMECVNDAVQTLLQGDDKLGK(18) }\end{array}$ & sp|P43609|RSC8_YEAST (409)-sp|P43609|RSC8_YEAST (406)/ & 4 & RSC8_YEAST & RSC8_YEAST & 409 & 406 & 0.77 \\
\hline $\begin{array}{l}\text { KLVLISEQVDSGIQLVEKDQEGDDEDGNTATGHGVKR(36)- } \\
\text { TKDHPPNKEMLR(8) }\end{array}$ & sp|P43609|RSC8_YEAST (529)-sp|P25632|RSC6_YEAST (71)/ & 2 & RSC8_YEAST & RSC6_YEAST & 529 & 71 & 0.47 \\
\hline EESATLGAEVAHKK(13)-AYKDTR(3) & sp|P43609|RSC8_YEAST (54)-sp|P43609|RSC8_YEAST (119)/ & 2 & RSC8_YEAST & RSC8_YEAST & 54 & 119 & 0.75 \\
\hline $\begin{array}{l}\text { KEFPVNLTIKKNVYDSAQDFNALQDESR(10)- } \\
\text { MLNDRLSLSKQILDLNK(10) }\end{array}$ & sp|P43609|RSC8_YEAST (231)-sp|P43609|RSC8_YEAST (477)/ & 1 & RSC8_YEAST & RSC8_YEAST & 231 & 477 & 0.73 \\
\hline $\begin{array}{l}\text { KLVLISEQVDSGIQLVEKDQEGDDEDGNTATGHGVK(18)- } \\
\text { VGKEGEEVGEGDSIAK(3) }\end{array}$ & sp|P43609|RSC8_YEAST (511)-sp|P43609|RSC8_YEAST (533)/ & 5 & RSC8_YEAST & RSC8_YEAST & 511 & 533 & 0.70 \\
\hline $\begin{array}{l}\text { KLVLISEQVDSGIQLVEKDQEGDDEDGNTATGHGVKR(36)- } \\
\text { DRKQSQATNATN(3) }\end{array}$ & sp|P43609|RSC8_YEAST (529)-sp|Q12406|ARP7_YEAST (468)/ & 2 & RSC8_YEAST & ARP7_YEAST & 529 & 468 & 0.40 \\
\hline $\begin{array}{l}\text { TKPSLIGPSFTGHFQVVLDTPQGLKPFLPENVIKQEVEGGDGAEPQ } \\
\text { VK(25)-EESATLGAEVAHKK(13) }\end{array}$ & sp|P43609|RSC8_YEAST (198)-sp|P43609|RSC8_YEAST (54)/ & 1 & RSC8_YEAST & RSC8_YEAST & 198 & 54 & 0.67 \\
\hline $\begin{array}{l}\text { LVLISEQVDSGIQLVEKDQEGDDEDGNTATGHGVKR(17)- } \\
\text { TKDHPPNKEMLR(8) }\end{array}$ & sp|P43609|RSC8_YEAST (511)-sp|P25632|RSC6_YEAST (71) & 3 & RSC8_YEAST & RSC6_YEAST & 511 & 71 & 0.38 \\
\hline $\begin{array}{l}\text { TKPSLIGPSFTGHFQVVLDTPQGLKPFLPENVIKQEVEGGDGAEPQ } \\
\text { VK(25)-KEFPVNLTIK(1) }\end{array}$ & sp|P43609|RSC8_YEAST (198)-sp|P43609|RSC8_YEAST (222)/ & 1 & RSC8_YEAST & RSC8_YEAST & 198 & 222 & 0.66 \\
\hline $\begin{array}{l}\text { KLVLISEQVDSGIQLVEKDQEGDDEDGNTATGHGVK(18)- } \\
\text { QILDLNKSLEELNVSK(7) }\end{array}$ & sp|P43609|RSC8_YEAST (511)-sp|P43609|RSC8_YEAST (484)/ & 1 & RSC8_YEAST & RSC8_YEAST & 511 & 484 & 0.60 \\
\hline $\begin{array}{l}\text { KLVLISEQVDSGIQLVEKDQEGDDEDGNTATGHGVKR(18)- } \\
\text { VGKEGEEVGEGDSIAK(3) }\end{array}$ & sp|P43609|RSC8_YEAST (511)-sp|P43609|RSC8_YEAST (533)/ & 5 & RSC8_YEAST & RSC8_YEAST & 511 & 533 & 0.54 \\
\hline KNVYDSAQDFNALQDESR(1)-TDLHSQTKR(8) & sp|P43609|RSC8_YEAST (232)-sp|Q06488|RSC2_YEAST (772)/ & 1 & RSC8_YEAST & RSC2 & 232 & 772 & 0.34 \\
\hline ALRFLAKQTHPVIIPSFASWFDISK (7)-ESEKMLNDR(4) & sp|P43609|RSC8_YEAST (75)-sp|P43609|RSC8_YEAST (467)/ & 1 & RSC8_YEAST & RSC8_YEAST & 75 & 467 & 0.46 \\
\hline $\begin{array}{l}\text { LVLISEQVDSGIQLVEKDQEGDDEDGNTATGHGVKR(35)- } \\
\text { SLEELNVSKK(9) }\end{array}$ & sp|P43609|RSC8_YEAST (529)-sp|P43609|RSC8_YEAST (493)/ & 1 & RSC8_YEAST & RSC8_YEAST & 529 & 493 & 0.46 \\
\hline $\begin{array}{l}\text { KNWSDQEMLLLLEGIEMYEDQWEKIADHVGGHKR(24)- } \\
\text { VGSPGAGGPLTVQGLKQHQLQR(16) }\end{array}$ & sp|P43609|RSC8_YEAST (336)-sp|Q06488|RSC2_YEAST (695)/ & 2 & RSC8_YEAST & RSC2_YEAST & 336 & 695 & 0.32 \\
\hline $\begin{array}{l}\text { ANKVSYYGNPTSK(3)-NQEDEKNLQR(6) } \\
\text { YDWYQPSKVSSNVQQPQQLGDMENNLEKYPFR(8)- }\end{array}$ & $\begin{array}{l}\text { sp|P53330|RT102_YEAST (12)-sp|P53330|RT102_YEAST (71)/ } \\
\text { sp|P53330|RT102_YEAST (34)-sp|P32597|STH1_YEAST (326)/ }\end{array}$ & $\begin{array}{c}6 \\
19\end{array}$ & $\begin{array}{l}\text { RT102_YEAST } \\
\text { RT102_YEAST }\end{array}$ & $\begin{array}{l}\text { RT102_YEAST } \\
\text { STH1 YEAST }\end{array}$ & $\begin{array}{l}12 \\
34\end{array}$ & $\begin{array}{c}71 \\
326\end{array}$ & $\begin{array}{l}4.78 \\
4.11\end{array}$ \\
\hline
\end{tabular}




\begin{tabular}{|c|c|c|c|c|c|c|c|}
\hline PEPTIDE & PROTEINS & CSM & PROTEIN1 & PROTEIN2 & R1 & $\mathbf{R 2}$ & $\mathbf{s}$ \\
\hline \multicolumn{8}{|l|}{ NLHLQKINSIIDFIK(6) } \\
\hline NQEDEKNLQR(6)-YKTWLR(2) & sp|P53330|RT102_YEAST (71)-sp|P53330|RT102_YEAST (61)/ & 15 & RT102_YEAST & RT102_YEAST & 71 & 61 & 3.76 \\
\hline ESCEDILDLKEFDRR(10)-KSLMTSHTK(1) & sp|P53330|RT102 YEAST (85)-sp|P53330|RT102 YEAST (94)/ & 32 & RT102 YEAST & RT102 YEAST & 85 & 94 & 3.76 \\
\hline VSSNVQQPQQQLGDMENNLEKYPFR(21)-TLNRK(1) & sp|P53330|RT102 YEAST (55)-sp|Q12406|ARP7 YEAST (2)/ & 12 & RT102 YEAST & ARP7 YEAST & 55 & $\begin{array}{c}94 \\
2\end{array}$ & 3.74 \\
\hline SLMTSHTKGDTSK(8)-LLDQTKDTR(6) & sp|P53330|RT102_YEAST (102)-sp|P32597|STH1_YEAST (397)/ & 2 & RT102_YEAST & STH1_YEAST & 102 & 397 & 3.44 \\
\hline VSYYGNPTSKESWR(10)-NQEDEKNLQR(6) & sp|P53330|RT102_YEAST (22)-sp|P53330|RT102_YEAST (71)/ & 4 & RT102_YEAST & RT102_YEAST & 22 & 71 & 3.42 \\
\hline GDTSKATGAPSANQGDEALSVDDIR(5)- & sp|P53330|RT102_YEAST (107)-sp|P53330|RT102_YEAST (149)/ & 1 & RT102_YEAST & RT102_YEAST & 107 & 149 & 3.31 \\
\hline GDTSKATGAPSANQGDEALSVDDIR(5)-KSLMTSHTK(1) & sp|P53330|RT102_YEAST (107)-sp|P53330|RT102_YEAST (94)/ & 27 & RT102_YEAST & RT102_YEAST & 107 & 94 & 3.21 \\
\hline ESCEDILDLKEFDR(10)-KSLMTSHTK(1) & sp|P53330|RT102_YEAST (85)-sp|P53330|RT102_Y'EAST (94)// & 32 & RT102_YEAST & RT102_YEAST & 85 & 94 & 3.15 \\
\hline YDWYQPSKVSSNVQQPQQQLGDMENNLEKYPFR(8)- & sp|P53330|RT102_YEAST (34)-sp|P32597|STH1_YEAST (326)/ & 19 & RT102_YEAST & STH1_YEAST & 34 & 326 & 2.98 \\
\hline ESCEDILDLKEFDRR(10)-SLMTSHTKGDTSK(8) & sp|P53330|RT102_YEAST (85)-sp|P53330|RT102_YEAST (102)/ & 1 & RT102_YEAST & RT102_YEAST & 85 & 102 & 2.75 \\
\hline $\begin{array}{l}\text { GAVGNSEAIPGLSAGVNNDNTKESKDVK(25)- } \\
\text { SLMTSHTKGDTSK(8) }\end{array}$ & sp|P53330|RT102_YEAST (152)-sp|P53330|RT102_YEAST (102)/ & 1 & RT102_YEAST & RT102_YEAST & 152 & 102 & 2.57 \\
\hline $\begin{array}{l}\text { YDWYQPSKVSSNVQQPQQQLGDMENNLEK(8)- } \\
\text { ERNLHLQKINSIIDFIK(8) }\end{array}$ & sp|P53330|RT102_YEAST (34)-sp|P32597|STH1_YEAST (326)/ & 19 & RT102_YEAST & STH1_YEAST & 34 & 326 & 2.55 \\
\hline $\begin{array}{l}\text { YDWYQPSKVSSNVQQPQQQLGDMENNLEKYPFR(8)- } \\
\text { ERNLHLQKINSIIDFIK( } 8 \text { ) }\end{array}$ & sp|P53330|RT102_YEAST (34)-sp|P32597|STH1_YEAST (326)/ & 19 & RT102_YEAST & STH1_YEAST & 34 & 326 & 2.30 \\
\hline GDTSKATGAPSANQGDEALSVDDIR(5)-EKTDYYEVAHR(2) & sp|P53330|RT102_YEAST (107)-sp|P32597|STH1_YEAST (444)/ & 1 & RT102_YEAST & STH1_YEAST & 107 & 444 & 2.10 \\
\hline $\begin{array}{l}\text { ESCEDILDLKEFDRR(10)-KSLMTSHTKGDTSK(1) } \\
\text { YDWYQPSKVSSNVQQPQQQLGDENNLEK(8)- }\end{array}$ & $\begin{array}{l}\text { sp|P53330|RT102_YEAST (85)-sp|P53330|RT102_YEAST (94)/ } \\
\text { sp|P53330|RT102 YEAST (34)-sp|P32597|STH1 YEAST (326)/ }\end{array}$ & $\begin{array}{l}32 \\
19\end{array}$ & $\begin{array}{l}\text { RT102_YEAST } \\
\text { RT102 YEAST }\end{array}$ & $\begin{array}{l}\text { RT102_YEAST } \\
\text { STH1_YEAST }\end{array}$ & $\begin{array}{l}85 \\
34\end{array}$ & $\begin{array}{c}94 \\
326\end{array}$ & $\begin{array}{l}1.94 \\
1.88\end{array}$ \\
\hline $\begin{array}{l}\text { NLHLQKINSIIDFIK(6) } \\
\text { GDTSKATGAPSANQGDEALSVDDIR(5)-QDGSYTYHSTNKDNK(12) }\end{array}$ & $\begin{array}{l}\text { sp|P53330|RT102_YEAST (34)-sp|P32597|STH1_YEAST (326)/ } \\
\text { sp|P53330|RT102_YEAST (107)-sp|Q05123|ARP9 YEAST (58)/ }\end{array}$ & 3 & RT102 YEAST & ARP9 YEAST & 107 & 58 & 1.56 \\
\hline ESWRYDWYQPSSVSSNVQQPQQQLGDMENNLEKYPFR(12)- & sp|P53330|RT102_YEAST (34)-sp|P32597|STH1_YEAST (326)/ & 19 & RT102_YEAST & STH1_YEAST & 34 & 326 & 1.35 \\
\hline VSSNVQQPQQQLGDMENNLEKYPFR(21)-NLHLQKINSIIDFIK(6) & sp|P53330|RT102_YEAST (55)-sp|P32597|STH1_YEAST (326)/ & 1 & RT102_YEAST & STH1_YEAST & 55 & 326 & 1.28 \\
\hline SLMTSHTKGDTSK(8)-GQKVK(3) & sp|P53330|RT102_YEAST (102)-sp|Q05123|ARP9_YEAST (270)/ & 3 & RT102_YEAST & ARP9_YEAST & 102 & 270 & 1.17 \\
\hline GAVGNSEAIPGLSAGVNNDNTKESKDVK(22)- & sp|P53330|RT102_YEAST (149)-sp|P25632|RSC6_YEAST (307)/ & 1 & RT102_YEAST & RSC6_YEAST & 149 & 307 & 1.02 \\
\hline $\begin{array}{l}\text { DKPELGEVKLDSLLQK(2) } \\
\text { GDTSKATGAPSANQGDEALSVDDIR(5)-VKNVK(2) }\end{array}$ & sp|P53330|RT102 YEAST (107)-sp|Q05123|ARP9 YEAST (272)/ & 4 & RT102 YEAST & ARP9 YEAST & 107 & 272 & 0.91 \\
\hline ESWRYDWYQPSKVSSNVQQPQQQLGDMENNLEK(12)- & sp|P53330|RT102_YEAST (34)-sp|P32597|STH1_YEAST (326)/ & 19 & RT102_YEAST & STH1_YEAST & 34 & 326 & 0.83 \\
\hline GDTSKATGAPSANQGDEALSVDDIR(5)-ESCEDILDLKEFDRR(10) & sp|P53330|RT102 YEAST (107)-sp|P53330|RT102 YEAST (85)/ & 2 & RT102 YEAST & RT102 YEAST & 107 & 85 & 1.03 \\
\hline VSYYGNPTSKESWR(10)-TLNRK(1) & sp|P53330|RT102_YEAST (22)-sp|Q12406|ARP7_YEAST (2)/ & 1 & RT102_YEAST & ARP7_YEAST & 22 & 2 & 0.76 \\
\hline SLMTSHTKGDTSK (8)-VKNVK(2) & sp|P53330|RT102 YEAST (102)-sp|Q05123|ARP9 YEAST (272)/ & 1 & RT102 YEAST & ARP9 YEAST & 102 & 272 & 0.76 \\
\hline GDTSKATGAPSANQGDEALSVDDIR(5)-LLDQTKDTR(6) & sp|P53330|RT102_YEAST (107)-sp|P32597|STH1_YEAST (397)/ & 1 & RT102_YEAST & STH1_YEAST & 107 & 397 & 0.73 \\
\hline YDWYQPSKVSSNVQQPQQQLGDMENNLEKYPFR(8)-TLNRK(1) & sp|P53330|RT102_YEAST (34)-sp|Q12406|ARP7_YEAST (2)/ & 4 & RT102_YEAST & ARP7_YEAST & 34 & 2 & 0.60 \\
\hline KSLMTSHTK(1)-GQKVK(3) & sp|P53330|RT102 YEAST (94)-sp|Q05123|ARP9 YEAST (270)/ & 1 & RT102 YEAST & ARP9 ${ }^{-}$YEAST & 94 & 270 & 0.54 \\
\hline $\begin{array}{l}\text { SLMTSHTKGDTSK (8)-ERGQKVK(5) } \\
\text { SLRTKG }\end{array}$ & sp|P53330|RT102 YEAST (102)-sp|Q05123|ARP9 YEAST (270)/ & 3 & RT102 YEAST & ARP9 YEAST & 102 & 270 & 0.50 \\
\hline GDTSKATGAPSANQGDEALSVDDIR & p|P53330|RT102_YEAST (152)/ & 1 & RT102_YEAST & RT102_YEAST & 107 & 152 & 0.54 \\
\hline GGKGLGK(3)-GGAKR(4) & P62799|H4 XENLA (16)/ & 9 & H4 XENLA & H4 XENLA & 8 & 16 & 12.37 \\
\hline GLGKGGAK(4)-GKGGK(2) & sp|P62799|H4_XENLA (12)-sp|P62799|H4__XENLA (5)/ & 6 & H4_XENLA & H4_XENLA & 12 & 5 & 4.92 \\
\hline GLGKGGAKR(4)-GKGGK(2) & sp|P62799|H4 XENLA (12)-sp|P62799|H4 XENLA (5)/ & 6 & H4 XENLA & H4 XENLA & 12 & 5 & 4.86 \\
\hline DAVTYTEHAKR(10)-GKGGK(2) & sp|P62799|H4_XENLA (77)-s & 2 & H4_XENLA & H4_XENLA & 77 & 5 & 3.42 \\
\hline DAVTYTEHAKRK(10)-EIAQDFKTDLR(7) & sp|P62799|H4 ${ }^{-}$XENLA (77)-sp|P84233|H32 XENLA (79)/ & 6 & H4 ${ }^{-}$XENLA & H32 XENLA & 77 & 79 & 2.56 \\
\hline TVTAMDVVYALKR(12)-KESYAIYVYK(1) & sp|P62799|H4_XENLA (91)-sp|P02281|H2B11_XENLA (31)/ & 3 & H4_XENLA & H2B11_XENLA & 91 & 31 & 2.49 \\
\hline TVTAMDVVYALKR(12)-KGNYAER(1) & sp|P62799|H4 ${ }^{-}$XENLA (91)-tr|Q6AZJ8|H2A XE ENLA (36)/ & 2 & H4 ${ }^{-}$XENLA & H2A XENLA & 91 & 36 & 2.35 \\
\hline KVLRDNIQGITKPAIR(1)-GKGGK(2) & sp|P62799|H4_XE & 8 & & H4_XENLA & 20 & 5 & 2.24 \\
\hline KVLRDNIQGITKPAIR(1)-GLGKGGAKR(4) & sp|P62799|H4 $4^{-}$XENLA (20)-sp|P62799|H4 ${ }^{-}$XENLA (12)/ & 12 & H4 ${ }^{-}$XENLA & H4 $4^{-}$XENLA & 20 & 12 & 1.90 \\
\hline
\end{tabular}




\begin{tabular}{|c|c|c|c|c|c|c|c|}
\hline PEPTIDE & PROTEINS & CSM & PROTEIN1 & PROTEIN2 & R1 & R2 & $\mathbf{s}$ \\
\hline KVLRDNIQGITKPAIR(1)-GLGKGGAK(4) & sp|P62799|H4_XENLA (20)-sp|P62799|H4_XENLA (12)/ & 12 & H4_XENLA & H4_XENLA & 20 & 12 & 1.63 \\
\hline KTVTAMDVVYALKR(1)-DAVTYTEHAKR(10) & sp|P62799|H4_XENLA (79)-sp|P62799|H4_XENLA (77)/ & 1 & H4_XENLA & H4_XENLA & 79 & 77 & 1.48 \\
\hline KVLRDNIQGITKPAIR(1)-GGKGLGK(3) & sp|P62799|H4_XENLA (20)-sp|P62799|H4_XENLA (8)/ & 5 & H4_XENLA & H4_XENLA & 20 & 8 & 1.33 \\
\hline GVLKVFLENVIR(4)-GGAKR(4) & sp|P62799|H4_XENLA (59)-sp|P62799|H4_XENLA (16)/ & 1 & H4_XENLA & H4_XENLA & 59 & 16 & 1.25 \\
\hline TVTAMDVVYALKR(12)-YYKEQADIYAK(3) & sp|P62799|H4_XENLA (91)-sp|Q12406|ARP7_YYAST (251)/ & 1 & H4_XENLA & ARP7_YEAST & 91 & 251 & 0.53 \\
\hline KVLRDNIQGITKPAIR(1)-NDEELNKLLGR(7) & sp|P62799|H4_XENLA (20)-tr|Q6AZJ8|H2A_XENLA (95)/ & 1 & H4_XENLA & H2A_X $\mathrm{X}$ ENLA & 20 & 95 & 0.48 \\
\hline DAVTYTEHAKR(10)-GLGKGGAK(4) & sp|P62799|H4_XENLA (77)-sp|P62799|H4_XENLA (12)/ & 1 & H4_XENLA & H4_X̄ENLA & 77 & 12 & 0.56 \\
\hline GVLKVFLENVIR(4)-GLGKGGAK(4) & sp|P62799|H4_XENLA (59)-sp|P62799|H4_XENLA (12)/ & 1 & H4_XENLA & H4_XENLA & 59 & 12 & 0.48 \\
\hline $\begin{array}{l}\text { ISGLIYEETRGVLKVFLENVIRDAVTYTEHAK(14)- } \\
\text { KTVTAMDVVYALKR(1) }\end{array}$ & sp|P62799|H4_XENLA (59)-sp|P62799|H4_XENLA (79)/ & 1 & H4_XENLA & H4_XENLA & 59 & 79 & 0.47 \\
\hline $\begin{array}{l}\text { KTVTAMDVVYALKR(1) } \\
\text { KVLRDNIQGITKPAIR(1)-GGAKR(4) }\end{array}$ & sp|P62799|H4_XENLA (20)-sp|P62799|H4_XENLA (16)/ & 1 & H4_XENLA & $\mathrm{H} 4$ XENLA & 20 & 16 & 0.45 \\
\hline KVLRDNIQGITKPAIR(1)-GKGGKGLGK(5) & sp|P62799|H4_XENLA (20)-sp|P62799|H4_XENLA (8)/ & 5 & H4_XENLA & H4_XENLA & 20 & 8 & 0.43 \\
\hline KQLATK(1)-KSTGGK(1) & sp|P84233|H32 XENLA (18)-sp|P84233|H32 XENLA (9)/ & 8 & $\mathrm{H} 3 \overline{2}$ XENLA & $\mathrm{H} 3 \overline{2}$ XENLA & 18 & 9 & 16.83 \\
\hline TKQTAR(2)-KQLATK(1) & sp|P84233|H32_XENLA (4)-sp|P84233|H32_X XENLA (18)/ & 14 & H32_XENLA & H32_XENLA & 4 & 18 & 10.84 \\
\hline STGGKAPR(5)-TKQTAR(2) & sp|P84233|H32_XENLA (14)-sp|P84233|H32_XENLA (4)/ & 8 & H32_XENLA & H32_XENLA & 14 & 4 & 9.58 \\
\hline KSAPATGGVK(1)-KQLATK(1) & sp|P84233|H32_XENLA (27)-sp|P84233|H32_XENLA (18)/ & 22 & H32_XENLA & H32_XENLA & 27 & 18 & 8.05 \\
\hline KSAPATGGVK(1)-KSTGGK(1) & sp|P84233|H32_XENLA (27)-sp|P84233|H32_XENLA (9)/ & 7 & H32_XENLA & H32_XENLA & 27 & 9 & 6.73 \\
\hline YQKSTELLIR(3)-KSAPATGGVK(1) & sp|P84233|H32_XENLA (56)-sp|P84233|H32_XENLA (27)/ & 9 & H32_XENLA & H32_XENLA & 56 & 27 & 6.48 \\
\hline KQLATKAAR(6)-KSAPATGGVK(1) & sp|P84233|H32_XENLA (23)-sp|P84233|H32_XENLA (27)/ & 41 & H32_XENLA & H32_XENLA & 23 & 27 & 6.48 \\
\hline KQLATKAAR(6)-KSTGGK(1) & sp|P84233|H32_XENLA (23)-sp|P84233|H32_XENLA (9)/ & 5 & H32_XENLA & H32_XENLA & 23 & 9 & 5.46 \\
\hline KSAPATGGVK(1)-STGGKAPR(5) & sp|P84233|H32_XENLA (27)-sp|P84233|H32_XENLA (14)/ & 10 & H32_XENLA & H32_XENLA & 27 & 14 & 4.85 \\
\hline STGGKAPR(5)-KQLATK(1) & sp|P84233|H32_XENLA (14)-sp|P84233|H32_XENLA (18)/ & 16 & H32_XENLA & H32_XENLA & 14 & 18 & 4.65 \\
\hline KSAPATGGVK(1)-QLATKAAR(5) & sp|P84233|H32_XENLA (27)-sp|P84233|H32_XENLA (23)/ & 23 & H32_XENLA & H32_XENLA & 27 & 23 & 4.47 \\
\hline KSTGGKAPR(1)-TKQTAR(2) & sp|P84233|H32_XENLA (9)-sp|P84233|H32_X̄ENLA (4)/ & 5 & H32_XENLA & H32_XENLA & 9 & 4 & 4.27 \\
\hline EIAQDFKTDLR(7)-GKGGK(2) & sp|P84233|H32-XENLA (79)-sp|P62799|H4_XENLA (5)/ & 6 & H32-XENLA & $\mathrm{H} 4$ X XENLA & 79 & 5 & 4.25 \\
\hline YQKSTELLIR(3)-KSTGGK(1) & sp|P84233|H32_XENLA (56)-sp|P84233|H32_XENLA (9)/ & 2 & H32_XENLA & H32_XENLA & 56 & 9 & 4.14 \\
\hline EIAQDFKTDLR(7)-DAVTYTEHAKR(10) & sp|P84233|H32_XENLA (79)-sp|P62799|H4_XENLA (77)/ & 5 & H32_XENLA & H4_XEENLA & 79 & 77 & 4.05 \\
\hline KSTGGKAPR(1)-KQLATK(1) & sp|P84233|H32_XENLA (9)-sp|P84233|H32_XENLA (18)/ & 5 & H32_XENLA & $\mathrm{H} 3 \overline{2} \_\mathrm{XENLA}$ & 9 & 18 & 4.04 \\
\hline KSAPATGGVKKPHR(1)-KQLATKAAR(6) & sp|P84233|H32_XENLA (27)-sp|P84233|H32_XENLA (23)/ & 23 & H32_XENLA & H32_XENLA & 27 & 23 & 3.93 \\
\hline KSAPATGGVKKPHR(10)-KTESSK(1) & sp|P84233|H32_XENLA (36)-tr|Q6AZJ8|H2A_XENLA (119)/ & 8 & H32_XENLA & H2A_XENLA & 36 & 119 & 3.83 \\
\hline KSAPATGGVK(1)-KSTGGKAPR(6) & sp|P84233|H32_XENLA (27)-sp|P84233|H32_XENLA (14)/ & 10 & H32_XENLA & H32_XENLA & 27 & 14 & 3.61 \\
\hline KSAPATGGVKKPHR(1)-KQLATK(1) & sp|P84233|H32_XENLA (27)-sp|P84233|H32_XENLA (18)/ & 22 & H32_XENLA & H32_XENLA & 27 & 18 & 3.57 \\
\hline KSAPATGGVKKPHR(1)-KSTGGKAPR(1) & sp|P84233|H32_XENLA (27)-sp|P84233|H32_XENLA (9)/ & 7 & H32_XENLA & H32_XENLA & 27 & 9 & 3.45 \\
\hline $\begin{array}{l}\text { RYQKSTELLIR(4)-KQLATK(1) } \\
\text { R }\end{array}$ & sp|P84233|H32_XENLA (56)-sp|P84233|H32_XENLA (18)/ & 7 & H32_XENLA & H32_XENLA & 56 & 18 & 3.43 \\
\hline TKQTAR(2)-KSTGGK(1) & sp|P84233|H32_XENLA (4)-sp|P84233|H32_XEENLA (9)/ & 2 & H32_XENLA & H32_XENLA & 4 & 9 & 3.42 \\
\hline KSAPATGGVKKPHR(1)-KQLATKAAR(1) & sp|P84233|H32_XENLA (27)-sp|P84233|H32_XENLA (18)/ & 22 & H32_XENLA & H32_XENLA & 27 & 18 & 3.16 \\
\hline KQLATKAAR(6)-STGGKAPR(5) & sp|P84233|H32_XENLA (23)-sp|P84233|H32_XENLA (14)/ & 10 & H32_XENLA & H32_XENLA & 23 & 14 & 3.09 \\
\hline YQKSTELLIR(3)-KQLATK(1) & sp|P84233|H32 XENLA (56)-sp|P84233|H32 XENLA (18)/ & 7 & H32 XENLA & H32 XENLA & 56 & 18 & 3.06 \\
\hline YQKSTELLIR(3)-KTESSK(1) & sp|P84233|H32_XENLA (56)-tr|Q6AZJ8|H2A_XENLA (119)/ & 5 & H32_XENLA & H2A_XENLA & 56 & 119 & 2.87 \\
\hline KSTGGKAPR(6)-QLATKAAR(5) & sp|P84233|H32_XENLA (14)-sp|P84233|H32_XENLA (23)/ & 2 & H32_XENLA & H32_XENLA & 14 & 23 & 2.85 \\
\hline YQKSTELLIR(3)-TKQTAR(2) & sp|P84233|H32_XENLA (56)-sp|P84233|H32_XENLA (4)/ & 8 & H32_XENLA & H32_XENLA & 56 & 4 & 2.73 \\
\hline KSTGGKAPR(6)-KQLATK(1) & sp|P84233|H32 XENLA (14)-sp|P84233|H32_XENLA (18)/ & 16 & H32 XENLA & H32 XENLA & 14 & 18 & 2.56 \\
\hline QLATKAAR(5)-TKQTAR(2) & sp|P84233|H32_XENLA (23)-sp|P84233|H32_XENLA (4)/ & 3 & H32_XENLA & H32_XENLA & 23 & 4 & 2.55 \\
\hline KSAPATGGVKKPHR(1)-RYQKSTELLIR(4) & sp|P84233|H32_XENLA (27)-sp|P84233|H32_XENLA (56)/ & 3 & H32_XENLA & $\mathrm{H} 32_{-}^{-} \mathrm{XENLA}$ & 27 & 56 & 2.52 \\
\hline KQLATKAAR(6)-LWKTPDGK(3) & sp|P84233|H32_XENLA (23)-sp|Q06488|RSC2_YEAST (437)/ & 4 & H32_XENLA & RSC2_YEAST & 23 & 437 & 2.34 \\
\hline SAPATGGVKKPHR(9)-YQKSTELLIR(3) & sp|P84233|H32_XENLA (36)-sp|P84233|H32_XENLA (56)/ & 10 & H32_XENLA & H32_XENLA & 36 & 56 & 2.26 \\
\hline SAPATGGVKKPHR(9)-KQLATK(1) & sp|P84233|H32_XENLA (36)-sp|P84233|H32_XENLA (18)/ & 3 & H32_XENLA & H32_XENLA & 36 & 18 & 2.18 \\
\hline KSAPATGGVKKPHR(10)-KQLATK(1) & sp|P84233|H32_XENLA (36)-sp|P84233|H32_XENLA (18)/ & 3 & H32_XENLA & H32_XENLA & 36 & 18 & 2.16 \\
\hline YQKSTELLIR(3)-STGGKAPR(5) & sp|P84233|H32- XENLA (56)-sp|P84233|H32-XENLA (14)/ & 6 & H32-XENLA & H32-XENLA & 56 & 14 & 2.06 \\
\hline SAPATGGVKKPHR(9)-KQLATKAAR(6) & sp|P84233|H32 XENLA (36)-sp|P84233|H32 XENLA (23) & 6 & $\mathrm{H} 32^{-}$XENLA & $\mathrm{H} 32^{-}$XENLA & 36 & 23 & 2.04 \\
\hline
\end{tabular}




\begin{tabular}{|c|c|c|c|c|c|c|c|}
\hline PEPTIDE & PROTEINS & CSM & PROTEIN1 & PROTEIN2 & R1 & R2 & $\mathbf{s}$ \\
\hline QLATKAAR(5)-STGGKAPR(5) & sp|P84233|H32 XENLA (23)-sp|P84233|H32 XENLA (14)/ & 10 & H32 XENLA & H32 XENLA & 23 & $\frac{14}{14}$ & 1.96 \\
\hline KSAPATGGVKKPHR(1)-KSTGGKAPR(6) & sp|P84233|H32_XENLA (27)-sp|P84233|H32_XENLA (14)/ & 10 & H32_XENLA & H32_XENLA & 27 & 14 & 1.85 \\
\hline KSTGGKAPR(6)-TKQTAR(2) & sp|P84233|H32_XENLA (14)-sp|P84233|H32_XENLA (4)/ & 8 & H32 XENLA & H32 XENLA & 14 & 4 & 1.79 \\
\hline SAPATGGVKKPHR(9)-KSTGGK(1) & sp|P84233|H32_XENLA (36)-sp|P84233|H32_XENLA (9)/ & 2 & H32_XENLA & H32_XENLA & 36 & 9 & 1.66 \\
\hline KSAPATGGVKKPHR(1)-YQKSTELLIR(3) & sp|P84233|H32 XENLA (27)-sp|P84233|H32 XENLA (56)/ & 3 & H32 XENLA & H32 XENLA & 27 & 56 & 1.60 \\
\hline RYQKSTELLIR(4)-SAPATGGVKKPHR(9) & sp|P84233|H32_XENLA (56)-sp|P84233|H32_XENLA (36)/ & 5 & H32_XENLA & H32_XENLA & 56 & 36 & 1.56 \\
\hline $\begin{array}{l}\text { YQKSTELLIR(3)-KLPFQR(1) } \\
\text { Yor }\end{array}$ & sp|P84233|H32 XENLA (56)-sp|P84233|H32 XENLA (64)/ & 1 & H32 XENLA & H32 XENLA & 56 & 64 & 1.53 \\
\hline KQLATKAAR(6)-KSTGGKAPR(1) & sp|P84233|H32_XENLA (23)-sp|P84233|H32_XENLA (9)/ & 5 & H32_XENLA & H32_XENLA & 23 & 9 & 1.49 \\
\hline $\begin{array}{l}\text { KSAPATGGVKKPHR(10)-KTESSKSAK(1) } \\
\text { KSTEMTE }\end{array}$ & sp|P84233|H32 XENLA (36)-tr|Q6AZJ8|H2A- XENLA (119)/ & 8 & H32 XENLA & H2A XENLA & 36 & 119 & 1.46 \\
\hline RYQKSTELLIR(4)-KSTGGK(1) & sp|P84233|H32_XENLA (56)-sp|P84233|H32_XENLA (9)/ & 2 & H32_XENLA & H32_XENLA & 56 & 9 & 1.31 \\
\hline KSAPATGGVK(1)-TKQTAR(2) & sp|P84233|H32 XENLA (27)-sp|P84233|H32 XENLA (4)/ & 1 & H32 XENLA & H32 XENLA & 27 & 4 & 1.30 \\
\hline KSAPATGGVKKPHR(10)-KTESSKSAK(6) & sp|P84233|H32_XENLA (36)-tr|Q6AZJ8|H2A_XENLA (124)/ & 3 & H32_XENLA & H2A_XENLA & 36 & 124 & 1.05 \\
\hline SAPATGGVKKPHR(9)-KTESSK(1) & sp|P84233|H32 XENLA (36)-tr|Q6AZJ8|H2A XENLA (119)/ & 8 & H32 XENLA & H2A XENLA & 36 & 119 & 1.00 \\
\hline EIAQDFKTDLR(7)-GLGKGGAK(4) & sp|P84233|H32_XENLA (79)-sp|P62799|H4_XENLA (12)/ & 2 & H32_XENLA & H4_XENLA & 79 & 12 & 0.95 \\
\hline EIAQDFKTDLR(7)-GGKGLGK(3) & sp|P84233|H32 XENLA (79)-sp|P62799|H4 ${ }^{-}$XENLA (8)/ & 3 & H32 XENLA & H4 XENLA & 79 & 8 & 0.87 \\
\hline KSAPATGGVK(1)-KDGKKR(5) & sp|P84233|H32_XENLA (27)-sp|P02281|H2B11_XENLA (25)/ & 1 & H32_XENLA & H2B11_XENLA & 27 & 25 & 0.72 \\
\hline KSAPATGGVKKPHR(10)-KSTGGK(1) & sp|P84233|H32 XENLA (36)-sp|P84233|H32 XENLA (9)/ & 2 & H32 XENLA & H32 XENLA & 36 & 9 & 0.96 \\
\hline YQKSTELLIR(3)-DNKKTR(4) & sp|P84233|H32_XENLA (56)-tr|Q6AZJ8|H2A_XENLA (75)/ & 1 & H32_XENLA & H2A_XENLA & 56 & 75 & 0.57 \\
\hline YQKSTELLIR(3)-SAKSK(3) & sp|P84233|H32 XENLA (56)-tr|Q6AZJ8|H2A- XENLA (127)/ & 1 & H32 XENLA & H2A XENLA & 56 & 127 & 0.54 \\
\hline KSAPATGGVK(1)-KSTGGKAPR(1) & sp|P84233|H32_XENLA (27)-sp|P84233|H32_XENLA (9)/ & 7 & H32_XENLA & H32_XENLA & 27 & 9 & 0.88 \\
\hline SAPATGGVKKPHR(9)-KSTGGKAPR(6) & sp|P84233|H32 XENLA (36)-sp|P84233|H32 XENLA (14)/ & 1 & H32 XENLA & H32 XENLA & 36 & 14 & 0.84 \\
\hline YQKSTELLIRK(3)-SAPATGGVKKPHR(9) & sp|P84233|H32_XENLA (56)-sp|P84233|H32_XENLA (36)/ & 5 & H32_XENLA & H32_XENLA & 56 & 36 & 0.84 \\
\hline QTARKSTGGK(5)-AKSAPAPK(1) & sp|P84233|H32 XENLA (9)-sp|P02281|H2B11 XENLA (1)/ & 1 & H32 XENLA & H2B11 XENLA & 9 & 1 & 0.50 \\
\hline YQKSTELLIR(3)-SDTEKDK(1) & sp|P84233|H32_XENLA (56)-sp|P43609|RSC8_YEAST (2)/ & 1 & H32_XENLA & RSC8_YEAST & 56 & 2 & 0.47 \\
\hline KSTGGKAPR(1)-QLATKAAR(5) & sp|P84233|H32 XENLA (9)-sp|P84233|H32 XENLA (23)/ & 1 & H32 XENLA & H32 XENLA & 9 & 23 & 0.80 \\
\hline KQLATK(1)-GGAKR(4) & sp|P84233|H32 XENLA (18)-sp|P62799|H4 XENLA (16)/ & 1 & H32 XENLA & H4 XEENLA & 18 & 16 & 0.46 \\
\hline KQLATKAAR(6)-TKQTAR(2) & sp|P84233|H32 XENLA (23)-sp|P84233|H32 XENLA (4)/ & 3 & H32 XENLA & H32 XENLA & 23 & 4 & 0.70 \\
\hline QLATKAARK(5)-KSAPATGGVK(1) & sp|P84233|H32_XENLA (23)-sp|P84233|H32_XENLA (27)/ & 41 & H32_XENLA & H32_XENLA & 23 & 27 & 0.64 \\
\hline KSAPATGGVKKPHR(1)-KSTGGKAPRK(6) & sp|P84233|H32 XENLA (27)-sp|P84233|H32 XENLA (14)/ & 10 & H32 XENLA & H32 XENLA & 27 & 14 & 0.50 \\
\hline $\begin{array}{l}\text { KQLATKAAR(6)-KTESSKSAK(6) } \\
\text { KATKR(0) }\end{array}$ & sp|P84233|H32 XENLA (23)-tr|Q6AZJ8|H2A XENLA (124)/ & 1 & H32 XENLA & H2A XENLA & 23 & $\begin{array}{c}14 \\
124\end{array}$ & 0.36 \\
\hline $\begin{array}{l}\text { EIAQDFKTDLR(7)-GKGGKGLGK(5) } \\
\text { IASTER }\end{array}$ & 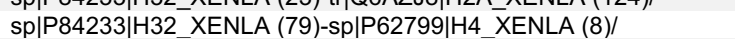 & 3 & H32 XENLA & H4 X XNLA & 79 & 8 & 0.34 \\
\hline KLIAKPETVQSEVKNER(1)-VKVLLVDQQGLR(2) & sp|Q02206|RSC4 YEAST (386)-sp|P32832|NPL6 YEAST (340)/ & 25 & RSC4 YEAST & NPL6 YEAST & 386 & 340 & 8.83 \\
\hline KLIAKPETVQSEVK(1)-VKVLLVDQQGLR(2) & sp|Q02206|RSC4-YEAST (386)-sp|P32832|NPL6_YEAST (340)/ & 25 & RSC4_YEAST & NPL6_YEAST & 386 & 340 & 8.36 \\
\hline STTSDIEKTNSLESEHLK(8)-NEDKLVLTTINKR(4) & sp|Q02206|RSC4 YEAST (410)-sp|Q07979|RSC58 YEAST (70)/ & $\begin{array}{c}20 \\
5\end{array}$ & RSC4 YEAST & RSC58 YEAST & $\begin{array}{l}300 \\
410\end{array}$ & 70 & $\begin{array}{l}0.30 \\
4.98\end{array}$ \\
\hline KLIAKPETVQSEVKNER(1)-KNFIAVSAANR(1) & sp|Q02206|RSC4_YEAST (386)-sp|Q06488|RSC2_YEAST (905)/ & 3 & RSC4_YEAST & RSC2 YEAST & 386 & 905 & 4.90 \\
\hline STTSDIEKTNSLESEHLK(8)-KLIAKPETVQSEVKNER(1) & sp|Q02206|RSC4_YEAST (410)-sp|Q02206|RSC4_YEAST (386)/ & 10 & RSC4_YEAST & RSC4_YEAST & 410 & 386 & 4.63 \\
\hline 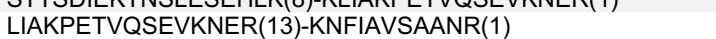 & sp|Q02206|RSC4_YEAST (399)-sp|Q06488|RSC2_YEAST (905)/ & 5 & RSC4_YEAST & RSC2 YEAST & 399 & 905 & 4.60 \\
\hline SMQKEMQSLSEQHTMEYKPYK(4)-STTSDIEKTNSLESEHLK (8) & sp|Q02206|RSC4 YEAST (432)-sp|Q02206|RSC4 YEAST (410)/ & 4 & RSC4 YEAST & RSC4 YEAST & 432 & 410 & $\begin{array}{l}4.60 \\
4.54\end{array}$ \\
\hline KLIAKPETVQSEVKNER(1)-KINYEQEAQKLEEK(1) & sp|Q02206|RSC4_YEAST (386)-sp|(P43609|RSC8-YYEAST (55)/ & 4 & RSC4_YEAST & RSC8 YEAST & 386 & 55 & 4.18 \\
\hline KLIAKPETVQSEVKNER(1)-EESATLGAEVAHKK(13) & sp|Q02206|RSC4_YEAST (386)-sp|P43609|RSC8_YEAST (54)/ & 8 & RSC4_YEAST & RSC8_YEAST & 386 & 54 & 4.18 \\
\hline SLLTEDYLLNPNNFKK(15)-VKVLLVDQQGLR(2) & sp|Q02206|RSC4-YEAST (385)-sp|P32832|NPL6_YEAST (340)/ & 4 & RSC4-YEAST & NPL6_YEAST & 385 & 340 & 4.17 \\
\hline KLIAKPETVQSEVK(1)-RIPGLINPWKR(10) & sp|Q02206|RSC4 YEAST (386)-sp|Q06488|RSC2 YEAST $(900) /$ & $\begin{array}{l}4 \\
9\end{array}$ & RSC4 YEAST & RSC2 YEAST & $\begin{array}{l}300 \\
386\end{array}$ & 900 & 4.15 \\
\hline 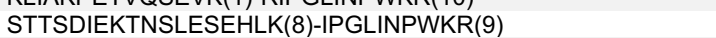 & sp|Q02206|RSC4_YEAST (410)-sp|Q06488|RSC2_YEAST (900)/ & 43 & RSC4-YEAST & RSC2 YEAST & 410 & 900 & 3.89 \\
\hline $\begin{array}{l}\text { LIAKPETVQSEVKNER(4)-KINYEQEAQKLEEK(1) } \\
\text { LINERER }\end{array}$ & sp|Q02206|RSC4 YEAST (390)-sp|P43609|RSC8 YEAST (55)/ & $\begin{array}{c}40 \\
5\end{array}$ & RSC4 YEAST & RSC8 YEAST & 390 & 55 & $\begin{array}{l}3.09 \\
3.86\end{array}$ \\
\hline 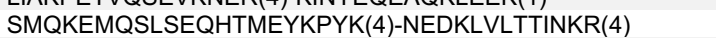 & sp|Q02206|RSC4_YEAST (432)-sp|Q07979|RSC58_YEAST (70)/ & 8 & RSC4-YEAST & RSC58_YEAST & 432 & 70 & 3.85 \\
\hline 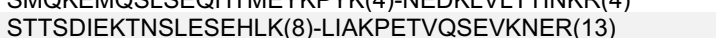 & sp|Q02206|RSC4 YEAST (410)-sp|Q02206|RSC4 YEAST (399)/ & 28 & RSC4-YEAST & RSC4 YEAST & 410 & 399 & $\begin{array}{l}3.05 \\
3.80\end{array}$ \\
\hline LIAKPETVQSEVKNER(4)-KINYEQEAQK(1) & sp|Q02206|RSC4_YEAST (390)-sp|P43609|RSC8 YEAST (55)/ & 5 & RSC4 YEAST & RSC8 YEAST & 390 & 55 & 3.54 \\
\hline KFHPQYYYKIQQPMSINEIK(9)-NQEKTPHVDYNAPLNPK(4) & sp|Q02206|RSC4_YEAST (95)-sp|Q02206|RSC4_YEAST (32)/ & 3 & RSC4_YEAST & RSC4_YEAST & 95 & 32 & 3.49 \\
\hline STTSDIEKTNSLESEHLK(8)-NEDKLVLTTINKR(12) & sp|Q02206|RSC4 YEAST (410)-sp|Q07979|RSC58 YEAST (78)/ & 1 & RSC4 YEAST & RSC58 YEAST & 410 & 78 & 3.46 \\
\hline SII TFDYY I NPNNFKK (15)-IIAKPFTVOSFVYKNFR (4) & 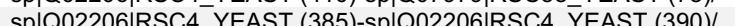 & 16 & RSCA YFAST & RSC4 $\bar{Y}$ FAST & 385 & 390 & 338 \\
\hline
\end{tabular}




\begin{tabular}{|c|c|c|c|c|c|c|c|}
\hline PEPTIDE & PROTEINS & CSM & PROTEIN1 & PROTEIN2 & R1 & $\mathbf{R 2}$ & $\mathbf{s}$ \\
\hline NQEKTPHVDYNAPLNPK(4)-GKQGGK(2) & sp|Q02206|RSC4_YEAST (32)-tr|Q6AZJ8|H2A XENLA (5)/ & 6 & RSC4_YEAST & H2A XENLA & 32 & 5 & 3.38 \\
\hline STTSDIEKTNSLESEHLK(8)-KNFIAVSAANR(1) & sp|Q02206|RSC4_YEAST (410)-sp|Q06488|RSC2_YEAST (905)/ & 8 & RSC4_YEAST & RSC2_YEAST & 410 & 905 & 3.37 \\
\hline YNVIKSMQK(5)-FIKNR(3) & sp|Q02206|RSC4_YEAST (428)-sp|Q07979|RSC58_YEAST (56)/ & 13 & RSC4_YEAST & RSC58_YEAST & 428 & 56 & 3.22 \\
\hline STTSDIEKTNSLESEHLK(8)-KLIAKPETVQSEVK(1) & sp|Q02206|RSC4_YEAST (410)-sp|Q02206|RSC4_Y̌EAST (386)/ & 10 & RSC4_YEAST & RSC4_Y̌EAST & 410 & 386 & 3.16 \\
\hline STTSDIEKTNSLESEHLKIPK(8)-LIAKPETVQSEVKNER(13) & sp|Q02206|RSC4_YEAST (410)-sp|Q02206|RSC4_YEAST (399)/ & 28 & RSC4_YEAST & RSC4_YEAST & 410 & 399 & 3.06 \\
\hline STTSDIEKTNSLESEHLKIPK(8)-KNFIAVSAANR(1) & sp|Q02206|RSC4_YEAST (410)-sp|Q06488|RSC2_YEAST (905)/ & 8 & RSC4_YEAST & RSC2 YEAST & 410 & 905 & 3.03 \\
\hline LIAKPETVQSEVKNER(13)-KINYEQEAQKLEEK(1) & sp|Q02206|RSC4_YEAST (399)-sp|P43609|RSC8_YEAST (55)/ & 3 & RSC4_YEAST & RSC8_YEAST & 399 & 55 & 2.96 \\
\hline KLIAKPETVQSEVK(1)-EESATLGAEVAHKK(13) & sp|Q02206|RSC4_YEAST (386)-sp|P43609|RSC8_YEAST (54)/ & 8 & RSC4_YEAST & RSC8_YEAST & 386 & 54 & 2.92 \\
\hline KLIAKPETVQSEVK(1)-KNFIAVSAANR(1) & sp|Q02206|RSC4_YEAST (386)-sp|Q06488|RSC2_YEAST (905)/ & 3 & RSC4_YEAST & RSC2 YEAST & 386 & 905 & 2.90 \\
\hline NQEKTPHVDYNAPLNPK(4)-KAVTK(1) & sp|Q02206|RSC4 YEAST (32)-sp|P02281|H2B11 XENLA (13)/ & 4 & RSC4-YEAST & H2B11 XENLA & 32 & 13 & 2.88 \\
\hline STTSDIEKTNSLESEHLK(8)-KLIAKPETVQSEVKNER(14) & sp|Q02206|RSC4_YEAST (410)-sp|Q02206|RSC4_YEAST (399)/ & 28 & RSC4_YEAST & RSC4_YEAST & 410 & 399 & 2.88 \\
\hline NQEKTPHVDYNAPLNPK(4)-KGSKK(1) & sp|Q02206|RSC4_YEAST (32)-sp|P02281|H2B11_XENLA (9)/ & 3 & RSC4_YEAST & H2B11_XENLA & 32 & 9 & 2.79 \\
\hline LIAKPETVQSEVKNER(4)-VKVLLVDQQGLR(2) & sp|Q02206|RSC4_YEAST (390)-sp|P32832|NPL6_YEAST (340)/ & 2 & RSC4_YEAST & NPL6_Y̌EAST & 390 & 340 & 2.78 \\
\hline 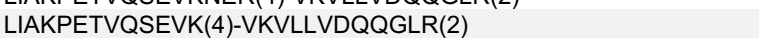 & sp|Q02206|RSC4_YEAST (390)-sp|P32832|NPL6_YEAST (340)/ & 2 & RSC4_YEAST & NPL6_YEAST & 390 & 340 & 2.53 \\
\hline LIAKPETVQSEVKNER(4)-EESATLGAEVAHKK(13) & sp|Q02206|RSC4_YEAST (390)-sp|P43609|RSC8_YEAST (54)/ & 3 & RSC4_YEAST & RSC8_YEAST & 390 & 54 & 2.51 \\
\hline KLIAKPETVQSEVK(1)-IPGLINPWKR(9) & sp|Q02206|RSC4_YEAST (386)-sp|Q06488|RSC2_YEAST (900)/ & 9 & RSC4_YEAST & RSC2_YEAST & 386 & 900 & 2.51 \\
\hline STTSDIEKTNSLESEHLK(8)-LIAKPETVQSEVKNER(4) & sp|Q02206|RSC4_YEAST (410)-sp|Q02206|RSC4_YEAST (390)/ & 3 & RSC4_YEAST & RSC4_YEAST & 410 & 390 & 2.48 \\
\hline TNSLESEHLKIPK(10)-KNFIAVSAANR(1) & sp|Q02206|RSC4_YEAST (420)-sp|Q06488|RSC2_YEAST (905)/ & 12 & RSC4_YEAST & RSC2 YEAST & 420 & 905 & 2.45 \\
\hline STTSDIEKTNSLESEHLK(8)-RIPGLINPWKR(10) & sp|Q02206|RSC4_YEAST (410)-sp|Q06488|RSC2_YEAST (900)/ & 43 & RSC4_YEAST & RSC2_YEAST & 410 & 900 & 2.37 \\
\hline INQALLGASSPKNLDDKVK(12)-LVDATEKK(7) & sp|Q02206|RSC4_YEAST (201)-sp|Q02206|RSC4_YEAST (188)/ & 6 & RSC4_YEAST & RSC4_YEAST & 201 & 188 & 2.36 \\
\hline STTSDIEKTNSLESEHLKIPK(8)-IPGLINPWKR(9) & sp|Q02206|RSC4_YEAST (410)-sp|Q06488|RSC2_YEAST (900)/ & 43 & RSC4_YEAST & RSC2_YEAST & 410 & 900 & 2.29 \\
\hline LIAKPETVQSEVK(4)-KNFIAVSAANR(1) & sp|Q02206|RSC4_YEAST (390)-sp|Q06488|RSC2_YEAST (905)/ & 1 & RSC4_YEAST & RSC2 YEAST & 390 & 905 & 2.04 \\
\hline NQEKTPHVDYNAPLNPK(4)-KLATEAGGSDERPK(1) & sp|Q02206|RSC4_YEAST (32)-sp|Q02206|RSC4_Y̌EAST (7)/ & 1 & RSC4_YEAST & RSC4_YEAST & 32 & 7 & 2.00 \\
\hline TNSLESEHLKIPK(10)-NEDKLVLTTINK(4) & sp|Q02206|RSC4_YEAST (420)-sp|Q07979|RSC5̄8_YEAST (70)/ & 4 & RSC4_YEAST & RSC50__YEAST & 420 & 70 & 1.99 \\
\hline SLLTEDYLLNPNNFKK(15)-LIAKPETVQSEVKNER(13) & sp|Q02206|RSC4_YEAST (385)-sp|Q02206|RSC4_YEAST (399)/ & 8 & RSC4_YEAST & RSC4_Y̌EAST & 385 & 399 & 1.99 \\
\hline 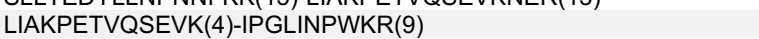 & sp|Q02206|RSC4_YEAST (390)-sp|Q06488|RSC2_YYAST (900)/ & 5 & RSC4_YEAST & RSC2_YEAST & 390 & 900 & 1.94 \\
\hline KLATEAGGSDERPK(1)-VVKKR(4) & sp|Q02206|RSC4_YEAST (7)-sp|Q02206|RSC4_YEAST (5)/ & 1 & RSC4_YEAST & RSC4_YEAST & 7 & 5 & 1.92 \\
\hline SMQKEMQSLSEQHTMEYKPYK(4)-NEDKLVLTTINK(4) & sp|Q02206|RSC4_YEAST (432)-sp|Q07979|RSC̄58_YEAST (70)/ & 8 & RSC4_YEAST & RSC $5 \overline{8}$ YYEAST & 432 & 70 & 1.87 \\
\hline STTSDIEKTNSLESEHLKIPK(8)-KLIAKPETVQSEVKNER(14) & sp|Q02206|RSC4_YEAST (410)-sp|Q02206|RSC4_Y YEAST (399)/ & 28 & RSC4_YEAST & RSC4_Y̌EAST & 410 & 399 & 1.84 \\
\hline STTSDIEKTNSLESEHLK(8)-IPGLINPWKRR(9) & sp|Q02206|RSC4_YEAST (410)-sp|Q06488|RSC2_YYAST (900)/ & 43 & RSC4_YEAST & RSC2_YEAST & 410 & 900 & 1.73 \\
\hline STTSDIEKTNSLESEHLKIPK(8)-KLIAKPETVQSEVKNER(1) & sp|Q02206|RSC4_YEAST (410)-sp|Q02206|RSC4_YEAST (386)/ & 10 & RSC4_YEAST & RSC4_YEAST & 410 & 386 & 1.68 \\
\hline STTSDIEKTNSLESEHLK(8)-LIAKPETVQSEVK(4) & sp|Q02206|RSC4_YEAST (410)-sp|Q02206|RSC4_YEAST (390)/ & 3 & RSC4_YEAST & RSC4_YEAST & 410 & 390 & 1.64 \\
\hline LIQQIYIFSSKNLYSQATKPLLGSR(11)-NSEAKR(5) & sp|Q02206|RSC4_YEAST (460)-sp|P38210|LDB7_YEAST (149)/ & 1 & RSC4_YEAST & LDB7_YEAST & 460 & 149 & 1.64 \\
\hline SLLTEDYLLNPNNFKK(15)-IPGLINPWKR(9) & sp|Q02206|RSC4_YEAST (385)-sp|Q06488|RSC2_YYAST (900)/ & 3 & RSC4_YEAST & RSC2_YEAST & 385 & 900 & 1.59 \\
\hline LIAKPETVQSEVKNER(13)-TNSLESEHLKIPK(10) & sp|Q02206|RSC4_YEAST (399)-sp|Q02206|RSC4_YEAST (420)/ & 2 & RSC4_YEAST & RSC4_YEAST & 399 & 420 & 1.52 \\
\hline LIAKPETVQSEVKNER(13)-IPGLINPWKR(9) & sp|Q02206|RSC4_YEAST (399)-sp|Q06488|RSC2_YYAST (900)/ & 8 & RSC4_YEAST & RSC2_YEAST & 399 & 900 & 1.45 \\
\hline SLLTEDYLLNPNNFKK(15)-LIAKPETVQSEVK(4) & sp|Q02206|RSC4_YEAST (385)-sp|Q02206|RSC4_YEAST (390)/ & 16 & RSC4_YEAST & RSC4_YEAST & 385 & 390 & 1.41 \\
\hline $\begin{array}{l}\text { LIAKPETVQSEVKNER(13)-RIPGLINPWKR(10) } \\
\text { LIPKR }\end{array}$ & sp|Q02206|RSC4_YEAST (399)-sp|Q06488|RSC2_YEAST (900)/ & 8 & RSC4_YEAST & RSC2-YEAST & 399 & 900 & 1.27 \\
\hline SMQKEMQSLSEQHTMEYKPYK(4)-FIKNR(3) & sp|Q02206|RSC4_YEAST (432)-sp|Q07979|RSC58_YEAST (56)/ & 3 & RSC4_YEAST & RSC58_YEAST & 432 & 56 & 1.24 \\
\hline TNSLESEHLKIPK(10)-YNVIKSMQK(5) & sp|Q02206|RSC4_YEAST (420)-sp|Q02206|RSC4_YEAST (428)/ & 1 & RSC4_YEAST & RSC4_YEAST & 420 & 428 & 1.21 \\
\hline STTSDIEKTNSLESEHLK(8)-SGREEMFPWKK(10) & sp|Q02206|RSC4_YEAST (410)-sp|Q06488|RSC2_YEAST (783)/ & 1 & RSC4_YEAST & RSC2_YEAST & 410 & 783 & 1.20 \\
\hline LIAKPETVQSEVKNER(4)-GGAKR(4) & sp|Q02206|RSC4_YEAST (390)-sp|P62799|H4_XENLA (16)/ & 3 & RSC4_YEAST & H4 XENLA & 390 & 16 & 1.17 \\
\hline STTSDIEKTNSLESEHLKIPK(8)- & sp|Q02206|RSC4_YEAST (410)-sp|Q06488|RSC2_YEAST (900)/ & 43 & RSC4_YEAST & RSC2_YEAST & 410 & 900 & 1.16 \\
\hline LIAKPETVQSEVKNER(4)-GKGGK(2) & sp|Q02206|RSC4_Y_YEAST (390)-sp|P62799|H4_XĒNLA (5)/ & 1 & RSC4_YEAST & H4_XĒNLA & 390 & 5 & 1.13 \\
\hline $\begin{array}{l}\text { TSNVNSNLSQPQQQENDVIGNDTKQDIENLTIGGGNNNDIVGNDND } \\
\text { KR(47)-IGIALTQTNNIKSR(12) }\end{array}$ & sp|Q02206|RSC4_YEAST (583)-sp|Q07979|RSC58_YEAST (149)/ & 1 & RSC4_YEAST & RSC58_YEAST & 583 & 149 & 1.03 \\
\hline LIAKPETVQSEVK(4)-RIPGLINPWKR(10) & sp|Q02206|RSC4_YEAST (390)-sp|Q06488|RSC2_YEAST (900)/ & 5 & RSC4_YEAST & RSC2 YEAST & 390 & 900 & 1.03 \\
\hline KLIAKPETVQSEVK(1)-GGAKR(4) & sp|Q02206|RSC4_YEAST (386)-sp|P62799|H4_XEENLA (16)/ & 2 & RSC4_YEAST & H4_XĒNLA & 386 & 16 & 0.87 \\
\hline STTSDIEKTNSLESEHLKIPK(8)-NEDKLVLTTINKR(4) & sp|Q02206|RSC4_YEAST (410)-sp|Q07979|RSC58_YEAST (70)/ & 5 & RSC4_YEAST & RSC58_YEAST & 410 & 70 & 0.77 \\
\hline LIAKPETVQSEVKNER(4)-INYEQEAQKLEEK(9) & sp|Q02206|RSC4_YEAST (390)-sp|P43609|RSC8_YEAST (64)/ & 1 & RSC4_YEAST & RSC8_Y̌YAST & 390 & 64 & 0.75 \\
\hline
\end{tabular}




\begin{tabular}{|c|c|c|c|c|c|c|c|}
\hline PEPTIDE & PROTEINS & CSM & PROTEIN1 & PROTEIN2 & R1 & R2 & $\mathbf{s}$ \\
\hline $\begin{array}{l}\text { KLIAKPETVQSEVKNER(1)-RIPGLINPWKR(10) } \\
\end{array}$ & sp|Q02206|RSC4_YEAST (386)-sp|Q06488|RSC2_YEAST (900)/ & 9 & RSC4_YEAST & RSC2_YEAST & 386 & 900 & 0.66 \\
\hline LIAKPETVQSEVKNER(4)-RIPGLINPWKR(10) & sp|Q02206|RSC4_YEAST (390)-sp|Q06488|RSC2_YEAST (900)/ & 5 & RSC4_YEAST & RSC2_YEAST & 390 & 900 & 0.64 \\
\hline LIAKPETVQSEVKNER(13)-NEDKLVLTTINKR(4) & sp|Q02206|RSC4_YEAST (399)-sp|Q07979|RSC58_YEAST (70)/ & 1 & RSC4_YEAST & RSC58_YEAST & 399 & 70 & 0.62 \\
\hline LIAKPETVQSEVKNER(13)-IPGLINPWKRR(9) & sp|Q02206|RSC4_YEAST (399)-sp|Q06488|RSC2_Y̌EAST (900)/ & 8 & RSC4_YEAST & RSC2_Y̌YEAST & 399 & 900 & 0.62 \\
\hline YNVIKSMQK(5)-SPAKK(4) & sp|Q02206|RSC4_YEAST (428)-sp|P32597|STH1_YEAST (1235)/ & 1 & RSC4_YEAST & STH1_YEAST & 428 & 1235 & 0.58 \\
\hline TNSLESEHLKIPK(10)-RIPGLINPWKR(10) & sp|Q02206|RSC4_YEAST (420)-sp|Q06488|RSC2_YEAST (900)/ & 4 & RSC4_YEAST & RSC2_YEAST & 420 & 900 & 0.56 \\
\hline LVDATEKK(7)-NLDDKVK(5) & sp|Q02206|RSC4_YEAST (188)-sp|Q02206|RSC4_YEAST (206)/ & 2 & RSC4_YEAST & RSC4_YEAST & 188 & 206 & 0.89 \\
\hline KLATEAGGSDERPK(1)-VVKKR(3) & sp|Q02206|RSC4_YEAST (7)-sp|Q02206|RSC4_YEAST (4)/ & 1 & RSC4_YEAST & RSC4_YEAST & 7 & 4 & 0.86 \\
\hline SLLTEDYLLNPNNFKK(15)-KEFPVNLTIK(1) & sp|Q02206|RSC4_YEAST (385)-sp|P43609|RSC8_YEAST (222)/ & 1 & RSC4_YEAST & RSC8_YEAST & 385 & 222 & 0.51 \\
\hline INQALLGASSPKNLDDK(12)-LVDATEKK(7) & sp|Q02206|RSC4_YEAST (201)-sp|Q02206|RSC4_YEAST (188)/ & 6 & RSC4_YEAST & RSC4_YEAST & 201 & 188 & 0.81 \\
\hline LIQQIYIFSSKNLYSQATK(11)-YPKIR(3) & sp|Q02206|RSC4_YEAST (460)-sp|Q06168|SFH1_YEAST (170)/ & 1 & RSC4_YEAST & SFH1_YEAST & 460 & 170 & 0.49 \\
\hline LIAKPETVQSEVKNER(13)-LENNGNSVKK(9) & sp|Q02206|RSC4_YEAST (399)-sp|P43609|RSC8_YEAST (312)/ & 1 & RSC4_YEAST & RSC8_YEAST & 399 & 312 & 0.46 \\
\hline TNSLESEHLKIPK(10)-IPGLINPWKR(9) & sp|Q02206|RSC4_YEAST (420)-sp|Q06488|RSC2_YEAST (900)/ & 4 & RSC4_YEAST & RSC2_YEAST & 420 & 900 & 0.45 \\
\hline TNSLESEHLKIPK(10)-LVLTTINKR(8) & sp|Q02206|RSC4_YEAST (420)-sp|Q07979|RSC58_YEAST (78)/ & 1 & RSC4_YEAST & RSC58_YEAST & 420 & 78 & 0.37 \\
\hline SLLTEDYLLNPNNFKK(15)-GGAKR(4) & sp|Q02206|RSC4_YEAST (385)-sp|P62799|H4_XENLLA (16)/ & 1 & RSC4_YEAST & H4_XENTLA & 385 & 16 & 0.35 \\
\hline LIAKPETVQSEVKNER(13)-EESATLGAEVAHKK(13) & sp|Q02206|RSC4_YEAST (399)-sp|P43609|RSC8_YEAST (54)/ & 1 & RSC4_YEAST & RSC8_YEAST & 399 & 54 & 0.33 \\
\hline FVIKGIQPR(4)-SKFLNDSK(2) & sp|Q03124|RSC9 YEAST (470)-sp|Q03124|RSC9 YEAST (498)/ & 16 & RSC9 YEAST & RSC9 ${ }^{-}$YEAST & 470 & 498 & 15.49 \\
\hline ESQVPISALKSK(10)-FVIKGIQPR(4) & sp|Q03124|RSC9_YEAST (496)-sp|Q03124|RSC9_YEAST (470)/ & 26 & RSC9_YEAST & RSC9_YEAST & 496 & 470 & 12.63 \\
\hline SKECSLILEATLPNLLSYNIATPDYHLLQKHK(30)- & sp|Q03124|RSC9_YEAST (358)-sp|P25632|RSC6_YEAST (2)/ & 14 & RSC9_YEAST & RSC6_YEAST & 358 & 2 & 7.24 \\
\hline $\begin{array}{l}\text { VTQTNPVPVTYPTDAYIPTYLPDDK(1) } \\
\text { SKFLNDSKEITPAR(2)-LKPPAPK(2) }\end{array}$ & sp|Q03124|RSC9 YEAST (498)-sp|Q03124|RSC9 YEAST (368)/ & 31 & RSC9 YEAST & RSC9 YEAST & 498 & 368 & 5.40 \\
\hline $\begin{array}{l}\text { SKFLNDSKEITPAR(2)-LKPPAPK(2) } \\
\text { KLLPAVEFIK(1)-AKVSKPK(2) }\end{array}$ & sp|Q03124|RSC9_YEAST (434)-Sp|P38210|LDB7_YEAST (54)/ & 12 & RSC9_YEAST & LDB7_YEAST & 434 & 54 & 4.92 \\
\hline $\begin{array}{l}\text { LKPPAPKEPPNLSEDLFQQLFK(7)- } \\
\text { NVSNAFNNAAIVITDPVTGKK(21) }\end{array}$ & sp|Q03124|RSC9_YEAST (373)-sp|Q03124|RSC9_YEAST (464)/ & 7 & RSC9_YEAST & RSC9_YEAST & 373 & 464 & 4.01 \\
\hline FKALGIADGER(2)-LKPPAPK(2) & sp|Q03124|RSC9 YEAST (477)-sp|Q03124|RSC9 YEAST (368)/ & 29 & RSC9 YEAST & RSC9 YEAST & 477 & 368 & 3.99 \\
\hline KLLPAVEFIK(1)-VSKPKR(5) & sp|Q03124|RSC9_YEAST (434)-sp|P38210|LDB7_YEAST (59)/ & 6 & RSC9_YEAST & LDB7_YEAST & 434 & 59 & 3.93 \\
\hline FLNDSKEITPAR(6)-ESQVPISALKSK(10) & sp|Q03124|RSC9_YEAST (504)-sp|Q03124|RSC9-YEAST (496)/ & 23 & RSC9_YEAST & RSC9_YEAST & 504 & 496 & 3.76 \\
\hline SKFLNDSK(2)-LKPPAPK(2) & sp|Q03124|RSC9_YEAST (498)-sp|Q03124|RSC9_YEAST (368)/ & 31 & RSC9_YEAST & RSC9_YEAST & 498 & 368 & 3.72 \\
\hline ALGIADGERESQVPISALKSK(19)-FLNDSKEITPAR(6) & sp|Q03124|RSC9_YEAST (496)-sp|Q03124|RSC9_YEAST (504)/ & 5 & RSC9-YEAST & RSC9_YEAST & 496 & 504 & 3.58 \\
\hline SKANEESAADNLDHK(2)-QQLLEKR(6) & sp|Q03124|RSC9_YEAST (274)-sp|P32597|STH1_YEAST (315)/ & 18 & RSC9_YEAST & STH1_YEAST & 274 & 315 & 3.54 \\
\hline LKPPAPKEPPNLSEDLFQLFK(7)-SKFLNDSKEITPAR(2) & sp|Q03124|RSC9_YEAST (373)-sp|Q03124|RSC9_YEAST (498)/ & 13 & RSC9_YEAST & RSC9_YEAST & 373 & 498 & 3.48 \\
\hline LKPPAPKEPPNLSEDLFQQLFK(7)-LQPQVYKPWSL(7) & sp|Q03124|RSC9_YEAST (373)-sp|P43609|RSC8_YEAST (553)/ & 2 & RSC9_YEAST & RSC8_YEAST & 373 & 553 & 3.40 \\
\hline FKALGIADGER(2)-SKFLNDSK(2) & sp|Q03124|RSC9_YEAST (477)-sp|Q03124|RSC9_YEAST (498)/ & 7 & RSC9-YEAST & RSC9 YEAST & 477 & 498 & 3.33 \\
\hline FVIKGIQPR(4)-LKPPAPK(2) & sp|Q03124|RSC9_YEAST (470)-sp|Q03124|RSC9_YEAST (368)/ & 9 & RSC9_YEAST & RSC9_YEAST & 470 & 368 & 3.28 \\
\hline SKFLNDSK(2)-RLKPPAPK(3) & sp|Q03124|RSC9_YEAST (498)-sp|Q03124|RSC9_YEAST (368)/ & 31 & RSC9_YEAST & RSC9_YEAST & 498 & 368 & 3.25 \\
\hline ESQVPISALKSK(10)-FKALGIADGER(2) & sp|Q03124|RSC9_YEAST (496)-sp|Q03124|RSC9_YEAST (477)/ & 7 & RSC9_YEAST & RSC9_YEAST & 496 & 477 & 3.13 \\
\hline SKECSLILEATLPNLLSYNIATPDYHLLQKHK(30)- & sp|Q03124|RSC9_YEAST (358)-sp|P25632|RSC6_YEAST (2)/ & 14 & RSC9_YEAST & RSC6_YEAST & 358 & 2 & 3.01 \\
\hline $\begin{array}{l}\text { VTQTNPVPVTYPTDAYIPTYLPDDKVSNLADLK(1) } \\
\text { FLNDSKEITPAR(6)-LKPPAPK(2) }\end{array}$ & sp|Q03124|RSC9_YEAST (504)-sp|Q03124|RSC9_YEAST (368)/ & 21 & RSC9_YEAST & YEAST & 504 & 368 & 2.99 \\
\hline KLLPAVEFIK(1)-KQNDENNK(1) & sp|Q03124|RSC9_YEAST (434)-sp|Q07979|RSC58_YEAST (343)/ & 1 & RSC9_YEAST & RSC58_YEAST & 434 & 343 & 2.92 \\
\hline FLNDSKEITPAR(6)-LKPLGMGK(2) & sp|Q03124|RSC9_YEAST (504)-sp|P25632|RSC6 Y̌ YEAST (135)/ & 20 & RSC9-YEAST & RSC6 YEAST & 504 & 135 & 2.87 \\
\hline ESQVPISALKSK(10)-LKPLGMGK(2) & sp|Q03124|RSC9_YEAST (496)-sp|P25632|RSC6_YEAST (135)/ & 10 & RSC9_YEAST & RSC6_YEAST & 496 & 135 & 2.73 \\
\hline FLNDSKEITPAR(6)-LKPLGMGKK(2) & sp|Q03124|RSC9_YEAST (504)-sp|P25632|RSC6_YEAST (135)/ & 20 & RSC9_YEAST & RSC6_YEAST & 504 & 135 & 2.65 \\
\hline FKALGIADGER(2)-RLKPPAPK(3) & sp|Q03124|RSC9 YEAST (477)-sp|Q03124|RSC9 YEAST (368)/ & 29 & RSC9 YEAST & RSC9 ${ }^{-}$YEAST & 477 & 368 & 2.62 \\
\hline SKFLNDSKEITPAR(2)-FKALGIADGER(2) & sp|Q03124|RSC9_YEAST (498)-sp|Q03124|RSC9_YEAST (477)/ & 1 & RSC9-YEAST & RSC9-YEAST & 498 & 477 & 2.57 \\
\hline KLLPAVEFIK(1)-GIEGSENSKAK(9) & sp|Q03124|RSC9_YEAST (434)-sp|P38210|LDB7_YEAST (52)/ & 4 & RSC9_YEAST & LDB7_YEAST & 434 & 52 & 2.56 \\
\hline ESQVPISALKSK(10)-GKVDGKR(2) & sp|Q03124|RSC9 YEAST (496)-sp|P38781|RSC30 YEAST (258)/ & 3 & RSC9 YEAST & RSC 30 YEAST & 496 & 258 & 2.44 \\
\hline LKPPAPKEPPNLSEDLFQQLFK(7)-FLNDSKEITPAR(6) & sp|Q03124|RSC9_YEAST (373)-sp|Q03124|RSC9_YEAST (504)/ & 2 & RSC9_YEAST & RSC9_Y̌YEAST & 373 & 504 & 2.42 \\
\hline FLNDSKEITPAR(6)-FKALGIADGER(2) & sp|Q03124|RSC9 YEAST (504)-sp|Q03124|RSC9 YEAST (477)/ & 7 & RSC9 YEAST & RSC9 ${ }^{-}$YEAST & 504 & 477 & 2.40 \\
\hline ESQVPISALKSK(10)-LKPPAPK(2) & sp|Q03124|RSC9_YEAST (496)-sp|Q03124|RSC9_YEAST (368)/ & 19 & RSC9-YEAST & RSC9_YEAST & 496 & 368 & 2.40 \\
\hline
\end{tabular}




\begin{tabular}{|c|c|c|c|c|c|c|c|}
\hline PEPTIDE & PROTEINS & CSM & PROTEIN1 & PROTEIN2 & R1 & $\mathbf{R 2}$ & $\mathbf{s}$ \\
\hline KLLPAVEFIK(1)-VSKPK(3) & sp|Q03124|RSC9_YEAST (434)-sp|P38210|LDB7_YEAST (57)/ & 4 & RSC9_YEAST & LDB7_YEAST & 434 & 57 & 2.39 \\
\hline ECSLILEATLPNLLSYNIATPDYHLLQKHK(28)- & sp|Q03124|RSC9_YEAST (358)-sp|P25632|RSC6_YEAST (2)/ & 14 & RSC9_YEAST & RSC6_YEAST & 358 & 2 & 2.38 \\
\hline LKPPAPKEPPNLSEDLFQQLFK(2)-ESQVPISALKSK(10) & sp|Q03124|RSC9_YEAST (368)-sp|Q03124|RSC9_YEAST (496)/ & 1 & RSC9_YEAST & RSC9_YEAST & 368 & 496 & 2.12 \\
\hline $\begin{array}{l}\text { SIHYSLANFNVFQSLPKETAR(17)- } \\
\text { VGSPGAGGPLTVQGLKQHQLQR(16) }\end{array}$ & sp|Q03124|RSC9_YEAST (73)-sp|Q06488|RSC2_YEAST (695)/ & 5 & RSC9_YEAST & RSC2_YEAST & 73 & 695 & 2.04 \\
\hline LKPPAPKEPPNLSEDLFQQLFK(7)-SKFLNDSK(2) & sp|Q03124|RSC9_YEAST (373)-sp|Q03124|RSC9_YEAST (498)/ & 13 & RSC9_YEAST & RSC9_YEAST & 373 & 498 & 2.04 \\
\hline SKFLNDSKEITPAR(2)-FVIKGIQPR(4) & sp|Q03124|RSC9_YEAST (498)-sp|Q03124|RSC9_YEAST (470)/ & 6 & RSC9_YEAST & RSC9_YEAST & 498 & 470 & 1.96 \\
\hline NVSNAFNNAAAIVITDPVTGKK(21)-SDSGTPADSGKTTWTLR(11) & sp|Q03124|RSC9_YEAST (464)-sp|P25632|RSC6_YEAST (98)/ & 2 & RSC9_YEAST & RSC6_YEAST & 464 & 98 & 1.88 \\
\hline $\begin{array}{l}\text { LKPPAPKEPPNLSEDLFQQLFK(2)-FKALGIADGERESQVPISALK(2) } \\
\text { (2) }\end{array}$ & sp|Q03124|RSC9 YEAST (368)-sp|Q03124|RSC9 YEAST (477)/ & 1 & RSC9 YEAST & RSC9 YEAST & 368 & 477 & 1.79 \\
\hline RFVIKGIQPR(5)-SKFLNDSK(2) & sp|Q03124|RSC9_YEAST (470)-sp|Q03124|RSC9_YEAST (498)/ & 16 & RSC9_YEAST & RSC9_YEAST & 470 & 498 & 1.65 \\
\hline LKPPAPKEPPNLSEDLFQQLFK(7)-ESQVPISALKSK(10) & sp|Q03124|RSC9_YEAST (373)-sp|Q03124|RSC9_YEAST (496)/ & 3 & RSC9-YEAST & RSC9_YEAST & 373 & 496 & 1.63 \\
\hline FLNDSKEITPAR(6)-LKPLGMGKK(8) & sp|Q03124|RSC9_YEAST (504)-sp|P25632|RSC6_YEAST (141)/ & 2 & RSC9_YEAST & RSC6_YEAST & 504 & 141 & 1.37 \\
\hline FVIKGIQPR(4)-QGKDNLR(3) & sp|Q03124|RSC9 YEAST (470)-sp|P25632|RSC6 YEAST (213)/ & 3 & RSC9 YEAST & RSC6 YEAST & 470 & 213 & 1.34 \\
\hline SKFLNDSKEITPAR(8)-FKALGIADGER(2) & sp|Q03124|RSC9_YEAST (504)-sp|Q03124|RSC9_YEAST (477)/ & 7 & RSC9_YEAST & RSC9_YEAST & 504 & 477 & 1.33 \\
\hline SKFLNDSKEITPAR(8)-LKPPAPK(2) & sp|Q03124|RSC9_YEAST (504)-sp|Q03124|RSC9_YEAST (368)/ & 21 & RSC9-YEAST & RSC9_YEAST & 504 & 368 & 1.28 \\
\hline ESQVPISALKSK(10)-TGENSKK(6) & sp|Q03124|RSC9_YEAST (496)-sp|P38781|RSC30_YEAST (255)/ & 2 & RSC9_YEAST & RSC30_YEAST & 496 & 255 & 1.09 \\
\hline ESQVPISALKSK(10)-LKPLGMGKK(2) & sp|Q03124|RSC9_YEAST (496)-sp|P25632|RSC6_YEAST (135)/ & 10 & RSC9-YEAST & RSC6 YEAST & 496 & 135 & 0.90 \\
\hline SKFLNDSK(2)-LKPLGMGK(2) & sp|Q03124|RSC9_YEAST (498)-sp|P25632|RSC6_YEAST (135)/ & 1 & RSC9 YEAST & RSC6_YEAST & 498 & 135 & 0.86 \\
\hline EPPNLSEDLFQQLFKLNEPLR(15)-FSNATKTALGDPDTEIR(6) & sp|Q03124|RSC9_YEAST (388)-sp|P32597|STH1_YEAST (183)/ & 1 & RSC9-YEAST & STH1_YEAST & 388 & 183 & 0.76 \\
\hline QNSIPEVKFPQELSDVSK(8)-ESQVPISALKSK(10) & sp|Q03124|RSC9_YEAST (518)-sp|Q03124|RSC9_YEAST (496)/ & 1 & RSC9_YEAST & RSC9_YEAST & 518 & 496 & 0.96 \\
\hline ALGIADGERESQVPISALKSK(19)-FVIKGIQPR(4) & sp|Q03124|RSC9_YEAST (496)-sp|Q03124|RSC9_YEAST (470)/ & 26 & RSC9-YEAST & RSC9_YEAST & 496 & 470 & 0.95 \\
\hline MEMALLSGIPEEIKWSLKK(18)-YLTYSNKAPYMISLR(7) & sp|Q03124|RSC9_YEAST (102)-sp|Q03124|RSC9_YEAST (110)/ & 1 & RSC9_YEAST & RSC9_YEAST & 102 & 110 & 0.93 \\
\hline ESQVPISALKSK(10)-RLKPPAPK(3) & sp|Q03124|RSC9_YEAST (496)-sp|Q03124|RSC9_YEAST (368)/ & 19 & RSC9_YEAST & RSC9_YEAST & 496 & 368 & 0.84 \\
\hline FLNDSKEITPAR(6)-RLKPPAPK(3) & sp|Q03124|RSC9_YEAST (504)-sp|Q03124|RSC9_YEAST (368)/ & 21 & RSC9_YEAST & RSC9_YEAST & 504 & 368 & 0.79 \\
\hline ESQVPISALKSK(10)-NQEEFLTIKK(9) & sp|Q03124|RSC9_YEAST (496)-sp|Q06639|RSC3_YEAST (231)/ & 2 & RSC9_YEAST & RSC3_YEAST & 496 & 231 & 0.46 \\
\hline SKANEESAADNLDHK(2)-RKKER(2) & sp|Q03124|RSC9_YEAST (274)-sp|P32597|STH1_YEAST (317)/ & 1 & RSC9_YEAST & STH1_YEAST & 274 & 317 & 0.43 \\
\hline KLLPAVEFIK(1)-GRKMKK(3) & sp|Q03124|RSC9_YEAST (434)-sp|Q06639|RSC3_YEAST (7)/ & 1 & RSC9_YEAST & RSC3_YEAST & 434 & 7 & 0.40 \\
\hline $\begin{array}{l}\text { RLKPPAPKEPPNLSEDLFQQLFK(3)- } \\
\text { PFLPENVIKQEVEGGDGAEPQVK(9) }\end{array}$ & sp|Q03124|RSC9_YEAST (368)-sp|P43609|RSC8_YEAST (207)/ & 1 & RSC9_YEAST & RSC8_YEAST & 368 & 207 & 0.39 \\
\hline ALGIADGERESQVPISALKSK(19)-LKPI & sp|Q03124|RSC9 YEAST (496)-sp|Q03124|RSC9 YEAST (368)/ & 19 & RSC9 Y & Rs & 496 & 368 & 0.59 \\
\hline FKALGIADGERESQVPISALK(2)-LKPPAPK(2) & sp|Q03124|RSC9_YEAST (477)-sp|Q03124|RSC9_YEAST (368)/ & 29 & RSC9_YEAST & RSC9_YEAST & 477 & 368 & 0.56 \\
\hline FKALGIADGER(2)-IRLIKR(5) & sp|Q03124|RSC9_YEAST (477)-sp|Q03124|RSC9_YEAST (365)/ & 1 & RSC9_YEAST & RSC9_YEAST & 477 & 365 & 0.45 \\
\hline KFQCVATGDNKWQLYEGNATFFNELTHYTLDLMEAISSYIAPAMK(1 & sp|Q03124|RSC9_YEAST (204)-sp|Q03124|RSC9_YEAST (2)/ & 1 & RSC9_YEAST & RSC9_YEAST & 204 & 2 & 0.44 \\
\hline NVSNAFNNAAAIVITDPVTGKK(21)-YLTYSNKAPYMISLR(7) & sp|Q03124|RSC9_YEAST (464)-sp|Q03124|RSC9_YEAST (110)/ & 1 & RSC9_YEAST & RSC9_YEAST & 464 & 110 & 0.41 \\
\hline QRFQGCNNLIKNISNR(11)-KLLPQWDDDQIESLKK(1) & sp|Q05123|ARP9 YEAST (307)-sp|Q05123|ARP9 YEAST (200)/ & 2 & ARP9 YEAST & ARP9 YEAST & 307 & 200 & 5.86 \\
\hline KLLPQWDDDQIESLKK(1)-FQGCNNLIKNISNR(9) & sp|Q05123|ARP9 YEAST (200)-sp|Q05123|ARP9 YEAST (307)/ & 18 & ARP9 YEAST & ARP9 YEAST & 200 & 307 & 5.69 \\
\hline GNEIKVGK(5)-VKNVK(2) & sp|Q05123|ARP9_YEAST (293)-sp|Q05123|ARP9_YEAST (272)/ & 20 & ARP9_YEAST & ARP9 YEAST & 293 & 272 & 4.71 \\
\hline KSGYSEIIFLGAQIVSK(1)-KSLMTSHTK(1) & sp|Q05123|ARP9_YEAST (421)-sp|P53330|RT102_YEAST (94)/ & 9 & ARP9 YEAST & RT102_YEAST & 421 & 94 & 4.54 \\
\hline SPIFEVLSDDAKK(12)-VGKQR(3) & sp|Q05123|ARP9_YEAST (227)-sp|Q05123|ARP9_YEAST (296)/ & 8 & ARP9_YEAST & ARP9_YEAST & 227 & 296 & 4.38 \\
\hline QIFTHPKDTFYITR(7)-APFRQDSILIIYPR(1) & sp|Q05123|ARP9 YEAST (444)-sp|Q05123|ARP9 YEAST (2)/ & 13 & ARP9 YEAST & ARP9 YEAST & 444 & 2 & 4.19 \\
\hline $\begin{array}{l}\text { SKFMTNSTAFVPTIEYVQCPTVIK(2)-SVLPAATKK (8) } \\
\text { SKFMTIN }\end{array}$ & sp|Q05123|ARP9 YEAST (386)-sp|Q05123|ARP9 YEAST (382)/ & 12 & ARP9 YEAST & ARP9 YEAST & 386 & 382 & 4.16 \\
\hline FQGCNNLIKNISNR(9)-SPIFEVLSDDAKK(12) & sp|Q05123|ARP9_YEAST (307)-sp|Q05123|ARP9_YEAST (227)/ & 9 & ARP9_YEAST & ARP9 YEAST & 307 & 227 & 4.05 \\
\hline NSDLEFNTFWDEKGNEIKVGK(18)-VKNVK(2) & sp|Q05123|ARP9_YEAST (293)-sp|Q05123|ARP9_YEAST (272)/ & 20 & ARPS_YEAST & ARP9_YEAST & 293 & 272 & 2.77 \\
\hline YPDYFPEWKK(9)-LLDQTKDTR(6) & sp|Q05123|ARP9 YEAST (420)-sp|P32597|STH1 YEAST (397)/ & 13 & ARP9 YEAST & STH1 YEAST & 420 & 397 & 2.76 \\
\hline KLSSFDFGNENEDEDEGTLNVAEIITSGR(1)-FQGCNNLIKNISNR(9) & sp|Q05123|ARP9 YEAST (228)-s & 1 & ARP9 YEAST & ARP9 YEAST & 228 & 307 & 2.59 \\
\hline KSKFMTNSTAFVPTIEYVQCPTVIK(3)-SVLPAATKK(8) & sp|Q05123|ARP9_YEAST (386)-s & 12 & ARP9_YEAST & ARP9_YEAST & 386 & 382 & 2.57 \\
\hline SGYSEIIFLGAQIVSKQIFTHPK(16)-LGASLHNQMEKDEQKR(15) & sp|Q05123|ARP9 YEAST (437)-sp|P32597|STH1 YEAST (368)/ & 1 & ARP9 YEAST & STH1 YEAST & 437 & 368 & 2.45 \\
\hline LAKYPDYFPEWK(3)-MKNVMK(2) & sp|Q05123|ARP9 YEAST (411)-sp|Q06488|RSC2 YEAST (282)/ & 1 & ARP9 YEAST & RSC2 YEAST & 411 & 282 & 2.37 \\
\hline
\end{tabular}




\begin{tabular}{|c|c|c|c|c|c|c|c|}
\hline PEPTIDE & PROTEINS & CSM & PROTEIN1 & PROTEIN2 & R1 & $\mathbf{R 2}$ & $\mathbf{S}$ \\
\hline KSGYSEIIFLGAQIVSK(1)-LLDQTKDTR(6) & sp|Q05123|ARP9_YEAST (421)-sp|P32597|STH1_YEAST (397)/ & 9 & ARP9_YEAST & STH1_YEAST & 421 & 397 & 2.35 \\
\hline KLSSFDFGNENEDEDEGTLNVAEIITSGR(1)- & sp|Q05123|ARP9_YEAST (228)-sp|Q05123|ARP9_YEAST (355)/ & 1 & ARP9_YEAST & ARP9_YEAST & 228 & 355 & 2.26 \\
\hline YPDYFPEWKK(9)-GQKVK(3) & sp|Q05123|ARP9_YEAST (420)-sp|Q05123|ARP9_YEAST (270)/ & 4 & ARP9_YEAST & ARP9_YEAST & 420 & 270 & 2.16 \\
\hline KLLPQWDDDQIESLK(1)-FQGCNNLIKNISNR(9) & sp|Q05123|ARP9_YEAST (200)-sp|Q05123|ARP9_YEAST (307)/ & 18 & ARP9_YEAST & ARP9_YEAST & 200 & 307 & 2.11 \\
\hline KSGYSEIIFLGAQIVSK(1)-SLMTSHTKGDTSK(8) & sp|Q05123|ARP9_YEAST (421)-sp|P53330|RT102_YEAST (102)/ & 1 & ARP9_YEAST & RT102_YEAST & 421 & 102 & 2.06 \\
\hline SVLPAATKK(8)-GKQGGK(2) & sp|Q05123|ARP9_YEAST (382)-tr|Q6AZJ8|H2A_XENLA (5)/ & 1 & ARP9_YEAST & H2A_XENLA & 382 & 5 & 2.04 \\
\hline DHLIIEPEEEKSK(11)-EEEAKSVLPAATK(5) & sp|Q05123|ARP9_YEAST (366)-sp|Q05123|ARP9_YEAST (374)/ & 4 & ARP9_YEAST & ARPg__YEAST & 366 & 374 & 1.98 \\
\hline NVKNSDLEFNTFWDEKGNEIK(3)-ERGQKVK(5) & sp|Q05123|ARP9_YEAST (275)-sp|Q05123|ARP9_YEAST (270)/ & 1 & ARP9_YEAST & ARP9_YEAST & 275 & 270 & 1.97 \\
\hline EALLGQLLKDHLIIEPEEEK(9)-FQGCNNLIKNISNR(9) & sp|Q05123|ARP9 YEAST (355)-sp|Q05123|ARP9 YEAST (307)/ & 2 & ARP9 YEAST & ARP9 YEAST & 355 & 307 & 1.96 \\
\hline YPDYFPEWKK(9)-VKNVK(2) & sp|Q05123|ARP9_YEAST (420)-sp|Q05123|ARP9_YEAST (272)/ & 1 & ARP9_YEAST & ARP9_YEAST & 420 & 272 & 1.89 \\
\hline SGYSEIIFLGAQIVSKQIFTHPK(16)-LGASLHNQMEKDEQKR(11) & sp|Q05123|ARP9_YEAST (437)-sp|P32597|STH1_YEAST (364)/ & 1 & ARP9_YEAST & STH1_YEAST & 437 & 364 & 1.58 \\
\hline NSDLEFNTFWDEKGNEIKVGK(18)-SLMTSHTKGDTSK(8) & sp|Q05123|ARP9_YEAST (293)-sp|P53330|RT102_YEAST (102)/ & 4 & ARP9_YEAST & RT102_YEAST & 293 & 102 & 1.35 \\
\hline NSDLEFNTFWDEKGNEIKVGK(18)-ERGQKVK(5) & sp|Q05123|ARP9_YEAST (293)-sp|Q05123|ARP9_YEAST (270)/ & 3 & ARP9_YEAST & ARP9 YEAST & 293 & 270 & 1.08 \\
\hline KSGYSEIIFLGAQIVSK(1)-VKNVK(2) & sp|Q05123|ARP9_YEAST (421)-sp|Q05123|ARP9_YEAST (272)/ & 1 & ARP9_YEAST & ARP9_YEAST & 421 & 272 & 1.08 \\
\hline APFRQDSILIIYPR(1)-GKQGGK(2) & sp|Q05123|ARP9_YEAST (2)-tr|Q6AZJ8|H2A_XENLA (5)/ & 2 & ARP9_YEAST & H2A_X̄ENLA & 2 & 5 & 0.85 \\
\hline KLSSFDFGNENEDEDEGTLNVAEIITSGR(1)- & sp|Q05123|ARP9_YEAST (228)-sp|P25632|RSC6_YEAST (321)/ & 1 & ARP9_YEAST & RSC6_YEAST & 228 & 321 & 0.82 \\
\hline $\begin{array}{l}\text { LDSLLQKVLDTNAAHLPLMNVVQTVNK } \\
\text { QIFTHPKDTFYITR(7)-ILFDKIDKER(5) }\end{array}$ & sp|Q05123|ARP9 YEAST (444)-sp|P32597|STH1 YEAST (997)/ & 1 & ARP9 YEAST & STH1 YEAST & 444 & 997 & 0.77 \\
\hline FQGCNNLIKNISNR(9)-KSPIFEVLSDDAKK(13) & sp|Q05123|ARP9_YEAST (307)-sp|Q05123|ARP9_YEAST (227)/ & 9 & ARP9_YEAST & ARP9 YEAST & 307 & 227 & 0.95 \\
\hline YNMKGPAALWDVQF(4)-TLNRK(1) & sp|Q05123|ARP9 YEAST (457)-sp|Q12406|ARP7 YEAST (2)/ & 2 & ARP9 YEAST & ARP7 ${ }^{-}$YEAST & 457 & 2 & 0.45 \\
\hline EKYNMKGPAALWDVQF(6)-TLNRK(1) & sp|Q05123|ARP9_YEAST (457)-sp|Q12406|ARP7 Y YEAST (2)/ & 2 & ARP9_YEAST & ARP7 YEAST & 457 & 2 & 0.39 \\
\hline QIFTHPKDTFYITR(7)-EKYNMK(2) & sp|Q05123|ARP9_YEAST (444)-sp|Q05123|ARP9_YEAST (453)/ & 1 & ARP9_YEAST & ARP9_YEAST & 444 & 453 & 0.58 \\
\hline 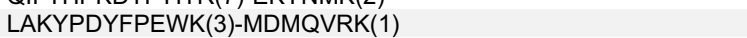 & sp|Q05123|ARPY_YEAST (411)-sp|P38781|RSC30_YEAST (2)/ & 1 & ARP9_YEAST & RSC30_YEAST & 411 & 2 & 0.37 \\
\hline KLLPQWDDDQIESLKK(1)-VGKQR(3) & sp|Q05123|ARP9_YEAST (200)-sp|Q05123|ARP9_Y'YAST (296)/ & 1 & ARP9_YEAST & ARP9_YEAST & 200 & 296 & 0.53 \\
\hline 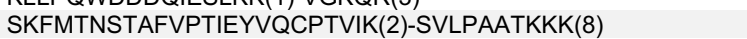 & sp|Q05123|ARP9_YEAST (386)-sp|Q05123|ARP9_YEAST (382)/ & 12 & ARP9_YEAST & ARP9_YEAST & 386 & 382 & 0.47 \\
\hline KSGYSEIIFLGAQIVSK(1)-QTARKSTGGK(5) & sp|Q05123|ARP9_YEAST (421)-sp|P84233|H32_XENLA (9)/ & 1 & ARP9_YEAST & H32_XENLA & 421 & 9 & 0.33 \\
\hline VEILTKEEIQKR(6)-HAVSEGTKAVTK(8) & Sp|Q06168|SFH1_YEAST (385)-Sp|P02281|H2B11_XENLA (113)/ & 3 & SFH1_YEAST & H2B11_XENLA & 385 & 113 & 2.56 \\
\hline EIEKER(4)-EEIQKR(5) & sp|Q06168|SFH1_YEAST (395)-sp|Q06168|SFH1_YEAST (390)/ & 1 & SFH1_YEAST & SFH1_Y̌EAST & 395 & 390 & 1.62 \\
\hline LNTDQETKANANSGEAIMIPITLDIEHMGHTIK(8)-IHEIEKR(6) & sp|Q06168|SFH1_YEAST (193)-sp|P43609|RSC8_YEAST (99)/ & 1 & SFH1_YEAST & RSC8_YEAST & 193 & 99 & 1.21 \\
\hline $\begin{array}{l}\text { YDAGSNMVVESLSGLNSNNNASNGPSNKAQAQDIGNAVLPDLQD } \\
\text { DHNPFN R(28)-OHKRAK(3) }\end{array}$ & sp|Q06168|SFH1_YEAST (141)-sp|Q06168|SFH1_YEAST (40)/ & 1 & SFH1_YEAST & SFH1_YEAST & 141 & 40 & 0.46 \\
\hline VEILTKEEIQKR(11)-NDEELNKLLGR(7) & sp|Q06168|SFH1 YEAST (390)-tr|Q6AZJ8|H2A XENLA (95)/ & 1 & SFH1 YEAST & H2A XENLA & 390 & 95 & 0.33 \\
\hline WFTTNKK(6)-TESVGGNK(1) & sp|Q06488|RSC2_YEAST (823)-sp|Q07979|RSC58_YEAST (2)/ & 8 & RSC2_YEAST & RSC58_YEAST & 823 & 2 & 9.53 \\
\hline KNFIAVSAANR(1)-TESVGGNK(1) & sp|Q06488|RSC2_YEAST (905)-sp|Q07979|RSC58_YEAST (2)/ & 8 & RSC2_YEAST & RSC58_YEAST & 905 & 2 & 7.79 \\
\hline IPGLINPWKR(9)-KNFIAVSAANR(1) & sp|Q06488|RSC2_YEAST (900)-sp|Q06488|RSC2_YEAST (905)/ & 31 & RSC2_YEAST & RSC2_YEAST & 900 & 905 & 5.87 \\
\hline TDLHSQTKR(8)-LWKTPDGK(3) & sp|Q06488|RSC2 YEAST (772)-sp|Q06488|RSC2 YEAST (437)/ & 6 & RSC2 YEAST & RSC2 YEAST & 772 & 437 & 5.77 \\
\hline WFTTNKKR(6)-TESVGGNK(1) & sp|Q06488|RSC2 YEAST (823)-sp|Q07979|RSC58 YEAST (2)/ & 8 & RSC2 YEAST & RSC5 8 YEAST & 823 & 2 & 5.47 \\
\hline LQQQQHQYQQQKR(12)-NQEEFLTIKK(9) & sp|Q06488|RSC2_YEAST (713)-sp|Q06639|RSC3_YEAST (231)/ & 13 & RSC2_YEAST & RSC3_YEAST & 713 & 231 & 5.38 \\
\hline SGREEMFPWKK(10)-TDLHSQTKR(8) & sp|Q06488|RSC2_YEAST (783)-sp|Q06488|RSC2_YEAST (772)/ & 33 & RSC2_YEAST & RSC2_YEAST & 783 & 772 & 5.32 \\
\hline IPGLINPWKR(9)-TDLHSQTKR(8) & sp|Q06488|RSC2_YEAST (900)-sp|Q06488|RSC2_YEAST (772)/ & 16 & RSC2_YEAST & RSC2_YEAST & 900 & 772 & 5.11 \\
\hline RIPGLINPWKR(10)-TESVGGNK(1) & sp|Q06488|RSC2_YEAST (900)-sp|Q07979|RSC58_YEAST (2)/ & 7 & RSC2_YEAST & RSC58_YEAST & 900 & 2 & 5.02 \\
\hline DDDGLEPDVENEKESLPGPF & sp|Q06488|RSC2_YEAST (857)-sp|Q06488|RSC2_YEAST (842)/ & 12 & RSC2_YEAST & RSC2_YEAST & 857 & 842 & 4.70 \\
\hline $\begin{array}{l}\text { KLEYEEVEETMEDVTGKDK(17) } \\
\text { KPLININPYTR(1)-KPLRDNR(1) }\end{array}$ & sp|Q06488|RSC2 YEAST (223)-sp|Q06488|RSC2 YEAST (234)/ & 21 & YEA & RS & 223 & 234 & 4.39 \\
\hline IPGLINPWKR(9)-TESVGGNK(1) & sp|Q06488|RSC2_YEAST (900)-sp|Q07979|RSC58_YEAST (2)/ & 7 & RSC2_YEAST & RSC58_YEAST & 900 & 2 & 4.27 \\
\hline IPGLINPWKR(9)-KEFPVNLTIK(1) & sp|Q06488|RSC2 YEAST (900)-sp|P43609|RSC8 YEAST (222)/ & 9 & RSC2 YEAST & RSC8 YEAST & 900 & 222 & 3.93 \\
\hline SGREEMFPWKK(10)-TKGEILWFR(2) & sp|Q06488|RSC2 YEAST (783)- & 14 & RSC2 YEAST & RSC2 YEAST & 783 & 786 & 3.83 \\
\hline EEMFPWKK(7)-TDLHSQTKR(8) & sp|Q06488|RSC2 YEAST (772)/ & 33 & RSC2 YEAST & RSC2 YEAST & 783 & 772 & 3.78 \\
\hline KNFIAVSAANR(1)-KEFPVNLTIK(1) & sp|Q06488|RSC2 YEAST (905)-sp|P43609|RSC8 YEAST (222)/ & 2 & RSC2 YEAST & RSC8 YEAST & 905 & 222 & 3.71 \\
\hline
\end{tabular}




\begin{tabular}{|c|c|c|c|c|c|c|c|}
\hline PEPTIDE & PROTEINS & CSM & PROTEIN1 & PROTEIN2 & R1 & $\mathbf{R 2}$ & $\mathbf{S}$ \\
\hline $\begin{array}{l}\text { VGSPGAGGPLTVQGLKQHQLQR(16)- } \\
\text { QKLINNVASQAHHNIPYLR(2) }\end{array}$ & sp|Q06488|RSC2_YEAST (695)-sp|P32597|STH1_YEAST (260)/ & 6 & RSC2_YEAST & STH1_YEAST & 695 & 260 & 3.71 \\
\hline KPLININPYTR(1)-LWKTPDGK(3) & sp|Q06488|RSC2_YEAST (223)-sp|Q06488|RSC2_YEAST (437)/ & 5 & RSC2_YEAST & RSC2_YEAST & 223 & 437 & 3.60 \\
\hline LQQQQHQYQQQKR(12)-VLKPTTEER(3) & sp|Q06488|RSC2_YEAST (713)-sp|Q07979|RSC58_YEAST (440)/ & 5 & RSC2_YEAST & RSC58_YEAST & 713 & 440 & 3.59 \\
\hline LQQQQHQYQQQKR(12)-VSKETAALLQK(3) & sp|Q06488|RSC2_YEAST (713)-sp|Q06639|RSC3_Y̌EAST (81)/ & 8 & RSC2_YEAST & RSC3_Y̌EAST & 713 & 81 & 3.45 \\
\hline TKGEILWFR(2)-TDLHSQTKR(8) & sp|Q06488|RSC2_YEAST (786)-sp|Q06488|RSC2_YEAST (772)/ & 10 & RSC2_YEAST & RSC2_YEAST & 786 & 772 & 3.43 \\
\hline NQNDPQKPIVGQIFR(7)-LFYKNEVMK(4) & sp|Q06488|RSC2_YEAST (426)-sp|Q06488|RSC2_YEAST (466)/ & 10 & RSC2_YEAST & RSC2_YEAST & 426 & 466 & 3.24 \\
\hline LWKTPDGK(3)-KQLATK(1) & sp|Q06488|RSC2_YEAST (437)-sp|P84233|H32_XENLA (18)/ & 3 & RSC2_YEAST & H32_XENLA & 437 & 18 & 3.23 \\
\hline LWKTPDGK(3)-KPLRDNR(1) & sp|Q06488|RSC2_YEAST (437)-sp|Q06488|RSC2_YEAST (234)/ & 11 & RSC2_YEAST & RSC2_YEAST & 437 & 234 & 3.09 \\
\hline WKKNFIAVSAANR(3)-TESVGGNK(1) & sp|Q06488|RSC2_YEAST (905)-sp|Q07979|RSC58_YEAST (2)/ & 8 & RSC2_YEAST & RSC58_YEAST & 905 & 2 & 3.08 \\
\hline $\begin{array}{l}\text { YIIRPNDSPEEGQVDIETGTITTNTPTANALPKTGYSSSK(33)- } \\
\text { VGSPGAGGPLTVQGLKQHQLQR(16) }\end{array}$ & sp|Q06488|RSC2_YEAST (644)-sp|Q06488|RSC2_YEAST (695)/ & 1 & RSC2_YEAST & RSC2_YEAST & 644 & 695 & 2.87 \\
\hline $\begin{array}{l}\text { ESEPGTDTNNDEDYEATDMDIDNPKDADFPDLIR(25)- } \\
\text { KPLININPYTR(1) }\end{array}$ & sp|Q06488|RSC2_YEAST (213)-sp|Q06488|RSC2_YEAST (223)/ & 1 & RSC2_YEAST & RSC2_YEAST & 213 & 223 & 2.80 \\
\hline NVMKVLK(4)-KVKTR(1) & sp|Q06488|RSC2_YEAST (286)-sp|Q06488|RSC2_YEAST (331)/ & 1 & RSC2_YEAST & RSC2_YEAST & 286 & 331 & 2.78 \\
\hline LWKTPDGK(3)-KSAPATGGVK(1) & sp|Q06488|RSC2_YEAST (437)-sp|P84233|H32_XENLA (27)/ & 1 & RSC2_YEAST & H32_XENLA & 437 & 27 & 2.72 \\
\hline RIPGLINPWKR(10)-KNFIAVSAANR(1) & sp|Q06488|RSC2_YEAST (900)-sp|Q06488|RSC2_YEAST (905)/ & 31 & RSC2_YEAST & RSC2_YEAST & 900 & 905 & 2.71 \\
\hline VGSPGAGGPLTVQGLKQHQLQR(16)-TDLHSQTKR(8) & sp|Q06488|RSC2_YEAST (695)-sp|Q06488|RSC2_YEAST (772)/ & 10 & RSC2_YEAST & RSC2_YEAST & 695 & 772 & 2.67 \\
\hline LWKTPDGK(3)-KSTGGK(1) & sp|Q06488|RSC2_YEAST (437)-sp|P84233|H32_XENLA (9)/ & 2 & RSC2_YEAST & H32_XENLA & 437 & 9 & 2.66 \\
\hline RIPGLINPWKR(10)-KEFPVNLTIKK(1) & sp|Q06488|RSC2_YEAST (900)-sp|P43609|RSC8_YEAST (222)/ & 9 & RSC2_YEAST & RSCE_YEAST & 900 & 222 & 2.65 \\
\hline DSGIYKYALVLEK(6)-YLKDTIYPNLK(3) & sp|Q06488|RSC2_YEAST (104)-sp|Q06488|RSC2_YEAST (114)/ & 5 & RSC2_YEAST & RSC2_YEAST & 104 & 114 & 2.62 \\
\hline VGSPGAGGPLTVQGLKQHQLQR(16)-TGYSSSKLSSLR(7) & sp|Q06488|RSC2_YEAST (695)-sp|Q06488|RSC2_YEAST (651)/ & 5 & RSC2_YEAST & RSC2_YEAST & 695 & 651 & 2.59 \\
\hline LWKTPDGK(3)-TQVKR(4) & sp|Q06488|RSC2_YEAST (437)-sp|Q06488|RSC2_YEAST (266)/ & 3 & RSC2_YEAST & RSC2_YEAST & 437 & 266 & 2.56 \\
\hline NEVMKTGQYR(5)-TQVKR(4) & sp|Q06488|RSC2_YEAST (471)-sp|Q06488|RSC2_YEAST (266)/ & 5 & RSC2_YEAST & RSC2_YEAST & 471 & 266 & 2.55 \\
\hline VGSPGAGGPLTVQGLKQHQLQR(16)-AKVSKPK(2) & sp|Q06488|RSC2_YEAST (695)-sp|P38210|LDB7_YEAST (54)/ & 2 & RSC2_YEAST & LDB7_YEAST & 695 & 54 & 2.53 \\
\hline VGSPGAGGPLTVQGLKQHQLQR(16)-LQQQQHQYQQQKR(12) & sp|Q06488|RSC2_YEAST (695)-sp|Q06488|RSC2_YEAST (713)/ & 10 & RSC2_YEAST & RSC2_YEAST & 695 & 713 & 2.39 \\
\hline KFFKYPSPIR(1)-MKNVMK(2) & sp|Q06488|RSC2_YEAST (550)-sp|Q06488|RSC2_YEAST (282)/ & 1 & RSC2_YEAST & RSC2_YEAST & 550 & 282 & 2.13 \\
\hline TKGEILWFR(2)-EEMFPWKK(7) & sp|Q06488|RSC2_YEAST (786)-sp|Q06488|RSC2_YEAST (783)/ & 7 & RSC2_YEAST & RSC2_YEAST & 786 & 783 & 2.13 \\
\hline IPGLINPWKRR(9)-KNFIAVSAANR(1) & sp|Q06488|RSC2_YEAST (900)-sp|Q06488|RSC2_YEAST (905)/ & 31 & RSC2_YEAST & RSC2_YEAST & 900 & 905 & 2.12 \\
\hline LQQQQHQYQQQKR(12)-TGYSSSKLSSLF & sp|Q06488|RSC2_YEAST (713)-sp|Q06488|RSC2_YEAST (651)/ & 4 & RSC2_YEAST & RSC2_YEAST & 713 & 651 & 2.10 \\
\hline VGSPGAGGPLTVQGLKQHQLQR(16)-AIQNHPKENKLEIK(7) & sp|Q06488|RSC2_YEAST (695)-sp|P32597|STH1_YEAST (70)/ & 4 & RSC2_YEAST & STH1_YEAST & 695 & 70 & 1.99 \\
\hline EKAEEVAR(2)-TSVKR(4) & sp|Q06488|RSC2_YEAST (152)-sp|Q06488|RSC2_YEAST (187)/ & 2 & RSC2_YEAST & RSC2_YEAST & 152 & 187 & 1.88 \\
\hline RIPGLINPWKR(10)-LENNGNSVKK(9) & sp|Q06488|RSC2_YEAST (900)-sp|P43609|RSC8_YEAST (312)/ & 2 & RSC2_YEAST & RSC8_YEAST & 900 & 312 & 1.74 \\
\hline KNFIAVSAANR(1)-TDLHSQTKR(8) & sp|Q06488|RSC2_YEAST (905)-sp|Q06488|RSC2_YEAST (772)/ & 3 & RSC2_YEAST & RSC2_YEAST & 905 & 772 & 1.69 \\
\hline VGSPGAGGPLTVQGLKQHQLQR(16)-LWKTPDGK(3) & sp|Q06488|RSC2_YEAST (695)-sp|Q06488|RSC2_YEAST (437)/ & 1 & RSC2_YEAST & RSC2_YEAST & 695 & 437 & 1.64 \\
\hline RIPGLINPWKR(10)-TDLHSQTKR(8) & sp|Q06488|RSC2_YEAST (900)-sp|Q06488|RSC2_YEAST (772)/ & 16 & RSC2_YEAST & RSC2_YEAST & 900 & 772 & 1.62 \\
\hline VGSPGAGGPLTVQGLKQHQLQR(16)-ETAALLQKNPELASLEQIR(8) & sp|Q06488|RSC2_YEAST (695)-sp|Q06639|RSC3_YEAST (89)/ & 3 & RSC2_YEAST & RSC3_YEAST & 695 & 89 & 1.56 \\
\hline VGSPGAGGPLTVQGLKQHQLQR(16)-VLKPTTEER(3) & sp|Q06488|RSC2_YEAST (695)-sp|Q07979|RSC58_YEAST (440)/ & 1 & RSC2_YEAST & RSC58_YEAST & 695 & 440 & 1.54 \\
\hline LQQQQHQYQQQKR(12)-ILNKVLKPTTEER(4) & sp|Q06488|RSC2_YEAST (713)-sp|Q07979|RSC58_YEAST (437)/ & 3 & RSC2_YEAST & RSC58_YEAST & 713 & 437 & 1.53 \\
\hline RIPGLINPWKR(10)-KEFPVNLTIK(1) & sp|Q06488|RSC2_YEAST (900)-sp|P43609|RSC8_Y̌EAST (222)/ & 9 & RSC2_YEAST & RSC8_ȲEAST & 900 & 222 & 1.51 \\
\hline KPLININPYTR(1)-TSVKR(4) & sp|Q06488|RSC2_YEAST (223)-sp|Q06488|RSC2_YEAST (187)/ & 4 & RSC2_YEAST & RSC2_YEAST & 223 & 187 & 1.47 \\
\hline YNESDKIFNK(6)-TQVKR(4) & sp|Q06488|RSC2_YEAST (520)-sp|Q06488|RSC2_YEAST (266)/ & 1 & RSC2_YEAST & RSC2-YEAST & 520 & 266 & 1.46 \\
\hline ISNILEKTFTSLAR(7)-KPLRDNR(1) & sp|Q06488|RSC2_YEAST (373)-sp|Q06488|RSC2_YEAST (234)/ & 1 & RSC2_YEAST & RSC2_YEAST & 373 & 234 & 1.46 \\
\hline VGSPGAGGPLTVQGLKQHQLQR(16)-TKDMDLFSSIISK(2) & 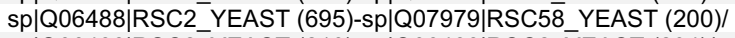 & 1 & RSC2_YEAST & 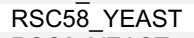 & 695 & 200 & 1.42 \\
\hline ESEPGTDTNNDEDYEATDMDIDNPKDADFPDLIR(25)-KPLRDNR(1) & sp|Q06488|RSC2_YEAST (213)-sp|Q06488|RSC2_Y̌EAST (234)/ & 1 & RSC2_YEAST & RSC2_Y̌YAST & 213 & 234 & 1.39 \\
\hline YNESDKIFNK(6)-NPVSFNTLKK(9) & sp|Q06488|RSC2_YEAST (520)-sp|Q06488|RSC2_YEAST (69)/ & 1 & RSC2_YEAST & RSC2_YEAST & 520 & 69 & 1.38 \\
\hline LQQQQHQYQQQKR(12)-ISNILEKTFTSLAR(7) & sp|Q06488|RSC2_YEAST (713)-sp|Q06488|RSC2_YEAST (373)/ & 3 & RSC2_YEAST & RSC2_YEAST & 713 & 373 & 1.35 \\
\hline $\begin{array}{l}\text { DDDGLEPDVENEKESLPGPFVLGLRPSAK(13)- } \\
\text { LEYEEVEETMEDVTGKDK(16) }\end{array}$ & sp|Q06488|RSC2_YEAST (857)-sp|Q06488|RSC2_YEAST (842)/ & 12 & RSC2_YEAST & RSC2_YEAST & 857 & 842 & 1.33 \\
\hline $\begin{array}{l}\text { LEYEEVEETMEDVTGKDK(16) } \\
\text { VGSPGAGGPLTVQGLKQHQLQR(16)-VSKETAALLQK(3) }\end{array}$ & sp|Q06488|RSC2 YEAST (695)-sp|Q06639|RSC3 YEAST (81)/ & 1 & RSC2 YEAST & RSC3 YEAST & 695 & 81 & 1.30 \\
\hline
\end{tabular}




\begin{tabular}{|c|c|c|c|c|c|c|c|}
\hline PEPTIDE & PROTEINS & CSM & PROTEIN1 & PROTEIN2 & R1 & R2 & $\mathbf{s}$ \\
\hline $\begin{array}{l}\text { LQQQQHQYQQQKR(12)-NQEEFLTIKKR(9) } \\
\end{array}$ & sp|Q06488|RSC2_YEAST (713)-sp|Q06639|RSC3_YEAST (231)/ & 13 & RSC2_YEAST & RSC3_YEAST & 713 & 231 & 1.29 \\
\hline NEVMKTGQYR(5)-TLNRK(1) & sp|Q06488|RSC2_YEAST (471)-sp|Q12406|ARP7_YEAST (2)/ & 1 & RSC2_YEAST & ARP7_YEAST & 471 & 2 & 1.23 \\
\hline KEVLDSGIGLTDLFERLPDR(1)-NVMKVLK(4) & sp|Q06488|RSC2_YEAST (290)-sp|Q06488|RSC2_YEAST (286)/ & 2 & RSC2_YEAST & RSC2_YEAST & 290 & 286 & 1.20 \\
\hline ISNILEKTFTSLAR(7)-TKDMDLFSSIISK(2) & sp|Q06488|RSC2_YEAST (373)-sp|Q07979|RSC58_YEAST (200)/ & 1 & RSC2_YEAST & RSC58_YEAST & 373 & 200 & 1.13 \\
\hline KISSSGALDYDIPTTASENLYFQ(1)-NEDKLVLTTINKR(4) & sp|Q06488|RSC2_YEAST (918)-sp|Q07979|RSC58_YEAST (70)/ & 3 & RSC2_YEAST & RSC58_YEAST & 918 & 70 & 0.99 \\
\hline $\begin{array}{l}\text { YLKDTIYPNLKEKYPQLVYPDLGPLPDEPGYEEFQQK(3)- } \\
\text { EIEKERNLRR(4) }\end{array}$ & sp|Q06488|RSC2_YEAST (114)-sp|Q06168|SFH1_YEAST (395)/ & 1 & RSC2_YEAST & SFH1_YEAST & 114 & 395 & 0.92 \\
\hline NQNDPQKPIVGQIFR(7)-VDRLFYKNEVMK(7) & sp|Q06488|RSC2_YEAST (426)-sp|Q06488|RSC2_YEAST (466)/ & 10 & RSC2_YEAST & RSC2_YEAST & 426 & 466 & 1.04 \\
\hline YNESDKIFNK(6)-QKSIR(2) & sp|Q06488|RSC2_YEAST (520)-sp|P32597|STH1_YEAST (255)/ & 1 & RSC2_YEAST & STH1_YEAST & 520 & 255 & 0.76 \\
\hline KISSSGALDYDIPTTASENLYFQ(1)-STTSDIEKTNSLESEHLK(8) & sp|Q06488|RSC2_YEAST (918)-sp|Q02206|RSC4_YEAST (410)/ & 1 & RSC2_YEAST & RSC4_YEAST & 918 & 410 & 0.68 \\
\hline KISSSGALDYDIPTTASENLYFQ(1)-IPGLINPWKR(9) & sp|Q06488|RSC2_YEAST (918)-sp|Q06488|RSC2_YEAST (900)/ & 3 & RSC2_YEAST & RSC2_YEAST & 918 & 900 & 0.96 \\
\hline VGSPGAGGPLTVQGLKQHQLQR(16)-AIQNHPKENK(7) & sp|Q06488|RSC2_YEAST (695)-sp|P32597|STH1_YEAST (70)/ & 4 & RSC2_YEAST & STH1_YEAST & 695 & 70 & 0.66 \\
\hline VGSPGAGGPLTVQGLKQHQLQR(16)-VSKPKR(5) & sp|Q06488|RSC2 YEAST (695)-sp|P38210|LDB7 YEAST (59)/ & 2 & RSC2_YEAST & LDB7_YEAST & 695 & 59 & 0.63 \\
\hline VGSPGAGGPLTVQGLKQHQLQR(16)-SYESKFGQPVR(5) & sp|Q06488|RSC2_YEAST (695)-sp|Q03124|RSC9_YEAST (423)/ & 1 & RSC2_YEAST & RSC9_YEAST & 695 & 423 & 0.55 \\
\hline EKYPQLVYPDLGPLPDEPGYEEFQQK(2)- & sp|Q06488|RSC2_YEAST (124)-sp|P43609|RSC8_YEAST (221)/ & 1 & RSC2_YEAST & RSC8_YEAST & 124 & 221 & 0.53 \\
\hline $\begin{array}{l}\text { QEVEGGDGAEPQVKKEFPVNLTIK(14) } \\
\text { YKTFQEFQNDFNLMLTNFR(2)-MDPQTLITKANK(9) }\end{array}$ & sp|Q06488|RSC2 YEAST (338)-sp|P53330|RT102 YEAST (9)/ & 1 & RSC2 YEAST & RT102 YEAST & 338 & 9 & 0.52 \\
\hline EKYPQLVYPDLGPLPDEPGYEEFQQK(2)- & sp|Q06488|RSC2_YEAST (124)-sp|P02281|H2B11_XENLA (54)/ & 1 & RSC2_YEAST & H2B11_XENLA & 124 & 54 & 0.52 \\
\hline $\begin{array}{l}\text { QVHPDTGISSKAMSIMNSFVNDVFER(11) } \\
\text { DKDDDGLEPDVENEKESLPGPFVLGLRPSA }\end{array}$ & & & & & & & \\
\hline $\begin{array}{l}\text { NPVSFNTLKKR(9) } \\
\text { (1) }\end{array}$ & sp|Q06488|RSC2_YEAST (857)-sp|Q06488|RSC2_YEAST (69)/ & 9 & RSC2_YEAST & RSC2_YEAST & 857 & 69 & 0.81 \\
\hline LFYKNEVMKTGQYR(9)-TQVKR(4) & sp|Q06488|RSC2_YEAST (471)-sp|Q06488|RSC2_YEAST (266)/ & 5 & RSC2_YEAST & RSC2_YEAST & 471 & 266 & 0.81 \\
\hline $\begin{array}{l}\text { MMPDDNSNSSTQNSSALYKDLR(19)- } \\
\text { PQLISDKFSPEDGLGPLMAK(7) }\end{array}$ & sp|Q06488|RSC2_YEAST (19)-sp|Q12406|ARP7_YEAST (322)/ & 1 & RSC2_YEAST & ARP7_YEAST & 19 & 322 & 0.49 \\
\hline WKKNFIAVSAANR(3)-KSTGGK(1) & sp|Q06488|RSC2 YEAST (905)-sp|P84233|H32 XENLA (9)/ & 1 & RSC2 YEAST & H32 XENLA & 905 & 9 & 0.44 \\
\hline VGSPGAGGPLTVQGLKQHQLQR(16)-ISNIL & sp|Q06488|RSC2_YEAST (695)-sp|Q06 & 1 & RSC2_YEAST & RSC2_YEAST & 695 & 373 & 0.73 \\
\hline EDSGLEISPIFNVLPPKKDYPDYYAVIK(17)-NPVSFNTLKKR(10) & sp|Q06488|RSC2_YEAST (49)-sp|Q06488|RSC2_Y̌EAST (70)/ & 1 & RSC2_YEAST & RSC2_YEAST & 49 & 70 & 0.69 \\
\hline $\begin{array}{l}\text { DKDDDGLEPDVENEKESLPGPFVLGLR(15)- } \\
\text { KLEYEEVEETMEDVTGKDK(17) }\end{array}$ & sp|Q06488|RSC2_YEAST (857)-sp|Q06488|RSC2_YEAST (842)/ & 12 & RSC2_YEAST & RSC2_YEAST & 857 & 842 & 0.66 \\
\hline LFYKNEVMKTGQYR(9)-KEFPVNLTIK(1) & sp|Q06488|RSC2_YEAST (471)-sp|P43609|RSC8_YEAST (222)/ & 1 & RSC2_YEAST & RSC8_YEAST & 471 & 222 & 0.38 \\
\hline $\begin{array}{l}\text { DKDDDGLEPDVENEKESLPGPFVLGLRPSAK(15)- } \\
\text { NPVSFNTLKKR(10) }\end{array}$ & sp|Q06488|RSC2_YEAST (857)-sp|Q06488|RSC2_YEAST (70)/ & 2 & RSC2_YEAST & RSC2_YEAST & 857 & 70 & 0.53 \\
\hline $\begin{array}{l}\text { YIIRPNDSPEEGQVDIETGTITTNTPTANALPKTGYSSSK(33)- } \\
\text { TKPSLIGPSFTGHFQVVLDTPQGLKPFLPENVIK(2) }\end{array}$ & sp|Q06488|RSC2_YEAST (644)-sp|P43609|RSC8_YEAST (175)/ & 1 & RSC2_YEAST & RSC8_YEAST & 644 & 175 & 0.35 \\
\hline $\begin{array}{l}\text { EDSGLEISPIFNVLPPKKDYPDYYAVIKNPVSFNTLK(17)- } \\
\text { NEVMKTGQYR(5) }\end{array}$ & sp|Q06488|RSC2_YEAST (49)-sp|Q06488|RSC2_YEAST (471)/ & 1 & RSC2_YEAST & RSC2_YEAST & 49 & 471 & 0.47 \\
\hline VGSPGAGGPLTVQGLKQHQLQR(16)-ISHRGDPESIKISNILEK(11) & sp|Q06488|RSC2_YEAST (695)-sp|Q06488|RSC2_YEAST (366)/ & 1 & RSC2_YEAST & RSC2 YEAST & 695 & 366 & 0.45 \\
\hline $\begin{array}{l}\text { NEVMKTGQYRDHLVSNLVGKCYVIHFTR(20)- } \\
\text { EDSGLEISPIFNVLPPKK(17) }\end{array}$ & sp|Q06488|RSC2_YEAST (486)-sp|Q06488|RSC2_YEAST (49)/ & 1 & RSC2_YEAST & RSC2_YEAST & 486 & 49 & 0.45 \\
\hline KISSSGALDYDIPTTASENLYFQ(1)-KNFIAVSAANR(1) & sp|Q06488|RSC2_YEAST (918)-sp|Q06488|RSC2_YEAST (905)/ & 1 & RSC2_YEAST & RSC2_YEAST & 918 & 905 & 0.44 \\
\hline $\begin{array}{l}\text { YIKNLEENKVTTSADSNYSINNGFSGISAEQLIK(3)- } \\
\text { KNSTVSNGVLGAAAPVDSDANSDTFGLTK(1) }\end{array}$ & sp|Q06639|RSC3_YEAST (792)-sp|Q06639|RSC3_YEAST (845)/ & 11 & RSC3_YEAST & RSC3_YEAST & 792 & 845 & 16.08 \\
\hline $\begin{array}{l}\text { KNSTVSNGVLGAAAPVDSDANSDTFGLTKENFNEVFEAIRS(1)- } \\
\text { YIKNLEENKVTTSADSNYSINNGFSGISAEQLIK(3) }\end{array}$ & sp|Q06639|RSC3_YEAST (845)-sp|Q06639|RSC3_YEAST (792)/ & 10 & RSC3_YEAST & RSC3_YEAST & 845 & 792 & 14.87 \\
\hline KNSTVSNGVLGAAAPVDSDANSDTFGLTK(1)-IKNKNVIHLIINK(2) & sp|Q06639|RSC3_YEAST (845)-sp|Q06639|RSC3_YEAST (747)/ & 1 & RSC3_YEAST & RSC3 YEAST & 845 & 747 & 6.91 \\
\hline KITGVNLDLKQDSSAQMQSSHSNR(10)- & sp|Q06639|RSC3_YEAST (208)-sp|Q06639|RSC3_YEAST (234)/ & 5 & RSC3_YEAST & RSC3_YEAST & 208 & 234 & 5.63 \\
\hline $\begin{array}{l}\text { KLSEDGVTDGDGKPIPESER(1) } \\
\text { ITGVNLDLKQDSSAQMQSSHSNR(9)-LQQQQHQYQQQKR(12) }\end{array}$ & sp|Q06639|RSC3_YEAST (208)-sp|Q06488|RSC2_YEAST (713)/ & 6 & RSC3_YEAST & RSC2_YEAST & 208 & 713 & 5.53 \\
\hline $\begin{array}{l}\text { ITGVNLDLKRDSSAQMQSSHSNR(9)- } \\
\text { SLKEKSNLNADNTTANKINK(5) }\end{array}$ & sp|Q06639|RSC3_YEAST (208)-sp|P38781|RSC30_YEAST (234)/ & 13 & RSC3_YEAST & RSC30_YEAST & 208 & 234 & 5.36 \\
\hline
\end{tabular}




\begin{tabular}{|c|c|c|c|c|c|c|c|}
\hline PEPTIDE & PROTEINS & CSM & PROTEIN1 & PROTEIN2 & R1 & $\mathbf{R 2}$ & $\mathbf{s}$ \\
\hline $\begin{array}{l}\text { KITGVNLDLKQDSSAQMQSSHSNR(10)- } \\
\text { SLKEKSNLNADNTTANKINK(5) }\end{array}$ & sp|Q06639|RSC3_YEAST (208)-sp|P38781|RSC30_YEAST (234)/ & 13 & RSC3_YEAST & RSC30_YEAST & 208 & 234 & 5.24 \\
\hline KLSEDGVTDGDGKPIPESER(1)-KLLPAVEFIK(1) & sp|Q06639|RSC3_YEAST (234)-sp|Q03124|RSC9_YEAST (434)/ & 3 & RSC3_YEAST & RSC9_YEAST & 234 & 434 & 5.22 \\
\hline KLSEDGVTDGDGKPIPESER(1)-NQEEFLTIKK(9) & sp|Q06639|RSC3_YEAST (234)-sp|Q06639|RSC3_YEAST (231)/ & 25 & RSC3_YEAST & RSC3_YEAST & 234 & 231 & 4.76 \\
\hline NQEEFLTIKK(9)-KLLPAVEFIK(1) & sp|Q06639|RSC3_YEAST (231)-sp|Q03124|RSC9_YEAST (434)/ & 7 & RSC3_YEAST & RSC9_YEAST & 231 & 434 & 4.74 \\
\hline $\begin{array}{l}\text { ITGVNLDLKQDSSAQMQSSHSNR(9)- } \\
\text { KLSEDGVTDGDGKPIPESER(1) }\end{array}$ & sp|Q06639|RSC3_YEAST (208)-sp|Q06639|RSC3_YEAST (234)/ & 5 & RSC3_YEAST & RSC3_YEAST & 208 & 234 & 4.51 \\
\hline ITGVNLDLKQDSSAQMQSSHSNR(9)-EKSNLNADNTTANK(2) & sp|Q06639|RSC3 YEAST (208)-sp|P38781|RSC30 YEAST (234)/ & 13 & RSC3 YEAST & RSC30 YEAST & 208 & 234 & 4.46 \\
\hline DLDPQFLDTNKVFNVFNSAISEEGR(11)-KLLPAVEFIK(1) & sp|Q06639|RSC3_YEAST (272)-sp|Q03124|RSC9_YEAST (434)/ & 4 & RSC3_YEAST & RSC9_Y̌YEAST & 272 & 434 & 4.34 \\
\hline ITGVNLDLKQDSSAQMQSSHSNR(9)-NQEEFLTIKK(9) & sp|Q06639|RSC3_YEAST (208)-sp|Q06639|RSC3_YEAST (231)/ & 1 & RSC3_YEAST & RSC3_YEAST & 208 & 231 & 4.32 \\
\hline NSGISQILKFPSQSITQTLINK(9)-LLPIVEQLFPSNTINKPNSK(16) & sp|Q06639|RSC3 YEAST (354)-sp|Q06639|RSC3_YEAST (402)/ & 5 & RSC3 YEAST & RSC3 YEAST & 354 & 402 & 4.29 \\
\hline NQEEFLTIKKR(9)-LQPQVYKPWSL(7) & sp|Q06639|RSC3_YEAST (231)-sp|P43609|RSC8_YEAST (553)/ & 2 & RSC3_YEAST & RSC8_YEAST & 231 & 553 & 4.04 \\
\hline ITGVNLDLKQDSSAQMQSSHSNR(9)-SLKEKSNLNADNTTANK(5) & sp|Q06639|RSC3_YEAST (208)-sp|P38781|RSC30_YEAST (234)/ & 13 & RSC3 YEAST & RSC 30 YYEAST & 208 & 234 & 4.00 \\
\hline KITGVNLDLKQDSSAQMQSSHSNR(10)-EKSNLNADNTTANK(2) & sp|Q06639|RSC3_YEAST (208)-sp|P38781|RSC30_YEAST (234)/ & 13 & RSC3_YEAST & RSC30_YEAST & 208 & 234 & 3.94 \\
\hline KLSEDGVTDGDGKPIPESER(1)-SNLNADNTTANKINK(12) & sp|Q06639|RSC3_YEAST (234)-sp|P38781|RSC30_YEAST (246)/ & 1 & RSC3_YEAST & RSC30_YEAST & 234 & 246 & 3.74 \\
\hline $\begin{array}{l}\text { KNSTVSNGVLGAAAPVDSDANSDTFGLTK(1)- } \\
\text { TNDANEISEOTDIHSNNDNSKR(21) }\end{array}$ & sp|Q06639|RSC3_YEAST (845)-sp|Q06639|RSC3_YEAST (744)/ & 1 & RSC3_YEAST & RSC3_YEAST & 845 & 744 & 3.63 \\
\hline KLSEDGVTDGDGKPIPESER(1)-LQQQQHQYQQQKR(12) & sp|Q06639|RSC3_YEAST (234)-sp|Q06488|RSC2_YEAST (713)/ & 5 & RSC3_YEAST & RSC2_YEAST & 234 & 713 & 3.27 \\
\hline $\begin{array}{l}\text { RPHLNEFKDLDPQFLDTNKVFNVFNSAISEEGR(19)- } \\
\text { KLLPAVEFIK(1) }\end{array}$ & sp|Q06639|RSC3_YEAST (272)-sp|Q03124|RSC9_YEAST (434)/ & 4 & RSC3_YEAST & RSC9_YEAST & 272 & 434 & 3.18 \\
\hline $\begin{array}{l}\text { SVPSLESKPVTSANESSTPLNWVQGPAIFHMLTSPYTQDEIINHEM } \\
\text { NFLK(8)-VAVHGYLGGKVSLADAAQVEYEVGHSLGSYVPR(10) }\end{array}$ & sp|Q06639|RSC3_YEAST (144)-sp|P38210|LDB7_YEAST (70)/ & 1 & RSC3_YEAST & LDB7_YEAST & 144 & 70 & 3.13 \\
\hline KLSEDGVTDGDGKPIPESER(1)-KLSEDGVTDGDGKPIPESER(1) & o|Q06639|RSC3_YEAs & 2 & $\mathrm{RS}$ & RS & 234 & 234 & 2.89 \\
\hline KNSTVSNGVLGAAAPVDSDANSDTFGLTK(1)-YIKNLEENK(3) & sp|Q06639|RSC3_YEAST (845)-sp|Q06639|RSC3_YEAST (792)/ & 10 & RSC3_YEAST & RSC3_YEAST & 845 & 792 & 2.82 \\
\hline KNSTVSNGVLGAAAPVDSDANSDTFGLTK(1)-IKTISK(2) & sp|Q06639|RSC3_YEAST (845)-sp|Q06639|RSC3_YEAST (785)/ & 23 & RSC3_YEAST & RSC3_YEAST & 845 & 785 & 2.51 \\
\hline $\begin{array}{l}\text { PHLNEFKDLDPQFLDTNKVFNVFNSAISEEGR(7)- } \\
\text { KLSEDGVTDGDGKPIPESER(13) }\end{array}$ & sp|Q06639|RSC3_YEAST (261)-sp|Q06639|RSC3_YEAST (246)/ & 15 & RSC3_YEAST & RSC3_YEAST & 261 & 246 & 2.45 \\
\hline $\begin{array}{l}\text { RPHLNEFKDLDPQFLDTNKVFNVFNSAISEEGR(8)- } \\
\text { KLSEDGVTDGDGKPIPESER(1) }\end{array}$ & sp|Q06639|RSC3_YEAST (261)-sp|Q06639|RSC3_YEAST (234)/ & 1 & RSC3_YEAST & RSC3_YEAST & 261 & 234 & 2.43 \\
\hline NQEEFLTIKK(9)-VSKPKR(5) & sp|Q06639|RSC3_YEAST (231)-sp|P38210|LDB7_YEAST (59)/ & 5 & RSC3_YEAST & LDB7_YEAST & 231 & 59 & 2.39 \\
\hline KNSTVSNGVLGAAPVDSDANSDTFGLTK(1)- & sp|Q06639|RSC3_YEAST (845)-sp|Q06639|RSC3_YEAST (768)/ & 4 & RSC3_YEAST & RSC3_YEAST & 845 & 768 & 2.35 \\
\hline $\begin{array}{l}\text { IAMLLSDYTKNCK(10) } \\
\text { YIKNLEENK(3)-IKTISK(2) }\end{array}$ & sp|Q06639|RSC3_YEAST (792)-sp|Q06639|RSC3_YEAST (785)/ & 17 & RSC3 YEAST & RSC3 YEAST & 792 & 785 & 2.30 \\
\hline KLSEDGVTDGDGKPIPESER( & BYYEAS & 2 & 3-YE & 7-YE & & 104 & 2.27 \\
\hline YIKNLEENKVTTSADSNYSINNGFSGISAEQLIK(3)-IK & sp|Q06639|RSC3_YEAST (792)-sp|Q06639|RSC3_YEAST (785)/ & 17 & RSC3_YEAST & RSC3_YEAST & 792 & 785 & 2.26 \\
\hline PHLNEFKDLDPQFLDTNK(7)-LSEDGVTDGDGKPIPESER(12) & sp|Q06639|RSC3_YEAST (261)-sp|Q06639|RSC3_YEAST (246)/ & 15 & RSC3_YEAST & RSC3_YEAST & 261 & 246 & 2.17 \\
\hline RKLSEDGVTDGDGKPIPESER(2)-NQEEFLTIKKR(9) & sp|Q06639|RSC3_YEAST (234)-sp|Q06639|RSC3_YEAST (231)/ & 25 & RSC3_YEAST & RSC3_YEAST & 234 & 231 & 2.08 \\
\hline KLSEDGVTDGDGKPIPESER(1)-ESQVPISALKSK(10) & sp|Q06639|RSC3_YEAST (234)-sp|Q03124|RSC9_YEAST (496)/ & 1 & RSC3_YEAST & RSC9_YEAST & 234 & 496 & 1.95 \\
\hline LLELQEITGKK(10)-EIDFLKNK(6) & sp|Q06639|RSC3_YEAST (198)-sp|P38781|RSC30_YEAST (217)/ & 5 & RSC3_YEAST & RSC 30 Y YEAST & 198 & 217 & 1.94 \\
\hline $\begin{array}{l}\text { SVPSLESKPVTSANESSTPLNWVQGPAIFHMLTSPYTQDEIINHEM } \\
\text { NFIKGR(50)-KITGVNLDLK(1) }\end{array}$ & sp|Q06639|RSC3_YEAST (186)-sp|Q06639|RSC3_YEAST (199)/ & 1 & RSC3_YEAST & RSC3_YEAST & 186 & 199 & 1.82 \\
\hline IAMLLSDYTKNCKK(10)-IKTISK(2) & & 3 & & & 768 & 785 & \\
\hline KITGVNLDLKQDSSAQMQSSHSNR(10)-SNLNADNTTANKINK(12) & sp|Q06639|RSC3_YEAST (208)-sp|P38781|RSC30_YEAST (246)/ & 3 & RSC3_YEAST & RSC 30 YYEAST & 208 & 246 & 1.66 \\
\hline KNSTVSNGVLGAAAPVDSDANSDTFGLTK(1)-IKTISKYIK(6) & sp|Q06639|RSC3_YEAST (845)-sp|Q06639|RSC3_YEAST (789)/ & 7 & RSC3_YEAST & RSC3_YEEAST & 845 & 789 & 1.63 \\
\hline $\begin{array}{l}\text { KNSTVSNGVLGAAAPVDSDANSDTFGLTKENFNEVFEAIR(1)- } \\
\text { IKTISK(2) }\end{array}$ & sp|Q06639|RSC3_YEAST (845)-sp|Q06639|RSC3_YEAST (785)/ & 23 & RSC3_YEAST & RSC3_YEAST & 845 & 785 & 1.50 \\
\hline $\begin{array}{l}\text { KNSTVSNGVLGAAAPVDSDANSDTFGLTK(1)- } \\
\text { IAMLLSDYTKNCKK(10) }\end{array}$ & sp|Q06639|RSC3_YEAST (845)-sp|Q06639|RSC3_YEAST (768)/ & 4 & RSC3_YEAST & RSC3_YEAST & 845 & 768 & 1.49 \\
\hline KLSEDGVTDGDGKPIPESER(1)-NQEEFLTIKKR( & sp|Q06639|RSC3_YEAST (234)-sp|Q06639|RSC3_YEAST (231)/ & 25 & RSC3_YEAST & RSC3_YEAST & 234 & 231 & 1.46 \\
\hline VSKETAALLQK(3)-ADAKAQGLR(4) & sp|Q06639|RSC3_YEAST (81)-sp|P32597|STH1_YEAST (1011)/ & 1 & RSC3_YEAST & STH1_YEAST & 81 & 1011 & 1.37 \\
\hline
\end{tabular}




\begin{tabular}{|c|c|c|c|c|c|c|c|}
\hline PEPTIDE & PROTEINS & CSM & PROTEIN1 & PROTEIN2 & R1 & R2 & $\mathbf{s}$ \\
\hline NQEEFLTIKK(9)-AKVSKPK(2) & sp|Q06639|RSC3 YEAST (231)-sp|P38210|LDB7 YEAST (54)/ & 2 & RSC3 YEAST & LDB7 YEAST & 231 & 54 & 1.27 \\
\hline ITGVNLDLKQDSSAQMQSSHSNR(9)-SNLNADNTTANKINK(12) & sp|Q06639|RSC3 YEAST (208)-sp|P38781|RSC30 YEAST (246)/ & 3 & RSC3 YEAST & RSC30_YEAST & 208 & 246 & 1.26 \\
\hline IAMLLSDYTKNCK(10)-IKTISK(2) & sp|Q06639|RSC3_YEAST (768)-sp|Q06639|RSC3_YEAST (785)/ & 3 & RSC3_YEAST & RSC3_YEAST & 768 & 785 & 1.16 \\
\hline TNDANEISEQTDIHSNNDNSKR(21)-IKNKNVIHLIINK(2) & sp|Q06639|RSC3_YEAST (744)-sp|Q06639|RSC3_YEAST (747)/ & 1 & RSC3_YEAST & RSC3_YEAST & 744 & 747 & 1.15 \\
\hline $\begin{array}{l}\text { KNSTVSNGVLGAAAPVDSDANSDTFGLTKENFNEVFEAIRS(1)- } \\
\text { IKTISK(2) }\end{array}$ & sp|Q06639|RSC3_YEAST (845)-sp|Q06639|RSC3_YEAST (785)/ & 23 & RSC3_YEAST & RSC3_YEAST & 845 & 785 & 1.10 \\
\hline KLSEDGVTDGDGKPIPESER(13)-AKVSKPK(2) & sp|Q06639|RSC3_YEAST (246)-sp|P38210|LDB7_YEAST (54)/ & 1 & RSC3_YEAST & LDB7_YEAST & 246 & 54 & 1.06 \\
\hline ETAALLQKNPELASLEQIR(8)-TDLHSQTKR(8) & sp|Q06639|RSC3_YEAST (89)-sp|Q06488|RSC2_YYEAST (772)/ & 1 & RSC3_YEAST & RSC2_YEAST & 89 & 772 & 1.04 \\
\hline ITGVNLDLKQDSSAQMQSSHSNR(9)-SLKEK(3) & sp|Q06639|RSC3_YEAST (208)-sp|P38781|RSC30_YEAST (232)/ & 3 & RSC3 YEAST & RSC30_YEAST & 208 & 232 & 0.86 \\
\hline PHLNEFKDLDPQFLDTNK(7)-KLSEDGVTDGDGKPIPESER(13) & sp|Q06639|RSC3_YEAST (261)-sp|Q06639|RSC3_YEAST (246)/ & 15 & RSC3_YEAST & RSC3_YEAST & 261 & 246 & 1.05 \\
\hline KLSEDGVTDGDGKPIPESER(13)-NQEEFLTIKK(9) & $\begin{array}{l}\text { sp|Q06639|RSC3_YEAST (246)-sp|Q06639|RSC3_YEAST (231)/ }\end{array}$ & 2 & RSC3_YEAST & RSC3_YEAST & 246 & 231 & 1.03 \\
\hline $\begin{array}{l}\text { PHLNEFKDLDPQFLDTNKVFNVFNSAISEEGR(7)- } \\
\text { (SFGVTRG }\end{array}$ & sp|Q06639|RSC3_YEAST (261)-sp|Q06639|RSC3_YEAST (246)/ & 15 & RSC3_YEAST & RSC3_YEAST & 261 & 246 & 0.98 \\
\hline $\begin{array}{l}\text { NQEEFLTIKK (9)-VSKPK(3) } \\
\text { NG(12) }\end{array}$ & spl006639|RSC3 YEAST (231)-sp|P38210|LDB7 YEAST (57)/ & 1 & RSC3 YEAST & LDB7 YEAST & 231 & 57 & 0.58 \\
\hline KLSEDGVTDGDGKPIPESER(1)-GKVDGKR(6) & sp|Q06639|RSC3_YEAST (234)-sp|P38781|RSC30_YEAST (262)/ & 1 & RSC3_YEAST & RSC30_YEAST & 234 & 262 & 0.53 \\
\hline KLSEDGVTDGDGKPIPESER(1)-INKTGENSK(3) & sp|Q06639|RSC3_YEAST (234)-sp|P38781|RSC30_YEAST (249)/ & 1 & RSC3_YEAST & RSC30_YEAST & 234 & 249 & 0.52 \\
\hline $\begin{array}{l}\text { KNSTVSNGVLGAAAPVDSDANSDTFGLTKENFNEVFEAIRS(1)- } \\
\text { YIKNLEENK(3) }\end{array}$ & sp|Q06639|RSC3_YEAST (845)-sp|Q06639|RSC3_YEAST (792)/ & 10 & RSC3_YEAST & RSC3_YEAST & 845 & 792 & 0.79 \\
\hline KLSEDGVTDGDGKPIPESER(1)-AKVSKPKR(7) & sp|Q06639|RSC3_YEAST (234)-sp|P38210|LDB7_YEAST (59)/ & 2 & RSC3_YEAST & LDB7_YEAST & 234 & 59 & 0.44 \\
\hline KLSEDGVTDGDGKPIPESER(1)-VSKPKR(5) & sp|Q06639|RSC3_YEAST (234)-sp|P38210|LDB7_YEAST (59)/ & 2 & RSC3_YEAST & LDB7_YEAST & 234 & 59 & 0.44 \\
\hline KITGVNLDLKQDSSAQMQSSHSNR(10)-TGENSKK(6) & sp|Q06639|RSC3_YEAST (208)-sp|P38781|RSC30_YEAST (255)/ & 1 & RSC3_YEAST & RSC30_YEAST & 208 & 255 & 0.38 \\
\hline LSEDGVTDGDGKPIPESERRPHLNEFK(12)-NQEEFLTIKKRK(9) & sp|Q06639|RSC3_YEAST (246)-sp|Q06639|RSC3_YEAST (231)/ & 2 & RSC3-YEAST & RSC3 YEAST & 246 & 231 & 0.61 \\
\hline FEIEKR(5)-GRKMK(3) & sp|Q06639|RSC3_YEAST (479)-sp|Q06639|RSC3_YEAST (7)/ & 1 & RSC3_YEAST & RSC3_YEAST & 479 & 7 & 0.59 \\
\hline TNDANEISEQTDIHSNNDNSKR(21)-LNHELSKISESLIK(7) & sp|Q06639|RSC3_YEAST (744)-sp|Q06639|RSC3_YEAST (830)/ & 1 & RSC3_YEAST & RSC3_YEAST & 744 & 830 & 0.59 \\
\hline KNSTVSNGVLGAAAPVDSDANSDTFGLTK(1)-TISKYIK(4) & sp|Q06639|RSC3_YEAST (845)-sp|Q06639|RSC3_YEAST (789)/ & 7 & RSC3_YEAST & RSC3_YEAST & 845 & 789 & 0.49 \\
\hline PHLNEFKDLDPQFLDTNKVFNVFNSAISEEGR(18)- & sp|Q06639|RSC3_YEAST (272)-sp|Q06639|RSC3_YEAST (246)/ & 1 & RSC3_YEAST & RSC3_YEAST & 272 & 246 & 0.44 \\
\hline $\begin{array}{l}\text { LSEDGVTDGDGKPIPESER(12) } \\
\text { KNSTVSNGVLGAAAPVDSDANSDTFGLTKEN } \\
\text { TISKYIK (1) }\end{array}$ & sp|Q06639|RSC3_YEAST (845)-sp|Q06639|RSC3_YEAST (789)/ & 7 & RSC3_YEAST & RSC3_YEAST & 845 & 789 & 0.43 \\
\hline $\begin{array}{l}\text { TISKYIK(4) } \\
\text { KQNDENNKNVDGK(1)-KLLPAVEFIK(1) }\end{array}$ & sp|Q07979|RSC58 YEAST (343)-sp|Q03124|RSC9 YEAST (434)/ & & & RSC9 YEAST & 343 & 434 & 8.48 \\
\hline IGIALTQTNNIKSR(12)-TESVGGNK(1) & sp|Q07979|RSC58_YEAST (149)-sp|Q07979|RSC58_YEAST (2)/ & 4 & RSC58_YEAST & RSC58_YEAST & 149 & 2 & 7.84 \\
\hline KVKQEELLNTNEEGINR(1)-KQNDENNKNVDGK(1) & sp|Q07979|RSC58_YEAST (326)-sp|Q07979|RSC58_YEAST (343)/ & 8 & RSC58_YEAST & RSC58_YEAST & 326 & 343 & 7.32 \\
\hline KVKQEELLNTNEEGINRK(1)-EITKLALQLNSSAQK(4) & sp|Q07979|RSC58_YEAST (326)-sp|P25632|RSC6_Y̌EAST (404) $/$ & 2 & RSC58_YEAST & RSC6_Y̌YEAST & 326 & 404 & 5.74 \\
\hline KVKQEELLNTNEEGINRK(3)-AKSLGLESK(2) & sp|Q07979|RSC58_YEAST (328)-sp|P32597|STH1_YYEAST (170)/ & 25 & RSC58_YEAST & STH1_YEAST & 328 & 170 & 5.39 \\
\hline VKQEELLNTNEEGINR(2)-KQNDENNKNVDGK(1) & sp|Q07979|RSC58_YEAST (328)-sp|Q07979|RSC58_YEAST (343)/ & 22 & RSC58_YEAST & RSC58_YEAST & 328 & 343 & 5.13 \\
\hline KVKQEELLNTNNEEGINRK(1)-AKSLGLESK(2) & sp|Q07979|RSC58_YEAST (326)-sp|P32597|STH1_YYEAST (170)/ & 22 & RSC58_YEAST & STH1_YYEAST & 326 & 170 & 4.96 \\
\hline KQNDENNKNVDGK(1)-EVVGSTLNGKGGDSR(10) & sp|Q07979|RSC58_YEAST (343)-sp|P43609|RSC8_YEAST (375)/ & 5 & RSC58_YEAST & RSC8_YEAST & 343 & 375 & 4.85 \\
\hline VKQEELLNTNEEGINR(2)-KQNDENNK(1) & sp|Q07979|RSC58_YEAST (328)-sp|Q07979|RSC58_YEAST (343)/ & 22 & RSC58_YEAST & RSC58 YEAST & 328 & 343 & 4.80 \\
\hline NPDKIYESYCK(4)-FIKNR(3) & sp|Q07979|RSC58_YEAST (46)-sp|Q07979|RSC58_YेEAST (56)/ & 8 & RSC58_YEAST & RSC58_YEAST & 46 & 56 & 4.71 \\
\hline CHVVDESFPAKFFEK(11)-NEDKLVLTTINKR(4) & sp|Q07979|RSC58_YEAST (3 & 41 & RSC58_YEAST & RSC58 YEAST & 38 & 70 & 4.67 \\
\hline KVKQEELLNTNEEGINR(3)-KQNDENNKNVDGK(1) & sp|Q07979|RSC58_YEAST (328)-sp|Q07979|RSC58_YEAST (343)/ & 22 & RSC58_YEAST & RSC58_YEAST & 328 & 343 & 4.57 \\
\hline KVKQEELLNTNEEGINR(1)-AKSLGLESK(2) & sp|Q07979|RSC58_YEAST (326)-sp|P32597|STH1_YEAST (170)/ & 22 & RSC58_YEAST & STH1_YEAST & 326 & 170 & 4.43 \\
\hline ILNKVLKPT & Q07979|RSC58_YEAST (437)-sp|Q07979|RSC58_YEAST (343)/ & 5 & RSC58_YEAST & RSC58_YEAST & 437 & 343 & 4.33 \\
\hline VKQEELLN & $07979 \mid$ RSC58 YEAST (328) & 25 & & STH1_YEAST & 328 & 170 & 4.32 \\
\hline VKQEELLNTNEEGINRK(2)-AKVSKPK(2) & sp|Q07979|RSC58_YEAST (328)-sp|P38210|LDB7_YEAST (54)/ & 16 & RSC58_YEAST & LDB7_YEAST & 328 & 54 & 4.26 \\
\hline ILNKVLKPTTEER(4)-GIEGSENSKAK(9) & sp|Q07979|RSC58_YEAST (437)-sp|P38210|LDB7_YEAST (52)/ & 8 & RSC58_YEAST & LDB7_YEAST & 437 & 52 & 4.24 \\
\hline IGIALTQTNNIKSR(12)-LENNGNSVKK(9) & sp|Q07979|RSC58_YEAST (149)-sp|P43609|RSC8_YEAST (312)/ & 7 & RSC58_YEAST & RSC8_YEAST & 149 & 312 & 4.24 \\
\hline VKQEELLNTNEEGINR(2)-AKSLGLESK(2) & sp|Q07979|RSC58_YEAST (328)-sp|P32597|STH1_YEAST (170)/ & 25 & 58_YEAST & STH1_YEAST & 328 & 170 & 4.17 \\
\hline NEDKLVLTTINKR(12)-TNSLESEHLKIPK(10) & sp|Q07979|RSC58_YEAST (78)-sp|Q02206|RSC4_YEAST (420)/ & 9 & RSC58 YEAST & RSC4_YEAST & 78 & 420 & 4.06 \\
\hline ILNKVLKPTTEER(4)-AKVSKPKR(2) & sp|Q07979|RSC58 YEAST (437)-sp|P38210|LDB7 YEAST (54)/ & 46 & RSC58 YEAST & LDB7 YEAST & 437 & 54 & 4.05 \\
\hline
\end{tabular}




\begin{tabular}{|c|c|c|c|c|c|c|c|}
\hline PEPTIDE & PROTEINS & CSM & PROTEIN1 & PROTEIN2 & R1 & R2 & $\mathbf{s}$ \\
\hline IGIALTQTNNIKSR(12)-NPDKIYESYCK(4) & sp|Q07979|RSC58_YEAST (149)-sp|Q07979|RSC58_YEAST (46)/ & 8 & RSC58_YEAST & RSC58_YEAST & 149 & 46 & 3.83 \\
\hline $\begin{array}{l}\text { SNLDKRPHELPNTNFK(5)-FIKNR(3) } \\
\text { SN) }\end{array}$ & 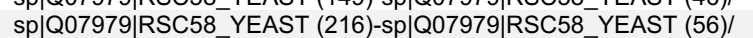 & 8 & RSC58-YEAST & RSC58 YEAST & 216 & 56 & 3.56 \\
\hline KVKQEELLNTNEEGINRK(1)-FSNATKTALGDPDTEIR(6) & sp|Q07979|RSC58_YEAST (326)-sp|P32597|STH1_YYEAST (183)/ & 7 & RSC58_YEAST & STH1_YEAST & 326 & 183 & 3.56 \\
\hline IGIALTQTNNIKSR(12)-WFTTNKK(6) & sp|Q07979|RSC58_YEAST (149)-sp|Q06488|RSC2_YEAST (823)/ & 2 & RSC58_YEAST & RSC2_YEAST & 149 & 823 & 3.55 \\
\hline NGTPDKLVSDSLLKLK(14)-KQNDENNKNVDGK(1) & sp|Q07979|RSC58_YEAST (425)-sp|Q07979|RSC58_YEAST (343)/ & 12 & RSC58_YEAST & RSC58_YEAST & 425 & 343 & 3.54 \\
\hline NEDKLVLTTINKR(4)-YNVIKSMQK(5) & sp|Q07979|RSC58_YEAST (70)-sp|Q02206|RSC4_YEAST (428)/ & 37 & RSC58_YEAST & RSC4_Y̌EAST & 70 & 428 & 3.52 \\
\hline SGKLLSGNEMDEYDDDDATELDKIISYDFIK(3)- & sp|Q07979|RSC58_YEAST (154)-sp|Q07979|RSC58_YEAST (149)/ & 7 & RSC58_YEAST & RSC58_YEAST & 154 & 149 & 3.49 \\
\hline $\begin{array}{l}\text { IGIALTQTNNIKSR(12) } \\
\text { NEDKLVLTTINKR(4)-TNSLESEHLKIPK(10) }\end{array}$ & sp|Q07979|RSC58_YEAST (70)-sp|Q02206|RSC4_YEAST (420)/ & 16 & RSC58 YEAST & RSC4 YEAST & 70 & 420 & 3.49 \\
\hline KQNDENNKNVDGK(1)-LVSDSLLKLKR(8) & sp|Q07979|RSC58_YEAST (343)-sp|Q07979|RSC5̄8_YEAST (425)/ & 4 & RSC58_YEAST & RSC58_YEAST & 343 & 425 & 3.45 \\
\hline $\begin{array}{l}\text { RPHELPNTNFKINNVLPQTDIENEAPR(11)- } \\
\text { EMQSLSEQHTMEYKPYK(14) }\end{array}$ & sp|Q07979|RSC58_YEAST (227)-sp|Q02206|RSC4_YEAST (446)/ & 4 & RSC58_YEAST & RSC4_YEAST & 227 & 446 & 3.44 \\
\hline $\begin{array}{l}\text { SNGVQDDGGDNDNDATIASANSESTENKEQFIIK(28)- } \\
\text { MLQEQSELMSTVMNNTPTTVAALAAVAAASETNGK(1) }\end{array}$ & sp|Q07979|RSC58_YEAST (383)-sp|P32597|STH1_YEAST (1)/ & 2 & RSC58_YEAST & STH1_YEAST & 383 & 1 & 3.35 \\
\hline ILNKVLKPTTEER(4)-AKVSKPK(2) & sp|Q07979|RSC58_YEAST (437)-sp|P38210|LDB7_YEAST (54)/ & 46 & RSC58_YEAST & LDB7_YEAST & 437 & 54 & 3.33 \\
\hline $\begin{array}{l}\text { NVDGKSNGVQDDGGDNDNDATIASANSESTENKEQFIIK(5)- } \\
\text { KQNDENNK(1) }\end{array}$ & sp|Q07979|RSC58_YEAST (355)-sp|Q07979|RSC58_YEAST (343)/ & 5 & RSC58_YEAST & RSC58_YEAST & 355 & 343 & 3.28 \\
\hline SNSEGLIRNEDKLVLTTINKR(12)-CHVVDESFPAKFFEK(11) & sp|Q07979|RSC58_YEAST (70)-sp|Q07979|RSC58_YEAST (38)/ & 3 & RSC58_YEAST & RSC58_YEAST & 70 & 38 & 3.21 \\
\hline QNDENNKNVDGK(7)-KLLPAVEFIK(1) & sp|Q07979|RSC58_YEAST (350)-sp|Q03124|RSC9_YEAST (434)/ & 6 & RSC58_YEAST & RSC9_Y̌YEAST & 350 & & 3.14 \\
\hline NEDKLVLTTINK(4)-YNVIKSMQK(5) & sp|Q07979|RSC58_YEAST (70)-sp|Q02206|RSC4_YEAST (428)/ & 37 & RSC58_YEAST & RSC4_YEAST & 70 & 428 & 3.00 \\
\hline IGYMDLYEKNEKK(12)-KQNDENNKNVDGK(1) & sp|Q07979|RSC58_YEAST (325)-sp|Q07979|RSC58_YEAST (343)/ & 2 & RSC58_YEAST & RSC58_YEAST & 325 & 343 & 2.94 \\
\hline NGTPDKLVSDSLLKLKR(14)-KQNDENNKNVDGK(1) & sp|Q07979|RSC58_YEAST (425)-sp|Q07979|RSC58_YEAST (343)/ & 12 & RSC58_YEAST & RSC58 YEAST & 425 & 343 & 2.93 \\
\hline IGYMDLYEKNEKK(9)-AKSLGLESK(2) & sp|Q07979|RSC58_YEAST (322)-sp|P32597|STH1_YEAST (170)/ & 14 & RSC58_YEAST & STH1_YEAST & 322 & 170 & 2.90 \\
\hline KVKQEELLNTNEEGINR(3)-AKSLGLESK(2) & sp|Q07979|RSC58_YEAST (328)-sp|P32597|STH1_YEAST (170)/ & 25 & RSC58_YEAST & STH1_YEAST & 328 & 170 & 2.82 \\
\hline IGYMDLYEKNEKK(12)-AKSLGLESK(2) & sp|Q07979|RSC58_YEAST (325)-sp|P32597|STH1_YEAST (170)/ & 7 & RSC58_YEAST & STH1_YEAST & 325 & 170 & 2.73 \\
\hline CHVVDESFPAKFFEKNPDKIYESYCK(11)-NEDKLVLTTINKR(4) & Sp|Q07979|RSC58_YEAST (38)-sp|Q07979|RSC58_YEAST (70)/ & 41 & RSC58_YEAST & RSC58_YEAST & 38 & 70 & 2.64 \\
\hline CHVVDESFPAKFFEK(11)-NEDKLVLTTINK(4) & sp|Q07979|RSC58_YEAST (38)-sp|Q07979|RSC58_YEAST (70)/ & 41 & RSC58_YEAST & RSC58_YEAST & 38 & 70 & 2.62 \\
\hline $\begin{array}{l}\text { SNGVQDDGGDNDNDATIASANSESTENKEQFIIK(28)- } \\
\text { ENKLEIKAIEDTFR(7) }\end{array}$ & sp|Q07979|RSC58_YEAST (383)-sp|P32597|STH1_YEAST (77)/ & 4 & RSC58_YEAST & STH1_YEAST & 383 & 77 & 2.61 \\
\hline VKQEELLNTNEEGINRK(2)-FSNATKTALGDPDTEIR(6) & sp|Q07979|RSC58_YEAST (328)-sp|P32597|STH1_YEAST (183)/ & 12 & RSC58_YEAST & STH1_YEAST & 328 & 183 & 2.58 \\
\hline VKQE & sp|Q07979|RSC58_YEAST (328)-sp|P43609|RSC8_YEAST (375)/ & 2 & 3_YEAST & 8_YEAST & 328 & 375 & 2.55 \\
\hline KVKQEELLNTNEEGINRK(3)-FSNATKTALGDPDTEIR(6) & sp|Q07979|RSC58_YEAST (328)-sp|P32597|STH1_YYAST (183)/ & 12 & RSC58_YEAST & STH1_YEAST & 328 & 183 & 2.54 \\
\hline VKQEELLNTNEEGINR(2)-FSNATKTALGDPDTEIR(6) & sp|Q07979|RSC58_YEAST (328)-sp|P32597|STH1_YEAST (183)/ & 12 & RSC58_YEAST & STH1_YEAST & 328 & 183 & 2.49 \\
\hline IGYMDLYEKNEKK(12)-TKDMDLFSSIISK(2) & sp|Q07979|RSC58 YEAST (325)-sp|Q07979|RSC58 YEAST (200)/ & 4 & RSC58 YEAST & RSC58 YEAST & 325 & 200 & 2.46 \\
\hline IGYMDLYEKNEK(9)-AKSLGLESK(2) & sp|Q07979|RSC58_YEAST (322)-sp|P32597|STH1_YEAST (170)/ & 14 & RSC58_YEAST & STH1_YEAST & 322 & 170 & 2.43 \\
\hline VKQEELLNTNEEGINRK(2)-KLLPAVEFIK(1) & sp|Q07979|RSC58_YEAST (328)-sp|Q03124|RSC9_YEAST (434)/ & 1 & RSC58_YEAST & RSC9_YEAST & 328 & 434 & 2.35 \\
\hline KVKQEELLNTNEEGINRK(1)-QNDENNKNVDGK(7) & sp|Q07979|RSC58 YEAST (326)-sp|Q07979|RSC58 YEAST (350)/ & 3 & RSC58 YEAST & RSC58 YEAST & 326 & 350 & 2.33 \\
\hline NPDKIYESYCKFIK(11)-IGIALTQTNNIKSR(12) & sp|Q07979|RSC58 YEAST (53)-sp|Q07979|RSC58 YEAST (149)/ & 1 & RSC58 YEAST & RSC58 YEAST & 53 & 149 & 2.33 \\
\hline KVKQEELLNTNEEGINRK(1)-IGYMDLYEKNEK(9) & sp|Q07979|RSC58_YEAST (326)-sp|Q07979|RSC58_YEAST (322)/ & 5 & RSC58_YEAST & RSC58_YEAST & 326 & 322 & 2.32 \\
\hline $\begin{array}{l}\text { QNDENNKNVDGKSNGVQDDGGDNDNDATIASANSESTENKEQFII } \\
\text { K(12)-SSQVVFSATELQQLTQQSHATDKGIEGSENSK(23) }\end{array}$ & sp|Q07979|RSC58_YEAST (355)-sp|P38210|LDB7_YEAST (43)/ & 2 & RSC58_YEAST & LDB7_YEAST & 355 & 43 & 2.26 \\
\hline KQNDENNKNVDGK(8)-KLLPAVEFIK(1) & sp|Q07979|RSC58_YEAST (350)-sp|Q03124|RSC9_YEAST (434)/ & 6 & RSC58_YEAST & RSC9_YEAST & 350 & 434 & 2.19 \\
\hline $\begin{array}{l}\text { RPHELPNTNFKINNVLPQTDIENEAPR(11)-DTETLLTINKEPVK(10) } \\
\text { (1) }\end{array}$ & sp|Q07979|RSC58_YEAST (227)-sp|Q02206|RSC4_YEAST (530)/ & 2 & RSC58_YEAST & RSC4_YEAST & 227 & 530 & 2.18 \\
\hline KVKQEELLNTNEEGINRK(1)-KPIKISADFNAK(4) & sp|Q07979|RSC58_YEAST (326)-sp|P32597|STH1_YEAST (160)/ & 4 & RSC58_YEAST & STH1_YEAST & 326 & 160 & 2.18 \\
\hline KVKQEELLNTNEEGINRK(3)-AKVSKPK(2) & sp|Q07979|RSC58_YEAST (328)-sp|P38210|LDB7_YEAST (54)/ & 16 & RSC58_YEAST & LDB7_YEAST & 328 & 54 & 2.18 \\
\hline ERILNKVLKPTTEER(6)-AKVSKPK(2) & sp|Q07979|RSC58 YEAST (437)-sp|P38210|LDB7 YEAST (54)/ & 46 & RSC58 YEAST & LDB7 YEAST & 437 & 54 & 2.14 \\
\hline KQNDENNKNVDGK(1)-AKVSKPK(2) & sp|Q07979|RSC58 YEAST (343)-sp|P38210|LDB7 YEAST (54)/ & 3 & RSC58 YEAST & LDB7 YEAST & 343 & 54 & 2.03 \\
\hline KVKQEELLNTNEEGINR(3)-FSNATKTALGDPDTEIR(6) & sp|Q07979|RSC58_YEAST (328)-sp|P32597|STH1_YYEAST (183)/ & 12 & RSC58_YEAST & STH1_YEAST & 328 & 183 & 2.00 \\
\hline $\begin{array}{l}\text { SNGVQDDGGDNDNDATIASANSESTENKEQFIIK(28)- } \\
\text { LEIKAIEDTFR(4) }\end{array}$ & sp|Q07979|RSC58_YEAST (383)-sp|P32597|STH1_YEAST (77)/ & 4 & RSC58_YEAST & STH1_YEAST & 383 & 77 & 1.86 \\
\hline
\end{tabular}




\begin{tabular}{|c|c|c|c|c|c|c|c|}
\hline PEPTIDE & PROTEINS & CSM & PROTEIN1 & PROTEIN2 & R1 & R2 & $\mathbf{S}$ \\
\hline VKQEELLNTNEEGINRK(2)-KQNDENNKNVDGK(1) & sp|Q07979|RSC58_YEAST (328)-sp|Q07979|RSC58_YEAST (343)/ & 22 & RSC58_YEAST & RSC58_YEAST & 328 & 343 & 1.85 \\
\hline $\begin{array}{l}\text { SNGVQDDGGDNDNDATIASANSESTENKEQFIIK(28)- } \\
\text { AlONHPKENKLEIKAIEDTFR(14) }\end{array}$ & sp|Q07979|RSC58_YEAST (383)-sp|P32597|STH1_YEAST (77)/ & 4 & RSC58_YEAST & STH1_YEAST & 383 & 77 & 1.82 \\
\hline 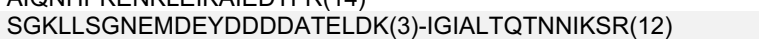 & sp|Q07979|RSC58_YEAST (154)-sp|Q07979|RSC58_YEAST (149)/ & 7 & RSC58_YEAST & RSC58_YEAST & 154 & 149 & 1.82 \\
\hline VKQEELLNTNEEGINRK(2)-QNDENNKNVDGK(7) & $\begin{array}{l}\text { sp|Q07979|RSC58 YEAST (328)-sp|Q07979|RSC58 YEAST (350)/ }\end{array}$ & 1 & RSC58 YEAST & RSC58 YEAST & 328 & 350 & 1.81 \\
\hline KVKQEELLNTNEEGINR(3)-AKVSKPK(2) & sp|Q07979|RSC58_YEAST (328)-sp|P38210|LDB7_YEAST (54)/ & 16 & RSC58 YEAST & LDB7 YEAST & 328 & 54 & 1.76 \\
\hline CHVVDESFPAKFFEK(11)-NEDKLVLTTINKR(12) & sp|Q07979|RSC58_YEAST (38)-sp|Q07979|RSC58_YEAST (78)/ & 2 & RSC58_YEAST & RSC58_YEAST & 38 & 78 & 1.73 \\
\hline KVKQEELLNTNEEGINRK(3)-EVVGSTLNGKGGDSR(10) & sp|Q07979|RSC58_YEAST (328)-sp|P43609|RSC8_YEAST (375)/ & 2 & RSC58_YEAST & RSC8_YEAST & 328 & 375 & 1.71 \\
\hline SNLDKRPHELPNTNFK(5)-IGIALTQTNNIKSR(12) & sp|Q07979|RSC58_YEAST (216)-sp|Q07979|RSC58_YEAST (149)/ & 6 & RSC58_YEAST & RSC58_YEAST & 216 & 149 & 1.66 \\
\hline ILNKVLKPTTEER(4)-VSKPKR(5) & sp|Q07979|RSC58_YEAST (437)-sp|P38210|LDB7_YEAST (59)/ & 6 & RSC58_YEAST & LDB7_YEAST & 437 & 59 & 1.62 \\
\hline $\begin{array}{l}\text { SNGVQDDGGDNDNDATIASANSESTENKEQFIIK(28)- } \\
\text { AlQNHPKENKLEIK(10) }\end{array}$ & sp|Q07979|RSC58_YEAST (383)-sp|P32597|STH1_YEAST (73)/ & 6 & RSC58_YEAST & STH1_YEAST & 383 & 73 & 1.46 \\
\hline VKQEELLNTNEEGINR(2)-AKVSKPK(2) & sp|Q07979|RSC58_YEAST (328)-sp|P38210|LDB7_YEAST (54)/ & 16 & RSC58_YEAST & LDB7_YEAST & 328 & 54 & 1.41 \\
\hline $\begin{array}{l}\text { QNDENNKNVDGKSNGVQDDGGDNDNDATIASANSESTENKEQFII } \\
\text { K(12)-NGTPDKLVSDSLLKLK(14) }\end{array}$ & sp|Q07979|RSC58_YEAST (355)-sp|Q07979|RSC58_YEAST (425)/ & 1 & RSC58_YEAST & RSC58_YEAST & 355 & 425 & 1.34 \\
\hline KVKQEELLNTNEEGINR(3)-IGYMDLYEKNEK(9) & sp|Q07979|RSC58_YEAST (328)-sp|Q07979|RSC58_YEAST (322)/ & 15 & RSC58_YEAST & RSC58_YEAST & 328 & 322 & 1.33 \\
\hline SNLDKRPHELPNTNFKINNVLPQTDIENEAPR(16)- & sp|Q07979|RSC58_YEAST (227)-sp|Q02206|RSC4_YEAST (530)/ & 2 & RSC58_YEAST & RSC4_YEAST & 227 & 530 & 1.26 \\
\hline $\begin{array}{l}\text { IGIALTQTNNIKSR(12)-FIKNR(3) } \\
\text { I I I T }\end{array}$ & sp|Q07979|RSC58_YEAST (149)-sp|Q07979|RSC58_YEAST (56)/ & 3 & RSC58_YEAST & RSC58_YEAST & 149 & 56 & 23 \\
\hline KVKQEELLNTNEEGINRK(3)-IGYMDLYEKNEK(9) & sp|Q07979|RSC58_YEAST (328)-sp|Q07979|RSC58_YEAST (322)/ & 15 & RSC58_YEAST & RSC58_YEAST & 328 & 322 & 1.21 \\
\hline QNDENNKNVDGKSNGVQDDGGDNDNDATIASANSESTENKEQFII & sp|Q07979|RSC58_YEAST (355)-sp|Q07979|RSC58_YEAST (328)/ & 1 & RSC58_YEAST & RSC58_YEAST & 355 & 328 & 1.19 \\
\hline 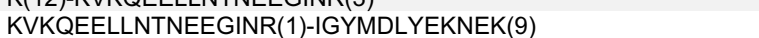 & sp|Q07979|RSC58_YEAST (326)-sp|Q07979|RSC58_YEAST (322)/ & 5 & RSC58_YEAST & RSC58_YEAST & 326 & 322 & 1.17 \\
\hline NGTPDKLVSDSLLKLKR(14)-QNDENNKNVDGK(7) & sp|Q07979|RSC58_YEAST (425)-sp|Q07979|RSC58_YEAST (350)/ & 1 & RSC58_YEAST & RSC58_YEAST & 425 & 350 & 1.16 \\
\hline RPHELPNTNFKINNVLPQTDIENEAPR(11)-KSTGGK(1) & sp|Q07979|RSC58_YEAST (227)-sp|P84233|H32_XENLA (9)/ & 2 & RSC58_YEAST & H32_XĒNLA & 227 & 9 & 1.01 \\
\hline $\begin{array}{l}\text { SNGVQDDGGDNDNDATIASANSESTENKEQFIIK(28)- } \\
\text { DQDIYETKLDTLRK(8) }\end{array}$ & sp|Q07979|RSC58_YEAST (383)-sp|P32597|STH1_YEAST (96)/ & 2 & RSC58_YEAST & STH1_YEAST & 383 & 96 & 0.90 \\
\hline $\begin{array}{l}\text { QNDENNKNVDGKSNGVQDDGGDNDNDATIASANSESTENKEQFII } \\
\text { K(12)-KLLPAVEFIK(1) }\end{array}$ & sp|Q07979|RSC58_YEAST (355)-sp|Q03124|RSC9_YEAST (434)/ & 1 & RSC58_YEAST & RSC9_YEAST & 355 & 434 & 0.87 \\
\hline VKQEELLNTNEEGINRK(2)-VSKPKR(5) & sp|Q07979|RSC58_YEAST (328)-sp|P38210|LDB7_YEAST (59)/ & 2 & RSC58_YEAST & LDB7_YEAST & 328 & 59 & 0.86 \\
\hline ILNKVLKPTTEER(4)-AKVSK(2) & sp|Q07979|RSC58_YEAST (437)-sp|P38210|LDB7_YEAST (54)/ & 46 & RSC58_YEAST & -YEAST & 437 & 54 & 0.81 \\
\hline ILNKVLKPTTEER(4)-VSKPK(3) & sp|Q07979|RSC58_YEAST (437)-sp|P38210|LDB7_YEAST (57)/ & 5 & RSC58_YEAST & LDB7_YEAST & 437 & 57 & 0.79 \\
\hline 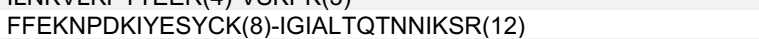 & |Q07979|RSC58_YEAST (149)/ & 1 & RSC58_YEAST & RSC58_YEAST & 46 & 149 & 1.02 \\
\hline SNGVQDDGGDNDNDATIASANSESTENKEQFIIK(28)-ENKLEIK(3) & sp|Q07979|RSC58_YEAST (383)-sp|P32597|STH1_YEAST (73)/ & 6 & RSC58_YEAST & STH1_Y̌EAST & 383 & 73 & 0.77 \\
\hline KVKQEELLNTNEEGINRK(1)-EVVGSTLNGKGGDSR(10) & sp|Q07979|RSC58_YEAST (326)-sp|P43609|RSC8_YEAST (375)/ & 1 & RSC58_YEAST & RSC8_YEAST & 326 & 375 & 0.77 \\
\hline $\begin{array}{l}\text { SNLDKRPHELPNTNFKINNVLPQTDIENEAPR(16)- } \\
\text { EMOSLSEQHTMEYKPYK(14) }\end{array}$ & sp|Q07979|RSC58_YEAST (227)-sp|Q02206|RSC4_YEAST (446)/ & 4 & RSC58_YEAST & RSC4_YEAST & 227 & 446 & 0.76 \\
\hline SGKLLSGNEMDEYDDDDATELDKIISYDFIK(3)-LENNGNSVKK(9) & sp|Q07979|RSC58_YEAST (154)-sp|P43609|RSC8_YEAST (312)/ & 1 & RSC58_YEAST & RSC8_YEAST & 154 & 312 & 0.75 \\
\hline IGIAL & 8_YEA & & RS & Rs & 149 & 42 & 0.91 \\
\hline SNLDKRPHELPNTNF & 07979|RSC58_YEAST (216)-sp|Q07979|RSC58_YEAST (46)/ & 1 & RSC58_YEAST & RSC58_YEAST & 216 & 46 & 0.88 \\
\hline $\begin{array}{l}\text { ILNKVLKPTTEER(4)-VSKPKR(3) } \\
\text { ILNKTH }\end{array}$ & sp|Q07979|RSC58_YEAST (437)-sp|P38210|LDB7_YEAST (57)// & 5 & RSC58_YEAST & LDB7_YEAST & 437 & 57 & 0.48 \\
\hline ILKE & 07979|RSC58_YEAST (45 & 1 & RSC58_YEAST & RSC8_YE & & 443 & 0.43 \\
\hline IEKNEKK(12)-KEVEK(1) & C58_YEAST $(325$ & 1 & RSC58_YEAST & YEA & 325 & 700 & 0.41 \\
\hline KVKQEELLNTNEEGINRK & sp|Q07979|RSC58_YEAST (328 & 1 & RSC58_YEAST & YEAST & 328 & 234 & 0.40 \\
\hline $\begin{array}{l}\text { IGYMDLYEKNEK(9)-KQN } \\
\text { IGMLE }\end{array}$ & $\begin{array}{l}\text { sp|Q07979|RSC58_YEAST (322)-sp|Q07979|RSC58 YEAST (343)/ }\end{array}$ & 1 & RSC58_YEAST & RSC58_YEAST & 322 & 343 & 0.57 \\
\hline ENNKNVDGK(8) & sp|Q07979|RSC58_YEAST (326 & 3 & RSC58_YEAST & RSC58_YEAST & 326 & 350 & 0.45 \\
\hline MTESVGGNK(1)-SNLDKR(5) & 88_YEAST (1)-sp|Q0797 & 1 & 58_YEAST & & 1 & 216 & 0.43 \\
\hline STMLQVSEKDLFELER(9)-IIKELSIR(3) & $\begin{array}{l}\text { sp|Q12406|ARP7_YEAST (241)-sp|Q12406|ARP7_YEAST (410)/ } \\
\text { (A) }\end{array}$ & 105 & ARP7_Y'YEAST & ARP7_Y̌YEAST & 241 & 410 & 15.00 \\
\hline VSPEELPLVITMPATNGKPDN & sp|Q12406|ARP7_YEAST (114)-sp|Q12406|ARP7_YEAST (339)/ & 71 & ARP7_YE & ARP7_YEAST & 114 & 339 & 14.41 \\
\hline WYSKEDYETLKR(11)-KQSQATNATN( & 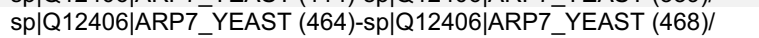 & 7 & ARP7_YEAST & ARP7_YEAST & 464 & 468 & 7.04 \\
\hline
\end{tabular}




\begin{tabular}{|c|c|c|c|c|c|c|c|}
\hline PEPTIDE & PROTEINS & CSM & PROTEIN1 & PROTEIN2 & R1 & R2 & $\mathbf{s}$ \\
\hline WYSKEDYETLKR(4)-DRKQSQATNATN(3) & sp|Q12406|ARP7_YEAST (457)-sp|Q12406|ARP7_YEAST (468)/ & 14 & ARP7_YEAST & ARP7_YEAST & 457 & 468 & 5.06 \\
\hline WYSKEDYETLKR(4)-KQSQATNATN(1) & sp|Q12406|ARP7_YEAST (457)-sp|Q12406|ARP7_YEAST (468)/ & 14 & ARP7_YEAST & ARP7_YEAST & 457 & 468 & 4.99 \\
\hline STMLQVSEKDLFELER(9)-IIKELSIRFPQYK(3) & sp|Q12406|ARP7_YEAST (241)-sp|Q12406|ARP7_YEAST (410)/ & 105 & ARP7_YEAST & ARP7_YEAST & 241 & 410 & 4.81 \\
\hline $\begin{array}{l}\text { SSAFVIDIGASGCNVTPIIDGIVVKNAVVR(25)- } \\
\text { VSPEELPLVITMPATNGKPDMAILER(18) }\end{array}$ & sp|Q12406|ARP7_YEAST (176)-sp|Q12406|ARP7_YEAST (114)/ & 2 & ARP7_YEAST & ARP7_YEAST & 176 & 114 & 4.72 \\
\hline VSPEELPLVITMPATNGKPDMAILER(18)-NLHLOKINSIIDFIK (6) & 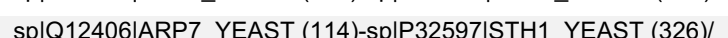 & 7 & ARP7 YEAST & STH1 YEAST & 114 & 326 & 3.79 \\
\hline YYKEQADIYAKQQEQLK(11)-KSAPATGGVK(1) & sp|Q12406|ARP7_YEAST (259)-sp|P84233|H32_X XENLA (27)/ & 1 & ARP7_YEAST & H32 XENLA & 259 & 27 & 3.66 \\
\hline VSPEELPLVITMPATNGKPDMAILER(18)-SKFGGDFLDFQVHER(2) & sp|Q12406|ARP7_YEAST (114)-sp|Q12406|ARP7_YEAST (183)/ & 3 & ARP7_YYEAST & ARP7_YEAST & 114 & 183 & 3.60 \\
\hline VSPEELPLVITMPATNGKPDMAILER(18)-NLHLQKINSIIDFIKER(6) & sp|Q12406|ARP7_YEAST (114)-sp|P32597|STH1_YEAST (326)/ & 7 & ARP7_YEAST & STH1_YEAST & 114 & 326 & 3.58 \\
\hline SSAFVIDIGASGCNVTPIIDGIVVKNAVVR(25)-NLHLQKINSIIDFIK(6) & sp|Q12406|ARP7_YEAST (176)-sp|P32597|STH1_YEAST (326)/ & 2 & ARP7_YEAST & STH1_YEAST & 176 & 326 & 3.36 \\
\hline VSPEELPLVITMPATNGKPDMAILER(18)-VGKEGEEVGEGDSIAK(3) & sp|Q12406|ARP7_YEAST (114)-sp|P43609|RSC8_YEAST (533)/ & 2 & ARP7_YEAST & RSC8 YEAST & 114 & 533 & 3.22 \\
\hline STMLQVSEKDLFELER(9)-KSAPATGGVK(1) & sp|Q12406|ARP7_YEAST (241)-sp|P84233|H32_XENLA (27)/ & 5 & ARP7_YEAST & H32_XENLA & 241 & 27 & 3.18 \\
\hline IIKELSIR(3)-KSAPATGGVK(1) & sp|Q12406|ARP7_YEAST (410)-sp|P84233|H32 XENLA (27)/ & 2 & ARP7_YEAST & H32 XENLA & 410 & 27 & 3.12 \\
\hline FSPEDGLGPLMAKSVK(13)-KAGASINSMK(1) & sp|Q12406|ARP7_YEAST (335)-sp|Q12406|ARP7_YEAST (339)/ & 14 & ARP7_YEAST & ARP7_YEAST & 335 & 339 & 2.97 \\
\hline YYKEQADIYAK(3)-SVLPAATKK(8) & sp|Q12406|ARP7_YEAST (251)-sp|Q05123|ARP9_YEAST (382)/ & 1 & ARP7_YEAST & ARP9_YEAST & 251 & 382 & 2.48 \\
\hline $\begin{array}{l}\text { VSPEELPLVITMPATNGKPDMAILER(18)- } \\
\text { DQEGDDEDGNTATGHGVKR(18) }\end{array}$ & sp|Q12406|ARP7_YEAST (114)-sp|P43609|RSC8_YEAST (529)/ & 2 & ARP7_YEAST & RSC8_YEAST & 114 & 529 & 2.38 \\
\hline $\begin{array}{l}\text { ECYQFAEYLFKPQLISDKFSPEDGLGPLMAKSVK(31)- } \\
\text { KAGASINSMK(1) }\end{array}$ & sp|Q12406|ARP7_YEAST (335)-sp|Q12406|ARP7_YEAST (339)/ & 14 & ARP7_YEAST & ARP7_YEAST & 335 & 339 & 2.37 \\
\hline QQEQLKQMDQQLQYTALTGSPNNPLVQK(6)-SVLPAATKK(8) & sp|Q12406|ARP7_YEAST (265)-sp|Q05123|ARP9_YEAST (382)/ & 8 & ARP7_YEAST & ARP9_YEAST & 265 & 382 & 2.32 \\
\hline QQEQLKQMDQQLQYTALTGSPNNPLVQKK(6)-SVLPAATKK(8) & sp|Q12406|ARP7_YEAST (265)-sp|Q05123|ARPY_YEAST (382)/ & 8 & ARP7_YEAST & ARP9_YEAST & 265 & 382 & 2.22 \\
\hline QMDQQLQYTALTGSPNNPLVQKK(22)-SVLPAATKK(8) & sp|Q12406|ARP7_YEAST (287)-sp|Q05123|ARP9_YEAST (382)/ & 2 & ARP7_YEAST & ARP9_YEAST & 287 & 382 & 2.15 \\
\hline $\begin{array}{l}\text { ECYQFAEYLFKPQLISDKFSPEDGLGPLMAKSVK(31)- } \\
\text { SKFGGDFLDFQVHER(2) }\end{array}$ & sp|Q12406|ARP7_YEAST (335)-sp|Q12406|ARP7_YEAST (183)/ & 3 & ARP7_YEAST & ARP7_YEAST & 335 & 183 & 2.09 \\
\hline QQEQLKQMDQQLQYTALTGSPNNPLVQKK(6)-GKQGGK(2) & sp|Q12406|ARP7_YEAST (265)-tr|Q6AZJ8|H2A_XENLA (5)/ & 1 & ARP7_YEAST & H2A_XENLA & 265 & 5 & 1.81 \\
\hline SKFGGDFLDFQVHER(2)-VGKEGEEVGEGDSIAK(3) & sp|Q12406|ARP7_YEAST (183)-sp|P43609|RSC8_YEAST (533)/ & 1 & ARP7_YEAST & RSC8_YEAST & 183 & 533 & 1.36 \\
\hline SKFGGDFLDFQVHER(2)-FSPEDGLGPLMAKSVK(13) & sp|Q12406|ARP7_YEAST (183)-sp|Q12406|ARP7_YEAST (335)/ & 1 & ARP7_YEAST & ARP7_YEAST & 183 & 335 & 1.33 \\
\hline VSPEELPLVITMPATNGKPDMAILER(18)-KQSQATNATN(1) & sp|Q12406|ARP7_YEAST (114)-sp|Q12406|ARP7_YEAST (468)/ & 1 & ARP7_YEAST & ARP7_YEAST & 114 & 468 & 1.09 \\
\hline KQSQATNATN(1)-EDYETLKR(7) & sp|Q12406|ARP7_YEAST (468)-sp|Q12406|ARP7_YEAST (464)/ & 3 & ARP7_YEAST & ARP7_YEAST & 468 & 464 & 1.09 \\
\hline $\begin{array}{l}\text { STMLQVSEKDLFELER(9)-STGGKAPR(5) } \\
\text { STGEG }\end{array}$ & sp|Q12406|ARP7_YEAST (241)-sp|P84233|H32_XENLA (14)/ & 2 & ARP7_YEAST & H32_XENLA & 241 & 14 & 0.97 \\
\hline SVKKAGASINSMK(3)-ILFDKIDK(5) & sp|Q12406|ARP7_YEAST (338)-sp|P32597|STH1_YEAST (997)/ & 2 & ARP7_YEAST & STH1_YEAST & 338 & 997 & 0.91 \\
\hline STMLQVSEKDLFELER(9)-KQLATKAAR(6) & sp|Q12406|ARP7_YEAST (241)-sp|P84233|H32_X̄ENLA (23)/ & 1 & ARP7_YEAST & H32_XENLA & 241 & 23 & 0.80 \\
\hline DRKQSQATNATN(3)-EDYETLKR(7) & sp|Q12406|ARP7_YEAST (468)-sp|Q12406|ARP7_YEAST (464)/ & 3 & ARP7_YEAST & ARP7_YEAST & 468 & 464 & 1.03 \\
\hline IIKELSIR(3)-KSTGGKAPR(1) & sp|Q12406|ARP7_YEAST (410)-sp|P84233|H32_XENLA (9)/ & 1 & ARP7_YEAST & H32_X̄ENLA & 410 & 9 & 0.71 \\
\hline $\begin{array}{l}\text { ECYQFAEYLFKPQLISDKFSPEDGLGPLMAK(11)- } \\
\text { IAMLLSDYTKNCKKQNK(13) }\end{array}$ & sp|Q12406|ARP7_YEAST (315)-sp|Q06639|RSC3_YEAST (771)/ & 1 & ARP7_YEAST & RSC3_YEAST & 315 & 771 & 0.60 \\
\hline $\begin{array}{l}\text { STDVWYEASTWIQQFKSTMLQVSEKDLFELER(16)- } \\
\text { LAPLIKEENDMENMADEOK(6) }\end{array}$ & sp|Q12406|ARP7_YEAST (232)-sp|Q12406|ARP7_YEAST (202)/ & 1 & ARP7_YEAST & ARP7_YEAST & 232 & 202 & 0.92 \\
\hline STMLQVSEKDLFELER(9)-KQLATK(1) & sp|Q12406|ARP7_YEAST (241)-sp|P84233|H32_XENLA (18)/ & 1 & ARP7_YEAST & H32_XENLA & 241 & 18 & 0.47 \\
\hline STMLQVSEKDLFELERYYKEQADIYAK(9)-IIKELSIRFPQYK(3) & sp|Q12406|ARP7_YEAST (241)-sp|Q12406|ARP7_YEAST (410)/ & 105 & ARP7_YEAST & ARP7_YE & 241 & 410 & 0.71 \\
\hline VSPEELPLVITMPATNGKPDMAILER(18)-QQLLEKR(6) & sp|Q12406|ARP7_YEAST (114)-sp|P32597|STH1_YEAST (315)/ & 1 & ARP7_YEAST & STH1_YEAST & 114 & 315 & 0.40 \\
\hline $\begin{array}{l}\text { ECYQFAEYLFKPQLISDKFSPEDGLGPLMAK(11)- } \\
\text { NSRQIHKVYICHTCGNESINVRYHNLR(7) }\end{array}$ & sp|Q12406|ARP7_YEAST (315)-sp|P43609|RSC8_YEAST (256)/ & 1 & ARP7_YEAST & RSC8_YEAST & 315 & 256 & 0.39 \\
\hline $\begin{array}{l}\text { RTDEGGEAEFIFGTYNMIDAAAEKR(24)- } \\
\text { LAPLIKEENDMENMADEQKR(19) }\end{array}$ & sp|Q12406|ARP7_YEAST (61)-sp|Q12406|ARP7_YEAST (215)/ & 1 & ARP7_YEAST & ARP7_YEAST & 61 & 215 & 0.67 \\
\hline AYNNVTLKNLTAFQLLSQR(8)-SLGLESKFSNATK(7) & sp|Q9URQ5|HTL1_YEAST (22)-sp|P32597|STH1_YEAST (177)/ & 9 & HTL1_YEAST & STH1_YEAST & 22 & 177 & 3.95 \\
\hline KNER(6)-MSLEEMKK(7) & sp|Q9URQ5|HTL1_YEAST (73)-sp|Q9URQ5|HTL1_YEAST (66)/ & 6 & HTL1_YEAST & HTL1_YEAST & 73 & 66 & 2.35 \\
\hline SQNNTISSMNPER(1)-MSLEEMKK(7) & sp|Q9URQ5|HTL1-YEAST (2)-sp|Q9URQ5|HTL1 YEAST (66)/ & 2 & HTL1 YEAST & HTL1 YEAST & 2 & 66 & 0.83 \\
\hline NDEELNKLLGR(7)-EEIQKR(5) & tr|Q6AZJ8|H2A_XENLA (95)-sp|Q06168|SFH1_YEAST (390)/ & 11 & H2A_X XENLA & SFH1_YEAST & 95 & 390 & 4.25 \\
\hline NDEELNKLLGR(7)-GKGGK(2) & tr|Q6AZJ8|H2A XENLA (95)-sp|P62799|H4 XĒNLA (5)/ & 4 & H2A XENLA & H4 XĒENA & 95 & 5 & 3.30 \\
\hline
\end{tabular}




\begin{tabular}{|c|c|c|c|c|c|c|c|}
\hline PEPTIDE & PROTEINS & CSM & PROTEIN1 & PROTEIN2 & R1 & R2 & $\mathbf{s}$ \\
\hline QGGKTR(4)-YTSAK(5) & tr|Q6AZJ8|H2A XENLA (9)-sp|P02281|H2B11 XENLA (122)/ & 2 & H2A XENLA & H2B11 XENLA & 9 & 122 & 3.02 \\
\hline NDEELNKLLGR(7)-GGKGLGK(3) & 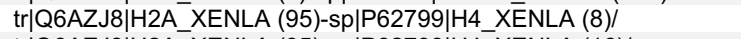 & 4 & H2A_XENLA & H4_XENELA & 95 & 8 & 2.59 \\
\hline NDEELNKLLGR(7)-GGAKR(4) & $\operatorname{tr} \mid$ Q6AZJ8|H2A_XENLA (95)-sp|P62799|H4_XENLA (16)/ & 4 & H2A_XENLA & H4_XENLA & 95 & 16 & 2.19 \\
\hline NDEELNKLLGR(7)-TKQTAR(2) & $\operatorname{tr|}|\mathrm{Q} 6 \mathrm{ZJJ} 8| \mathrm{H} 2 \mathrm{~A}$ XENLA (95)-sp|P84233|H32_XENLA (4)/ & 2 & H2A_XENLA & $\mathrm{H} 3 \overline{2}_{-} \mathrm{XENLA}$ & 95 & 4 & 2.12 \\
\hline VTIAQGGVLPNIQSVLLPKK(19)-RGGVKR(5) & tr|Q6AZJ8|H2A_XENLA (118)-sp|P62799|H4_XENLA (44)/ & 3 & H2A_XENLA & H4_XEENLA & 118 & 44 & 1.58 \\
\hline GKQGGK(2)-KAVTK(1) & tr|Q6AZJ8|H2A_ XENLA (5)-sp|P02281|H2B11 XENLA (13)/ & 1 & H2A XENLA & H2B 11 XENLA & 5 & 13 & 1.41 \\
\hline NDEELNKLLGR(7)-SAKSK(3) & 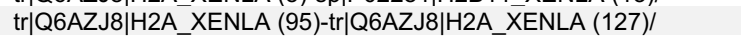 & 2 & H2A XENLA & H2A XENLA & 95 & 127 & 1.22 \\
\hline HLQLAVRNDEELNKLLGR(14)-LLLPGELAKHAVSEGTK(9) & 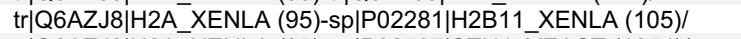 & 1 & H2A_XENLA & H2B11__XENLA & 95 & 105 & 0.99 \\
\hline NDEELNKLLGR(7)-IRQKK(4) & tr|Q6AZJ8|H2A_XENLA (95)-sp|P32597|STH1_YEAST (1054)/ & 2 & H2A_XENLA & STH1_YEEAST & 95 & 1054 & 0.91 \\
\hline NDEELNKLLGR(7)-MKDLK(2) & 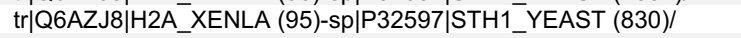 & 1 & H2A_XENLA & STH1__YEAST & 95 & 830 & 0.67 \\
\hline VTIAQGGVLPNIQSVLLPKK(19)-SAKSK(3) & $\operatorname{tr|} \mid$ 6AZJ8|H2A_XENLA (118)-tr|Q6AZJ8|H2A_XENLA (127)/ & 2 & $\mathrm{H} 2 \mathrm{~A}$ XENLA & H2A X̄ENLA & 118 & & \\
\hline SAPAPKK(6)-KAVTK(1) & sp|P02281|H2B11_XENLA (8)-sp|P02281|H2B11_XENLA (13)/ & 12 & H2B $\overline{1} 1$ XENLA & H2B1̄1_XENLA & 8 & 13 & 14.09 \\
\hline TQKKDGK(3)-KAVTK(1) & sp|P02281|H2B11_XENLA (20)-sp|P02281|H2B11_XENLA (13)/ & 2 & H2B11_XENLA & H2B11_XENLA & 20 & 13 & 8.01 \\
\hline KESYAIYVYK(1)-KGNYAER(1) & sp|P02281|H2B11_XENLA (31)-tr|Q6AZJ8|H2A XENLA (36)/ & 14 & H2B11_XENLA & H2A XENLA & 31 & 36 & 5.49 \\
\hline QVHPDTGISSKAMSIMNSFVNDVFER(11)-KESYAIYVYK(1) & sp|P02281|H2B11_XENLA (54)-sp|P02281|H2B11_XENLA (31)/ & 8 & H2B11_XENLA & H2B11_XENLA & 54 & 31 & 5.21 \\
\hline AVTKYTSAK(4)-GKQGGK(2) & sp|P02281|H2B11_XENLA (117)-tr|Q6AZJ8|H2A_X̄ENLA (5)/ & 2 & H2B11_XENLA & 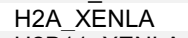 & 117 & 5 & 5.18 \\
\hline SAPAPKKGSK(7)-KAVTK(1) & sp|P02281|H2B11_XENLA (9)-sp|P02281|H2B11_XENLA (13)/ & 1 & H2B11_XENLA & H2B11_XENLA & 9 & 13 & 4.14 \\
\hline SAPAPKKGSK(6)-KAVTK(1) & sp|P02281|H2B11_XENLA (8)-sp|P02281|H2B11_XENLA (13)/ & 12 & H2B11_XENLA & H2B11_XENLA & 8 & 13 & 3.72 \\
\hline AKSAPAPK(2)-KAVTK(1) & sp|P02281|H2B11_XENLA (2)-Sp|P02281|H2B11_XENLA (13)/ & 1 & H2B11_XENLA & H2B11_XENLA & 2 & 13 & 3.51 \\
\hline HAVSEGTKAVTK(8)-YTSAK(5) & sp|P02281|H2B11- XENLA (113)-sp|P02281|H2B̄11 XENLA (122)/ & 6 & H2B11_XENLA & H2B11_XENLA & 113 & 122 & 3.24 \\
\hline QVHPDTGISSKAMSIMNSFVNDVFER(11)-TRKESYAIYVYK(3) & sp|P02281|H2B11_XENLA (54)-sp|P02281|H2B11_X̌XENLA (31)/ & 8 & H2B11_XENLA & H2B11_XENLA & 54 & 31 & 3.05 \\
\hline QVHPDTGISSKAMSIMNSFVNDVFER(11)-AKSAPAPK(1) & sp|P02281|H2B11_XENLA (54)-sp|P02281|H2B11_XENLA (1)/ & 2 & H2B11_XENLA & H2B11_XENLA & 54 & 1 & 2.96 \\
\hline LAHYNKR(6)-KGNYAER(1) & sp|P02281|H2B11_XENLA (82)-tr|Q6AZJ8|H2A_XENNLA (36)/ & 3 & H2B11_XENLA & H2A_XENLA & 82 & 36 & 2.31 \\
\hline LLLPGELAKHAVSEGTK(9)-NDEELNKLLGR(7) & sp|P02281|H2B11_XENLA (105)-tr|Q6AZJ8|H2A_XENLA (95)/ & 37 & H2B11_XENLA & H2A_XENLA & 105 & 95 & 2.28 \\
\hline HAVSEGTKAVTK(8)-LAGDPIDKSAR(8) & sp|P02281|H2B11_XENLA (113)-sp|P32832|NPL6_YEAST (267)/ & 5 & H2B11_XENLA & NPLE__YEAST & 113 & 267 & 2.18 \\
\hline TQKKDGK(4)-KAVTK(1) & sp|P02281|H2B11_XENLA (21)-sp|P02281|H2B11_XENLA (13)/ & 1 & H2B11_XENLA & H2B11_XENLA & 21 & 13 & 2.18 \\
\hline QVHPDTGISSKAMSIMNSFVNDVFER(11)-TKQTAR(2) & sp|P02281|H2B11_XENLA (54)-sp|P84233|H32_XENLA (4)/ & 3 & H2B11_XENLA & H32_XĒNLA & 54 & 4 & 2.08 \\
\hline LLLPGELAKHAVSEGTK(9)-GLGKGGAKR(4) & sp|P02281|H2B11_XENLA (105)-sp|P62799|H4_XENLA (12)/ & 6 & H2B11_XENLA & H4_X̄ENLA & 105 & 12 & 1.99 \\
\hline KAVTKTQK(5)-KGSKK(1) & sp|P02281|H2B11_XENLA (17)-Sp|P02281|H2B11_XENLA (9)/ & 1 & H2B11_XENLA & H2B 111 XENLA & 17 & 9 & 1.83 \\
\hline LLLPGELAKHAVSEGTK(9)-TKQTAR & sp|P02281|H2B11_XENLA (105)-sp|P84233|H32_XENLA (4)/ & 8 & H2B11_XENLA & H32_XĒNLA & 105 & 4 & 1.69 \\
\hline LLLPGELAKHAVSEGTK(9)-GKGGK(2) & sp|P02281|H2B11_XENLA (105)-sp|P62799|H4_XENLA (5)/ & 13 & H2B11_XENLA & H4_XEENLA & 105 & 5 & 1.64 \\
\hline LLLPGELAKHAVSEGTK(9)-ADAKAQGLR(4) & sp|P02281|H2B11_XENLA (105)-sp|P32597|STH1_YEAST (1011)/ & 4 & H2B11_XENLA & STH1_YEAST & 105 & 1011 & 1.54 \\
\hline TRKESYAIYVYK(3)-KGNYAER(1) & sp|P02281|H2B11_XENLA (31)-tr|Q6AZJ8|H2A_XĒNLA (36)/ & 14 & H2B11_XENLA & H2A_X̄ENLA & 31 & 36 & 1.50 \\
\hline QVHPDTGISSKAMSIMNSFVNDVFER(11)-KLPFQR( & sp|P02281|H2B11_XENLA (54)-sp|P84233|H32_XENLA (64)/ & 13 & H2B11_XENLA & H32_XENLA & 54 & 64 & 1.39 \\
\hline QVHPDTGISSKAMSIMNSFVNDVFER(11)-KSTGGK(1) & sp|P02281|H2B11_XENLA (54)-sp|P84233|H32_XENLA (9)/ & 2 & H2B11_XENLA & H32_XENLA & 54 & 9 & 1.37 \\
\hline AVTKTQK(4)-SAPAPKK(6) & sp|P02281|H2B11_XENLA (17)-sp|P02281|H2B11_XENLA (8)/ & 2 & H2B11_XENLA & H2B11_XENLA & 17 & 8 & 1.26 \\
\hline LLLPGELAKHAVSEGTK(9)-VEILTKEEIQKR(6) & sp|P02281|H2B11_XENLA (105)-sp|Q06168|SFH1_YEAST (385)/ & 1 & H2B11_XENLA & SFH1_Y YEAST & 105 & 385 & 1.14 \\
\hline $\begin{array}{l}\text { HAVSEGTKAVTK(8)-AKVSKPKR(2) } \\
\text { HeTI) }\end{array}$ & sp|P02281|H2B11_XENLA (113)-sp|P38210|LDB7_YYEAST (54)/ & 1 & H2B11_XENLA & LDB7_YEAST & 113 & 54 & 1.06 \\
\hline LLLPGELAKHAVSEGTK(9)-EIAQDFKTDLR(7) & sp|P02281|H2B11_XENLA (105)-sp|P84233|H32_X̄ENLA (79)/ & 6 & H2B11_XENLA & H32_X̄ENLA & 105 & 79 & 1.04 \\
\hline 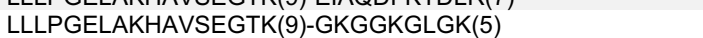 & sp|P02281|H2B11_XENLA (105)-sp|P62799|H4_XENLA (8)/ & 10 & H2B11_XENLA & $\mathrm{H} 4$ XENLA & 105 & 8 & 0.88 \\
\hline LLLPGELAKHAVSEGTK(9)-GGAKR(4) & sp|P02281|H2B11_XENLA (105)-sp|P62799|H4_XENLA (16)/ & 7 & H2B11_XENLA & H4_XENLA & 105 & 16 & 0.78 \\
\hline LLLPGELAKHAVSEGTK(9)-KPLRDNR(1) & sp|P02281|H2B11_XENLA (105)-sp|Q06488|RSC2_YEAST (234)/ & 2 & H2B11_XENLA & RSC2_YEAST & 105 & 234 & 0.77 \\
\hline LLLPGELAKHAVSEGTK(9)-GKGGKGLGK(2) & sp|P02281|H2B11_XENLA (105)-sp|P62799|H4_XENLA (5)/ & 13 & H2B11_XENLA & H4_XĒNLA & 105 & 5 & 0.71 \\
\hline AVTKYTSAK(4)-AKAKTR(2) & sp|P02281|H2B11_XENLA (117)-tr|Q6AZJ8|H2A_ XENLA (13)/ & 2 & H2B11_XENLA & H2A XENLA & 117 & 13 & 0.67 \\
\hline LLLPGELAKHAVSEGTK(9)-IRQKK(4) & sp|P02281|H2B11_XENLA (105)-sp|P32597|STH1_YEAST (1054)/ & 3 & H2B11_XENLA & STH1_YEAST & 105 & 1054 & 0.66 \\
\hline
\end{tabular}




\section{APPENDIX}

\section{List of abbreviations}

\begin{tabular}{|c|c|c|c|}
\hline$\AA$ & ångstrom & $\mathrm{m}$ & milli \\
\hline $\mathrm{ADP}$ & adenosine diphosphate & M & mol \\
\hline $\mathrm{ADP}-\mathrm{BeF}_{3}$ & ADP beryllium fluoride & MES & 2-(N-morpholino)ethanesulfonic acid \\
\hline Amp & ampicillin & MOPS & $\begin{array}{l}\text { 3-(N-morpholino)propanesulfonic } \\
\text { acid }\end{array}$ \\
\hline armadillo & armadillo repeat fold & MWCO & molecular weight cut-off \\
\hline ATP & adenosine triphosphate & $\mathrm{N}_{2}$ & nitrogen \\
\hline $\mathrm{Au}$ & gold & $\begin{array}{l}\text { Na- } \\
\text { HEPES }\end{array}$ & HEPES pH adjusted with $\mathrm{NaOH}$ \\
\hline BAH & bromo-adjacent homology & $\mathrm{NaCl}$ & sodium chloride \\
\hline $\mathrm{bp}$ & base pair & $\mathrm{NaOH}$ & sodium hydroxide \\
\hline bromo & bromodomain & Ni-NTA & nickel-nitrilotriacetic acid \\
\hline BS3 & bis(sulfosuccinimidyl)suberate & $\mathrm{OD}_{600}$ & optical density at $600 \mathrm{~nm}$ \\
\hline BSA & bovine serum albumin & PAGE & polyacrylamide gel electrophoresis \\
\hline CBP & calmodulin binding protein & PBS & phosphate buffered saline \\
\hline cDNA & complementary DNA & PCR & polymerase chain reaction \\
\hline coiled coil & $\begin{array}{l}\text { C-terminal helix forming coiled } \\
\text { coil-like structure }\end{array}$ & PI & protein inhibitor \\
\hline CTF & contrast transfer function & PIC & pre-initiation complex \\
\hline $\mathrm{Cu}$ & copper & PMSF & phenylmethylsulfonyl fluoride \\
\hline dATP & deoxyadenosine triphosphate & $\mathrm{rcf}$ & relative centrifuagal force \\
\hline dCTP & deoxycytidine triphosphate & RFX & DNA-binding RFX-type winged-helix \\
\hline dGTP & deoxyguanosine triphosphate & $\mathrm{rpm}$ & rounds per minute \\
\hline DIM & DNA-interaction module & RPT & repeat \\
\hline DMSO & dimethyl sulfoxide & SANT & Swi3 Ada N-Cor TFIIIB \\
\hline dNTP & deoxynucleoside triphosphate & SDS & sodium dodecyl sulfate \\
\hline DTT & dithiothreitol & $\mathrm{SnAC}$ & Snf2 ATP coupling \\
\hline EDTA & ethylenediaminetetraacetic acid & SWIRM & Swi3 Rsc8 Moira \\
\hline EGTA & $\begin{array}{l}\text { ethylene glycol-bis( } \beta \text {-aminoethyl } \\
\text { ether)- } N, N, N^{\prime}, N^{\prime} \text {-tetraacetic acid }\end{array}$ & TAE & tris-acetate-EDTA \\
\hline
\end{tabular}




\begin{tabular}{|c|c|c|c|}
\hline EM & electron microscopy & TAP & tandem affinity purification \\
\hline g & gram & TBE & tris-borate-EDTA \\
\hline GA & glutaraldehyde & TCEP & $\begin{array}{l}\text { tris(2-carboxyethyl)phosphine } \\
\text { hydrochloride }\end{array}$ \\
\hline GC & Guanine Cytosine & $\mathrm{TE}$ & tris-EDTA \\
\hline GeFi & $\begin{array}{l}\text { gel filtration, size exclusion } \\
\text { chromatography }\end{array}$ & Tet & tetracyclin \\
\hline Gen & gentamycin & TEV & tobacco etch virus \\
\hline GFP & green fluorescent protein & $\mathrm{Tm}$ & melting temperature \\
\hline GraFix & gradient fixation & Tris & tris(hydroxymethyl)aminomethane \\
\hline $\mathrm{H}_{2} \mathrm{O}$ & water & TSS & transcription start site \\
\hline HEPES & $\begin{array}{l}\text { 2-[4-(2-hydroxyethyl)piperazin- } \\
\text { 1- yl]ethanesulfonic acid }\end{array}$ & $\mathrm{v} / \mathrm{v}$ & volume per volume \\
\hline HRP & horseradish peroxidase & $\mathrm{w} / \mathrm{v}$ & weight per volume \\
\hline HSA & helicase-SANT-associated & $\mathrm{X}-\mathrm{Gal}$ & $\begin{array}{l}\text { 5-bromo-4-chloro-3-indolyl- } \beta \text {-D- } \\
\text { galactopyranoside }\end{array}$ \\
\hline $\operatorname{IgG}$ & immune globulin G & $\mathrm{xg}$ & times gravity \\
\hline IPTG & $\begin{array}{l}\text { isopropyl- } \beta \text {-D-1- } \\
\text { thiogalactopyranoside }\end{array}$ & XL-MS & cross-linking-mass spectrometry \\
\hline K-HEPES & HEPES $\mathrm{pH}$ adjusted with $\mathrm{KOH}$ & YPD & yeast extract peptone dextrose \\
\hline KAc & potassium acetate & $\mathrm{Zn}$ & zinc finger \\
\hline Kan & kanamycin & $\mathrm{ZZ}$ & ZZ-type zinc finger \\
\hline $\mathrm{KOH}$ & potassium hydroxide & $\beta-\mathrm{ME}$ & $\beta$-mercaptoethanol \\
\hline LB & $\begin{array}{l}\text { lysogeny broth/Luria-Bertani } \\
\text { broth }\end{array}$ & $\mu$ & micro \\
\hline LiAc & lithium acetate & & \\
\hline
\end{tabular}




\section{List of figures}

Figure 1 | Assembly pathway and architecture of the nucleosome. ............................................. 3

Figure 2 | ATPase domains of the four chromatin remodeller families. ......................................... 6

Figure 3 | Schematic overview of remodelling mechanism............................................................

Figure 4 | Two cryo-EM studies on the RSC complex. ............................................................... 14

Figure 5 | Preparation of endogenous Rsc2-containing isoform of the RSC complex............. 44

Figure 6 | Negative stain electron microscopy of the RSC complex............................................ 45

Figure 7 | Remodelling activity of RSC in a genome-wide reconstitution assay....................... 47

Figure 8 | Assembly of the RSC-nucleosome complex.................................................................... 48

Figure 9 | Cryo-EM analysis of the RSC-nucleosome complex....................................................... 50

Figure 10 | Cryo-EM analysis of the free RSC complex.................................................................. 52

Figure 11 | Structure of RSC-nucleosome complex....................................................................... 54

Figure 12 | Cryo-EM densities for selected RSC regions as examples of map quality............. 55

Figure 13 | Cross-linking network of the RSC nucleosome complex........................................... 56

Figure 14 | Structure of RSC body and arm modules. ....................................................................... 57

Figure 15 | Course of polypeptide chains of architectural subunits Sth1, Rsc8 and Rsc58.. 58

Figure 16 | ATPase-nucleosome interactions.

Figure 17 | Position of H2A and H3 histone tails. ............................................................................ 61

Figure 18 | RSC sandwiches the nucleosome................................................................................... 62

Figure 19 | Density representing the finger helix at the acidic patch of the nucleosome.... 63

Figure 20 | Interactions of the Sfh1 finger helix with the acidic patch of the nucleosome... 64

Figure 21 | Interaction of RSC with the nucleosome is sterically impaired by H2Bub.......... 65

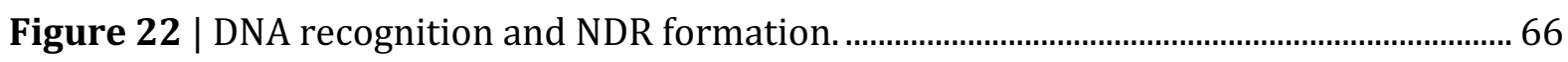

Figure 23 | Model of RSC remodelling at a promoter................................................................... 68

Figure 24 | Conservation between SWI/SNF complexes RSC (yeast) and PBAF (human)... 69

Figure 25 | Sequence alignments for the Sth1 ATPase domain and HSA region...................... 70

Figure 26 | Comparison of overall structure of RSC with complexes of CHD and INO80

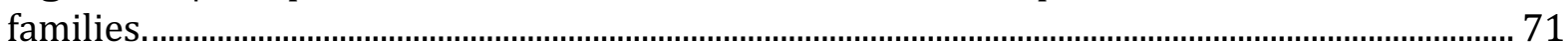

Figure 27 | Schematic representation of the ARP module of the RSC complex and the IN080 complex. 


\section{List of tables}

Table 1 | Subunit composition of RSC and related chromatin remodelling complexes. ........ 15

Table 2 | List of chemicals and consumables. .............................................................................. 18

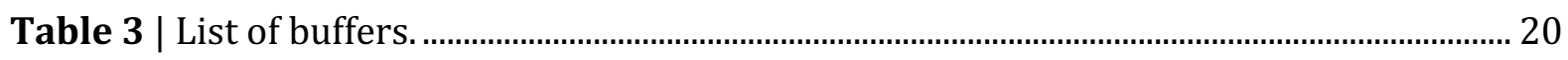

Table 4 | List of growth media for E. coli and S. cerevisiae cells................................................. 20

Table 5 | List of supplements and antibiotics for S. cerevisiae and E. coli cultures. ................ 21

Table 6 | Bacterial and yeast strains used in this study. ............................................................... 21

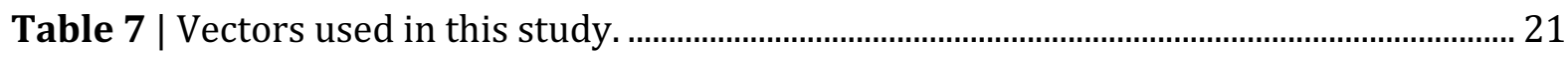

Table 8 | Antibodies used in this study....................................................................................... 22

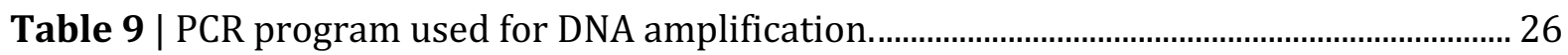

Table 10 | Cryo-EM data collection, refinement and validation statistics.................................... 53

Table S11 | RSC subunit modelling. ............................................................................................107

Table S12 | List of all BS3 cross-links detected for the RSC-nucleosome complex. ………....113 